Prepared in cooperation with the Western Association of Fish and Wildlife Agencies and the Bureau of Land Management

\title{
Range-wide Greater Sage-Grouse Hierarchical Monitoring Framework: Implications for Defining Population Boundaries, Trend Estimation, and a Targeted Annual Warning System
}

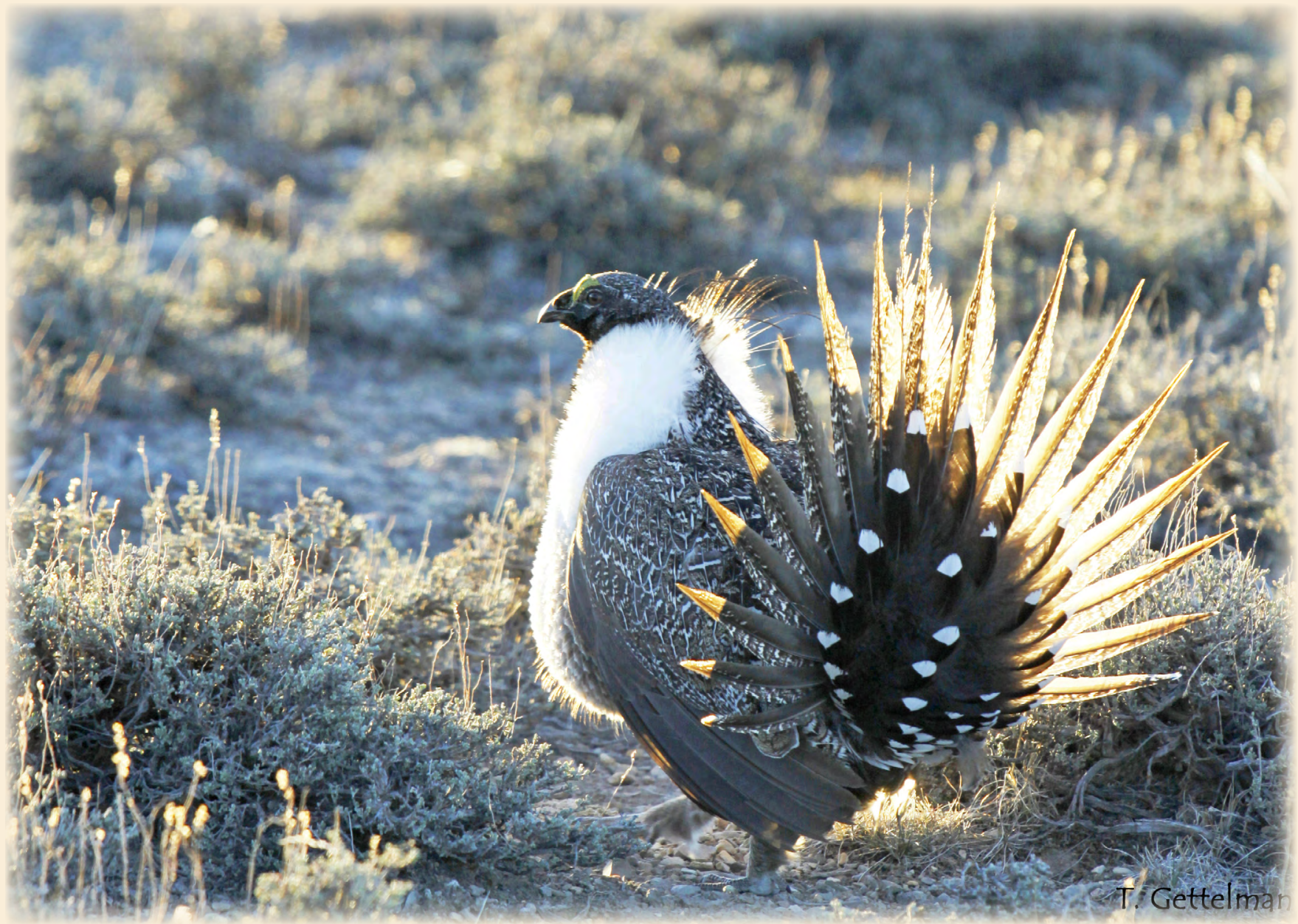

Open-File Report 2020-1154 
Cover Photos: (Main) Male sage-grouse displaying on a traditional breeding ground (lek). Photograph by Tatiana Gettelman, U.S. Geological Survey, April 21, 2012.

(Bottom) Ruby Mountains in northeastern Nevada. Photograph by Tatiana Gettelman, U.S. Geological Survey, March 30, 2012. 


\section{Range-wide Greater Sage-Grouse Hierarchical Monitoring Framework: Implications for Defining Population Boundaries, Trend Estimation, and a Targeted Annual Warning System}

By Peter S. Coates, Brian G. Prochazka, Michael S. O'Donnell, Cameron L. Aldridge, David R. Edmunds, Adrian P. Monroe, Mark A. Ricca, Gregory T. Wann, Steve E. Hanser, Lief A. Wiechman, and Michael P. Chenaille

Prepared in cooperation with the Western Association of Fish and Wildlife Agencies and the Bureau of Land Management

Open-File Report 2020-1154 


\section{U.S. Geological Survey, Reston, Virginia: 2021}

For more information on the USGS - the Federal source for science about the Earth, its natural and living resources, natural hazards, and the environment—visit https://www.usgs.gov or call 1-888-ASK-USGS.

For an overview of USGS information products, including maps, imagery, and publications, visit https://store.usgs.gov/.

Any use of trade, firm, or product names is for descriptive purposes only and does not imply endorsement by the U.S. Government.

Although this information product, for the most part, is in the public domain, it also may contain copyrighted materials as noted in the text. Permission to reproduce copyrighted items must be secured from the copyright owner.

Suggested citation:

Coates, P.S., Prochazka, B.G., O’Donnell, M.S., Aldridge, C.L., Edmunds, D.R., Monroe, A.P., Ricca, M.A., Wann, G.T., Hanser, S.E., Wiechman, L.A., and Chenaille, M.P., 2021, Range-wide greater sage-grouse hierarchical monitoring framework - Implications for defining population boundaries, trend estimation, and a targeted annual warning system: U.S. Geological Survey Open-File Report 2020-1154, 243 p., https://doi.org/10.3133/ofr20201154.

ISSN 2331-1258 (online) 


\section{Preface}

This study was completed to provide timely scientific information regarding a hierarchical monitoring and modeling framework for greater sage-grouse (Centrocercus urophasianus). Findings herein fill a prominent information gap to help inform current assessments of sage-grouse population trends at nested spatial and temporal scales for the Western Association of Fish and Wildlife Agencies. This study also highlights a 'targeted annual warning system' (TAWS) solution for managers that could be used to identify where and when management action is likely to benefit declining populations of sage-grouse at the appropriate spatial scale. The TAWS developed from this collaborative study is readily usable on an annual basis and can be modified to evaluate effectiveness of conservation efforts. Findings are also intended to provide timely scientific information for state and federal land use plans and conservation credit systems. 


\section{Acknowledgments}

We conducted this project in close consultation with the Bureau of Land Management, Columbian Sharp-tailed Grouse and Sage-Grouse Technical Team for the Western Association of Fish and Wildlife Agencies, and the U.S. Fish and Wildlife Service. We thank R. Arkle (U.S. Geological Survey), L. Schreiber (Wyoming Game and Fish Department) for helpful comments in reviewing the report in its entirety. We are also thankful to K. Doherty (U.S. Fish and Wildlife Service) for thoughts and contributions on the pilot efforts to the TAWS and helpful reviews of previous versions. We appreciate the efforts of S. Mathews, B. Brussee, I. Dwight, J. Mintz, S. O'Neil, M. Meyerpeter, and C. Roth (U.S. Geological Survey) for providing thoughtful edits on various sections; E. Tyrrell (U.S. Geological Survey) for helping to compile data and build tables; J. Atkinson (U.S. Geological Survey) for assisting with report preparation; and D. Nahhas and K. Engelking (U.S. Geological Survey) for editing, formating, and final production of this report. We extend gratitude for the cooperation of personnel from 11 western state wildlife agencies, who provided feedback at various stages on uses of lek data, modeling methods, and constructive reviews at various stages of production. Specifically, we value the contributions from T. Remington (Western Association of Fish and Wildlife Agencies), S. Stiver (Western Association of Fish and Wildlife Agencies), S. Espinosa (Nevada Department of Wildlife), K. Griffin (Colorado Parks and Wildlife), K. Miller (California Department of Fish and Wildlife), A. Moser (Idaho Department of Fish and Game), A. Cook (Utah Division of Wildlife Resources), L. Foster (Oregon Department of Fish and Wildlife), J. Kolar (North Dakota Game and Fish Department), T. Runia (South Dakota Department of Game, Fish and Parks), M. Schroeder (Washington Department of Fish and Wildlife), N. Whitford (Wyoming Game and Fish Department), C. Wightman (Montana Fish, Wildlife \& Parks), B. Wakeling (Montana Fish, Wildlife \& Parks), S. Vold (Oegon Department of Fish and Wildlife), and M. Cline (Oregon Department of Fish and Wildlife). We thank K. McGowan, K. Andrle, M. Magaletti, A. Kosic, P. Winters (Bureau of Land Management), J. Tull (U.S. Fish and Wildlife Service), K. Borland, M. Nelson (U.S. Forest Service), S. Abele (U.S. Fish and Wildlife Service), S. Gardner, and B. Ehler (California Department of Fish and Wildlife) for their input throughout the initial components of this study. We thank the many researchers and state wildlife agencies for providing or allowing access to data or generating results from evaluating sage-grouse movements among clusters and across cluster levels (hierarchical population monitoring framework; see Objective 2). Specifically, we thank Colorado Parks and Wildlife researchers and biologists for evaluating clusters in northwest Colorado, including A. Apa, M. Cowardin, B. Holmes, L. Rossi, and B. Walker; Idaho Department of Fish and Game and Bureau of Land Management researchers and biologists for providing data across the species range in Idaho, including E. Ellsworth (Bureau of Land Management-Idaho), V. Guyer (Bureau of Land Management-Idaho), S. Norman, J. Rabon, B. Schoeberl; Oregon State University researchers for evaluating clusters in southeastern Oregon, including C. Anthony, C. Hagen, and Oregon Department of Fish and Wildlife; Washington State University researchers providing data in Washington, including P.J. Olsoy, D. Thornton, and M. Schroeder of Washington Department of Fish and Wildlife; J. Taylor (currently, U.S. Department of Agriculture), J. Dinkins (currently, Oregon State University), and Wyoming Game and Fish Department for evaluating clusters in the bighorn basin of Wyoming; U.S. Geological Survey and Bureau of Land Management researchers for providing data in South Dakota, including R. Newton, A. Johnston, R. Diel, E. Beever; South Dakota Game, Fish and Parks researchers and biologists for providing data in South Dakota, including C. Sink, J. Gehrt, K. Norton, L. Bichoff, S. Hone; Nevada Department of Wildlife and California Department of Fish and Wildlife for granting permits to the U.S. Geological Survey for marking and tracking sage-grouse. This project could not have been completed without the financial support of the Bureau of Land Management, U.S. Geological Survey, and the funds for the pilot efforts that were provided by the Bureau of Land Management-Nevada and Nevada Department of Wildlife. 


\section{Contents}

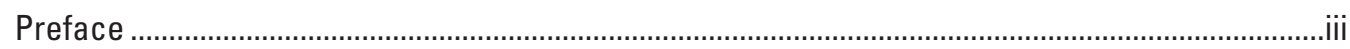

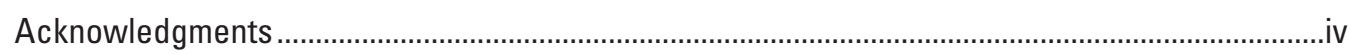

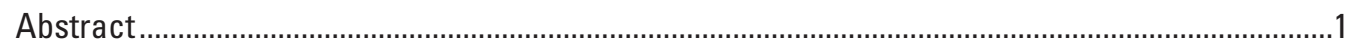

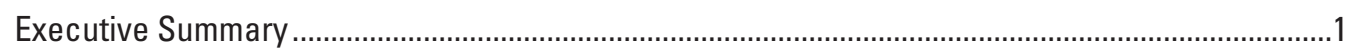

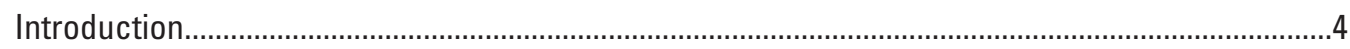

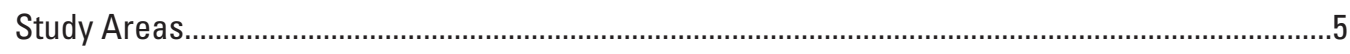

Objective 1. Database for Sage-grouse Lek Counts …….........................................................

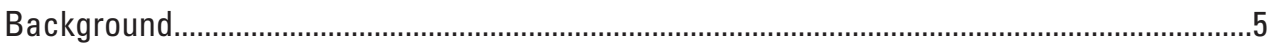

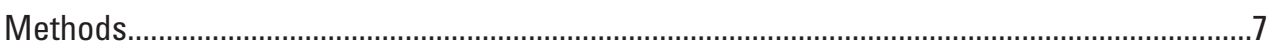

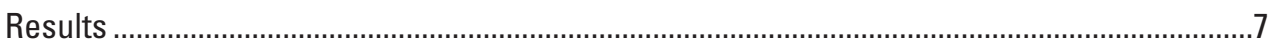

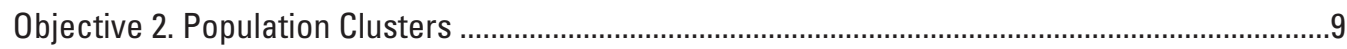

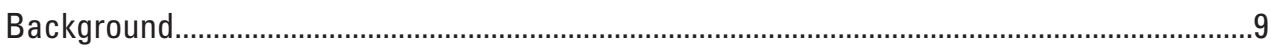

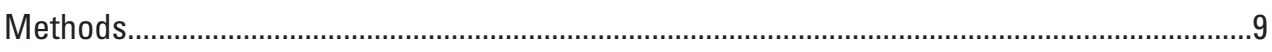

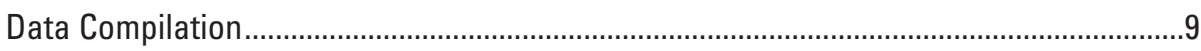

Least Cost Path Analysis ............................................................................................

Clustering Algorithm.......................................................................................................

Selecting Cluster Levels to Support Population Modeling ...........................................12

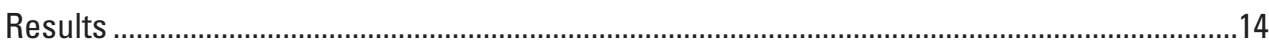

Objective 3. Spatiotemporal Patterns of Sage-Grouse Population Abundance Trends .................26

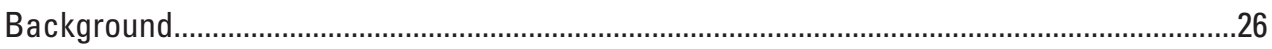

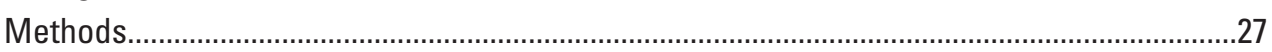

Defining Spatial and Temporal Scales ...................................................................27

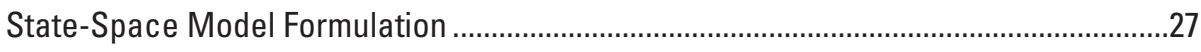

Model Diagnostics and Tests of Predictiveness ...........................................................28

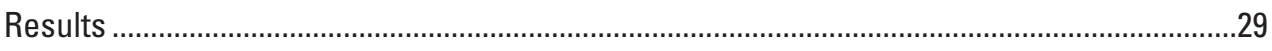

Range-wide Population Trends ....................................................................................29

Cluster Level Population Trends..............................................................................29

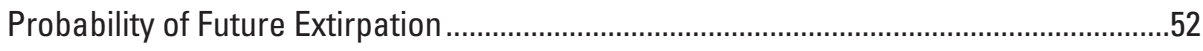

Objective 4. Targeted Annual Warning System .......................................................................56

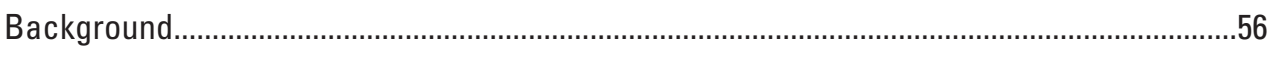

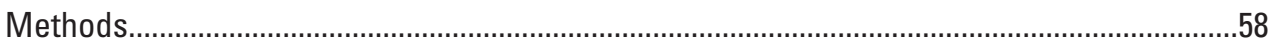

Evaluation of Posterior Probability Distributions...........................................................58

Developing Thresholds for Targeted Annual Warning System ........................................58

Evaluation of a Targeted Annual Warning System..........................................................5

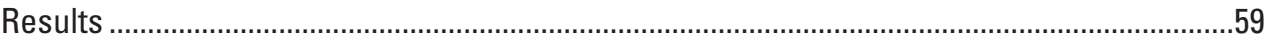

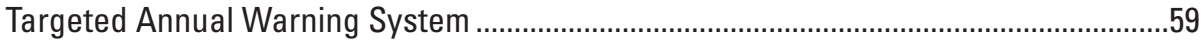

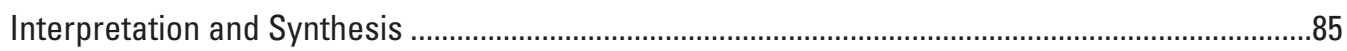

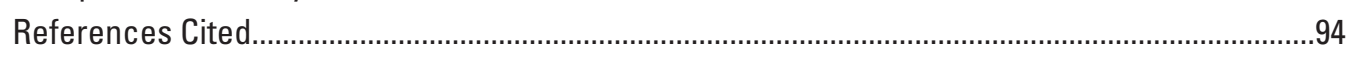

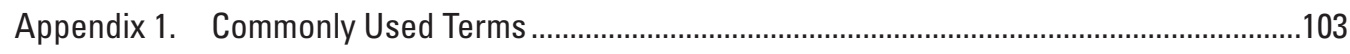

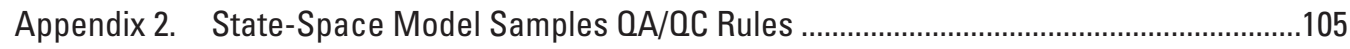

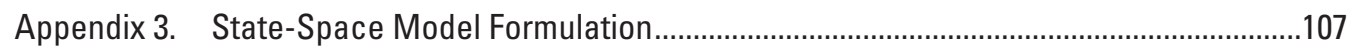

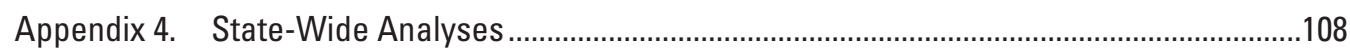

Appendix 5. Simulation to Establish Thresholds for a Targeted Annual Warning System and Omission and Commission Errors ............................................................................231 
Appendix 6. Case Comparison of a State-Space Model and N-Mixture estimates...................238

Appendix 7. Example of Targeted Annual Warning System at Pueblo Fire in

Southeast Oregon

Appendix 8. Development of Targeted Annual Warning System for Climate Cluster Stability..240

\section{Figures}

1. Map showing greater sage-grouse distribution within the sagebrush biome across 11 states in the western United States.

2. Graph showing proportion of greater sage-grouse leks sampled between 1960 and 2019 for different population climate clusters after removing observations defined in Objective 1 and Objective 3

3. Map showing study locations where greater sage-grouse research projects were conducted from which we acquired very high frequency and global positioning systems data

4. Graph showing results from evaluating greater sage-grouse hierarchical population monitoring framework across different nested population clusters using very high frequency and global positioning systems data from multiple research studies across the sage-grouse range.

5. Box and whiskers plot showing results from evaluating greater sage-grouse relationship between precipitation during late brood-rearing period and population rate of change across different nested population clusters within a hierarchical monitoring framework

6. Map showing greater sage-grouse hierarchical population monitoring framework for neighborhood cluster in the western United States

7. Map showing greater sage-grouse hierarchical population monitoring framework for climate cluster in the western United States

8. Map showing greater sage-grouse hierarchical population monitoring framework for neighborhood clusters within climate cluster A

9. Map showing greater sage-grouse hierarchical population monitoring framework for neighborhood clusters within climate cluster B

10. Map showing greater sage-grouse hierarchical population monitoring framework for neighborhood clusters within climate cluster $\mathrm{C}$

11. Map showing greater sage-grouse hierarchical population monitoring framework for neighborhood clusters within the southern section of climate cluster D

12. Map showing greater sage-grouse hierarchical population monitoring framework for neighborhood clusters within the northern section of climate cluster D

13. Map showing greater sage-grouse hierarchical population monitoring framework for neighborhood clusters within the southern section of climate cluster $\mathrm{E}$

14. Map showing greater sage-grouse hierarchical population monitoring framework for neighborhood clusters within the northern section of climate cluster $\mathrm{E}$

15. Map showing greater sage-grouse hierarchical population monitoring framework for neighborhood clusters within climate cluster $F$.

16. Diagram depicting six complete oscillations from nadir to nadir for a population that increases and decreases in abundance over time. 
17. Diagram depicting population trends of increasing, neutral, and decreasing trend scenarios across six complete oscillations..

18. Graphs showing abundance index, intrinsic rate of population change, and median estimate of abundance trend across temporal scales.

19. Images showing range-wide spatial estimates of average annual rate of change in abundance of greater sage-grouse across six temporal scales based on periods of oscillation within climate clusters

20. Images showing range-wide spatial estimates of average annual rate of change in abundance of greater sage-grouse across six temporal scales based on periods of oscillation within neighborhood clusters.

21. Graphs showing abundance index, intrinsic rate of population change of greater sage-grouse within climate cluster A from lek observations used to model population trends during 1960-2019, and median estimate of abundance trend across temporal scales based on periods of oscillation...

22. Images showing spatial estimates of average annual population rate of change in abundance of greater sage-grouse at neighborhood clusters across six temporal scales based on periods of oscillation within climate cluster $A$.

23. Images showing spatial estimates of average annual population rate of change in abundance of greater sage-grouse at leks across six temporal scales based on periods of oscillation within climate cluster $A$.

24. Graphs showing abundance index, intrinsic rate of population change of greater sage-grouse within B from lek observations used to model population trends during 1960-2019, and median estimate of abundance trend across temporal scales based on periods of oscillation.

25. Images showing spatial estimates of average annual population rate of change in abundance of greater sage-grouse at neighborhood clusters across six temporal scales based on periods of oscillation within climate cluster $B$.

26. Images showing spatial estimates of average annual population rate of change in abundance of greater sage-grouse at leks across six temporal scales based on periods of oscillation within climate cluster $B$

27. Graphs showing abundance index, intrinsic rate of population change of greater sage-grouse within climate cluster $C$ from lek observations used to model population trends during 1989-2019, and median estimate of abundance trend across temporal scales based on periods of oscillation.

28. Images showing spatial estimates of average annual population rate of change in abundance of greater sage-grouse at neighborhood clusters across six temporal scales based on periods of oscillation within climate cluster $\mathrm{C}$.

29. Images showing spatial estimates of average annual population rate of change in abundance of greater sage-grouse at leks across six temporal scales based on periods of oscillation within climate cluster $\mathrm{C}$.

30. Graphs showing abundance index, intrinsic rate of population change of greater sage-grouse within $D$ from lek observations used to model population trends during 1960-2019, and median estimate of abundance trend across temporal scales based on periods of oscillation

31. Images showing spatial estimates of average annual population rate of change in abundance of greater sage-grouse at neighborhood clusters across six temporal scales based on periods of oscillation within climate cluster $D$.

32. Images showing spatial estimates of average annual population rate of change in abundance of greater sage-grouse at leks across six temporal scales based on periods of oscillation within climate cluster $D$ 
33. Graphs showing abundance index, intrinsic rate of population change of greater sage-grouse within $\mathrm{E}$ from lek observations used to model population trends during 1960-2019, and median estimate of abundance trend across temporal scales based on periods of oscillation

34. Images showing spatial estimates of average annual population rate of change in abundance of greater sage-grouse at neighborhood clusters across six temporal scales based on periods of oscillation within climate cluster $\mathrm{E}$.

35. Images showing spatial estimates of average annual population rate of change in abundance of greater sage-grouse at leks across six temporal scales based on periods of oscillation within climate cluster $\mathrm{E}$

36. Graphs showing abundance index, intrinsic rate of population change of greater sage-grouse within $\mathrm{F}$ from lek observations used to model population trends during 1960-2019, and median estimate of abundance trend across temporal scales based on periods of oscillation.

37. Images showing spatial estimates of average annual population rate of change in abundance of greater sage-grouse at neighborhood clusters across six temporal scales based on periods of oscillation within climate cluster $F$.

38. Images showing spatial estimates of average annual population rate of change in abundance of greater sage-grouse at leks across six temporal scales based on periods of oscillation within climate cluster $F$.

39. Graph showing posterior predictive probability distribution from state-space models for greater sage-grouse population growth on a holdout sample using a restricted dataset compared to estimates over the same time frame using a full dataset

40. Histograms depicting the distribution of model predicted extirpation probabilities for greater sage-grouse at leks, over the same respective temporal scales, based on average period of oscillation within the western United States

41. Image showing spatial model predictions of extirpation probabilities for greater sage-grouse at leks across different temporal scales, based on average period of oscillation within the western United States.

42. Image showing spatial model predictions of extirpation probabilities for greater sage-grouse at neighborhood clusters across different temporal scales, based on average period of oscillation within the western United States.

43. Illustrations displaying four hypothesized outcomes in comparing trends between a finer scale and a broader scale

44. Diagrams depicting intersection of population rate of change posterior probability distributions for scale of interest and climate cluster in relation to neutral growth

45. Image showing spatial and temporal depiction of range-wide watches and warnings of greater sage-grouse population declines at the lek scale within the western United States from 1990 to 2019.

46. Graphs showing proportion of warnings and watches of greater sage-grouse annual declines at the lek and neighborhood cluster scale across each climate cluster within the western United States

47. Image showing spatial and temporal depiction of watches and warnings of greater sage-grouse population declines at the lek scale within climate cluster A from 1990 to 2019 
48. Image showing spatial and temporal depiction of watches and warnings of greater sage-grouse population declines at the lek scale within climate cluster B from 1990 to 2019

49. Image showing spatial and temporal depiction of watches and warnings of greater sage-grouse population declines at the lek scale within climate cluster C from 1990 to 2019 .

50. Image showing spatial and temporal depiction of watches and warnings of greater sage-grouse population declines at the lek scale within climate cluster D from 1990 to 2019

51. Image showing spatial and temporal depiction of watches and warnings of greater sage-grouse population declines at the lek scale within climate cluster E from 1990 to 2019

52. Image showing spatial and temporal depiction of watches and warnings of greater sage-grouse population declines at the lek scale within climate cluster F from 1990 to 2019

53. Image showing spatial and temporal depiction of range-wide watches and warnings of greater sage-grouse population declines at the neighborhood cluster scale within the western United States from 1990 to 2019.

54. Image showing spatial and temporal depiction of watches and warnings of greater sage-grouse population declines at the neighborhood cluster scale within climate cluster A from 1990 to 2019

55. Image showing spatial and temporal depiction of watches and warnings of greater sage-grouse population declines at the neighborhood cluster scale within climate cluster D from 1990 to 2019

56. Image showing spatial and temporal depiction of watches and warnings of greater sage-grouse population declines at the neighborhood cluster scale within climate cluster E from 1990 to 2019

57. Image showing spatial and temporal depiction of watches and warnings of greater sage-grouse population declines at the neighborhood cluster scale within climate cluster F from 1990 to 2019

58. Image showing spatial and temporal depiction of range-wide watches and warnings of greater sage-grouse population declines at the lek scale within the western United States during 2019

59. Image showing spatial and temporal depiction of watches and warnings of greater sage-grouse population declines at the lek scale within climate cluster A during 2019

60. Image showing spatial and temporal depiction of watches and warnings of greater sage-grouse population declines at the lek scale within climate cluster D during 2019

61. Image showing spatial and temporal depiction of watches and warnings of greater sage-grouse population declines at the lek scale within climate cluster E during 2019

62. Image showing spatial and temporal depiction of watches and warnings of greater sage-grouse population declines at the lek scale within climate cluster F during 2019

63. Image showing spatial and temporal depiction of watches and warnings of greater sage-grouse population declines at the neighborhood cluster scale within climate cluster D during 2019 
64. Image showing spatial and temporal depiction of watches and warnings of greater sage-grouse population declines at the neighborhood cluster scale within climate cluster E during 2019

65. Image showing spatial and temporal depiction of watches and warnings of greater sage-grouse population declines at the neighborhood cluster scale within climate cluster $\mathrm{F}$ during 2019

66. Graphs showing proportion of warnings at leks, watches at leks, warnings at neighborhood clusters, and watches at neighborhood clusters of greater sage-grouse population annual declines across each climate cluster in the western United States

67. Graphs showing relationship between breeding season precipitation and intrinsic rate of population change, and breeding season precipitation and proportion of leks signaled within climate clusters for greater sage-grouse in the western United States.

\section{Tables}

1. Identified conservation status values developed for standardized greater sage-grouse lek databases.

2. Candidate habitat covariates identified for the Spatial "K"luster Analysis by Tree Edge Removal clustering algorithm to develop a greater sage-grouse hierarchical population monitoring framework of lekking locations

3. Constraint-based rules used to inform clustering of greater sage-grouse lek locations based on distance rules and landscape features

4. Constraint-based rules used to inform range-wide clustering of greater sage-grouse lek locations based on a range of leks to group per cluster level.

5. Identified years of population abundance nadirs used to define temporal scales of population trend estimates across different climate clusters and range-wide for greater sage-grouse in the western United States.

6. Greater sage-grouse average annual rate of population change across six different temporal scales for each climate cluster within the western United States...31

7. Greater sage-grouse average annual rate of increase in abundance at neighborhood clusters across six different temporal scales that were based on complete periods of oscillation of population abundance within the western United States external file

8. Model predictions of extirpation probabilities for greater sage-grouse leks and neighborhood clusters, summarized in terms of mean and standard error, at different temporal scales based on an average period of oscillation within the western United States

9. Watches and warnings identified at greater sage-grouse leks across climate clusters using state-space model estimates within a targeted annual warning system in the western United States during 1990-2019.

10. Watches and warnings identified at the neighborhood cluster scale across different climate clusters by state-space model estimates using a targeted annual warning system framework for greater sage-grouse across their range in the western United States during 1990-2019. 
11. Watches and warnings identified at the lek scale across different climate clusters by state-space model estimates using a targeted annual warning system framework for greater sage-grouse across their range in the western United States during 2019

12. Watches and warnings identified at the neighborhood cluster scale across different climate clusters using state-space model estimates in a targeted annual warning system framework for greater sage-grouse across their range in the western United States during 2019

\section{Conversion Factors}

International System of Units to U.S. customary units

\begin{tabular}{lcl}
\hline \multicolumn{1}{c}{ Multiply } & \multicolumn{1}{c}{ By } & \multicolumn{1}{c}{ To obtain } \\
\hline & Length & \\
\hline meter $(\mathrm{m})$ & 3.281 & foot $(\mathrm{ft})$ \\
kilometer $(\mathrm{km})$ & 0.6214 & mile $(\mathrm{mi})$ \\
\hline & Area & \\
\hline hectare $($ ha) & 2.471 & acre \\
square hectometer $\left(\mathrm{hm}^{2}\right)$ & 2.471 & acre \\
square kilometer $\left(\mathrm{km}^{2}\right)$ & 0.3861 & square mile $\left(\mathrm{mi}^{2}\right)$ \\
square kilometer $\left(\mathrm{km}^{2}\right)$ & 247.1 & acre \\
\hline
\end{tabular}

\section{Datum}

Vertical coordinate information is referenced to the North American Vertical Datum of 1988 (NAVD 88).

Horizontal coordinate information is referenced to the World Geodetic System (WGS 84).

Elevation, as used in this report, refers to distance above the vertical datum. 


\section{Abbreviations}

$\lambda \quad$ finite rate of population change

$\hat{\lambda} \quad$ estimated finite rate of population change

$\hat{r} \quad$ estimated intrinsic rate of population change

$N \quad$ abundance

$\hat{N} \quad$ estimated abundance

BLM Bureau of Land Management

CC climate cluster

CRI credible interval

ESA Endangered Species Act

IPM integrated population model

LC-MST least-cost minimum spanning tree

PD posterior probability distributions

LOR log odds ratio

NC neighborhood cluster

$R \& R \quad$ resilience and resistance

SMZ Sage-Grouse Management Zones

SKATER Spatial 'K'luster Analysis by Tree Edge Removal

SSM state-space model

TAWS targeted annual warning system

Tech Team Sage-Grouse and Columbian Sharp-tailed Grouse Technical Team

USFWS U.S. Fish and Wildlife Service

WAFWA Western Association for Fish and Wildlife Agencies 


\title{
Range-wide Greater Sage-Grouse Hierarchical Monitoring Framework: Implications for Defining Population Boundaries, Trend Estimation, and a Targeted Annual Warning System
}

\author{
By Peter S. Coates, ${ }^{1}$ Brian G. Prochazka, ${ }^{1}$ Michael S. O'Donnell,2,3 Cameron L. Aldridge,2,3 \\ David R. Edmunds,2,3 Adrian P. Monroe,2,3 Mark A. Ricca, 1,4 Gregory T. Wann,1 Steve E. Hanser,2,5 \\ Lief A. Wiechman, ${ }^{5}$ and Michael P. Chenaille ${ }^{1}$
}

\begin{abstract}
Incorporating spatial and temporal scales into greater sage-grouse (Centrocercus urophasianus) population monitoring strategies is challenging and rarely implemented. Sage-grouse populations experience fluctuations in abundance that lead to temporal oscillations, making trend estimation difficult. Accounting for stochasticity is critical to reliably estimate population trends and investigate variation related to deterministic factors on the landscape, which are amenable to management action. Here, we describe a novel, rangewide hierarchical monitoring framework for sage-grouse centered on four objectives: (1) create a standardized database of lek counts, (2) develop spatial population structures by clustering leks, (3) estimate spatial trends at different temporal extents based on abundance nadirs (troughs), and (4) develop a targeted annual warning system to help inform management decisions. Using automated and repeatable methods (software), we compiled a lek database (as of 2019) that contained 262,744 counts and 8,421 unique lek locations from disparate state data. The hierarchical population units (clusters) included 13 nested levels, identifying biologically relevant units and population structure that minimized intercluster sage-grouse movements. With these products, we identified spatiotemporal variation in trends in population abundance using Bayesian state-space models. We estimated 37.0, 65.2, and 80.7-percent declines in abundance rangewide during short (17 years), medium (33 years), and long (53 years) temporal scales, respectively. However, some areas exhibited evidence of increasing trends in abundance in
\end{abstract}

\footnotetext{
1U.S. Geological Survey, Dixon, California

${ }^{2}$ U.S. Geological Survey, Fort Collins, Colorado

${ }^{3}$ Colorado State University, Fort Collins, Colorado

${ }^{4}$ U.S. Geological Survey, Corvallis, Oregon

${ }^{5}$ U.S. Geological Survey, Reston, Virginia
}

recent decades. Models predicted 12.3, 19.2, and 29.6 percent of populations (defined as clusters of neighboring leks) consisted of over 50-percent probability of extirpation at 19 , 38 , and 56-year projections from 2019, respectively, based on averaged annual rate of change in apparent abundance across two, four, and six oscillations (average period of oscillation is 9.4 years). At the lek level, models predicted 45.7, 60.1, and 78.0 percent of leks with over 50-percent extirpation probabilities over the same time periods, respectively, mostly located on the periphery of the species' range. The targeted annual warning system automates annual identification of local populations exhibiting asynchronous decline relative to regional population patterns using simulated management actions and an optimization algorithm for evaluating range-wide stabilization of population abundance. In 2019 , approximately 3.2 percent of leks and 2.0 percent of populations were identified by the targeted annual warning system for management intervention range-wide.

\section{Executive Summary}

Managing greater sage-grouse (Centrocercus urophasianus) populations is often challenging because of their extensive spatial distribution (overlapping multiple regulatory jurisdictions), disparities and imperfections in counts at breeding grounds (leks) that index population sizes, and uncertainties in estimating trends in abundance given inter-annual fluctuations. These challenges could be mitigated with a decision support framework based on long-term monitoring data from across the range and applying advanced population models that account for demographic and environmental stochasticity, movement dynamics, ecological productivity, and errors in counting sage-grouse on their leks. However, even when population data are collected with relatively standard methodologies, logistical hurdles first must be cleared to compile disparate databases of sage-grouse abundance into consistent formats for analysis. 
Next, relevant spatial scales need to be delineated to correctly identify factors influencing population trends. It follows that associated complexes of sub-populations at more local spatial scales would respond similarly to changes in environmental conditions (for example, precipitation and drought) that affect resource availability across larger areas. However, when negative population rates of change at local scales deviate from rates occurring at larger scales, these differences could indicate that local disturbances are contributing to poor population performance. Applying these concepts to standardized datasets in a unified, objective, and repeatable framework could inform more targeted management actions for this species and avoid costly misapplication.

Researchers with the U.S. Geological Survey and Colorado State University, in close cooperation with multiple state and federal resource agency partners, extended a hierarchical population monitoring framework across the entire greater sage-grouse range in the United States. This framework was initially tested at state-wide levels (California, Nevada, and Wyoming), and research needs were identified by state (Western Association for Fish and Wildlife Agencies) and federal (Bureau of Land Management, U.S. Fish and Wildlife Service) natural resource agencies. Herein, we report results of four primary study objectives from this hierarchical population monitoring framework, namely:

1. Create a range-wide database for sage-grouse lek counts:

Purpose: Eleven western state wildlife agencies have identified the need to compile lek count and location data collected by wildlife agencies into a single comprehensive lek database.

Methods: We compiled all digitized field observations of sage-grouse leks from state wildlife agencies within any state that monitored sage-grouse populations between 1953 and 2019 into a single database. We worked with each agency to ensure the fullest understanding of the data to maximize the number of appropriate records kept in the database, we addressed spatial errors, and we reviewed all data products with state wildlife members of the Sage and Columbian Sharptailed Grouse Technical Team.

Results and interpretation: The resulting range-wide lek database contained 262,744 counts and 8,421 unique lek locations with data standardized by coordinate reference system, field names and types, and conservation status definitions. These data informed the remaining three objectives.

\section{Develop nested population lek clusters:}

Purpose: Population rates of change are affected by environmental factors that operate on multiple spatial and temporal scales that follow ecological, rather than geopolitical, boundaries. Hence, examining population trends across biologically relevant and hierarchically nested units can improve the detection of factors driving change across various spatial scales.

Methods: We grouped sage-grouse lekking sites into hierarchical, nested clusters, or populations. We used least-cost minimum spanning trees, a clustering algorithm, and a suite of relevant spatial products (biotic and abiotic) to group leks into multiple cluster levels. We selected two cluster levels to represent a fine (neighborhood cluster, NC) and broad spatial scale (climate cluster, CC) in subsequent trend analyses. We used movement data from radio and global positioning system marked sage-grouse to inform the NC scale, and relationships between precipitation and rate of change in population abundance to inform the $\mathrm{CC}$ scale.

Results and interpretation: We produced 13 hierarchically nested cluster levels comprising population groupings of sage-grouse leks from fine to coarse scales. Each cluster level was evaluated for migration potential to ensure that changes in abundance were more likely to be driven by changes in demographic rates as opposed to movement of individuals into or out of the population. Cluster levels 2 and 13 represented $\mathrm{NC}$ and $\mathrm{CC}$, respectively, which were used in trend and targeted annual warning system analyses (Objectives 3 and 4). Neighborhood clusters represent amalgamations of leks that likely experience similar landscape-level impacts (for example, wildfire), whereas CCs more closely represent large-scale populations from leks governed by similar climatic and habitat conditions at regional extents. Population dynamics at the $\mathrm{NC}$ and $\mathrm{CC}$ scales reflect changes in demographic rates because migration between clusters at these scales were negligible, whereas at the lek level, changes in demographic rates could not be disentangled from emigration and immigration.

3. Estimate spatiotemporal trends in population abundance:

Purpose: Sage-grouse generally exhibit inter-annual variation in population abundance with consecutive years of increases or decreases that reflect oscillations over approximately 10 -year periods. Accounting for oscillating patterns of abundance is critical to obtaining reliable estimates of population trends across different temporal scales. Otherwise, estimates can be biased by short-term fluctuations in abundance and yield misleading results, regardless of the duration of inference. Analytical methods that permit abundance estimation across nadirs (that is, troughs within oscillations) can produce greater accuracy in trends, as opposed to different points along the oscillations (for example, nadir to apex or apex to nadir). Examining trends across more than one period (that is, complete oscillation) is also important, given their variability (that is, number of years between a single set of nadirs) and amplitude (that is, displacement from mid-point to nadir or apex). 
Methods: We used a state-space model (SSM) informed by lek count data, collected across approximately six decades, to estimate population trends in relative abundance across three nested spatial scales (leks, NCs, and CCs) and six different temporal scales that reflected complete oscillations. Specifically, we identified six abundance nadirs from model outputs and calculated averaged annual rate of change $(\hat{\lambda})$ in abundance relative to 2019. If populations continue to decline in subsequent years, $\hat{\lambda}$ estimates reported here will be overestimated based on the assumption that 2019 represents the most recent abundance nadir. To facilitate interpretation, we graphically illustrate spatial trends at short (two oscillations), medium (four oscillations), and long (six oscillations) temporal scales.

Results and interpretation: State-space models revealed six different abundance nadirs (1966, 1975, 1986, 1996, 2002, and 2013) at the range-wide scale, with slight variations in the timing of these nadirs occurring across CCs. We reported declines in range-wide estimates of average annual $\hat{\lambda}$ (evaluated at median value) across all temporal scales. For example, over the course of relatively short (17 years), medium (33 years), and long (53 years) temporal scales from three abundance nadirs $(2002,1986$, and 1966) to 2019, we report that range-wide sage-grouse populations have declined 37.0, 65.2, and 80.7 percent, respectively. We also estimated median $\hat{\lambda}$ to be less than 1.0 for $81.3,88.4$, and 94.3 percent of NCs across short, medium, and long temporal scales, respectively. A relatively small percentage of decline annually can result in substantial cumulative losses in $\hat{N}$ across temporal scales. Climate cluster B (Washington area) experienced the greatest overall declines across long and medium temporal scales. The only $\mathrm{CC}$ with estimated slight growth was F (western Wyoming area; average annual median $\hat{\lambda}>1.0$ ), where growth was observed during the most recent oscillation and the last three oscillations combined (short/ medium temporal scale). We found spatiotemporal variation in $\hat{\lambda}$ across the range. For example, declines strengthened in recent oscillations for CC-E (Great Basin area) which are likely attributed to increases in cumulative impacts of wildfire and invasive grass, conifer expansion, and changes in predator composition. On the contrary, declines weakened through time for CC-D (eastern area) which might reflect recent conservation action strategies that may, in part, offset the primary threats in this portion of the range, including cropland conversion and energy exploration and development.

Additionally, we estimated over 50-percent extirpation probability for $45.7(\mathrm{n}=2,347), 60.1(\mathrm{n}=3,084)$, and 78.0 $(\mathrm{n}=4,001)$ percent of leks based on 19,38, and 56-year projections of population growth projected from 2019, respectively. We also predicted over 50-percent extirpation probability for $12.3(n=52), 19.2(n=81)$, and $29.6(n=125)$ percent of populations, defined as neighboring clustered leks (NC), over the same projected time frames. Spatial depictions revealed that most lek and $\mathrm{NC}$ extirpations were likely to occur within peripheral populations that generally were subjected to landscape disturbances (for example, burned areas and anthropogenic infrastructure) coupled with a high degree of environmental stochasticity. For climate clusters, we found that CC-C (Jackson Hole, Wyoming, area) had the highest extirpation probability.

4. Develop a targeted annual warning system (TAWS) to signal declining leks and lek clusters:

Purpose: A framework for identifying population declines that likely are attributable to disturbances on the landscape versus environmental stochasticity or intrinsic factors across broader regions could help immediately inform when and where increased monitoring or direct management intervention may be needed and also reduce the chance for costly misallocation of limited management resources. The key components to detecting changes in population trends include (1) identifying when declining trends at nested local scales are below estimated trends at broader CC-scales which are ostensibly governed by less manageable climatic factors and (2) targeting leks or NCs early enough to allow management intervention to be more effective at thwarting declines. We developed TAWS to signal when and where trends of individual leks and NCs are declining below trends at the $\mathrm{CC}$ scale, resulting from local disturbance rather than fluctuations driven by environmental stochasticity.

Methods: The TAWS used two categories for multi-year signaling events referred to as 'watches' and 'warnings.' We assigned watches to populations that exhibited evidence of population decline below those of the CC (slow signal) over 2 consecutive years. We assigned warnings to populations that had slow signals in 3 out of 4 consecutive years or a relatively strong magnitude (fast signal) of evidence for 2 out of 3 consecutive years. Watches may identify the need for intensive monitoring whereas warnings may identify the need for management intervention aimed at stabilizing populations. Declines in abundance over multiple years may result in consecutive watches and warnings (for example, multiple years following disturbance). Repeat watches and warnings provide valuable information about chronic effects on the same population that is associated with a disturbance. Thus, we report proportion of leks with first and repeated events of watches and warnings. Collectively, these rules facilitate quantification of population decline that distinguishes localized adverse impacts from broad-scale environmental stochasticity and provide valuable information to potentially and proactively manage populations.

Because populations can demonstrate poor performance in successive years as a result of the same perturbation, or over multiple years from multiple perturbations, we present summary statistics of watches and warnings in terms of first and repeat events. Unless stated otherwise, watches and warnings are summarized in terms of first events, which represent the first time a population experienced a watch or warning (that is, no previous evidence of declining below the CC). Populations that decline over multiple years may experience consecutive watches and warnings (for example, several years following fire). Repeat watches and warnings provide valuable information about the chronic effects on the same population that is associated with a perturbation. 
Results and interpretation: We estimated 63.4 and 46.7 percent of sage-grouse leks across the range experienced at least one watch and at least one warning, respectively, from 1990 to 2019. On an annual basis, we found that 2.5 (repeat $=6.0$ ) percent of leks range-wide activated watches and 1.9 (repeat $=5.8$ ) percent of leks range-wide activated warnings. The $\mathrm{CC}$ with the greatest number of watches and warnings was CC-E (Great Basin area), with 1,163 (repeat $=2,834$ ) watches and 830 (repeat $=2,712$ ) warnings, representing 61.0 (watches) and 43.5 (warnings) percent of total leks activated since 1990. The greatest proportion of watches and warnings activated at the NC scale occurred within climate clusters CC-F (western Wyoming area), CC-E (Great Basin area), and CC-D (eastern area). Watches and warnings have increased substantially through time range-wide, with the greatest increases also found in these three climate clusters since the mid- to late-2000s. Increases in watches and warnings through time is likely a function of more disturbances on the landscape (for example, increased anthropogenic developments and frequency of wildfire). Although our models were robust for missing lek count data by imputing missing values, increased sampling effort in recent years may also increase frequency of watches and warnings based on greater precision in parameter estimation and rules that exclude leks without recent count histories. Therefore, improvements in standardized lek-count protocols that aim to minimize the number of years between lek counts for known leks and conducting periodic searches to discover previously unknown leks would be highly beneficial to refine estimates in trends and inform the TAWS using this SSM framework, especially if the goal is to annually identify when and where management actions will be most effective at stabilizing or reversing declines associated with disturbances on the landscape.

\section{Introduction}

Greater sage-grouse (Centrocercus urophasianus; hereafter, sage-grouse) are a sagebrush obligate bird that currently occupy most sagebrush ecosystems across 11 U.S. states and 2 Canadian Provinces in western North America (Patterson, 1952; Schroeder and others, 2004).

Sage-grouse are of high conservation concern and their habitat needs are now central to guiding land-management action and policies across most of the western United States. At the turn of the twenty-first century, sage-grouse occupied roughly half of their former historical range (Schroeder and others, 2004; Miller and others, 2011), and over the past three to five decades have demonstrated apparent population declines in many parts of their current range (Garton and others, 2011, 2015; Western Association of Fish and Wildlife Agencies, [WAFWA], 2015), which largely have been attributed to sagebrush loss and environmental stressors (Connelly and others, 2004; Schroeder and others, 2004; Doherty and others,
2016). Since 1999, sage-grouse have been petitioned for legal protection under the Endangered Species Act (ESA) of 1973 on nine occasions. However, an unprecedented level of conservation effort and planning among federal (for example, Bureau of Land Management [BLM], 2015), state, and private stakeholders was identified as the primary driver for a decision by the U.S. Fish and Wildlife Service (USFWS) that sage-grouse did not warrant listing in the most recent status assessment (U.S. Fish and Wildlife Service, 2015).

Sage-grouse are considered an indicator of the integrity of sagebrush ecosystems, as well as an umbrella species for the protection of other sagebrush-obligate or semi-obligate species given their near complete dependence on sagebrush ecosystems throughout their life history (Rich and Altman, 2001; Rich and others, 2005; Rowland and others, 2006; Hanser and Knick, 2011). Specifically, in recent years, the resource needs of sage-grouse helped to guide management actions aimed at improving conditions in sagebrush ecosystems, and resultant practices are thought to carry over onto other sagebrush-dependent species (Rowland and others, 2006; Hanser and Knick, 2011; Dinkins and others, 2019), though some less associated species may not be well covered by the sage-grouse umbrella (Carlisle and others, 2018; Pilliod and others, 2020). Importantly, several federal resource management plan amendments accompanying the 'not warranted' 2015 ESA listing determination called for greater integration of adaptive management into land-use planning, and specifically, identifying how to implement adaptive management.

The U.S. Geological Survey (USGS), Bureau of Land Management (BLM) and Colorado State University (CSU; Natural Resources Ecology Laboratory), in collaboration with the Sage-Grouse and Columbian Sharp-tailed Grouse Technical Team (hereafter, Tech Team) under the WAFWA, are developing standardized protocols for monitoring sage-grouse populations and estimating changes in population size through time. Typically, population trends of sage-grouse are estimated using standardized count survey techniques at lek sites (traditional breeding grounds; Connelly and others, 2003). If lek count data are collected in a similar manner across space and time, then they can be useful as an index to identify population changes and associated drivers (Connelly and others, 2003; Connelly and Schroeder, 2007).

Our primary goal was to develop a population monitoring and modeling framework applied to sage-grouse populations range-wide, under which population relative abundance and trends are estimated readily across different nested spatiotemporal scales by using lek count data. However, further work will likely be needed to fully understand the relationship between count indices and true population sizes. Our framework also builds upon concepts that were tested previously (Coates and others, 2017, 2019a; O'Donnell and others, 2019) by comparing annual changes in relative abundance among different spatial scales to signal when and where populations are in decline. 
It relies on standardized annual lek counts to immediately and systematically streamline scientific findings into the management decision-making process (Walters, 1986). Thus, this framework can be used to inform population status of sage-grouse at different spatiotemporal scales, as well as identify areas for monitoring within these highly stochastic ecosystems, affording managers opportunities to understand and act upon mechanisms behind changes in population growth. The specific objectives were:

1. Collaborate with personnel from state wildlife agencies to compile a standardized lek count dataset across the sage-grouse geographic range. We sought to make the database easy to update through open-source software.

2. Define range-wide hierarchical population units of active sage-grouse leks. The hierarchical framework is intended to reflect biologically relevant groupings of leks (that is, clusters) characterized by similar environmental features and sage-grouse movement ecology. We identify multiple nested levels of clusters where the number of grouped leks increased with each increasing level.

3. Estimate spatiotemporal trends in sage-grouse population abundance throughout their range using a Bayesian state-space modeling framework of annual maximum lek counts from survey data. Time series lek count data were compiled into a single comprehensive database (Objective 1) and spatial scales were delineated from lek clustering methods (Objective 2).

4. Independent of Objective 3, we developed and implemented a TAWS that can be readily applied on an annual basis and identifies where and when declines in sage-grouse population abundance occur while accounting for inter-annual variation that typically is associated with broad-scale climatic conditions. Specifically, we identified temporal and spatial thresholds that, when crossed, activate signals for leks or populations (such as, clusters of leks) that most likely decline in response to localized perturbations. Such signaling can inform when and where localized monitoring (for example, telemetry studies) or management intervention is needed.

\section{Study Areas}

Our study extent represents the sagebrush biome occurring across western North America and extending east from the Sierra Nevada/Cascade Mountain ranges to the western regions of the Great Plains of the United States (fig. 1). These areas also include forests at higher elevations, salt deserts in the Great Basin and Colorado Plateau, and a mixture of different types of grasslands in the western Great Plains (Pyke and others, 2015; Shinneman, 2020). On average, the sagebrush biome receives 118-1,380 millimeters (mm) of precipitation per year ( $\bar{x}=331.2 \mathrm{~mm}$ per year), maintains cold, wet winters and hot, dry summers $(\bar{x}=49.9 \mathrm{~mm}$ during wettest month and $\bar{x}=11.2 \mathrm{~mm}$ during driest month; PRISM Climate Group, Oregon State University, 2015), and is characterized by shrubs interspersed with grasses and forbs. The vegetation communities vary with changes in precipitation, temperature, soils, topographic position, and elevation (Miller and others, 2011). The most abundant shrub species include sagebrush (Artemisia spp.) with less abundant non-sagebrush species of rabbitbrush (Chrysothamnus spp.), horsebrush (Tetradymia spp.), greasewood (Sarcobatus spp.), common snowberry (Symphoricarpos spp.), serviceberry (Amelanchier spp.), fourwing saltbush (Atriplex spp.), and bitterbrush (Purshia spp.). The primary herbaceous species include wheatgrass (Agropyron spp.), fescue (Festuca spp.), bluegrass (Poa spp.), needlegrass (Stipa spp.), bromegrass (Bromus spp.), and squirreltail (Sitanion spp.), whereas less abundant forb species include phlox (Phlox spp.), milk-vetch (Astragalus spp.), and fleabane (Erigeron spp.).

\section{Objective 1. Database for Sage-grouse Lek Counts}

\section{Background}

The largest challenge associated with a range-wide analysis of sage-grouse population trends is the lack of a single comprehensive lek count database serving as the foundation for an inter-agency monitoring program. Many limitations can exist when using long-term monitoring data because methods of monitoring can change over time, multiple organizations collect data differently, and data are managed independently among data stewards. Sage-grouse long-term monitoring data exist for 11 states within the western United States. State wildlife agencies collect survey counts of males on leks annually (lek datasets), but differences in the data collection and management require that we standardize these data to develop a range-wide lek database. Further details for standardizing the state lek databases and associated software are described in O'Donnell and others (unpub. data, U.S. Geological Survey, 2020). The purpose of this objective was to develop a standardized sage-grouse lek database across the species' range-wide distribution to inform the creation of a hierarchical population monitoring framework (Objective 2), spatiotemporal patterns in population trends (Objective 3), and a TAWS (Objective 4). 


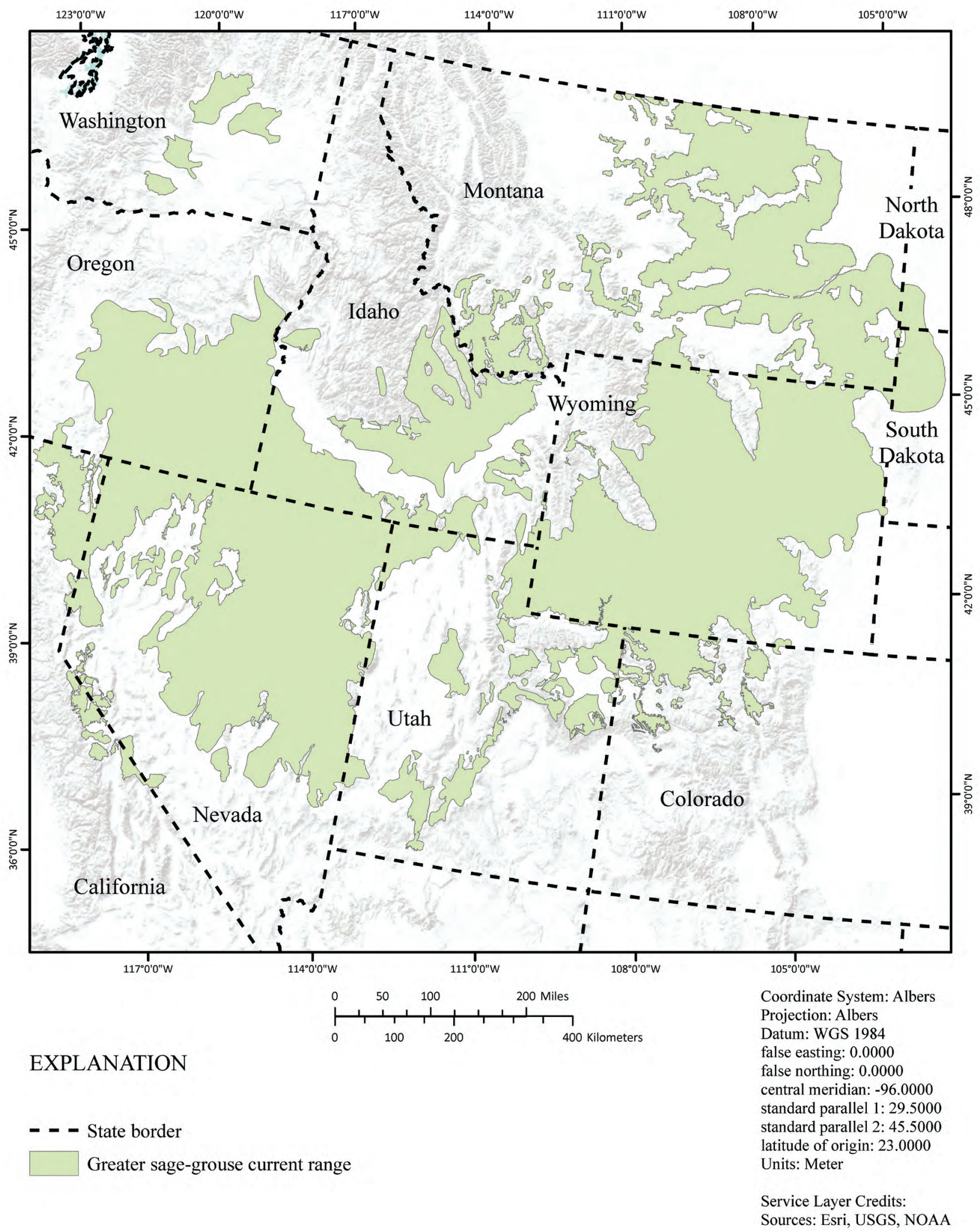

Figure 1. Greater sage-grouse (Centrocercus urophasianus) distribution within the sagebrush biome across 11 states in the western United States. Map image is the intellectual property of Esri and is used herein under license. Copyright (C) 2020 Esri and its licensors. All rights reserved. 


\section{Methods}

Researchers at the U.S. Geological Survey and Colorado State University (CSU; Natural Resources Ecology Laboratory) worked with WAFWA to obtain data-sharing agreements, which allowed the use of state sage-grouse lek data for multiple objectives. The database contained all digitized field observations of males counted on leks and lek locations from each state as of 2019. We used an automated, systematic, and repeatable method for standardizing and cleaning up long-term monitoring data by creating open-source software that automated compilation of state databases. We programmatically generated a new NoSQL (not only Structured Query Language) database that maximized the inclusion of data, standardized all content and terminology, and retained information needed to inform future population trend analyses. For database development and management, we used Python $\AA$ 3.7.7 (Python Software Foundation) and numerous well-supported third-party Python libraries in a Windows ${ }^{\circledR}$ (Microsoft Corporation) operating system environment.

We developed a two-phase software workflow to arrive at a standardized, multi-state lek database. The first phase of our software package addressed state-specific modifications necessary to achieve a crosswalk to an initial standardized database schema. Here, we standardized coordinate reference systems, addressed spatial errors (for example, null geometry and missing coordinates), standardized field names and types (for example, integers, date, and strings), and populated standardized survey methods. The second phase of the workflow addressed differences in definitions of lek types, monitoring methods, and similar components. During this phase, we removed records that did not adhere to a standardized definition of a lek (greater than or equal to two males observed for greater than or equal to 2 years), and those with no recorded year or a number that reflected a count (a count of 0 males was included). We also aggregated male counts for leks within 500 meters (m) of each other, as most states were already implementing. We then thoroughly reviewed the resulting database with representatives of the Tech Team.

After developing clusters (Objective 2), and before assessing population trends and developing a TAWS, we identified field observations within the sage-grouse lek database that maximized detection probabilities of lek attendance and minimized errors in counting of males associated with survey methods. We selected observations that were done during March 1 to May 31 (Rule 1; breeding season), within 30 minutes before and 90 minutes after local sunrise (Rule 2; sunrise), and were surveyed using one of the following survey methods (Rule 3; survey method): ground (count), aerial helicopter camera HD/IR (count), aerial helicopter (count), ground route (count), aerial fixed-wing (count), aerial unknown, ground unknown, and aerial fixed-wing camera HD/IR (count). Within the state of Wyoming, we also included observations that were collected according to the state defined survey method "ground (survey)," based on the rigorous criteria required to achieve that categorical assignment and the ensuing adequacy of those data for trend estimation. In the absence of a recorded date, time, or survey method, we assumed the observations were done near sunrise, during the breeding season, and were conducted with one of the survey methods mentioned previously. We also aggregated within-year repeat counts by maintaining the maximum count per lek per year (Rule 4; max count). This was because not all states reported repeated counts and some reported only maximum values. Thus, in this framework, as currently implemented, detection probability is not accounted for and modeled estimates are considered relative abundance. At this point, we bifurcated our dataset sub-setting criteria according to whether the dataset would be applied to Objectives 3 or 4. For Objective 3, we removed counts that were recorded before 1960 (Rule 5a; post 1960) and removed leks that had fewer than 5 years of active counts (greater than or equal to two males) during the 60-year time series (Rule 6a; five active counts). The rule of 5 years was determined based on preliminary investigations of spurious inferences of intrinsic rate of population change $(\hat{r}$; Coates and others, 2019a). The post 1960 rule was used because data were too sporadic for our model during the 1950s. For Objective 4, we reduced the dataset by removing counts collected before 1990 because we did not require extensive count histories and recent counts are most relevant to current management applications, thereby restricting to a period when leks were more frequently counted (Rule 5b; post 1990). Similar to Objective 3, we removed leks that had fewer than 5 years of active counts through the time series (Rule 6b; five active counts).

\section{Results}

The standardized and complete range-wide lek database (observation and location, as of 2019) contained 262,744 counts and 8,421 unique lek locations, with most of the states collecting survey data beginning in 1953. Using the standardized definitions of conservation status, we observed 5,542 active, 1,341 historic, 1,079 inactive, 342 pending new, and 117 pending old leks (for lek type definitions, see table 1). Range-wide, the mean number of males displaying on all leks with counts was 12.3 (median=6.0, [standard deviation] $\mathrm{SD}=6.2$ ). The standardized range-wide data included a total of 185,915 within-year repeat count observations. We removed 68,808 observations from the originating data mostly because of an absence of male counts (for example, record blank or coded by state as NA; 57,220) and observations where the definition of a lek was not met $(6,257$; where greater than or equal to two males observed for 2 years was not met). As a result of removing these observations, as well as leks with missing coordinates, we removed 3,742 lek locations. Our workflow maximized the inclusion of data and addressed inconsistencies, such as standardizing field names and content within fields, deleting erroneous records (for example, blank records and duplicate records), and addressing typing errors. 
Table 1. Identified conservation status values developed for standardized greater sage-grouse (Centrocercus urophasianus) lek databases.

\begin{tabular}{|c|c|}
\hline Lek type & Definition \\
\hline Active & $\begin{array}{l}\text { A lek that has greater than or equal to two males per } 2 \text { observations occurring on different years that were recorded in last } \\
10 \text { years. }\end{array}$ \\
\hline Historic & $\begin{array}{l}\text { A lek that has greater than or equal to two males per } 2 \text { observations occurring on different years that were recorded longer than } \\
20 \text { years ago and is not considered active or inactive. }\end{array}$ \\
\hline $\begin{array}{l}\text { Pending } \\
\text { old }\end{array}$ & $\begin{array}{l}\text { A lek with insufficient observations to classify as Active, Inactive, Historic, and Pending New. We required that there was one } \\
\text { observation greater than or equal to two males recorded 11-20 years ago and at least one observation of greater than or equal to } \\
\text { two males longer than } 20 \text { years ago. }\end{array}$ \\
\hline
\end{tabular}

The standardized and complete range-wide lek database incurred additional data sub-setting rules (Rules 1-6), which produced lek datasets for Objectives 3 and 4 and resulted in the sequential removal of approximately 0.0 percent of leks and 0.4 percent of observations (Rule 1; breeding season), 0.3 percent of leks and 8.3 percent of observations (Rule 2; sunrise), 1.0 percent of leks and 11.9 percent of observations (Rule 3; survey method), 1.0 percent of leks and 54.7 percent of observations (Rule 4; max count), 1.1 percent of leks and 55.4 percent of observations (Rule 5a; post 1960), 39.1 percent of leks and 63.7 percent of observations (Rule 6a; five active counts), 1.6 percent of leks and 63.4 percent of observations (Rule 5b; post 1990), and 46.8 percent of leks and 73.1 percent of observations (Rule $6 \mathrm{~b}$; five active counts) range-wide. State-specific summaries of number and percentage of leks and observations retained following the sequential application of Rules 1-6 are listed in appendix 2 (tables 2.1-2.4). Using all the aforementioned rules for selecting data appropriate to population modeling, we retained 95,297 observations across 5,131 leks (Objective 3) and 70,646 observations across 4,478 leks (Objective 4). The proportion of leks counted increased substantially through time for every CC (fig. 2). Climate cluster A (Bi-State area) had the most robust sampling across the time series; more than double the sampling effort observed in most other areas of the range in earlier years. The next highest sampling effort occurred within climate cluster B (Washington area). All sampling efforts increased drastically (approximately doubling) across all CCs from 2000 to 2010. The predominant survey method for both datasets was ground (count) and represented approximately 39 percent and 43 percent of the observations, for Objectives 3 and 4 , respectively. We classified approximately 30 percent and 23 percent of observations in the database as "No Data" because count method information was missing. Less than 3 percent of observations fell within any of the Aerial type survey methods across both datasets. Additionally, approximately 63 percent and 72 percent of observations lacked a date for Objectives 3 and 4, respectively, and a time of survey was absent in approximately 44 percent and 54 percent of observations, respectively.

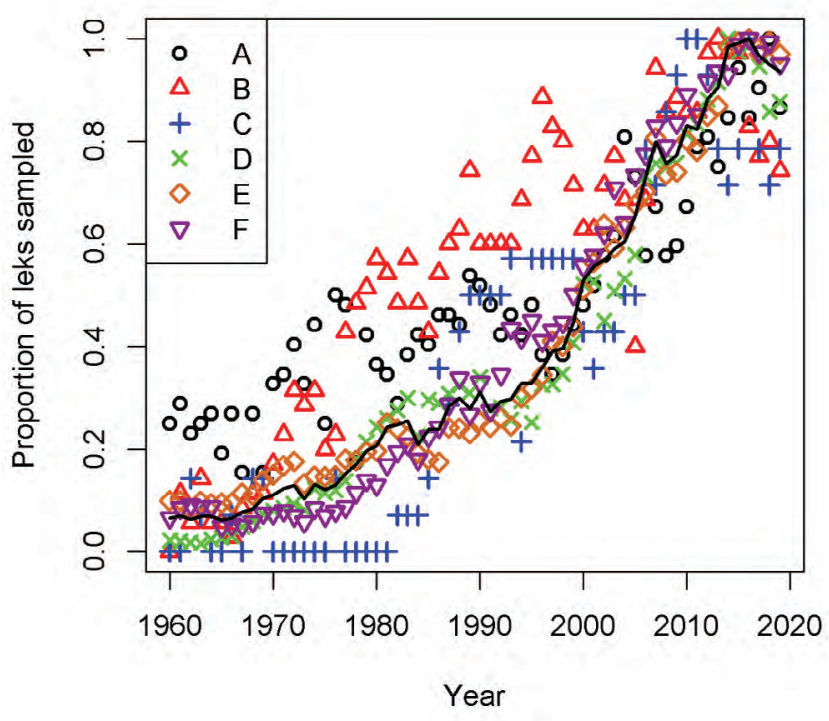

Figure 2. Proportion of greater sage-grouse (Centrocercus urophasianus) leks sampled between 1960 and 2019 for different population climate clusters $(\mathrm{A}=\mathrm{Bi}$-State area; $\mathrm{B}=$ Washington area; $C$ = Jackson Hole, Wyoming, area; $D=$ eastern area; $\mathrm{E}=$ Great Basin area; and $\mathrm{F}=$ western Wyoming area; see Objective 2 for climate cluster delineation) after removing observations defined in Objective 1 and Objective 3. Solid black line indicates the average across climate clusters. 


\section{Objective 2. Population Clusters}

\section{Background}

Demographic properties, such as population rates of change, are affected by multiple environmental and intrinsic factors that operate on different spatial and temporal scales (Gurevitch and others, 2016), and evaluations of demographics at a single scale risk missing important scale-dependent patterns. Therefore, studying population trends across biologically relevant and hierarchically nested units can improve the detection of factors driving change, which can operate at different spatial scales (Sadoul, 1997; DeSante and others, 2001; Wallace and others, 2010). The smallest scale of measurement is a lek site, which is a traditional breeding ground (for example, revisited for purposes of reproduction annually). Changes in lek abundance may be a function of changes in demographic rates and movements to and from leks. However, clustering leks into hierarchical levels represents populations in which changes in abundance are more likely driven by changes in demographic rates and less influenced by migration. Thus, we clustered sage-grouse leks across the western United States to develop a multi-scale hierarchical population structure that can guide appropriate conservation and management actions informed from population assessments. We developed range-wide lek clusters (based on O'Donnell and others, 2019), which we used to assess population trends (Objective 3) and develop a TAWS (Objective 4).

\section{Methods}

\section{Data Compilation}

For this spatial clustering objective, we only considered leks with a conservation status of active or pending new (table 1) to develop the hierarchical population monitoring framework using the standardized range-wide lek database (Objective 1). We omitted inactive and historic leks because their current habitat represented in spatial data may not have accurately represented previous habitat conditions during past use by lekking sage-grouse. However, the clusters captured leks of all conservation status and the cluster polygons appropriately grouped the inactive and historic leks based on inspections of the data (O’Donnell and others, 2019).

\section{Least Cost Path Analysis}

We developed a least-cost minimum spanning tree (LC-MST) from selected lek locations to inform the development of a hierarchical population monitoring framework. A least-cost path represents a path with the smallest cost of moving between two areas (Etherington, 2016). We created the LC-MST using a resistance surface that reflected the cost of sage-grouse movement across the landscape and between lekking sites. The resistance surface included gradients of habitat suitability, where larger resistance values penalized movements relative to smaller values (Liu and others, 2018). The resistance surface was composed of terrain ruggedness, tree canopy cover, water bodies greater than or equal to 5,120 acres, a digital elevation model, and sagebrush fractional cover (see table 2 for data source references). We used elevation as a baseline for all pixel values and accumulated penalties by assigning larger values to pixels with greater ruggedness, tree canopy cover, and water bodies that would restrict movement. The inclusion of large water bodies (approximated at $>5,120$ acres) was intended to capture reservoirs with higher human activity that may have increased associations with predators such as ravens and other affiliated disturbances (for example, boat ramps, campgrounds, and garbage). We then developed least-cost paths (Etherington, 2016), which represented a distance-weighted minimum cost of moving through an n-dimensional cost array (resistance surface). Therefore, the cost paths were informed from distances between each lek and all neighboring leks, as well as the cost to travel across the landscape (resistance surface). The resulting paths defined a collection of spanning trees (graphs) where we assigned the cost of travel to each edge of the graph. We then derived the total LC-MST of all spanning trees using the Prim pruning algorithm (Prim, 1957). We worked with state members of the Tech Team to review the LC-MST to ensure it properly represented the known movement behavior of sage-grouse. We modified 13 paths from the fully connected LC-MST based on state-specific comments.

\section{Clustering Algorithm}

We used a graph-based clustering algorithm (Spatial "K"luster Analysis by Tree Edge Removal; SKATER; AssunÇão and others, 2006) in program R (R Development Core Team, 2017) to cluster leks. The algorithm was informed from the LC-MST, habitat covariates at multiple scales surrounding leks, and constraint-based rules. The candidate habitat characteristics (covariates) included shrubland fractional cover, topographic indices, and bioclimatic variables at multiple scales (table 2). The constraint-based rules accounted for varying movement distances observed from sage-grouse studies, interstate highways obstructions due to high traffic volumes (table 3 ) and incrementing numbers of leks grouped per cluster level (table 4). The constraint-based rules informed five tiers of LC-MSTs (table 3), which helped define a spatially balanced framework. Using the fully connected LC-MST and constraint-based rules, we developed graphs for each tier, which were composed of subgraphs. This required removing edges from the LC-MST that did not adhere to the constraint-based rules for a specific tier. Each of the five different tiers of graphs informed clustering rules for two cluster levels, and each cluster level used an increasing number of leks. 
Table 2. Candidate habitat covariates identified for the Spatial "K"luster Analysis by Tree Edge Removal clustering algorithm to develop a greater sage-grouse (Centrocercus urophasianus) hierarchical population monitoring framework of lekking locations (historic to 2019). For moving windows, cells denoted as not applicable (NA) were not developed, and cells marked with an " $x$ " were created using the specified scale.

[For moving windows, cells denoted as not applicable (NA) were not developed, and cells marked with an " $\mathrm{x}$ " were created using the specified scale. Abbreviations: $\mathrm{m}$, meter; - , no data; ${ }^{\circ} \mathrm{C}$, degrees Celsius]

\begin{tabular}{|c|c|c|c|c|c|c|c|c|c|c|c|c|c|}
\hline \multirow[t]{2}{*}{ Covariate } & \multirow[t]{2}{*}{ Ground date } & \multirow{2}{*}{$\begin{array}{l}\text { Source spatial } \\
\text { resolution } \\
\text { (m) }\end{array}$} & \multicolumn{4}{|c|}{$\begin{array}{l}\text { Moving window dimensions } \\
\text { (cells) }\end{array}$} & \multicolumn{7}{|c|}{$\begin{array}{l}\text { Moving window dimensions } \\
\text { (radius meters) }\end{array}$} \\
\hline & & & Cell & 3-cell & 10-cell & 25-cell & 500 & 1,000 & 1,500 & 2,200 & 3,000 & 4,700 & 6,400 \\
\hline Digital elevation model (DEM) ${ }^{1}$ & Current data & 10 & $\mathrm{x}$ & NA & NA & NA & NA & NA & NA & NA & NA & NA & NA \\
\hline Hydrologically corrected DEM² & Current data & 10 & $\mathrm{x}$ & NA & NA & NA & $\mathrm{x}$ & $\mathrm{x}$ & $\mathrm{x}$ & $\mathrm{x}$ & $\mathrm{x}$ & $\mathrm{x}$ & $\mathrm{x}$ \\
\hline Compound topographic index weighted by annual precipitation ${ }^{3}$ & Current data & 10 & NA & $\mathrm{x}$ & NA & NA & $\mathrm{x}$ & $\mathrm{x}$ & $\mathrm{x}$ & $\mathrm{x}$ & $\mathrm{x}$ & $\mathrm{x}$ & $\mathrm{x}$ \\
\hline Heat load index ${ }^{4}$ & Current data & 10 & $\mathrm{x}$ & NA & NA & NA & $\mathrm{x}$ & $\mathrm{x}$ & $\mathrm{x}$ & $\mathrm{x}$ & $\mathrm{x}$ & $\mathrm{x}$ & $\mathrm{x}$ \\
\hline Topographic position index 5 & Current data & 10 & NA & $\mathrm{x}$ & $\mathrm{x}$ & $\mathrm{x}$ & $\mathrm{x}$ & $\mathrm{x}$ & $\mathrm{x}$ & $\mathrm{x}$ & $\mathrm{x}$ & $\mathrm{x}$ & $\mathrm{x}$ \\
\hline Vector ruggedness index ${ }^{6}$ & Current data & 10 & NA & NA & NA & NA & $\mathrm{x}$ & $\mathrm{x}$ & $\mathrm{x}$ & $\mathrm{x}$ & $\mathrm{x}$ & $\mathrm{x}$ & $\mathrm{x}$ \\
\hline Percent cover bare ground 7 & 2015,2016 & 30 & $\mathrm{x}$ & NA & NA & NA & $\mathrm{x}$ & $\mathrm{x}$ & $\mathrm{x}$ & $\mathrm{x}$ & $\mathrm{x}$ & $\mathrm{x}$ & $\mathrm{x}$ \\
\hline Percent cover big sagebrush ${ }^{7}$ & 2015,2016 & 30 & $\mathrm{x}$ & NA & NA & NA & $\mathrm{x}$ & $\mathrm{x}$ & $\mathrm{x}$ & $\mathrm{x}$ & $\mathrm{x}$ & $\mathrm{x}$ & $\mathrm{x}$ \\
\hline Percent cover herbaceous 7 & 2015,2016 & 30 & $\mathrm{x}$ & NA & NA & NA & $\mathrm{x}$ & $\mathrm{x}$ & $\mathrm{x}$ & $\mathrm{x}$ & $\mathrm{x}$ & $\mathrm{x}$ & $\mathrm{x}$ \\
\hline Percent cover all sagebrush ${ }^{7}$ & 2015,2016 & 30 & $\mathrm{x}$ & NA & NA & NA & $\mathrm{x}$ & $\mathrm{x}$ & $\mathrm{x}$ & $\mathrm{x}$ & $\mathrm{x}$ & $\mathrm{x}$ & $\mathrm{x}$ \\
\hline Percent cover sagebrush height ${ }^{7}$ & 2015,2016 & 30 & $\mathrm{x}$ & NA & NA & NA & $\mathrm{x}$ & $\mathrm{x}$ & $\mathrm{x}$ & $\mathrm{x}$ & $\mathrm{x}$ & $\mathrm{x}$ & $\mathrm{x}$ \\
\hline Percent cover all shrub 7 & 2015,2016 & 30 & $\mathrm{x}$ & NA & NA & NA & $\mathrm{x}$ & $\mathrm{x}$ & $\mathrm{x}$ & $\mathrm{x}$ & $\mathrm{x}$ & $\mathrm{x}$ & $\mathrm{x}$ \\
\hline Percent cover shrub height ${ }^{7}$ & 2015,2016 & 30 & $\mathrm{x}$ & NA & NA & NA & $\mathrm{x}$ & $\mathrm{x}$ & $\mathrm{x}$ & $\mathrm{x}$ & $\mathrm{x}$ & $\mathrm{x}$ & $\mathrm{x}$ \\
\hline Percent non-big (other) sagebrush ${ }^{8}$ & 2015,2016 & 30 & $\mathrm{x}$ & NA & NA & NA & $\mathrm{x}$ & $\mathrm{x}$ & $\mathrm{x}$ & $\mathrm{x}$ & $\mathrm{x}$ & $\mathrm{x}$ & $\mathrm{x}$ \\
\hline Percent non-sagebrush shrub ${ }^{8}$ & 2015,2016 & 30 & $\mathrm{x}$ & NA & NA & NA & $\mathrm{x}$ & $\mathrm{x}$ & $\mathrm{x}$ & $\mathrm{x}$ & $\mathrm{x}$ & $\mathrm{x}$ & $\mathrm{x}$ \\
\hline Percent perennial grass ${ }^{8}$ & - & 30 & $\mathrm{x}$ & NA & NA & NA & $\mathrm{x}$ & $\mathrm{x}$ & $\mathrm{x}$ & $\mathrm{x}$ & $\mathrm{x}$ & $\mathrm{x}$ & $\mathrm{x}$ \\
\hline Bio1: The annual mean temperature $\left({ }^{\circ} \mathrm{C}\right)^{9}$ & $1981-2010$ & 800 & NA & NA & NA & NA & NA & $\mathrm{x}$ & $\mathrm{x}$ & $\mathrm{x}$ & $\mathrm{x}$ & $\mathrm{x}$ & $\mathrm{x}$ \\
\hline Bio8: Mean temperature $\left({ }^{\circ} \mathrm{C}\right)$ of wettest season ${ }^{9}$ & $1981-2010$ & 800 & NA & NA & NA & NA & NA & $\mathrm{x}$ & $\mathrm{x}$ & $\mathrm{x}$ & $\mathrm{x}$ & $\mathrm{x}$ & $\mathrm{x}$ \\
\hline Bio10: Mean temperature $\left({ }^{\circ} \mathrm{C}\right)$ of warmest quarter 9 & $1981-2010$ & 800 & NA & NA & NA & NA & NA & $\mathrm{x}$ & $\mathrm{x}$ & $\mathrm{x}$ & $\mathrm{x}$ & $\mathrm{x}$ & $\mathrm{x}$ \\
\hline Bio12: Annual precipitation totals & $1981-2010$ & 800 & NA & NA & NA & NA & NA & $\mathrm{x}$ & $\mathrm{x}$ & $\mathrm{x}$ & $\mathrm{x}$ & $\mathrm{x}$ & $\mathrm{x}$ \\
\hline Bio15: Precipitation seasonality 9 & 1981-2010 & 800 & NA & NA & NA & NA & NA & $\mathrm{x}$ & $\mathrm{x}$ & $\mathrm{x}$ & $\mathrm{x}$ & $\mathrm{x}$ & $\mathrm{x}$ \\
\hline
\end{tabular}

1Digital elevation model (DEM; U.S. Geological Survey, 2018).

${ }^{2}$ Hydrologically corrected DEM derived using methods and software defined by Soille (2004; v. 1.5.1).

${ }^{3}$ Compound topographic index (Gessler and others, 1995) weighted with 30-year climate normal (PRISM Climate Group Oregon State University, 2015).

${ }_{4}^{4}$ Heat load index (McCune and Keon, 2002) equation 3 for mid-latitudes.

5Topographic position index (Weiss, 2001).

${ }^{6}$ Vector ruggedness measure defined by Sappington and others (2007).

${ }^{7}$ Shrubland (Rigge and others, 2020).

${ }^{8}$ Derived from shrubland data products.

9Bioclimatic variables based on methods from O'Donnell and Ignizio (2012) and 30-year climate normal (PRISM Climate Group Oregon State University, 2015). 
Table 3. Constraint-based rules used to inform clustering of greater sage-grouse (Centrocercus urophasianus) lek locations based on distance rules and landscape features (resistance rule). The landscape features included annual average daily traffic (AADT). We defined multiple rules based on sage-grouse movement distances and features on the landscape that attenuate movements to inform least-cost minimum spanning trees.

[ID, identification; $\geq$, greater than or equal to; km, kilometer; >, greater than; NW, northwest; CO, Colorado; ID, Idaho, MT, Montana; ND, North Dakota; $\mathrm{SD}$, South Dakota; , approximately; <, less than; kV, kilovolt; +, plus]

\begin{tabular}{|c|c|c|c|}
\hline ID & Rule & Description and supporting information & $\begin{array}{l}\text { Count of } \\
\text { dropped edges }\end{array}$ \\
\hline \multicolumn{4}{|c|}{ Distance rule } \\
\hline 2 & $\geq 30 \mathrm{~km}$ & Seasonal movements in MT > 20 km (Tack and others, 2012) and NW CO (Dunn and Braun, 1986). & 44 \\
\hline 3 & $\geq 50 \mathrm{~km}$ & $\begin{array}{l}\text { Breeding dispersals in ID, MT, ND, SD and Alberta } \sim 50 \mathrm{~km} \text { (Cross and others, 2017). Individual } \\
\text { movements of } 50 \mathrm{~km} \text { to winter habitat in }<2 \text { days (Carpenter and others, 2010). }\end{array}$ & 12 \\
\hline \multicolumn{4}{|c|}{ Resistance rule } \\
\hline 5 & Edge intersection & $\begin{array}{l}\geq 4,000-7,000 \mathrm{AADT}: \text { these transportation routes frequently co-exist with } 220 \mathrm{kV} \text { transmission } \\
\text { lines }(\geq 220 \mathrm{kV}), \text { agriculture, and other disturbance types. }\end{array}$ & 50 \\
\hline & Tier 1 (level 1,2) & Incorporates rules 1,5 , and 6. & 331 \\
\hline & Tier $2($ level 3,4$)$ & Incorporates rules 2,5 , and 6. & 64 \\
\hline & Tier $3($ level 5, 6) & Incorporates rules 3 and 6. & 35 \\
\hline & Tier 4 (level 7,8) & Incorporates rules 4. & 10 \\
\hline & Tier 5 (level 9+) & Incorporates no rules (fully connected graph). & 3 \\
\hline & & Total edge count & $5,831(5,828)^{1}$ \\
\hline
\end{tabular}

1Remaining edges after isolating Washington, Bi-state (California-Nevada), and Jackson Hole due to genetic isolation.

Table 4. Constraint-based rules used to inform range-wide clustering of greater sage-grouse (Centrocercus urophasianus) lek locations based on a range of leks to group per cluster level.

\begin{tabular}{cc}
\hline Cluster levels & Range of leks \\
\hline 1 & $10-20$ \\
2 & $20-30$ \\
3 & $30-45$ \\
4 & $45-65$ \\
5 & $65-90$ \\
6 & $90-120$ \\
7 & $120-155$ \\
8 & $155-205$ \\
9 & $205-245$ \\
10 & $245-305$ \\
11 & $305-445$ \\
12 & $445-705$ \\
13 & $705-1,245$ \\
\hline
\end{tabular}

The SKATER algorithm used a LC-MST graph, with a cost to travel assigned to each edge, leks with associated habitat covariates, and constraints on number of leks to group. These three factors used in the algorithm maximized similarities of leks within each group and maximized dissimilarities among groupings of leks. We aggregated leks using an agglomerative clustering approach (smallest populations to largest populations) until we reached eight groupings of populations across the range, which we believed accurately captured the large-scale population structure. We intentionally isolated three of the eight populations (Bi-state region in Nevada/California, the state of Washington, and Jackson Hole in Wyoming) because of known genetic isolation (Oyler-McCance and others, 2005; Schulwitz and others, 2014). We reviewed all clustering results with state wildlife representatives of the Tech Team. Further details for developing the sage-grouse hierarchical population monitoring framework, and associated software, are described in O'Donnell and others (unpub. data, 2020). 


\section{Selecting Cluster Levels to Support Population Modeling}

We evaluated each cluster level using data collected from radio-marked sage-grouse to determine the smallest clusters that we could use for assessing "fine scale" population trends (NC level; Objective 3) and a TAWS (Objective 4). The selection of a NC reflected the smallest cluster level that could represent a closed population unit that minimizes immigration and emigration. Movements of sage-grouse between leks vary geographically and by age (Wann and others, 2019), and we accounted for these movements by incorporating distance thresholds (15-km inter-lek movement distance) applied to cluster level 2 (see the "Methods" section in O'Donnell and others, 2019). Identification of small clusters (polygons) also was important for supporting land-management actions that could occur at reasonable geographic extents with fewer leks. We included data from independent research studies (see the "Acknowledgments" section for data providers) across sage-grouse range that used both very high frequency (VHF) and global positioning systems (GPS) devices to maximize the sample size (fig. 3). The GPS and VHF data included both males and females and varying ages (juvenile [hatch year], yearling [second year], and adult [greater than second year]). The temporal frequency for GPS collections varied among and within studies, but when provided or known, they ranged between 1 and 3 hours. We assessed all locations based on a biological year beginning at breeding season (March 1). For evaluating clusters with VHF and GPS data, we defined a home cluster for each bird and biological year (for example, Bird A locations from March 1, 2012 to March 1, 2013) as the cluster (polygon) that contained the maximum number of locations (VHF) or maximum amount of time (GPS). To evaluate locations identified with VHF telemetry, we calculated the proportion of locations of each bird falling outside its home cluster, which we assessed across all biological years and for each cluster level. For evaluating clusters with GPS data, we used the dynamic Brownian bridge movement model (dBBMM; Kranstauber and others, 2012) in program R (R Development Core Team, 2018) with library move (function move and brownian.bridge.dyn; Kranstauber and others, 2012). The dBBMM defined the home range (utilization distribution [UD]) by accounting for the temporal information of the GPS data and allowing the bridge to expand and contract based on the length of time between successive locations and the Brownian motion variance. Like the VHF evaluation, we assessed GPS dBBMM UD across all biological years and for each cluster level to assess the amount of time birds spent outside their home cluster.

Our objective in selecting which level of cluster should represent a CC (broad scale) was to maximize the magnitude of the relationship between the population rates of change at the lek level and a climate variable (for example, precipitation) measured at a larger spatial extent. To accomplish this goal, we first needed to select a climate variable that had been linked to population performance across the range. Based on a literature review, we decided that cumulative precipitation during the late brood-rearing period (Blomberg and others, 2014; Gibson and others, 2017; Peebles and others, 2017) would provide strong predictability of population rates of change at the lek level, when summarized at larger spatial extents. In some portions of sage-grouse range, precipitation has demonstrated a negative effect with chick survival, which could be due, in part, to exposure during the early stages of brood-rearing when independent mechanisms for thermoregulation may not have been fully developed (Guttery and others, 2013). For that reason, we chose a precipitation variable (monthly precipitation values; PRISM Climate Group, 2020) that extended beyond the early stages of brood-rearing (June-August) when the cumulative effects of chick exposure were less of a concern. Precipitation also has been shown to have a strong relationship with rangeland productivity (Campbell and others, 1997; Izaurralde and others, 2011), which during late growing seasons can explain the predominantly positive relationship with brood response, given the high degree of herbivory during latter stages of chick development (Blomberg and others, 2013).

To estimate population growth at the lek level, we used the same dataset that was developed for Objective 4. We estimated intrinsic rates of population change from those data using a state-space model (SSM). We fit correlated random slopes and intercepts (Kéry and Schaub, 2012) to the mean hyperparameter within the state process equation of the SSM. The random slope coefficients related the change in abundance from year $t$ to year $t+1$ using the log transformed cumulative precipitation recorded between June and August of year $t$. The number of groups estimated for each random slope and intercept were defined by the cluster level modeled (cluster levels 3-13, with level 2 defining the NC), with the greatest number of groups estimated for cluster level three, and the fewest number for cluster level 13. A total of 11 models were specified, 1 per cluster level (cluster level 3-13). The log-transformed cumulative precipitation (JuneAugust) value was calculated by cluster level group and year. For example, cluster level 13 had 174 precipitation values calculated based on 6 groups and 29 years. The magnitude of the relationship between population rates of change at the lek level and precipitation at the cluster level were evaluated using the median estimate of the mean hyperparameter of the random slope effect. We removed cluster levels from consideration when the 95 percent credible interval (CRI) of the mean hyperparameter overlapped 0 . We otherwise did not consider the precision of that estimate when selecting the most appropriate level as CC, largely because we assumed that the response varied more substantially as the number of leks in groupings increased. 


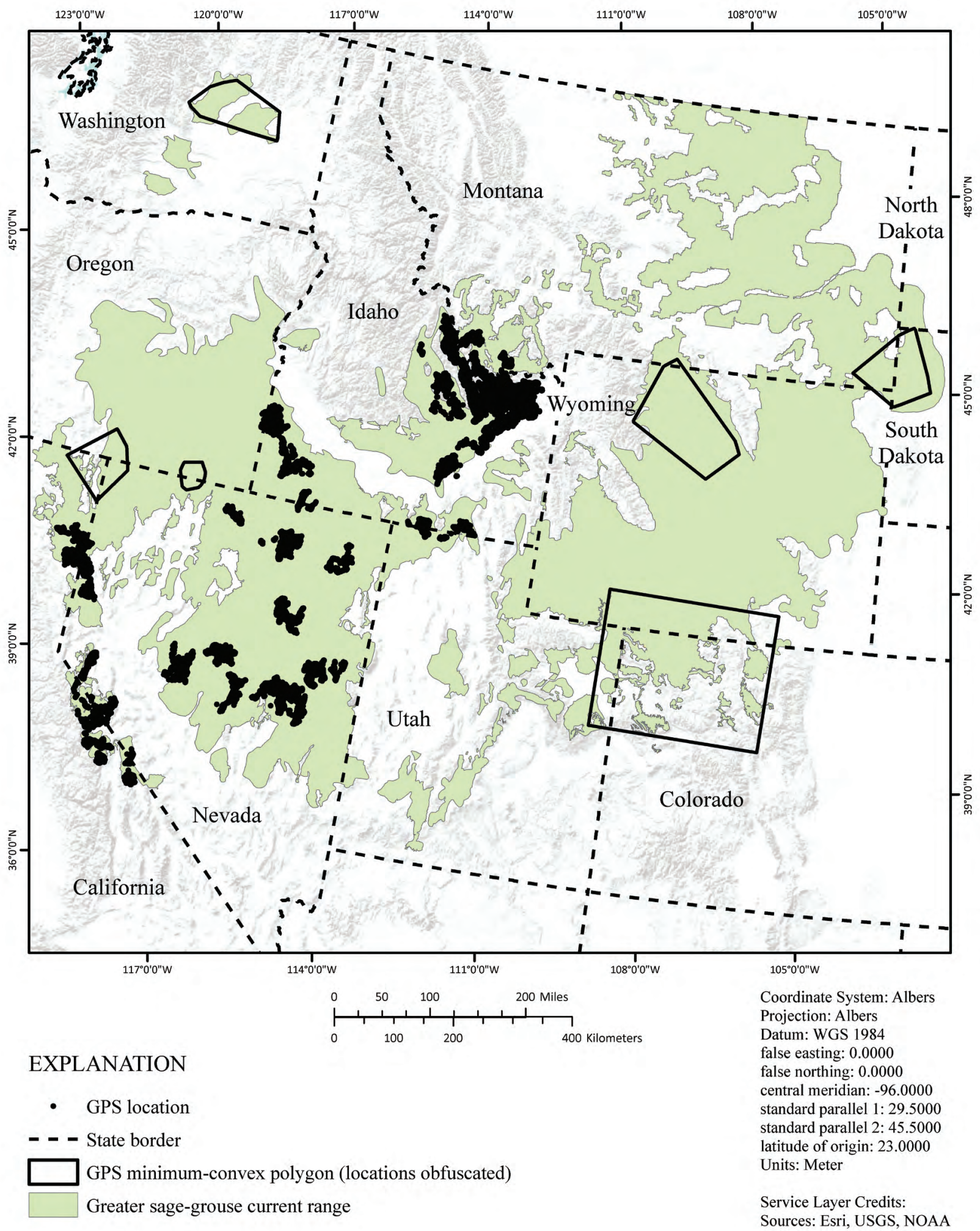

Figure 3. Study locations where greater sage-grouse (Centrocercus urophasianus) research projects (see the "Acknowledgments" section for contributions) were conducted from which we acquired very high frequency (VHF) and global positioning systems (GPS) data. We used the data to evaluate the hierarchical population monitoring framework with respect to selecting an appropriate neighborhood cluster (fine scale). Map image is the intellectual property of Esri and is used herein under license. Copyright (C) 2020 Esri and its licensors. All rights reserved. 


\section{Results}

We produced 13 hierarchically nested cluster levels, each level containing groupings, or populations, of sage-grouse leks. The finest scale level (level 1) represented a conditioning period for the clustering process and was not intended for use in population assessments. With our neighborhood evaluation of the cluster levels, we used 1,551 (GPS) and 1,270 (VHF) unique birds, with collections spanning between 2006 and 2020, and we analyzed 1,685,443 (GPS) and 31,731 (VHF) observations. Using dBBMMs from the GPS data, we found that sage-grouse spent greater than 92 percent of their time within their home cluster for cluster levels 2 through 13 . Similarly, we found greater than 95 percent of sage-grouse occurrence was within clusters 2 through 13 using VHF locations (fig. 4).

Concerning the $\mathrm{CC}$ evaluation, the median value of the mean hyperparameter for the random slope effect was positive for all cluster levels (fig. 5). The magnitude of the relationship between population growth and precipitation had a general and moderate upward trend from cluster levels 3-9, at which point that trend reversed with the magnitude of the relationship decreasing from cluster levels 9-11. Cluster level 13 had the highest median value $(0.652$; 95 -percent $\mathrm{CRI}=0.215-1.050)$ for the mean hyperparameter.

Figures 6 and 7 reflected cluster levels 2 (NC) and 13 (CC), respectively, which we selected for the development of population trend assessments (Objective 3) and a TAWS
(Objective 4). Cluster boundaries were defined based on midpoint distances between peripheral leks between adjacent clusters by developing Thiessen polygons. Clustering algorithms resulted in $11,4,2,169,241$, and $56 \mathrm{NCs}$ within A, $\mathrm{B}, \mathrm{C}, \mathrm{D}, \mathrm{E}$, and F climate cluster, respectively, while the mean (and standard deviation) number of leks per NC by CC was 9 (11.4), 35.5 (26.7), 8.5 (6.5), 20.6 (19.3), 18.5 (17.9), and 28.8 (16.8), respectively. Climate clusters A-C reflect genetic clusters but also identify with distinct characteristics. Jackson Hole, Wyoming, area (CC-C) and Bi-State area (CC-A) are both at higher elevations. Climate cluster $\mathrm{C}$ included leks at higher elevations isolated by distance and surrounded by significantly dense forests. Washington area (CC-B) is isolated by distance (greater than $200 \mathrm{~km}$ ) and lacked intact corridors of connected habitats with Oregon. The populations occurred on the leeward side of the northern Cascades above the Columbia River and have substantial anthropogenic and natural barriers further isolating it from Oregon populations. Southwestern Wyoming, which we refer to as the western Wyoming area (CC-F), represented a high-elevation plateau relative to eastern Wyoming, which was located within the eastern area (CC-D). Climate cluster D captured the transition into grasslands and reflected the west-to-east zones of precipitation (PRISM Climate Group Oregon State University, 2015). Figures $8-15$ provide labels of NCs by each CC that correspond with tables of trends, watches, and warnings described in Objectives 3 and 4. 


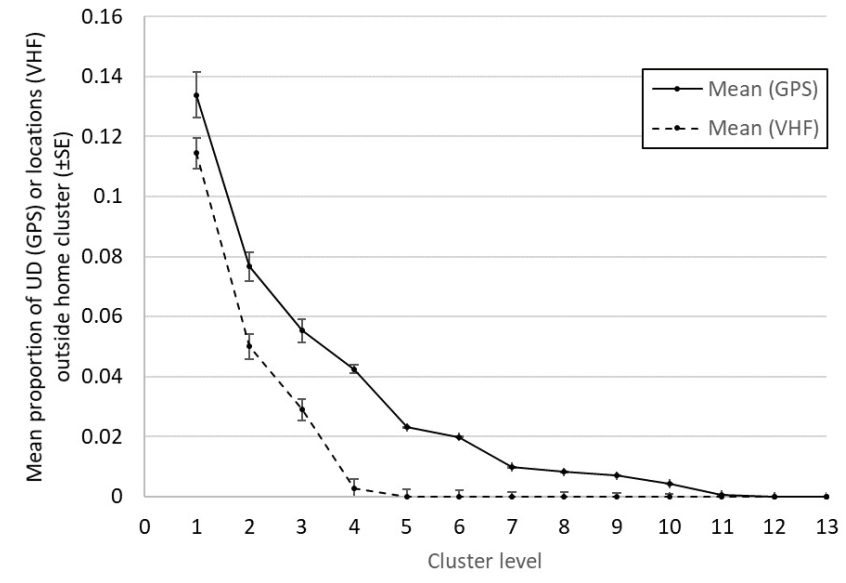

Figure 4. Results from evaluating greater sage-grouse (Centrocercus urophasianus) hierarchical population monitoring framework across different nested population clusters (levels 1-13) using very high frequency (VHF) and global positioning systems (GPS) data from multiple research studies across the sage-grouse range (see the "Acknowledgments" section for contributions). Our assessment of sage-grouse movements applied to individual birds by biological year (March 1-May 31). We evaluated VHF data by calculating the proportion of a bird's locations falling outside its home cluster for each cluster level and biological year. We evaluated GPS data by using the dynamic Brownian bridge movement model to calculate a bird's utilization distribution (UD), which is used to calculate the proportion of time a bird occurred outside its home cluster.

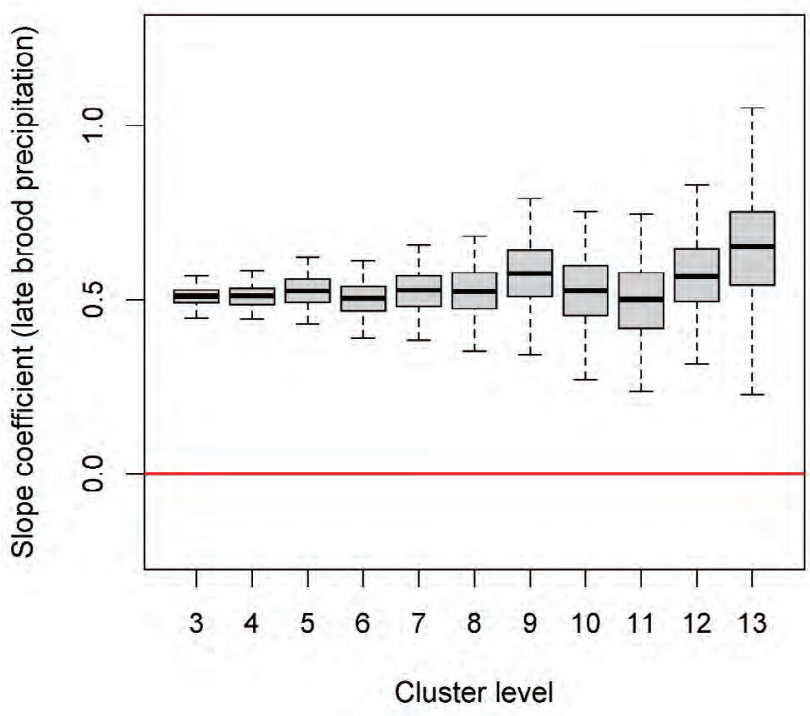

Figure 5. Results from evaluating greater sage-grouse (Centrocercus urophasianus) relationship (slope coefficient) between precipitation during late brood-rearing period (JuneAugust of year $t$ ) and population rate of change (year $t$ to year $t+1$ ) across different nested population clusters (levels $3-13$ ) within a hierarchical monitoring framework. Positive values revealed that increased precipitation leads to population growth and greater values reflect stronger evidence. Cluster level 13 (largest spatial scale) revealed the strongest evidence and credible intervals did not overlap zero. 


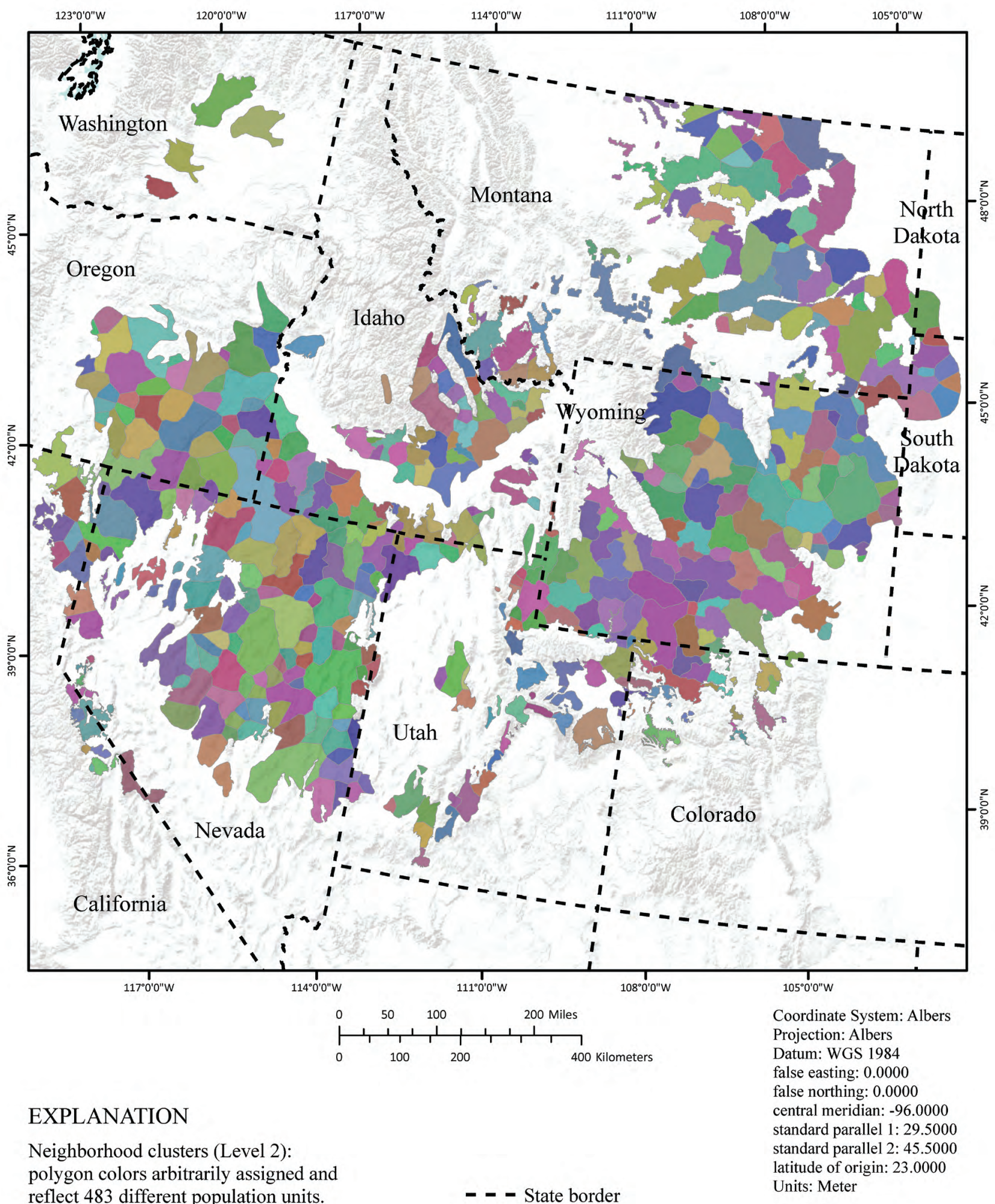

Service Layer Credits:

Sources: Esri, USGS, NOAA

Figure 6. Greater sage-grouse (Centrocercus urophasianus) hierarchical population monitoring framework for neighborhood clusters (level 2) in the western United States. Map image is the intellectual property of Esri and is used herein under license. Copyright $(C 2020$ Esri and its licensors. All rights reserved. 


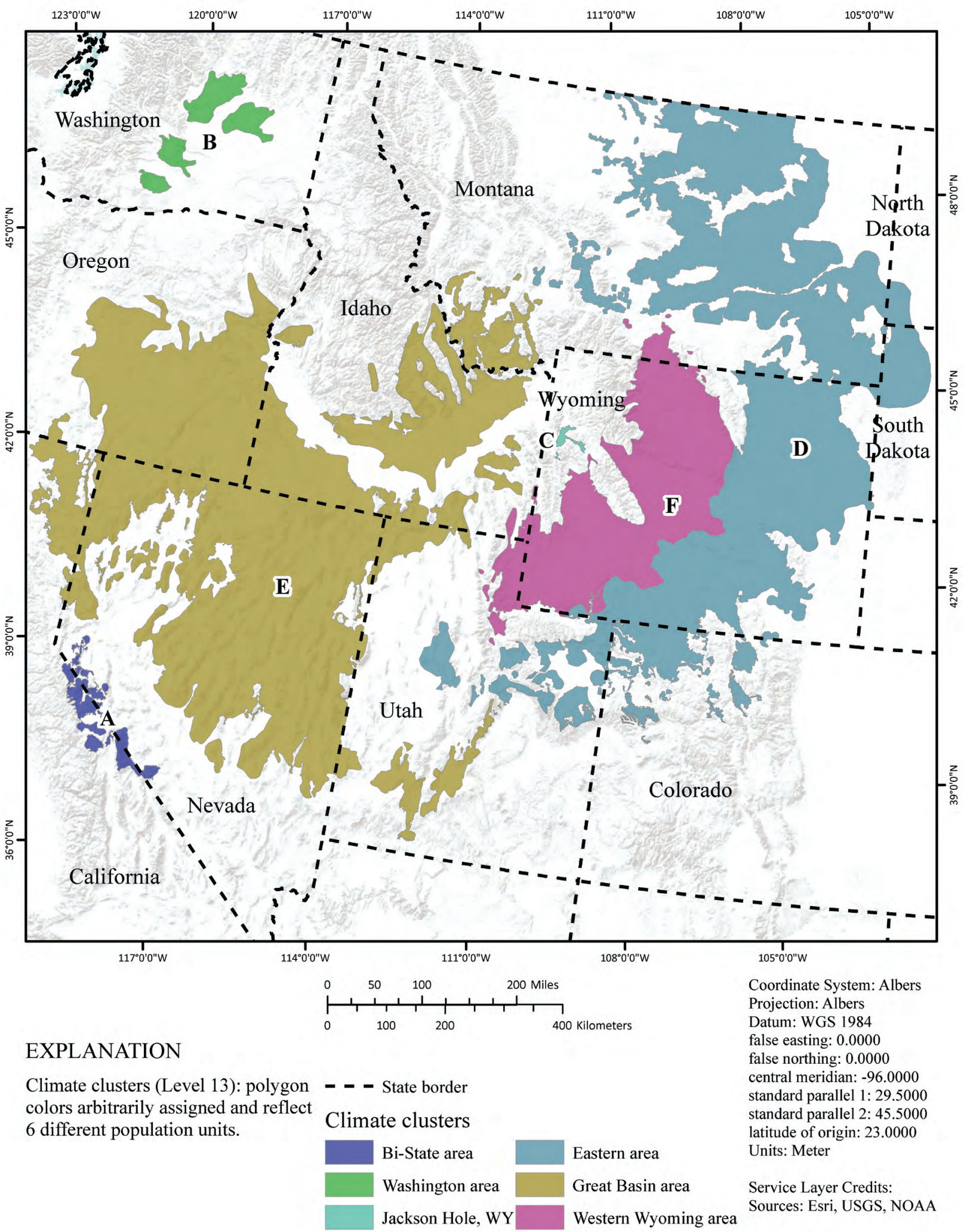

Figure 7. Greater sage-grouse (Centrocercus urophasianus) hierarchical population monitoring framework for climate clusters (level 13) in the western United States. A = Bi-State area, B = Washington area, C = Jackson Hole, Wyoming, area, D = eastern area, $\mathrm{E}=$ Great Basin area, $\mathrm{F}=$ western Wyoming area. Map image is the intellectual property of Esri and is used herein under license. Copyright @ 2020 Esri and its licensors. All rights reserved. 


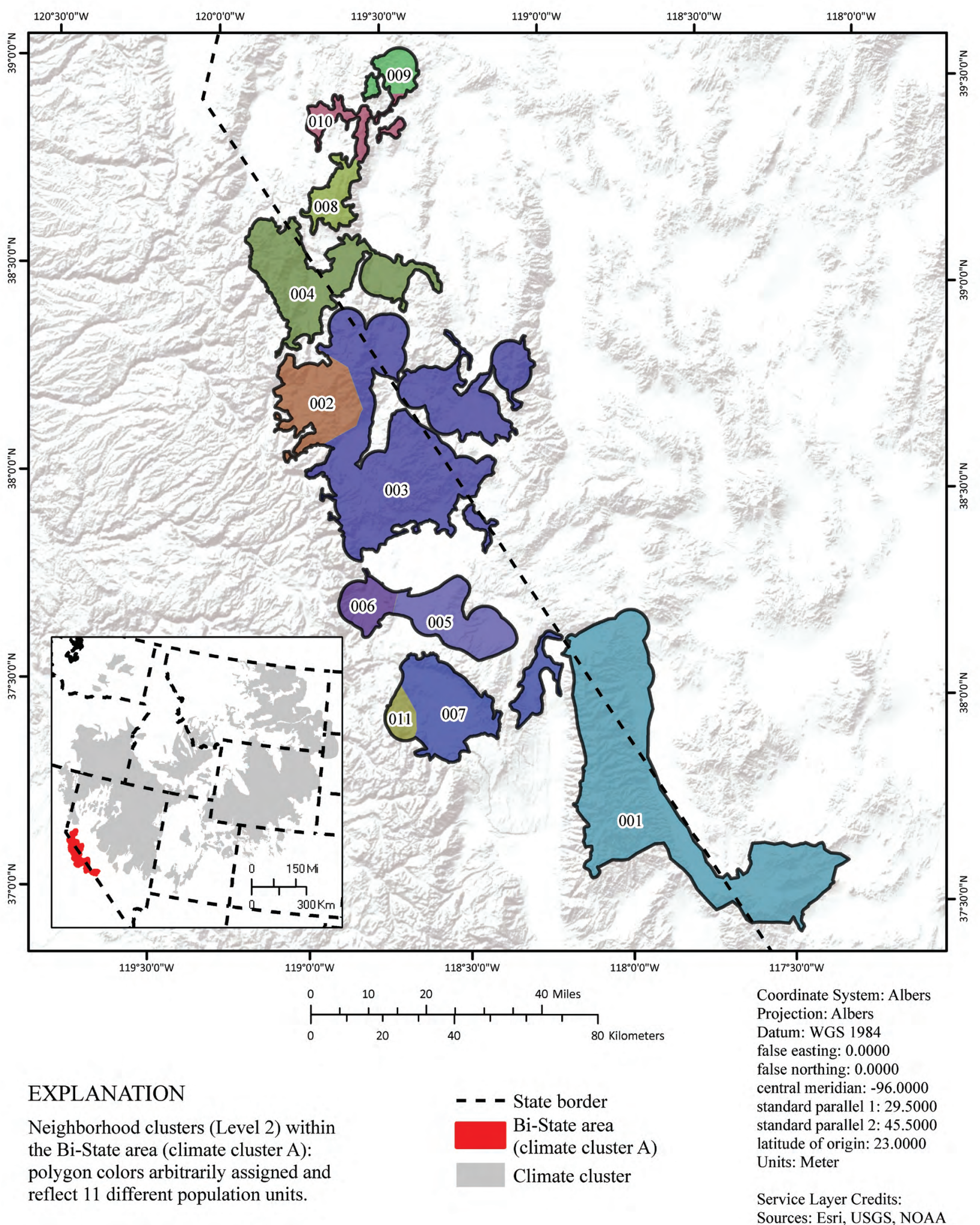

Figure 8. Greater sage-grouse (Centrocercus urophasianus) hierarchical population monitoring framework for neighborhood clusters within climate cluster A (Bi-State area). Three-digit numbers located inside neighborhood cluster boundaries serve as neighborhood cluster identifications. Map image is the intellectual property of Esri and is used herein under license. Copyright () 2020 Esri and its licensors. All rights reserved. 


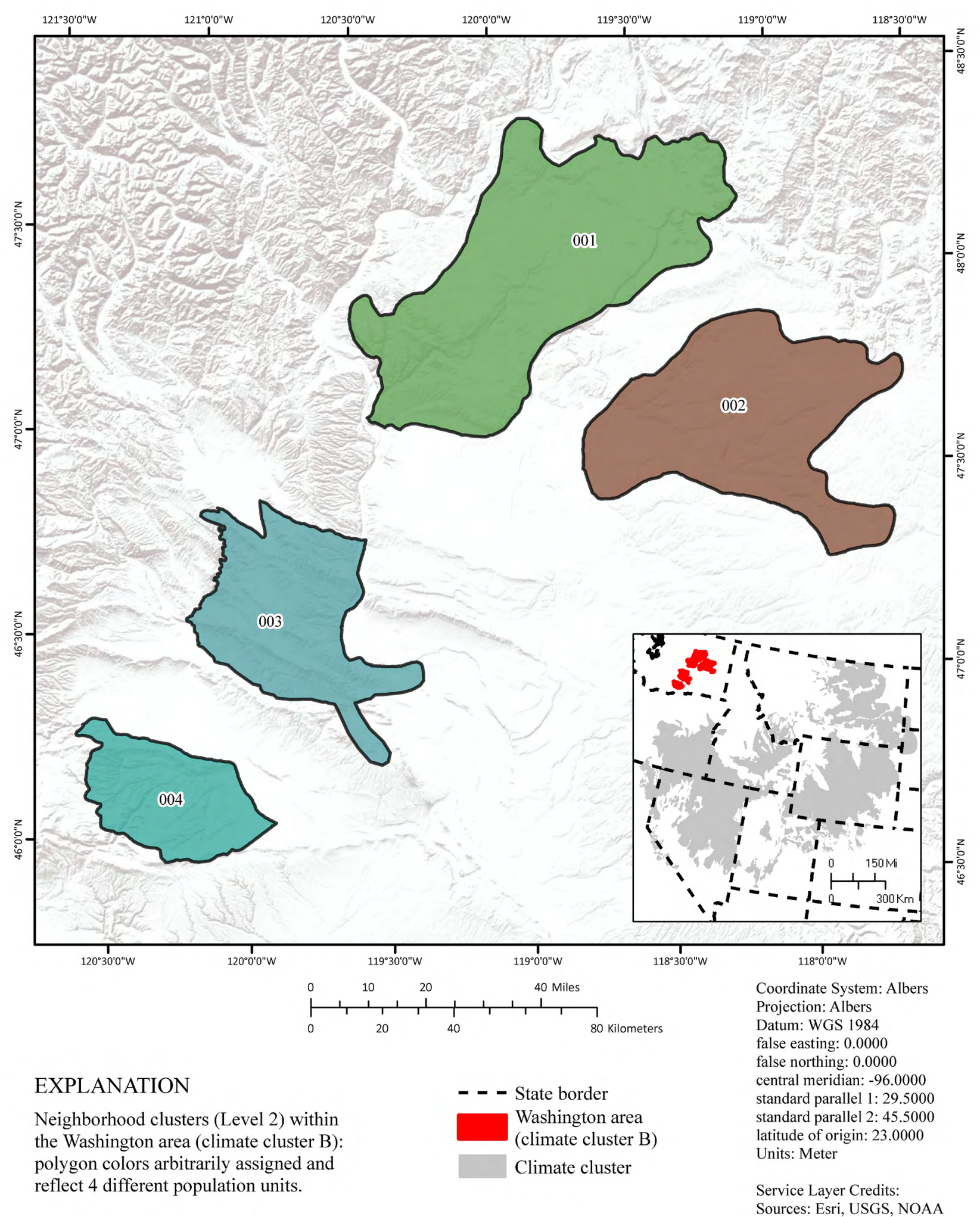

Figure 9. Greater sage-grouse (Centrocercus urophasianus) hierarchical population monitoring framework for neighborhood clusters within climate cluster B (Washington area). Three-digit numbers located inside neighborhood cluster boundaries serve as neighborhood cluster identifications. Map image is the intellectual property of Esri and is used herein under license. Copyright (C) 2020 Esri and its licensors. All rights reserved. 


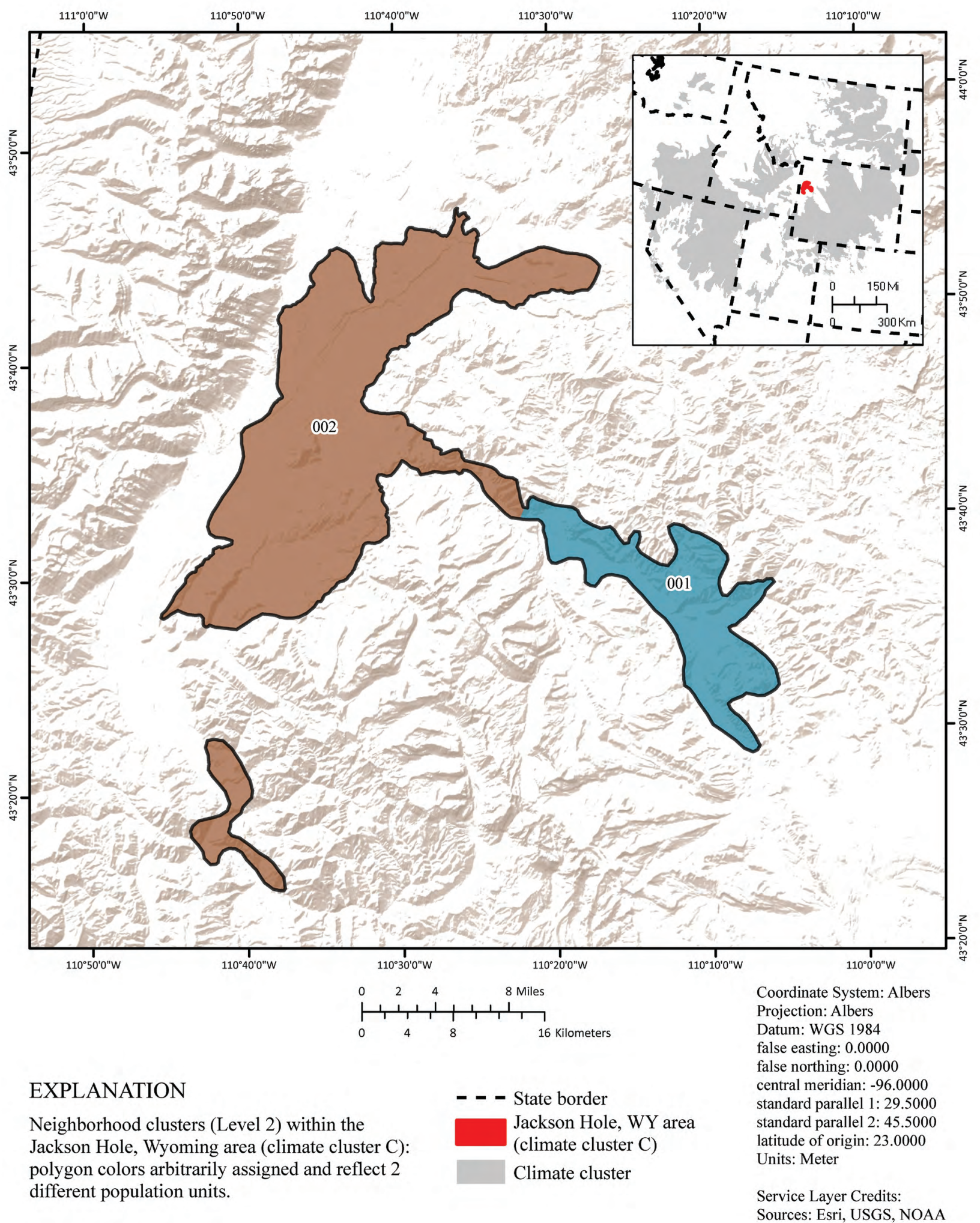

Figure 10. Greater sage-grouse (Centrocercus urophasianus) hierarchical population monitoring framework for neighborhood clusters within climate cluster C (Jackson Hole, Wyoming, area). Three-digit numbers located inside neighborhood cluster boundaries serve as neighborhood cluster identifications. Map image is the intellectual property of Esri and is used herein under license. Copyright (C) 2020 Esri and its licensors. All rights reserved. 


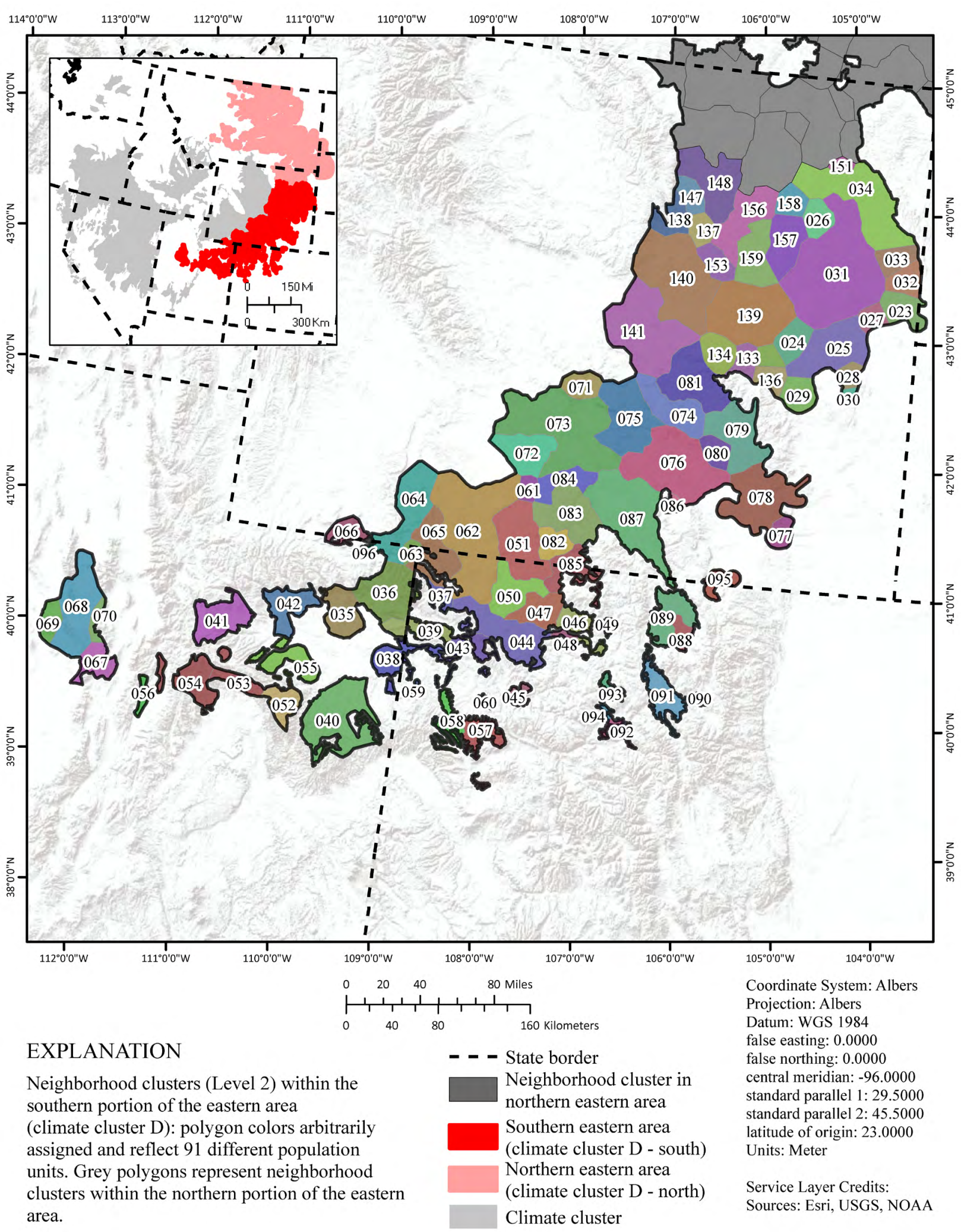

Figure 11. Greater sage-grouse (Centrocercus urophasianus) hierarchical population monitoring framework for neighborhood clusters within the southern section of climate cluster D (eastern area). Three-digit numbers located inside or adjacent to neighborhood cluster boundaries serve as neighborhood cluster identifications. Map image is the intellectual property of Esri and is used herein under license. Copyright () 2020 Esri and its licensors. All rights reserved. 


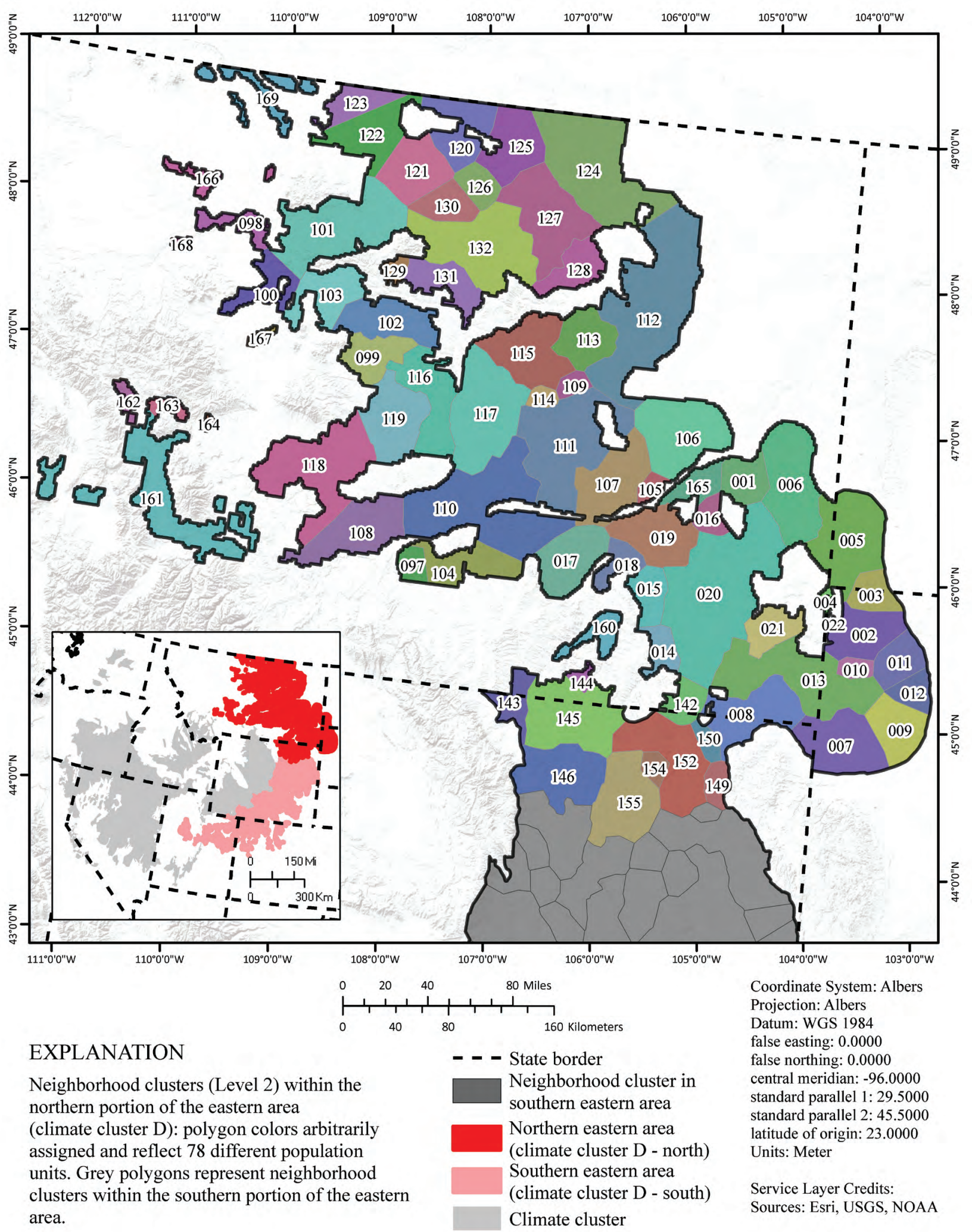

Figure 12. Greater sage-grouse (Centrocercus urophasianus) hierarchical population monitoring framework for neighborhood clusters within the northern section of climate cluster $D$ (eastern area). Three-digit numbers located inside or adjacent to neighborhood cluster boundaries serve as neighborhood cluster identifications. Map image is the intellectual property of Esri and is used herein under license. Copyright (C 2020 Esri and its licensors. All rights reserved. 


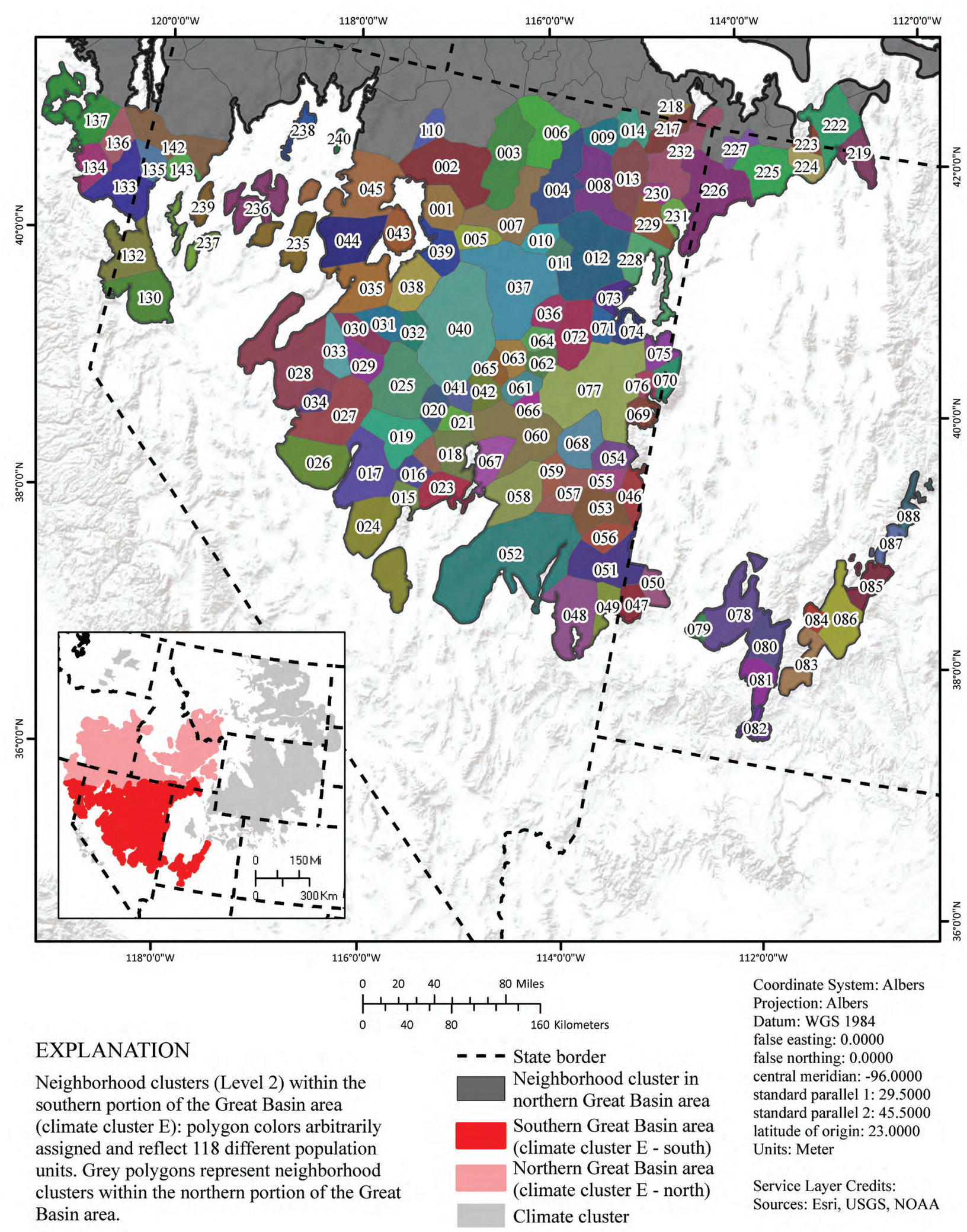

Figure 13. Greater sage-grouse (Centrocercus urophasianus) hierarchical population monitoring framework for neighborhood clusters within the southern section of climate cluster E (Great Basin area). Three-digit numbers located inside or adjacent to neighborhood cluster boundaries serve as neighborhood cluster identifications. Map image is the intellectual property of Esri and is used herein under license. Copyright () 2020 Esri and its licensors. All rights reserved. 


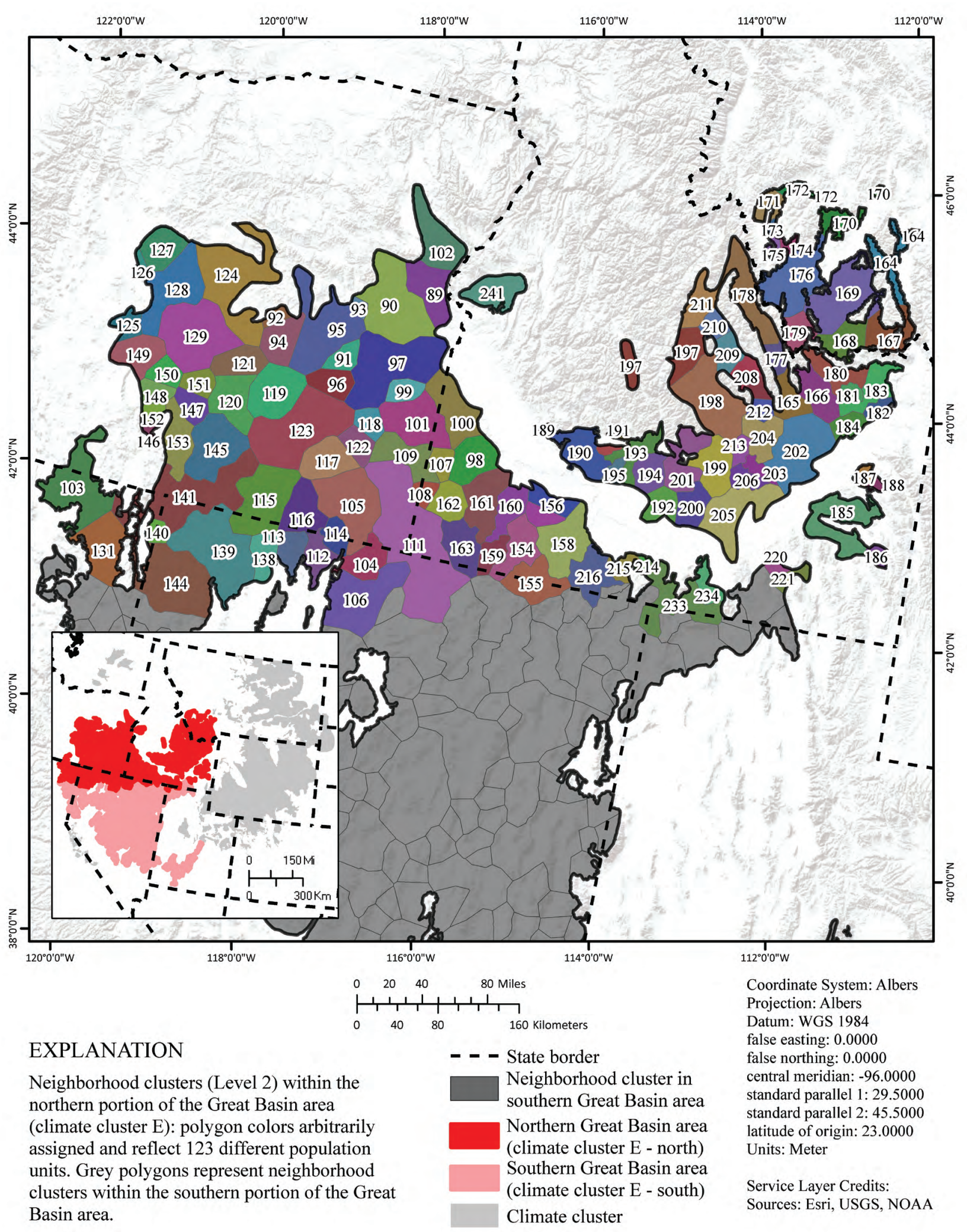

Figure 14. Greater sage-grouse (Centrocercus urophasianus) hierarchical population monitoring framework for neighborhood clusters within the northern section of climate cluster E (Great Basin area). Three-digit numbers located inside or adjacent to neighborhood cluster boundaries serve as neighborhood cluster identifications. Map image is the intellectual property of Esri and is used herein under license. Copyright (C 2020 Esri and its licensors. All rights reserved. 


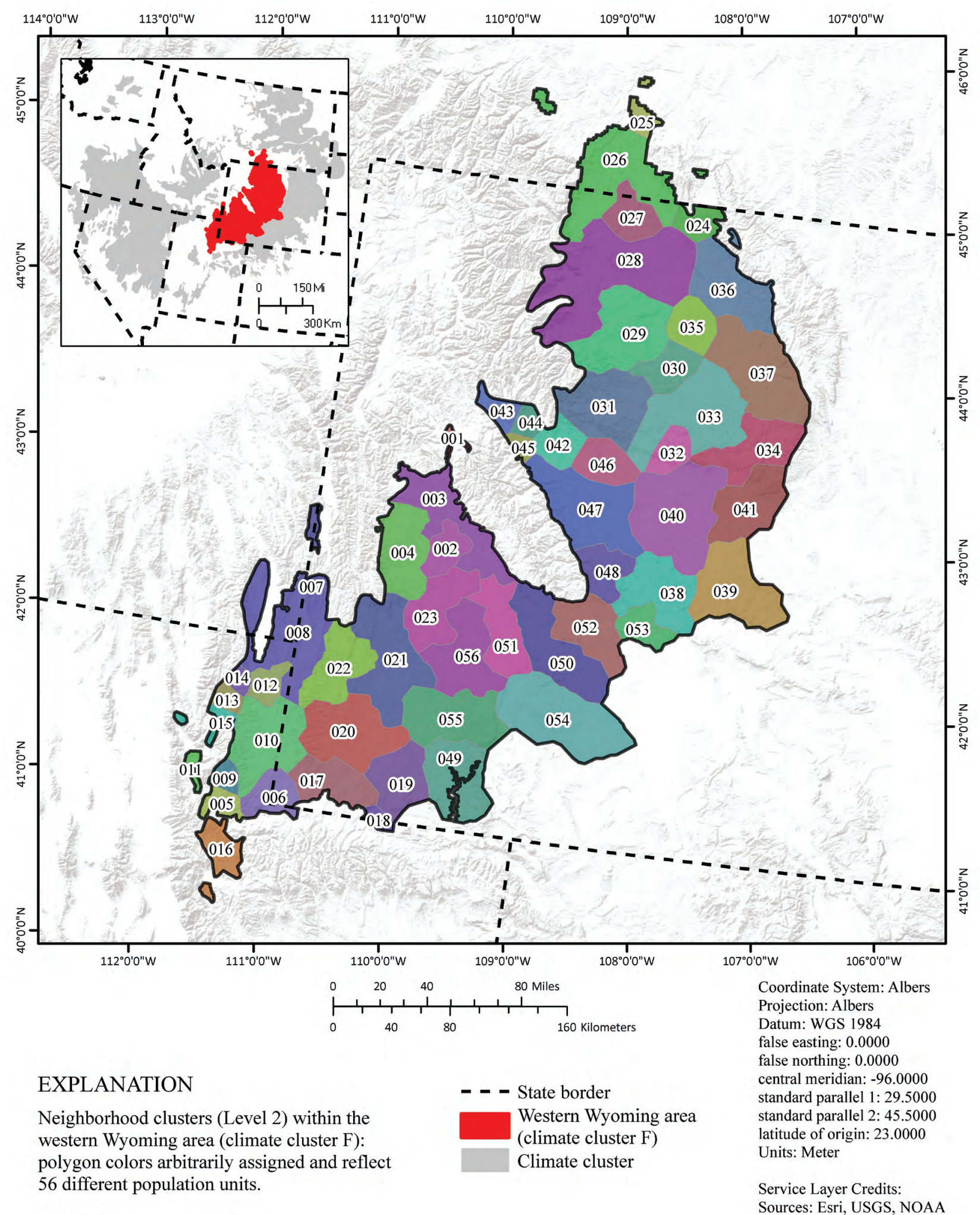

Figure 15. Greater sage-grouse (Centrocercus urophasianus) hierarchical population monitoring framework for neighborhood clusters within climate cluster $\mathrm{F}$ (western Wyoming area). Three-digit numbers located inside or adjacent to neighborhood cluster boundaries serve as neighborhood cluster identifications. Map image is the intellectual property of Esri and is used herein under license. Copyright (C) 2020 Esri and its licensors. All rights reserved. 


\section{Objective 3. Spatiotemporal Patterns of Sage-Grouse Population Abundance Trends}

\section{Background}

A major challenge in estimating long-term trends in population abundance is accounting for environmental stochasticity that often manifests as patterns of oscillation in abundance ( $\hat{N}$; Morris and Doak, 2002), which is typically caused by abiotic (for example, climatic conditions that influence resource availability; Ranta and others, 1995; Lindström and others, 1996) and biotic effects (for example, predator-prey relationships; Archibald, 2014; Blasius and others, 2020). Sage-grouse populations exhibit inter-annual variation in abundance that reflect periods of oscillation (Rich, 1985; Fedy and Aldridge, 2011; Garton and others, 2015; Row and Fedy, 2017) and in some regions can be highly influenced by climatic variation at broader spatial scales. For example, such fluctuations have been linked to climatic conditions such as consecutive years of drought in the Great Basin (Blomberg and others, 2012; Coates and others, 2016, 2018). This leads to upward, downward, or neutral oscillating or cyclic trends (hereafter, for consistency, oscillations) with high and low abundances occurring at varying intervals of 6-12 years (Fedy and Aldridge, 2011; Row and Fedy, 2017). Because sage-grouse occupy large geographic extents and experience a high degree of spatial heterogeneity in biotic and abiotic effects across their range (Doherty and others, 2016), it is reasonable to hypothesize that sage-grouse exhibit regional variability in functional responses to broad scale climatic effects. A population modeling framework that accounts for effects of environmental stochasticity on sage-grouse population abundance could provide managers with more robust trend estimates across different temporal scales.

Given inter-annual variation in sage-grouse population abundance that reflect patterns of oscillation through time, conclusions drawn from modeling approaches of trend can be highly sensitive to start and stop dates that define the temporal scale of inference (fig. 16). For example, failure to account for inter-annual variation can yield significant under- or overestimation of population trajectories using multi-year data (Mathews and others, 2018; Coates and others, 2019a). Inferences based on temporal scales defined by nadirs (troughs) as start and stop points across multiple oscillations, will be less prone to misleading results than those that span nadir to apex or apex to nadir. Estimates of average annual rate of change $(\hat{\lambda})$ in abundance across multiple years also could vary depending on the number of complete oscillations, as well as oscillation period (length of time between nadirs) and amplitude (displacement of nadirs and apexes from the mean; Row and Fedy, 2017). Additionally, populations consistency in annual changes in abundance through time would not require nadir-to-nadir inference approaches because trend estimates are therefore not sensitive to start-stop years. Thus, we restrict inferences to complete oscillations, similar to the "Methods" section in Coates and others (2019a), and we examine population trends using multiple time periods, each containing a different number of complete oscillations during the past 60 years. We used population abundance nadirs, versus apexes, to define start-stop temporal scales of inference because (1) variability in nadirs was lower compared to apexes and thus likely to better represent realized trends and (2) populations are most at risk of local- or broadscale extirpation when abundance reaches a minimum and vulnerabilities to stochastic factors increase (Morris and Doak, 2002; Melbourne and Hastings, 2008); therefore, using nadirs in trend modeling is more conservative than using apexes and provides better insight into population vulnerabilities.

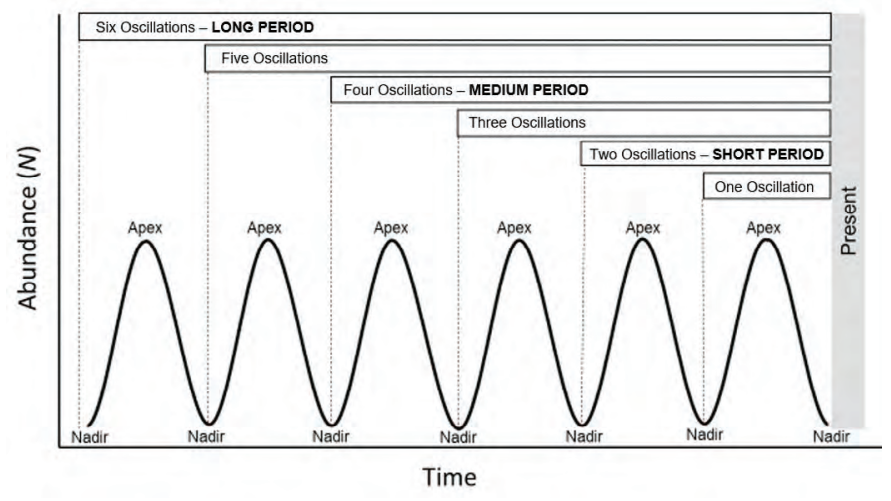

Figure 16. Diagram depicting six complete oscillations from nadir (trough) to nadir for a population that increases and decreases in abundance $(N)$ over time. 
A second substantial challenge facing trend estimation is limitations in time series data associated with individual leks. Although multiple studies have reported population trend estimates at regional and range-wide spatial scales (Garton and others, 2011, 2015; Western Association of Fish and Wildlife Agencies, 2015), shortcomings associated with the exclusion of leks with missing data from irregular count histories can limit inferences. Sampling biases based on increasing efforts in counting leks through time also can mislead apparent trends. Modeling approaches that allow information sharing across aggregations of spatially structured (and thus similar) leks allow finer resolution estimates and avoid potential biases from analyses with a restricted sample of leks. Thus, our goal was to estimate relative abundance where data were missing in a probabilistic manner (that is, impute) using a Markovian process while taking advantage of spatiotemporal relationships by fitting nested random effect structures that better account for variable sampling effort over time, as well as accounting for uncertainty in our lek counts by assuming an underlying observation error. Pairing a range-wide database of lek counts (Objective 1) with a nested population structure (Objective 2) and employing hierarchical state-space models provides a novel framework to estimate population trends at different spatiotemporal scales, and thereby readily and consistently produce empirical population assessments for state and federal resource agencies.

\section{Methods}

\section{Defining Spatial and Temporal Scales}

We grouped sage-grouse lek locations into distinct clusters across hierarchical spatial scales based on similar landscape and climatic factors known to influence spatial connectivity among sage-grouse populations, as described in Objective 2. The resulting spatially nested scales provided a way to partition local, neighborhood, and broad scale population trends. Specifically, the different scales were derived from (1) the single lek, (2) a neighborhood of leks clustered together where sage-grouse movements to surrounding clusters were negligible (NCs, fig. 6), and (3) more regional groups of 2-241 neighborhood clusters that shared similar climatic and vegetation conditions (CCs, fig. 7). The NCs represented population dynamics that are likely governed mostly by births and deaths within a closed biological unit (in other words, local drivers of population change). Thus, NCs were thought of as a network of leks that function as a meta-population, whereas higher-order broad scale clusters are likely to more closely represent dynamics governed by similar climatic and habitat conditions (in other words, regional drivers of population change).

\section{State-Space Model Formulation}

We took advantage of recent advances in ecological models (Kéry and Schaub, 2012) to account for differences in population dynamics across spatial and temporal scales. We developed a Bayesian state-space model (SSM) that relies on Markov chain Monte Carlo (MCMC) sampling using a database of maximum lek counts developed in Objective 1 to estimate the intrinsic rate of population change $(\hat{r}$; Kéry and Schaub, 2012; Green and others, 2017; Monroe and others, 2017). State-space models use a hierarchical structure to separate process variance (that is, environmental flux) from observation error by partitioning each variance component (Kéry and Schaub, 2012). In SSMs, the true abundance state $(N)$ is represented by a first-order Markov process and observed through maximum lek counts. Although these lek count data did not permit direct estimation of detection probability, SSMs can yield an unbiased $\hat{r}$ when detection error is constant or random over time (Monroe and others, 2019).

We fit a SSM to maximum lek count data to estimate trends at leks and across different geographic cluster boundaries (described in Objective 2) using a nested hierarchical modeling framework. This spatially explicit framework allowed a single model to be fit for the entire range-wide dataset with specified nested random effect structures. This spatially nested approach allowed us to derive posterior probability distributions (PD) of $\hat{r}$ for each lek, as well as higher-order spatial extents (in other words, $\mathrm{NC}$ and $\mathrm{CC}$ scales), during each year of the time series. Specifically, leks were nested within NCs and NCs were nested within CCs. The nested hierarchical model structure permitted the sharing of information among lower levels within higher-order delineations to inform estimation of trends in years with missing count data, facilitating analysis of numerous leks with sporadic count histories. Due to the hierarchical structure of the model and its inherent information sharing properties, we make an important assumption about how to deal with historic and inactive leks, which were those that were confirmed absent and did not receive visits/counts in subsequent years. For leks that exhibited such count histories, we adopted similar assumptions as those made by state agencies regarding their classification of historic and inactive. Specifically, leks with observations of zero for greater than or equal to 2 years and listed as inactive or historic (for instance, missing values for remainder of time series) received zero values for the remainder of the count history. Populating that specific type of missing value with zero values was a numerical extension of the assumption made by state agencies to cease sampling at those leks. This allowed for the inclusion of leks that were extirpated, such that monitoring had concluded, and helped reduce sampling bias in estimating $\hat{r}$. The full model specification is reported in appendix 3. Rules that were applied to the full database to meet criteria for samples to be used in the SSM model were described in Objective 1 and final samples sizes are reported in appendix 2 . 
We used annual median abundance estimates $(\hat{N}$ ) from the SSM to visually identify abundance nadirs along the time series aggregated at the range-wide and CC spatial scales. These nadirs were then used to define the different temporal scales for population trend inference. We did not define nadirs at the NC scale for two reasons: (1) CCs more accurately represent nadirs from oscillations governed by precipitation (see Objective 2) and (2) NCs did not always possess enough samples (for example, NCs with fewer than two leks). However, where data were robust at the NC scale, inspection of trends aligned well with those at the CC scale. Furthermore, evidence of associations between precipitation and changes in population abundance was compelling at the NC scale (see Objective 2). Thus, we conclude that NCs typically track CCs in their trends because factors driving oscillations in abundance operate at broad spatial scales. We did not define nadirs at other boundaries, such as states, because they were not biologically based. We averaged posteriors of $\hat{r}$ across each temporal scale (nadir year to 2019) and transformed those values to finite rate of change $\hat{\lambda}=\exp (\hat{r})$. It is important to note that we assumed 2019 represented a population nadir. However, it is possible that relative abundance decreases further in subsequent years. The two possible scenarios are (1) $\hat{N}$ decreases further during the current oscillation period and trend estimates also decrease or (2) $\hat{N}$ increases, ending the current period, and our assumption of 2019 representing a nadir will be validated. We report the annual median $\hat{\lambda}$ (trend) for each temporal scale (one to six complete oscillations) by NC and CC. We calculated the percent increase or decrease from each abundance nadir back in time to 2019. Thus, this modeling framework and method for inference provided empirical evaluation of increasing, neutral, or decreasing trends across different temporal scales for populations that experience interannual variation in abundance (fig. 17). We also estimated the mean number of years between each population abundance nadir with 95-percent confidence intervals. For illustrative

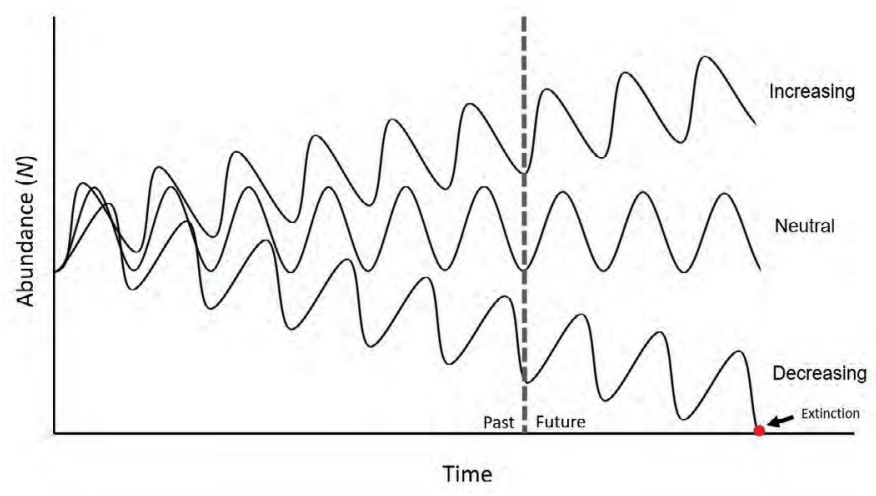

Figure 17. Diagram depicting population trends of increasing, neutral, and decreasing trend scenarios across six complete oscillations. Right side of dashed vertical line represents predictions across three additional oscillations with extinction identified on the declining scenario. purposes, we plotted abundance index through time, where the index reflected abundance relativized to the averaged abundance across the entire data set.

Expanding on Objective 3 , we projected $\hat{N}$ for each lek and NC across three temporal scales that reflected two- (short), four- (medium), and six-periods (long) of oscillation by using the same model and dataset. We used the mean oscillation period (9.4 years) based on estimated $\hat{N}$ from SSM results. We then calculated the proportion of the posterior probability distribution of $\hat{N}$ that was less than two males (minimum number to represent a lek) for the last prediction year of each temporal scale. Although this value is not true extirpation (zero or one bird), we refer to it as extirpation to align with state definitions of lek inactivity. Thus, this proportion of the distribution represented the probability of extirpation for each lek and $\mathrm{NC}$ at a nadir, approximately at short, medium, and long temporal scales into the future. Extirpation of leks within an $\mathrm{NC}$ was thought to reflect a loss of a meta-population as a result of reduced demographic rates.

\section{Model Diagnostics and Tests of Predictiveness}

We fit the SSM model in Just Another Gibbs sampler (JAGS) version 4.3 (Plummer, 2017) and R version 3.4.0 (R Core Team, 2017). For the MCMC settings, we specified 3 chains of 120,000 iterations each and discarded the first 100,000 iterations from each chain. Parameter inference was based on a subsample of the Markov chains, whereby every twentieth sample was kept and the rest were discarded. We inspected traceplots to assess chain mixing and model convergence, as well as calculated the potential scale reduction factor (PSRF) to assess the degree of convergence between and within chains (Gelman and others, 2004). We used the process variance hyperparameters of the random effects for assessing chain convergence and we did not observe a PSRF greater than 1.2 among those parameters.

Because we were interested in future uses of predictions under the fitted model, we calculated a Bayesian (or posterior predictive) $p$ value (Gelman and others, 2004) from predictions and observations, where $p=0.5$ represents good model fit. We took additional steps to directly assess predictive ability of our model given the oscillations in sage-grouse abundance through time. First, we developed a training dataset that excluded all lek data from 2019 to a population nadir that extended over the past three periods of oscillation (for instance, covering period 1,2, and 3). We then generated a PD of annual average $\hat{r}$ for years of missing data by averaging across the three periods and compared it to a PD of average annual $\hat{r}$ from the same model run on the full (that is, validation) dataset over the same period. Lastly, we developed a predictive probability $p$ value in a similar fashion to methods that compare predictions to observed data by calculating discrepancy between the $\hat{r}$ values generated from models fit to training and validation datasets using Pearson's chi-squared diagnostic (Gelman and others, 2004). These discrepancy measures were restricted to the years associated with the most recent three oscillations (that is, validation years). 


\section{Results}

\section{Range-wide Population Trends}

Overall, our model fit the observed data well (Bayesian p value $=0.51$ ). Median $\hat{N}$ estimates from the SSM revealed six distinct range-wide population nadirs across the 60 years of data, which were: 1966, 1975, 1986, 1996, 2002, and 2013 The range-wide, across-years, mean male count was 18.4 per lek $(\mathrm{SD}=24.2)$ based on data restricted to trend analyses. The number of years for complete oscillation periods (nadir to nadir) was relatively consistent across periods (average $=9.4$; 95 -percent confidence interval $=6.3-11.0$ ). Nadirs also were relatively consistent across each CC (table 5). Model estimates revealed evidence of range-wide decline, on average, from every historic abundance nadir to 2019 (fig. 18A; table 6). For example, the average annual $\hat{\lambda}$ for short (17 years, two oscillations), medium (33 years, four oscillations), and long (53 years, six oscillations) temporal scales were 0.973 (median; 95-percent CRI=0.971-0.975), 0.969 (median; 95-percent CRI $=0.963-0.973$ ), and 0.969 (median; 95-percent CRI $=0.967-0.974$ ), respectively. These trends imply declines of $37.0,65.2$, and 80.7 percent, relative to initial population sizes, over the associated time periods of 17,33 , and 53 years, respectively. The only spatiotemporal combination at the $\mathrm{CC}$

Table 5. Identified years of population abundance nadirs (troughs) used to define temporal scales (recent to long-term) of population trend estimates across different climate clusters $(\mathrm{A}-\mathrm{F}$ ) and range-wide for greater sage-grouse (Centrocercus urophasianus) in the western United States. Range-wide estimated abundance nadirs were 1966, 1975, 1986, 1996, 2002, and 2013 that reflect Long, Medium/Long, Medium, Short/ Medium, Short, and Recent, respectively, temporal scales for inferencing trends.

[CC, climate cluster; A, Bi-state area; B, Washington area; C, Jackson Hole, Wyoming area; D, eastern area; E, Great Basin area; F, Wyoming]

\begin{tabular}{lcccccc}
\hline \multicolumn{1}{c}{ CC } & Long & $\begin{array}{c}\text { Medium/ } \\
\text { Long }\end{array}$ & Medium & $\begin{array}{c}\text { Short/ } \\
\text { Medium }\end{array}$ & Short & Recent \\
\hline A & 1969 & 1977 & 1983 & 1995 & 2002 & 2008 \\
B & 1964 & 1976 & 1987 & 1996 & 2001 & 2008 \\
C & 1963 & 1973 & 1984 & 1999 & 2003 & 2011 \\
D & 1966 & 1977 & 1986 & 1997 & 2004 & 2014 \\
E & 1967 & 1975 & 1985 & 1996 & 2002 & 2013 \\
F & 1966 & 1973 & 1987 & 1996 & 2002 & 2013 \\
Range & 1966 & 1975 & 1986 & 1996 & 2002 & 2013 \\
\hline
\end{tabular}

scale that estimated a positive rate of change (median $\hat{\lambda}>1$ ) was CC-F (western Wyoming area) during recent (6 years, one oscillation) and short/medium temporal scales. Climate cluster $\mathrm{F}$ demonstrated higher averaged annual growth in abundance, compared to all other CCs, during every temporal scale except the medium and medium/long (fig. 19; table 6). We estimated median $\hat{\lambda}$ to be less than 1.0 for $81.3,88.4$, and 94.3 percent of NCs across short, medium, and long temporal scales, respectively (fig. 20; table 7), throughout the sagegrouse range. We report CC scale trends in the next paragraph, whereas trends estimated using state boundaries can be found in appendix 4. However, trends estimated within state boundaries are less grounded in biological rationale.

\section{Cluster Level Population Trends}

Climate cluster A (CC-A; Bi-State area) consisted of 11 NCs that encompassed 726,907 hectares (ha; table 7). Two NCs did not have sufficient lek data to estimate trends. Climate cluster A consisted of 84 leks, representing approximately 1.0 percent of the range-wide database. After quality assurance and quality control (QA/QC), 55 leks met criteria for use in the SSM (table 7), totaling 1,510 field observations. Mean male count was 21.3 (95-percent confidence interval=19.9-22.8).

For CC-A, we estimated six population abundance nadirs that dated back to 1960 and included nadirs of 1969,1977 , 1983, 1995, 2002, and 2008 (table 5; fig. 21A). We estimated $\hat{\lambda}$ at the short (2002-2019, two periods of oscillation over 17 years), medium (1983-2019, four periods over 36 years), and long temporal scales (1969-2019, six periods over 50 years) as 0.973 (median, 95-percent $\mathrm{CRI}=0.964-0.981$ ), 0.990 (median, 95-percent CRI $=0.974-1.000$ ), and 0.978 (median, 95-percent CRI=0.965-0.988), respectively (fig. 21B; table 6). Over the past 17,36, and 50 years, sage-grouse populations have declined 36.9, 29.6, and 67.0 percent, respectively. We estimated median $\hat{\lambda}$ to be less than 1.0 for all NCs across short, medium, and long temporal scales, respectively (fig. 22). We reported spatiotemporal variation in average annual $\hat{\lambda}$ across different NCs (fig. 22) and leks (fig. 23).

Climate cluster B (CC-B; Washington area) consisted of four NCs that encompassed 726,907-ha (table 7). One NC did not have sufficient lek data to estimate trends. Climate cluster B consisted of 108 leks, representing approximately 1.3 percent of the lek database. After QA/QC, 70 leks met criteria for use in the state-space trend model (table 7), totaling 1,141 field observations. Mean male count was 14.0 (95-percent confidence interval=13.2-14.9). 

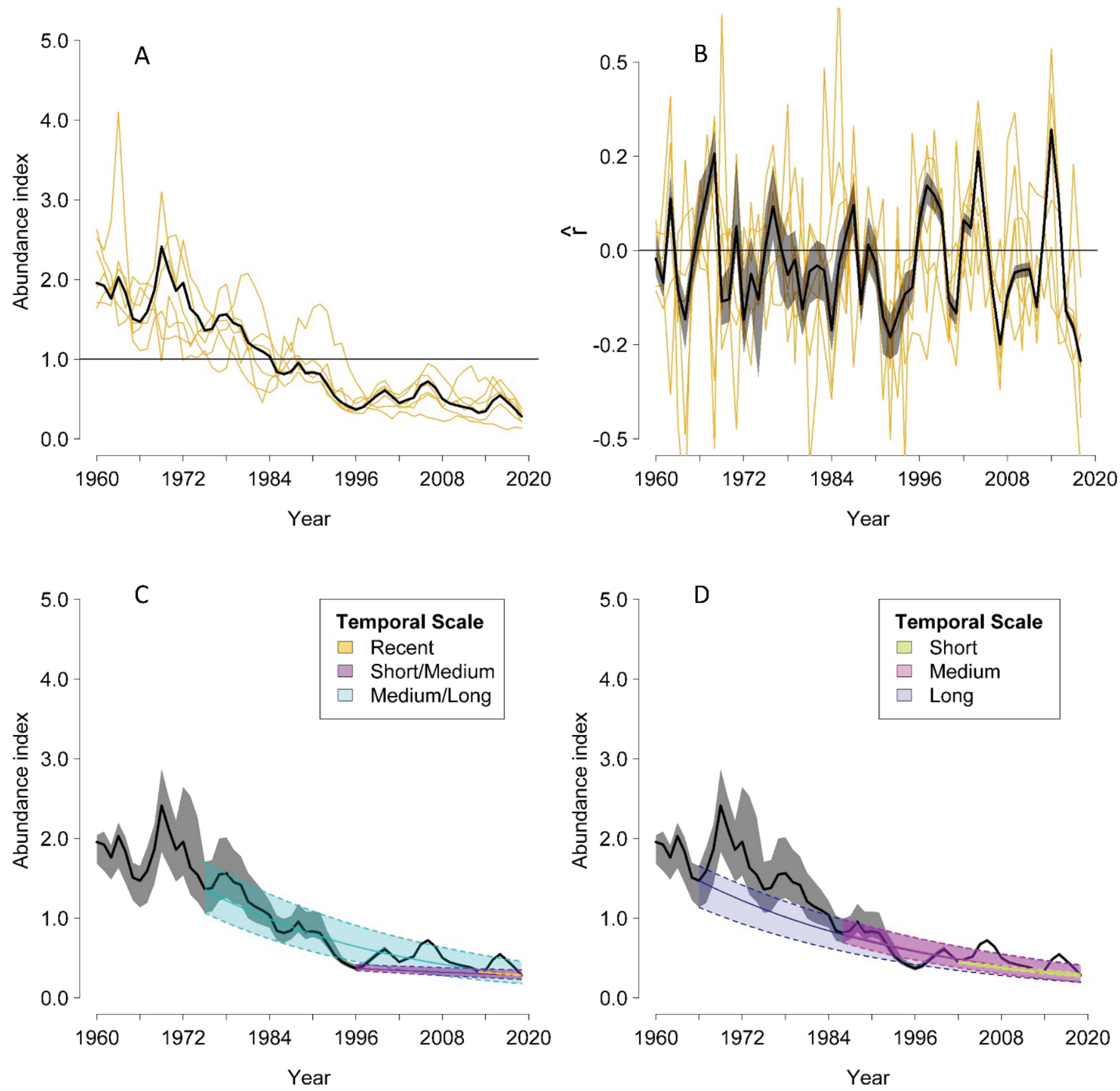

Figure 18. A, Abundance index (calculated as $\hat{N}$ divided by 60-year mean of $\hat{N}$ ); $B$, intrinsic rate of population change $(\hat{r})$ of greater sage-grouse (Centrocercus urophasianus) across their range from lek observations used to model population trends during 1960-2019; $C$, Median estimate of abundance trend (colored lines) across temporal scales: Recent (one period), Short/Medium (three periods), and Medium/Long (five periods); and $D$, Short (two periods), Medium (four periods), and Long (six periods), right to left. Black trend line represents median estimates and yellow lines represent median values for climate clusters $(A$ and $B)$. Horizontal thin black line represents $(A)$ rescaled long-term mean and $(B)$ neutral population growth. Colored areas represent 95-percent credible limits of trend estimates. Grey shaded areas represent 95-percent credible limits on abundance index $(C$ and $D)$ and $\hat{r}(B)$. 
Table 6. Greater sage-grouse (Centrocercus urophasianus) average annual rate of population change $(\hat{\lambda})$ across six different temporal scales which corresponded to differing periods of oscillation (Recent [one period], Short [two periods], Short/Medium [three periods], Medium [four periods], Medium/Long [five periods], Long [six periods]) for each climate cluster within the western United States (see table 5).

[CC, climate cluster; A, Bi-state area; B, Washington area; C, Jackson Hole, Wyoming area; D, eastern area; E, Great Basin area; F, Wyoming]

\begin{tabular}{|c|c|c|c|c|c|c|c|c|}
\hline \multirow{2}{*}{ CC } & \multicolumn{6}{|c|}{ Temporal scales 1} & \multirow{2}{*}{$\begin{array}{l}\text { Number } \\
\text { of leks }\end{array}$} & \multirow{2}{*}{$\begin{array}{c}\text { Average } \\
\text { count/lek }{ }^{3}\end{array}$} \\
\hline & Long & Medium/Long & Medium & Short/Medium & Short & Recent & & \\
\hline A & $\begin{array}{c}0.978 \\
(0.965-0.988)\end{array}$ & $\begin{array}{c}0.978 \\
(0.962-0.986)\end{array}$ & $\begin{array}{c}0.990 \\
(0.974-1.000)\end{array}$ & $\begin{array}{c}0.995 \\
(0.979-1.005)\end{array}$ & $\begin{array}{c}0.973 \\
(0.964-0.981)\end{array}$ & $\begin{array}{c}0.981 \\
(0.969-0.993)\end{array}$ & $\begin{array}{c}84 \\
(55)\end{array}$ & $\begin{array}{c}21.3 \\
(19.9-22.8)\end{array}$ \\
\hline B & $\begin{array}{c}0.956 \\
(0.944-0.973)\end{array}$ & $\begin{array}{c}0.949 \\
(0.928-0.968)\end{array}$ & $\begin{array}{c}0.946 \\
(0.907-0.966)\end{array}$ & $\begin{array}{c}0.956 \\
(0.909-0.981)\end{array}$ & $\begin{array}{c}0.966 \\
(0.936-0.998)\end{array}$ & $\begin{array}{c}0.957 \\
(0.932-1.000)\end{array}$ & $\begin{array}{l}108 \\
(70)\end{array}$ & $\begin{array}{c}14.0 \\
(13.2-14.9)\end{array}$ \\
\hline $\mathrm{C}$ & $\begin{array}{c}0.966 \\
(0.951-0.982)\end{array}$ & $\begin{array}{c}0.963 \\
(0.941-0.980)\end{array}$ & $\begin{array}{c}0.972 \\
(0.942-0.997)\end{array}$ & $\begin{array}{c}0.970 \\
(0.948-0.989)\end{array}$ & $\begin{array}{c}0.962 \\
(0.942-0.981)\end{array}$ & $\begin{array}{c}0.935 \\
(0.905-0.966)\end{array}$ & $\begin{array}{c}17 \\
(14)\end{array}$ & $\begin{array}{c}14.1 \\
(12.3-15.9)\end{array}$ \\
\hline $\mathrm{D}$ & $\begin{array}{c}0.963 \\
(0.960-0.968)\end{array}$ & $\begin{array}{c}0.956 \\
(0.946-0.960)\end{array}$ & $\begin{array}{c}0.967 \\
(0.960-0.972)\end{array}$ & $\begin{array}{c}0.983 \\
(0.975-0.989)\end{array}$ & $\begin{array}{c}0.963 \\
(0.959-0.967)\end{array}$ & $\begin{array}{c}0.980 \\
(0.972-0.989)\end{array}$ & $\begin{array}{c}2,944 \\
(1,831)\end{array}$ & $\begin{array}{c}16.7 \\
(16.5-16.9)\end{array}$ \\
\hline $\mathrm{E}$ & $\begin{array}{c}0.971 \\
(0.967-0.976)\end{array}$ & $\begin{array}{c}0.973 \\
(0.967-0.978)\end{array}$ & $\begin{array}{c}0.974 \\
(0.963-0.979)\end{array}$ & $\begin{array}{c}0.986 \\
(0.981-0.990)\end{array}$ & $\begin{array}{c}0.968 \\
(0.964-0.971)\end{array}$ & $\begin{array}{c}0.949 \\
(0.944-0.955)\end{array}$ & $\begin{array}{c}4,015 \\
(2,187)\end{array}$ & $\begin{array}{c}17.3 \\
(17.1-17.5)\end{array}$ \\
\hline $\mathrm{F}$ & $\begin{array}{c}0.980 \\
(0.975-0.987)\end{array}$ & $\begin{array}{c}0.976 \\
(0.969-0.984)\end{array}$ & $\begin{array}{c}0.976 \\
(0.966-0.980)\end{array}$ & $\begin{array}{c}1.003 \\
(0.997-1.008)\end{array}$ & $\begin{array}{c}0.991 \\
(0.988-0.995)\end{array}$ & $\begin{array}{c}1.016 \\
(1.011-1.023)\end{array}$ & $\begin{array}{l}1,253 \\
(974)\end{array}$ & $\begin{array}{c}23.7 \\
(23.3-24.2)\end{array}$ \\
\hline Range & $\begin{array}{c}0.969 \\
(0.967-0.974)\end{array}$ & $\begin{array}{c}0.965 \\
(0.960-0.970)\end{array}$ & $\begin{array}{c}0.969 \\
(0.963-0.973)\end{array}$ & $\begin{array}{c}0.989 \\
(0.983-0.992)\end{array}$ & $\begin{array}{c}0.973 \\
(0.971-0.975)\end{array}$ & $\begin{array}{c}0.977 \\
(0.973-0.981)\end{array}$ & $\begin{array}{c}8,421 \\
(5,131)\end{array}$ & $\begin{array}{c}18.4 \\
(18.3-18.6)\end{array}$ \\
\hline
\end{tabular}

${ }^{1}$ Lengths of temporal scales were defined by population abundance nadirs that varied for each climate cluster (see table 5). Estimates for each period represent median $\hat{\lambda}$ with 95-percent credible intervals in parentheses.

${ }^{2}$ Number of leks in database and number that were used analysis in parentheses.

${ }^{3}$ Average number of males counted on leks used in analysis and 95-percent confidence intervals in parentheses. 

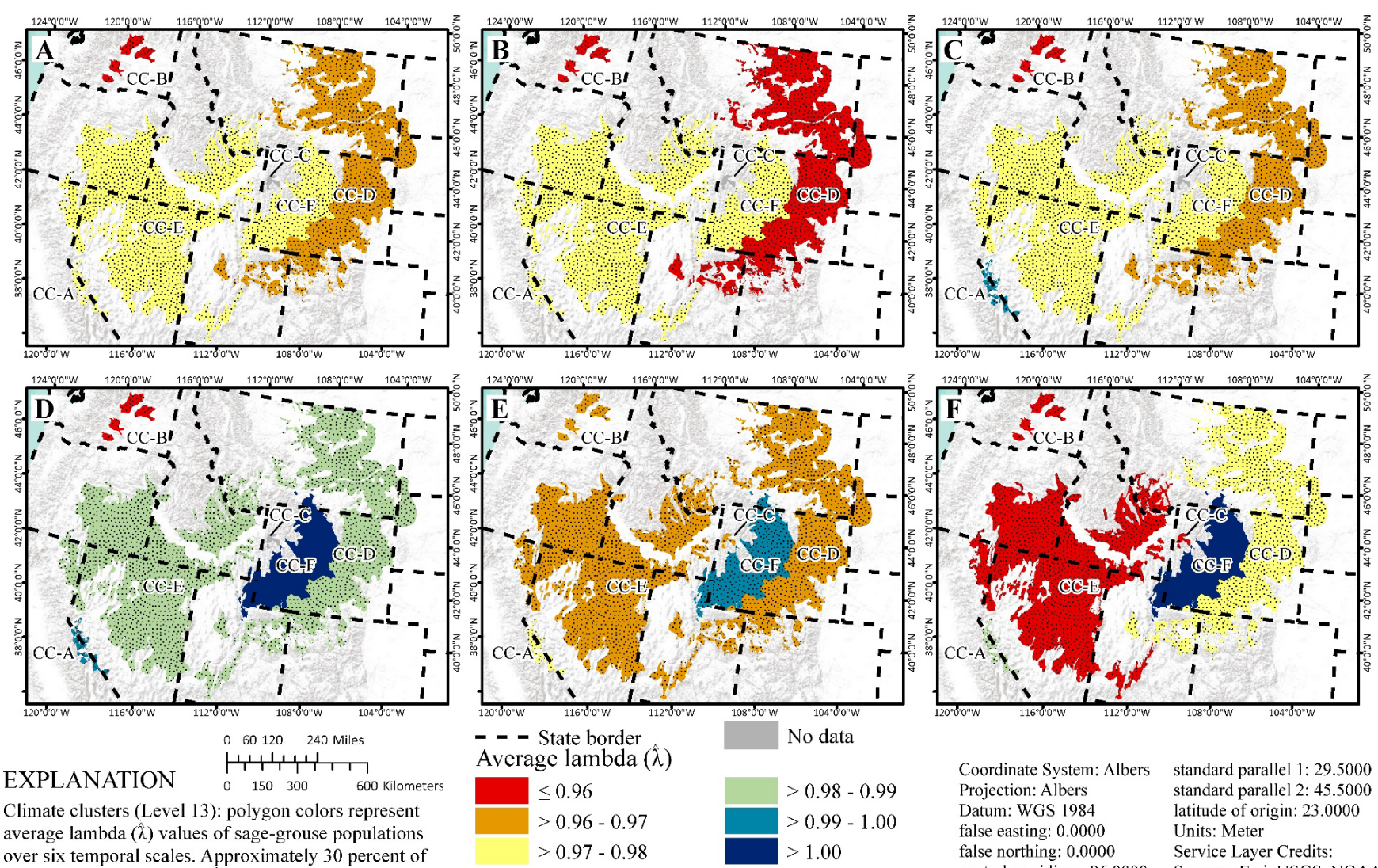

Climate clusters (Level 13): polygon colors represent average lambda $(\lambda)$ values of sage-grouse populations over six temporal scales. Approximately 30 percent of the distribution of average $\lambda$ are within the upper and

Proportion of posterior distribution representing decline lower color classes.

$\geq 0.95<0.95-0.80<0.80-0.50<0.50 \cdot 0.20<0.20$

Coordinate System: Albers standard parallel 1:29.5000 Projection: Albers $\quad$ standard parallel 2: 45.5000 Datum: WGS 1984 false easting: 0.0000 false northing: 0.0000 latitude of origin: 23.0000 central meridian: -96.0000 Sources: Esri, USGS, NOAA

Figure 19. Range-wide spatial estimates of average annual rate of change $(\hat{\lambda})$ in abundance of greater sage-grouse (Centrocercus urophasianus) across six temporal scales based on periods of oscillation: $A$, Long (six periods); $B$, Medium/Long (five periods); $C$, Medium (four periods); $D$, Short/Medium (three periods); $E$, Short (two periods); and $F$, Recent (one period) within climate clusters (A-F). Map images are the intellectual property of Esri and are used herein under license. Copyright (c) 2020 Esri and its licensors. All rights reserved.

Table 7. Greater sage-grouse (Centrocercus urophasianus) average annual rate of increase $(\hat{\lambda})$ in abundance at neighborhood clusters across six different temporal scales that were based on complete periods of oscillation (Recent [one period], Short [two periods], Short/Medium [three periods], Medium [four periods], Medium/Long [five periods], and Long [six periods]) of population abundance within the western United States. (Available for download from https://doi.org/10.3133/ofr20201154). 

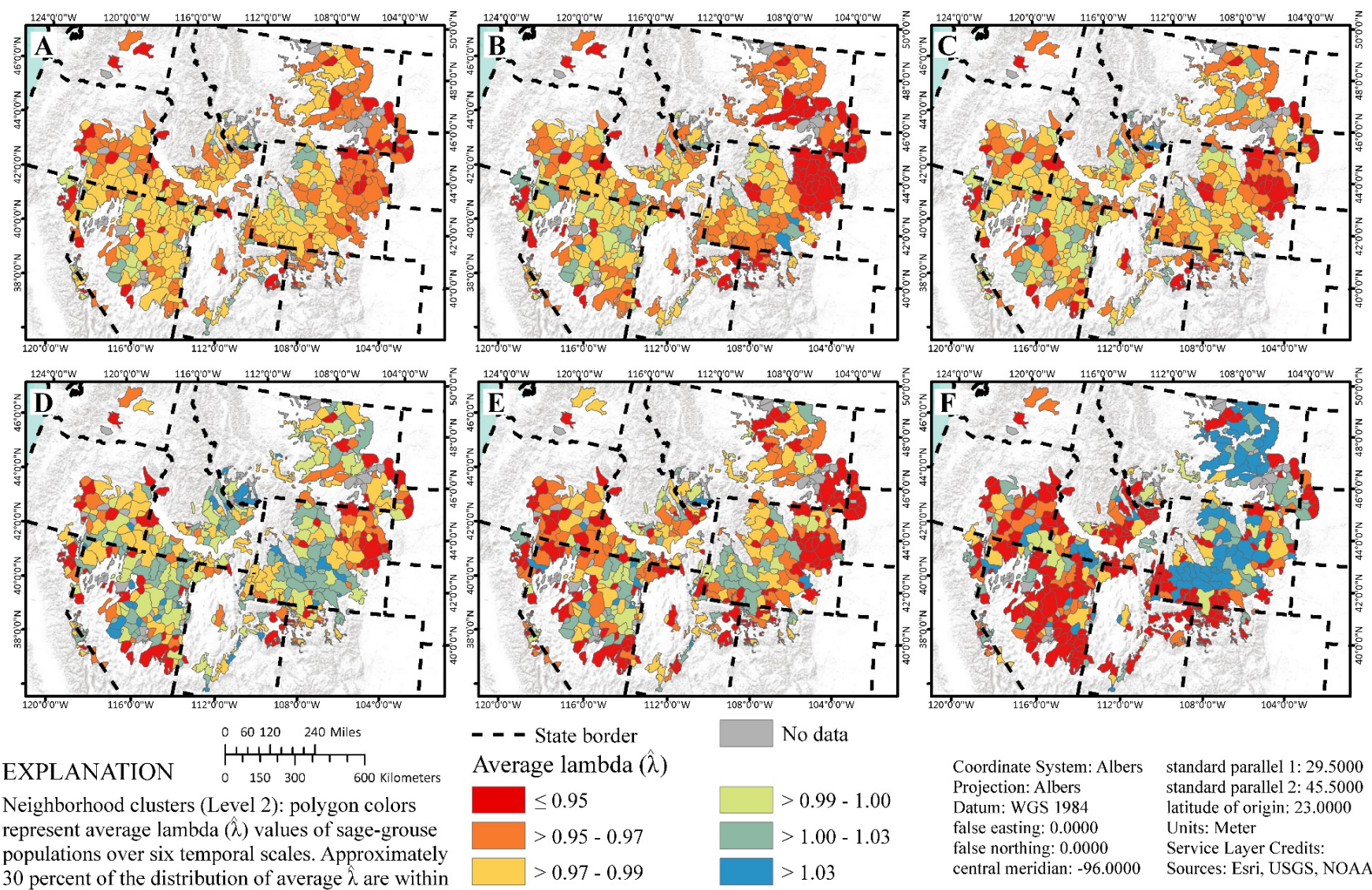

Neighborhood clusters (Level 2): polygon colors represent average lambda $(\hat{\lambda})$ values of sage-grouse populations over six temporal scales. Approximately 30 percent of the distribution of average $\hat{\lambda}$ are within the upper and lower color classes.

Coordinate System: Albers standard parallel 1:29.5000 Projection: Albers $\quad$ standard parallel 2: 45.5000 Datum: WGS $1984 \quad$ latitude of origin: 23.0000 false easting: $0.0000 \quad$ Units: Meter false northing: $0.0000 \quad$ Service Layer Credits: central meridian: - 96.0000 Sources: Esri, USGS, NOAA

Figure 20. Range-wide spatial estimates of average annual rate of change $(\hat{\lambda})$ in abundance of greater sage-grouse (Centrocercus urophasianus) across six temporal scales based on periods of oscillation: $A$, Long (six periods); $B$, Medium/Long (five periods); $C$, Medium (four periods); $D$, Short/Medium (three periods); $E$, Short (two periods); and $F$, Recent (one period) within neighborhood clusters. Map images are the intellectual property of Esri and are used herein under license. Copyright (C) 2020 Esri and its licensors. All rights reserved. 

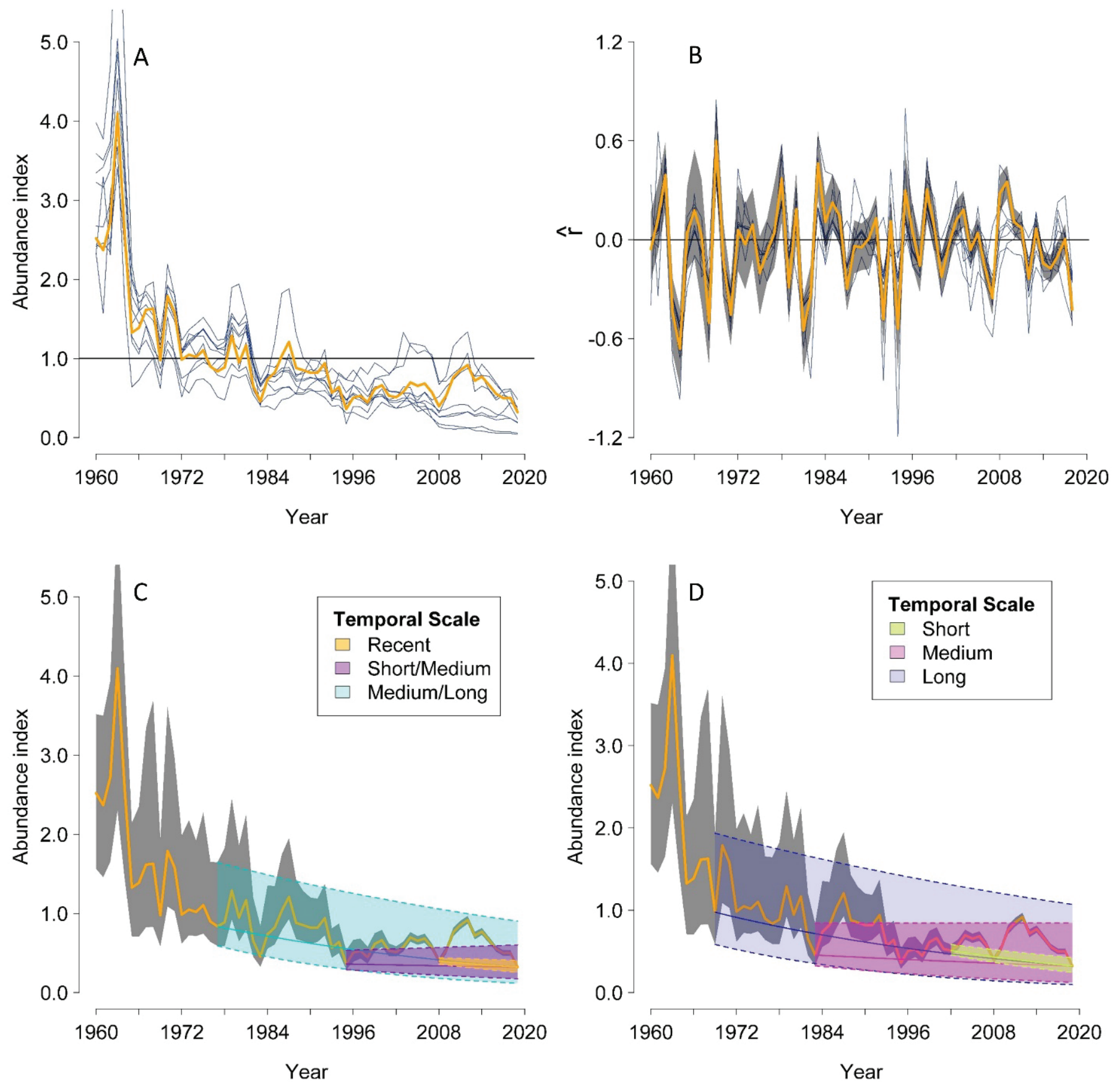

Figure 21. A, Abundance index (calculated as $\hat{N}$ divided by 60-year mean of $\hat{N}$ ); $B$, intrinsic rate of population change $(\hat{r})$ of greater sage-grouse (Centrocercus urophasianus) within climate cluster A (CC-A; Bi-State area) from lek observations used to model population trends during 1960-2019; C, Median estimate of abundance trend (colored lines) across temporal scales based on periods of oscillation: Recent (one period), Short/Medium (three periods), and Medium/Long (five periods); and $D$, Short (two periods), Medium (four periods), and Long (six periods), right to left. Yellow line represents median estimates and blue thin lines represent median values for neighborhood clusters $(A$ and $B)$. Horizontal thin black line represents $(A)$ rescaled long-term mean and $(B)$ neutral population growth. Colored areas represent 95-percent credible limits of trend estimates. Grey shaded areas represent 95-percent credible limits on abundance index $(C$ and $D)$ and $\hat{r}(B)$. 

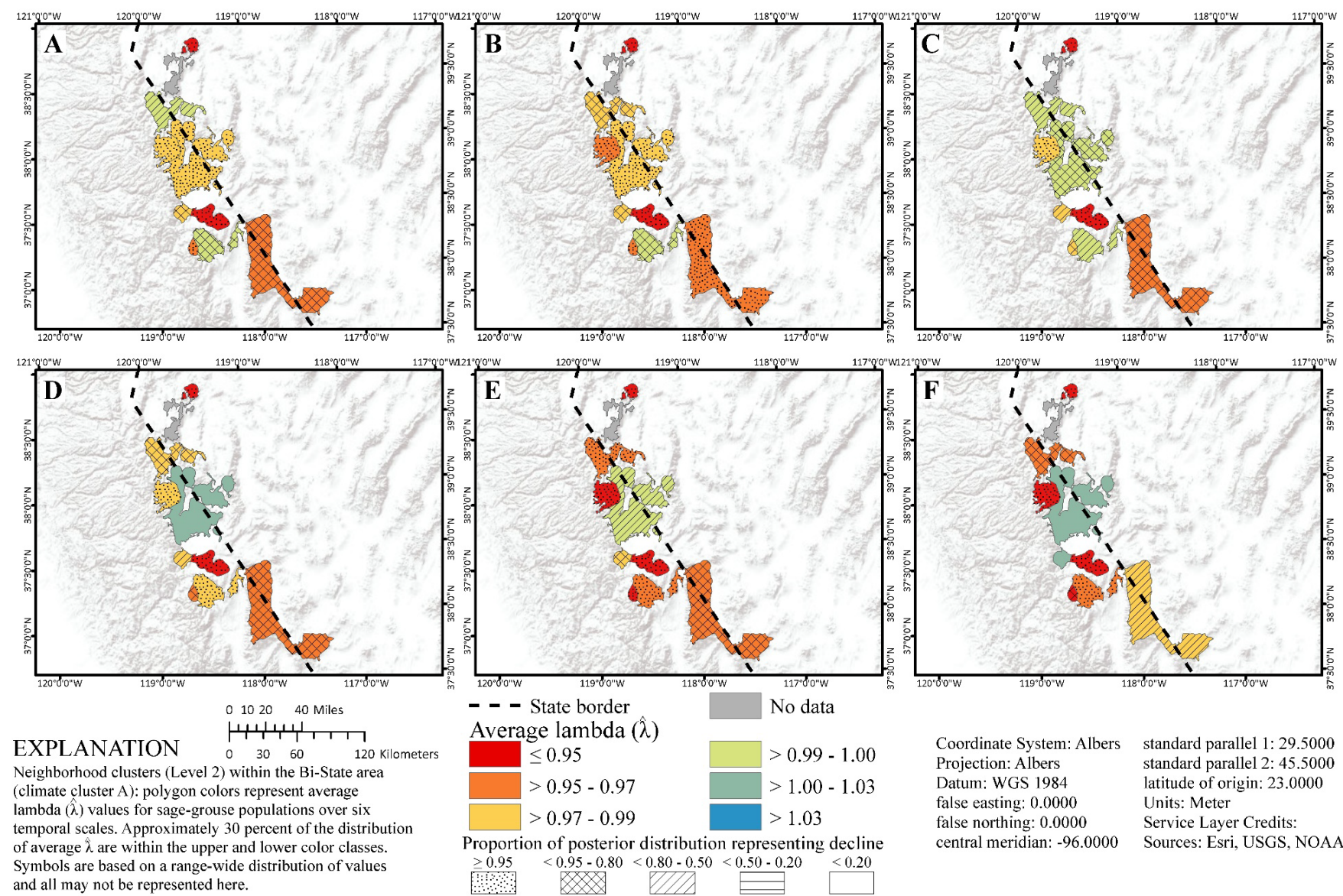

- - - State border

Average lambda $(\hat{\lambda})$

$\leq 0.95$

$>0.95-0.97$

$>0.97-0.99$

Proportion of posterior distribution representing decline $\geq 0.95<0.95-0.80<0.80-0.50<0.50-0.20<0.20$
No data

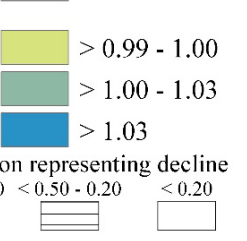

Coordinate System: Albers Projection: Albers Datum: WGS 1984 false easting: 0.0000 false northing: 0.0000 central meridian: -96.0000 standard parallel 1:29.5000 standard parallel 2: 45.5000 latitude of origin: 23.0000 Units: Meter Service Layer Credits: Sources: Esri, USGS, NOAA

Figure 22. Spatial estimates of average annual population rate of change $(\hat{\lambda})$ in abundance of greater sage-grouse (Centrocercus urophasianus) at neighborhood clusters across six temporal scales based on periods of oscillation: $A$, Long (six periods); $B$, Medium/ Long (five periods); $C$, Medium (four periods); $D$, Short/Medium (three periods); $E$, Short (two periods); and $F$, Recent (one period) within climate cluster A (CC-A; Bi-State area). Map images are the intellectual property of Esri and are used herein under license. Copyright (C) 2020 Esri and its licensors. All rights reserved. 

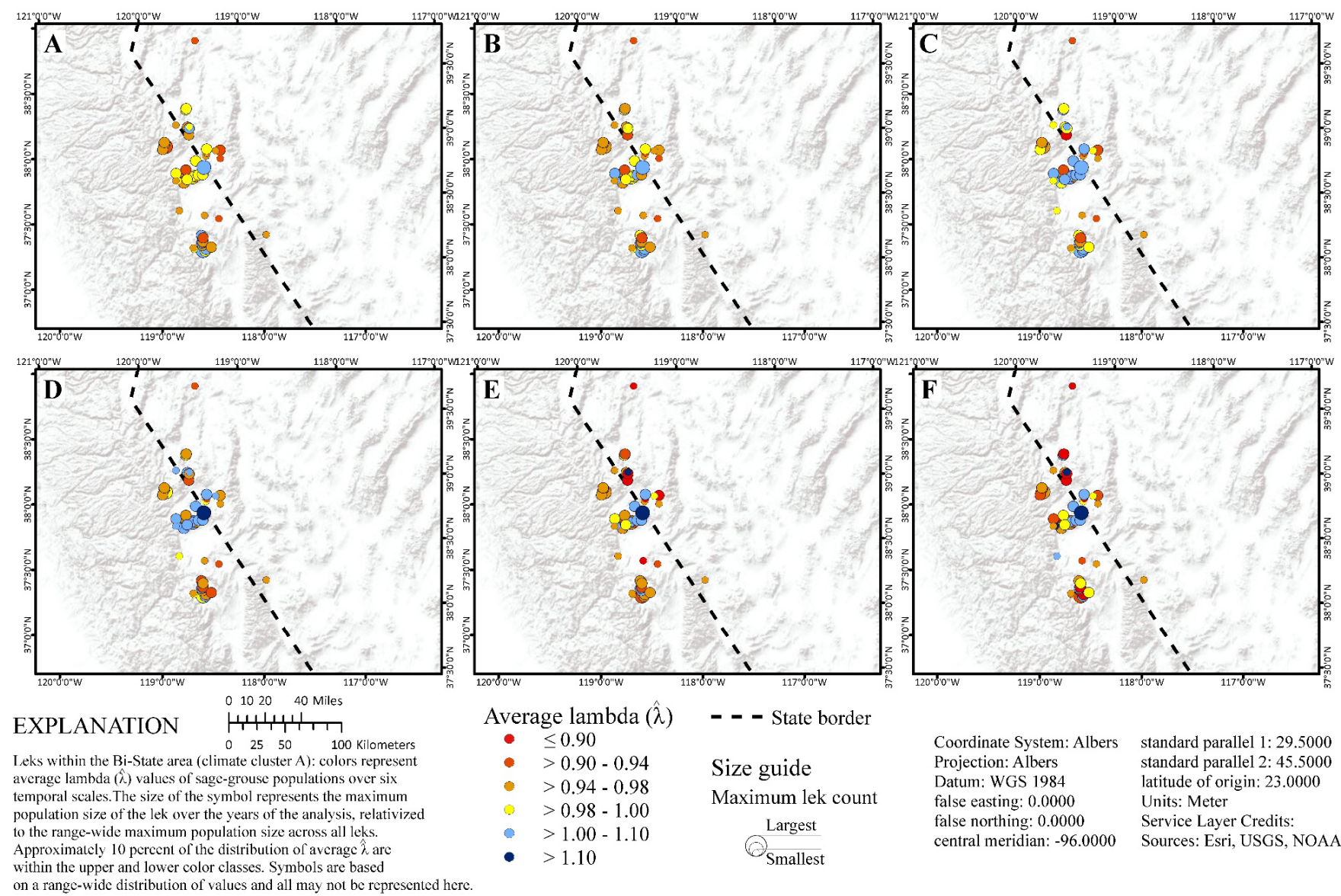

\begin{tabular}{|c|c|c|}
\hline \multicolumn{2}{|c|}{ Average lambda $(\lambda)$} & - - - State border \\
\hline & $\leq 0.90$ & \\
\hline - & $>0.90-0.94$ & Size guide \\
\hline$c$ & $>0.94-0.98$ & Maximum lek count \\
\hline 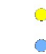 & $\begin{array}{l}>0.98-1.00 \\
>1.00-1.10\end{array}$ & 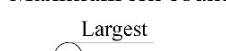 \\
\hline . & $>1.10$ & Smallest \\
\hline
\end{tabular}

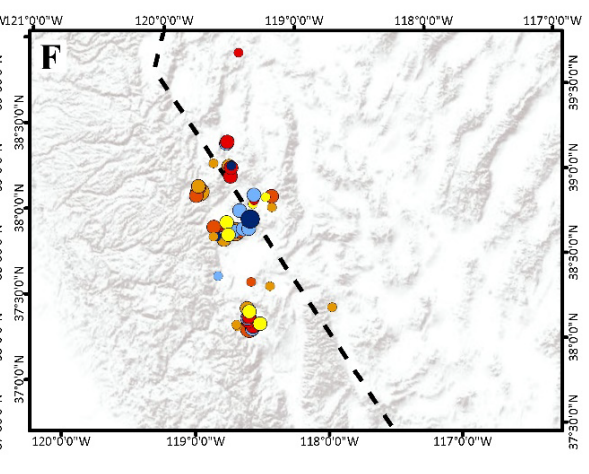

Coordinate System: Albers Projection: Albers Datum: WGS 1984 false easting: 0.0000 false northing: 0.0000 central meridian: -96.0000 standard parallel $1: 29.5000$ standard paralle1 2: 45.5000 latitude of origin: 23.0000 Units: Meter Service Layer Credits: Sources: Esri, USGS, NOAA

Figure 23. Spatial estimates of average annual population rate of change $(\hat{\lambda})$ in abundance of greater sage-grouse (Centrocercus urophasianus) at leks across six temporal scales based on periods of oscillation: $A$, Long (six periods); $B$, Medium/Long (five periods); $C$, Medium (four periods); $D$, Short/Medium (three periods); $E$, Short (two periods); and $F$, Recent (one period) within climate cluster $A$ (CC-A; Bi-State area). Map images are the intellectual property of Esri and are used herein under license. Copyright $(2020$ Esri and its licensors. All rights reserved.

For CC-B, we estimated six population abundance nadirs that dated back to 1960 and included 1964, 1976, 1987, 1996, 2001, and 2008 (table 5; fig. 24A). We estimated $\hat{\lambda}$ at the short (2001-2019, two periods of oscillation over 18 years), medium (1987-2019, four periods over 32 years), and long-temporal scales (1964-2019, six periods over 55 years) as 0.966 (95-percent $\mathrm{CRI}=0.936-0.998), 0.946$ (95-percent $\mathrm{CRI}=0.907-0.966$ ), and 0.956 (95-percent CRI $=0.944-0.973$ ), respectively (fig. 24B; table 6). Over the past 18,32, and 55 years, sage-grouse populations have declined 46.4, 83.1, 91.4 percent, respectively. We estimated median $\hat{\lambda}$ to be less than 1.0 for all NCs across short, medium, and long temporal scales, respectively (fig. 25). We reported spatial and temporal variation in average annual $\hat{\lambda}$ across different NCs (fig. 25) and leks (fig. 26).

Climate cluster C (CC-C; Jackson Hole, Wyoming, area) consisted of two NCs that encompassed 66,733 ha (table 7). Climate cluster $\mathrm{C}$ consisted of 17 leks, representing approximately 0.2 percent of lek database. After QA/QC, 14 leks met criteria for use in the SSM (table 7), totaling 311 field observations. Mean male count was 14.1 (95-percent confidence interval=12.3-15.9).

For CC-C, we estimated population abundance nadirs during 1963, 1973, 1984, 1999, 2003, and 2011 (table 5; fig. 27A). We estimated $\hat{\lambda}$ at the short (2003-2019, two periods of oscillation over 16 years), medium (1984-2019, four periods over 35 years), and long-temporal scales (1963-2019, six periods over 56 years) as 0.962 (95-percent CRI $=0.942-0.981$ ), 0.972 (95-percent CRI $=0.942-0.997$ ), and 0.966 (95-percent CRI $=0.951-0.982$ ), respectively. Over the past 16,35 , and 56 years, sage-grouse populations declined $45.8,63.6$, and 85.2 percent, respectively (fig. $27 B$; table 6 ). We estimated median $\hat{\lambda}$ to be less than 1.0 for all NCs across this temporal scale (fig. 28). We reported spatiotemporal variation in average annual $\hat{\lambda}$ across different NCs (fig. 28) and leks (fig. 29). 

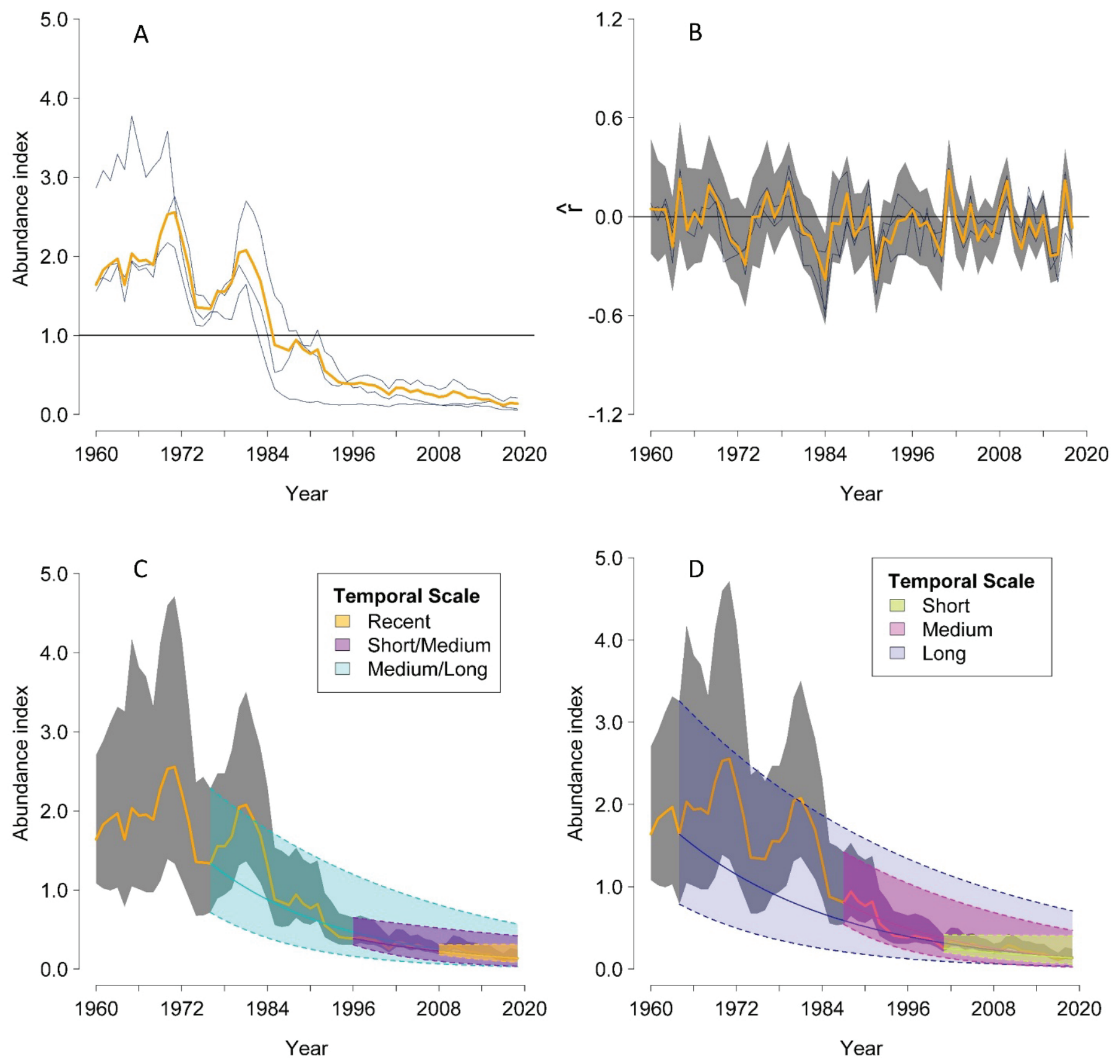

Figure 24. A, Abundance index (calculated as $\hat{N}$ divided by 60-year mean of $\hat{N}$ ); $B$, intrinsic rate of population change $(\hat{r})$ of greater sage-grouse (Centrocercus urophasianus) within B (CC-B; Washington area) from lek observations used to model population trends during 1960-2019; C, Median estimate of abundance trend (colored lines) across temporal scales based on periods of oscillation: Recent (one period), Short/Medium (three periods), and Medium/Long (five periods); and D, Short (two periods), Medium (four periods), and Long (six periods), right to left. Yellow line represents median estimates and blue thin lines represent median values for neighborhood clusters $(A$ and $B$ ). Horizontal thin black line represents $(A)$ rescaled long-term mean and $(B)$ neutral population growth. Colored areas represent 95-percent credible limits of trend estimates. Grey shaded areas represent 95-percent credible limits on abundance index $(C$ and $D)$ and $\hat{r}(B)$. 

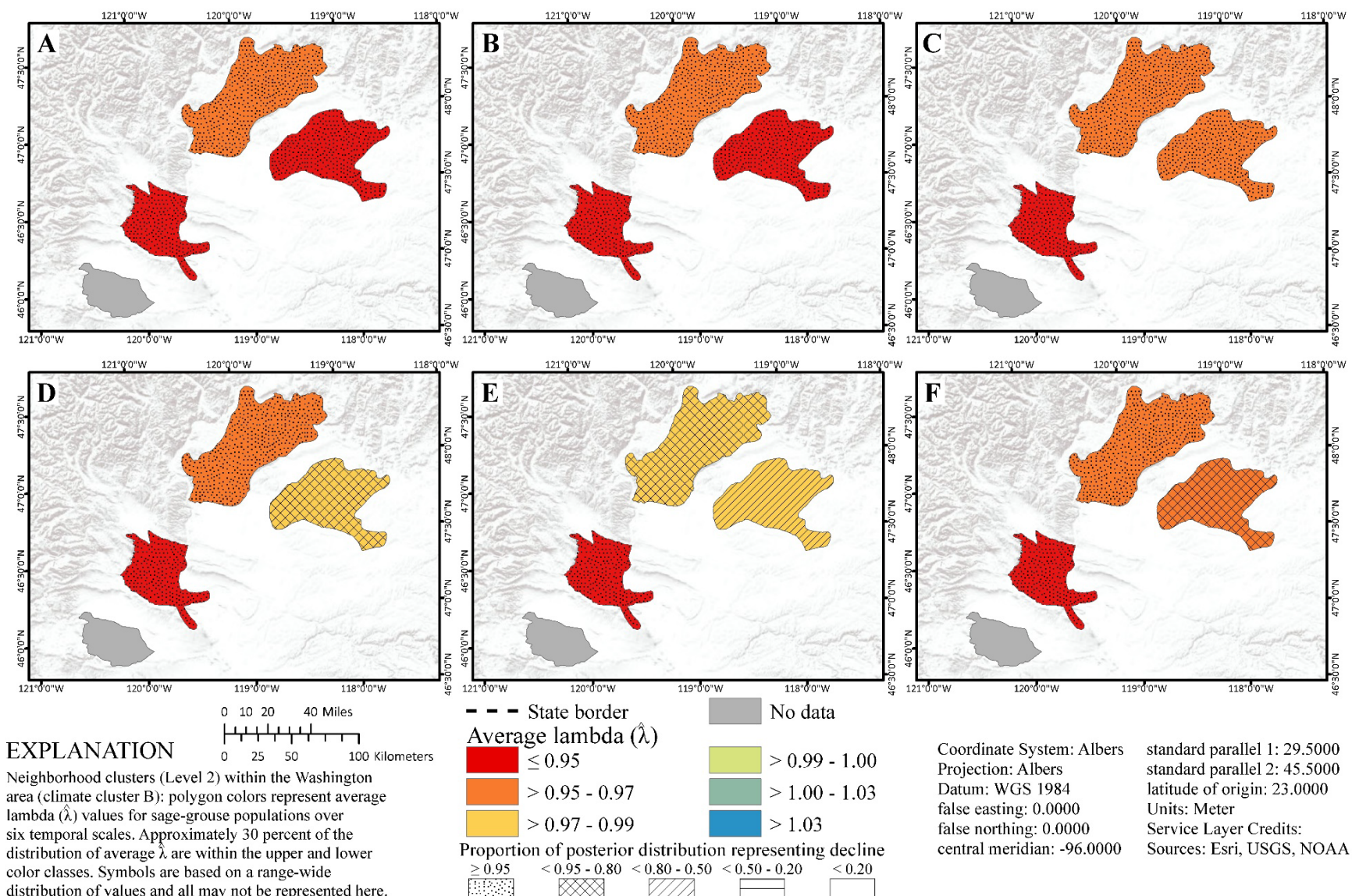
area (climate cluster $B)$ : polygon colors represent average lambda $(\hat{\lambda})$ values for sage-grouse populations over
six temporal scales. Approximately 30 percent of the

distribution of average $\lambda$ are within the upper and lower

color classes. Symbols are based on a range-wide

$\geq 0.95<0.95-0.80<0.80-0.50<0.50-0.20<0.20$

Figure 25. Spatial estimates of average annual population rate of change $(\hat{\lambda})$ in abundance of greater sage-grouse (Centrocercus urophasianus) at neighborhood clusters across six temporal scales based on periods of oscillation: $A$, Long (six periods); $B$, Medium/ Long (five periods); $C$, Medium (four periods); $D$, Short/Medium (three periods); $E$, Short (two periods); and $F$, Recent (one period) within climate cluster B (CC-B; Washington area). Map images are the intellectual property of Esri and are used herein under license. Copyright (C) 2020 Esri and its licensors. All rights reserved. 

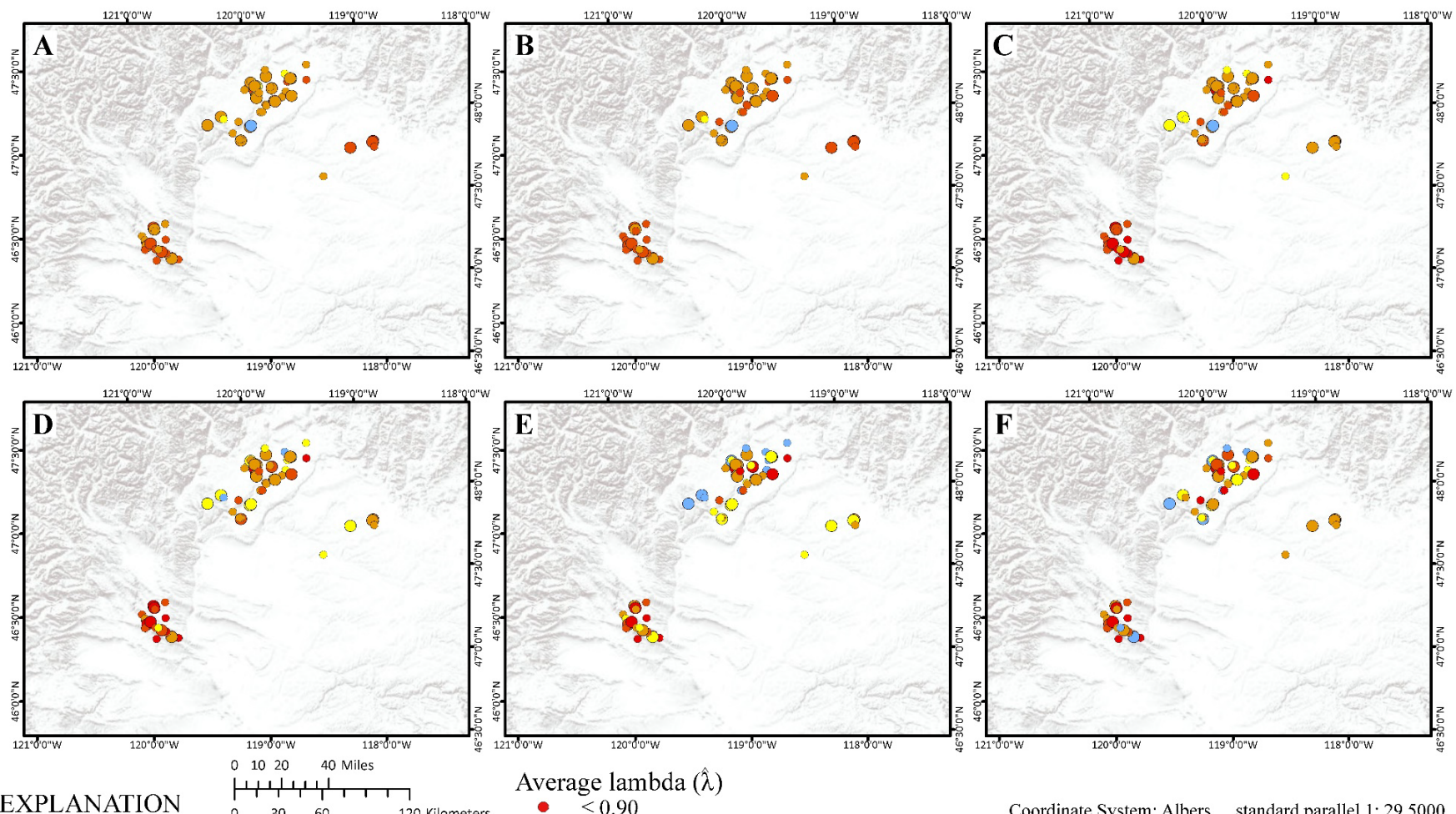

EXPLANATION 0 30 60

Leks within the Washington area (climate eluster B):colors represent average lambda $\hat{\lambda}$ ) values of sage-grouse populations over six temporal scales. The size of the symbol represents the maximum population size of the lek over the years of the analysis, relativized to the rangewide maximum population size across all leks. Approximately 30 percent of the distribution of average $\hat{\lambda}$ are within the upper and lower color classes.Symbols are based on a range-wide
distribution of values and all may not be represented here.
Average lambda $(\hat{\lambda})$

- $\leq 0.90$

- $>0.90-0.94$

$>0.94-0.98$

$>0.98-1.00$

$>1.00-1.10$

- $>1.10$
Size guide Maximum lek count Largest $\mathrm{O}_{\text {Smallest }}$
Coordinate System: Albers

Projection: Albers

Datum: WGS 1984 false easting: 0.0000

false northing: 0.0000

central meridian: $-\mathbf{9 6 . 0 0 0 0}$ standard parallel 1:29.5000 standard parallel 2: 45.5000 latitude of origin: 23.0000 Units: Meter

Service Layer Credits:

Sources: Esri, USGS, NOAA

Figure 26. Spatial estimates of average annual population rate of change $(\hat{\lambda})$ in abundance of greater sage-grouse (Centrocercus urophasianus) at leks across six temporal scales based on periods of oscillation: $A$, Long (six periods); $B$, Medium/Long (five periods); $C$, Medium (four periods); $D$, Short/Medium (three periods); $E$, Short (two periods); and $F$, Recent (one period) within climate cluster $B$ (CC-B; Washington area). Map images are the intellectual property of Esri and are used herein under license. Copyright (C) 2020 Esri and its licensors. All rights reserved. 

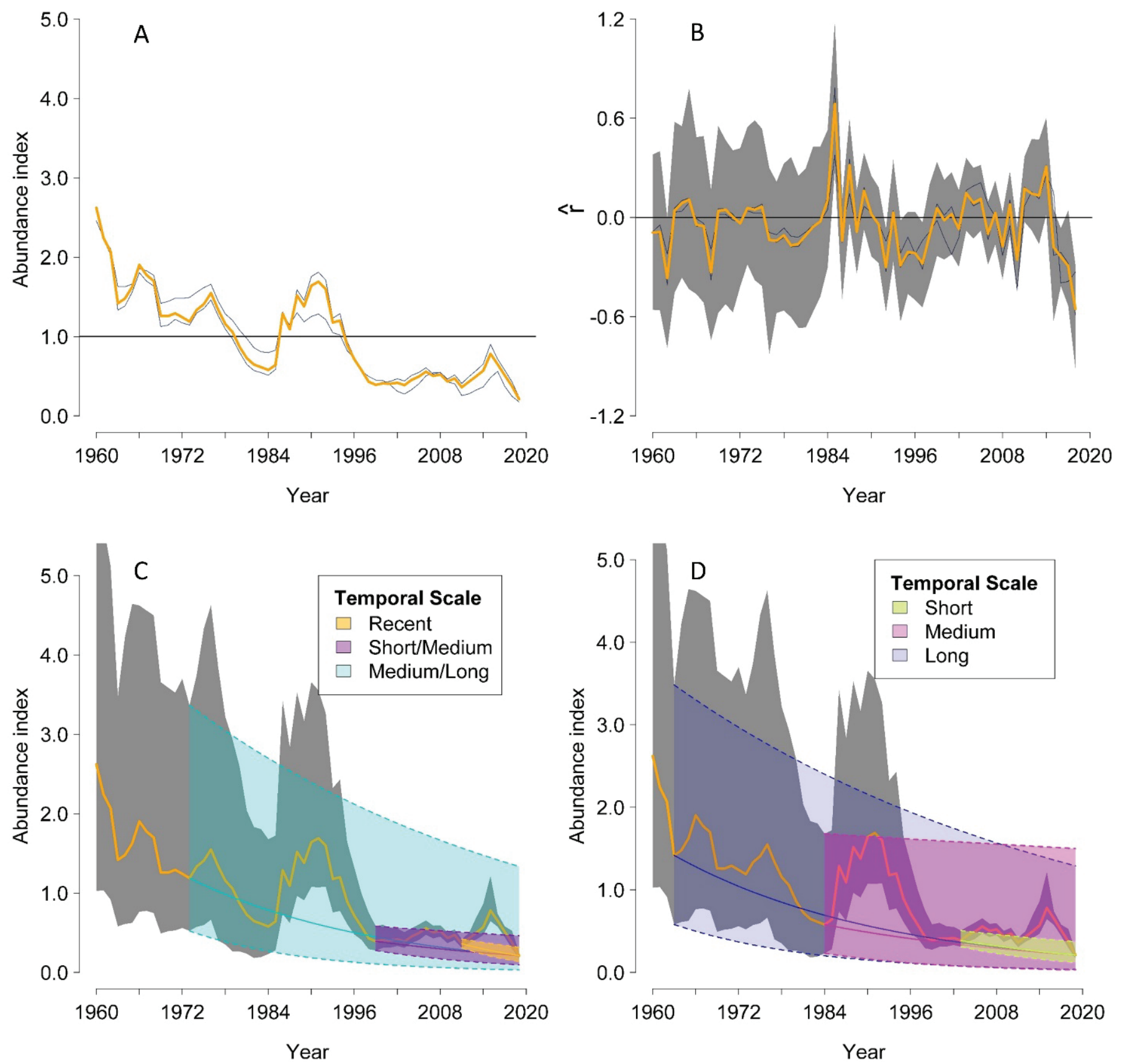

Figure 27. A, Abundance index (calculated as $\hat{N}$ divided by 31-year mean of $\hat{N})$; $B$, intrinsic rate of population change $(\hat{r})$ of greater sage-grouse (Centrocercus urophasianus) within climate cluster C (CC-C; Jackson Hole, Wyoming, area) from lek observations used to model population trends during 1989-2019; $C$, Median estimate of abundance trend (colored lines) across temporal scales based on periods of oscillation: Recent (one period), Short/Medium (three periods), and Medium/Long (five periods); and $D$, Short (two periods), Medium (four periods), and Long (six periods), right to left. Yellow line represents median estimates and blue thin lines represent median values for neighborhood clusters $(A$ and $B$ ). Horizontal thin black line represents $(A)$ rescaled long-term mean and $(B)$ neutral population growth. Colored areas represent 95-percent credible limits of trend estimates. Grey shaded areas represent 95-percent credible limits on abundance index $(C$ and $D)$ and $\hat{r}(B)$. 

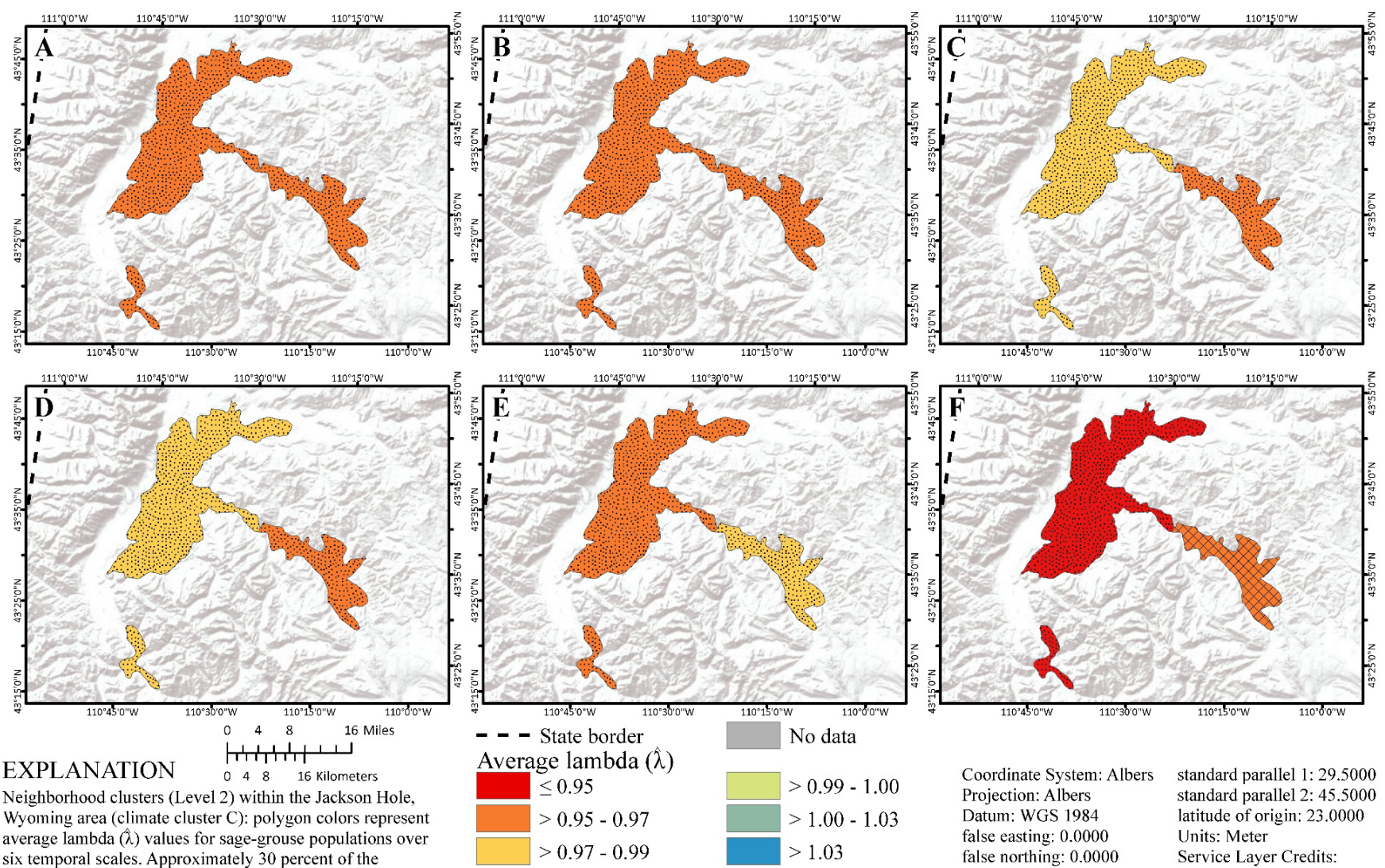

Neighborhood clusters (Level 2) within the Jackson Hole, Wyoming area (climate cluster $\mathrm{C}$ ): polygon colors represent average lambda $(\hat{\lambda})$ values for sage-grouse populations over six temporal scales. Approximately 30 percent of the distribution of average $\lambda$ are within the upper and lower color classes.Symbols are based on a range-wide distribution of values and all may not be represented here.

Proportion of posterior distribution representing decline $\geq 0.95<0.95-0.80<0.80-0.50<0.50 \cdot 0.20<0.20$

Coordinate System: Albers standard parallel 1:29.5000 Projection: Albers Datum: WGS 1984 false easting: 0.0000 false northing: 0.0000 central meridian: -96.0000 standard parallel 2: 45.5000 latitude of origin: 23.0000 Units: Meter Service Layer Credits: Sources: Esri, USGS, NOAA

Figure 28. Spatial estimates of average annual population rate of change $(\hat{\lambda})$ in abundance of greater sage-grouse (Centrocercus urophasianus) at neighborhood clusters across six temporal scales based on periods of oscillation: $A$, Long (six periods); $B$, Medium/ Long (five periods); $C$, Medium (four periods); $D$, Short/Medium (three periods); $E$, Short (two periods); and $F$, Recent (one period) within climate cluster C (CC-C; Jackson Hole, Wyoming, area). Map images are the intellectual property of Esri and are used herein under license. Copyright $@ 2020$ Esri and its licensors. All rights reserved. 

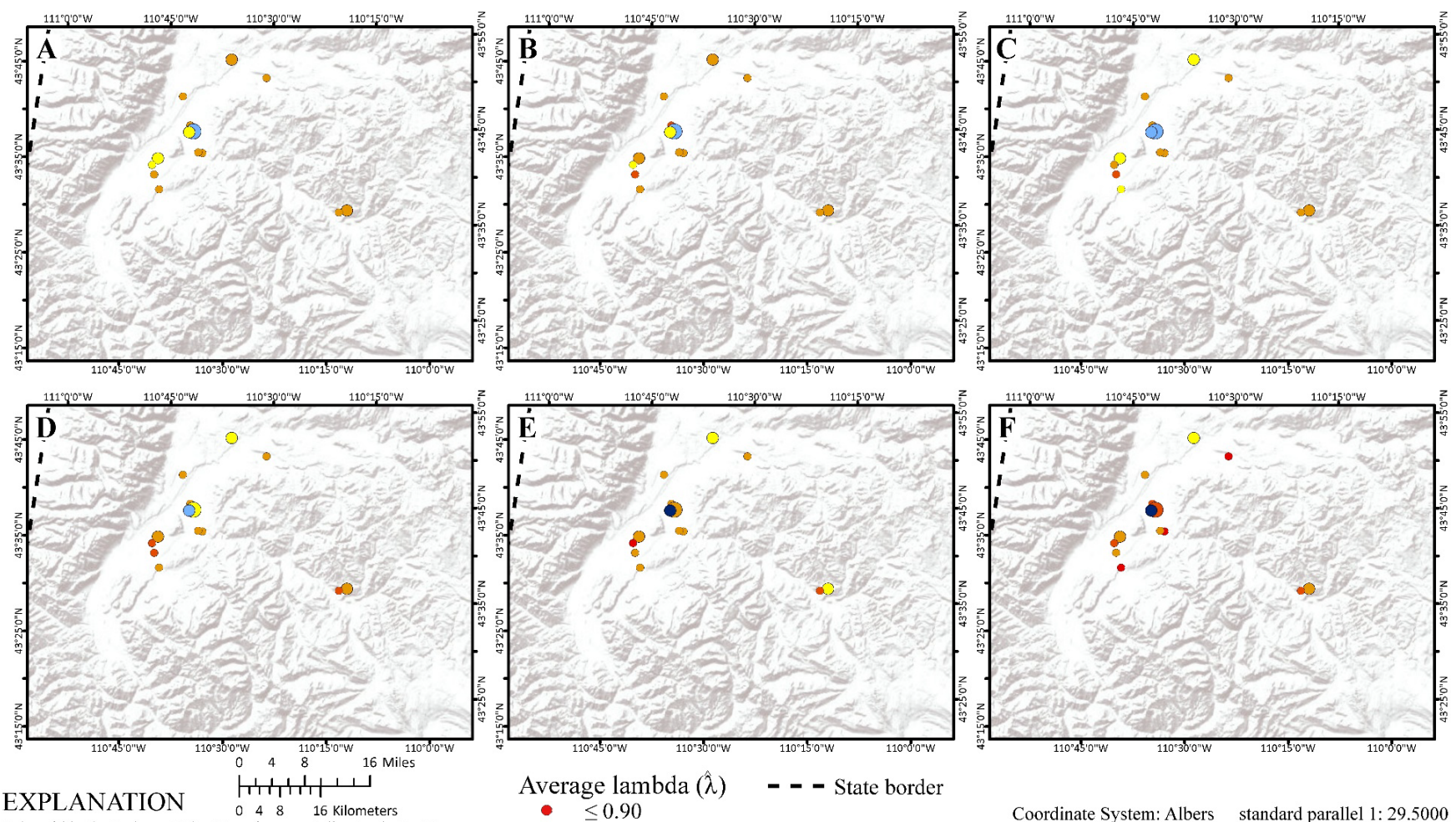

Leks within the Jackson Hole, Wyoming area (climate cluster C): colors represent average lambda $(\hat{\text {. }})$ values of sage-grouse populations over six temporal scales. The size of the symbol represents the maximum population size of the lek over the years of the analysis, relativized to the rangc-wide maximum population size across all lcks. Approximately 10 percent of the distribution of average $\hat{\lambda}$ are within the upper and lower color classes. Symbols are based on are within the upper and lower color classes. Symbols are based on

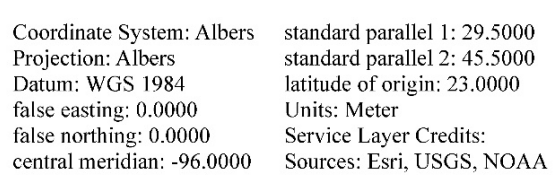

Figure 29. Spatial estimates of average annual population rate of change $(\hat{\lambda})$ in abundance of greater sage-grouse (Centrocercus urophasianus) at leks across six temporal scales based on periods of oscillation: $A$, Long (six periods); $B$, Medium/Long (five periods); $C$, Medium (four periods); $D$, Short/Medium (three periods); $E$, Short (two periods); and $F$, Recent (one period) within climate cluster $C$ (CC-C; Jackson Hole, Wyoming, area). Map images are the intellectual property of Esri and are used herein under license. Copyright $(\subset$ 2020 Esri and its licensors. All rights reserved.

Climate cluster D (CC-D; eastern area) consisted of 169 NCs encompassing 25,920,530-ha (table 7). Twenty-six NCs did not have sufficient lek data for trend estimates. Climate cluster D consisted of 2,944 leks, representing approximately 35.0 percent of the lek database. After QA/QC, 1,831 leks met criteria for use in the SSM (table 7) and totaled 33,535 field observations. Mean male count was 16.7 (95-percent confidence interval=16.5-16.9).

For CC-D, we estimated six population abundance nadirs that dated back to 1960 and included 1966, 1977, 1986, 1997, 2004, and 2014 (table 5; fig. 30A). We estimated $\hat{\lambda}$ at the short (2004-2019, two periods of oscillation over 15 years), medium (1986-2019, four periods over 33 years), and long temporal scales (1966-2019, six periods over 53 years) as 0.963 (95-percent CRI $=0.959-0.967$ ), 0.967 (95-percent $\mathrm{CRI}=0.960-0.972$ ), and 0.963 (95-percent $\mathrm{CRI}=0.960-0.968$ ), respectively (fig. 30B; table 6). Over the past 15,32 , and 53 years, sage-grouse populations declined 43.2, 66.7, and 86.8 percent, respectively. We estimated median $\hat{\lambda}$ to be less than 1.0 for $90.9,93.7$, and 99.3 percent of NCs across short, medium, and long temporal scales, respectively (fig. 31). We reported spatiotemporal variation in average annual $\hat{\lambda}$ across different NCs (fig. 31) and leks (fig. 32).

Climate cluster E (CC-E; Great Basin area) consisted of 241 NCs that encompassed 34,627,182-ha (table 7). Twenty-eight NCs lacked sufficient data to estimate trends. Climate cluster E consisted of 4,015 leks representing approximately 47.7 percent of the lek database. After QA/ QC, 2,187 leks met criteria for use in the SSM (table 7) and totaled 38,950 field observations. Mean male count was 17.3 (95-percent confidence interval=17.1-17.5). 

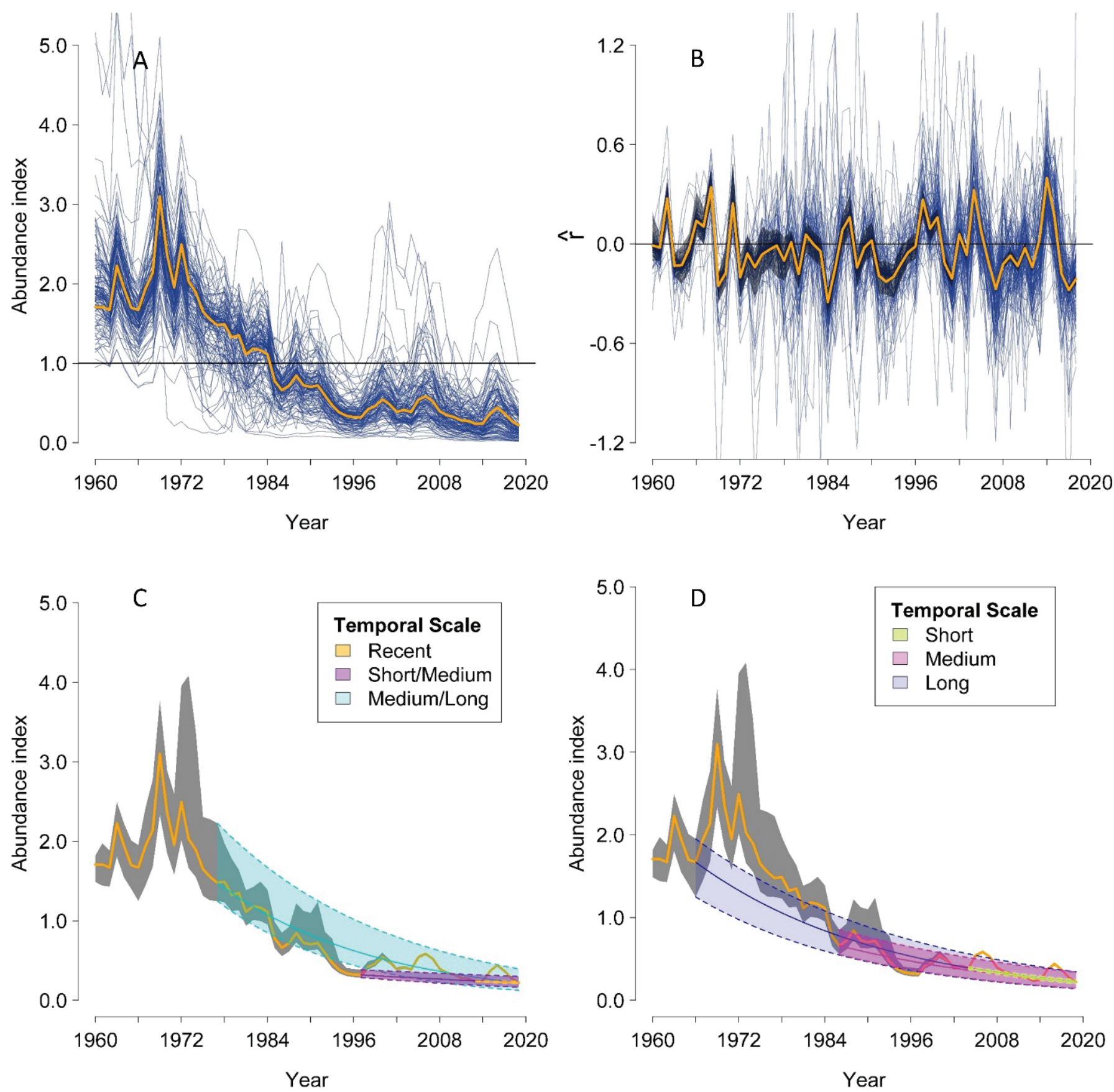

Figure 30. A, Abundance index (calculated as $\hat{N}$ divided by 60-year mean of $\hat{N}$ ); $B$, intrinsic rate of population change $(\hat{r})$ of greater sage-grouse (Centrocercus urophasianus) within D (CC-D; eastern area) from lek observations used to model population trends during 1960-2019; $C$, Median estimate of abundance trend (colored lines) across temporal scales based on periods of oscillation: Recent (one period), Short/Medium (three periods), and Medium/Long (five periods); and D, Short (two periods), Medium (four periods), and Long (six periods), right to left. Yellow line represents median estimates and blue thin lines represent median values for neighborhood clusters $(A$ and $B)$. Horizontal thin black line represents $(A)$ rescaled long-term mean and $(B)$ neutral population growth. Colored areas represent 95-percent credible limits of trend estimates. Grey shaded areas represent 95-percent credible limits on abundance index (C and $D)$ and $\hat{r}(B)$. 

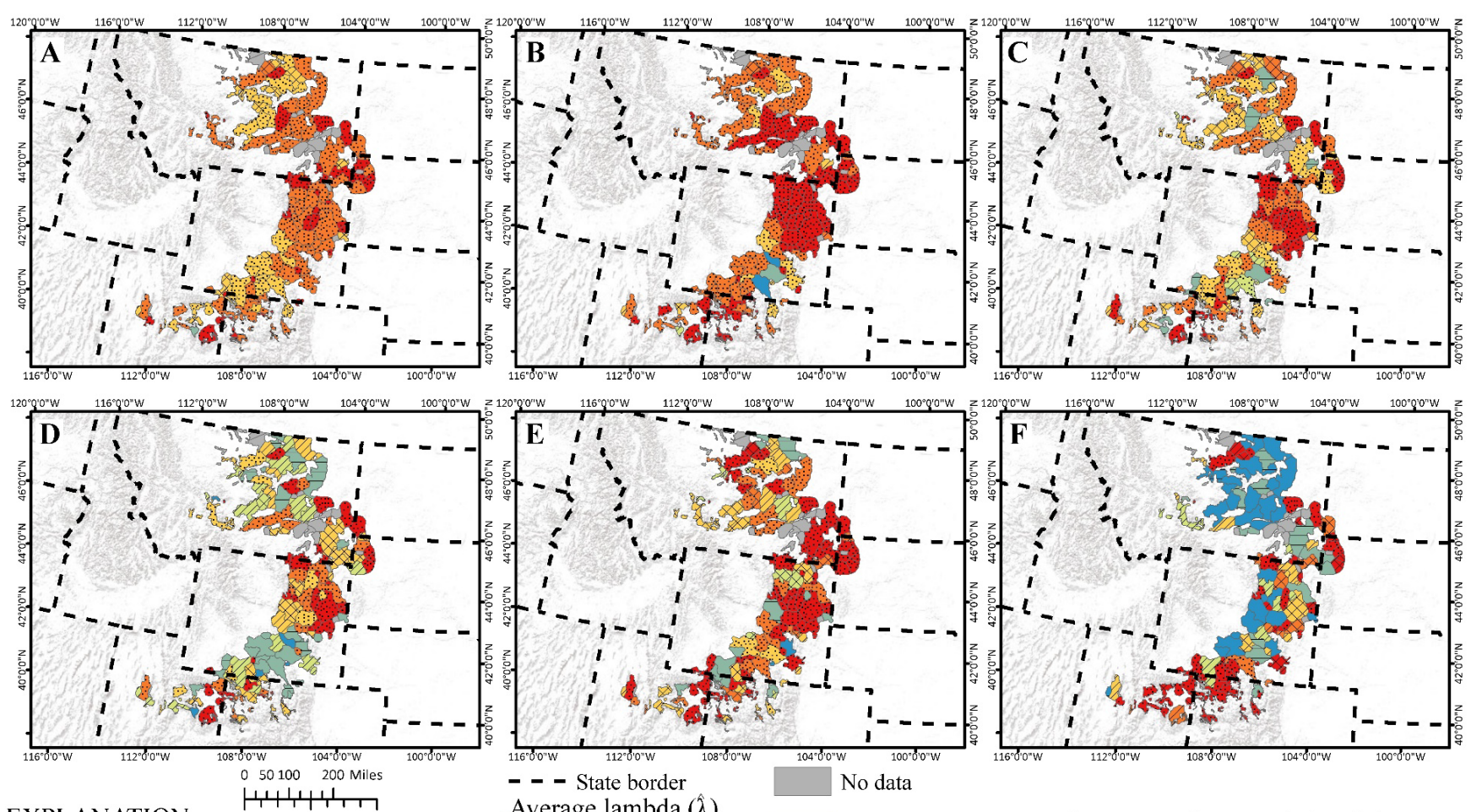

EXPLANATION

1
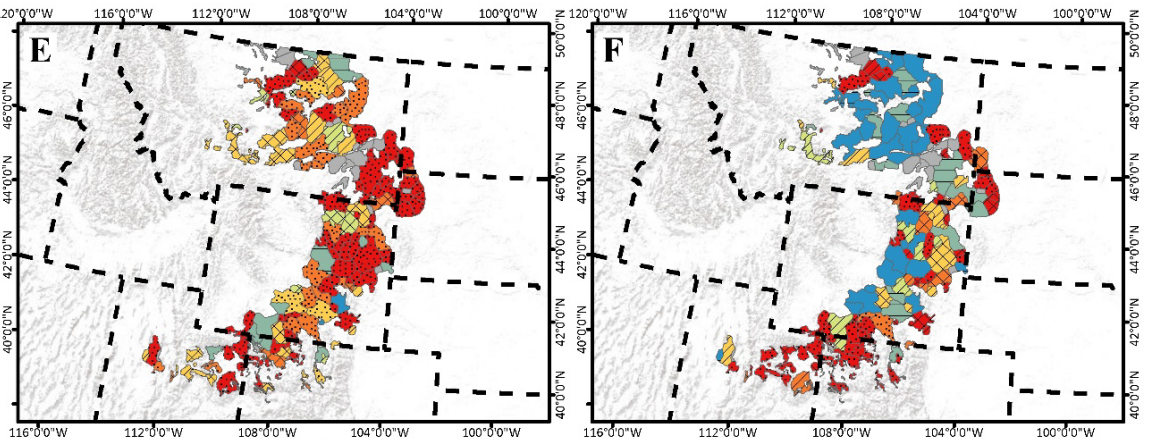

- - - State border Average lambda $(\hat{\lambda})$

$\leq 0.95$

$$
>0.95-0.97
$$

$>0.97-0.99$

No data

Proportion of posterior distribution representing decline $\geq 0.95<0.95-0.80<0.80-0.50<0.50-0.20<0.20$

$>0.99-1.00$

$>1.00-1.03$

1.03
Neighborhood clusters (Level 2) the within the eastern area (climate cluster D): polygon colors represent average lambda $(\hat{\lambda})$ values for sage-grouse populations over six temporal scales. Approximately 30 percent of the distribution of average $\lambda$ are with in the upper and lower color classes. Symbols are based on a range-wide distribution of values and all may not be represented here.

Figure 31. Spatial estimates of average annual population rate of change $(\hat{\lambda})$ in abundance of greater sage-grouse (Centrocercus urophasianus) at neighborhood clusters across six temporal scales based on periods of oscillation: $A$, Long (six periods); $B$, Medium/ Long (five periods); $C$, Medium (four periods); $D$, Short/Medium (three periods); $E$, Short (two periods); and $F$, Recent (one period) within climate cluster D (CC-D; eastern area). Map images are the intellectual property of Esri and are used herein under license. Copyright (C) 2020 Esri and its licensors. All rights reserved. 

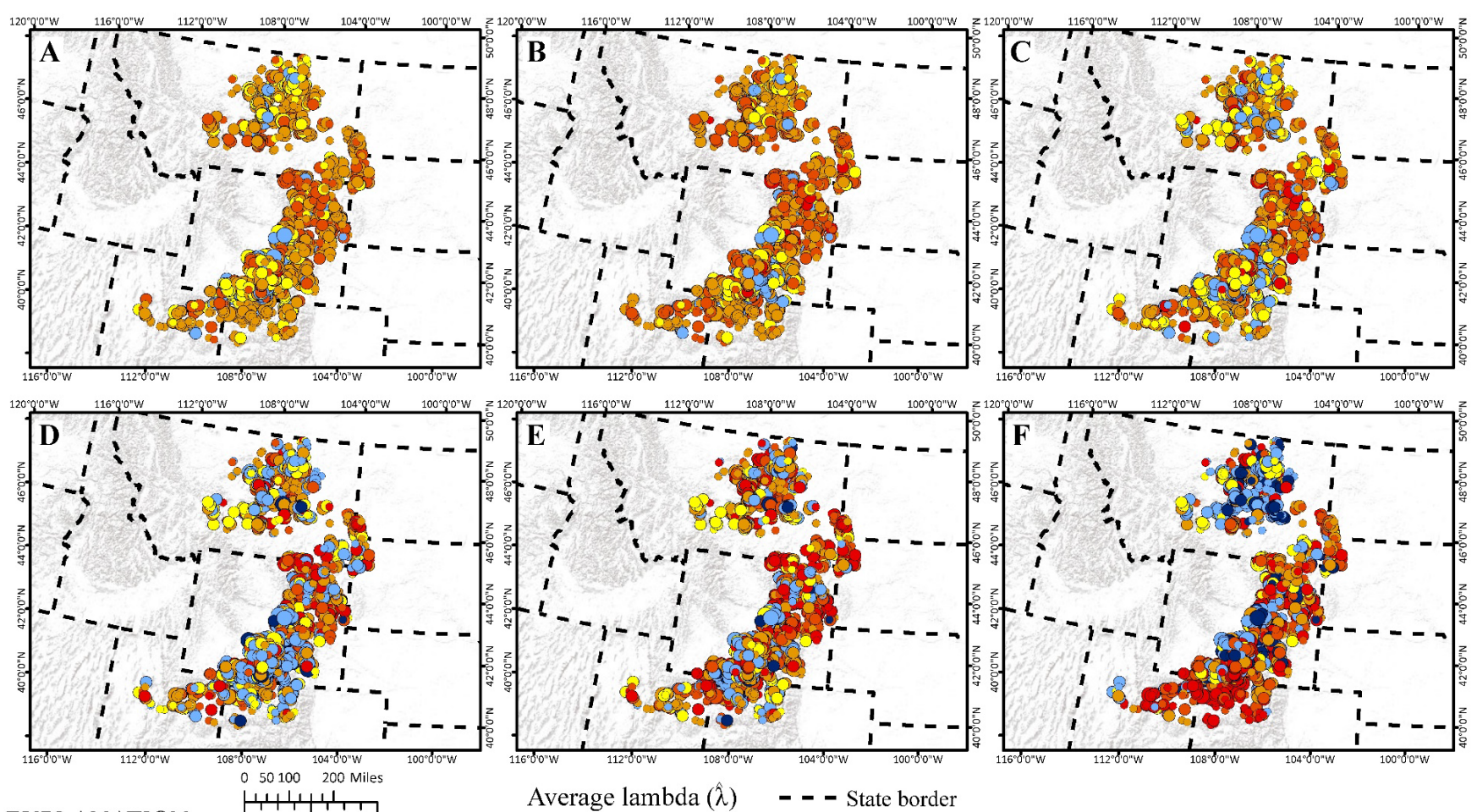

EXPLANATION

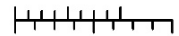
Leks within the eastern area (climate cluster D): colors represent average lambda $(\lambda)$ values of sage-grouse populations over six temporal scales. The size of the symbol represents the maximum
population size of the lek over the years of the analysis, relativized population size of the lek over the years of the analysis, relativized
to the range-wide maximum population size across all leks. to the range-wide maximum population size across all leks.
Approximately 10 percent of the distribution of average $\hat{\lambda}$ are $\Lambda$ pproximately 10 percent of the distribution of average $\lambda$ are
within the upper and lower color classes. Symbols are based on a range-wide distribution of values and all may not be represented here.

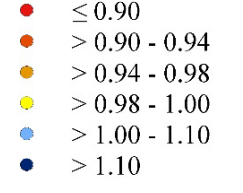

Size guide Maximum lek count Largest $Q_{\text {Smallest }}$
Coordinate System: Albers standard parallel 1:29.5000 Projection: Albers standard parallel 2: 45.5000 Datum: WGS 1984 false easting: 0.0000 false northing: 0.0000 central meridian: -96.0000 latitude of origin: 23.0000 Units: Meter Service Layer Credits: Sources: Esri, USGS, NOAA

Figure 32. Spatial estimates of average annual population rate of change $(\hat{\lambda})$ in abundance of greater sage-grouse (Centrocercus urophasianus) at leks across six temporal scales based on periods of oscillation: $A$, Long (six periods); $B$, Medium/Long (five periods); $C$, Medium (four periods); $D$, Short/Medium (three periods); E, Short (two periods); and $F$, Recent (one period) within climate cluster D (CC-D; eastern area). Map images are the intellectual property of Esri and are used herein under license. Copyright @ 2020 Esri and its licensors. All rights reserved.

For CC-E, we estimated six population abundance nadirs that dated back to 1960 and included 1967, 1975, 1985, 1996, 2002, and 2013 (table 5; fig. $33 A$ ). We estimated $\hat{\lambda}$ at the short (2002-2019, two periods of oscillation over 17 years), medium (1985-2019, four periods over 34 years), and long temporal scales (1967-2019, six periods over 52 years) as 0.968 (95-percent CRI $=0.964-0.971), 0.974$ (95-percent CRI $=0.963-0.979$ ), and 0.971 (95-percent CRI $=0.967-0.976$ ), respectively (fig. 33B; table 6). Over the past 17, 34, and 52 years, sage-grouse populations have declined 42.1, 58.9, and 78.0 percent, respectively. We estimated median $\hat{\lambda}$ to be less than 1.0 for 77.0, 85.0, and 92.5 percent of NCs across short, medium, and long temporal scales, respectively (fig. 34). We reported spatiotemporal variation in average annual $\hat{\lambda}$ across different NCs (fig. 34) and leks (fig. 35). 

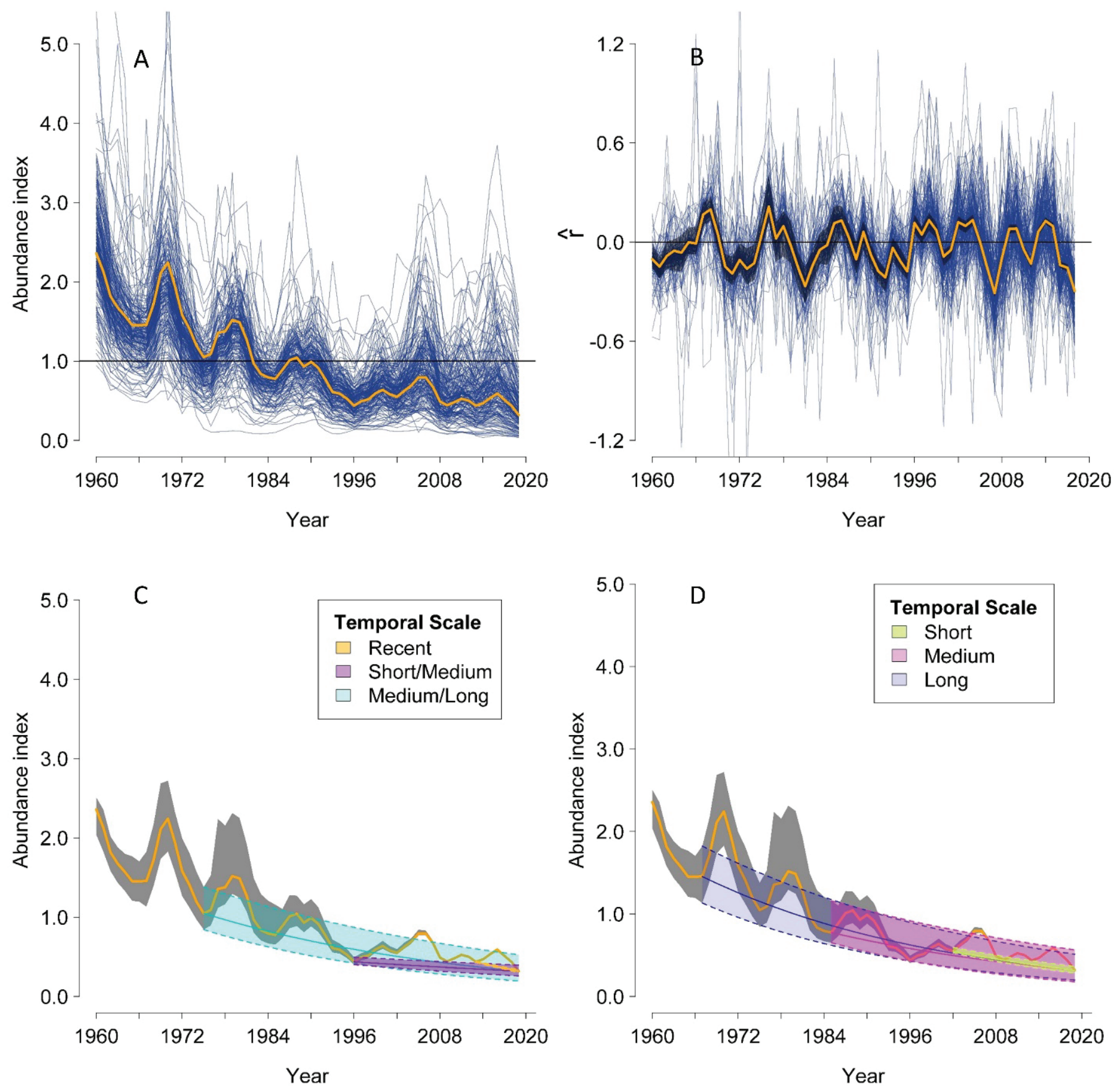

Figure 33. A, Abundance index (calculated as $\hat{N}$ divided by 60 -year mean of $\hat{N}$ ); $B$, intrinsic rate of population change $(\hat{r})$ of greater sage-grouse (Centrocercus urophasianus) within E (CC-E; Great Basin area) from lek observations used to model population trends during 1960-2019; C, Median estimate of abundance trend (colored lines) across temporal scales based on periods of oscillation: Recent (one period), Short/Medium (three periods), and Medium/Long (five periods); and $D$, Short (two periods), Medium (four periods), and Long (six periods), right to left. Yellow line represents median estimates and blue thin lines represent median values for neighborhood clusters $(A$ and $B)$. Horizontal thin black line represents $(A)$ rescaled long-term mean and $(B)$ neutral population growth. Colored areas represent 95-percent credible limits of trend estimates. Grey shaded areas represent 95-percent credible limits on abundance index $(C$ and $D)$ and $\hat{r}(B)$. 

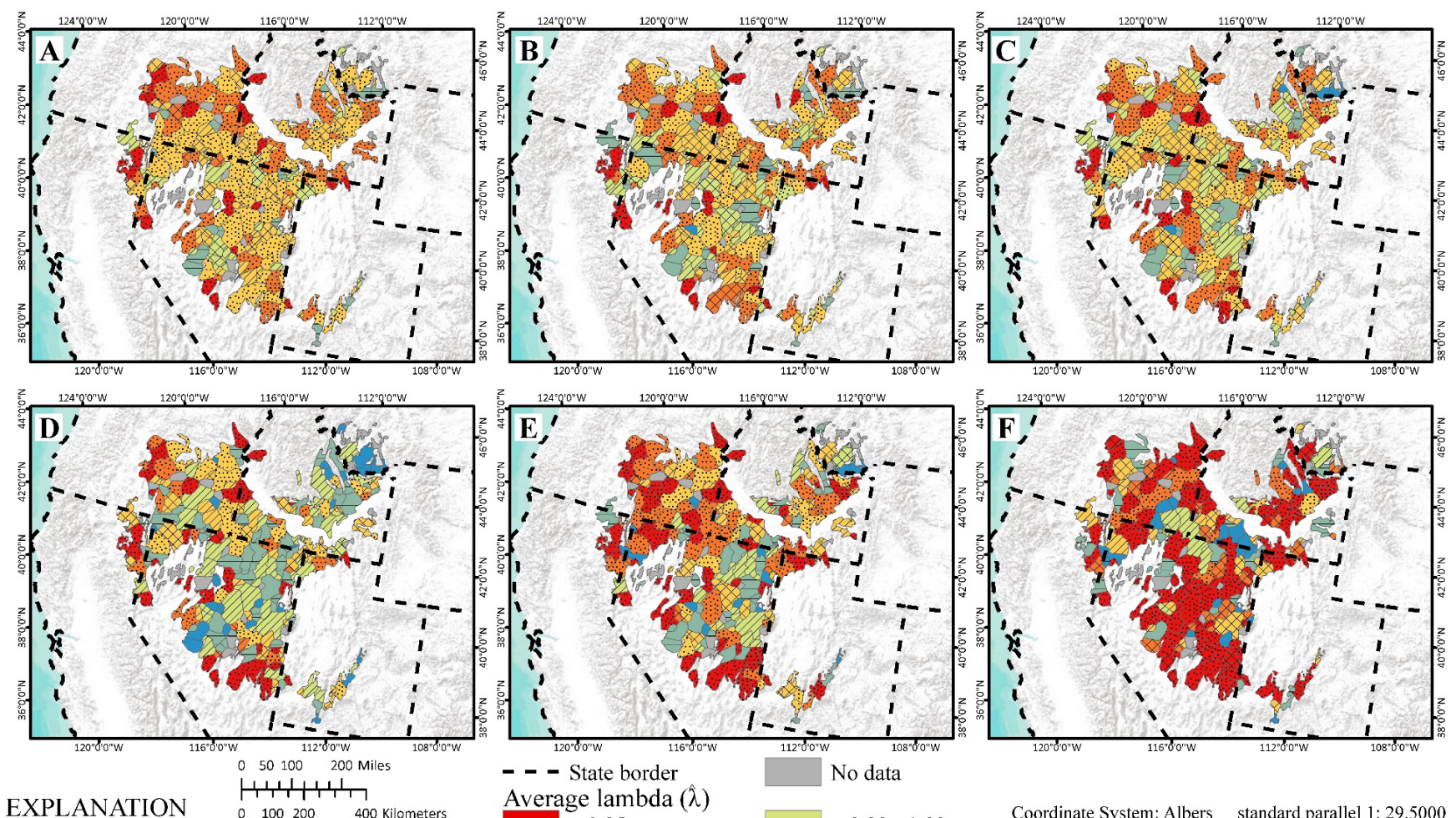

EXPLANATION

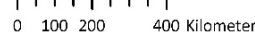

Neighborhood clusters (Level 2) within the Great Basin area (climate cluster E): polygon colors represent average lambda values $(\hat{\lambda})$ for sage-grouse populations over six temporal scales. Approximately 30 percent of the distribution of average $\hat{\lambda}$ are within the upper and lower color classes. ymbols are based on a range-wide distribution of values and all may not be represented here.

\section{- - - State border}

Average lambda $(\hat{\lambda})$

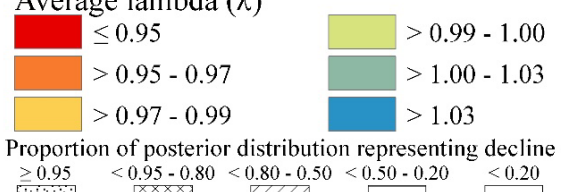

Coordinate System: Albers standard parallel 1: 29.5000 Projection: Albers standard parallel 2: 45.5000 Datum: WGS 1984 latitude of origin: 23.0000 false easting: $0.0000 \quad$ Units: Meter false northing: $0.0000 \quad$ Service Layer Credits: central meridian: - $96.0000 \quad$ Sources: Esri, USGS, NOAA

Figure 34. Spatial estimates of average annual population rate of change $(\hat{\lambda})$ in abundance of greater sage-grouse (Centrocercus urophasianus) at neighborhood clusters across six temporal scales based on periods of oscillation: $A$, Long (six periods); $B$, Medium/ Long (five periods); $C$, Medium (four periods); $D$, Short/Medium (three periods); $E$, Short (two periods); and $F$, Recent (one period) within climate cluster E (CC-E; Great Basin area). Map images are the intellectual property of Esri and are used herein under license. Copyright $(2020$ Esri and its licensors. All rights reserved. 

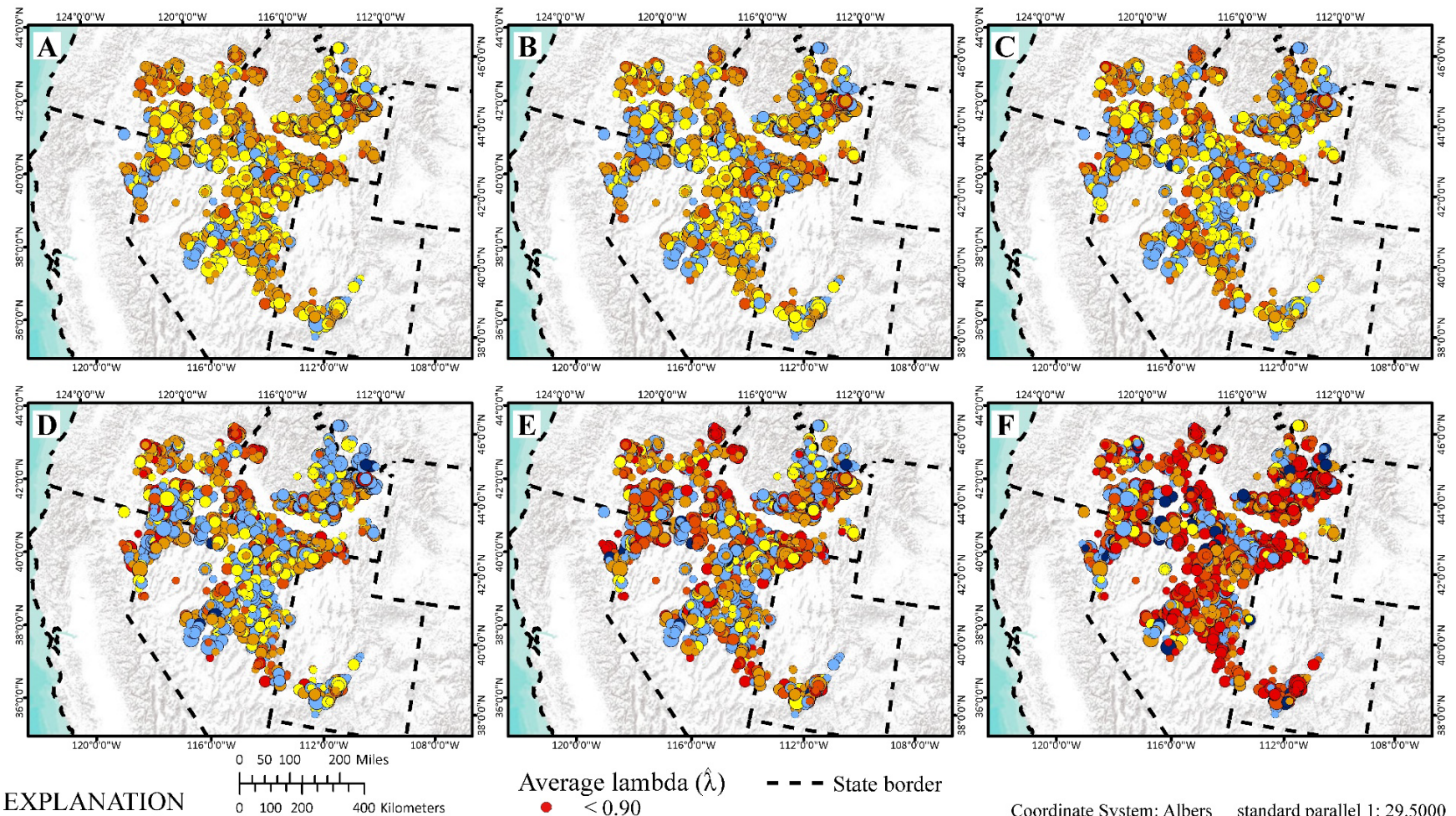

EXPLANATION $\quad 0 \quad 100200 \quad 400$ kilometers

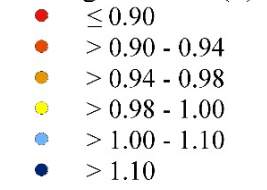

Size guide

Maximum lek count

Coordinate System: Albers Projection: Albers Datum: WGS 1984 false easting: 0.0000 false northing: 0.0000 central meridian: -96.0000

standard parallel 1:29.5000 standard parallel 2: 45.5000 average lambda (2) values of sage-grouse populations over six population size of the lek over the years of the analysis, relativized to the rangc-wide maximum population size across all leks. Approximately 10 percent of the distribution of average $\lambda$ are within the upper and lower color classes.Symbols are based on a rangewide distribution of values and all may not be represented here.

Figure 35. Spatial estimates of average annual population rate of change $(\hat{\lambda})$ in abundance of greater sage-grouse (Centrocercus urophasianus) at leks across six temporal scales based on periods of oscillation: $A$, Long (six periods); $B$, Medium/Long (five periods); $C$, Medium (four periods); $D$, Short/Medium (three periods); $E$, Short (two periods); and $F$, Recent (one period) within climate cluster $E$ (CC-E; Great Basin area). Map images are the intellectual property of Esri and are used herein under license. Copyright (C) 2020 Esri and its licensors. All rights reserved.

Climate cluster F (CC-F; western Wyoming area) consisted of $56 \mathrm{NCs}$ that encompassed 8,899,755-ha (table 7). Four NCs lacked sufficient data to estimate trends. Climate cluster F consisted of 1,253 leks and represented approximately 14.9 percent of the lek database. After QA/QC, 974 leks met criteria for inclusion in the SSM (table 7) and totaled 19,850 field observations. Mean male count was 23.7 (95-percent confidence interval=23.3-24.2).

For CC-F, we estimated six population abundance nadirs that dated back to 1960 and included 1966, 1973, 1987, 1996, 2002, and 2013 (table 5; fig. 36A). We estimated $\hat{\lambda}$ at the short (2002-2019, two periods of oscillation over 17 years), medium (1987-2019, four periods over 32 years), and long temporal scales (1966-2019, six periods over 53 years) as 0.991 (95-percent CRI=0.988-0.995), 0.976 (95-percent $\mathrm{CRI}=0.966-0.980)$, and 0.980 (95-percent $\mathrm{CRI}=0.975-0.987)$, respectively (fig. 36B; table 6). Over the past 17, 32, and 53 years, sage-grouse populations have declined 13.9, 54.7, and 66.0 percent, respectively. We estimated median $\hat{\lambda}$ to be less than 1.0 for $67.3,84.6$, and 86.5 percent of $\mathrm{NCs}$ across short, medium, and long temporal scales, respectively (fig. 37). We reported spatiotemporal variation in average annual $\hat{\lambda}$ across different NCs (fig. 37) and leks (fig. 38). 

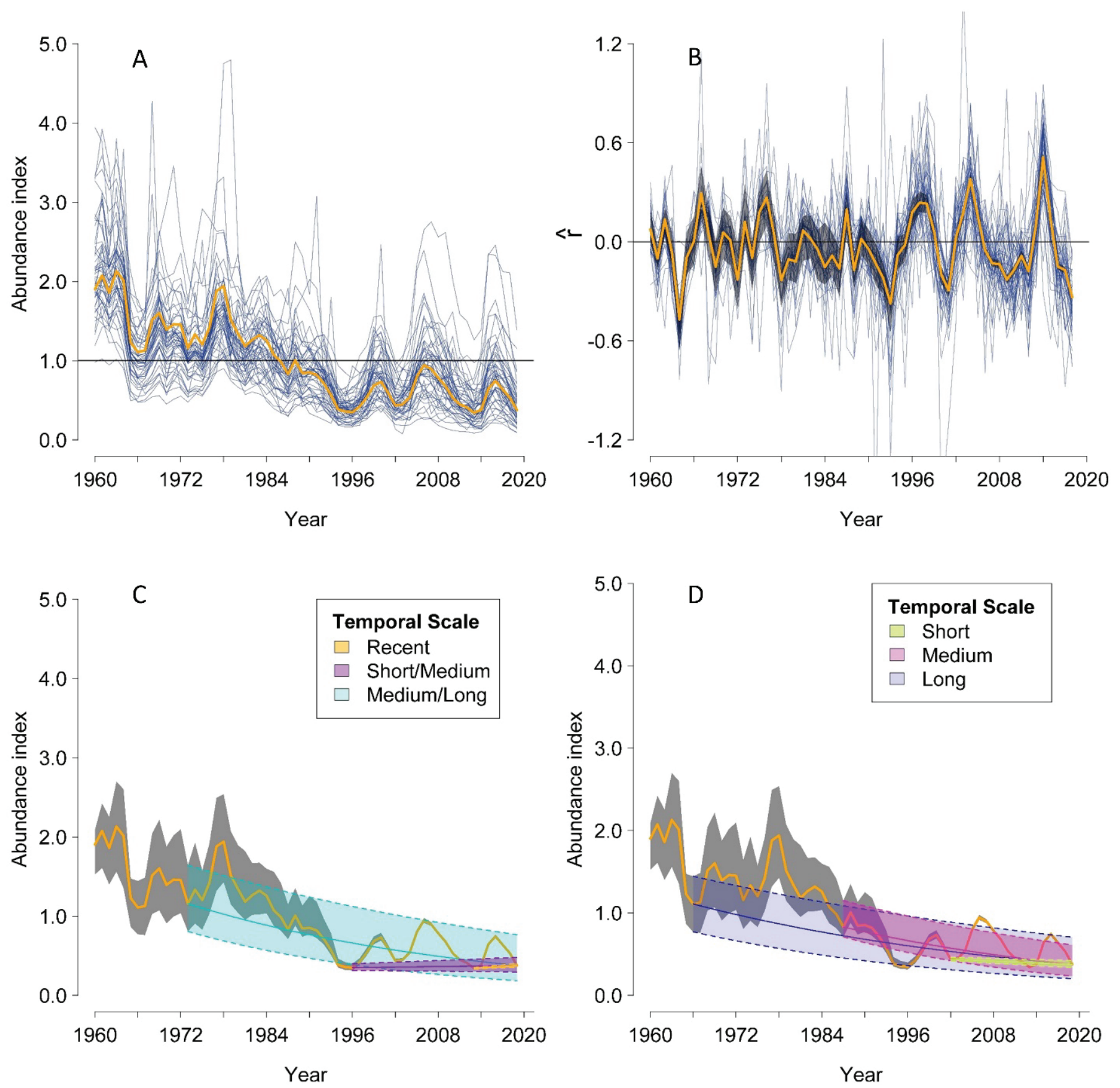

Figure 36. A, Abundance index (calculated as $\hat{N}$ divided by 60-year mean of $\hat{N}$ ); $B$, intrinsic rate of population change $(\hat{r})$ of greater sage-grouse (Centrocercus urophasianus) within F (CC-F; western Wyoming area) from lek observations used to model population trends during 1960-2019; C, Median estimate of abundance trend (colored lines) across temporal scales based on periods of oscillation: Recent (one period), Short/Medium (three periods), and Medium/Long (five periods); and D, Short (two periods), Medium (four periods), and Long (six periods), right to left. Yellow line represents median estimates and blue thin lines represent median values for neighborhood clusters $(A$ and $B)$. Horizontal thin black line represents $(A)$ rescaled long-term mean and $(B)$ neutral population growth. Colored areas represent 95-percent credible limits of trend estimates. Grey shaded areas represent 95-percent credible limits on abundance index $(C$ and $D)$ and $\hat{r}(B)$. 

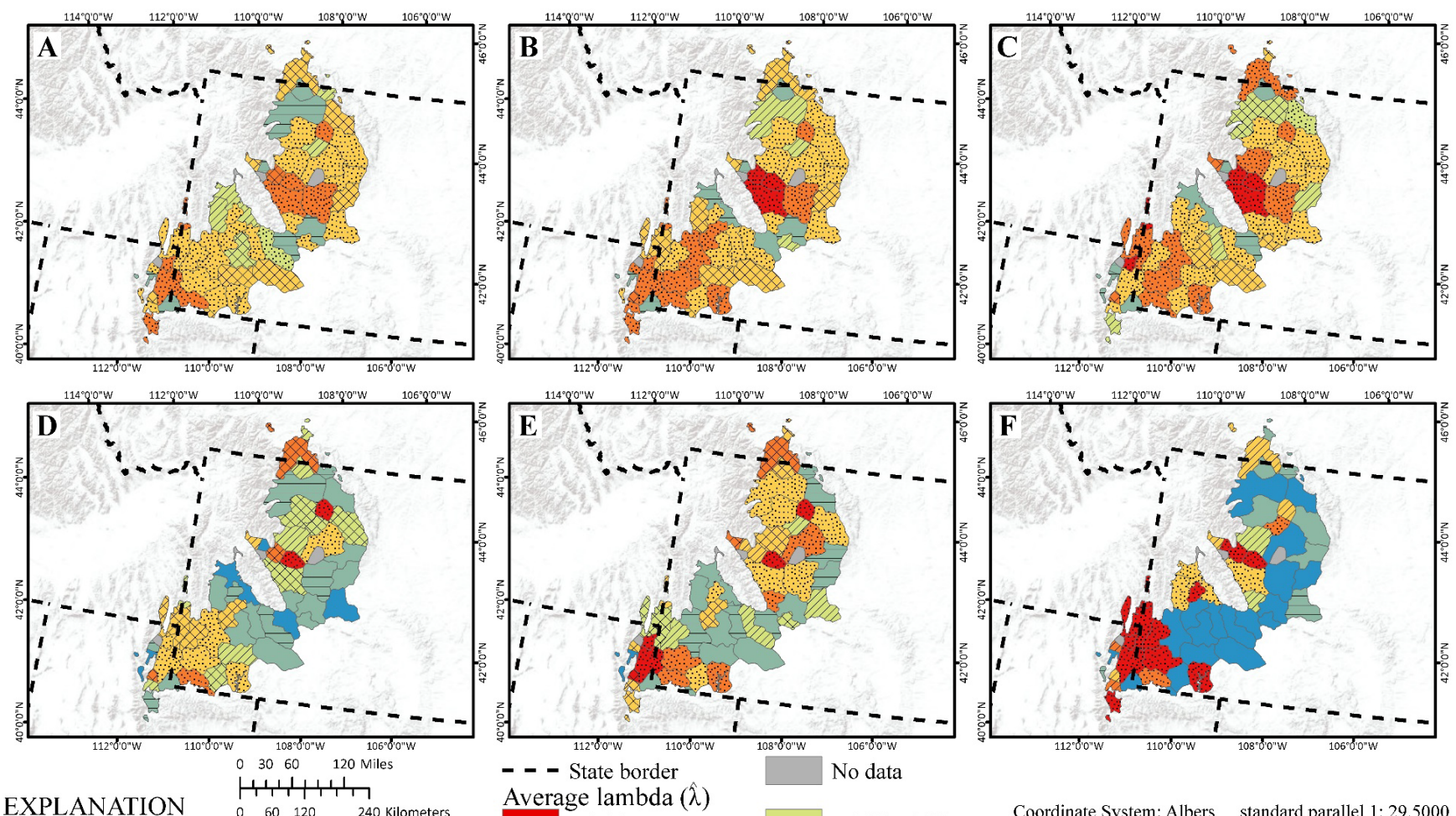

\section{EXPLANATION}

$\begin{array}{llll}0 & 60 & 120 & 240 \text { Kilometers }\end{array}$

Neighborhood clusters (Level 2) within the western Wyoming area (climate cluster F): polygon colors represent average lambda ( $\hat{\lambda})$ values for sage-grouse populations six temporal scales. Approximately 30 percent of the distribution of average $\hat{\lambda}$ are within the upper and lower color classes. Symbols are based on a range-

- - - State border Average lambda $(\hat{\lambda})$

$\leq 0.95$ $>0.95-0.97$ $>0.97-0.99$

Proportion of posterior distribution representing decline $>0.95<0.95-0.80<0.80 \cdot 0.50<0.50 \cdot 0.20<0.20$

No data

$>0.99-1.00$

$>1.00-1.03$

1.03

$x$

Figure 37. Spatial estimates of average annual population rate of change $(\hat{\lambda})$ in abundance of greater sage-grouse (Centrocercus urophasianus) at neighborhood clusters across six temporal scales based on periods of oscillation: $A$, Long (six periods); $B$, Medium/ Long (five periods); $C$, Medium (four periods); $D$, Short/Medium (three periods); $E$, Short (two periods); and $F$, Recent (one period) within climate cluster F (CC-F; western Wyoming area). Map images are the intellectual property of Esri and are used herein under license. Copyright (C) 2020 Esri and its licensors. All rights reserved. 

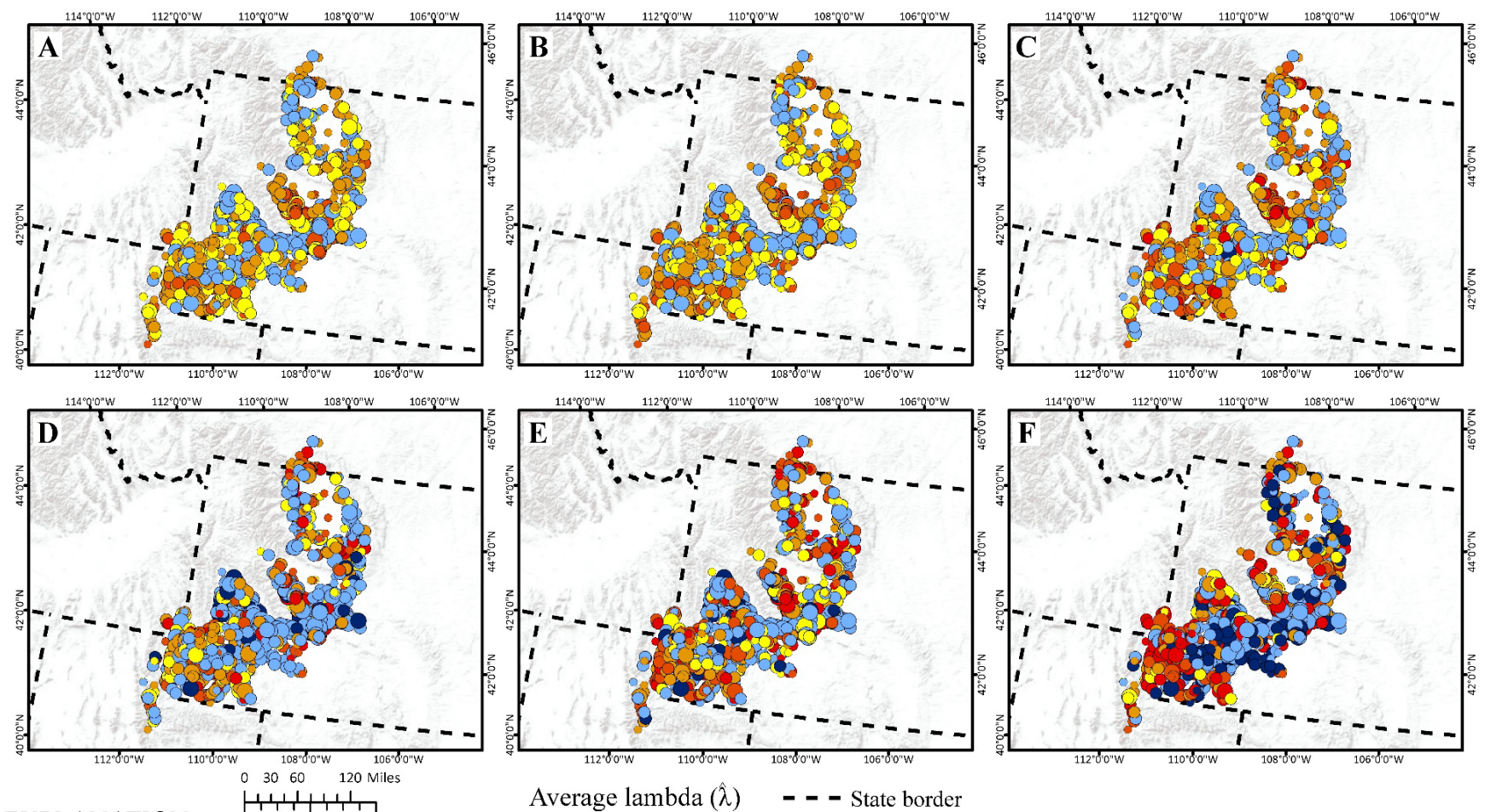

EXPLANATION

Leks with in the western Wroming are (climute cluster represent average lambda $(\hat{\lambda})$ values of sage-grouse populations over six temporal scales. The size of the symbol represents the

maximum population size of the lek over the years of the analysis, relativized to the range-wide maximum population size across all leks. Approximately 10 pereent of the distribution of average $\lambda$ are within the upper and lower color classes. Symbols are based on a
rangc-wide distribution of values and all may not be represented here.
Average lambda $(\hat{\lambda})$
$>0.90-0.94$
$>0.94-0.98$
$>0.98-1.00$
$>1.00-1.10$
$->1.10$
- - - State border
Size guide
Maximum lek count
Largest

Coordinate System: Albers Projection: Albers Datum: WGS 1984 false easting: 0.0000 false northing: 0.0000 central meridian: -96.0000 standard parallel 1: 29.5000 standard parallel 2: 45.5000 latitude of origin: 23.0000 Units: Meter

Service Layer Credits: Sources: Esri, USGS, NOAA

Figure 38. Spatial estimates of average annual population rate of change $(\hat{\lambda})$ in abundance of greater sage-grouse (Centrocercus urophasianus) at leks across six temporal scales based on periods of oscillation: $A$, Long (six periods); $B$, Medium/Long (five periods); $C$, Medium (four periods); $D$, Short/Medium (three periods); $E$, Short (two periods); and $F$, Recent (one period) within climate cluster $F$ (CC-F; western Wyoming area). Map images are the intellectual property of Esri and are used herein under license. Copyright (C) 2020 Esri and its licensors. All rights reserved. 


\section{Probability of Future Extirpation}

We reported substantial overlap in the posterior distributions of $\hat{r}$ for models run on training and validation datasets (fig. 39), which indicated a good measure of predictability across the three oscillations (posterior predictive $p$ value $=0.55$ ). The mean extirpation probability calculated across all leks was $0.48(\mathrm{SD}=0.26), 0.58(\mathrm{SD}=0.20)$, and $0.64(\mathrm{SD}=0.16)$ for short, medium, and long temporal scales, respectively. However, predicted extirpation rates for leks appeared to take on a bimodal distribution with peak probabilities located around 0.25 and 0.83 for the short temporal scale (fig. 40A), 0.41 and 0.83 for the medium temporal scale (fig. 40C), and 0.57 and 0.85 for the long temporal scale (fig. 40E). Approximately 46, 60, and 78 percent of leks possessed an extirpation probability greater than 0.5 for short, medium, and long temporal scales, respectively. There was substantial variation in the mean extirpation rate across $\mathrm{CC}$ when summarized at both the lek and NC scale (table 8). The spatial distribution of extirpation probabilities demonstrated higher concentrations of higher probabilities at the edges of the species range (fig. 41).

The mean extirpation probability calculated across all NCs was $0.16(\mathrm{SD}=0.26), 0.27(\mathrm{SD}=0.26)$, and $0.37(\mathrm{SD}=0.25)$ for short, medium, and long temporal scales, respectively. Approximately 12, 19, and 30 percent of NCs possessed an extirpation probability greater than 0.5 for short, medium, and long temporal scales, respectively (fig. 40B). In terms of spatial distribution, the highest probabilities of NC scale extirpation appeared to occur more often at the periphery of the species range (fig. 42). Conversely, interior NCs consisted primarily of low extirpation probabilities ranging from 0 to 0.25 .

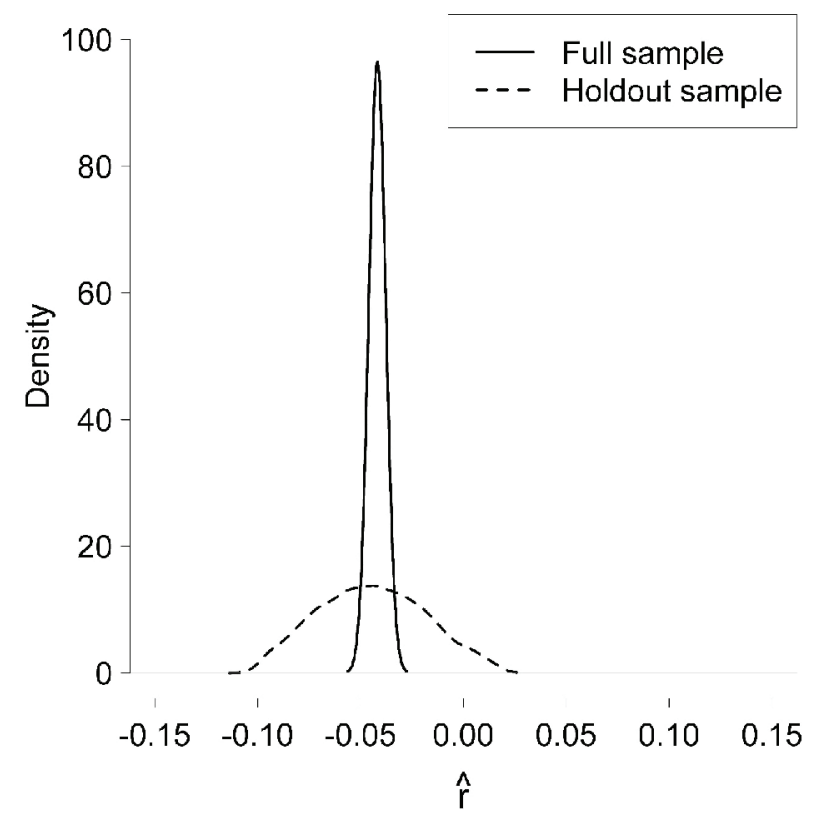

Figure 39. Posterior predictive probability distribution from state-space models for greater sage-grouse (Centrocercus urophasianus) population growth on a holdout sample (1987-2019; three full oscillations) using a restricted dataset (1960-1986) compared to estimates over the same time frame using a full (validation) dataset (1960-2019).

Table 8. Model predictions of extirpation probabilities for greater sage-grouse (Centrocercus urophasianus) leks and neighborhood clusters (NC), summarized in terms of mean and standard error, at different temporal scales (Short, [two periods, approximately 19 years], Medium [four periods, approximately 38 years], and Long [six periods, approximately 56 years]) based on an average period of oscillation (9.4 years) within the western United States.

[CC, climate cluster; A, Bi-state area; B, Washington area; C, Jackson Hole, Wyoming area; D, eastern area; E, Great Basin area; F, Wyoming]

\begin{tabular}{|c|c|c|c|c|c|c|}
\hline \multirow{3}{*}{ CC } & \multicolumn{6}{|c|}{ Temporal scales } \\
\hline & \multicolumn{2}{|c|}{$\begin{array}{c}\text { Short } \\
\text { (two oscillations) }\end{array}$} & \multicolumn{2}{|c|}{$\begin{array}{c}\text { Medium } \\
\text { (four oscillations) }\end{array}$} & \multicolumn{2}{|c|}{$\begin{array}{c}\text { Long } \\
\text { (six oscillations) }\end{array}$} \\
\hline & Lek & NC & Lek & NC & Lek & NC \\
\hline A & $0.5480(0.0322)$ & $0.4149(0.1194)$ & $0.6534(0.0226)$ & $0.5602(0.1051)$ & $0.7176(0.0174)$ & $0.6650(0.0865)$ \\
\hline B & $0.6742(0.0313)$ & $0.1038(0.0914)$ & $0.7664(0.0197)$ & $0.2806(0.1546)$ & $0.8187(0.0141)$ & $0.4379(0.1553)$ \\
\hline $\mathrm{C}$ & $0.6021(0.0587)$ & $0.1585(0.1165)$ & $0.6974(0.0387)$ & $0.3708(0.1582)$ & $0.7447(0.0311)$ & $0.5285(0.1358)$ \\
\hline $\mathrm{D}$ & $0.5284(0.00615)$ & $0.2258(0.0258)$ & $0.6203(0.00445)$ & $0.3397(0.0246)$ & $0.6797(0.0035)$ & $0.4465(0.0221)$ \\
\hline E & $0.4721(0.00545)$ & $0.1290(0.0148)$ & $0.5712(0.00396)$ & $0.2415(0.0156)$ & $0.6300(0.00312)$ & $0.3463(0.0148)$ \\
\hline $\mathrm{F}$ & $0.4107(0.00857)$ & $0.0846(0.0255)$ & $0.5058(0.00654)$ & $0.1503(0.0297)$ & $0.5668(0.00526)$ & $0.2246(0.0302)$ \\
\hline
\end{tabular}



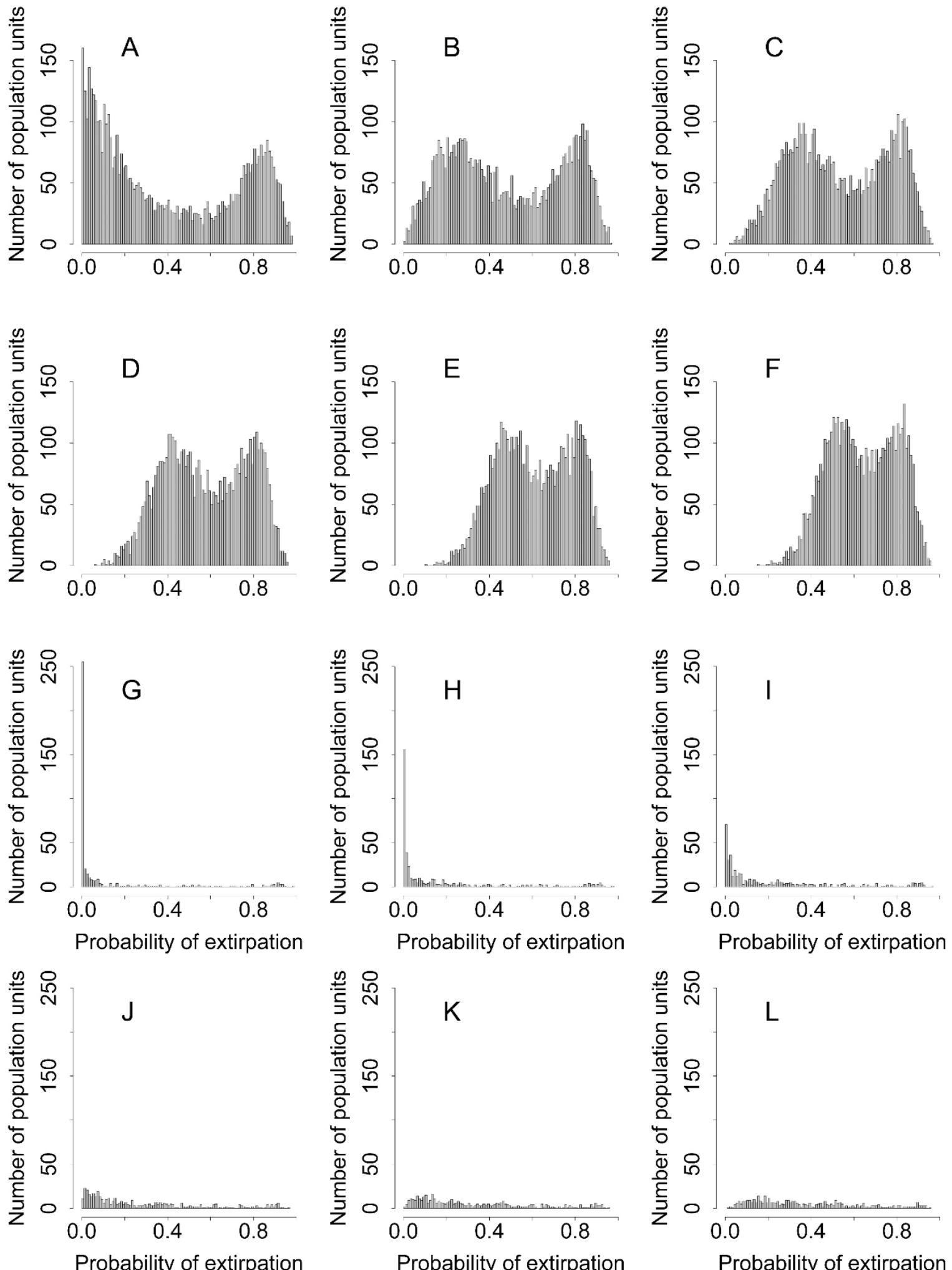

Figure 40. Distribution of model predicted extirpation probabilities for greater sage-grouse (Centrocercus urophasianus) at leks for $A$, Predicted Recent (one period, approximately 10 years); $B$, Predicted Short (two periods, approximately 19 years); $C$, Predicted Short/ Medium (three periods, approximately 29 years); $D$, Medium (four periods, approximately 38 years); $E$, Medium/Long (five periods, approximately 48 years); and $F$, Long (six periods, approximately 56 years), and neighborhood clusters $(G-L)$, over the same respective temporal scales, based on average period of oscillation within the western United States. Extirpation probabilities were calculated as the proportion of posterior samples with less than two sage-grouse. 

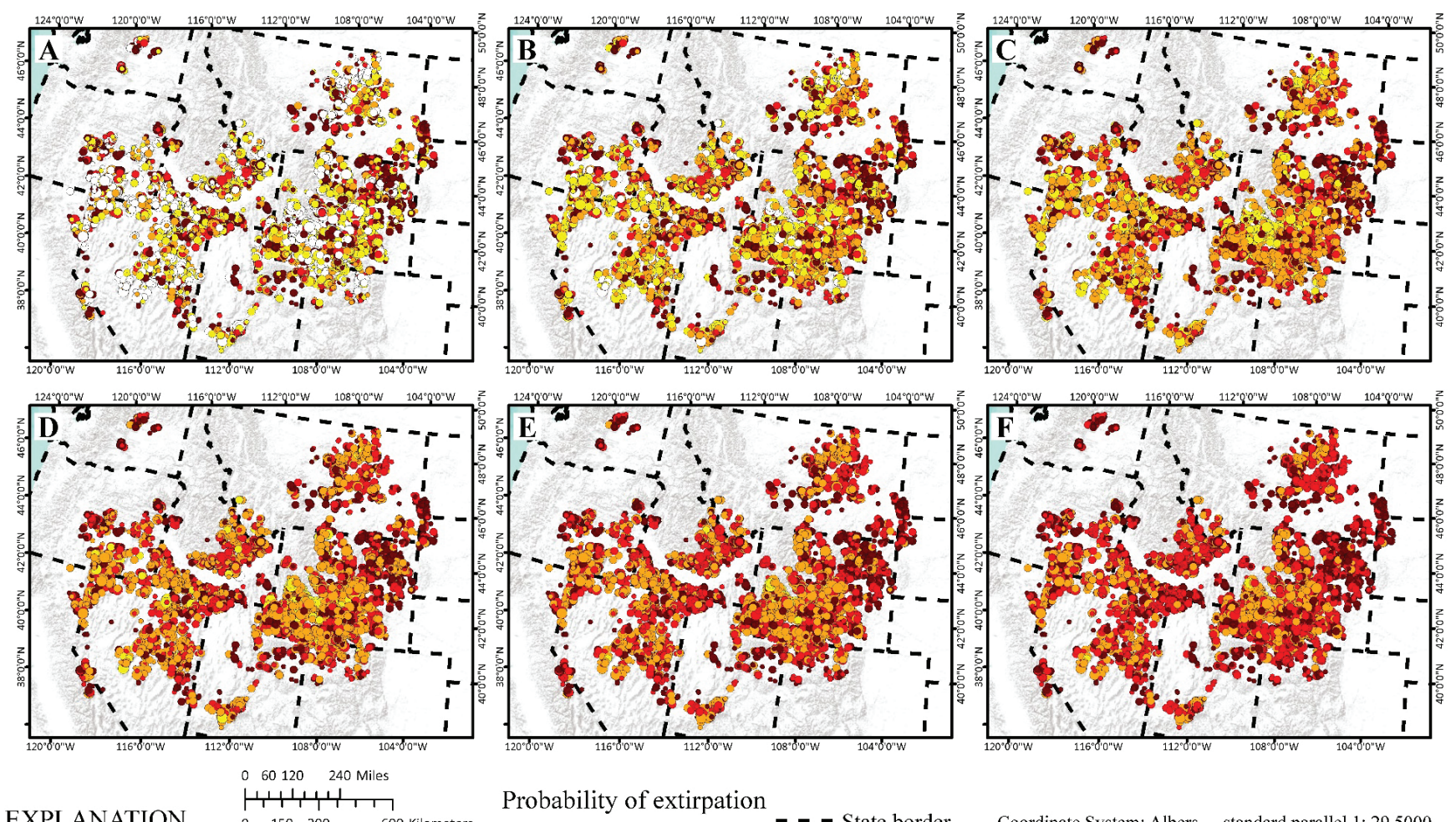

EXPLANATION

Leks: colors represent the probability of extirpation over six temporal scales. Darker colors correspond to a higher probability. The size of the symbol represents the maximum population size of the lek, relativized to the range-wide maximum population size across all leks.

Probability of extirpation

$\begin{array}{ll}0-0.10 & -- \text { State border } \\ >0.10-0.25 & \text { Size guide } \\ >0.25-0.50 & \text { Maximum lek count } \\ \quad>0.50-0.75 & \text { Largest } \\ \quad>0.75 & \text { Smallest }\end{array}$

$\begin{array}{ll}\text { Coordinate System: Albers } & \text { standard parallel 1: } 29.5000 \\ \text { Projection: Albers } & \text { standard parallel 2: } 45.5000 \\ \text { Datum: WGS 1984 } & \text { latitude of origin: } 23.0000 \\ \text { false easting: 0.0000 } & \text { Units: Meter } \\ \text { false northing: 0.0000 } & \text { Service Layer Credits: } \\ \text { central meridian: }-96.0000 & \text { Sources: Esri, USGS, NOAA }\end{array}$

Figure 41. Spatial model predictions of extirpation probabilities for greater sage-grouse (Centrocercus urophasianus) at leks across different temporal scales for $A$, Predicted Recent (one period, approximately 10 years); $B$, Predicted Short (two periods, approximately 19 years); $C$, Predicted Short/Medium (three periods, approximately 29 years); $D$, Medium (four periods, approximately 38 years); $E$, Medium/Long (five periods, approximately 48 years); and $F$, Long (six periods, approximately 56 years), based on average period of oscillation within the western United States. Extirpation probabilities were calculated as the proportion of posterior samples with less than two sage-grouse. Map images are the intellectual property of Esri and are used herein under license. Copyright @ 2020 Esri and its licensors. All rights reserved. 

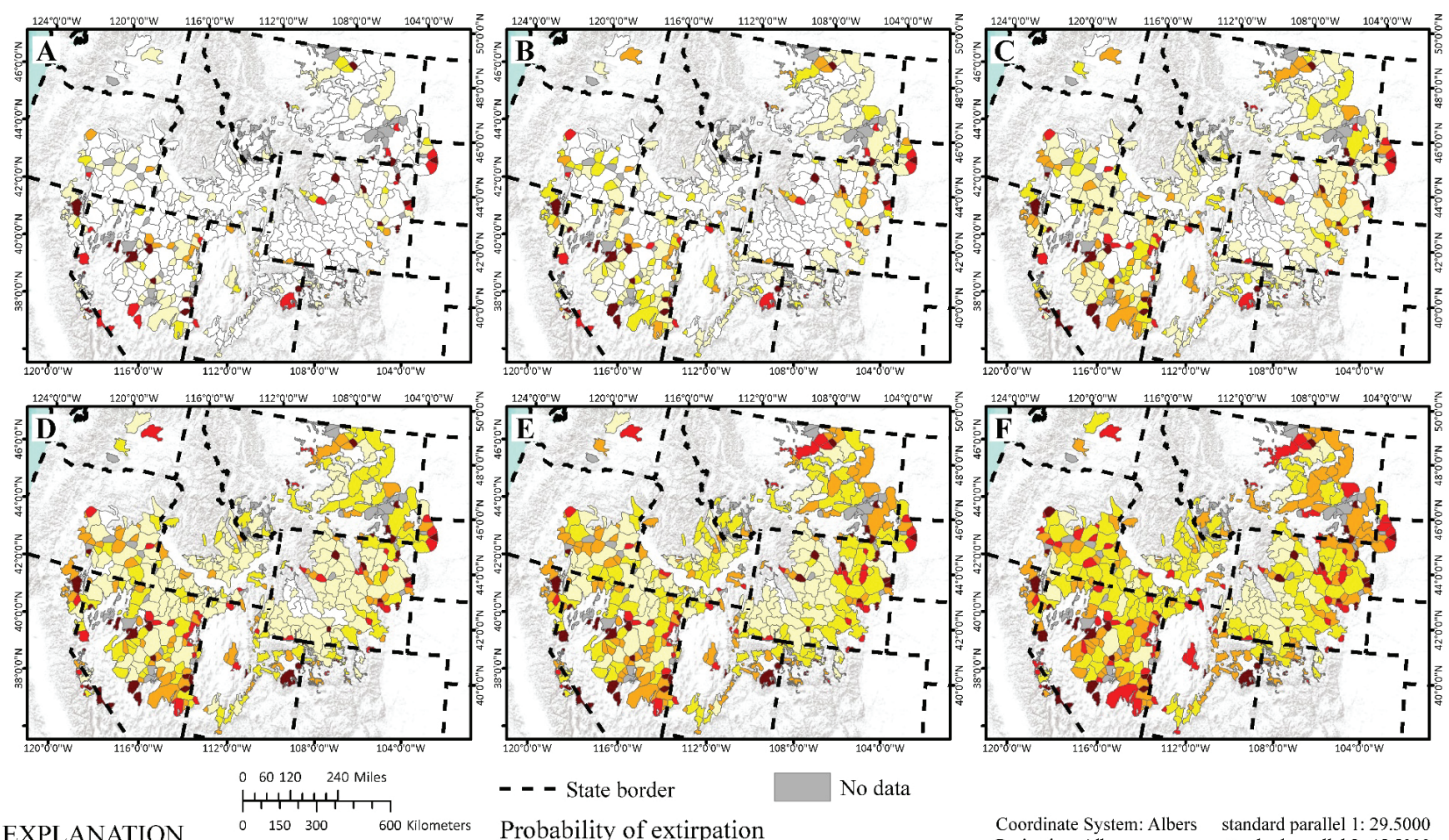

\section{EXPLANATION}

Probability of extirpation

Neighborhood clusters (Level 2): polygon colors represent the probability of extirpation over six temporal scales. Darker colors correspond to a higher probability.

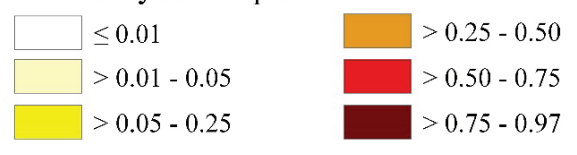

Figure 42. Spatial model predictions of extirpation probabilities for greater sage-grouse (Centrocercus urophasianus) at neighborhood clusters across different temporal scales for $A$, Predicted Recent (one period, approximately 10 years); $B$, Predicted Short (two periods, approximately 19 years); $C$, Predicted Short/Medium (three periods, approximately 29 years); $D$, Medium (four periods, approximately 38 years); $E$, Medium/Long (five periods, approximately 48 years); and $F$, Long (six periods, approximately 56 years), based on average period of oscillation within the western United States. Extirpation probabilities were calculated as the proportion of posterior samples with less than two sage-grouse. Map images are the intellectual property of Esri and are used herein under license. Copyright (C) 2020 Esri and its licensors. All rights reserved. 


\section{Objective 4. Targeted Annual Warning System}

\section{Background}

Population ecologists have long recognized that a fundamental component of population dynamics involves aligning demographic processes to the appropriate scale across space and time (Levin, 1992), where intrinsic and extrinsic factors influence those processes. For example, short-term fluctuations in population abundance are caused by environmental and demographic stochasticity (Morris and Doak, 2002), whereas climatic variation, particularly in productivity-limited systems, tends to influence longer-term cyclic patterns across larger spatial scales in the absence of local perturbations (Ranta and others, 1995; Lindström and others, 1996). Moreover, wildlife populations are spatially structured, meaning that local and broad scale relationships among interconnected populations require delineation, and failure to account for scale-specific processes (spatial and temporal) can result in significant misinterpretation of observed patterns in population growth and decline (Sadoul, 1997; Bissonette, 2017). Incorporating such complexity into tractable frameworks for targeted management actions is challenging, and often absent in traditional population monitoring programs (Nichols and Williams, 2006).

Nevertheless, management actions can be better facilitated if monitoring programs become rooted in a priori hypotheses based on how populations are expected to perform in response to environmental conditions that drive trends at larger scales while accounting for underlying demographic process and ecosystem productivity (Yoccoz and others, 2001; Nichols and Williams, 2006; Williams, 2011). For effective conservation, it is crucial to understand whether declines in abundance are associated with environmental stochasticity or landscape disturbances. Deterministic factors, like habitat loss associated with disturbance, are likely more amenable to direct land management actions compared to stochastic factors like climatic conditions.

Furthermore, the contribution of intrinsic factors responsible for regulating density-dependent or extrinsic environmental and anthropogenic factors that limit populations often differ when measured at varying spatial extents; thus, understanding such differences provides key information on the mechanisms underlying changes in population abundance (Fuhlendorf and others, 2002; Bissonette, 2017; Miguet and others, 2016). When comparative trends at local scales deviate significantly from larger scale estimates, it can be a signal for management actions directed at stemming population declines at relevant scales (Williams and Johnson, 1995; Sauer and Link, 2002).

A hierarchical monitoring strategy that evaluates $\hat{\lambda}$ and contrasts estimates across nested spatial scales on an annual basis can act as a powerful analytical tool to help target when and where to carry out management actions, which we refer to as a targeted annual warning system (TAWS). As identified in Objective 3, estimates of population trends are sensitive to start and stop years and require nadir-to-nadir inferences, which limits the ability to assess trends during years when abundance is not at a nadir. This is especially problematic because periods of oscillation are on average 9.4 years in length (Objective 3). However, the TAWS provides immediate assessment of populations at different spatial scales during any point in time, largely by making intra-annual comparisons of $\hat{\lambda}$ across multiple spatial scales and relating those comparisons to evidence of decline. The first step of the TAWS was an implementation of a lek clustering and sampling framework that provided a hierarchical partitioning (division of the landscape) of populations based on biotic and abiotic habitat characteristics, as well as population connectivity (O'Donnell and others, 2019). Comparisons of annual $\hat{\lambda}$ across nested lek clusters facilitated separation of scale-dependent factors that influence population trajectories and identified local scale declines associated with perturbations on the landscape (Coates and others, 2017, 2019a). Until recently, implementation of this type of strategy has remained elusive for sage-grouse management and other species that occupy expansive geographical ranges (Lindenmayer and Linkens, 2010). However, recent efforts for sage-grouse piloted at state and regional levels (Coates and others, 2017, 2019a; O'Donnell and others, 2019) have paved the way for range-wide efforts. The TAWS shares and contrasts information within and among spatial scales to identify where and when declines occur and could be used to investigate the corresponding local or regional drivers responsible for such population changes (for example, Wallace and others, 2010). 
It follows that meeting spatial criteria for 'signaling' under the TAWS will provide an effective framework to identify when and where management actions can be implemented to neutralize or reverse negative impacts of local perturbations on sage-grouse populations. These criteria include (1) showing statistically significant evidence of a declining trend at the local spatial scale that also exhibits (2) lower $\hat{\lambda}$ relative to the trend occurring at the broader spatial scale governed ostensibly by less manageable factors, such as climatic conditions. Moreover, temporal thresholds required for activation of TAWS signals can be evaluated on an annual basis, unlike estimation of trends over one or more population oscillations that are sensitive to start and stop points (see Objective 3). This is largely because the TAWS uses intra-annual comparisons of rates of change in abundance among nested scales. Thus, regardless of when comparisons are conducted during an oscillation, if annual $\hat{\lambda}$ is below one (that is, evidence of decline) and evidenced as declining at faster rates compared to those at the larger spatial scale driven primarily by climate, an activated signal can alert managers. Increased temporal sensitivity therefore allows earlier signaling for possible management action by the TAWS compared to methods informed solely by long-term trend monitoring across multiple oscillations.

Similar to trend inferences in Objective 3, we used leks, NCs, and CCs as the underlying spatial scales for the TAWS framework. We estimate annual $\hat{\lambda}$ at leks and NC scales and compared those estimates to $\hat{\lambda}$ at the CC scale within which they were nested. We illustrate the different scenarios of comparison among scales in figure 43. Evidence of repeated $\hat{\lambda}$ below CC helps identify areas with potential local deterministic factors driving population changes. These populations may need further assessments or targeted management actions, especially those aimed at reversing population declines. Because the TAWS explicitly accounts for nested spatiotemporal relationships, declines driven by broad-scale stochasticity, such as climatic conditions (Coates and others, 2018), should not lead to signaling for immediate management action. Lastly, the TAWS described herein accounts for underlying variation among ecological conditions influencing sagebrush recovery times and risks of exotic grass invasion following disturbance processes that are well-documented and form the basis for contemporary sagebrush ecosystem management (see appendix 5; Pyke and others, 2015; Chambers and others, 2017). This conceptual framework is particularly important given widespread wildfire and annual grass invasions altering disturbance regimes and state-transitions across much of the sagebrush biome (Chambers and others, 2019). These complexities also motivate better accounting of asynchronies between faster sage-grouse population dynamics and slower sagebrush ecosystem recovery processes in decision support tools, such as the TAWS (Coates and others, 2016; Ricca and Coates, 2020).

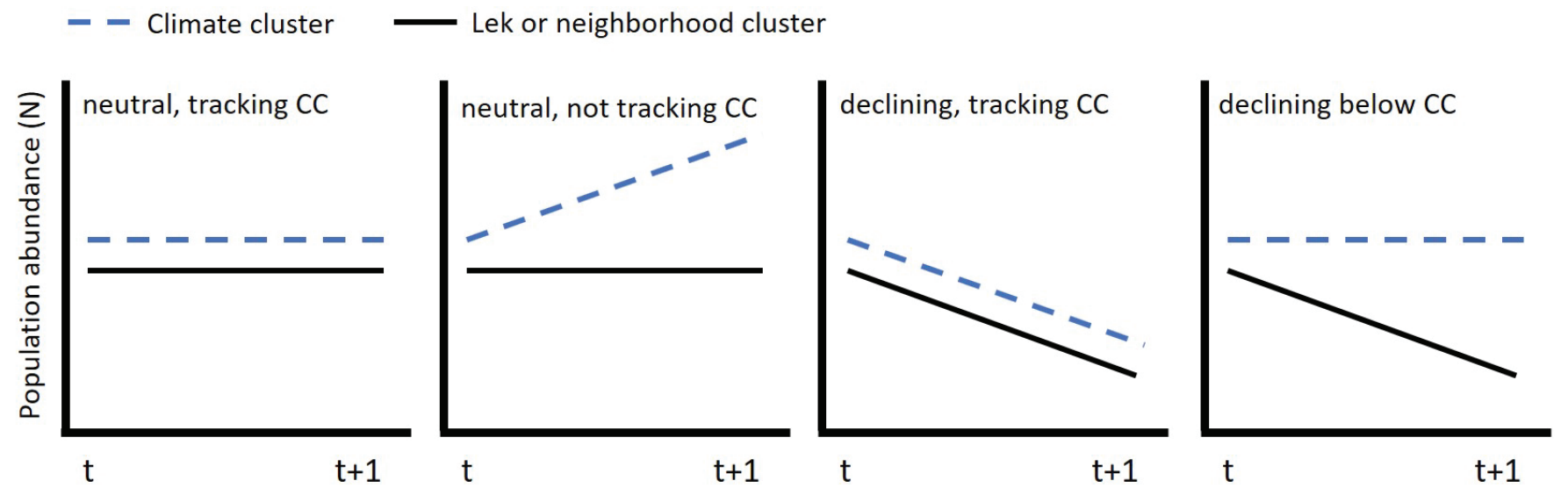

Figure 43. Four hypothesized outcomes in comparing trends between a finer scale (black solid line) and a broader scale (blue dashed line). 


\section{Methods}

\section{Evaluation of Posterior Probability Distributions}

We used an identical SSM structure for the TAWS described previously (see Objective 3 ) to estimate $\hat{N}$ and derive $\hat{r}$ across nested hierarchical spatial scales. However, because the purpose of TAWS was to achieve and compare intra-annual estimates across nested spatial scales rather than estimate trends across different temporal scales, and relatively more frequent lek sampling was required, we imposed a rule that excluded data collected prior to 1990 because sampling was much less frequent (see Objective 1). The SSMs produced PD of annual $\hat{\lambda}$ for each lek, NC, and CC. These PDs formed the foundation for evaluating evidence of leks and NCs that were declining and misaligned with the CC, which provides early indication of potential need for management intervention. After estimating the PD for annual $\hat{\lambda}$ at each spatial scale, we calculated the proportional density of the PD for the lek and the NC that was (1) below the CC $\hat{\lambda}$ PD and demonstrated evidence of declining annual trend $(\hat{\lambda}<1.0$; hereafter, "declining below CC"); (2) similar to the CC $\hat{\lambda} \mathrm{PD}$, decreasing, but less than the median of the CC $\hat{\lambda} \mathrm{PD} ;(3)$ similar, decreasing, and greater than the median of the $\mathrm{CC} \hat{\lambda} \mathrm{PD}$; and (4) stable or increasing. This process was carried out for each lek $\hat{\lambda}$ PD compared to their respective $\mathrm{CC} \hat{\lambda} \mathrm{PD}$ and each NC $\hat{\lambda}$ PD compared to their respective $\mathrm{CC} \hat{\lambda} \mathrm{PD}$. An example of how the four different areas under the curve can result in three scenarios is illustrated in figure 44. Using these areas calculated for every lek and $\mathrm{NC}$, we developed a ratio of the area that represented decline below $\mathrm{CC}$ against all other outcomes. We then took the natural log of the odds ratio (hereafter, log-odds) and established thresholds for log-odds that represented significant evidence of decline below the $\mathrm{CC}$ based on a simulation approach that spanned the entire sage-grouse range (described in appendix 5).

\section{Developing Thresholds for Targeted Annual Warning System}

We used 30 continuous years (1990-2019) of annual lek count data range-wide to inform retrospective simulation analyses designed to estimate a threshold that simultaneously identified (1) significant annual population decline by contrasting $\hat{r}(\log [\hat{\lambda}])$ at the scale of interest relative to $\hat{r}=$ zero (stable population) and (2) populations at smaller scales that negatively fell out of synchrony with those at the $\mathrm{CC}$ scale by contrasting proportional differences in $\hat{r}$ across nested scales. Because rates of decline at levels below the $\mathrm{CC}$ can occur gradually or precipitously, we developed separate thresholds for each scenario: (1) a slow threshold, which identified leks (or NC) likely to experience a gradual decline with $\hat{r}$ below estimates for the CC and (2) a fast threshold, which focused on leks (or NC) with relatively high likelihood of near-term extirpation from a precipitous decline also with estimates below CC.
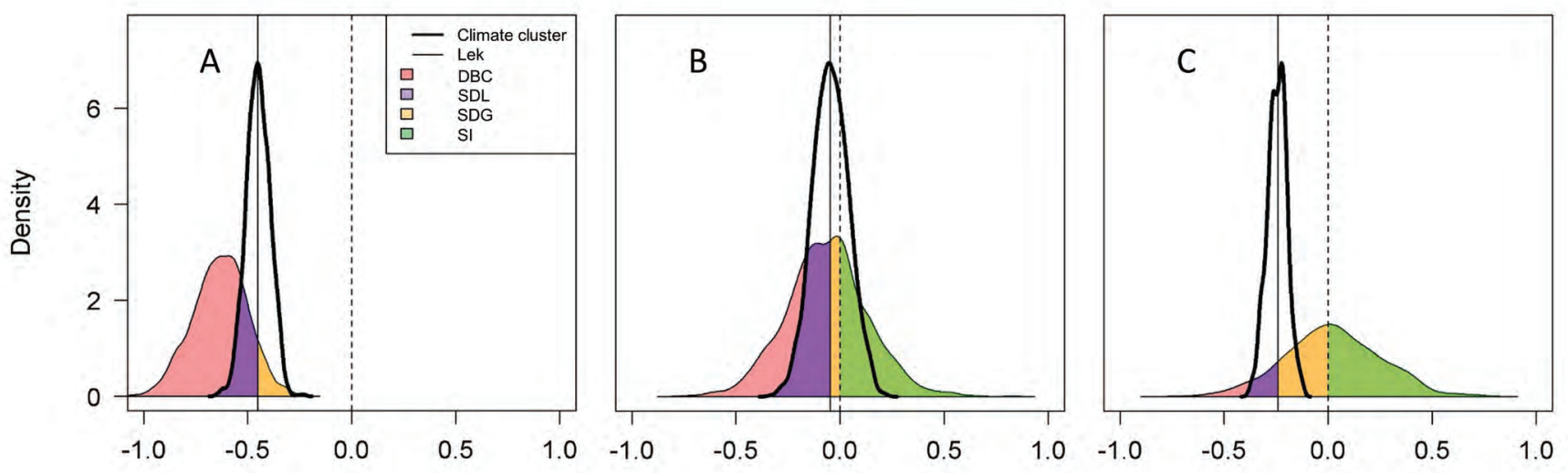

$\hat{\mathbf{r}}$

Figure 44. Intersection of population rate of change $(\hat{r})$ posterior probability distributions for scale of interest (lek or neighborhood cluster; thin distribution line) and climate cluster (CC; thick distribution line) in relation to neutral growth. $A$, Strong evidence of $\hat{r}$ at scale of interest declining at rates below the CC; $B$, similar to $C C$ and neutral; and $C$, above CC scale and neutral. Solid vertical line represents median $\hat{r}$ of $C$, and dashed vertical line represents $\hat{r}=0$ (no increase or decrease). DBC=declining below CC, SDL=similar and decreasing and less than median of $\hat{r}$ of the $\mathrm{CC}, \mathrm{SDG}=$ similar and decreasing and greater than median of $\hat{r}$ of the CC, SI=stable or increasing. 
We used data at a range-wide extent to estimate these generalizable thresholds by evaluating performance of all possible combinations. Specifically, we estimated PDs of $\hat{r}$ annually for each lek and used annual estimates of $\hat{N}$ from leks within a NC to derive $\hat{N}$ and $\hat{r}$ at the NC scale. Similarly, we used annual estimates of $\hat{N}$ at leks across CCs to derive $\hat{N}$ and $\hat{r}$ at the CC scale. We then developed a method to describe the relationship of $\hat{r}$ between the two extents (for instance, lek to $\mathrm{CC}$ and $\mathrm{NC}$ to $\mathrm{CC}$ ) based on log-odds ratios. The comparisons in $\hat{r}$ across these extents were made on an annual basis. If a threshold was exceeded (that is, the calculated log-odds was greater than the threshold that was established from an ancillary simulation analysis [see appendix 5], indicating evidence of decline below CC), then the lek (or NC) would be assigned a 'signal.' Slow or fast signals at a particular scale and year were activated if, and only if, log-odds for sage-grouse populations at the lek (or NC) exceeded the slow or fast threshold, respectively. The log-odds ratio extends on the use of $\hat{r}$ alone by including comparison in trend with that of the CC. For example, a population that is declining but tracking the larger climate cluster that is largely governed by broad-scale climatic conditions will not cross the threshold and thus not activate a signal. Considering that calculation of the log-odds ratio takes into account evidence of declining at a rate below the $\mathrm{CC}$, our process provides spatial safeguard against implementing immediate management action in response to local population declines that are most likely tracking unfavorable environmental conditions affecting the CC.

We also employed temporal safeguards against prematurely implementing actions owing to short-term population dynamics, such as those arising from a single poor year of demographic performance or errors in lek counts. Accordingly, we developed two categories for multi-year signaling events referred to as 'watches' and 'warnings.' We assigned watches to populations that had slow signals over 2 consecutive years. We assigned warnings to populations that had slow signals in 3 out of 4 consecutive years or fast signals in 2 out of 3 consecutive years. Watches may identify the need for intensive monitoring whereas warnings may identify the need for management intervention aimed at stabilizing populations. Collectively, these rules facilitate detection of population declines that are distinguished from the adverse impacts associated with wider-reaching environmental stochasticity.

\section{Evaluation of a Targeted Annual Warning System}

Because we established an empirically supported relationship between precipitation and $\hat{\lambda}$ at the CC scale previously (Objective 2), we tested the efficacy of the TAWS by evaluating the relationship between precipitation and TAWS signals. If TAWS signals were largely associated with precipitation patterns, we could conclude that our method did not effectively disentangle local scale impacts from broader climatic conditions. Conversely, if a relationship between TAWS signals and precipitation patterns could not be identified, then we can conclude that the declines in populations at smaller spatial scales (leks and NCs) were likely a function of deterministic factors associated with those sites and not because of broad-scale precipitation patterns. We used a simple linear model, where intrinsic population rate of change $(\hat{r})$ was fitted as a function of precipitation and proportion of leks signaled.

\section{Results}

\section{Targeted Annual Warning System}

Similar to the trend analysis, thousands of historic lek surveys underwent $\mathrm{QA} / \mathrm{QC}$ as described in the results of Objective 1 for the TAWS analysis. During 1990-2019, we estimated 0.634 and 0.467 proportion of sage-grouse leks experienced watches and warnings (table 9; fig. 45), respectively, across the entire range. We calculated a mean annual proportion of leks that underwent watches and warnings to be 0.025 (with repeated activation included in calculation [hereafter, repeat] $=0.060$ ) and 0.019 (repeat $=0.058$ ), respectively (fig. 46), which is approximately $114($ repeat $=269)$ and $84($ repeat $=261)$ leks each year, respectively. Spatial and temporal depiction of watches and warnings through time for leks within each CC are shown in figures 47-52. The CC with greatest proportion of activated watches at leks across the 29 years was CC-F (western Wyoming area), where 0.698 of total leks activated one or more times over 29 years (table 9), which represented over half the leks for this CC but spread throughout the region (fig. 51). Conversely, CC-E (Great Basin area) consisted of the greatest number of watches compared to other clusters (number of first watches $=1,163$; number of repeat watches $=2,834$; table 9). The CC with the least proportion of watches was CC-C (Jackson Hole, Wyoming, area) at 0.143 (number of first and repeat watches $=2$ and 2 , respectively; table 9 ). The $\mathrm{CC}$ with the greatest proportion of warnings was CC-F (0.515), whereas CC-E had the greatest number of warnings (first $=830$ and repeat $=2,712$ ). The only $\mathrm{CC}$ with no lek warnings over the course of 29 years was CC-C, although this cluster had two watches. The second highest proportion of watches $(0.653)$ and warnings $(0.502)$ was CC-D (eastern area), where we estimated 1,023 (repeat $=2,458)$ and 786 (repeat $=2,423$ ), respectively, over this time frame. 
Table 9. Watches and warnings identified at greater sage-grouse (Centrocercus urophasianus) leks across climate clusters (A-F) using state-space model estimates within a targeted annual warning system in the western United States during 1990-2019. Number of watches and warnings that include repeat $(r)$, only first time $(f)$, and proportion of leks $(p)$ are reported.

[CC, climate cluster; A, Bi-state area; B, Washington area; C, Jackson Hole, Wyoming area; D, eastern area; E, Great Basin area; F, Wyoming]

\begin{tabular}{lrrlrrrr}
\hline \multicolumn{1}{c}{ CC } & r.watch & f.watch & p.watch & r.warning & f.warning & p.warning & Leks \\
\hline A & 27 & 21 & 0.438 & 22 & 11 & 0.229 & 48 \\
B & 15 & 8 & 0.16 & 16 & 6 & 0.12 & 50 \\
C & 2 & 2 & 0.143 & 0 & 0 & 0 & 14 \\
D & 2,458 & 1,023 & 0.653 & 2,423 & 786 & 0.502 & 1,566 \\
E & 2,834 & 1,163 & 0.61 & 2,712 & 830 & 0.435 & 1,908 \\
F & 1,398 & 623 & 0.698 & 1,341 & 459 & 0.515 & 892 \\
Total & 6,734 & 2,840 & 0.634 & 6,514 & 2,092 & 0.467 & 4,478 \\
\hline
\end{tabular}




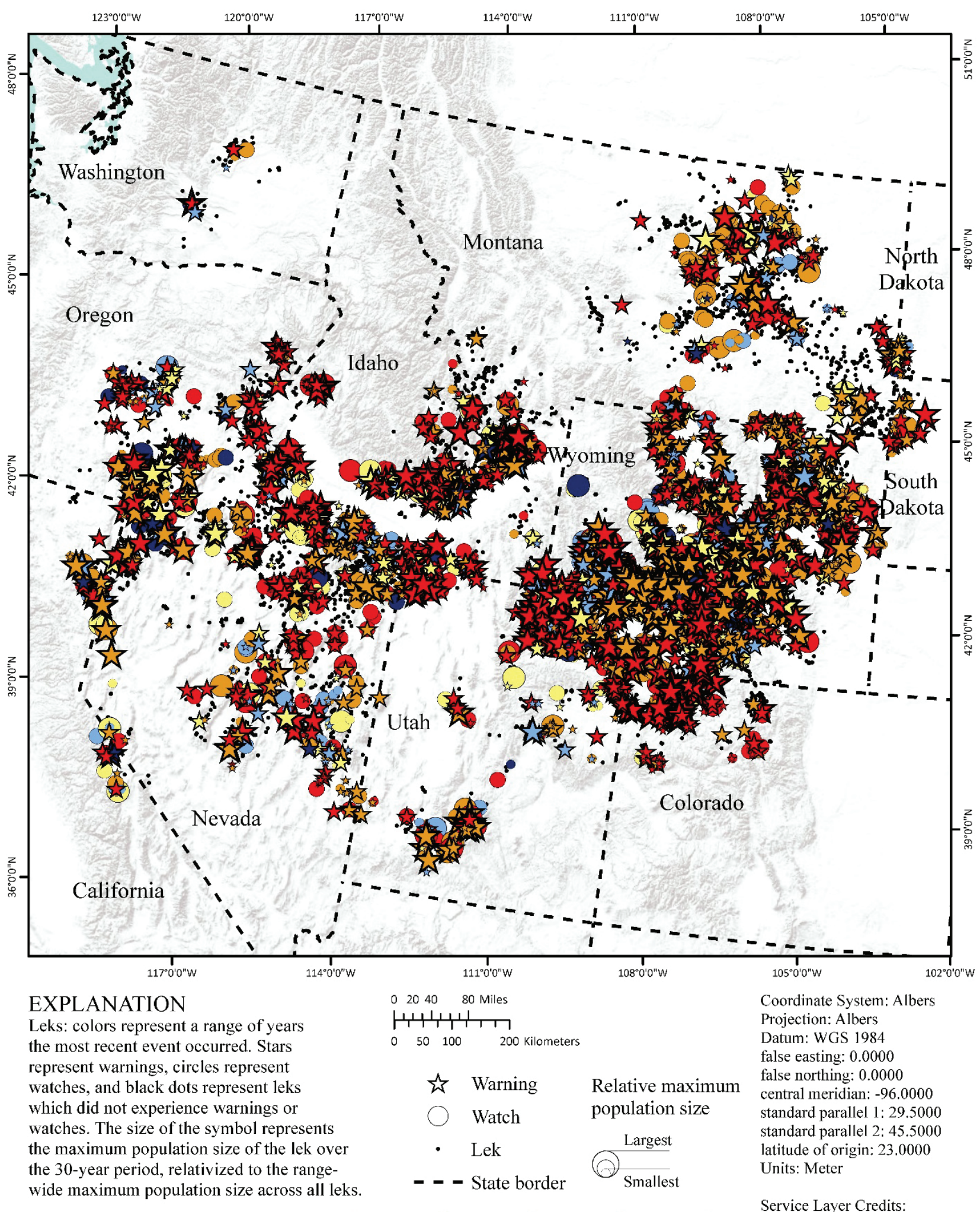

1995 -1999 2000-2004 2005-2009 2010-2014 2015-2019

Service Layer Credits: Sources: Esri, USGS, NOAA

Figure 45. Spatial and temporal depiction of range-wide watches and warnings of greater sage-grouse (Centrocercus urophasianus) population declines at the lek scale within the western United States from 1990 to 2019. Map image is the intellectual property of Esri and is used herein under license. Copyright $@ 2020$ Esri and its licensors. All rights reserved. 

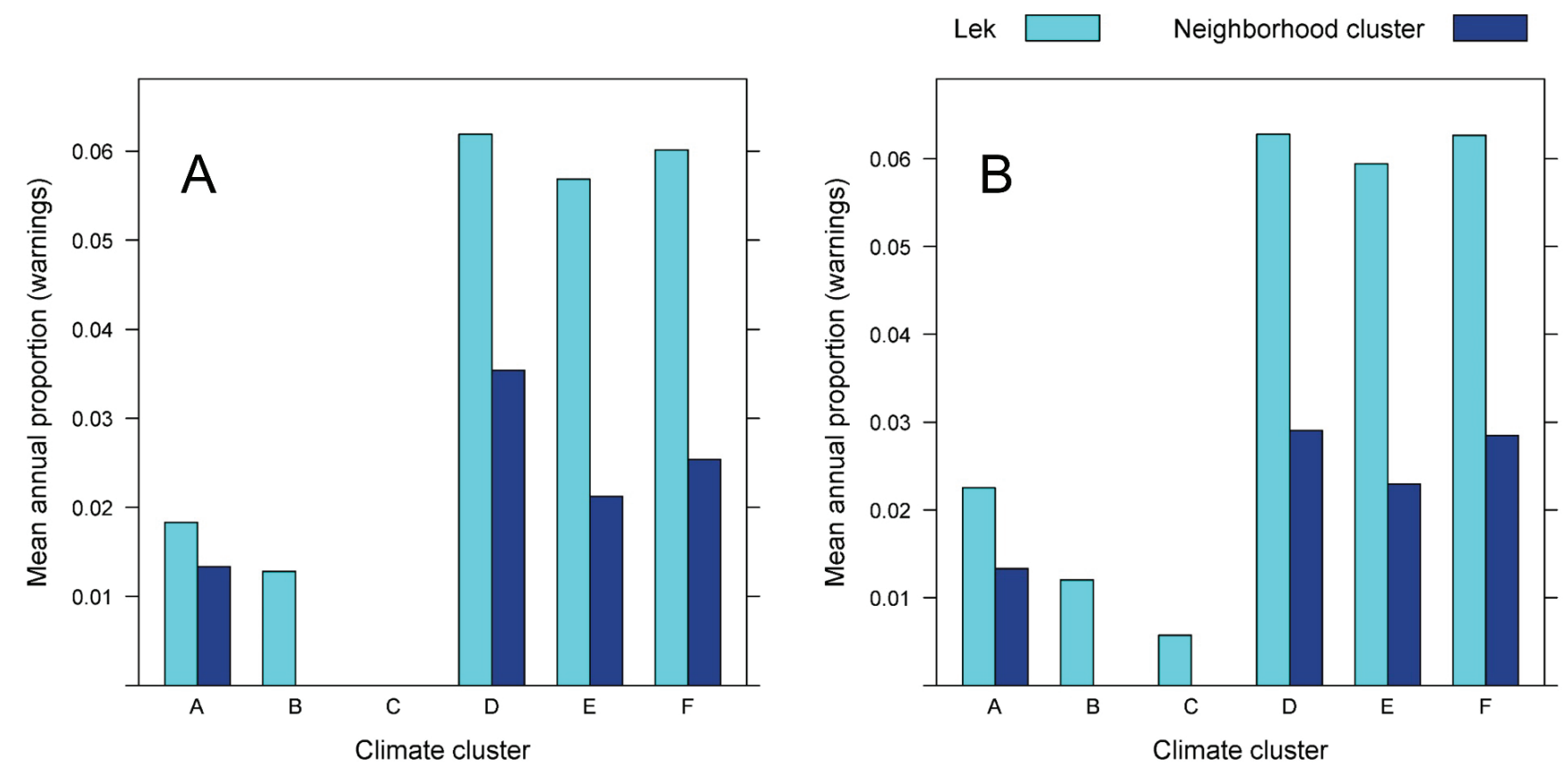

Figure 46. Proportion of $A$, warnings; and $B$, watches of greater sage-grouse (Centrocercus urophasianus) annual declines at the lek and neighborhood cluster scale across each climate cluster within the western United States. 


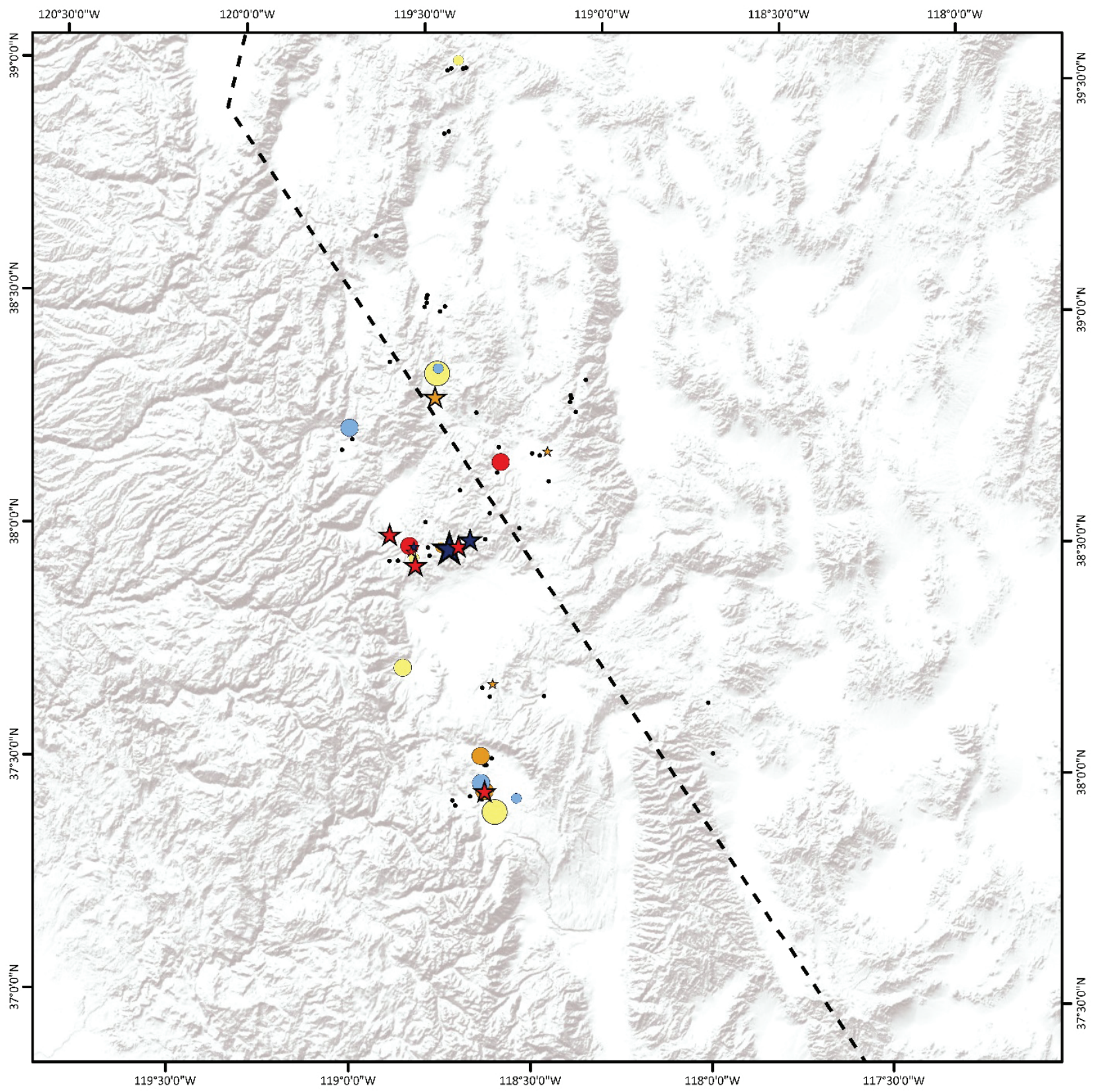

EXPLANATION

Leks in the Bi-State area (climate cluster A): colors represent a range of years the most recent event occurred. Stars represent warnings, circles represent watches, and black dots represent leks which did not experience warnings or watches. The size of the symbol represents the maximum population size of the lek over the 30-year period, relativized to the range-wide maximum population size across all leks. Symbols are based on a range-wide distribution of values and all may not be represented here.

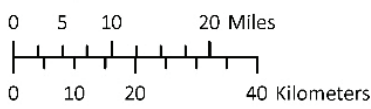

Coordinate System: Albers Projection: Albers Datum: WGS 1984 th Warning Relative maximum false easting: 0.0000 Watch population size central meridian: -96.0000 standard parallel $1: 29.5000$ standard parallel 2: 45.5000 latitude of origin: 23.0000 Units: Meter

Service Layer Credits: Sources: Esri, USGS, NOAA

Figure 47. Spatial and temporal depiction of watches and warnings of greater sage-grouse (Centrocercus urophasianus) population declines at the lek scale within climate cluster A (CC-A; Bi-State area) from 1990 to 2019. Map image is the intellectual property of Esri and is used herein under license. Copyright $@ 2020$ Esri and its licensors. All rights reserved. 


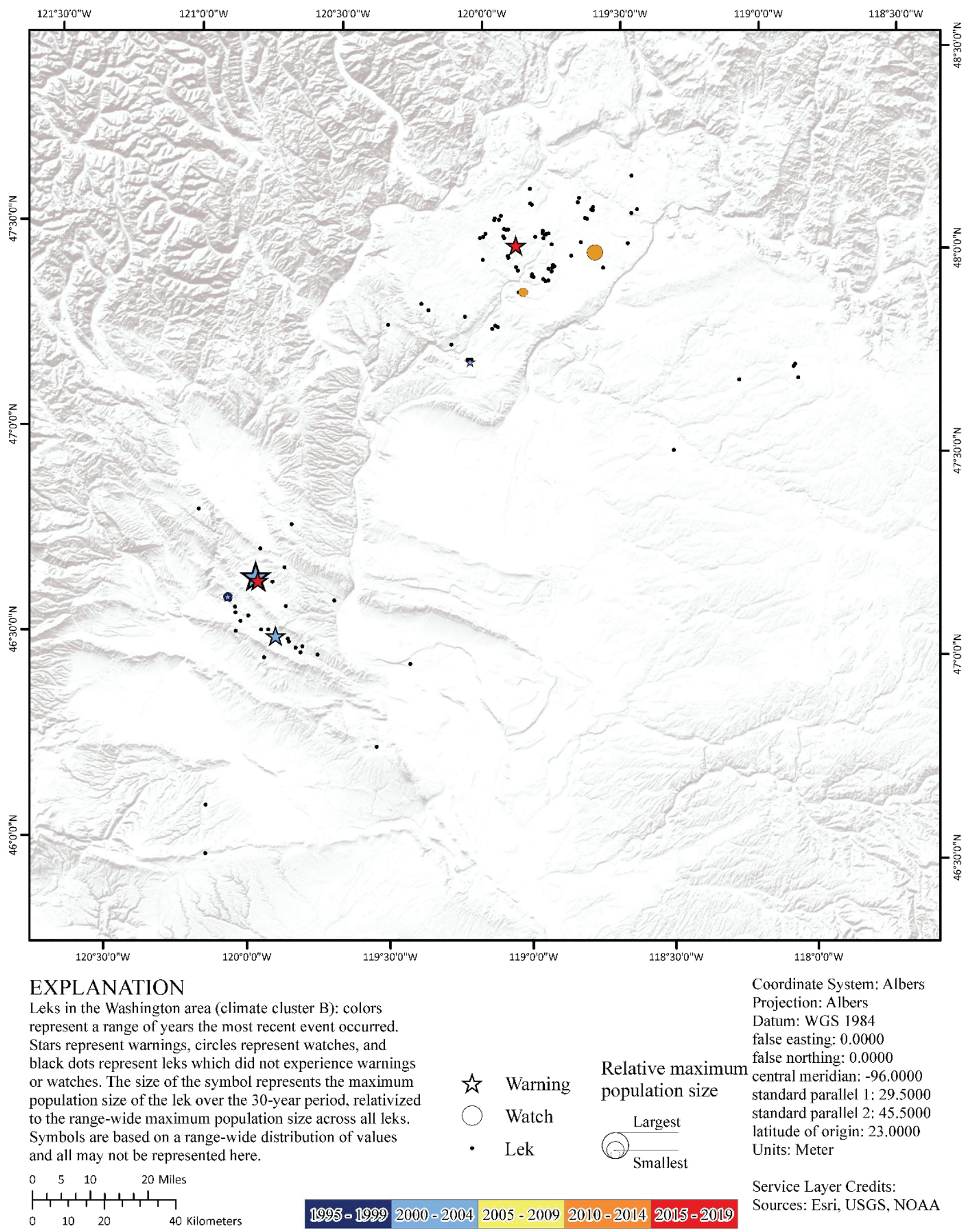

Figure 48. Spatial and temporal depiction of watches and warnings of greater sage-grouse (Centrocercus urophasianus) population declines at the lek scale within climate cluster B (CC-B; Washington area) from 1990 to 2019. Map image is the intellectual property of Esri and is used herein under license. Copyright $@ 2020$ Esri and its licensors. All rights reserved. 


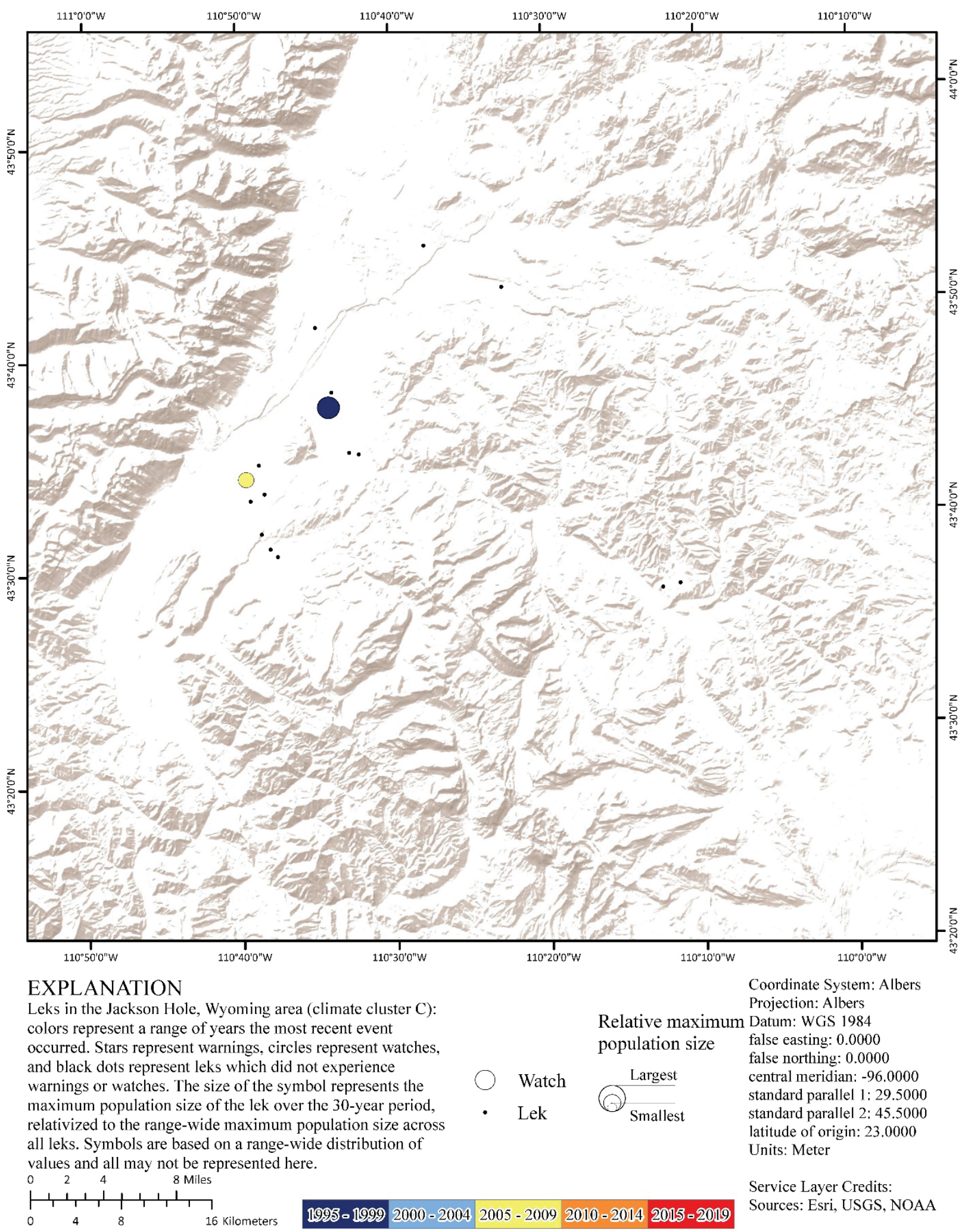

Figure 49. Spatial and temporal depiction of watches and warnings of greater sage-grouse (Centrocercus urophasianus) population declines at the lek scale within climate cluster C (CC-C; Jackson Hole, Wyoming, area) from 1990 to 2019. Map image is the intellectual property of Esri and is used herein under license. Copyright $(\subset) 2020$ Esri and its licensors. All rights reserved. 


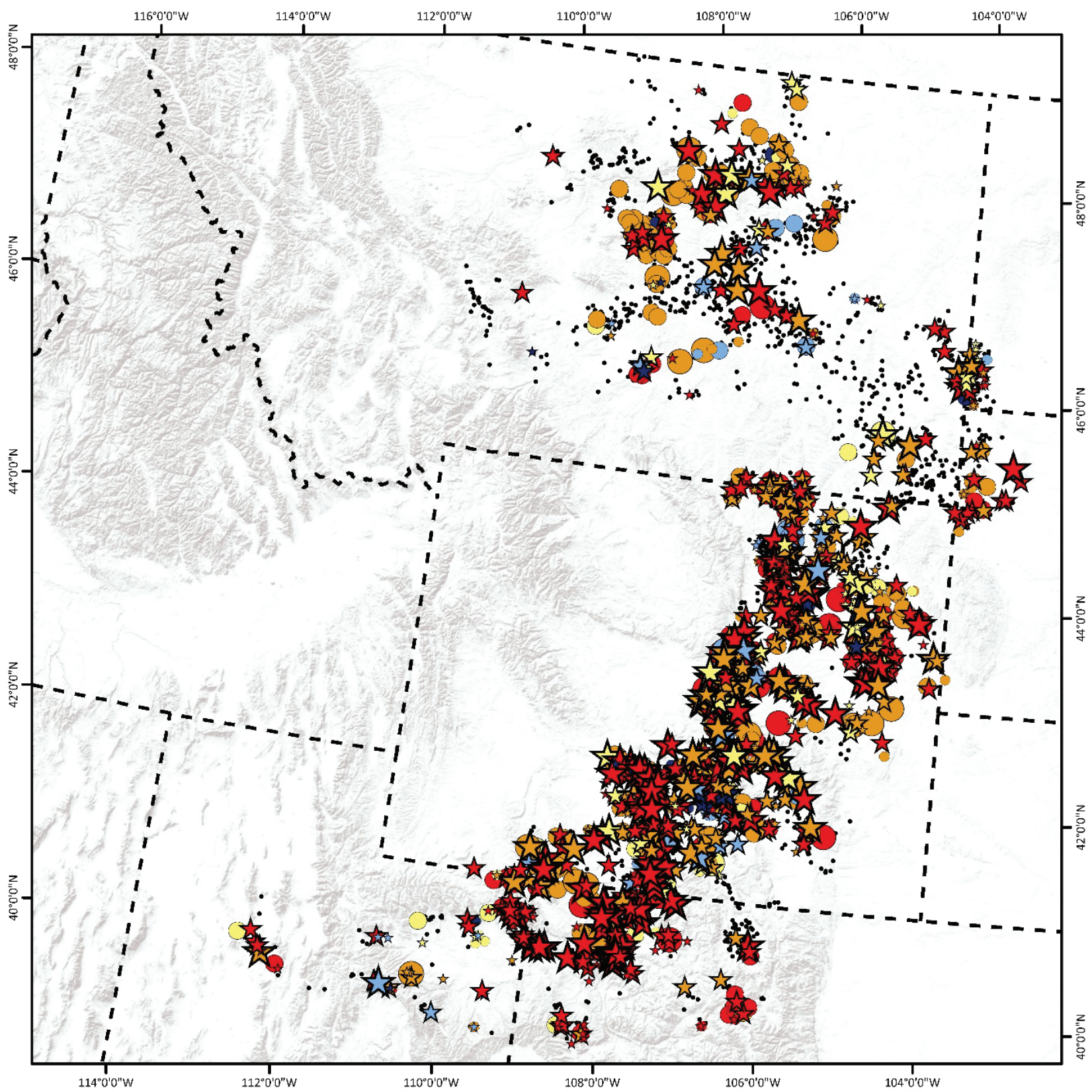

\section{EXPLANATION}

Leks in the eastern area (climate cluster D): colors represent a range of years the most recent event occurred. Stars represent warnings, circles represent watches, and black dots represent leks which did not experience warnings or watches. The size of the symbol represents the maximum population size of the lek over the 30-year period, relativized to the range-wide maximum population size across all leks. Symbols are based on a range-wide distribution of values and all maynot be represented here. $20 \quad 40 \quad 80$ Miles 200 Kilometers
Coordinate System: Albers Projection: Albers Datum: WGS 1984

负 Warning Relative maximum false easting: 0.0000 $\bigcirc$ Watch

- Lek

- - - State border central meridian: -96.0000 standard parallel 1: 29.5000 standard parallel 2: 45.5000 latitude of origin: 23.0000 Units: Meter

Service Layer Credits: Sources: Esri, USGS, NOAA

Figure 50. Spatial and temporal depiction of watches and warnings of greater sage-grouse (Centrocercus urophasianus) population declines at the lek scale within climate cluster D (CC-D; eastern area) from 1990 to 2019. Map image is the intellectual property of Esri and is used herein under license. Copyright $(\subset 2020$ Esri and its licensors. All rights reserved. 


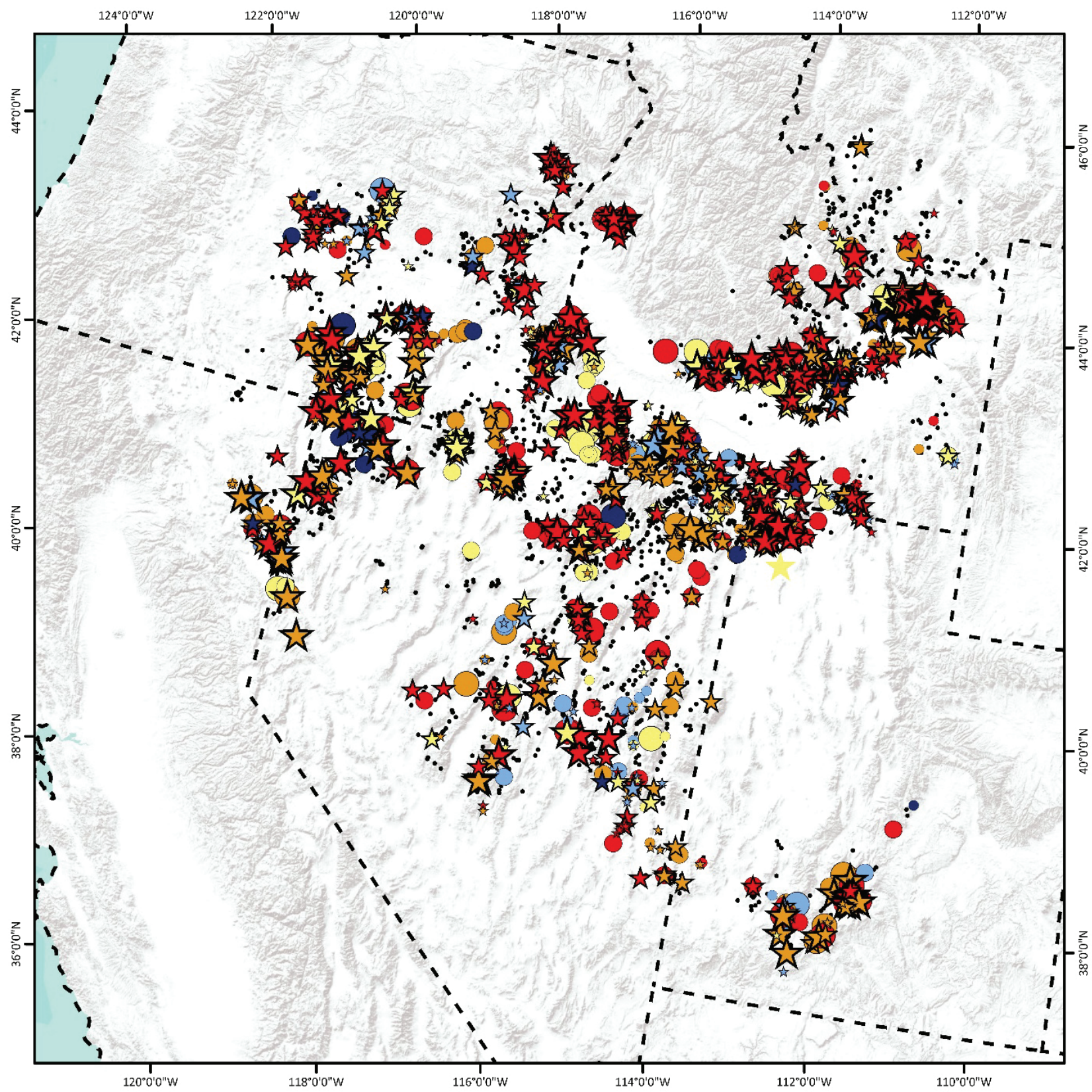

EXPLANATION

Leks in the Great Basin area (climate cluster E): colors represent a range of years the most recent event occurred. Stars represent warnings, circles represent watches, and black dots represent leks which did not experience warnings or watches. The size of the symbol represents the maximum population size of the lek over the 30 -year period, relativized to the range-wide maximum population size across all leks. Symbols are based on a range-wide distribution of values and all may not be represented here.

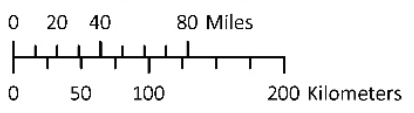

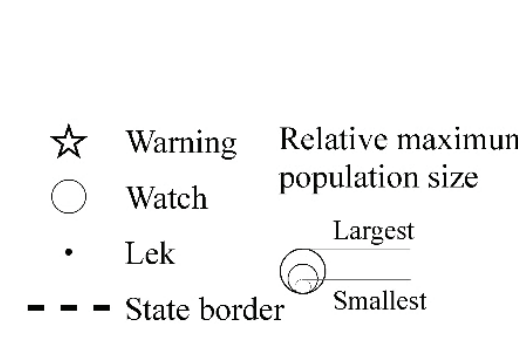

Coordinate System: Albers Projection: Albers Datum: WGS 1984 false easting: 0.0000 false northing: 0.0000 central meridian: -96.0000 standard parallel 1: 29.5000 standard parallel 2: 45.5000 latitude of origin: 23.0000 Units: Meter

Service Layer Credits: Sources: Esri, USGS, NOAA

Figure 51. Spatial and temporal depiction of watches and warnings of greater sage-grouse (Centrocercus urophasianus) population declines at the lek scale within climate cluster E (CC-E; Great Basin area) from 1990 to 2019. Map image is the intellectual property of Esri and is used herein under license. Copyright (C) 2020 Esri and its licensors. All rights reserved. 


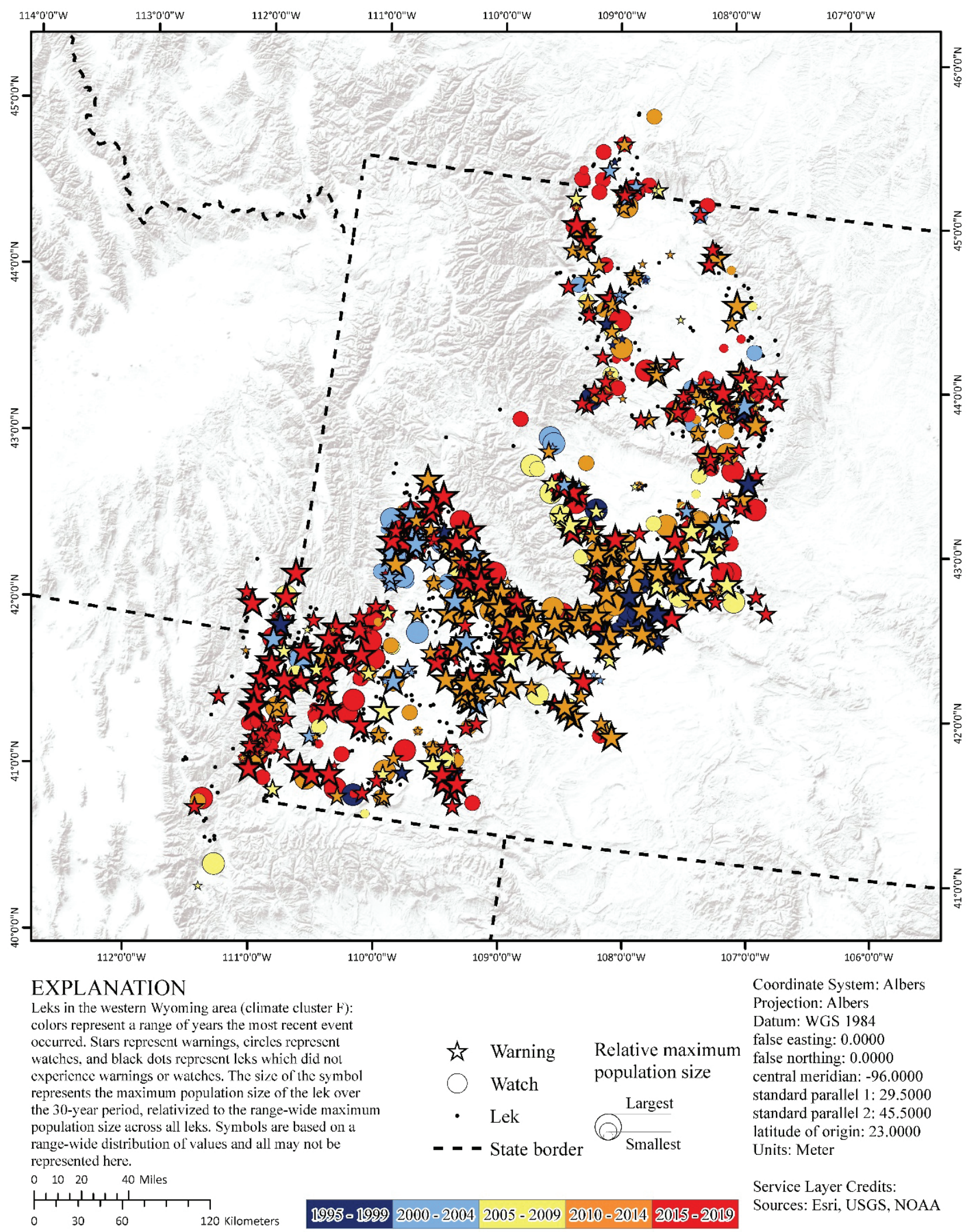

Figure 52. Spatial and temporal depiction of watches and warnings of greater sage-grouse (Centrocercus urophasianus) population declines at the lek scale within climate cluster F (CC-F; western Wyoming area) from 1990 to 2019. Map image is the intellectual property of Esri and is used herein under license. Copyright $(\subset) 2020$ Esri and its licensors. All rights reserved. 
We estimated 0.418 and 0.276 proportion of $\mathrm{NCs}$ were activated as watches and warnings (table 10; fig. 53), respectively, at NCs range-wide during 1990-2019. This calculated to an average of 0.025 (first $=0.017$ ) and 0.026 (first $=0.011$ ) proportion of clusters activating, respectively, per year (fig. 46), which was approximately 10.5 (first=7.0) and 10.8 (first=4.6) clusters. Spatial and temporal depiction of watches and warnings through time for NCs within each CC are shown in figures 54-57. Climate clusters not shown in figures had no neighborhood warnings across the 29 -year time frame. We reported CC-F (western Wyoming area) had the greatest proportion (0.519) of watches while CC-E (Great Basin area) had the greatest number (first $=82$ and repeat $=121$ ) of watches across the 29-year time frame (fig. 51). For warnings, CC-E had the greatest number (first=49 and repeat=112) and CC-D (eastern area) had the greatest proportion ( 0.345 ; table 10 ; fig. 50$)$. Climate cluster B (Washington area) and CC-C (Jackson Hole, Wyoming, area) did not have any $\mathrm{NC}$ warnings, and $\mathrm{CC}-\mathrm{A}$ (Bi-State area) only experienced two (repeat $=3$ ) across the 29 years.

During 2019, we estimated 0.020 and 0.032 proportion of leks experienced watches and warnings, respectively, range-wide (table 11), which resulted in 90 and 143 lek activations, respectively, (fig. 58). Spatial and temporal depiction of watches and warnings by $\mathrm{CC}$ are illustrated in figures 59-62 (leks) and figures 63-65 (NCs). During 2019, the greatest proportion of watches (0.029) and warnings (0.052) were within CC-F, which were 26 watches and
46 warnings in 2019 (table 11). Climate cluster-A and CC-E had relatively similar proportions of leks activated as warnings during this year and no warnings were activated at CC-B and CC-C (table 11). We estimated 0.014 and 0.019 proportion of neighborhoods experienced watches and warnings, respectively, range-wide (table 12). Climate cluster $\mathrm{F}$ experienced the greatest proportion of $\mathrm{NC}$ watches and warnings, in 2019 (fig. 66).

Most CCs exhibited increases in watches and warnings through time (fig. 66). Most notably, CC-D, CC-E, and CC-F exhibited major increases in watches and warnings across both scales (leks and NCs) following the mid- to late-2000s. At the lek scale, years with the greatest proportion of warnings within CC-D were 2009 and 2014. Within CC-E, we reported peaks at 2007 and 2010. Climate cluster-B exhibited multiple lek warnings in early years (1996-1999) and again during 2013-2015. More recently, in 2014, CC-A experienced one of two peaks of proportion of lek warnings across the time series, and the other high peak was in 2008.

Lastly, our model did not support a relationship between precipitation and signals at the CC scale (fig. 67). However, evidence of an association between $\hat{\lambda}$ and precipitation at this scale supports the concept that declines identified using TAWS were associated with deterministic factors and were not driven by precipitation and other stochastic factors. State-wide warning and watches can be found in appendix 3 .

Table 10. Watches and warnings identified at the neighborhood cluster scale across different climate clusters (A-F) by state-space model estimates using a targeted annual warning system framework for greater sage-grouse (Centrocercus urophasianus) across their range in the western United States during 1990-2019. Number of watches and warnings that include repeat (r), only first time (f), and proportion of leks (p) are reported.

[CC, climate cluster; A, Bi-state area; B, Washington area; C, Jackson Hole, Wyoming area; D, eastern area; E, Great Basin area; F, Wyoming]

\begin{tabular}{lccccccc}
\hline CC & r.watch & f.watch & p.watch & r.warning & f.warning & p.warning & Clusters \\
\hline A & 3 & 2 & 0.222 & 3 & 2 & 0.222 & 9 \\
B & 0 & 0 & 0 & 0 & 0 & 0 & 3 \\
C & 0 & 0 & 0 & 0 & 0 & 0 & 2 \\
D & 101 & 63 & 0.453 & 123 & 48 & 0.345 & 139 \\
E & 121 & 82 & 0.389 & 112 & 49 & 0.232 & 211 \\
F & 37 & 27 & 0.519 & 33 & 16 & 0.308 & 52 \\
Total & 262 & 174 & 0.418 & 271 & 115 & 0.276 & 416 \\
\hline
\end{tabular}




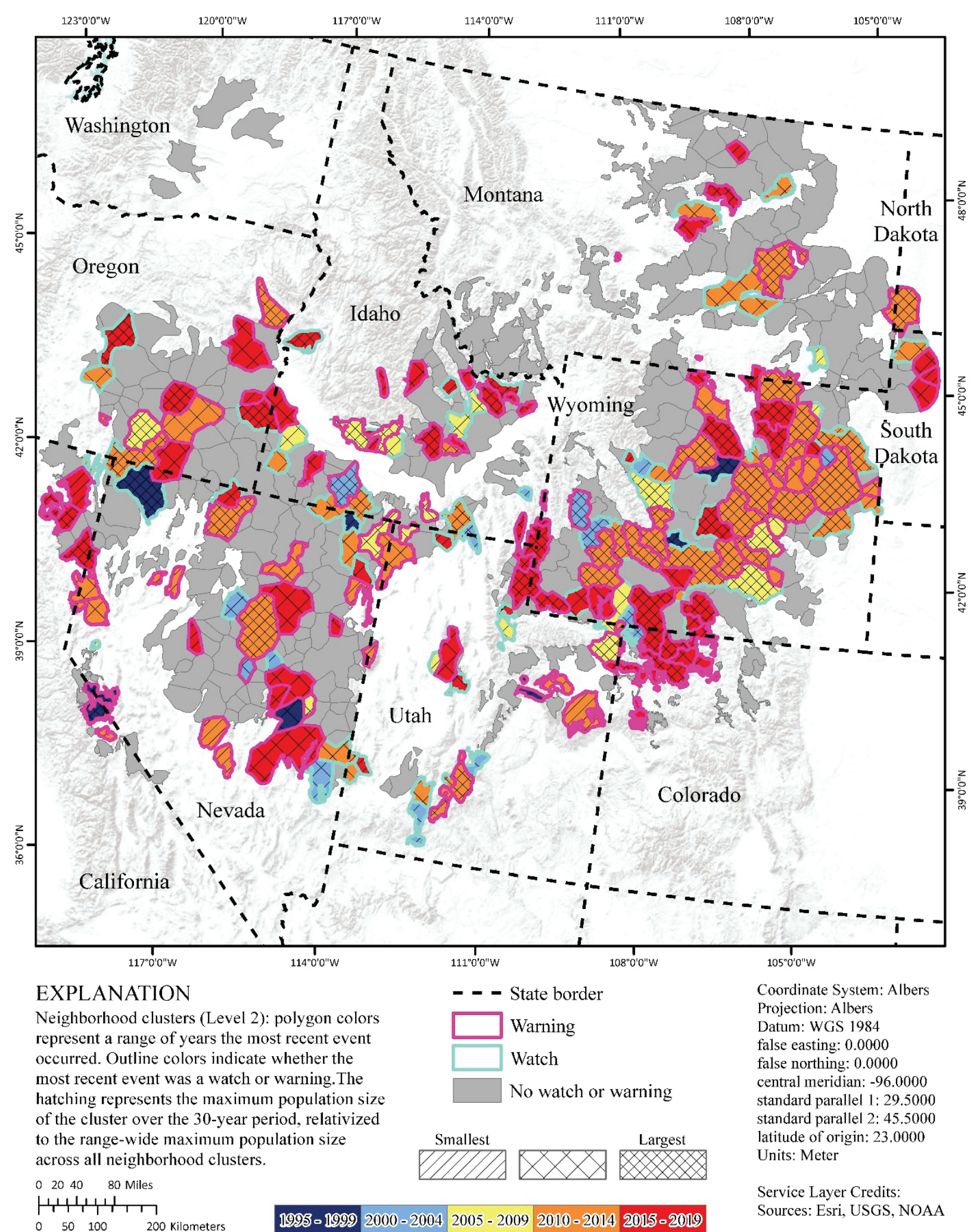

Figure 53. Spatial and temporal depiction of range-wide watches and warnings of greater sage-grouse (Centrocercus urophasianus) population declines at the neighborhood cluster scale within the western United States from 1990 to 2019. Map image is the intellectual property of Esri and is used herein under license. Copyright $(\subset) 2020$ Esri and its licensors. All rights reserved. 


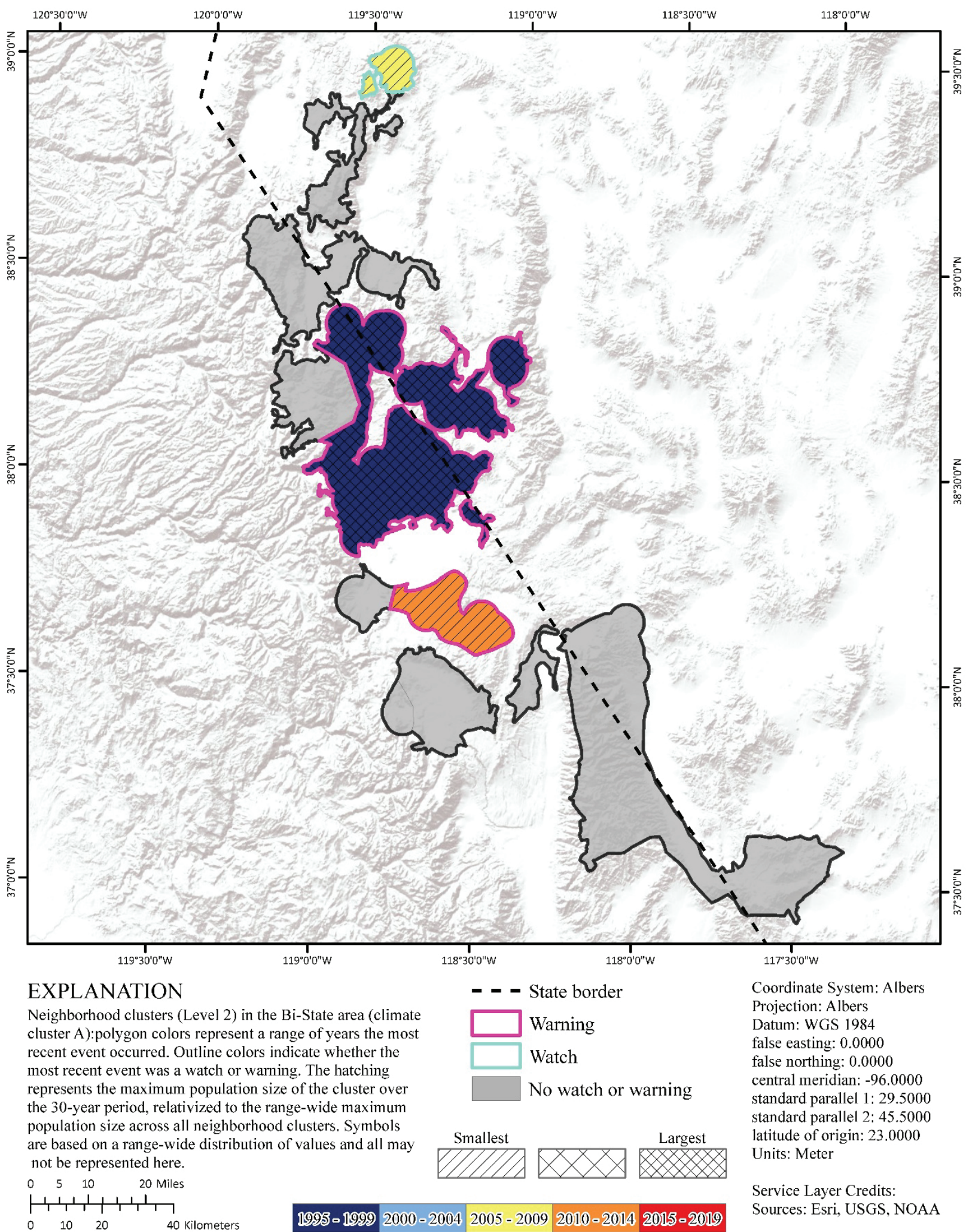

Figure 54. Spatial and temporal depiction of watches and warnings of greater sage-grouse (Centrocercus urophasianus) population declines at the neighborhood cluster scale within climate cluster A (CC-A; Bi-State area) from 1990 to 2019. Map image is the intellectual property of Esri and is used herein under license. Copyright $(\subset) 2020$ Esri and its licensors. All rights reserved. 


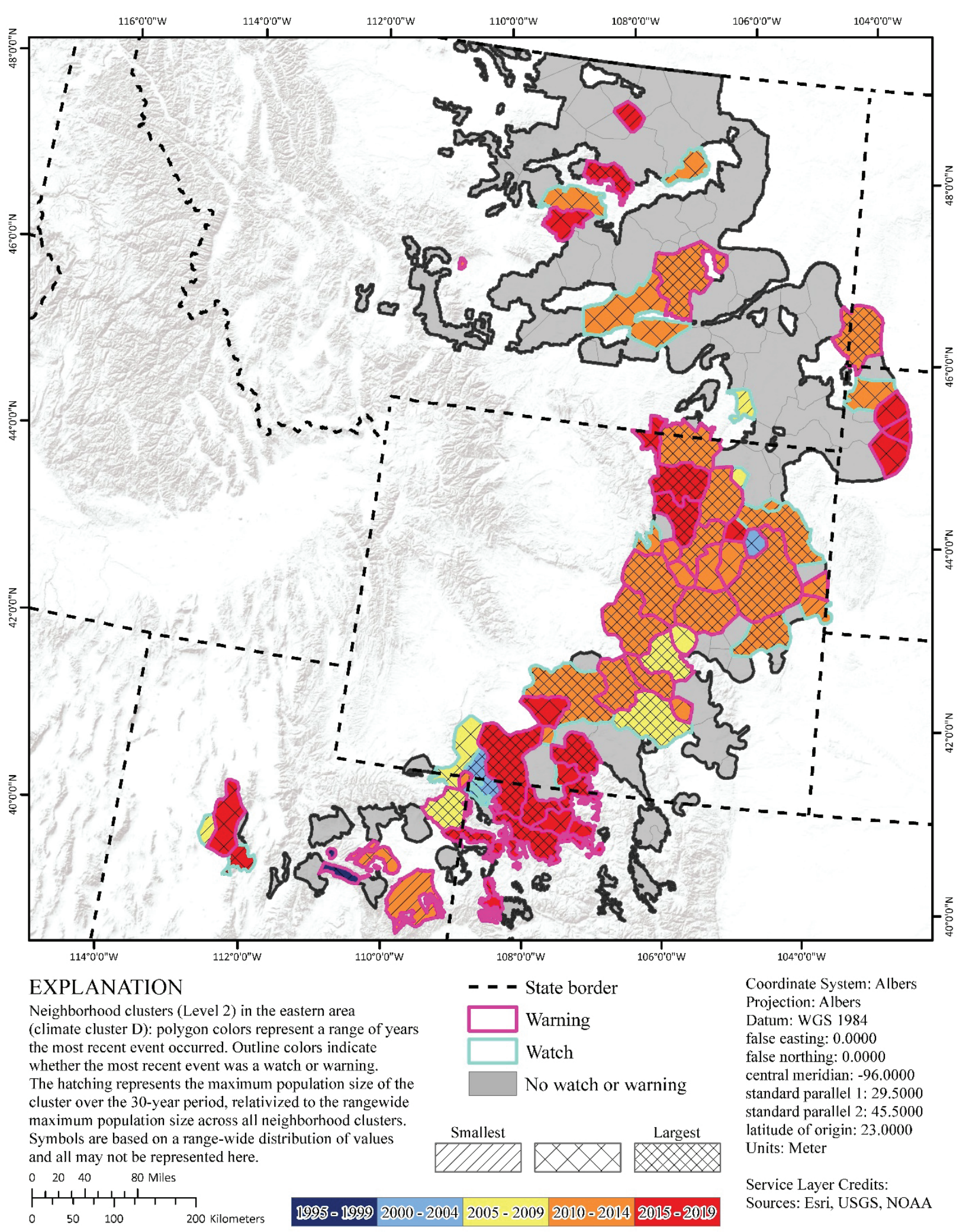

Figure 55. Spatial and temporal depiction of watches and warnings of greater sage-grouse (Centrocercus urophasianus) population declines at the neighborhood cluster scale within climate cluster D (CC-D; eastern area) from 1990 to 2019. Map image is the intellectual property of Esri and is used herein under license. Copyright (C) 2020 Esri and its licensors. All rights reserved. 


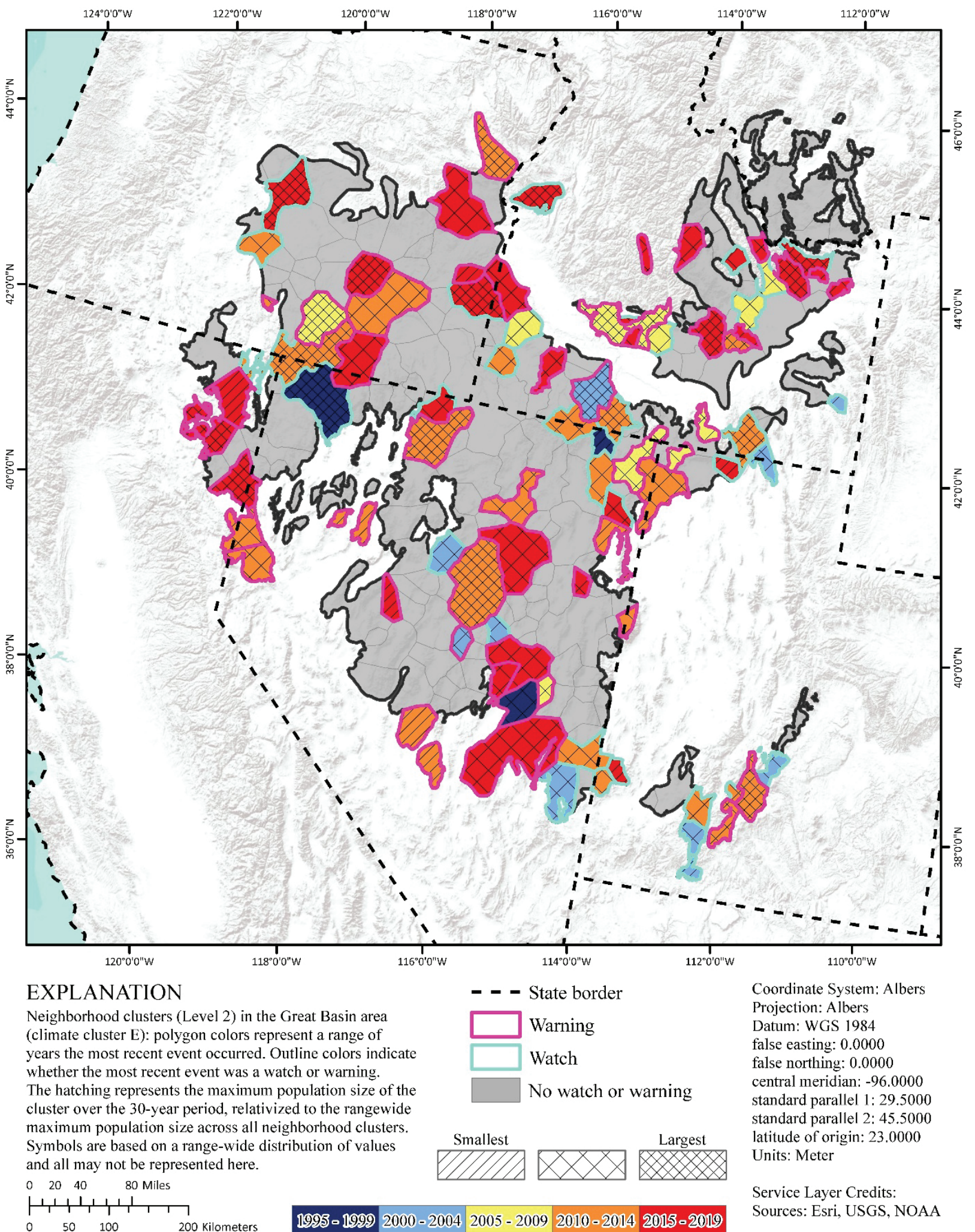

Figure 56. Spatial and temporal depiction of watches and warnings of greater sage-grouse (Centrocercus urophasianus) population declines at the neighborhood cluster scale within climate cluster E (CC-E; Great Basin area) from 1990 to 2019. Map image is the intellectual property of Esri and is used herein under license. Copyright $(\subset) 2020$ Esri and its licensors. All rights reserved. 


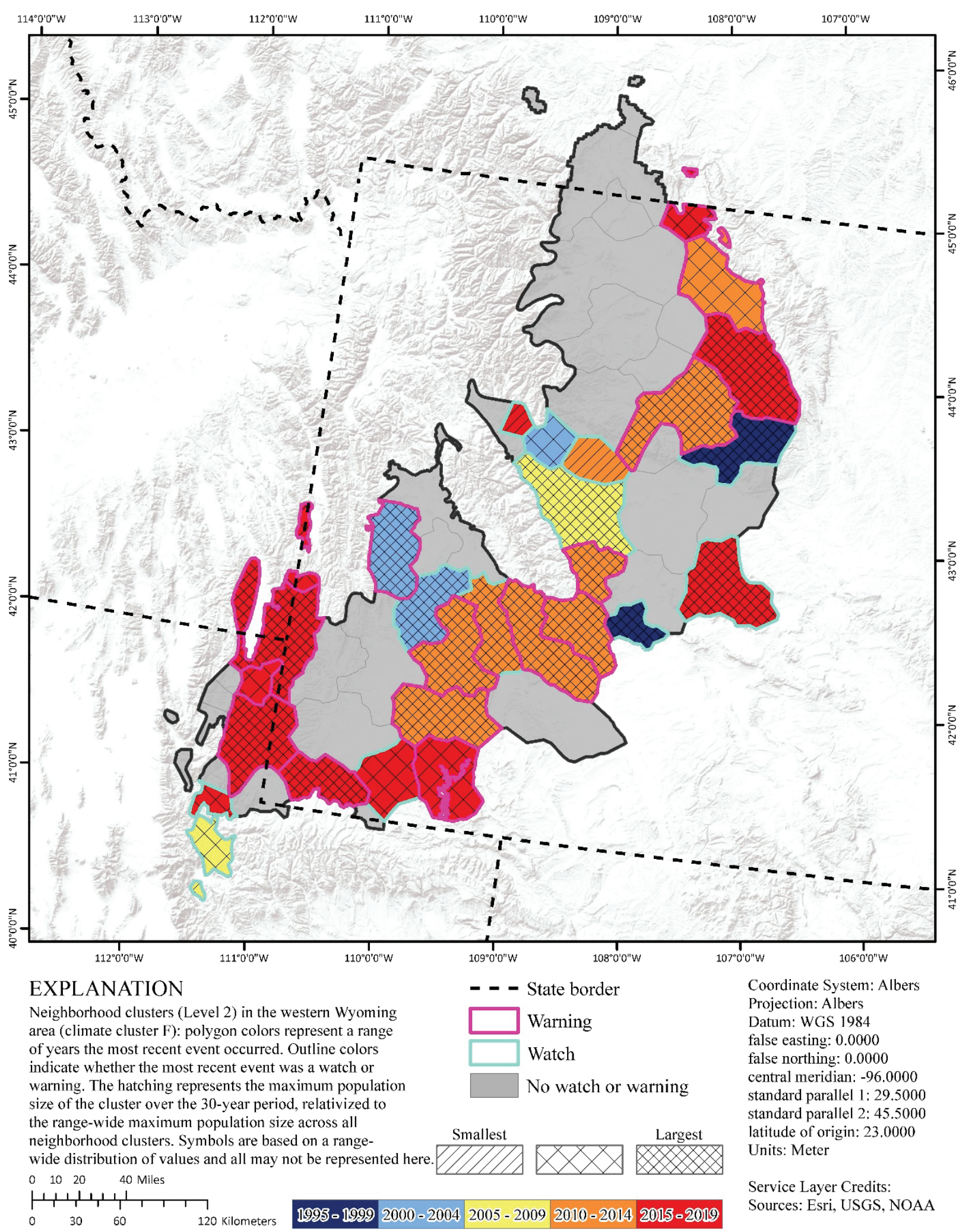

Figure 57. Spatial and temporal depiction of watches and warnings of greater sage-grouse (Centrocercus urophasianus) population declines at the neighborhood cluster scale within climate cluster F (CC-F; western Wyoming area) from 1990 to 2019. Map image is the intellectual property of Esri and is used herein under license. Copyright $(\subset) 2020$ Esri and its licensors. All rights reserved. 
Table 11. Watches and warnings identified at the lek scale across different climate clusters (A-F) by state-space model estimates using a targeted annual warning system framework for greater sage-grouse (Centrocercus urophasianus) across their range in the western United States during 2019. Number of watches and warnings that include repeat ( $r)$, only first time (f), and proportion of leks (p) are reported.

[CC, climate cluster; A, Bi-state area; B, Washington area; C, Jackson Hole, Wyoming area; D, eastern area; E, Great Basin area; F, Wyoming]

\begin{tabular}{lccccccr}
\hline CC & r.watch & f.watch & p.watch & r.warning & f.warning & p.warning & Leks \\
\hline A & 2 & 1 & 0.021 & 2 & 1 & 0.021 & 48 \\
B & 0 & 0 & 0 & 0 & 0 & 0 & 50 \\
C & 0 & 0 & 0 & 0 & 0 & 0 & 14 \\
D & 203 & 27 & 0.017 & 182 & 62 & 0.040 & 1,566 \\
E & 115 & 36 & 0.019 & 130 & 34 & 0.018 & 1,908 \\
F & 95 & 26 & 0.029 & 103 & 46 & 0.052 & 892 \\
Total & 415 & 90 & 0.020 & 417 & 143 & 0.032 & 4,478 \\
\hline
\end{tabular}

Table 12. Watches and warnings identified at the neighborhood cluster scale across different climate clusters (A-F) using state-space model estimates in a targeted annual warning system framework for greater sage-grouse (Centrocercus urophasianus) across their range in the western United States during 2019. Number of watches and warnings that include repeat (r), only first time (f), and proportion of leks (p) are reported.

[CC, climate cluster; A, Bi-state area; B, Washington area; C, Jackson Hole, Wyoming area; D, eastern area; E, Great Basin area; F, Wyoming]

\begin{tabular}{lccccccc}
\hline CC & r.watch & f.watch & p.watch & r.warning & f.warning & p.warning & Clusters \\
\hline A & 0 & 0 & 0 & 0 & 0 & 0 & 9 \\
B & 0 & 0 & 0 & 0 & 0 & 0 & 3 \\
C & 0 & 0 & 0 & 0 & 0 & 0 & 2 \\
D & 7 & 2 & 0.014 & 9 & 5 & 0.036 & 139 \\
E & 2 & 2 & 0.009 & 10 & 1 & 0.005 & 211 \\
F & 4 & 2 & 0.038 & 6 & 2 & 0.038 & 52 \\
Total & 13 & 6 & 0.014 & 25 & 8 & 0.019 & 416 \\
\hline
\end{tabular}




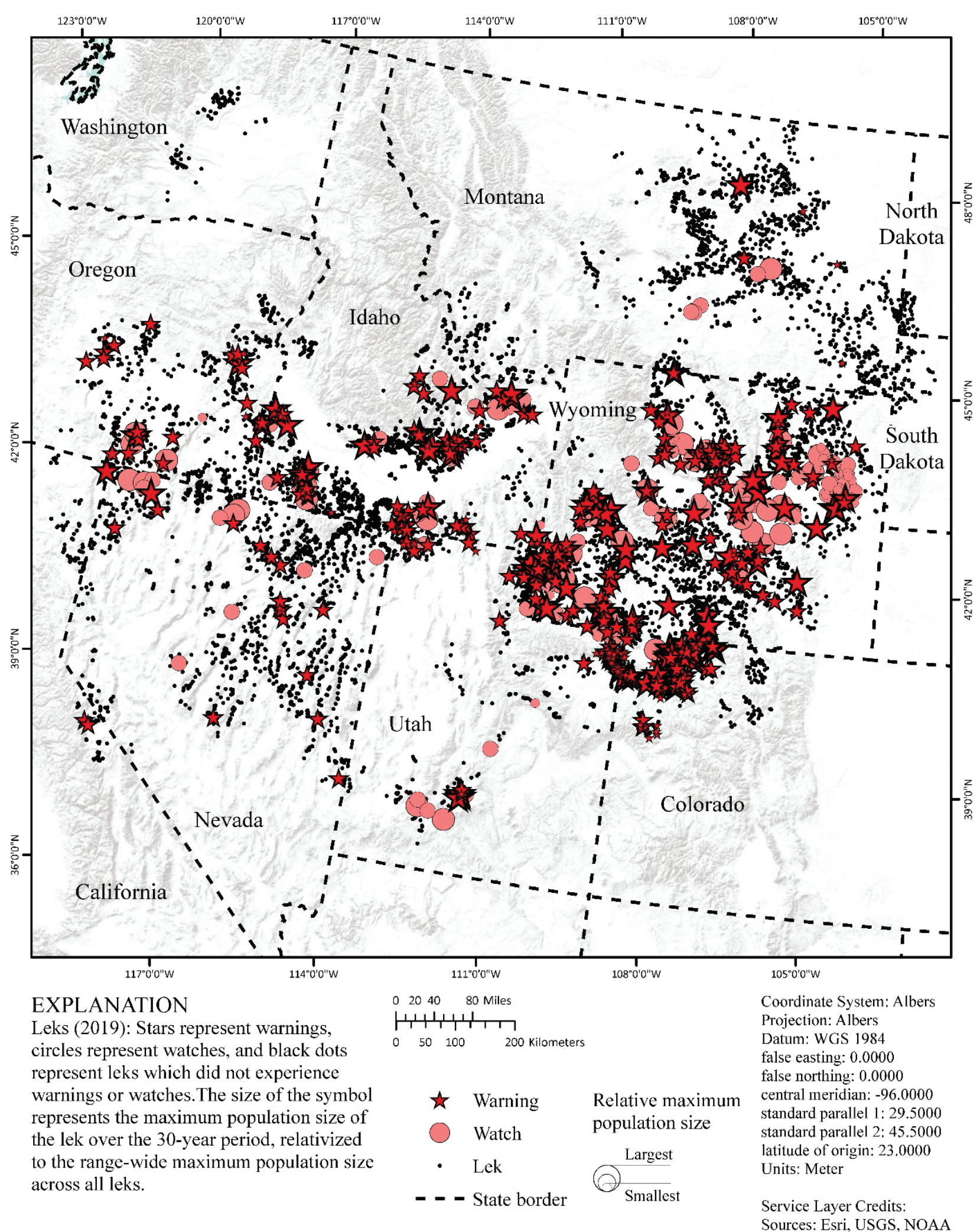

Figure 58. Spatial and temporal depiction of range-wide watches and warnings of greater sage-grouse (Centrocercus urophasianus) population declines at the lek scale within the western United States during 2019. Map image is the intellectual property of Esri and is used herein under license. Copyright () 2020 Esri and its licensors. All rights reserved. 


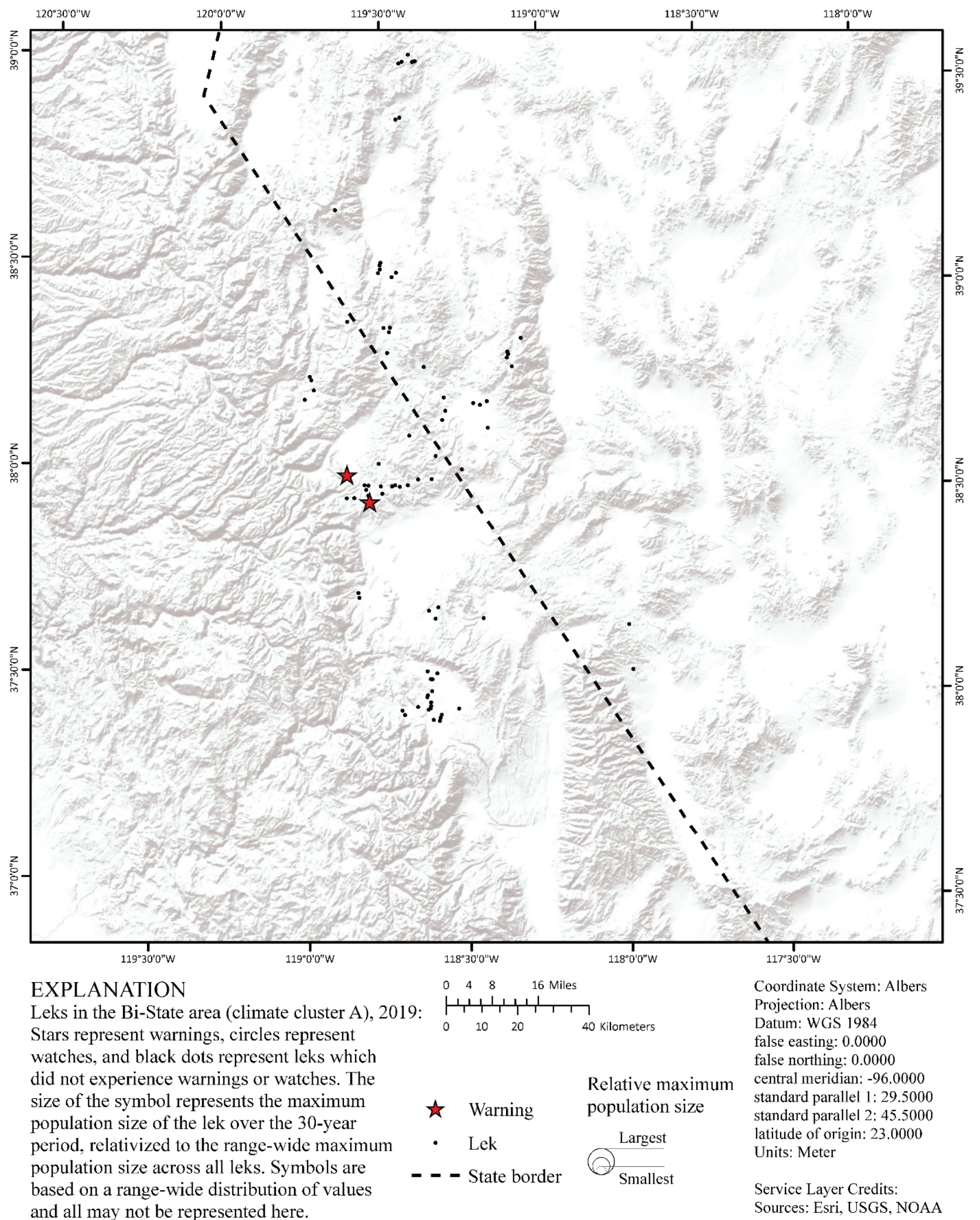

Figure 59. Spatial and temporal depiction of watches and warnings of greater sage-grouse (Centrocercus urophasianus) population declines at the lek scale within climate cluster A (CC-A; Bi-State area) during 2019. Map image is the intellectual property of Esri and is used herein under license. Copyright (C) 2020 Esri and its licensors. All rights reserved. 


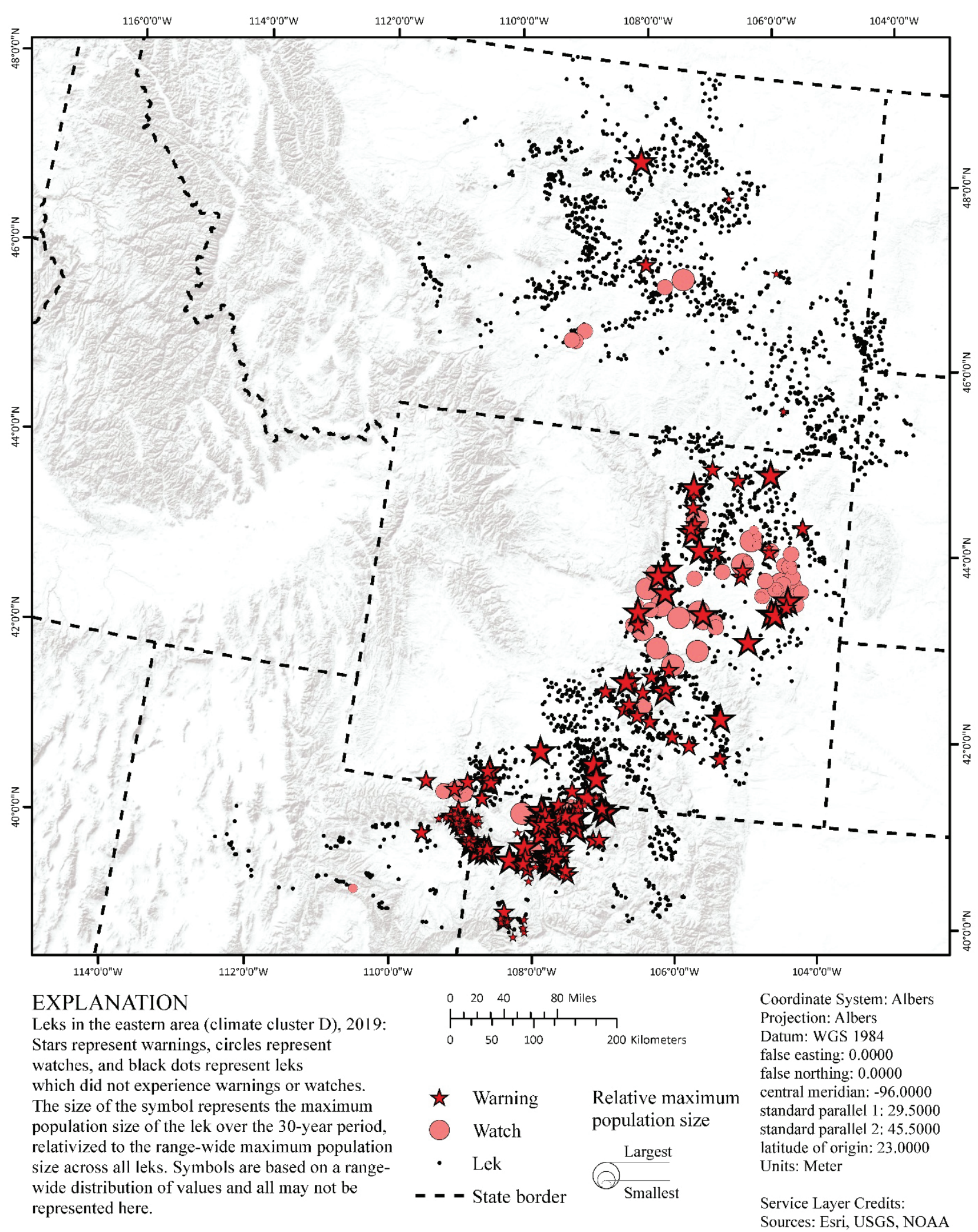

Figure 60. Spatial and temporal depiction of watches and warnings of greater sage-grouse (Centrocercus urophasianus) population declines at the lek scale within climate cluster D (CC-D; eastern area) during 2019. Map image is the intellectual property of Esri and is used herein under license. Copyright (C) 2020 Esri and its licensors. All rights reserved. 


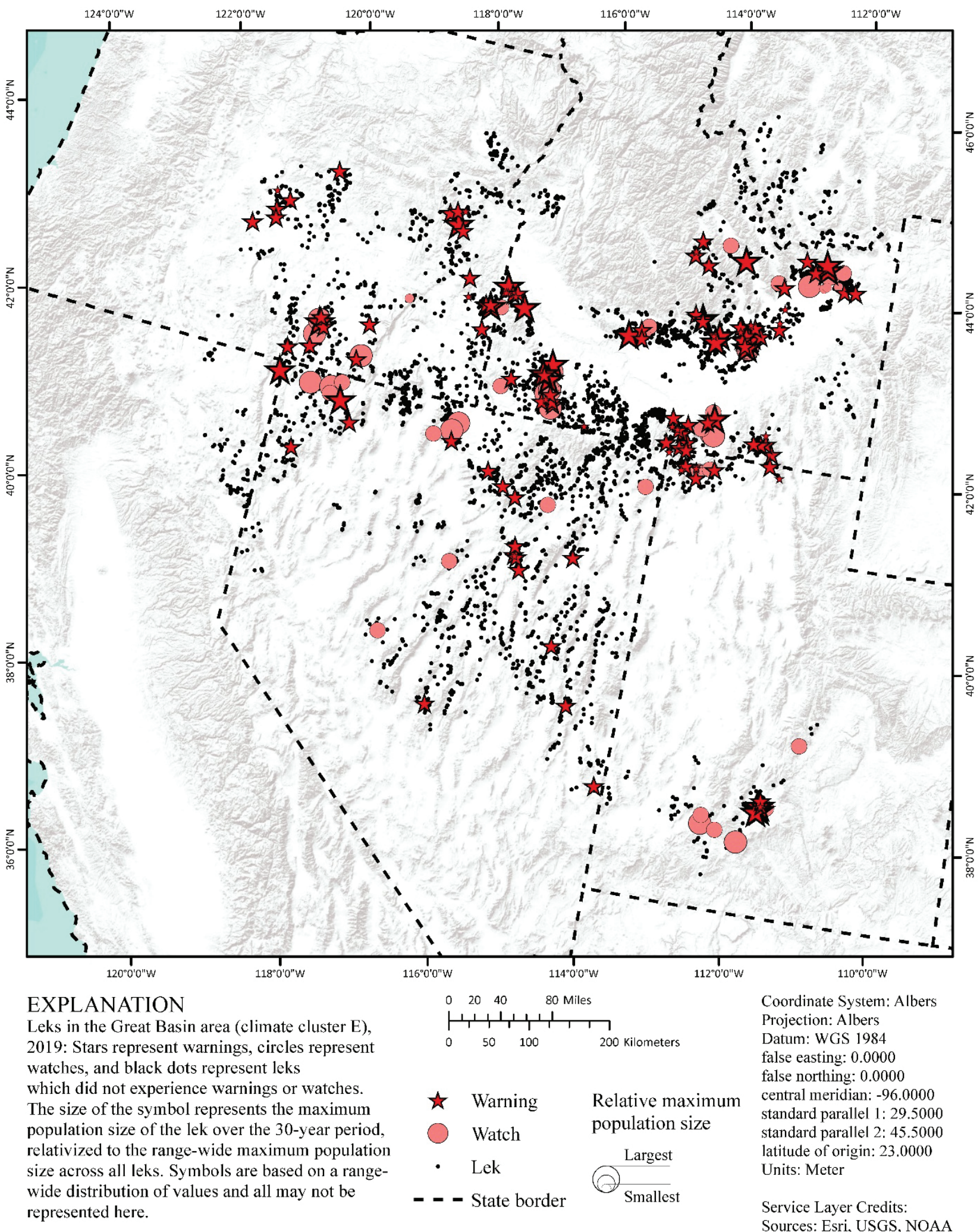

Figure 61. Spatial and temporal depiction of watches and warnings of greater sage-grouse (Centrocercus urophasianus) population declines at the lek scale within climate cluster E (CC-E; Great Basin area) during 2019. Map image is the intellectual property of Esri and is used herein under license. Copyright (C) 2020 Esri and its licensors. All rights reserved. 


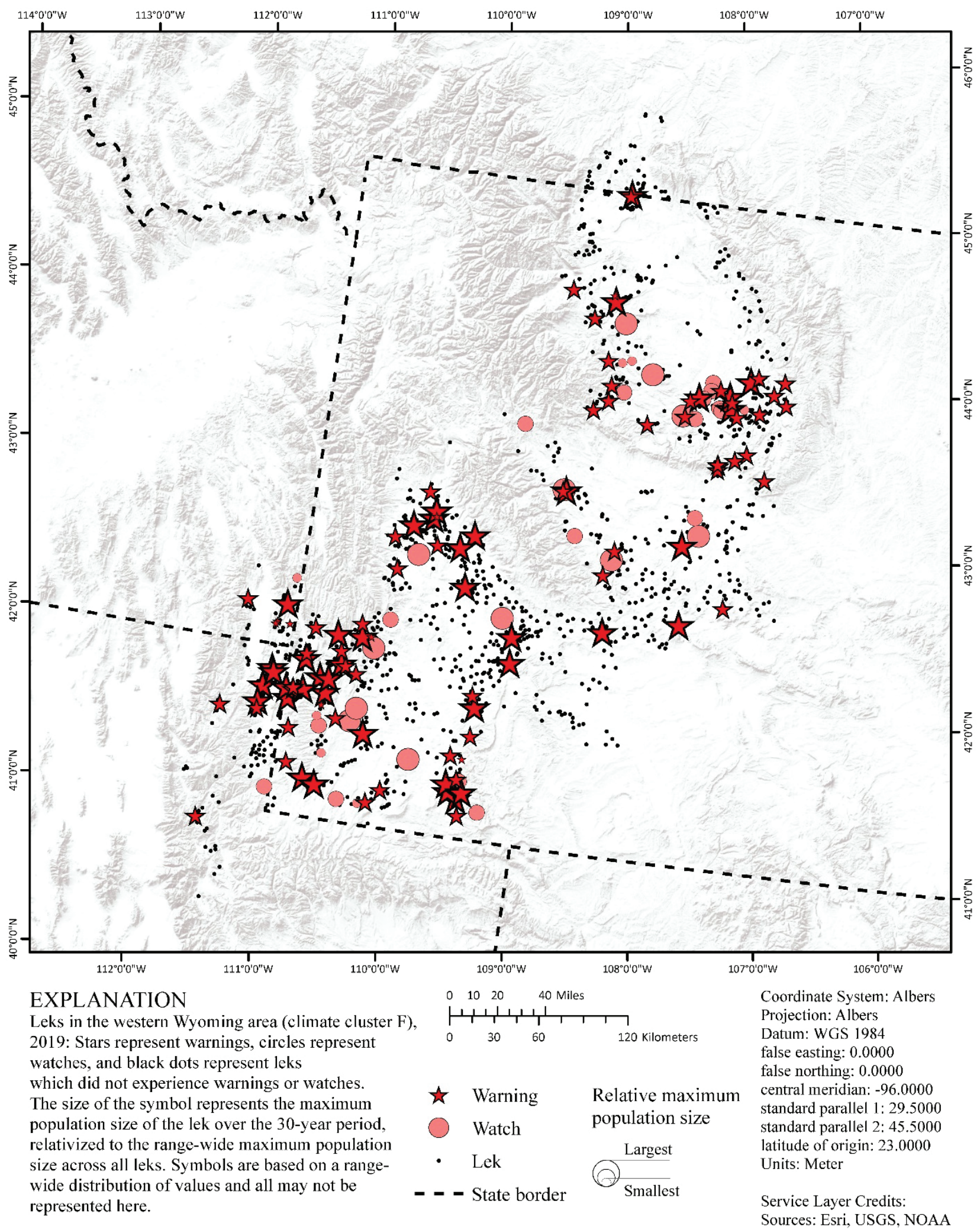

Figure 62. Spatial and temporal depiction of watches and warnings of greater sage-grouse (Centrocercus urophasianus) population declines at the lek scale within climate cluster F (CC-F; western Wyoming area) during 2019. Map image is the intellectual property of Esri and is used herein under license. Copyright $(\subset) 2020$ Esri and its licensors. All rights reserved. 


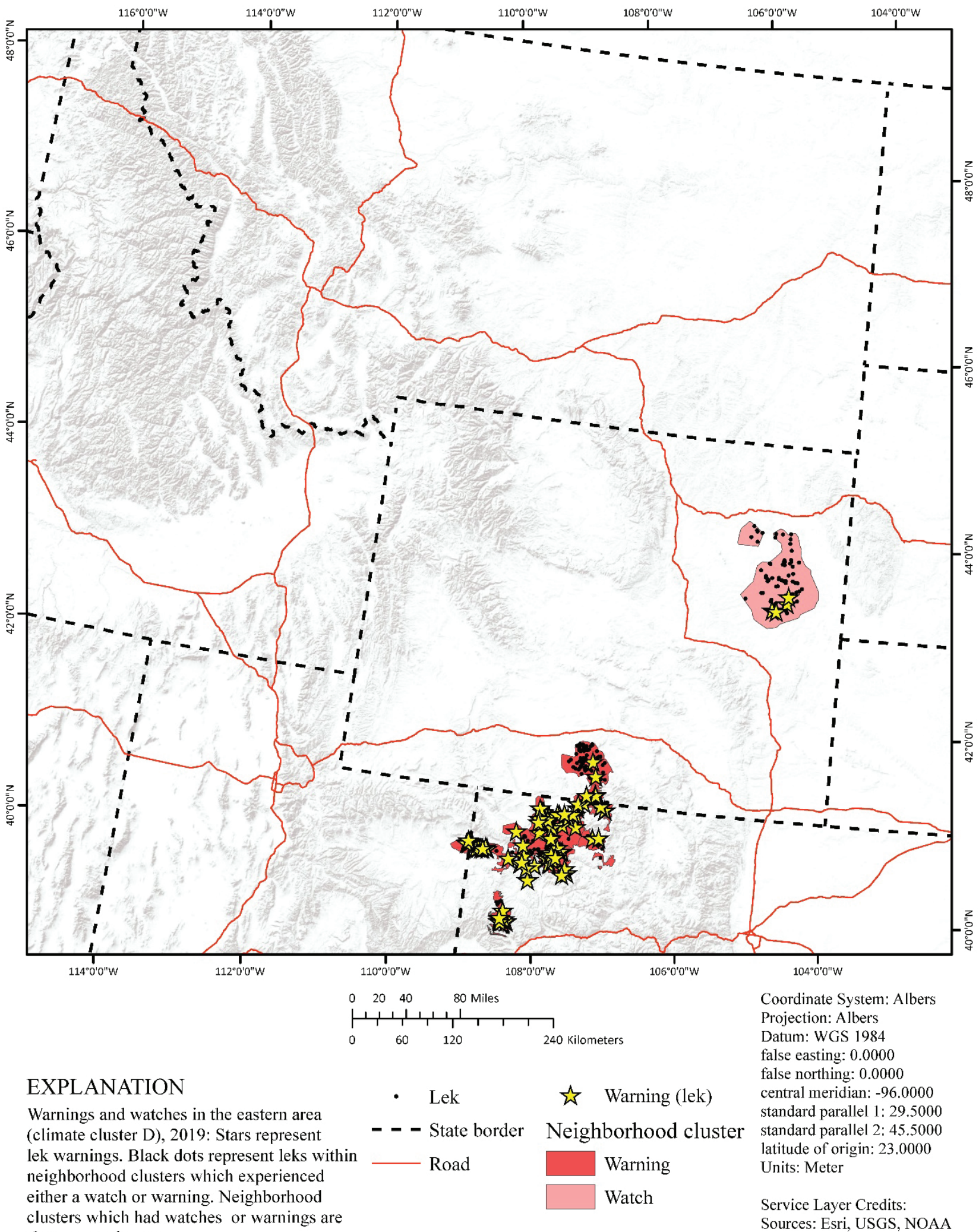
Sources: Esri, USGS, NOAA

Figure 63. Spatial and temporal depiction of watches and warnings of greater sage-grouse (Centrocercus urophasianus) population declines at the neighborhood cluster scale within climate cluster D (CC-D; eastern area) during 2019. Map image is the intellectual property of Esri and is used herein under license. Copyright $@ 2020$ Esri and its licensors. All rights reserved. 


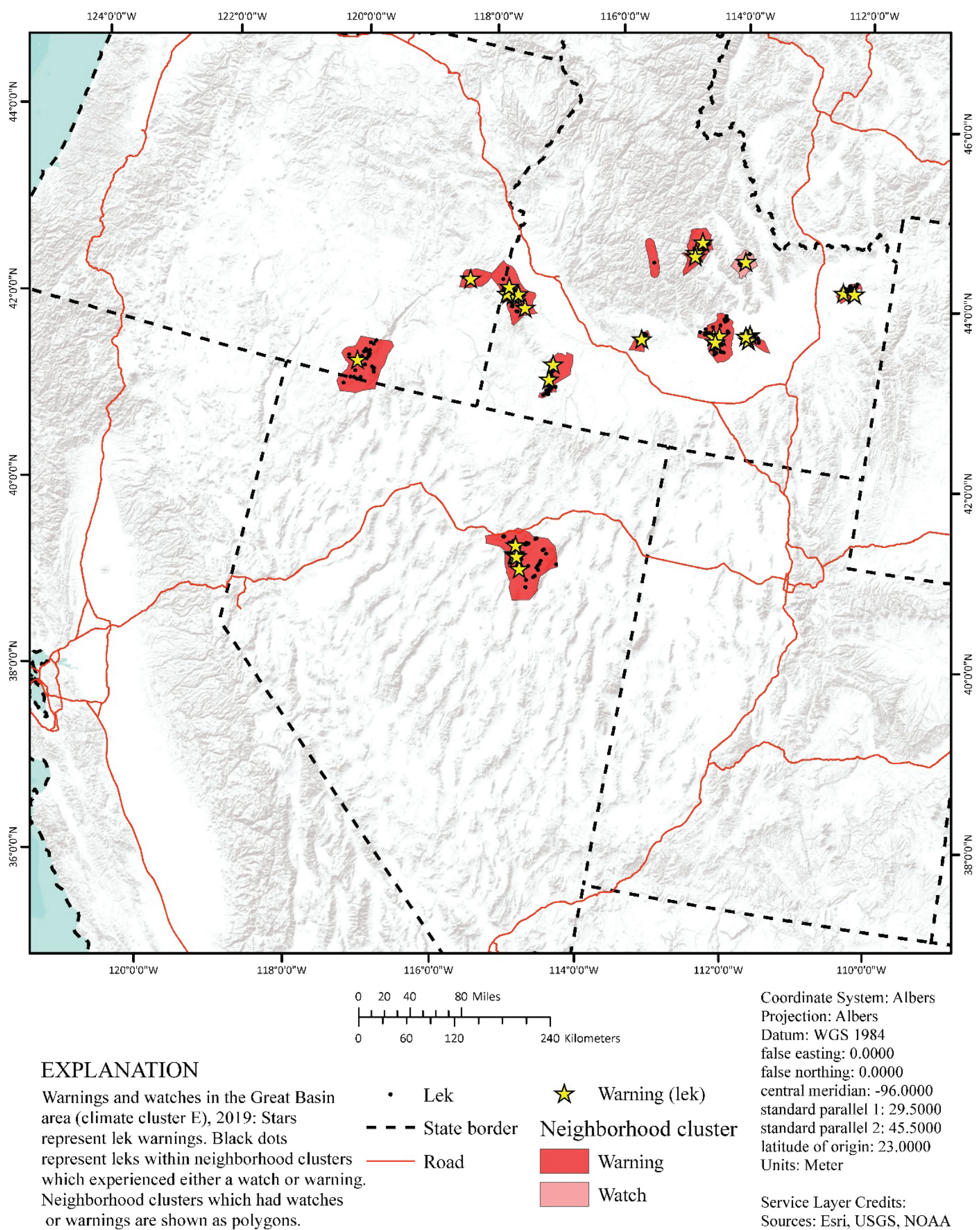

Figure 64. Spatial and temporal depiction of watches and warnings of greater sage-grouse (Centrocercus urophasianus) population declines at the neighborhood cluster scale within climate cluster E (CC-E; Great Basin area) during 2019. Map image is the intellectual property of Esri and is used herein under license. Copyright (C) 2020 Esri and its licensors. All rights reserved. 


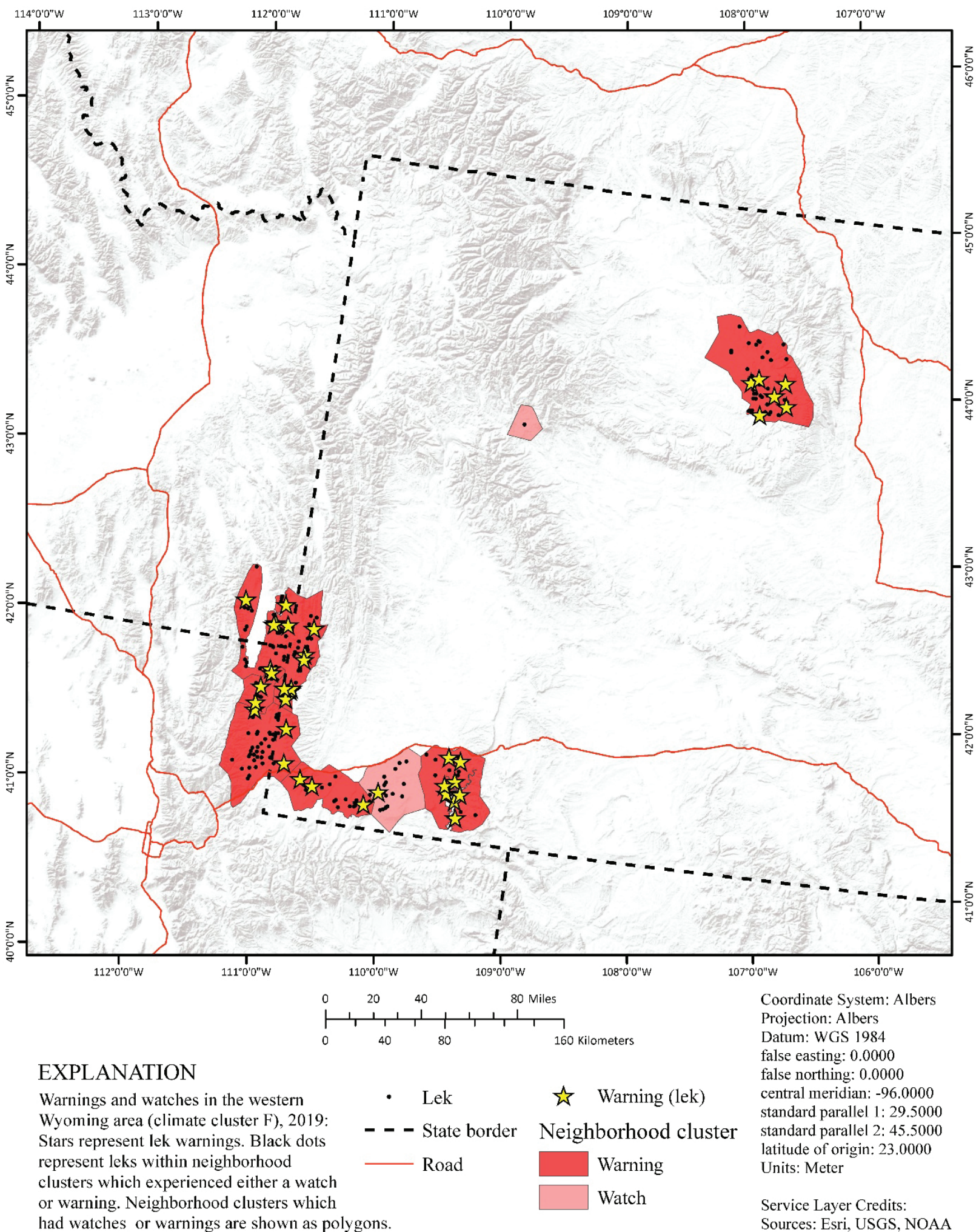

Figure 65. Spatial and temporal depiction of watches and warnings of greater sage-grouse (Centrocercus urophasianus) population declines at the neighborhood cluster scale within climate cluster F (CC-F; western Wyoming area) during 2019. Map image is the intellectual property of Esri and is used herein under license. Copyright $(\subset) 2020$ Esri and its licensors. All rights reserved. 

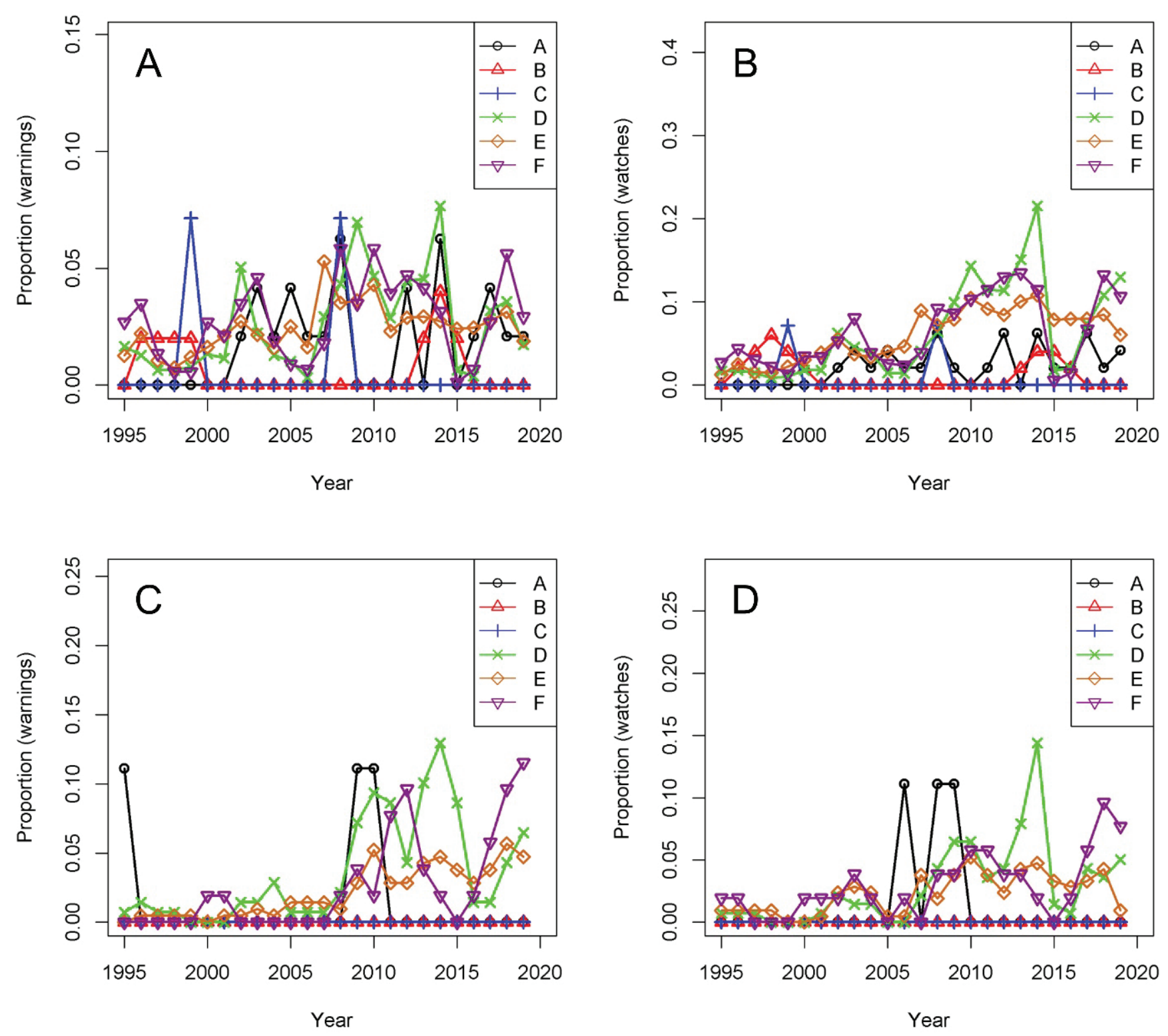

Figure 66. Proportion of $A$, warnings at leks; $B$, watches at leks; $C$, warnings at neighborhood clusters; and $D$, watches at neighborhood clusters of greater sage-grouse (Centrocercus urophasianus) population annual declines across each climate cluster (A-F) in the western United States. Years 1990-94 are excluded from the plot based on a temporal window of 5 years, which was used to evaluate minimum data requirements for reaching watches or warnings through the targeted annual warning system. 

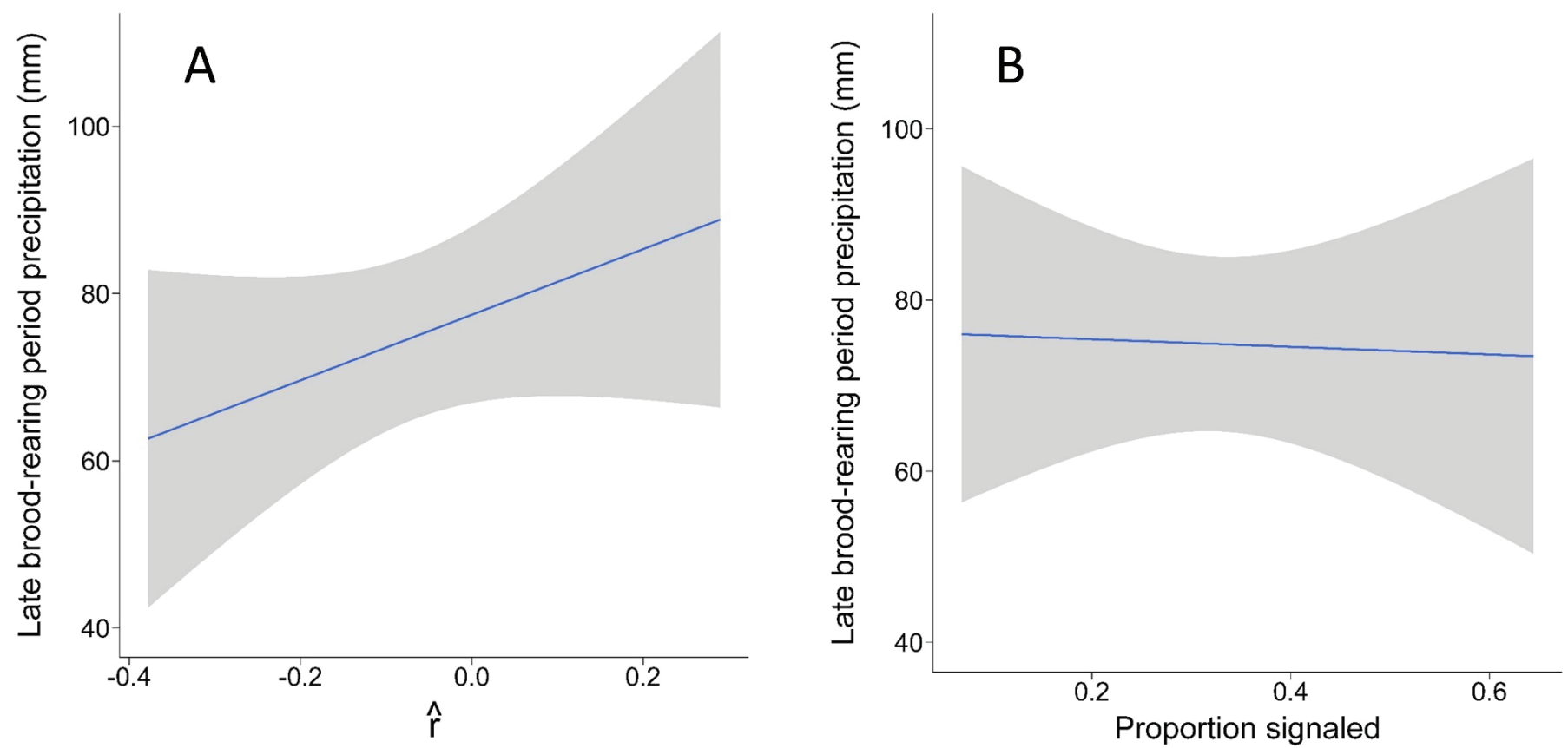

Figure 67. Relationship between $A$, breeding season precipitation and intrinsic rate of population change $(\hat{r})$; and $B$, breeding season precipitation and proportion of leks signaled within climate clusters for greater sage-grouse (Centrocercus urophasianus) in the western United States.

\section{Interpretation and Synthesis}

We presented a novel and integrated framework for evaluating sage-grouse population status and trends across the species' range in the United States. The first step involved compiling a wealth of sage-grouse count data previously stored in disparate databases by individual states into a single range-wide database of lek counts and locations that spanned six decades. Following methods by O'Donnell and others (2019), we then classified sage-grouse leks into spatially nested population clusters across the entire species' range that were derived from ecological covariates specific to sage-grouse ecology and habitat rather than geopolitical boundaries. These hierarchical cluster levels facilitated analyses of trends across multiple spatiotemporal scales dating back to the 1960s, as well as development of a targeted annual warning system (TAWS) that identifies declines in abundance at leks and populations that are likely attributed to local disturbances or habitat loss rather than those that operate at broad spatial scales governed by environmental stochasticity. Here, we provide interpretation of our results for our four study objectives and point out important caveats and limitations.

Objective 1-Lek Count Database. Synthesizing a standardized, range-wide sage-grouse database of field observations with recorded male lek counts, dates, times, and survey methods was vital to develop a hierarchical population monitoring framework, assess population trends, and create a TAWS. We used an open-source software solution for standardizing and compiling disparate sage-grouse lek count databases across states, which allowed for repeatable results that can better support scientific integrity. However, the approach sometimes required manual pre-processing of data inputs and quality-control steps of resulting data products due to the complex and often differing methods of data management implemented by states across six decades. The current lek databases, available at the time of this effort, included data gaps for some states, such as a lack of repeated counts of males, missing documentation on survey method types, and non-digitized field observations. Although these data gaps are not extensive across the range, they highlighted existing shortcomings and the need to compile data for the greatest accuracy of population trend assessments. 
Past studies that made use of lek counts in databases managed by multiple states have been faced with a myriad of decisions to reconcile differences in how counts were recorded to make records comparable. For example, in practice, lek locations on the landscape are fixed by geographic coordinates but may vary in space across time, which can result in a single lek having multiple location records that often include ephemeral coordinates for satellite locations with different lek names. Thus, information reported in Connelly and Braun (1997) was not sufficient to determine the decisions that went into combining lek count databases from nine states, but presumably some decision process took place to remove questionable records. Garton and others (2011) reported that they assumed most states followed the guidelines outlined by Connelly and others (2003) but found and removed instances of multiple locations representing the same lek, lek locations with no associated data, and additional records that invalidated use for their analysis. The Western Association of Fish and Wildlife Agencies (2015) used a hierarchical clustering algorithm to assign leks less than 1.2 kilometer $(\mathrm{km})$ apart to be combined into the same cluster, in an iterative procedure that proceeded until only a single cluster remained. Furthermore, they removed years with consecutive zero counts because they viewed the interpretation of zeros as often being ambiguous. Nielson and others (2015) combined lek count data across 11 states and adopted the same clustering and filtering procedures reported by Western Association of Fish and Wildlife Agencies (2015). Although there was likely considerable thought behind each of these data processing decisions, their influence on cross-study comparisons may be large and consequences are unknown.

We believe the lek count database used in our analysis is a major step forward for studies making use of range-wide lek count data. We acknowledge that our standardization practices did not fully eliminate problems associated with lek count data. For example, there is no ability to control for years when a lek went uncounted or records that lack a date or time of day for observations. Nonetheless, rules for lek count inclusion used in this study were described extensively in O'Donnell and others (unpub. data, 2020) and provide a common language describing how leks can be categorized (for instance, defined terms), summarized, and filtered. For cross-study comparisons, this is clearly a critical step to take that could result in less confusion when databases differ among studies. In other words, the use of a standardized lek database offers the opportunity to isolate differences among studies to factors independent of differing data inputs.

Objective 2 - Population Clusters. The development of population clusters to serve as a foundation for a hierarchically nested monitoring framework allows us to assess ecological patterns and processes acting on sage-grouse populations at multiple spatial scales, such as spatial patterns of abundance, habitat selection (Fedy and others, 2014; Rettie and Messier, 2000), and source-sink dynamics (Schumaker and others, 2014). The process of clustering leks at multiple spatial scales relied on graph linkages between high-fidelity lek sites, habitat covariates summarized at multiple spatial scales surrounding leks that can represent functional processes, and constraint-based rules of increasing distances that can reflect gradients of shorter, but more frequent, movements versus longer and less frequent movements. Spatial structure, movement constraints, and habitat conditions affect dispersal behavior (Campbell Grant and others, 2007; McRae and others, 2008), and incorporating these concepts into a clustering algorithm increased the likelihood of grouping leks shared by individuals across multiple spatial scales. The identification of lek adjacency and graph linkages, combined with habitat characteristics surrounding leks, aided in how we grouped leks and maximized the similarities of leks within groups while simultaneously maximizing dissimilarities between groups among different cluster levels.

We considered numerous caveats related to our hierarchical population monitoring framework. First, data gaps of missing or unknown leks could affect lek structure, and therefore, the derived fine-scaled clusters. As the number of leks grouped together increases, the effects of missing data are likely to dissipate quickly. Missing data would also need to occur in less dense areas of leks to affect the structure, as defined by the LC-MSTs. After we visually inspected nonactive leks excluded in the clustering algorithm, we did not find evidence that withholding those leks would have resulted in different clusters because they usually occurred adjacent to other active leks. Second, telemetry data describing sage-grouse movements could better inform lek structure and connectivity at local scales. Because such data was unavailable range-wide, for this analysis, we incorporated local expertise from state representatives to adjust LC-MSTs, which described prominent linkages between neighboring leks. Third, many clustering algorithms exist, but few exist for partitioning landscapes that can assess covariates, incorporate constraint-based rules, and account for structure (achieved by modifying LC-MSTs). After evaluating the capabilities of various clustering algorithms, we decided the SKATER algorithm was the best approach (see O'Donnell and others, 2019). Fourth, we did not incorporate population demographics (for example, trends or densities) as covariates within the clustering algorithm because these would conflict with the purpose of our clusters. For example, leks with higher population abundance could be in higher quality habitat, whereas lower abundance leks could be in lesser quality habitat. However, incorporating such relationships likely would lead to non-contiguous groupings instead of nested clusters. Fifth, we considered whether the inclusion of genetic data could inform the clustering of leks. We decided that these data were not appropriate because they reflect long temporal scales, and genetic flow does not directly correspond to habitat selection. Lastly, genetic data does not exist across the range at adequate spatial sampling corresponding to the fine scaled clusters. We refer readers to O'Donnell and others (2019) for further discussion of these caveats related to the sage-grouse hierarchical population monitoring framework. 
Objective 3 - Spatiotemporal Trends. We developed a single, novel SSM model that allowed estimation of trends in population abundance across different spatiotemporal scales using the range-wide lek count dataset. The advantage of this approach allowed hierarchical nested random effects based on spatial clustering to help inform missing data and disparate count histories while accounting for error associated with imperfect detection during lek counts. Furthermore, our approach reduced the large potential biases that are typically associated with sensitivity in start and stop years along abundances that fluctuate through time, specifically by inferencing trends at different temporal scales based on abundance nadirs.

We chose the lek and two different cluster levels for our trend analysis among the 13 levels derived from Objective 2. In our study, the lek was the finest unit of measurement of inference. Because sage-grouse are known to move between lek sites within and among years (Emmons and Braun, 1984; Walsh and others, 2002; Fremgen and others, 2017; Wann and others, 2019), population fluctuations at leks may reflect changes in simple shifting attendance between leks, immigration and emigration, as well as population vital rates (for example, reproduction and survival). It follows that observed reductions in population sizes at leks may not reflect reductions at larger spatial scales (for example, NCs) but instead reflect changes in local abundance and distributions within larger spatial scales, which can be informative at local levels. We chose cluster level two as the NC, our second unit of measurement, because it was likely the most suitable to account for groupings of leks with smaller inter-lek movements within NCs while minimizing movement between NCs. Although our model generated variation across the range in the number of leks included in level two clusters, we feel this spatial scale provided enough closure of populations (fig. 4) to attribute fluctuations in abundance to variation in population vital rates that are influenced primarily by local environmental conditions instead of sage-grouse movement patterns. Thus, trends that reflect declines at this scale more likely reflect changes in abundance at the meta-population level where actual loss of sage-grouse numbers are a result of abiotic and biotic factors that can dampen population vital rates. Furthermore, in consultation with land and wildlife managers, cluster level two collectively provided the most evidence of an appropriate scale for applicability of conservation actions aimed at sustaining sage-grouse populations. In comparison, our third unit of measurement, was the largest spatial scale where evidence indicated that population dynamics were most strongly governed by broadscale climatic conditions (for instance, precipitation; fig. 5). Specifically, estimated trends at this scale considered the greatest number of leks grouped together with no movement constraints and provided information about regional increases and decreases in numbers that operate at a meta-population level. In summary, the three units of measurements allowed comparison of trend estimates among temporal scales that were governed by processes acting at different spatial scales.
Several previously published studies evaluated range-wide trends in rates of population change based on peak male lek counts. For example, Connelly and Braun (1997) were the first to report large scale annual declines of sage-grouse based on long-term lek count data collected across nine states; they estimated variable population declines ranging from 17 to 47 percent over a time series that ranged from the 1950s and 1960s to 1994, which represented scales large enough to cover multiple oscillations. Connelly and others (2004) provided a more comprehensive analysis across sage-grouse range, which indicated sage-grouse declined at an annual rate of 2 percent per year from 1965 to 2003. Although these previous findings are not directly comparable to ours, owing to differences in spatial extent of analyses and temporal scales, the overall rates of decline range-wide identified by these earlier studies were less than those estimated here. Specifically, we found sage-grouse are declining annually at approximately 3.0 percent range-wide from 1965 to 2019 (figs. 18-20; table 6).

Similar to our framework, others have employed advanced modeling techniques to estimate trends at different spatial scales. For example, trends in peak male counts have been evaluated relative to seven Sage-Grouse Management Zones (SMZ) developed by WAFWA (Garton and others, 2011, 2015; Nielson and others, 2015; Western Association of Fish and Wildlife Agencies, 2015), which were delineated based on floristic characteristics thought to be biologically important to sage-grouse (Stiver and others, 2006). Our study expanded on these concepts by estimating trends across novel, empirically driven, and replicable lek clusters. These clusters required additional methods to identify nested spatial population structure most relevant to sage-grouse population ecology and landscape characteristics (see Objective 2). The inferences from a range-wide analysis of lek trends for SMZ revealed an average annual decline of 2.1 percent per year from 1965 to 2015 (Western Association of Fish and Wildlife Agencies, 2015), which equated to an average annual $\lambda$ of approximately 0.979 . This annual $\lambda$ value was approximately 1.0 -percent greater than the average annual values reported here $(\hat{\lambda}=0.969)$ across our longest temporal scale (1966-2019). However, it is important to recognize that a 1.0-percent difference on an annual basis results in profound cumulative changes in abundance over relatively long periods of time. For example, extrapolating Western Association of Fish and Wildlife Agencies (2015) estimates to 2019 indicated a 68.2-percent range-wide decline since 1965, which is less than the realized 80.7-percent decline we derived. For comparative purposes, we constrained our inferences from the SSM model to 1965-2015, matching Nielson and others (2015) years of trend assessment and derived a $\hat{\lambda}$ of approximately 0.977 , which was nearly identical to Nielson and others (2015). Importantly, the stop year of 2015 was near the oscillation apex, meaning estimating population trends from a start year nadir (1965) to an end year apex (2015) inevitably results in estimates that are biased high. Our trend assessment properly accounts for biases associated with oscillations in abundance such as sensitivity to start-stop years. 
The inferences from Garton and others $(2011,2015)$ also used SMZs and employed a population reconstruction modeling approach from successive paired counts across lek count histories, dating from 1965 to 2007 (Garton and others, 2011) and more recently from 2008 to 2015 (Garton and others, 2015). Garton and others (2015) reported substantial reduction in sage-grouse populations more recently, with a 56-percent decline in breeding males estimated over a 6 -year time frame from 2007 to 2013 , equating to average annual $\hat{\lambda}$ of approximately 0.872 which is much lower than the average annual trends reported here, even compared to average annual $\hat{\lambda}$ across the two most recent oscillations (past 17 years and past 6 years). After adjusting our inferences to 2007-2013 for comparison, we estimated an average annual $\hat{\lambda}$ of 0.893 , which is substantially greater than Garton and others (2015). The adjustment in range of years exemplifies the importance and sensitivity of any assessment to start and stop points, stressing the importance of consistency, such as basing any trend in abundance on population nadirs as we have done here. For example, data with initial years that start on apex and stop on nadirs only provide information about length of amplitude (that is, displacement) of oscillation and will bias $\hat{\lambda}$ low (Mathews and others, 2018). These start-stop sensitivities will lessen as more oscillations are included to estimate trend, but as shown with these comparisons, not accounting for such sensitivities could be misleading even after 60 years. Nevertheless, based on model estimates of minimum number of males in Garton and others (2015; see fig. 9) from 1965 to 2013, sage-grouse declined approximately 80 -percent, which reflects an average annual $\hat{\lambda}$ of 0.97 . These estimated trends were consistent with our findings of 80.7-percent declines and average annual $\hat{\lambda}$ of 0.970 from 1965 to 2019 . After adjusting the time frame of inference to stop at 2013, $\hat{\lambda}=0.97$, similar to estimates by Garton and others (2015).

Given the hierarchical cluster levels of our framework, trend comparisons with other published reports also can be made at the regional or state level. For example, an integrated population model (IPM) that relied on similar lek count data was carried out in the Bi-State Distinct Population Segment designated by the USFWS to help inform a listing status assessment (Coates and others, 2019a). Estimates in that study are comparable to ours because the spatial extent represented the same spatial boundaries as CC-A and both designs shared the nadir approach. Our estimates were slightly lower than Coates and others (2019a) across the different temporal scales. We attributed this to inclusion of lek counts collected during 2019, which were relatively low and influenced trends across the temporal scales. Additionally, Coates and others (2019a) included demographic information from telemetered sage-grouse in estimation of $\hat{N}$ and $\hat{r}$ using an IPM framework. However, both studies are advances on earlier published estimates from the Bi-State when years of study ranging from an apex to nadir resulted in lower trend estimates (Coates and others, 2018; Mathews and others, 2018), exemplifying the start-stop sensitivities to estimating trend. In the nadir-to-nadir studies, we found consistencies in relative patterns of stability in the central 'core' population of the Bi-State area and sharp declines in peripheral populations. In addition, studies consistently conclude that the Bi-State area demographically outperforms other areas of the species' range within the Great Basin, which can be explained by Bi-State populations being subjected to relatively fewer wildfires and located at higher elevations, also offering greater resilience to disturbance and lower susceptibility to drought conditions (Coates and others, 2019a).

At the state level, Montana estimated population size and trend using repeated count data (Montana Fish, Wildlife, and Parks, unpub. data, 2020) by using published $N$-mixture modeling approaches (McCaffery and others, 2016). These authors restricted the data from 2002 to 2020 based on criteria for repeated count data and modeled $\hat{N}$, which also resulted in annual average $\hat{\lambda}$ of 0.998 . Our model estimated a short-term annual averaged $\hat{\lambda}$ of 0.964 for Montana (see appendix 4), which differed from 0.998 . Montana primarily intersected CC-D (45.3 percent), of which the population abundance nadir was 2004. Because our analysis did not make inference to 2020, we instead constrained the Montana state $N$-mixture model estimates from 2004 to 2019 to make comparison with our results and reflect an unbiased trend of nadir to nadir. This constrained range of years resulted in an average annual $\hat{\lambda}$ of 0.966 for the Montana analysis, which was identical to our SSM estimate (0.966). In this case comparison, we conclude that SSM based on maximum counts provides very similar estimates as $N$-mixture models that rely on repeated count data, which can be expected if detection error is constant or random through time (Monroe and others, 2019). However, we recognize that $N$-mixture models allow for estimates of detection probability and are better suited for estimating true abundance. We provide graphical illustration of years of inference between an $N$-mixture model using repeated count data and our SSM model that relied on maximum count data for comparison (appendix 6, fig. 6.1).

We also note several additional considerations when comparing our results to previously published studies. First, data quality and preparation approaches differed substantially from the previous studies referenced, though our approaches were reasonable and the rules used and corrections made improved the quality of remaining data appropriate for our analyses (see earlier in text). Second, the way in which leks were aggregated to larger spatial extents (for instance, SMZ and CCs) differed, which may have implications for trend estimation. However, this hierarchical approach better matches the biology of sage-grouse and, therefore, the estimates are more likely to be relevant to populations and management. Finally, we used a SSM framework that included random effects for nested spatial structures to predict $\hat{N}$ and $\hat{\lambda}$ , which informed years of missing lek counts for newly found leks and allowed retention of leks even following their extirpation. In contrast, other studies employed different filtering methodologies to account for data collection inconsistencies and years when lek counts did not occur. 
Although a full investigation of biotic and abiotic factors that influenced spatiotemporal trends was beyond the scope of this study, some general patterns can be explained. In determining the most appropriate $\mathrm{CC}$ scale (Objective 2), our findings revealed an important association between precipitation and population growth (increased $\hat{\lambda}$ ) at broad spatial scales. This relationship corroborates recent studies conducted at smaller scales within sage-grouse range that employed other modeling approaches. For example, models revealed that in the absence of fire, a $100-\mathrm{mm}$ increase in precipitation from spring through fall was associated with a 4.4-percent increase in $\hat{\lambda}$ using lek count ratios in the Great Basin (Coates and others, 2016). Another study integrating lek and demographic data from telemetered sage-grouse in the Bi-State area reported that the most important driver was change in precipitation, such that a 50-percent increase in precipitation during this time frame resulted in 15.5-percent (95-percent CRI $=5.4-26.9$ ) increase in $\hat{\lambda}$ the following year (Coates and others, 2018). In the Great Basin, local studies have revealed mechanistic relationships between precipitation, and other climatic conditions, and population dynamics. For example, drought adversely affects population recruitment (Blomberg and others, 2012), such that chick survival is negatively influenced by water scarcity (Gibson and others, 2017). The prevailing explanation is that chicks rely on net primary productivity, which contains more moisture, provides food resources, and conceals chicks from predators during late summer months (Thompson and others, 2006; Aldridge and Boyce, 2008; Gregg and Crawford, 2009). Drought reduces above ground net primary production (Donnelly and others, 2018), and drought conditions substantially impact chick survival in sage-grouse (Aldridge and Boyce, 2008). Regional water scarcity (for instance, drought sensitivity) and resulting adverse lag effects on food resources act as abiotic and biotic factors that define ecological minimums that influence population carrying capacity (Donnelly and others, 2018). Thus, limitations in food and cover resources following multiple years of drought (Aldridge and Boyce, 2008) can act to reduce carrying capacity and lead to low demographic rates.

Of particular significance, most sage-grouse populations range-wide experienced above-average abundance during early periods of oscillation. Yet, moderate declines occurred from 1960 to the late-1980s, followed by a marked decline with steadily decreasing and below average abundance thereafter to present (2019; fig. 18). Across CCs, the estimated population abundance nadirs appeared to correspond to years of drought that dated back 60 years. The notable exception is CC-F, which experienced relatively high and consistent levels of annual precipitation from the 1960s to 1980s (see https://bit.ly/30h1agw, section 6.2.2 Instrumentation Record) that aligned with estimates of consistent levels of abundance (that is, little evidence of fluctuations). However, in the late 1980 s, our model estimates show a major reduction in abundance range-wide, which appeared to correspond to a widespread drought that spanned the western United States from 1986 through 2016 (Piechota and others, 2004; Williams and others, 2020) and included the western Wyoming area (CC-F). Following this decline, we observed fluctuations in abundance that corresponded to precipitation patterns for all CCs, including western Wyoming. These fluctuations were consistent with previous assessments (Fedy and Aldridge, 2011; Edmunds and others, 2018). Importantly, sage-grouse populations across their range were reduced to much smaller sizes apparently as a result of drought and local-scale disturbances and have not recovered to pre-1980s numbers. At small numbers, sage-grouse populations are less capable of recovering and more prone to extirpation (for example, the Allee effect), especially given both environmental and demographic stochasticity. Additionally, after the 1980s, many leks range-wide were likely extirpated and continue to decline or remain at low numbers. Further support for this phenomenon is that CC-F (western Wyoming area) consists of the largest population sizes based on greatest number of males per lek count, on average. Thus, ensuring that high-quality habitats will remain intact within these core areas could make populations less susceptible to abiotic impacts (for example, drought; Aldridge and Boyce, 2008), which will likely increase viability and resiliency to the effects of drought.

Furthermore, loss of habitat from disturbances on the landscape could interact with climate conditions to perpetuate long-term declines in population trajectories. Within the Great Basin (CC-E), wildfire frequency and size has dramatically increased in the past two decades (Brooks and others, 2015), which has contributed to conversion of vast areas from a shrubland state to one of annual grassland (Chambers and others, 2014; Pilliod and others, 2017). Consequently, CC-E has undergone rapid population declines over the past two periods of oscillation and recent evidence indicates that such long-term habitat loss from wildfire nullifies positive effects typically associated with years of increased precipitation (Coates and others, 2016). Thus, the vast spatial extent of wildfire and subsequent state-transitions to annual grasslands across much of this climate cluster may simply outpace implemented restoration efforts owing to spatial and temporal asynchronies between slower sagebrush recovery processes and more immediately reactive sage-grouse demographic responses (Ricca and Coates, 2020).

Similarly, we observed population fluctuations with continual and consistent declines at the medium temporal scale (four oscillations) in population abundance across the eastern area (CC-D). This time frame corresponds to widespread development of anthropogenic features associated with energy infrastructure and type conversion of sagebrush to agriculture. For example, declines within CC-D largely correspond to areas of oil and gas disturbance (Green and others, 2017), particularly in northeastern Wyoming and northwestern Colorado, as well as cropland conversion in areas of Montana (Aldridge and others, 2008; Smith and others, 2016). Therefore, sage-grouse population trends at broad scales, driven by stochastic climatic conditions, were likely stressed further by deterministic factors such as landscape disturbances that act to reduce the ability for populations to 
recover from drought years. However, declines across CC-D have weakened over the past 20 years and most notably in the past 10 years, perhaps a result of increased conservation efforts. For example, it is possible that management actions enacted to reverse impacts that limit surface disturbances in areas of high sage-grouse abundance, such as the Wyoming "core sage-grouse area" strategy, may have contributed to this phenomenon (Spence and others, 2017). However, research that investigates the efficacy of conservation efforts across different spatial scales would be beneficial because such actions are typically local and relatively recent. Additionally, in the absence of widespread disturbance and relatively large intact tracts of sagebrush landscapes, populations likely are more resilient and buffered against long-term declines albeit still experiencing stochasticity. For example, within western Wyoming (CC-F) populations experienced less severe declines and even slightly positive growth over the past three decades compared to longer time frames.

Our model predictions indicate that over 60 percent of leks range-wide have 38-year (approximately three complete oscillations) extirpation probabilities of over 50 percent, which is given the assumption that disturbances continue to persist on the landscape and are coupled with continued widespread drought throughout the western United States (Walsh and others, 2014; Crosby and others, 2015). The bimodal frequency distribution of extirpation probabilities supports the concept that leks have either relatively low (around 20 percent) or relatively high (around 80 percent) extirpation probabilities across the range. We hypothesized that increased probability of extirpation is a function of low lek abundance, high degree of inter-annual variation, decreasing population trend, and peripherality. A strong advantage to our SSM framework is that these conditions are inherently accounted for within the 30-year projection. Climate cluster F had the lowest extirpation probabilities for leks and NCs, supporting the hypothesis that robust populations within intact habitat have the lowest probability of extirpation despite relatively high stochasticity. Overall, spatial depictions of extirpation probabilities reflect increased vulnerability across scales at the periphery of sage-grouse range, supporting past findings (Aldridge and others, 2008). For example, CC-B (Washington area) had the highest lek extirpation probability (fig. 41; table 8), whereas CC-A (Bi-State area) had the highest NC scale extirpation probability (fig. 42; table 8 ). This should be expected because both regions represent isolated populations where $\mathrm{CC}-\mathrm{B}$ consists of the lowest average lek count (table 6) and CC-A consists of multiple NCs with the fewest leks per NC (table 7; lowest median value). It also is important to point out that virtually all NCs with greater than 50-percent extirpation probability are on the periphery of the range (fig. 42), which strongly reinforces the concept that maintaining large populations within intact, high quality habitats could help to buffer against environmental and demographic stochasticity (Aldridge and Boyce, 2008).

One of the key advantages of using SSMs to forecast extirpation probabilities is these models rely on a first-order Markov process. In our case, the error for each year of prediction was equal to the probability of observing the state in time $t$ (for example, $\hat{N}_{t}$ ), which was conditioned on the state in the previous time (for example, $\hat{N}_{t-1}$ ). If predictions are made across many years (for example, increased prediction intervals) and parameter uncertainty is high in the final intervals of observed data (for example, 2019), then predictions can become uninformative due to increased propagation of error. However, the increased precision of parameter estimates in recent years observed in our analysis, which was largely a function of increased sampling efforts through time, reduced the range of possible outcomes and magnitude of propagated uncertainty in prediction intervals. Nevertheless, multiple important caveats should be considered when interpreting extirpation probabilities. First, although we set the rule of extirpation as less than two males to align with an 'inactivity' status set by state agencies, we recognize that this criterion could lead to underestimation of actual lek extirpation probabilities as a result of Allee effects (Courchamp and others, 1999). Second, SSM estimates assumed that 2019 had reached an abundance nadir. However, if abundance continues to decline in subsequent years, then extirpation probabilities are underestimated and, thus, these probabilities could be interpreted as optimistic. Third, the lek level analysis did not account for recolonization based on meta-population dynamics or estimated changes in habitat that may lead to recovery. Fourth, at the NC scale, the sum of lek counts within a NC must be less than two for a NC to reach extirpation, which means every lek in the cluster must reach extirpation. Thus, our extirpation probabilities likely are conservative, given populations may be functionally extirpated at much larger numbers because 50-500 individuals may be required to maintain a minimally viable population (Lande, 1988). Similarly, NC extirpation probabilities may be near or at zero (that is, robust at metapopulation level) even though leks within the $\mathrm{NC}$ could have relatively high probabilities. 
Lastly, trend estimates from our SSM models at the relatively long temporal scales could be biased high based on potential effects of unequal sampling efforts through time (fig. 2). It is known that larger leks (sometimes referred to as 'trend' leks) were disproportionately favored for monitoring efforts in earlier years. Ancillary analyses that compared range-wide mean lek size (based on counts) to mean intrinsic rate of change in abundance $(\hat{r})$ revealed a positive correlation (Pearson's $r=0.11$ ), which suggests that larger leks experience higher growth in abundance on average. Although the SSM derived estimates of $\hat{N}$ for non-sampled leks back-in-time, the parameter that was linked through spatiotemporally nested random effects was $\hat{r}$. If smaller leks had been sampled proportionate to availability in early years, then estimated $\hat{r}$ at lek, NC, and CC scales during those periods would likely have been lower $(\hat{N}$ would have been higher) than reported here. Additionally, our model does not account for leks that reached extirpation before their discovery to be surveyed. It is unknown whether this is another source contributing to underestimation of $\hat{N}$ or if it is related to the previous sampling bias. It must be recognized that it is possible that newly discovered leks in recent years may not have existed back-in-time, although the model does impute $\hat{N}$ under the assumption that they did. Instances such as this may be offset, in part, as a result of missing leks that experienced extirpation before discovery. However, it is more likely to represent a spatial misallocation of sage-grouse, given that $\hat{N}$ is a derived parameter and $\hat{r}$, which is the scale-invariant information sharing parameter, has demonstrated decline across all temporal scales, and all sampling regimes. The potential for spatial misallocation of sage-grouse highlights the importance of closed populations at $\mathrm{NC}$ and CC scales.

Objective 4 - Targeted annual warning system. Our example TAWS was designed to reduce variability in sage-grouse population dynamics caused by climatic fluctuations, ultimately increasing the speed and precision in the ability to detect populations in need of local management intervention. Additionally, TAWS provides a standardized, range-wide assessment of potential management actions that can improve population stability, which did not previously exist as a decision-support tool for managers and across the species' range. In practice, the TAWS can reduce the time and energy that managers spend responding to populations that are declining as a result of less manageable climate-related effects. In highly dynamic cold-desert sagebrush ecosystems, where sage-grouse populations respond largely to shifts in precipitation driving primary production, understanding when populations respond naturally to climate-related patterns compared to more localized natural or anthropogenic drivers is critical so that management actions can align with the appropriate spatial scale of detected declines. This is of particular importance so that less-immediately manageable effects (for example, climate) can be partitioned from those arising from disturbances, which include wildfire and annual grass invasion (Blomberg and others, 2012; Coates and others,
2016), energy development (Doherty and others, 2016; Green and others, 2017), conifer expansion (Miller and Wigand, 1994; Miller and Tausch, 2001; Reinhardt and others, 2020), agricultural conversion (Aldridge and others, 2008; Smith and others, 2016), changes in predator composition (Coates and others, 2020), and overgrazing from livestock or free ranging equids (Beever and Aldridge, 2011; Davies and others, 2014; Monroe and others, 2017; Danvir, 2018; Davies and Boyd, 2019). Specifically, juxtaposing population $\hat{\lambda}$ assessed at two different scales (lek versus CC and $\mathrm{NC}$ versus $\mathrm{CC}$ ) allowed us to examine when local population trends diverged from regional population trends, which can then inform when and where management actions could most effectively improve local scale sage-grouse population growth and distribution. For example, insights can highlight areas on the landscape that require additional field investigation to understand why populations are declining and whether managers can increase those populations through varying management actions. Accordingly, the TAWS also helps fulfill information needs for sage-grouse populations identified under existing land-use planning amendments (Bureau of Land Management, 2015). This is a rigorous framework for adaptive management solutions tied directly to performance of identifiable population units. Importantly, the TAWS framework can be readily applied as a solution for other species of conservation concern that exhibit similar strong spatial structuring and population responses driven by large scale climatic variation in the absence of localized disturbances.

We found that CC-D (eastern area), CC-E (Great Basin area), and CC-F (western Wyoming area) were the largest of the climate clusters and exhibited the greatest proportion of watches and warnings activated at the $\mathrm{NC}$ and lek scales. Interestingly, CC-F differed from CC-E and CC-D in that this cluster does not exhibit declining trends (see Objective 3 ). This phenomenon may be explained by differences in trends across lek sizes. Averaged $\hat{\lambda}$ is largely affected by larger leks because those leks contribute more to $\hat{N}$ at the NC scale. Thus, if larger leks are more stable than smaller leks, we should expect warnings and watches to occur on the smaller leks while the climate cluster as a whole exhibits stability. It is important to recognize that watches and warnings are assumed to be activated by local-level perturbations and, thus, offsetting such disturbances may result in population growth. One hypothesis for CC-E exhibiting the highest percentage of watches and warnings at NCs and leks over the past 29 years compared to all other CCs is that this cluster experiences the most cumulative impacts at broad spatial scales. For example, populations in CC-E are impacted with increasing cheatgrass-wildfire cycle (Coates and others, 2016; Pilliod and others, 2017), conifer encroachment (Baruch-Mordo and others, 2013), and anthropogenic disturbances that increase abundances of generalist predators (Coates and others, 2020). In appendix 7, we provide an example of activation of watches and warnings at both the lek and neighborhood scale immediately following a wildfire in southeast Oregon within the Great Basin. Nevertheless, watches and warnings have 
increased substantially through time range-wide. Because our models were robust for missing lek count data, increases in watches and warnings through time were likely not a result of increased lek count efforts, rather likely due to increases in disturbances on the landscape (for example, increased anthropogenic developments and frequency of wildfire).

Like any decision-support tool, caveats associated with analytical methods and subsequent interpretation require careful examination. Several caveats were described previously by Coates and others (2017) for the initial TAWS technique that was piloted in Nevada and subsequently improved upon in this study, yet some remain. First, the TAWS relies upon a SSM to estimate missing counts from time series data based on prior variance of observed counts and sharing of information across leks using hyper-parameters (that is, the automatic selection of smoothing parameters), which increases the reliability of parameter estimates (Kéry and Schaub, 2012). The TAWS cannot evaluate leks that are infrequently counted or never counted. Standardized lek count protocols that minimize number of years between counts for leks of interest would be highly beneficial and further improve parameter estimation, especially if the goal is to target specific leks or NCs where investigation of immediate change is of high interest (for example, leks in recently burned areas or around new anthropogenic structures). Furthermore, the potential for spatial misallocation associated with newly discovered leks and leks that were extirpated prior to discovery, as discussed in objective 3, highlights the importance of (1) restricting datasets for TAWS to a period of more rigorous data collection and (2) adhering to rules that require the presence of lek count data during periods that inform watches and warnings.

Second, TAWS is a framework aimed at managers and land stewards to identify recent declines that have been disentangled from large scale climatic effects but does not provide information regarding the cause of decline. However, retrospective analyses using watches and warnings or the log-odds ratios can be evaluated as a function of environmental covariates in future analyses. Third, at the lek scale, TAWS cannot necessarily identify whether watches or warnings were related to redistribution of breeding sagegrouse away from lek sites versus reduced population vital rates. Studies indicate that adult sage-grouse exhibit strong site fidelity following disturbance (Holloran and others, 2010; O'Neil and others, 2020) but offspring may disperse away from affected areas, as has been shown for mining developments (Remington and Braun, 1991). Thus, activation of watches or warnings may be attributed to dispersal away from disturbances, possibly only by yearlings, or reduced reproductive rates at disturbed leks (Holloran and others, 2010). Further analyses could be carried out to investigate and compare log-odds of nearby leks to investigate evidence of distributional shifts and distinguish warnings associated with these different processes. Fourth, we built in temporal thresholds to guard against spurious watches and warnings as a result of major count errors (for example, major change in lek attendance from flush prior to count). However, these thresholds prevent watches from occurring immediately (for example, within-year), but managers and land stewards can obtain the signal data to explore a more immediate response. Fifth, CCs that consist of relatively few NCs, such as Washington area and Jackson Hole, Wyoming, area, will be less likely to activate $\mathrm{NC}$ warnings, especially if the majority of NCs are experiencing similar declines. This is because changes in $\hat{N}$ at a NC has relatively strong influences on the $\mathrm{CC}$ estimate, resulting in relatively smaller values of log-odds ratios less likely to cross signal thresholds. Thus, consistent declines across the small CCs of Washington area and Jackson Hole, Wyoming, area explain why no NCs were activated since the 1990s. However, the TAWS does continue to activate leks within these smaller CCs because enough variation exists at that scale to allow declines to exceed log-odds ratio thresholds. Although it has been demonstrated that activation of leks within small CCs is possible under the current version of the TAWS, we recognize areas of future improvement. Namely, further refinement of the simulation analysis to identify thresholds that vary by $\mathrm{CC}$, which would focus stability at the CC scale (for example, see appendix 8) as opposed to the range-wide scale. This refinement more likely supports fundamental conservation biology principles of resiliency, redundancy, and representation by activating watches and warnings across all declining areas of the species' range, as opposed to a broader goal that optimizes range-wide population level stability.

Lastly, identifying thresholds for watches and warnings required simulation of management action designed to stabilize populations that have declined below $\mathrm{CC}$ (appendix 5). The original framework (Coates and others, 2017) applied simulated actions that were uniformly effective and acknowledged that such an approach failed to consider variation in recovery processes driven by (now) well-understood underlying ecological site conditions (Chambers and others, 2020). The thresholds derived in this study address that shortcoming by accounting for variation in management effectiveness indexed by underlying resilience and resistance (R\&R) classes and subsequent differential sage-grouse population responses to disturbance. The revised simulations imposed lower probabilities of successful management action for populations inhabiting regions of low $R \& R$ compared to more productive regions of high $R \& R$.

We chose the wildfire-annual grass cycle as the disturbance regime to index owing to its ubiquity in the western portions of the species' range (Brooks and others, 2015; Coates and others, 2016), but recognize that sage-grouse respond differently to other forms of disturbance, such as energy development in the eastern portions of the species range. For example, both scenarios result in the removal 
of vegetation, but wildfires will disturb larger areas in a relatively short period (for example, $\geq$ day) compared to energy development that changes the landscape more slowly over time (for example, $\geq$ year), depending on socioeconomic drivers, technologies, and reserves available within an area. Wildfires often result in the introduction of invasive species (Chambers and others, 2019; Mahood and Balch, 2019), damage biological soil crusts (Root and others, 2018; Brianne and others, 2020), and alter fire return intervals (Pilliod and others, 2017; Ellsworth and others, 2020), often impacting large contiguous areas (for example, $>1,000$ acres) of vegetation. Activities to restore landscapes may begin immediately after a wildfire. In comparison, infrastructure associated with energy development requires direct removal of vegetation and can result in direct impacts associated with vehicle traffic, human activity, and noise pollution that persist for years after the initial disturbance event before land undergoes reclamation. We hypothesize that immediate loss of habitat associated with wildfire is likely to activate fast signals and more immediate warnings compared to slow signals that likely activate from incremental changes associated with point source disturbances like energy development. Although it was beyond the scope of our study, a retrospective analysis that investigates difference between slow and fast signals among different disturbance types would be beneficial. Such analyses could help inform ecological thresholds for different types of disturbances on the landscape that, when crossed, are likely to activate watches and warnings. Additionally, our framework can be amenable to evaluating efficacy of different conservation actions. For example, estimates of population responses to restoration efforts are often confounded by environmental stochasticity. Using similar comparisons and thresholds as TAWS, but instead annually evaluating realignment of $\hat{\lambda}$ among spatial scales, can act to disentangle changes in $\hat{N}$ associated with larger scale oscillations that are governed by climatic conditions and more accurately assess deterministic changes to sage-grouse habitat.

Advances in the Modeling Framework. Here, we describe a population modeling framework that spatially delineated population structures, estimated trends across different spatiotemporal scales, and provided warning of populations that have declined below $\mathrm{CC}$ on an annual basis. The advantage of this framework is that it relies on a single hierarchical model that can be readily updated with new lek count data given proper QA/QC procedures (Objective 1). Although the model accounts for uncertainty in count observations, it relies on maximum annual counts and does not fully account for detection probability and estimate true abundance. However, this modeling framework is highly flexible and future steps are underway to accommodate multiple sources of information (demographic data, repeat lek counts, and so forth) and multiple model types (for example, $\mathrm{N}$-mixture model, IPM, and so forth), where the end goal is improving precision of population parameter estimates, estimating true abundance, and correctly identifying population units in greatest need of close monitoring or management intervention within a single unified framework. Subsequent improvements also could involve incorporation of repeated count data (McCaffery and others, 2016; Monroe and others, 2019) combined with information on lek visitation rates (Fremgen and others, 2017; Wann and others 2019) and sage-grouse detection (Fremgen and others, 2016; Baumgardt and others, 2017; Coates and other, 2019b) to better estimate observation error. These additions could greatly improve potential population size estimation. For example, if repeated counts are collected within a single season at some leks, an $N$-mixture process can be used to model detection probability (McCaffery and others, 2016; Monroe and others, 2019) within the same framework. Our modeling framework could be adapted to implement this process, and we have already built in the capacity to do so. If repeated measures are not available and single lek counts within a season are accompanied by information such as survey date and time, counts can be adjusted based on parameters published from ancillary research to account for temporal and environmental effects on lek attendance and visitation rates (Coates and others, 2019b; Fremgen and others, 2019; Wann and others, 2019), or based on sources of observation error such as time of survey within a morning (Monroe and others, 2016). Such adjustment parameters likely will provide better population estimates than raw lek counts in the absence of $\mathrm{N}$-mixture processes that rely on repeated counts, largely because these estimates provide some information about variation in sightability and lek attendance. Additionally, in areas where demographic data (telemetry, mark recapture, brood surveys, hunter harvest, and so forth) and lek counts are available, procedures that rely on joint likelihood estimation (for example, IPM) can be implemented and incorporated into the SSM, similar to recent methods reported for the Bi-State area (Coates and others, 2019a). Thus, our hierarchical framework allows for integration of various forms of data across space and time by using advanced model structures as well as adjustment parameters derived from ancillary research where data collection may not be as robust.

Although coupling an evaluation of declining annual $\hat{\lambda}$ with separation in estimates between local and regional scales provides compelling evidence for unexpected population decline responsible for management concern, our results do not quantitatively investigate causal factors. Subsequent post-hoc analyses using spatiotemporal covariates not described in this study would allow evaluation of possible mechanisms responsible for these patterns, particularly 
those related to well documented drivers of changing habitat conditions described earlier in the text. In addition, more powerful analyses of changes in land cover will be facilitated by correlations with newly available 'back-in-time' mapped predictions of shrub, herbaceous, and bare ground components that spatially span the species' range and temporally span the Landsat satellite archive (over three decades; Rigge and others, 2019).

In the current structure, our trend and TAWS models do not partition density-dependent from density-independent effects that contribute to annual variation in sage-grouse abundance within and among spatial scales (Garton and others, 2011, 2015; Blomberg and others, 2017; Coates and others, 2018; Edmunds and others, 2018). In previous model investigations, we fitted density-dependent structures in the SSM but failed to achieve full model convergence for reliable parameter estimation. Although it is unlikely that density-dependent structure will have strong influences on the reported estimates, future methods that include density-dependence structures in SSMs will help refine parameter estimates.

Lastly, development of a user-friendly interface that will allow land and wildlife managers to input data (for example, lek counts) at different spatial extents and readily obtain estimated and derived parameters as well as watches and warnings is currently underway. We intend to build on the development of this range-wide hierarchical modeling framework as modeling procedures and web-based applications continue to advance. The ultimate goal is to provide solutions through user-friendly decision support tools whereby land and wildlife managers can periodically assess population status at different spatiotemporal scales and readily identify populations that are likely in most need of management intervention.

\section{References Cited}

Aldridge, C.L., and Boyce, M.S., 2008, Accounting for fitness - Combining survival and selection when assessing wildlife-habitat relationships: Israel Journal of Ecology and Evolution, v. 54, no. 3-4, p. 389-419, https://doi.org/10.1560/IJEE.54.3-4.389.
Aldridge, C.L., Nielsen, S.E., Beyer, H.L., Boyce, M.S., Connelly, J.W., Knick, S.T., and Schroeder, M.A., 2008, Range-wide patterns of greater sage-grouse persistence: Diversity and Distributions, v. 14, no. 6, p. 983-994, https://doi.org/10.1111/j.1472-4642.2008.00502.x.

Archibald, H.L., 2014, The enigma of the 10-year wildlife population cycle solved? Evidence that the periodicity and regularity of the cycle are driven by a lunar zeitgeber: Canadian Field-Naturalist, v. 128, no. 4, p. 327-340, https://doi.org/10.22621/cfn.v128i4.1626.

AssunÇão, R.M., Neves, M.C., Câmara, G., and Da Costa Freitas, C., 2006, Efficient regionalization techniques for socio-economic geographical units using minimum spanning trees: International Journal of Geographical Information Science, v. 20, no. 7, p. 797-811, https://doi.org/10.1080/13658810600665111.

Baruch-Mordo, S., Evans, J.S., Severson, J.P., Naugle, D.E., Maestas, J.D., Kiesecker, J.M., Falkowski, M.J., Hagen, C.A., and Reese, K.P., 2013, Saving sage-grouse from the trees-A proactive solution to reducing a key threat to a candidate species: Biological Conservation, v. 167, p. 233-241, https://doi.org/10.1016/j.biocon.2013.08.017.

Baumgardt, J.A., Reese, K.P., Connelly, J.W., and Garton, E.O., 2017, Visibility bias for sage-grouse lek counts: Wildlife Society Bulletin, v. 41, p. 461-470, https://doi.org/10.1002/wsb.800.

Beever, E.A., and Aldridge, C.L., 2011, Influences of freeroaming equids on sagebrush ecosystems, with focus on greater sage-grouse, in Knick, S.T., and Connelly, J.W., eds., Greater sage-grouse-Ecology and conservation of a landscape species and its habitats: Berkeley, Calif., University of California Press, Studies in Avian Biology, no. 38, p. 273-290, https://doi.org/10.1525/ california/9780520267114.003.0015.

Bissonette, J.A., 2017, Avoiding the scale sampling problem-A consistent solution: The Journal of Wildlife Management, v. 81, no. 2, p. 192-205, https://doi.org/10.1002/jwmg.21187. 
Blasius, B., Rudolf, L., Weithoff, G., Gaedke, U., and Fussmann, G.F., 2020, Long-term cyclic persistence in an experimental predator-prey system: Nature, v. 577 , no. 7789 , p. 226-230, https://doi.org/10.1038/s41586-019-1857-0.

Blomberg, E.J., Sedinger, J.S., Atamian, M.T., and Nonne, D.V., 2012, Characteristics of climate and landscape disturbance influence the dynamics of greater sage-grouse populations: Ecosphere, v. 3, no. 6, p. 1-20, https://doi.org/10.1890/ES11-00304.1.

Blomberg, E.J., Poulson, S.R., Sedinger, J.S., and Gibson, D., 2013, Prefledging diet is correlated with individual growth in greater sage-grouse (Centrocercus urophasianus): The Auk, v. 130, no. 4, p. 715-724, https://doi.org/10.1525/auk.2013.12188.

Blomberg, E.J., Sedinger, J.S., Gibson, D., Coates, P.S., and Casazza, M.L., 2014, Carryover effects and climatic conditions influence the post-fledging survival of greater sage-grouse: Ecology and Evolution, v. 4, no. 23, p. 4488-4499, https://doi.org/10.1002/ece3.1139.

Blomberg, E.J., Gibson, D., Atamian, M.T., and Sedinger, J.S., 2017, Variable drivers of primary versus secondary nesting; density-dependence and drought effects on greater sagegrouse: Journal of Avian Biology, v. 48, no. 6, p. 827-836, https://doi.org/10.1111/jav.00988.

Brianne, P., Rebecca, H., and David, L., 2020, The fate of biological soil crusts after fire-A meta-analysis: Global Ecology and Conservation, v. 24, p. 1-13, https://doi.org/10.1016/j.gecco.2020.e01380.

Brooks, M.L., Matchett, J.R., Shinneman, D.J., and Coates, P.S., 2015, Fire patterns in the range of the greater sage-grouse, 1984-2013-Implications for conservation and management: U.S. Geological Survey Open-File Report 2015-1167, 66 p., https://doi.org/10.3133/ofr20151167.

Bureau of Land Management, 2015, Notice of availability of the record of decision and approved resource management plan amendments for the Great Basin Region greater sage-grouse sub-regions of Idaho and southwestern Montana; Nevada and northeastern California; Oregon; and Utah-Department of the Interior, Bureau of Land Management: Federal Register, v. 80, p. 57633-57635, https://www.federalregister.gov/documents/2015/09/ 24/2015-24213/notice-of-availability-of-the-recordof-decision-and-approved-resource-management-planamendments.

Campbell, B.D., Stafford Smith, D.M., and McKeon, G.M., 1997, Elevated $\mathrm{CO}_{2}$ and water supply interactions in grasslands-A pastures and rangelands management perspective: Global Change Biology, v. 3, no. 3, p. 177-187, https://doi.org/10.1046/j.1365-2486.1997.00095.x.
Campbell Grant, E.H., Lowe, W.H., and Fagan, W.F., 2007, Living in the branches-Population dynamics and ecological processes in dendritic networks: Ecology Letters, v. 10, no. 2, p. 165-175, https://doi.org/10.1111/j.1461-0248.2006.01007.x.

Carlisle, J.D., Keinath, D.A., Albeke, S.E., and Chalfoun, A.D., 2018, Identifying holes in the greater sage-grouse conservation umbrella: The Journal of Wildlife Management, v. 82, no. 5, p. 948-957, https://doi.org/10.1002/jwmg.21460.

Carpenter, J., Aldridge, C., and Boyce, M.S., 2010, Sagegrouse habitat selection during winter in Alberta: The Journal of Wildlife Management, v. 74, no. 8, p. 1806-1814, https://doi.org/10.2193/2009-368.

Chambers, J.C., Pyke, D.A., Maestas, J.D., Pellant, M., Boyd, C.S., Campbell, S.B., Espinosa, S., Havlina, D.W., Mayer, K.E., and Wuenschel, A., 2014, Using resistance and resilience concepts to reduce impacts of invasive annual grasses and altered fire regimes on the sagebrush ecosystem and greater sage-grouse-A strategic multi-scale approach: Fort Collins, Colo., U.S. Department of Agriculture, Forest Service, Rocky Mountain Research Station, General Technical Report RMRS-GTR-326, 73 p., https://doi.org/10.2737/RMRS-GTR-326.

Chambers, J.C., Beck, J.L., Bradford, J.B., Bybee, J., Campbell, S., Carlson, J., Christiansen, T.J., Clause, K.J., Collins, G., Crist, M.R., Dinkins, J.B., Doherty, K.E., Edwards, F., Espinosa, S., Griffin, K.A., Griffin, P., Haas, J.R., Hanser, S.E., Havlina, D.W., Henke, K.F., Hennig, J.D., Joyce, L.A., Kilkenny, F.M., Kulpa, S.M., Kurth, L.L., Maestas, J.D., Manning, M., Mayer, K.E., Mealor, B.A., McCarthy, C., Pellant, M., Perea, M.A., Prentice, K.L., Pyke, D.A., Wiechman, L.A., and Wuenschel, A., 2017, Science framework for conservation and restoration of the sagebrush biome-Linking the Department of the Interior's integrated rangeland fire management strategy to long-term strategic conservation actions: Fort Collins, Colo., U.S Department of Agriculture, Forest Service, Rocky Mountain Research Station, General Technical Report RMRS-GTR-360, 213 p., https://www.fs.usda.gov/treesearch/pubs/53983.

Chambers, J.C., Brooks, M.L., Germino, M.J., Maestas, J.D., Board, D.I., Jones, M.O., and Allred, B.W., 2019, Operationalizing resilience and resistance concepts to address invasive grass-fire cycles: Frontiers in Ecology and Evolution, v. 7, no. 185, 25 p., https://doi.org/10.3389/fevo.2019.00185.

Chambers, J.C., Crist, M.R., Maestas, J.D., Prentice, K.L., and Pyke, D.A., 2020, A framework for sagebrush: The Wildlife Professional, p. 28-32, https://www.fs.fed.us/rm/pubs journals/2020/rmrs_2020_chambers_j002.pdf. 
Coates, P.S., Ricca, M.A., Prochazka, B.G., Brooks, M.L., Doherty, K.E., Kroger, T., Blomberg, E.J., Hagen, C.A., and Casazza, M.L., 2016, Wildfire, climate, and invasive grass interactions negatively impact an indicator species by reshaping sagebrush ecosystems: Proceedings of the National Academy of Sciences of the United States of America, v. 113, no. 45, p. 12745-12750, https://doi.org/10.1073/pnas.1606898113.

Coates, P.S., Prochazka, B.G., Ricca, M.A., Wann, G.T., Aldridge, C.L., Hanser, S.E., Doherty, K.E., O’Donnell, M.S., Edmunds, D.R., and Espinosa, S.P., 2017, Hierarchical population monitoring of greater sage-grouse (Centrocercus urophasianus) in Nevada and California-Identifying populations for management at the appropriate spatial scale: U.S. Geological Survey Open-File Report 2017-1089, 49 p., https://doi.org/10.3133/ofr20171089.

Coates, P.S., Prochazka, B.G., Ricca, M.A., Halstead, B.J., Casazza, M.L., Blomberg, E.J., Brussee, B.E., Wiechman, L., Tebbenkamp, J., Gardner, S.C., and Reese, K.P., 2018, The relative importance of intrinsic and extrinsic drivers to population growth vary among local populations of greater sage-grouse-An integrated population modeling approach: The Auk, v. 135 , no. 2, p. 240-261, https://doi.org/10.1642/AUK-17-137.1.

Coates, P.S., Ricca, M.A., Prochazka, B.G., O’Neil, S.T., Severson, J.P., Mathews, S.R., Espinosa, S., Gardner, S., Lisius, S., and Delehanty, D.J., 2019a, Population and habitat analyses for greater sage-grouse (Centrocercus urophasianus) in the bi-state distinct population segment - 2018 update: U.S. Geological Survey Open-File Report 2019-1149, 122 p., https://doi.org/10.3133/ofr20191149.

Coates, P.S., Wann, G.T., Gillette, G.L., Ricca, M.A., Prochazka, B.G., Severson, J.P., Andrle, K.M., Espinosa, S.P., Casazza, M.L., and Delehanty, D.J., 2019b, Estimating sightability of greater sage-grouse at leks using an aerial infrared system and N-mixture models: Wildlife Biology, v. 2019, no. 1, p. 1-11, https://doi.org/10.2981/wlb.00552.

Coates, P.S., O’Neil, S.T., Brussee, B.E., Ricca, M.A., Jackson, P.J., Dinkins, J.B., Howe, K.B., Moser, A.M., Foster, L.J., and Delehanty, D.J., 2020, Broad-scale impacts of an invasive native predator on a sensitive native prey species within the shifting avian community of the North American Great Basin: Biological Conservation, v. 243, 10 p., https://doi.org/10.1016/j.biocon.2020.108409.

Connelly, J.W., and Braun, C.E., 1997, Long-term changes in sage grouse Centrocercus urophasianus populations in western North America: Wildlife Biology, v. 3, no. 3-4, p. 229-234, https://doi.org/10.2981/wlb.1997.028.
Connelly, J.W., Browers, H.W., and Gates, R.J., 1988, Seasonal movements of sage grouse in southeastern Idaho: The Journal of Wildlife Management, v. 52, no. 1, p. 116122, https://www.jstor.org/stable/3801070?seq=1\#metadata info_tab_contents.

Connelly, J.W., Reese, K.P., and Schroeder, M.A., 2003, Monitoring of greater sage-grouse habitats and populations: College of Natural Resources Experimental Station Bulletin 80, 47 p., https://wdfw.wa.gov/sites/default/files/ publications/01316/wdfw01316.pdf.

Connelly, J.W., Knick, S.T., Schroeder, M.A., and Stiver, S.J., 2004, Conservation assessment of greater sage-grouse and sagebrush habitats: Cheyenne, Wyo., Western Association of Fish and Wildlife Agencies, 611 p., https://digitalcommons.usu.edu/cgi/viewcontent.cgi?article= 1079\&context=govdocs.

Connelly, J.W., and Schroeder, M.A., 2007, Historical and current approaches to monitoring greater sage-grouseMonitoring populations of sage-grouse: College of Natural Resources Experiment Station Bulletin, v. 88, p. 3-9.

Courchamp, F., Clutton-Brock, T., and Grenfell, B., 1999, Inverse density dependence and the Allee effect: Trends in Ecology \& Evolution, v. 14, no. 10, p. 405-410, https://doi.org/10.1016/S0169-5347(99)01683-3.

Crosby, M.K., Fan, Z., Spetich, M.A., Leininger, T., and Fan, X., 2015, Early indications of drought impacts on forests in the southeastern United States: The Forestry Chronicle, v. 91, no. 4, p. 376-383, https://doi.org/10.5558/tfc2015-067.

Cross, T.B., Naugle, D.E., Carlson, J.C., and Schwartz, M.K., 2017, Genetic recapture identifies long-distance breeding dispersal in Greater sage-grouse (Centrocercus urophasianus): The Condor, v. 119, no. 1, p. 155-166, https://doi.org/10.1650/CONDOR-16-178.1.

Danvir, R.E., 2018, Multiple-use management of western U.S. rangelands-Wild horses, wildlife, and livestock: Human-Wildlife Interactions, v. 12, no. 1, p. 5-17, https://doi.org/10.26077/cz0b-6261.

Davies, K.W., and Boyd, C.S., 2019, Ecological effects of free-roaming horses in North American rangelands: Bioscience, v. 69 , no. 7, p. 558-565, https://doi.org/10.1093/biosci/biz060.

Davies, K.W., Collins, G., and Boyd, C.S., 2014, Effects of feral free-roaming horses on semi-arid rangeland ecosystems-An example from the sagebrush steppe: Ecosphere, v. 5, no. 10, p. 1-14, https://doi.org/10.1890/ES14-00171.1. 
DeSante, D.F., Nott, M.P., and O'Grady, D.R., 2001, Identifying the proximate demographic cause(s) of population change by modeling spatial variation in productivity, survivorship, and population trends: Ardea, v. 89, p. 185-208, https://www.birdpop.org/docs/pubs/ DeSante_et_al_2001_Identifying_the_Proximate_ Demographic_Causes_of_Population_Change.pdf.

Dinkins, J.B., and Beck, J.L., 2019, Comparison of conservation policy benefits for an umbrella and related sagebrush-obligate species: Human-Wildlife Interactions, v. 13, no. 3, p. 447-458, https://doi.org/10.26077/4ypp-vj89.

Doherty, K.E., Evans, J.S., Coates, P.S., Juliusson, L.M., and Fedy, B.C., 2016, Importance of regional variation in conservation planning - A rangewide example of the greater sage-grouse: Ecosphere, v. 7, no. 10, p. 1-27, https://doi.org/10.1002/ecs2.1462.

Donnelly, J.P., Allred, B.W., Perret, D., Silverman, N.L., Tack, J.D., Dreitz, V.J., Maestas, J.D., and Naugle, D.E., 2018, Seasonal drought in North America's sagebrush biome structures dynamic mesic resources for sage-grouse: Ecology and Evolution, v. 8, no. 24, p. 12492-12505, https://doi.org/10.1002/ece3.4614.

Dunn, P.O., and Braun, C.E., 1986, Summer habitat use by adult female and juvenile sage grouse: Journal of Wildlife Management, v. 50, no. 2, p. 228-235, https://doi.org/10.2307/3801903.

Edmunds, D.R., Aldridge, C.L., O'Donnell, M.S., and Monroe, A.P., 2018, Greater sage-grouse population trends across Wyoming: The Journal of Wildlife Management, v. 82, no. 2, p. 397-412, https://doi.org/10.1002/jwmg.21386.

Ellsworth, L.M., Kauffman, J.B., Reis, S.A., Sapsis, D.B., and Moseley, K., 2020, Repeated fire altered succession and increased fire behavior in basin big sagebrush-native perennial grasslands: Ecosphere, v. 11, no. 5, p. 1-13, https://doi.org/10.1002/ecs2.3124.

Emmons, S.R., and Braun, C.E., 1984, Lek attendance of male sage grouse: The Journal of Wildlife Management, v. 48, no. 3, p. 1023-1028, https://doi.org/10.2307/3801461.

Etherington, T.R., 2016, Least-cost modelling and landscape ecology - Concepts, applications, and opportunities: Current Landscape Ecology Reports, v. 1, no. 1, p. 40-53, https://doi.org/10.1007/s40823-016-0006-9.

Fedy, B.C., and Aldridge, C.L., 2011, The importance of within-year repeated counts and the influence of scale on long-term monitoring of sage-grouse: The Journal of Wildlife Management, v. 75, no. 5, p. 1022-1033, https://doi.org/10.1002/jwmg.155.
Fedy, B.C., Doherty, K.E., Aldridge, C.L., O’Donnell, M.S., Beck, J.L., Bedrosian, B., Gummer, D., Holloran, M.J., Johnson, G.D., Kaczor, N.W., Kirol, C.P., Mandich, C.A., Marshall, D., Mckee, G., Olson, C., Pratt, A.C., Swanson, C.C., and Walker, B.L., 2014, Habitat prioritization across large landscapes, multiple seasons, and novel areas-An example using greater sage-grouse in Wyoming: Wildlife Monographs, v. 190, no. 1, p. 1-39, https://doi.org/10.1002/wmon.1014.

Fremgen, A.L., Rota, C.T., Hansen, C.P., Rumble, M.A., Gamo, R.S., and Millspaugh, J.J., 2017, Male greater sage-grouse movements among leks: The Journal of Wildlife Management, v. 81, no. 3, p. 498-508, https://doi.org/10.1002/jwmg.21208.

Fremgen, A.L., Hansen, C.P., Rumble, M.A., Gamo, R.S., and Millspaugh, J.J., 2019, Weather conditions and date influence male sage grouse attendance rates at leks: Ibis, v. 161, no. 1, p. 35-49, https://doi.org/10.1111/ibi.12598.

Fuhlendorf, S.D., Woodward, A.J.W., Leslie, D.M., and Shackford, J.S., 2002, Multi-scale effects of habitat loss and fragmentation on lesser prairie-chicken populations of the US Southern Great Plains: Landscape Ecology, v. 17, no. 7, p. 617-628, https://doi.org/10.1023/A:1021592817039.

Garton, E.O., Connelly, J.W., Horne, J.S., Hagen, C.A., Moser, A., and Schroeder, M., 2011, Greater sage-grouse population dynamics and probability of persistence, in Knick, S.T., and Connelly, J.W., eds., Greater sagegrouse-Ecology and conservation of a landscape species and its habitats: Berkeley, Calif., University of California Press, Studies in Avian Biology, no. 38, p. 292-382, https://doi.org/10.1525/california/9780520267114.003.0016.

Garton, E.O., Wells, A.G., Baumgardt, J.A., and Connelly, J.W., 2015, Greater sage-grouse population dynamics and probability of persistence: Final report to Pew Charitable Trusts, $90 \mathrm{p}$.

Gelman, A., Carlin, J.B., Stern, H.S., and Rubin, D.B., 2004. Bayesian data analysis-Texts in statistical science ( $2 \mathrm{~d}$ ed.): Chapman \& Hall, CRC, 689 p.

Gessler, P.E., Moore, I.D., McKenzie, N.J., and Ryan, P.J., 1995, Soil-landscape modelling and spatial prediction of soil attributes: International Journal of Geographical Information Systems, v. 9, no. 4, p. 421-432, https://doi.org/10.1080/02693799508902047.

Gibson, D., Blomberg, E.J., Atamian, M.T., and Sedinger, J.S., 2017, Weather, habitat composition, and female behavior interact to modify offspring survival in greater sagegrouse: Ecological Applications, v. 27, no. 1, p. 168-181, https://doi.org/10.1002/eap.1427. 
Green, A.W., Aldridge, C.L., and O’Donnell, M.S., 2017, Investigating impacts of oil and gas development on greater sage-grouse: The Journal of Wildlife Management, v. 81, no. 1, p. 46-57, https://doi.org/10.1002/jwmg.21179.

Gregg, M.A., and Crawford, J.A., 2009, Survival of greater sage-grouse chicks and broods in the northern Great Basin: The Journal of Wildlife Management, v. 73, no. 6, p. 904-913, https://doi.org/10.2193/2007-410.

Gurevitch, J., Fox, G.A., Fowler, N.L., and Graham, C.H., 2016, Landscape demography-Population change and its drivers across spatial scales: The Quarterly Review of Biology, v. 91, no. 4, p. 459-485, https://doi.org/10.1086/689560.

Guttery, M.R., Dahlgren, D.K., Messmer, T.A., Connelly, J.W., Reese, K.P., Terletzky, P.A., Burkepile, N., and Koons, D.N., 2013, Effects of landscape-scale environmental variation on greater sage-grouse chick survival: PLoS One, v. 8, no. 6, 11 p., https://doi.org/10.1371/journal.pone.0065582.

Hanser, S.E., and Knick, S.T., 2011, Greater sage-grouse as an umbrella species for shrubland passerine birds-A multiscale assessment, in Knick, S.T., and Connelly, J.W., eds., Greater sage-grouse-Ecology and conservation of a landscape species and its habitats: Berkeley, Calif., University of California Press, Studies in Avian Biology, v. 38 , p. 474-488, https://doi.org/10.1525/california/ 9780520267114.003.0020.

Holloran, M.J., Kaiser, R.C., and Hubert, W.A., 2010. Yearling greater sage-grouse response to energy development in Wyoming: The Journal of Wildlife Management, v. 74, no. 1, p. 65-72, https://doi.org/10.2193/2008-291.

Izaurralde, R.C., Thomson, A.M., Morgan, J.A., Fay, P.A., Polley, H.W., and Hatfield, J.L., 2011, Climate impacts on agriculture-Implications for forage and rangeland production: Agronomy Journal, v. 103, no. 2, p. 371-381, https://doi.org/10.2134/agronj2010.0304.

Kéry, M., and Schaub, M., 2012, Bayesian population analysis using WinBUGS - A hierarchical perspective: San Diego, Calif., Academic Press, 535 p.

Knick, S., and Connelly, J.W., eds., 2011, Greater sage-grouse-Ecology and conservation of a landscape species and its habitats: University of California Press, v. 38, 664 p., https://doi.org/10.1525/9780520948686.

Kranstauber, B., Kays, R., LaPoint, S.D., Wikelski, M., and Safi, K., 2012, A dynamic Brownian bridge movement model to estimate utilization distributions for heterogeneous animal movement: Journal of Animal Ecology, v. 81, no. 4, p. 738-746, https://doi.org/10.1111/j.1365-

2656.2012.01955.x.
Lande, R., 1988, Genetics and demography in biological conservation: Science, v. 241 , no. 4872 , p. 1455-1460, https://doi.org/10.1126/science.3420403.

Leonard, K.M., Reese, K.P., and Connelly, J.W., 2000, Distribution, movements and habitats of sage grouse Centrocercus urophasianus on the Upper Snake River Plain of Idaho - Changes from the 1950s to the 1990s: Wildlife Biology, v. 6, no. 1, p. 265-270, https://doi.org/10.2981/wlb.2000.025.

Levin, S.A., 1992, The problem of pattern and scale in ecology-The Robert H. MacArthur award lecture: Ecology, v. 73, no. 6, p. 1943-1967, https://doi.org/10.2307/1941447.

Lindenmayer, D.B., and Likens, G.E., 2010, The science and application of ecological monitoring: Biological Conservation, v. 143, no. 6 , p. 1317-1328, https://doi.org/10.1016/j.biocon.2010.02.013.

Lindström, J., Ranta, E., and Linden, H., 1996, Large-scale synchrony in the dynamics of capercaillie, black grouse and hazel grouse populations in Finland: Oikos, v. 76, no. 2, p. 221-227, https://doi.org/10.2307/3546193.

Liu, C., Newell, G., White, M., and Bennett, A.F., 2018, Identifying wildlife corridors for the restoration of regional habitat connectivity-A multispecies approach and comparison of resistance surfaces: PLoS One, v. 13, no. 11, 14 p., https://doi.org/10.1371/journal.pone.0206071.

Mahood, A.L., and Balch, J.K., 2019, Repeated fires reduce plant diversity in low-elevation Wyoming big sagebrush ecosystems (1984-2014): Ecosphere, v. 10, no. 2, p. 1-19, https://doi.org/10.1002/ecs2.2591.

Mathews, S.R., Coates, P.S., Prochazka, B.G., Ricca, M.A., Meyerpeter, M.B., Espinosa, S.P., Lisius, S., Gardner, S.C., and Delehanty, D.J., 2018, An integrated population model for greater sagegrouse (Centrocercus urophasianus) in the Bi State Distinct Population Segment, California and Nevada, 2003-17: U.S. Geological Survey Open-File Report 2018-1177, 89 p., https://doi.org/10.3133/ofr20181177.

McCaffery, R., Nowak, J.J., and Lukacs, P.M., 2016, Improved analysis of lek count data using N-mixture models: The Journal of Wildlife Management, v. 80, no. 6, p. 1011-1021, https://doi.org/10.1002/jwmg.21094.

McCune, B., and Keon, D., 2002, Equations for potential annual direct incident radiation and heat load: Journal of Vegetation Science, v. 13, no. 4, p. 603-606, https://doi.org/10.1111/j.1654-1103.2002.tb02087.x. 
McRae, B.H., Dickson, B.G., Keitt, T.H., and Shah, V.B., 2008, Using circuit theory to model connectivity in ecology, evolution, and conservation: Ecology, v. 89, no. 10, p. 2712-2724, https://doi.org/10.1890/07-1861.1.

Melbourne, B.A., and Hastings, A., 2008, Extinction risk depends strongly on factors contributing to stochasticity: Nature, v. 454, p. 100-103, https://doi.org/10.1038/nature06922.

Miguet, P., Jackson, H.B., Jackson, N.D., Martin, A.E., and Fahrig, L., 2016, What determines the spatial extent of landscape effects on species?: Landscape Ecology, v. 31, no. 6, p. 1177-1194, https://doi.org/10.1007/s10980-015-0314-1.

Miller, R.F., and Tausch, R.J., 2001, The role of fire in juniper and pinyon woodlands-A descriptive analysis in Galley, K.E.M., and Wilson, T.P., eds., Proceedings of the invasive species workshop-The role of fire in the control and spread of invasive species: Fire Conference 2000, Tallahassee, Fla., no. 11, p. 15-30, https://agsci.oregonstate.edu/sites/agscid7/files/eoarc/ attachments/460.pdf.

Miller, R.F., and Wigand, P.E., 1994, Holocene changes in semiarid pinyon-juniper woodlandsResponse to climate, fire, and human activities in the US Great Basin: Bioscience, v. 44, no. 7, p. 465-474, https://doi.org/10.2307/1312298.

Miller, R.F., Knick, S.T., Pyke, D.A., Meinke, C.W., Hanser, S.E., Wisdom, M.J., and Hild, A.L., 2011, Characteristics of sagebrush habitats and limitations to long-term conservation, in Knick, S.T., and Connelly, J.W., eds., Greater sage-grouse-Ecology and conservation of a landscape species and its habitats: Berkeley, Calif., University of California Press, Studies in Avian Biology, v. 38, p. 145-184, https://doi.org/10.1525/ california/9780520267114.003.0011.

Monroe, A.P., Edmunds, D.R., and Aldridge, C.L., 2016, Effects of lek count protocols on greater sagegrouse population trend estimates: The Journal of Wildlife Management, v. 80, no. 4, p. 667-678, https://doi.org/10.1002/jwmg.1050.

Monroe, A.P., Aldridge, C.L., Assal, T.J., Veblen, K.E., Pyke, D.A., and Casazza, M.L., 2017, Patterns in greater sage-grouse population dynamics correspond with public grazing records at broad scales: Ecological Applications, v. 27, no. 4, p. 1096-1107, https://doi.org/10.1002/eap.1512.

Monroe, A.P., Wann, G.T., Aldridge, C.L., and Coates, P.S., 2019, The importance of simulation assumptions when evaluating detectability in population models: Ecosphere, v. 10, no. 7, 16 p., https://doi.org/10.1002/ecs2.2791.
Morris, W.F., and Doak, D.F., 2002, Quantitative conservation biology - Theory and practice of population viability analysis: Sunderland, Mass., Sinauer Associates Inc., 480 p.

Nichols, J.D., and Williams, B.K., 2006, Monitoring for conservation: Trends in Ecology \& Evolution, v. 21, no. 12, p. 668-673, https://doi.org/10.1016/j.tree.2006.08.007.

Nielson, R.M., McDonald, L.L., Mitchell, J., Howlin, S., and LeBeau, C., 2015, Analysis of greater sage-grouse lek data-Trends in peak male counts 1965-2015: Cheyenne, Wyo., Western EcoSystems Technology, Inc., 43 p., https://ir.library.oregonstate.edu/concern/technical_reports/ $5 \mathrm{q} 47 \mathrm{rt} 895$.

O'Donnell, M.S., and Ignizio, D.A., 2012, Bioclimatic predictors for supporting ecological applications in the conterminous United States: Reston, Virginia, USA, U.S. Geological Survey Data Series 691, 10 p., https://pubs.usgs.gov/ds/691/.

O’Donnell, M.S., Edmunds, D.R., Aldridge, C.L., Heinrichs, J.A., Coates, P.S., Prochazka, B.G., and Hanser, S.E., 2019, Designing multi-scale hierarchical monitoring frameworks for wildlife to support management-A sage-grouse case study: Ecosphere, v. 10, no. 9, p. 1-34, https://doi.org/10.1002/ecs2.2872.

O’Neil, S.T., Coates, P.S., Brussee, B.E., Ricca, M.A., Espinosa, S.P., Gardner, S.C., and Delehanty, D.J., 2020, Wildfire and the ecological niche-Diminishing habitat suitability for an indicator species within semiarid ecosystems: Global Change Biology, v. 26, no. 11, p. 6296-6312, https://doi.org/10.1111/gcb.15300.

Oyler-McCance, S.J., Taylor, S.E., and Quinn, T.W., 2005, A multilocus population genetic survey of the greater sage-grouse across their range: Molecular Ecology, v. 14, no. 5, p. 1293-1310, https://doi.org/10.1111/j.1365-294X.2005.02491.x.

Patterson, R.L., 1952, The sage grouse in Wyoming: Denver, Colo., Wyoming Game and Fish Commission and Sage Books Inc., $341 \mathrm{p}$.

Peebles, L.W., Conover, M.R., and Dinkins, J.B., 2017, Adult sage-grouse numbers rise following raven removal or an increase in precipitation: Wildlife Society Bulletin, v. 41, no. 3, p. 471-478, https://doi.org/10.1002/wsb.788.

Piechota, T., Timilsena, J., Tootle, G., and Hidalgo, H., 2004, The western U.S. drought-How bad is it?: Washington, D.C., Eos, v. 85, no. 32, p. 301-304, https://doi.org/10.1029/2004EO320001. 
Pilliod, D.S., Welty, J.L., and Arkle, R.S., 2017, Refining the cheatgrass-fire cycle in the Great Basin-Precipitation timing and fine fuel composition predict wildfire trends: Ecology and Evolution, v. 7, no. 19, p. 8126-8151, https://doi.org/10.1002/ece3.3414.

Pilliod, D.S., Jeffries, M.I., Arkle, R.S., and Olson, D.H., 2020, Reptiles under the conservation umbrella of the greater sage-grouse: The Journal of Wildlife Management, v. 84, no. 3, p. 478-491, https://doi.org/10.1002/jwmg.21821.

Plummer, M., 2017, JAGS-A program for analysis of Bayesian graphical models using Gibbs sampling: version 4.3.0. software, http://mcmc-jags.sourceforge.net.

Prim, R.C., 1957, Shortest connection networks and some generalizations: The Bell System Technical Journal, v. 36, no. 6, p. 1389-1401, https://doi.org/10.1002/j.1538-7305.1957.tb01515.x.

PRISM Climate Group Oregon State University, 2015, Precipitation and temperature climate normals (1981-2010), accessed December 20, 2017, at http://prism.oregonstate.edu.

PRISM Climate Group, 2020, PRISM software, accessed March 25, 2020, at https://prism.oregonstate.edu.

Pyke, D.A., Chambers, J.C., Pellant, M., Knick, S.T., Miller, R.F., Beck, J.L., Doescher, P.S., Schupp, E.W., Roundy, B.A., Brunson, M., and McIver, J.D., 2015, Restoration handbook for sagebrush steppe ecosystems with emphasis on greater sage-grouse habitat-Part 1, Concepts for understanding and applying restoration: U.S. Geological Survey Circular 1416, 43 p., https://doi.org/10.3133/cir1416.

R Development Core Team, 2017, R-A language and environment for statistical computing v. 3.3.2.: Vienna, Austria, R Foundation for Statistical Computing, accessed January 4, 2021, http://www.R-project.org/.

R Development Core Team, 2018, R-A language and environment for statistical computing: Vienna, Austria, R Foundation for Statistical Computing, accessed February 2, 2018, at http://www.R-project.org/.

Ranta, E., Kaitala, V., Lindström, J., and Lindén, H., 1995, Synchrony in population dynamics: Proceedings of the Royal Society of London. Series B, Biological Sciences, v. 262, no. 1364, p. 113-118, https://doi.org/10.1098/rspb.1995.0184.

Reinhardt, J.R., Filippelli, S., Falkowski, M., Allred, B., Maestas, J.D., Carlson, J.C., and Naugle, D.E., 2020, Quantifying pinyon-juniper reduction within North America's sagebrush ecosystem: Rangeland Ecology and Management, v. 73, no. 3, p. 420-432, https://doi.org/10.1016/j.rama.2020.01.002.
Remington, T.E., and Braun, C.E., 1991, How surface coal mining affects sage grouse, North Park, Colorado, in Proceedings, Issues and Technology in the Management of Impacted Western Wildlife: Thorne Ecological Institute, v. 5, p. 128-132.

Rettie, W.J., and Messier, F., 2000, Hierarchical habitat selection by woodland caribou-Its relationship to limiting factors: Ecography, v. 23, no. 4, p. 466-478, https://doi.org/10.1111/j.1600-0587.2000.tb00303.x.

Ricca, M.A., and Coates, P.S., 2020, Integrating ecosystem resilience and resistance into decision support tools for multi-scale population management of a sagebrush indicator species: Frontiers in Ecology and Evolution, v. 7, no. 293, 22 p., https://doi.org/10.3389/fevo.2019.00493.

Rich, T., 1985, Sage grouse population fluctuations-Evidence for a 10-year cycle: U.S. Department of Interior, Bureau of Land Management, Idaho State Office, Technical Bulletin $85-1$, v. 85 , p. $1-20$.

Rich, T., and Altman, B., 2001, Under the sage-grouse umbrella: Bird Conservation, v. 14, 10 p.

Rich, T.D., Wisdom, M.J., and Saab, V.A., 2005, Conservation of sagebrush steppe birds in the interior Columbia Basin, General Technical Report PSW-GTR-191, in Ralph, C.J., Rich, T., and Long, L., eds., Proceedings of the third international partners in flight conference: Albany, Calif., U.S. Department of Agriculture, Forest Service, Pacific Southwest Research Station, p. 589-606.

Rigge, M., Shi, H., Homer, C., Danielson, P., and Granneman, B., 2019, Long-term trajectories of fractional component change in the Northern Great Basin, USA: Ecosphere, v. 10, no. 6, 24 p., https://doi.org/10.1002/ecs2.2762.

Rigge, M., Homer, C., Cleeves, L., Meyer, D.K., Bunde, B., Shi, H., Xian, G., Schell, S., and Bobo, M., 2020, Quantifying western U.S. rangelands as fractional components with multi-resolution remote sensing and in situ data: Remote Sensing, v. 12, no. 3, 26 p., https://doi.org/10.3390/rs12030412.

Root, H.T., Brinda, J.C., and Dodson, E.K., 2018, Biotic soil crust community composition 12-16 years after wildfires in Idaho, U.S.A: The Bryologist, v. 121, no. 3, p. 286-296, https://doi.org/10.1639/0007-2745-121.3.286.

Row, J.R., and Fedy, B.C., 2017, Spatial and temporal variation in the range-wide cyclic dynamics of greater sage-grouse: Oecologia, v. 185, no. 4, p. 687-698, https://doi.org/10.1007/s00442-017-3970-9. 
Rowland, M.M., Wisdom, M.J., Suring, L.H., and Meinke, C.W., 2006, Greater sage-grouse as an umbrella species for sagebrush-associated vertebrates: Biological Conservation, v. 129, no. 3 , p. 323-335, https://doi.org/10.1016/j.biocon.2005.10.048.

Sadoul, N., 1997, The importance of spatial scales in long-term monitoring of colonial Charadriiformes in southern France: Colonial Waterbirds, v. 20, no. 2, p. 330-338, https://doi.org/10.2307/1521701.

Sappington, J.M., Longshore, K.M., and Thompson, D.B., 2007, Quantifying landscape ruggedness for animal habitat analysis-A case study using bighorn sheep in the Mojave Desert: The Journal of Wildlife Management, v. 71, no. 5, p. 1419-1426, https://doi.org/10.2193/2005-723.

Sauer, J.R., and Link, W.A., 2002, Hierarchical modeling of population stability and species group attributes from survey data: Ecology, v. 83, no. 6, p. 1743-1751, https://doi.org/10.1890/0012-9658(2002)083[1743:HMOPS A]2.0.CO;2.

Schroeder, M.A., Aldridge, C.L., Apa, A.D., Bohne, J.R., Braun, C.E., Bunnell, S.D., Connelly, J.W., Deibert, P.A., Gardner, S.C., Hilliard, M.A., Kobriger, G.D., McAdam, S.M., McCarthy, C.W., McCarthy, J.J., Mitchell, D.L., Rickerson, E.V., and Stiver, S.J., 2004, Distribution of sage-grouse in North America: The Condor, v. 106, no. 2, p. 363-376, https://doi.org/10.1093/condor/106.2.363.

Schulwitz, S., Bedrosian, B., and Johnson, J.A., 2014, Low neutral genetic diversity in isolated greater sage-grouse (Centrocercus urophasianus) populations in northwest Wyoming: The Condor, v. 116, no. 4, p. 560-573, https://doi.org/10.1650/CONDOR-14-54.1.

Schumaker, N.H., Brookes, A., Dunk, J.R., Woodbridge, B., Heinrichs, J.A., Lawler, J.J., Carroll, C., and LaPlante, D., 2014, Mapping sources, sinks, and connectivity using a simulation model of northern spotted owls: Landscape Ecology, v. 29, no. 4, p. 579-592, https://doi.org/10.1007/s10980-014-0004-4.

Shinneman, D.J., 2020, North American sagebrush steppe and shrubland: Encyclopedia of the World's Biomes, p. 505-515, https://doi.org/10.1016/B978-0-12-4095489.11982-7.

Smith, J.T., Evans, J.S., Martin, B.H., Baruch-Mordo, S., Kiesecker, J.M., and Naugle, D.E., 2016, Reducing cultivation risk for at-risk species-Predicting outcomes of conservation easements for sage-grouse: Biological Conservation, v. 201, p. 10-19, https://doi.org/10.1016/j.biocon.2016.06.006.

Soille, P., 2004, Optimal removal of spurious pits in grid digital elevation models: Water Resources Research, v. 40, no. 12, p. 1-9, https://doi.org/10.1029/2004WR003060.
Spence, E.S., Beck, J.L., and Gregory, A.J., 2017, Probability of lek collapse is lower inside sage-grouse Core Areas-Effectiveness of conservation policy for a landscape species: PLoS One, v. 12, no. 11, 15 p., https://doi.org/10.1371/journal.pone.0185885.

Stiver, S.J., Apa, A.D., Bohne, J.R., Bunnell, P.A., Diebert, P.A., Gardner, S.C., Hilliard, M.A., McCarthy, C.W., and Schroeder, M.A., 2006, Greater sage-grouse comprehensive conservation strategy: Cheyenne, Wyoming, Western Association of Fish and Wildlife Agencies, https://www.fws.gov/greatersagegrouse/documents/ GreaterSage-grouseConservationStrategy2006.pdf.

Tack, J.D., Naugle, D.E., Carlson, J.C., and Fargey, P.J., 2012, Greater sage-grouse Centrocercus urophasianus migration links the USA and Canada - A biological basis for international prairie conservation: Oryx, v. 46, no. 1, p. 64-68, https://doi.org/10.1017/S003060531000147X.

Thompson, K.M., Holloran, M.J., Slater, S.J., Kuipers, J.L., and Anderson, S.H., 2006, Early brood-rearing habitat use and productivity of greater sage-grouse in Wyoming: Western North American Naturalist, v. 66, no. 3, p. 332342, https://doi.org/10.3398/1527-0904(2006)66[332:EBH $\mathrm{UAP}] 2.0 . \mathrm{CO} ; 2$.

U.S. Fish and Wildlife Service, 2015, Endangered and threatened wildlife and plants; 12-month finding on a petition to list greater sage-grouse (Centrocercus urophasianus) as an endangered or threatened species: Federal Register, v. 80, no. 191, p. 59857-59942, https://www.govinfo.gov/content/pkg/FR-2015-10-02/pdf/ 2015-24292.pdf.

U.S. Geological Survey, 2018, 1/3rd arc-second digital elevation models (DEMs): Reston, Virginia, USA, U.S. Geological Survey National Map 3DEP downloadable data collection, accessed April 4, 2014, at https://www.usgs.gov/core-science-systems/ngp/3dep/ about-3dep-products-services.

Wallace, B.P., DiMatteo, A.D., Hurley, B.J., Finkbeiner, E.M., Bolten, A.B., Chaloupka, M.Y., Hutchinson, B.J., Abreu-Grobois, F.A., Amorocho, D., Bjorndal, K.A., Bourjea, J., Bowen, B.W., Dueñas, R.B., Casale, P., Choudhury, B.C., Costa, A., Dutton, P.H., Fallabrino, A., Girard, A., Girondot, M., Godfrey, M.H., Hamann, M., López-Mendilaharsu, M., Marcovaldi, M.A., Mortimer, J.A., Musick, J.A., Nel, R., Pilcher, N.J., Seminoff, J.A., Troëng, S., Witherington, B., and Mast, R.B., 2010, Regional management units for marine turtles-A novel framework for prioritizing conservation and research across multiple scales: PLoS One, v. 5, no. 12, p. 1-11, https://doi.org/10.1371/journal.pone.0015465. 
Walsh, J.E., Kattsov, V.M., Chapman, W.L., Govorkova, V., and Pavlova, T., 2002, Comparison of Arctic climate simulations by uncoupled and coupled global models: Journal of Climate, v. 15, no. 12, p. 1429-1446, https://doi.org/10.1175/1520-0442(2002)015<1429:COACS $\mathrm{B}>2.0 . \mathrm{CO} ; 2$.

Walsh, J., Wuebbles, D., Hayhoe, K., Kossin, J., Kunkel, K., Stephens, G., Thorne, P., Vose, R., Wehner, M., Willis, J., and Anderson, D., 2014, Our changing climate, in Melillo, J.M., Richmond, T.T.C., and Yohe, G.W., eds., Climate change impacts in the United States-The Third National Climate Assessment: Washington, D.C., U.S. Global Change Research Program, p. 19-67, https://doi.org/10.7930/J0KW5CXT.

Walters, C.J., 1986, Adaptive management of renewable resources: New York, N.Y., MacMillan Publishing Company, 374 p., http://pure.iiasa.ac.at/id/eprint/2752/1/XB-86-702.pdf.

Wann, G.T., Coates, P.S., Prochazka, B.G., Severson, J.P., Monroe, A.P., and Aldridge, C.L., 2019, Assessing lek attendance of male greater sage-grouse using fine-resolution GPS data-Implications for population monitoring of lek mating grouse: Population Ecology, v. 61, no. 2, p. 183-197, https://doi.org/10.1002/1438-390X.1019.

Watts, M.J., and Wambolt, C.L., 1996, Long-term recovery of Wyoming big sagebrush after four treatments: Journal of Environmental Management, v. 46, no. 1, p. 95-102, https://doi.org/10.1006/jema.1996.0009.
Weiss, A.D., 2001, Topographic position and landforms analysis: Poster, ESRI User Conference, San Diego, California, $1 \mathrm{p}$.

Western Association of Fish and Wildlife Agencies, 2015, Greater sage-grouse population trends-An analysis of lek count databases 1965-2015: Cheyenne, Wyo., Western Association of Fish and Wildlife Agencies, 55 p., https://ir.library.oregonstate.edu/concern/technical_reports/ ng451p621.

Williams, B.K., 2011, Adaptive management of natural resources-Framework and issues: Journal of Environmental Management, v. 92, no. 5, p. 1346-1353, https://doi.org/10.1016/j.jenvman.2010.10.041.

Williams, B.K., and Johnson, F.A., 1995, Adaptive management and the regulation of waterfowl harvests: Wildlife Society Bulletin (1973-2006), v. 23, no. 3, p. $430-436$.

Williams, A.P., Cook, E.R., Smerdon, J.E., Cook, B.I., Abatzoglou, J.T., Bolles, K., Baek, S.H., Badger, A.M., and Livneh, B., 2020, Large contribution from anthropogenic warming to an emerging North American megadrought: Science, v. 368, no. 6488, p. 314-318, https://doi.org/10.1126/science.aaz9600.

Yoccoz, N.G., Nichols, J.D., and Boulinier, T., 2001, Monitoring of biological diversity in space and time: Trends in Ecology \& Evolution, v. 16, no. 8, p. 446-453, https://doi.org/10.1016/S0169-5347(01)02205-4. 


\section{Appendix 1 Commonly Used Terms}

Table 1.1. Definitions for commonly used terms in population clustering, spatiotemporal trends in population abundance (1960-2019), and a targeted annual warning system (1990-2019) for greater sage-grouse (Centrocercus urophasianus) across their range in western United States.

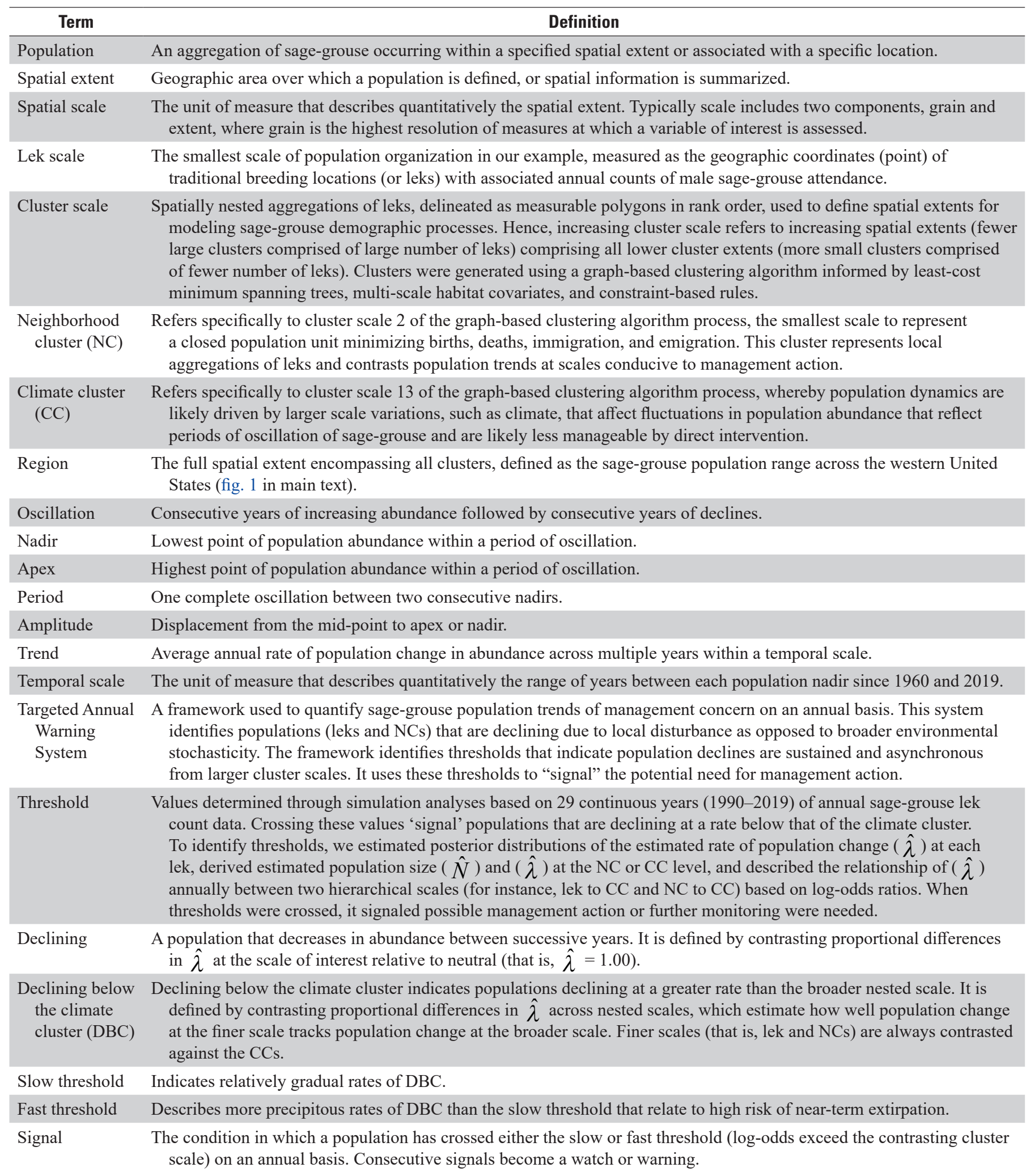


Table 1.1. Definitions for commonly used terms in population clustering, spatiotemporal trends in population abundance (1960-2019), and a targeted annual warning system (1990-2019) for greater sage-grouse (Centrocercus urophasianus) across their range in western United States.-Continued

\begin{tabular}{ll}
\hline \multicolumn{1}{c}{ Term } & \multicolumn{1}{c}{ Definition } \\
\hline Watch & $\begin{array}{l}\text { A watch is activated after } 2 \text { consecutive years of signal activation. This temporal requirement is intended to safeguard } \\
\text { against short-term population dynamics and to reflect the duration and magnitude of DBC. }\end{array}$ \\
Warning & $\begin{array}{c}\text { A warning is activated if a population signals at the slow threshold for } 3 \text { out of } 4 \text { consecutive years, or at the fast } \\
\text { threshold for } 2 \text { out of } 3 \text { consecutive years. }\end{array}$ \\
\hline
\end{tabular}




\section{Appendix 2 State-Space Model Samples Quality Assessment/Quality Control Rules}

Table 2.1. Number of leks retained for population analyses (Objectives 3 and 4) following sequential application of quality assessment and quality control $(\mathrm{QA} / \mathrm{QC})$ rules (1-6). Values under 'Rule $6 \mathrm{a}^{\prime}$ correspond to the number of leks used to estimate population trends (1960-2019) and 'Rule 6b' to inform a targeted annual warning system (1990-2019) for greater sage-grouse (Centrocercus urophasianus) across their range.

[Values under 'Rule 6a' correspond to the number of leks used to estimate population trends (1960-2019) and 'Rule 6b' to inform a targeted annual warning system (1990-2019) for greater sage-grouse (Centrocercus urophasianus) across their range. Abbreviations: CA, California; CO, Colorado; ID, Idaho; MT, Montana; ND, North Dakota; NV, Nevada; OR, Oregon; SD, South Dakota; UT, Utah; WA, Washington; WY, Wyoming]

\begin{tabular}{lrrrrrrrrr}
\hline State & No rules & Rule 1 & Rule 2 & Rule 3 & Rule 4 & Rule 5a & Rule 6a & Rule 5b & Rule 6b \\
\hline CA & 136 & 136 & 136 & 136 & 135 & 135 & 79 & 130 & 69 \\
CO & 322 & 322 & 322 & 322 & 322 & 322 & 213 & 322 & 213 \\
ID & 1,601 & 1,601 & 1,601 & 1,601 & 1,601 & 1,601 & 944 & 1,597 & 795 \\
MT & 1,272 & 1,272 & 1,251 & 1,219 & 1,219 & 1,219 & 564 & 1,218 & 464 \\
ND & 43 & 43 & 43 & 43 & 43 & 43 & 39 & 43 & 26 \\
NV & 1,369 & 1,369 & 1,363 & 1,339 & 1,340 & 1,340 & 618 & 1,337 & 526 \\
OR & 713 & 713 & 713 & 713 & 713 & 709 & 406 & 708 & 389 \\
SD & 59 & 59 & 59 & 59 & 59 & 59 & 40 & 57 \\
UT & 457 & 457 & 457 & 457 & 457 & 457 & 368 & 449 & 321 \\
\hline WA & 108 & 108 & 108 & 108 & 108 & 108 & 70 & 93 \\
WY & 2,341 & 2,341 & 2,341 & 2,341 & 2,341 & 2,340 & 1,790 & 2,332 & 1,593 \\
\hline Range & 8,421 & 8,421 & 8,394 & 8,338 & 8,338 & 8,333 & 5,131 & 8,286 & 4,478 \\
\hline
\end{tabular}

Table 2.2. Percent of leks retained for population analyses (Objectives 3 and 4) following sequential application of quality assessment and quality control (OA/OC) rules (1-6). Values under the 'No Rules' column correspond to the number of leks in the database prior to the application of $\mathrm{QA} / \mathrm{QC}$ rules. Values under 'Rule 6a' correspond to the percent of the 'No Rules' column value that were used to estimate population trends (1960-2019) and 'Rule 6b' to inform a targeted annual warning system (1990-2019) for greater sage-grouse (Centrocercus urophasianus) across their range.

[Values under the 'No Rules' column correspond to the number of leks in the database prior to the application of QA/QC rules. Values under 'Rule 6a' correspond to the percent of the 'No rules' column value that were used to estimate population trends (1960-2019) and 'Rule 6b' to inform a targeted annual warning system (1990-2019) for greater sage-grouse (Centrocercus urophasianus) across their range. Abbreviations: CA, California; CO, Colorado; ID, Idaho; MT, Montana; ND, North Dakota; NV, Nevada; OR, Oregon; SD, South Dakota; UT, Utah; WA, Washington; WY, Wyoming]

\begin{tabular}{|c|c|c|c|c|c|c|c|c|c|}
\hline State & No rules & Rule 1 & Rule 2 & Rule 3 & Rule 4 & Rule 5a & Rule 6a & Rule 5b & Rule 6b \\
\hline $\mathrm{CA}$ & 136 & 100.00 & 100.00 & 100.00 & 99.26 & 99.26 & 58.09 & 95.59 & 50.74 \\
\hline $\mathrm{CO}$ & 322 & 100.00 & 100.00 & 100.00 & 100.00 & 100.00 & 66.15 & 100.00 & 66.15 \\
\hline MT & 1,272 & 100.00 & 98.35 & 95.83 & 95.83 & 95.83 & 44.34 & 95.75 & 36.48 \\
\hline ND & 43 & 100.00 & 100.00 & 100.00 & 100.00 & 100.00 & 90.70 & 100.00 & 60.47 \\
\hline SD & 59 & 100.00 & 100.00 & 100.00 & 100.00 & 100.00 & 67.80 & 96.61 & 54.24 \\
\hline UT & 457 & 100.00 & 100.00 & 100.00 & 100.00 & 100.00 & 80.53 & 98.25 & 70.24 \\
\hline WA & 108 & 100.00 & 100.00 & 100.00 & 100.00 & 100.00 & 64.81 & 86.11 & 46.30 \\
\hline WY & 2,341 & 100.00 & 100.00 & 100.00 & 100.00 & 99.96 & 76.46 & 99.62 & 68.05 \\
\hline
\end{tabular}


Table 2.3. Number of lek count observations retained for population analyses (Objectives 3 and 4) following sequential application of quality assessment and quality control $(\mathrm{QA} / \mathrm{QC})$ rules $(1-6)$. Values under 'Rule $6 \mathrm{a}^{\prime}$ correspond to the number of lek count observations used to estimate population trends (1960-2019) and 'Rule 6b' to inform a targeted annual warning system (1990-2019) for greater sage-grouse (Centrocercus urophasianus) across their range.

[Values under 'Rule 6a' correspond to the number of lek count observations used to estimate population trends (1960-2019) and 'Rule 6b' to inform a targeted annual warning system (1990-2019) for greater sage-grouse (Centrocercus urophasianus) across their range. Abbreviations: CA, California; CO, Colorado; ID, Idaho; MT, Montana; ND, North Dakota; NV, Nevada; OR, Oregon; SD, South Dakota; UT, Utah; WA, Washington; WY, Wyoming]

\begin{tabular}{lrrrrrrrrr}
\hline State & No rules & \multicolumn{1}{c}{ Rule 1 } & Rule 2 & Rule 3 & Rule 4 & Rule 5a & Rule 6a & Rule 5b & Rule 6b \\
\hline CA & 6,424 & 6,338 & 6,338 & 6,338 & 2,550 & 2,423 & 2,080 & 1,745 & 1,425 \\
CO & 6,377 & 6,377 & 6,377 & 6,377 & 3,630 & 3,630 & 2,631 & 3,630 & 2,631 \\
ID & 46,559 & 46,513 & 43,575 & 43,575 & 23,191 & 22,523 & 17,554 & 18,217 & 12,388 \\
MT & 29,907 & 29,879 & 23,381 & 16,525 & 11,522 & 11,438 & 8,137 & 8,790 & 5,215 \\
ND & 1,364 & 1,364 & 1,364 & 1,364 & 1,363 & 1,363 & 1,317 & 802 & 634 \\
NV & 24,471 & 24,418 & 21,435 & 18,850 & 13,379 & 13,078 & 9,435 & 10,674 & 6,985 \\
OR & 19,264 & 19,246 & 18,573 & 18,573 & 8,490 & 8,328 & 6,708 & 7,531 & 5,824 \\
\hline SD & 1,761 & 1,758 & 1,570 & 1,568 & 862 & 862 & 717 & 705 & 522 \\
UT & 9,792 & 9,792 & 9,792 & 9,792 & 9,669 & 9,655 & 8,954 & 6,964 & 5,987 \\
WA & 8,831 & 8,178 & 8,178 & 8,178 & 1,316 & 1,314 & 1,141 & 917 & 722 \\
WY & 107,994 & 107,854 & 100,342 & 100,342 & 42,953 & 42,667 & 36,623 & 36,222 & 28,313 \\
\hline Range & 262,744 & 261,717 & 240,925 & 231,482 & 118,925 & 117,281 & 95,297 & 96,197 & 70,646 \\
\hline
\end{tabular}

Table 2.4. Percent of lek count observations retained for population analyses (Objectives 3 and 4) following sequential application of quality assessment and quality control $(\mathrm{OA} / \mathrm{OC})$ rules $(1-6)$. Values under the 'No Rules' column correspond to the number of lek count observations in the database prior to the application of $\mathrm{QA} / \mathrm{OC}$ rules. Values under 'Rule 6a' correspond to the percent of the 'No Rules' column value that were used to estimate population trends (1960-2019) and 'Rule $6 \mathrm{~b}$ ' to inform a targeted annual warning system (1990-2019) for greater sage-grouse (Centrocercus urophasianus) across their range.

[Values under the 'No rules' column correspond to the number of lek count observations in the database prior to the application QA/QC rules. Values under 'Rule $6 \mathrm{a}$ ' correspond to the percent of the 'No Rules' column value that were used to estimate population trends (1960-2019) and 'Rule 6b' to inform a targeted annual warning system (1990-2019) for greater sage-grouse (Centrocercus urophasianus) across their range. Abbreviations: CA, California; CO, Colorado; ID, Idaho; MT, Montana; ND, North Dakota; NV, Nevada; OR, Oregon; SD, South Dakota; UT, Utah; WA, Washington; WY, Wyoming]

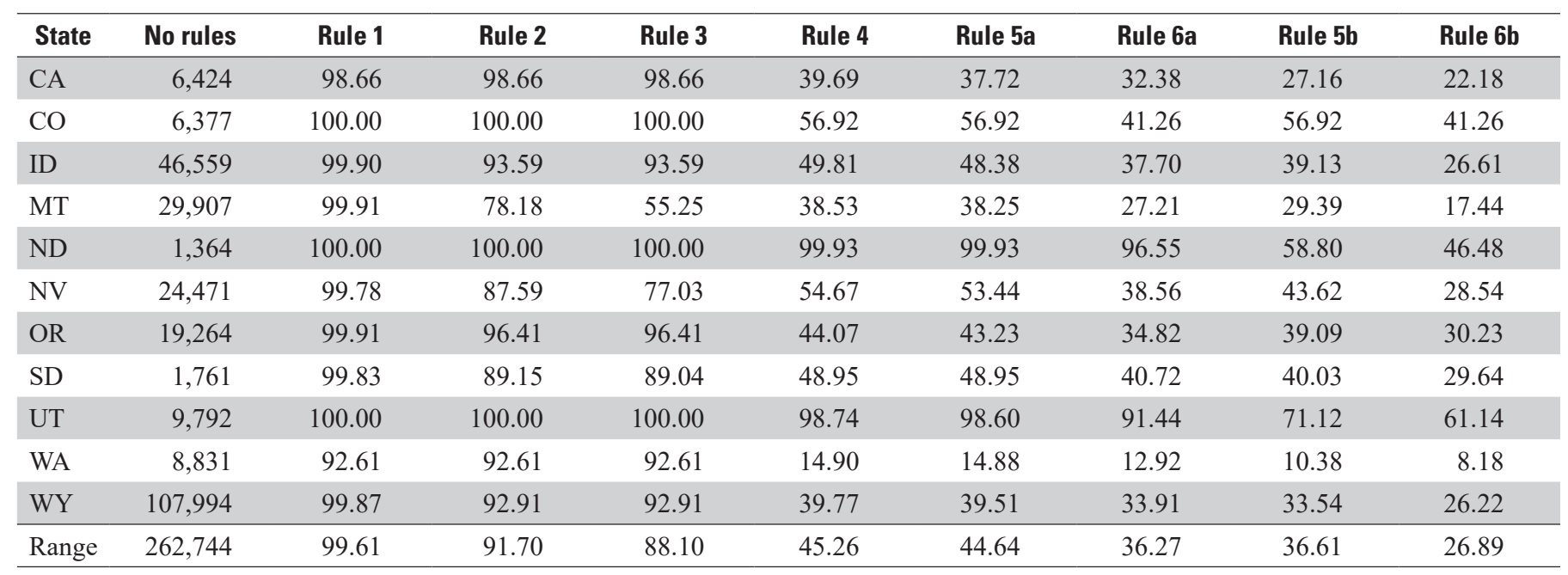




\section{Appendix 3 State-Space Model Formulation}

Posterior parameter distributions were estimated using a spatiotemporally nested design, and population dynamics were described using an exponential growth model on the $\log$ scale. We adopted the log-scale specification because it is more appropriate for populations that inhabit stochastic environments (Lande and others, 2003) and because it precludes the need to truncate distributions describing population size when extirpation is possible. State process equations took the form:

$$
\begin{gathered}
\log \left(\hat{N}_{l, t+1}\right)=\log \left(\hat{N}_{l, t}\right)+\hat{r}_{l, t} \\
\hat{r}_{l, t} \sim \operatorname{Normal}\left(\hat{r}_{n, t}, \hat{\sigma}_{r_{l}}\right)
\end{gathered}
$$

where $\hat{N}_{l, t}$ represents the unknown state (that is, population size) of lek $(l)$ in year $(t)$, and $\hat{r}_{l, t}$ the intrinsic (stochastic) rate of change in abundance, which describes changes in population size from one year to the next (that is, from $t$ to $t+1$ ). Rates of change at the lek were modeled as realizations of a normal random process with mean $\left(\hat{r}_{n, t}\right)$ and variance $\left(\hat{\sigma}_{r_{l}}\right)$. The mean hyperparameter $\left(\hat{r}_{n, t}\right)$ represents the intrinsic rate of change of neighborhood cluster (NC; $n$; to which lek $l$ is spatially nested) in year $(t)$, and the variance hyperparameter $\left(\hat{\sigma}_{r_{1}}\right)$ is a measure of inter-annual, environmental variability affecting lek-level rates of change. We assigned a weakly informative prior to $\hat{\sigma}_{r_{l}}$ which took the form:

$$
\hat{\sigma}_{r_{l}} \sim \operatorname{Gamma}(3,30)
$$

where hyperparameters $k$ and $\theta$ were assigned values 3 and 30 , respectively. We continued to propagate spatiotemporal information within the state-process using the equations:

$$
\begin{gathered}
\hat{r}_{n, t} \sim \operatorname{Normal}\left(\hat{r}_{c, t}, \hat{\sigma}_{r_{n}}\right) \\
\hat{\sigma}_{r_{n}} \sim \operatorname{Gamma}(3,30) \\
\hat{r}_{c, t} \sim \operatorname{Normal}\left(\hat{\mu}_{r_{c}}, \hat{\sigma}_{r}\right) \\
\hat{\sigma}_{r} \sim \operatorname{Gamma}(3,30)
\end{gathered}
$$

which represent an extension of equations 3.2-3.3, carried out at progressively larger spatial extents. Specifically, rates of change at the NC $\left(\hat{r}_{n, t}\right)$ were modeled as realizations of a normal random process with mean $\left(\hat{r}_{c, t}\right)$ and variance $\left(\hat{\sigma}_{r_{n}}\right)$. The mean hyperparameter $\left(\hat{r}_{c, t}\right)$ represents the intrinsic rate of change in abundance of climate cluster (CC; $c$; to which $\mathrm{NC} n$ is spatially nested) in year $(t)$, and the variance hyperparameter $\left(\hat{\sigma}_{r_{n}}\right)$ is a measure of inter-annual, environmental variability affecting NC-level rates of change. Rates at the CC $\left(\hat{r}_{c, t}\right)$ were modeled as realizations of a normal random process with mean $\left(\hat{\mu}_{r_{c}}\right)$ and variance $\left(\hat{\sigma}_{r_{c}}\right)$. The mean hyperparameter at the $\mathrm{CC}\left(\hat{\mu}_{r_{\mathrm{c}}}\right)$ represents the long-term intrinsic rate of population change of the $\mathrm{CC}(c)$, and the variance hyperparameter $\left(\hat{\sigma}_{r_{c}}\right)$ is a measure of inter-annual, environmental variability affecting CC-level rates of change. The long-term mean rate of change in abundance for each CC was assigned a vague, uniform prior:

$$
\hat{\mu}_{r_{c}} \sim \operatorname{Uniform}(-0.1,0.1)
$$

with upper and lower bounds of -0.1 and 0.1 , which equates to a long-term average percent change that can range from approximately -10 to 10 . These upper and lower bounds are well beyond the estimates reported by Garton and others (2011) for sage-grouse management zones between 1965 and 2015 , so we were comfortable that they would not truncate parameter estimation. The observation process:

$$
y_{l, t} \sim \operatorname{Poisson}\left(\hat{N}_{l, t}\right)
$$

mapped the true state of the process onto the observed data $\left(y_{l, t}\right)$, which, in this case, were individual maximum counts $(y)$ at a given lek $(l)$ and year $(t)$. Errors in the counts were modeled using a Poisson distribution with a mean equal to the variance. Use of a Poisson error structure assumed that observation error increased as the true number of birds present on the lek increased, which was a reasonable assumption for counts of sage-grouse at leks. The population size at each lek $(l)$ in the first year of our study $(t=1)$ was specified using a vague prior:

$$
\hat{N}_{l, 1} \sim \operatorname{Uniform}(2,500)
$$

where $\hat{N}_{l, 1}$ was assumed to arise from a uniform distribution with lower and upper bounds of 2 and 500, respectively. Here, the lower bound represents the fewest number of individuals meeting the definition of an active lek, whereas the upper bound represents a 4-percent increase over the maximum number of males observed across all leks between 1960 and 2019.

\section{References Cited}

Garton, E.O., Connelly, J.W., Horne, J.S., Hagen, C.A., Moser, A., and Schroeder, M.A., 2011, Greater sagegrouse population dynamics and probability of persistence, in Knick, S.T., and Connelly, J.W., eds., Greater sagegrouse-Ecology and conservation of a landscape species and its habitats: University of California Press, Studies in Avian Biology, no. 38, p. 292-382, https://doi.org/10.1525/ california/9780520267114.003.0016.

Lande, R., Engen, S., and Saether, B.E., 2003, Stochastic population dynamics in ecology and conservation: Oxford University Press on Demand, 698 p., https://doi.org/10.1093/acprof: oso/9780198525257.001.0001. 


\section{Appendix 4. State-Wide Analyses}

\section{California Results}

\section{California Lek Data and Clusters (Objectives 1 and 2)}

The state of California intersected 2 different climate clusters (CCs; CC-A and CC-E) and contained or intersected 21 different neighborhood clusters (NCs; fig. 4.1). The total area of the 21 NCs within California was 1,764,201 hectares (ha). These areas consisted of 272 leks, 136 of which were located within the California state boundary, representing 1.62 percent of the range-wide lek database. Following extensive quality assessment and quality control (QA/QC), we used 79 leks from California in the state-space model (SSM) to estimate population trends (tables 4.1 and 4.2), totaling 2,080 individual lek count surveys. Mean male count was 18.88 (95-percent confidence interval=18.33-19.43) for leks within NCs that were within or overlapped California boundaries. Mean male count for leks within California state boundaries was 20.08 ( 95 -percent confidence interval $=19.38-20.78$ ).

\section{California Population Trend Analysis (Objective 3)}

Most of the sage-grouse range within California (74.1 percent) fell within CC-E. For CC-E, we estimated six population abundance nadirs (troughs) that dated back to 1960. Each of these population abundance nadirs represent between one and six complete periods of oscillation. We used these nadirs to estimate population trends across three different temporal scales that represented two, four, and six complete periods of oscillation for the state (for instance, second, fourth, and sixth nadir). We estimated the average annual finite rate of population change $(\hat{\lambda})$ at different temporal scales that were based on periods of oscillation, which were: short (two periods), medium (four periods), and long (six periods) temporal scales as 0.963 (95-percent CRI $=0.953-0.970$ ), 0.974 (95-percent CRI $=0.962-0.981$ ), and 0.973 (95-percent $\mathrm{CRI}=0.963-0.982$ ), respectively (fig. 4.2). For all NCs that were modeled and intersected California, we estimated median $\hat{\lambda}$ to be less than 1.0 for $85.7,90.5$, and 95.2 percent across short, medium, and long temporal scales, respectively (fig. 4.3; table 4.2). We estimated median $\hat{\lambda}$ to be less than 1.0 for 81.0, 62.0, and 81.0 percent of all modeled leks within California across short, medium, and long temporal scales, respectively (fig. 4.4). We reported spatial and temporal variation in average annual $\hat{\lambda}$ across different neighborhood clusters (NCs; fig. 4.3) and leks (fig. 4.4).

\section{California Targeted Annual Warning System Analysis (Objective 4)}

During 1990-2019, a targeted annual warning system for sage-grouse populations in California activated a total of 41 and 32 leks as watches and warnings, respectively (fig. 4.5), which was 58.6 and 45.7 percent of the sampled leks used in the analysis. On average, across the 29 years, approximately 4.5 and 1.8 percent of leks per year experienced watches and warnings, respectively. The higher percentage for watches corresponds to repetitive activation. During this time frame, the TAWS also activated a total of five and five NCs as watches and warnings, respectively (fig. 4.6), which was 33.3 and 33.3 percent of the sampled clusters used in the analysis. On average, across the 29 years, approximately 2.9 and 1.3 percent of clusters per year experienced watches and warnings, respectively. The higher percentage for watches corresponds to repetitive activation. During 2019, the TAWS activated three (first $=1$ ) watches and three (first $=2$ ) warnings at leks (fig. 4.7) and zero watches and zero warnings at NCs. 


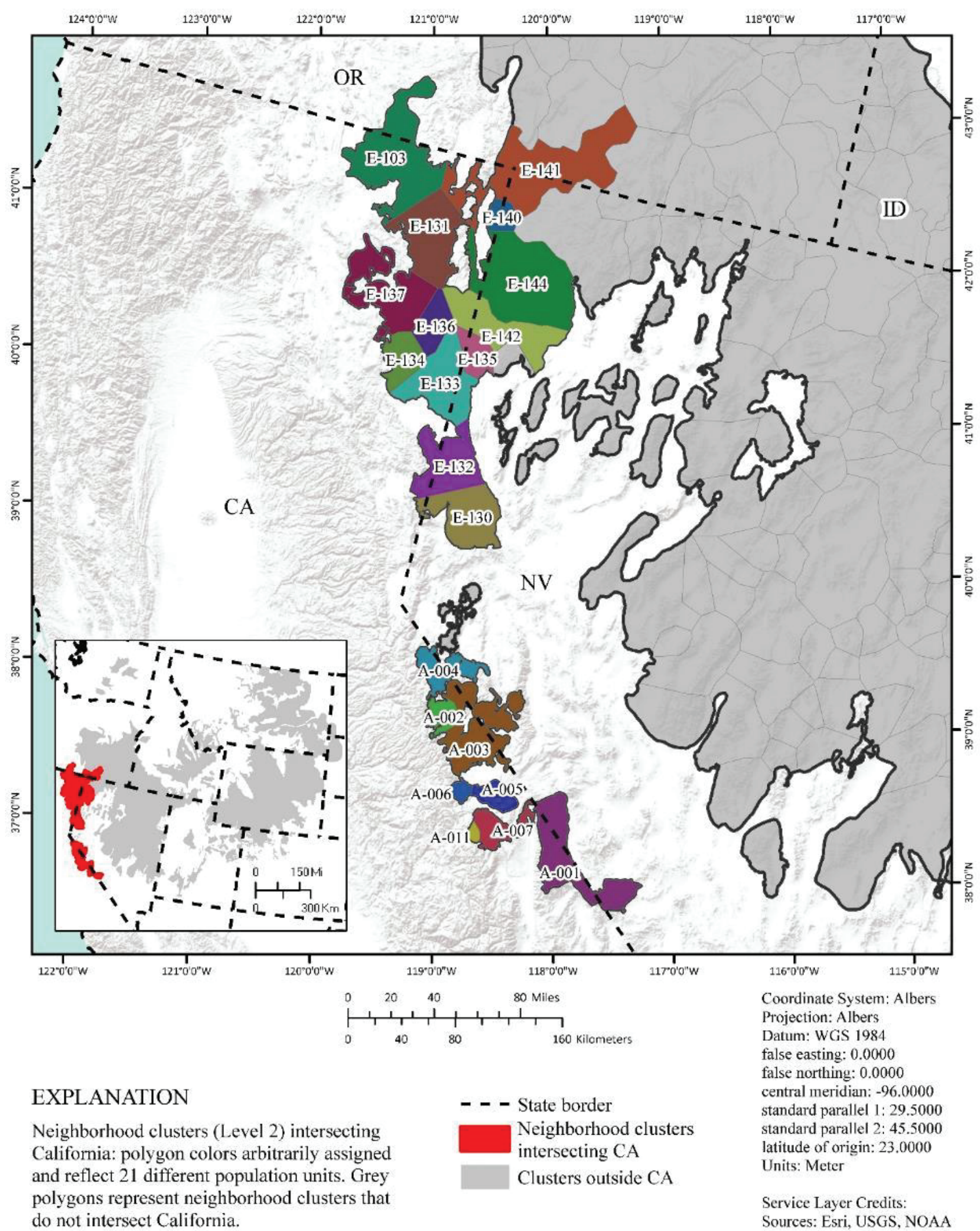

Figure 4.1. Greater sage-grouse (Centrocercus urophasianus) hierarchical population monitoring framework for neighborhood clusters that intersect the state of California. Map image is the intellectual property of Esri and is used herein under license. Copyright (C) 2020 Esri and its licensors. All rights reserved. 
Table 4.1. Table of greater sage-grouse (Centrocercus urophasianus) average annual rate of population change ( $\hat{\lambda})$ across six periods of oscillation in abundance that represent temporal scales for each climate cluster within the state of California. Estimates were derived from leks within the entire climate cluster.

[CC, climate cluster; A, Bi-state area; E, Great Basin area]

\begin{tabular}{|c|c|c|c|c|c|c|c|c|c|}
\hline \multirow{2}{*}{ CC } & \multirow{2}{*}{$\begin{array}{l}\text { Percent } \\
\text { CC1 }\end{array}$} & \multicolumn{6}{|c|}{ Temporal scales 2} & \multirow{2}{*}{$\begin{array}{c}\text { Number of } \\
\text { leks }^{3}\end{array}$} & \multirow{2}{*}{$\begin{array}{l}\text { Average } \\
\text { count/lek }\end{array}$} \\
\hline & & Long & Medium/Long & Medium & Short/Medium & Short & Recent & & \\
\hline A & 62.8 & $\begin{array}{c}0.978 \\
(0.965-0.988)\end{array}$ & $\begin{array}{c}0.978 \\
(0.962-0.986)\end{array}$ & $\begin{array}{c}0.990 \\
(0.974-1.000)\end{array}$ & $\begin{array}{c}0.995 \\
(0.979-1.005)\end{array}$ & $\begin{array}{c}0.973 \\
(0.964-0.981)\end{array}$ & $\begin{array}{c}0.981 \\
(0.969-0.993)\end{array}$ & $\begin{array}{c}84 \\
(55: 41,48: 36)\end{array}$ & $\begin{array}{c}21.3 \\
(19.9-22.8)\end{array}$ \\
\hline $\mathrm{E}$ & 3.8 & $\begin{array}{c}0.971 \\
(0.967-0.976)\end{array}$ & $\begin{array}{c}0.973 \\
(0.967-0.978)\end{array}$ & $\begin{array}{c}0.974 \\
(0.963-0.979)\end{array}$ & $\begin{array}{c}0.986 \\
(0.981-0.990)\end{array}$ & $\begin{array}{c}0.968 \\
(0.964-0.971)\end{array}$ & $\begin{array}{c}0.949 \\
(0.944-0.955)\end{array}$ & $\begin{array}{c}4,012 \\
(2,187: 38 \\
1,908: 33)\end{array}$ & $\begin{array}{c}17.3 \\
(17.1-17.5)\end{array}$ \\
\hline
\end{tabular}

1The percent of each climate cluster that intersects the state.

${ }^{2}$ Temporal scales were estimated from present to each major population abundance nadir (trough) since 1960. Number of temporal scales were used to estimate population trends across six different temporal scales from approximately 10 to approximately 60 years.

${ }^{3}$ Number of leks in database. In parentheses from left to right is (1) total number in cluster were used in trend analysis, (2) number used in trend analysis within state boundary, (3) total number in cluster were used in the targeted annual warning system (TAWS) analysis, and (4) number used in TAWS analysis within state boundary. 
Table 4.2. Table of greater sage-grouse (Centrocercus urophasianus) average annual rate of population change ( $\hat{\lambda}$ ) across six periods of oscillation in abundance that represent temporal scales for each neighborhood cluster within the state of California. Estimates were derived from leks within the entire neighborhood cluster.

[NC, neighborhood cluster; NA, not applicable]

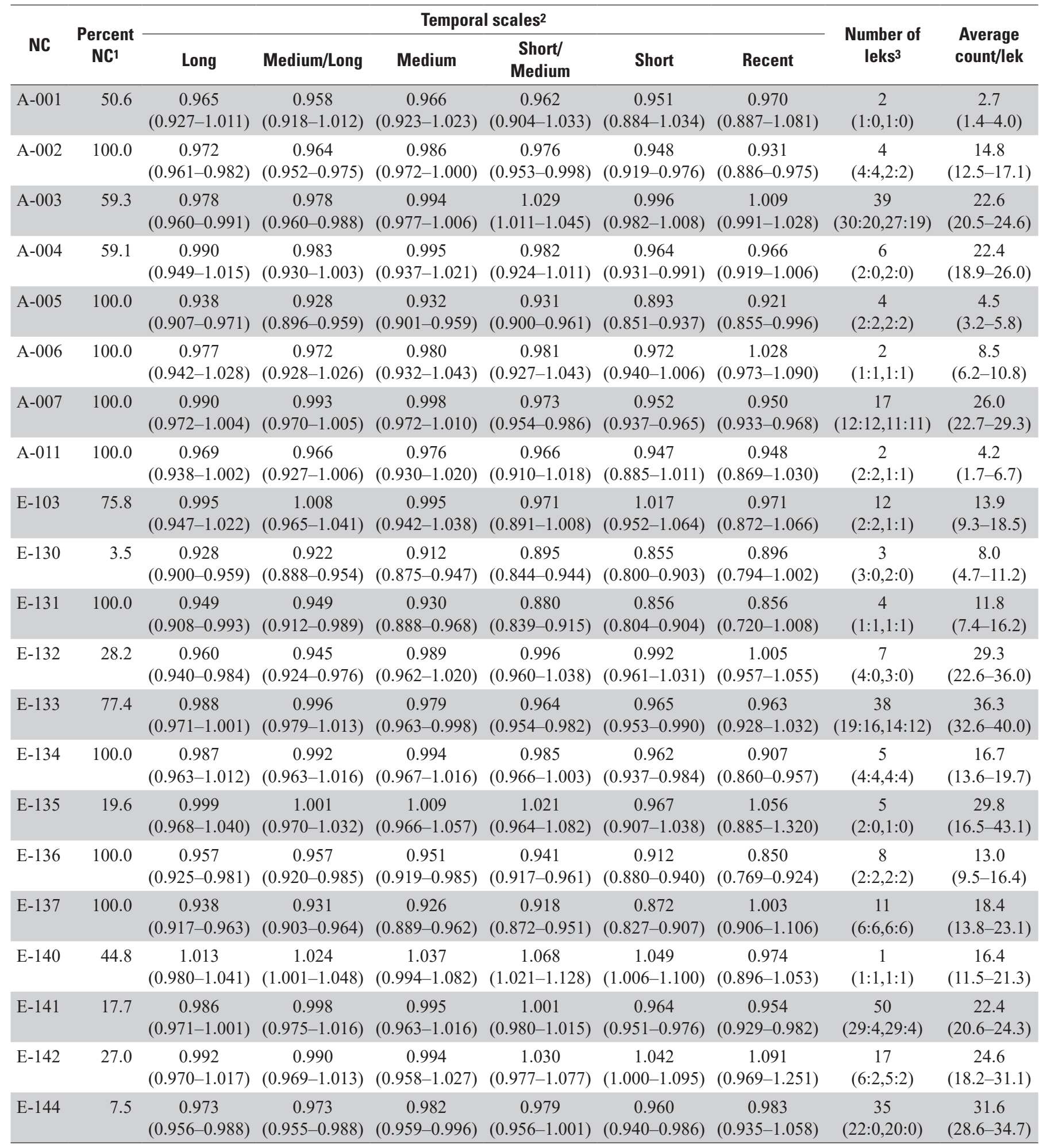

${ }^{1}$ The percent of each neighborhood cluster that intersects the state.

2Temporal scales were estimated from present to each major population abundance nadir (trough) since 1960. Number of temporal scales were used to estimate population trends across six different temporal scales from approximately 10 to approximately 60 years.

${ }^{3}$ Number of leks in database. In parentheses from left to right is (1) total number in cluster were used in trend analysis, (2) number used in trend analysis within state boundary, (3) total number in cluster were used in the targeted annual warning system (TAWS) analysis, and (4) number used in TAWS analysis within state boundary. 

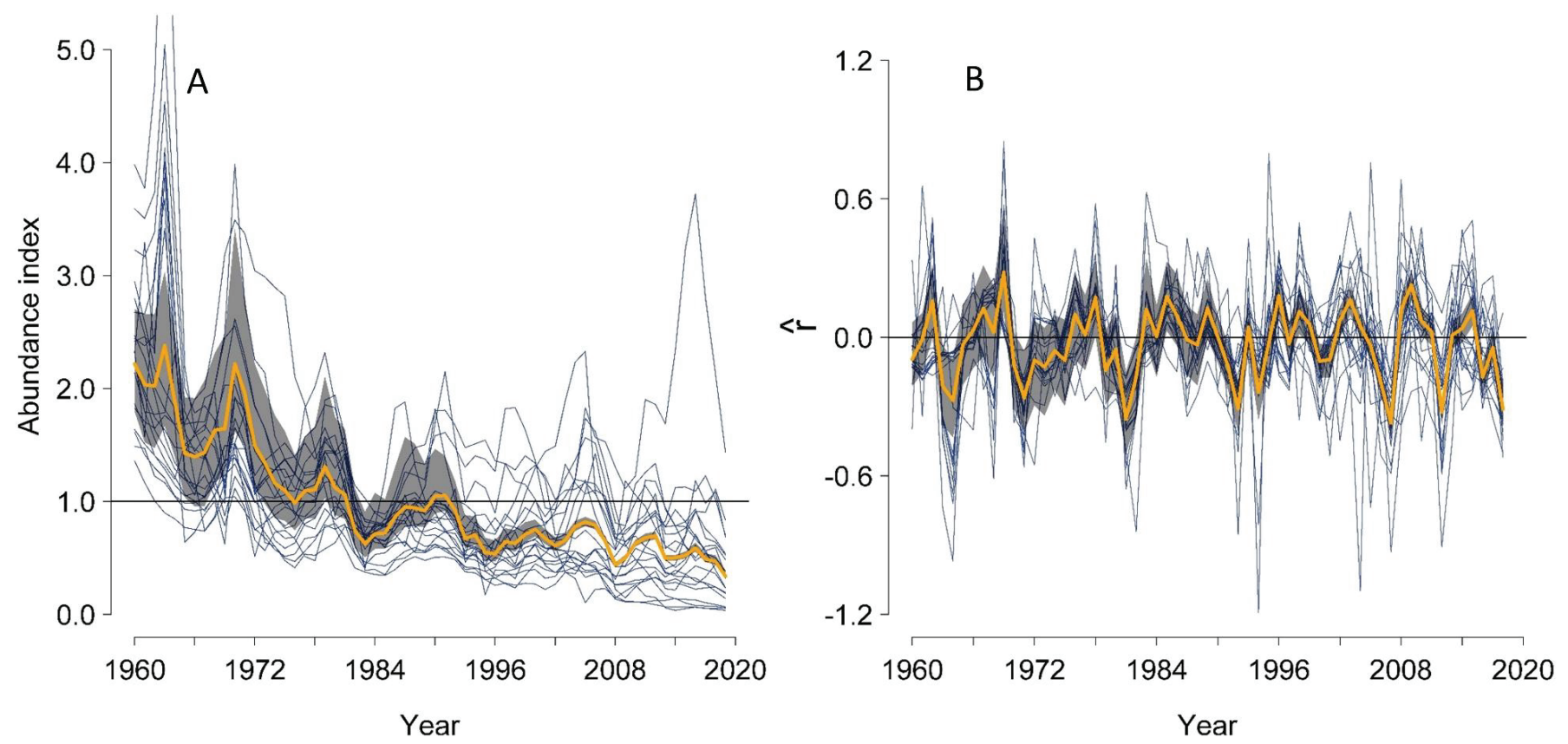

Figure 4.2. A, Abundance index (calculated as $\hat{N}$ divided by 60-year mean of $\hat{N}$ ); and $\mathrm{B}$, intrinsic rate of population change $(\hat{r})$ of greater sage-grouse (Centrocercus urophasianus) within the state of California from 1960 to 2019. Thick yellow line represents median estimates across all leks. Shaded areas represent 95-percent credible limits. Thin blue lines represent median values for neighborhood clusters. Black horizontal line (abundance index $=1.0$ ) represents 60 -year average. 


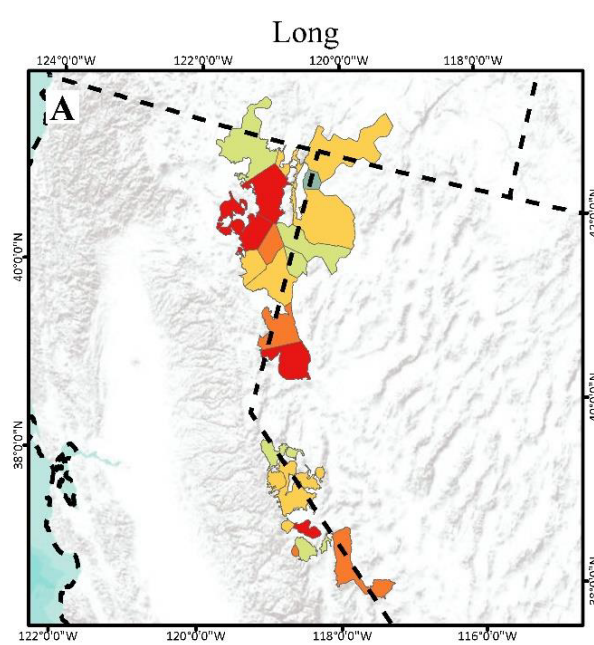

\section{EXPLANATION}

Neighborhood clusters (Level 2) intersecting California: polygon colors represent average lambda values for sage-grouse populations

over varying lengths of time (long, medium, short). Symbols are based on a range-wide distribution of values and all may not be represented here.
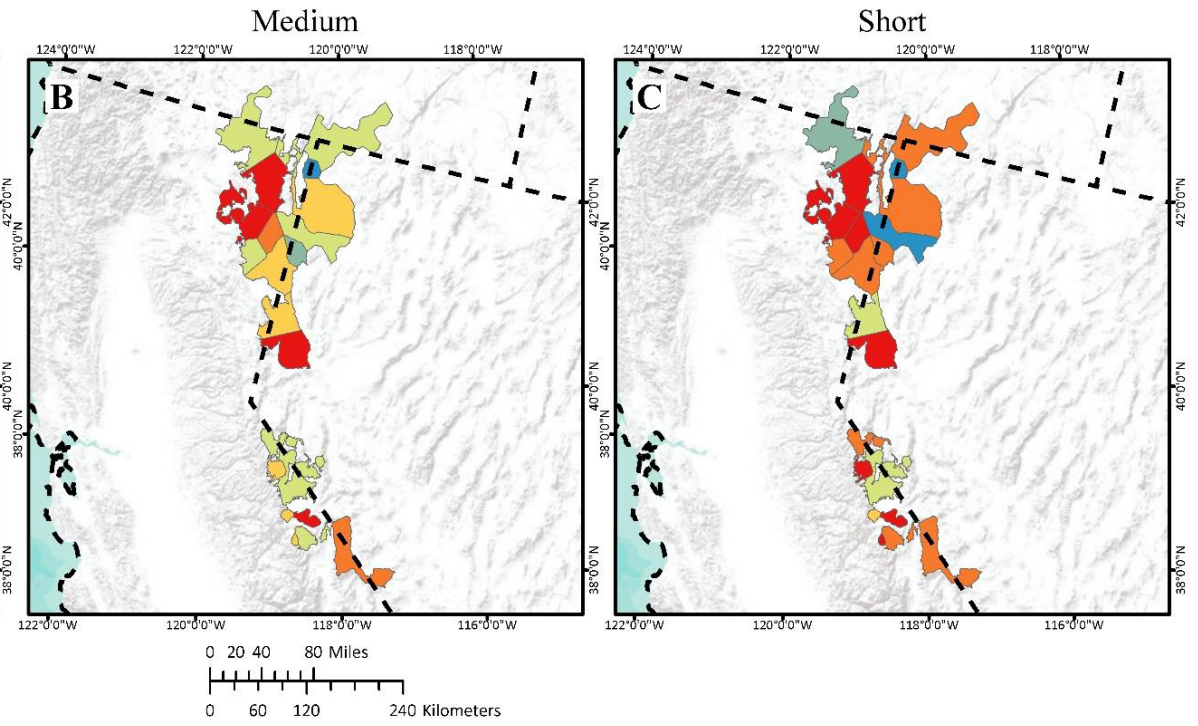

Average lambda $(\hat{\lambda})$

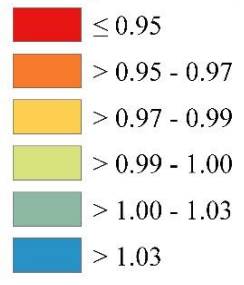

Coordinate System: Albers Projection: Albers Datum: WGS 1984 false easting: 0.0000 false northing: 0.0000 central meridian: -96.0000 standard parallel 1: 29.5000 standard parallel $2: 45.5000$ latitude of origin: 23.0000 Units: Meter

Service Layer Credits: Sources: Esri, USGS, NOAA

Figure 4.3. Spatial estimates of population trends across three temporal scales based on periods of oscillation (short, medium, and long) while accounting for periods of oscillation of greater sage-grouse (Centrocercus urophasianus) at different neighborhood clusters within the state of California. Map images are the intellectual property of Esri and are used herein under license. Copyright (C) 2020 Esri and its licensors. All rights reserved. 


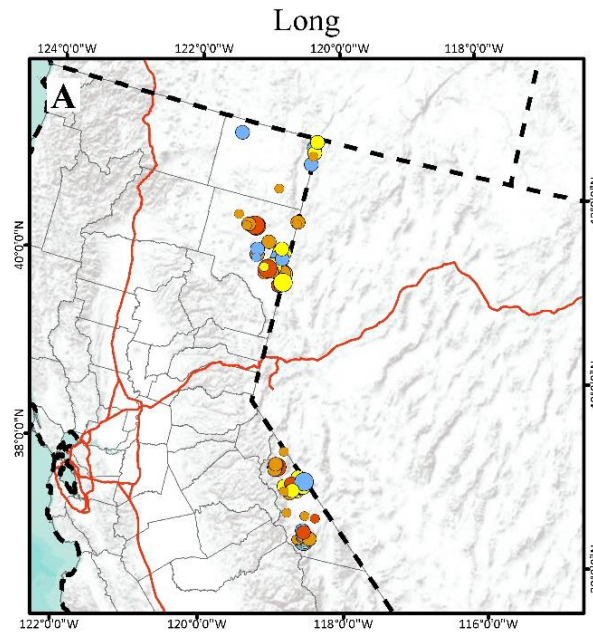

EXPLANATION

Leks within California: colors represent average lambda $(\hat{\lambda})$ values of sage-grouse populations over various time scales (long, medium, short). The size of the symbol represents the maximum population size of the lek over the years of the analysis, relativized to the range-wide maximum population size across all leks. Symbols are based on a range-wide distribution of values and all may not be represented here.
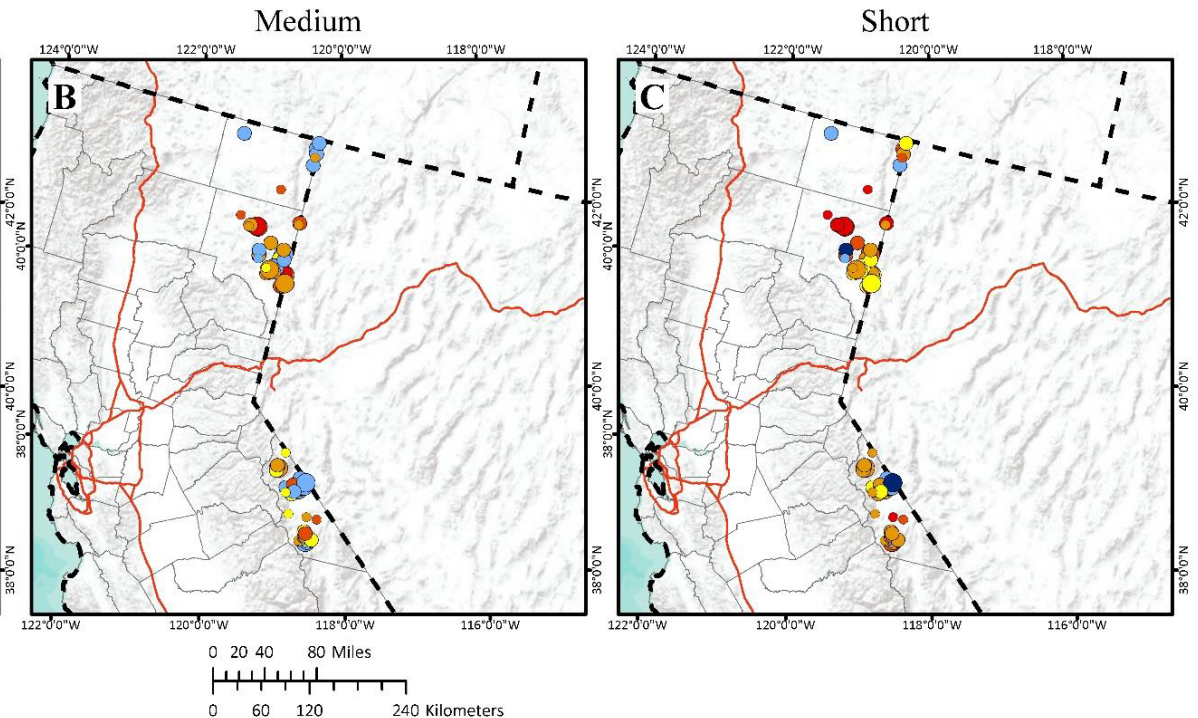

$\begin{array}{llll}0 & 60 & 120 & 240 \text { Kilometers }\end{array}$

$$
\begin{aligned}
\text { Average lambda }(\hat{\lambda}) \\
\quad \leq 0.90 \\
\quad>0.90-0.94 \\
\quad>0.94-0.98 \\
\quad>0.98-1.00 \\
\quad>1.00-1.10 \\
->1.10
\end{aligned}
$$

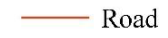

Coordinate System: Albers Projection: Albers Datum: WGS 1984 false easting: 0.0000 false northing: 0.0000 central meridian: -96.0000 standard parallel $1: 29.5000$ standard parallel $2: 45.5000$ latitude of origin: 23.0000 Units: Meter

Service Layer Credits: Sources: Esri, USGS, NOAA

Figure 4.4. Spatial estimates of population trends across three temporal scales based on periods of oscillation (short, medium, and long) while accounting for periods of oscillation of greater sage-grouse (Centrocercus urophasianus) at lek sites within the state of California. Map images are the intellectual property of Esri and are used herein under license. Copyright $(C 2020$ Esri and its licensors. All rights reserved. 


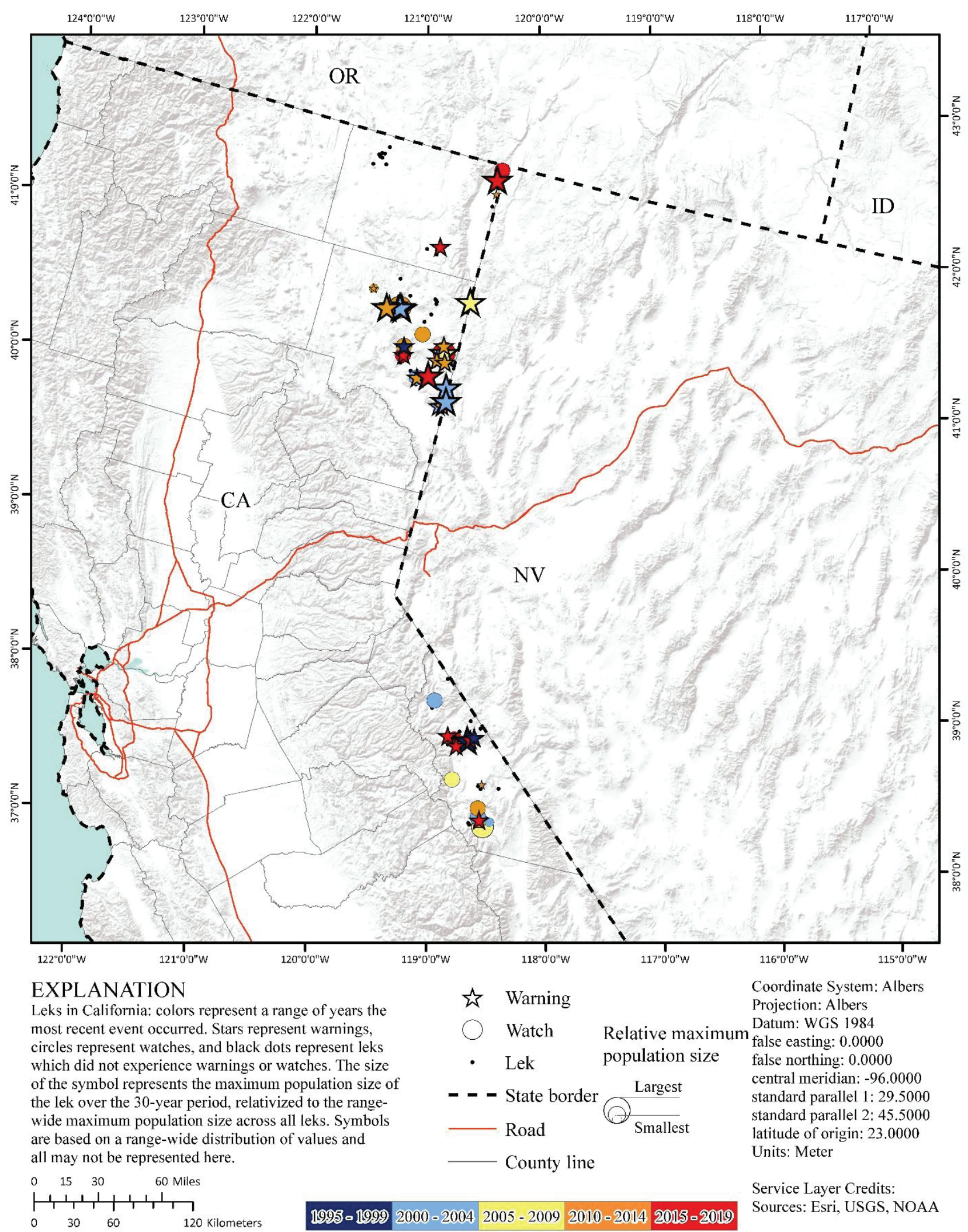

Figure 4.5. Spatial and temporal depiction of watches and warnings of greater sage-grouse (Centrocercus urophasianus) population declines at the lek scale within the state of California from 1990 to 2019. Map image is the intellectual property of Esri and is used herein under license. Copyright $@ 2020$ Esri and its licensors. All rights reserved. 


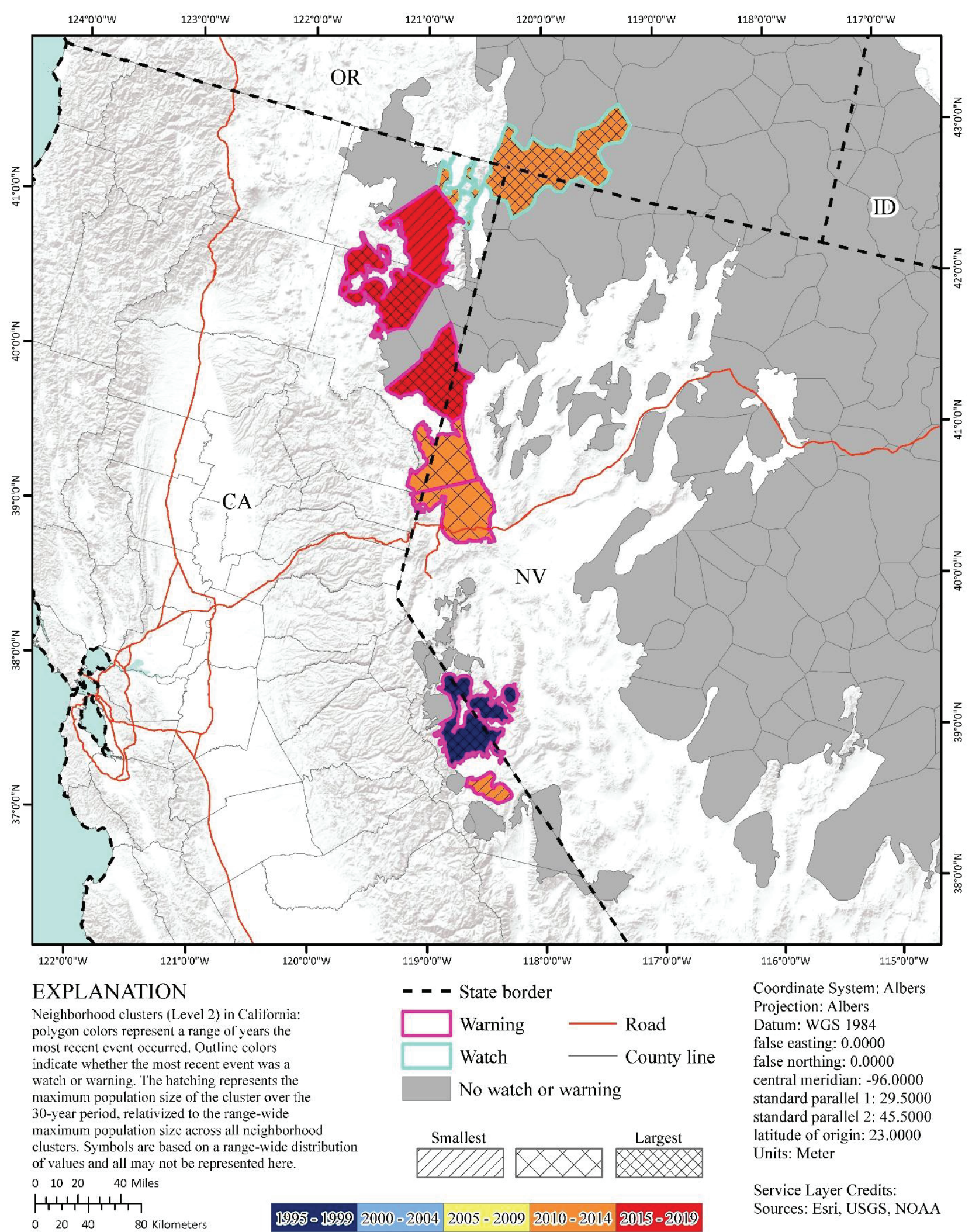

Figure 4.6. Spatial and temporal depiction of watches and warnings of greater sage-grouse (Centrocercus urophasianus) population declines at neighborhood cluster within the state of California from 1990 to 2019. Map image is the intellectual property of Esri and is used herein under license. Copyright $(C 2020$ Esri and its licensors. All rights reserved. 


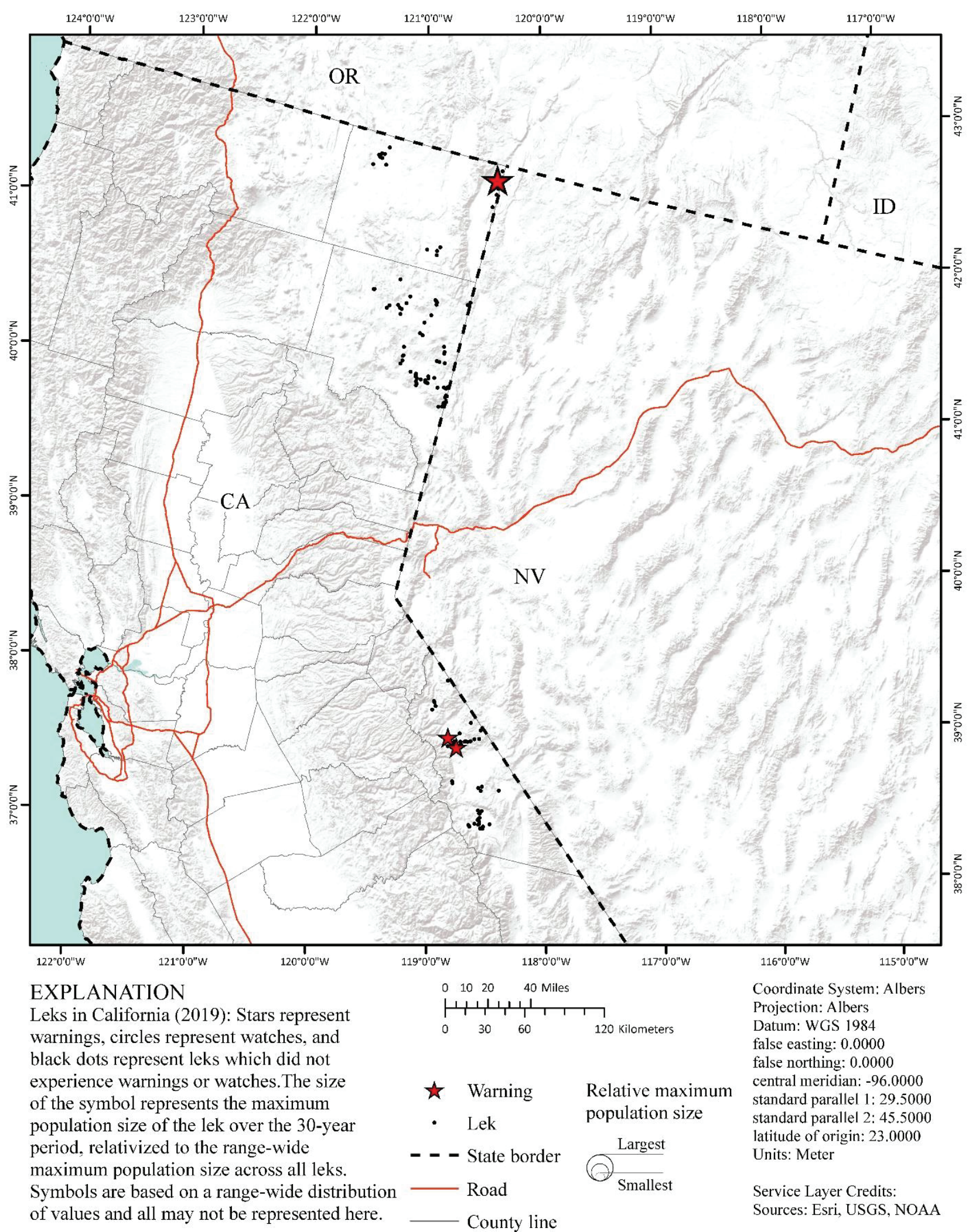

Figure 4.7. Spatial and temporal depiction of watches and warnings of greater sage-grouse (Centrocercus urophasianus) population declines at the lek scale within the state of California during 2019. Map image is the intellectual property of Esri and is used herein under license. Copyright () 2020 Esri and its licensors. All rights reserved. 


\section{Oregon Results}

\section{Oregon Lek Data and Clusters (Objectives 1 and 2)}

Oregon intersected CC-E and contained or intersected 47 different NCs (fig. 4.8). Total area of the $47 \mathrm{NCs}$ in Oregon was 7,620,928 ha. Forty-two of these clusters were used in the analysis after five were omitted because of limitations in sample sizes. These areas consisted of 936 leks, 713 of which were located within the Oregon state boundary and represented 8.47 percent of the range-wide lek database. Following extensive QA/QC, we used 406 leks from Oregon in the SSM to estimate population trends (tables 4.3 and 4.4), totaling 6,708 individual lek count surveys. Mean male count was 11.32 (95-percent confidence interval=11.11-11.54) for leks within NCs that were within or overlapped Oregon. Mean male count for leks in Oregon was 11.41 (95-percent confidence interval $=11.17-11.64$ ).

\section{Oregon Population Trend Analysis (Objective 3)}

For CC-E, we estimated six population abundance nadirs (troughs) that dated back to 1960. Each of these population abundance nadirs represent between one and six complete periods of oscillation. We used these nadirs to estimate population trends across three different temporal scales that represented two, four, and six complete periods of oscillation for the state (for instance, second, fourth, and sixth nadir). We estimated the average annual finite rate of population change $(\hat{\lambda})$ at the short (two periods), medium (four periods), and long (six periods) temporal scales as 0.949 (95-percent
$\mathrm{CRI}=0.943-0.954), 0.967$ (95-percent $\mathrm{CRI}=0.957-0.973$ ), and 0.966 (95-percent $\mathrm{CRI}=0.960-0.971$, respectively (fig. 4.9). Climate cluster estimates, which included leks in adjacent states to Oregon, were slightly different than estimates generated from leks only within Oregon. For all NCs that were modeled and intersected Oregon, we estimated median $\hat{\lambda}$ to be less than 1.0 for 85.7, 92.9, and 92.9 percent across short, medium, and long temporal scales, respectively (table 4.4), and estimated median $\hat{\lambda}$ to be less than 1.0 for $85.2,84.2$, and 94.6 percent of all modeled leks, respectively. We reported spatial and temporal variation in average annual $\hat{\lambda}$ across different NCs (fig. 4.10) and leks (fig. 4.11).

\section{Oregon Targeted Annual Warning System Analysis (Objective 4)}

During 1990-2019, the TAWS activated a total of 237 and 186 leks as watches and warnings, respectively (fig. 4.12), which represented 60.9 and 47.8 percent of the sampled leks used in the analysis. On average, across those 29 years, approximately 7.4 and 1.9 percent of leks per year experienced watches and warnings, respectively. The higher percentage for watches corresponds to repetitive activation. During this time frame, the TAWS activated a total of 13 and 8 NCs as watches and warnings, respectively (fig. 4.13), which was 34.2 and 21.1 percent of the sampled clusters used in the analysis. On average, across the 29 years, approximately 2.3 and 0.8 percent of clusters per year experienced watches and warnings, respectively. The higher percentage for watches corresponds to repetitive activation. During 2019, the TAWS activated 12 (first $=1$ ) watches and 24 (first $=2$ ) warnings at leks (fig. 4.14), and 0 (first $=0$ ) watches and 2 (first $=0$ ) warnings at NCs (fig. 4.15). 


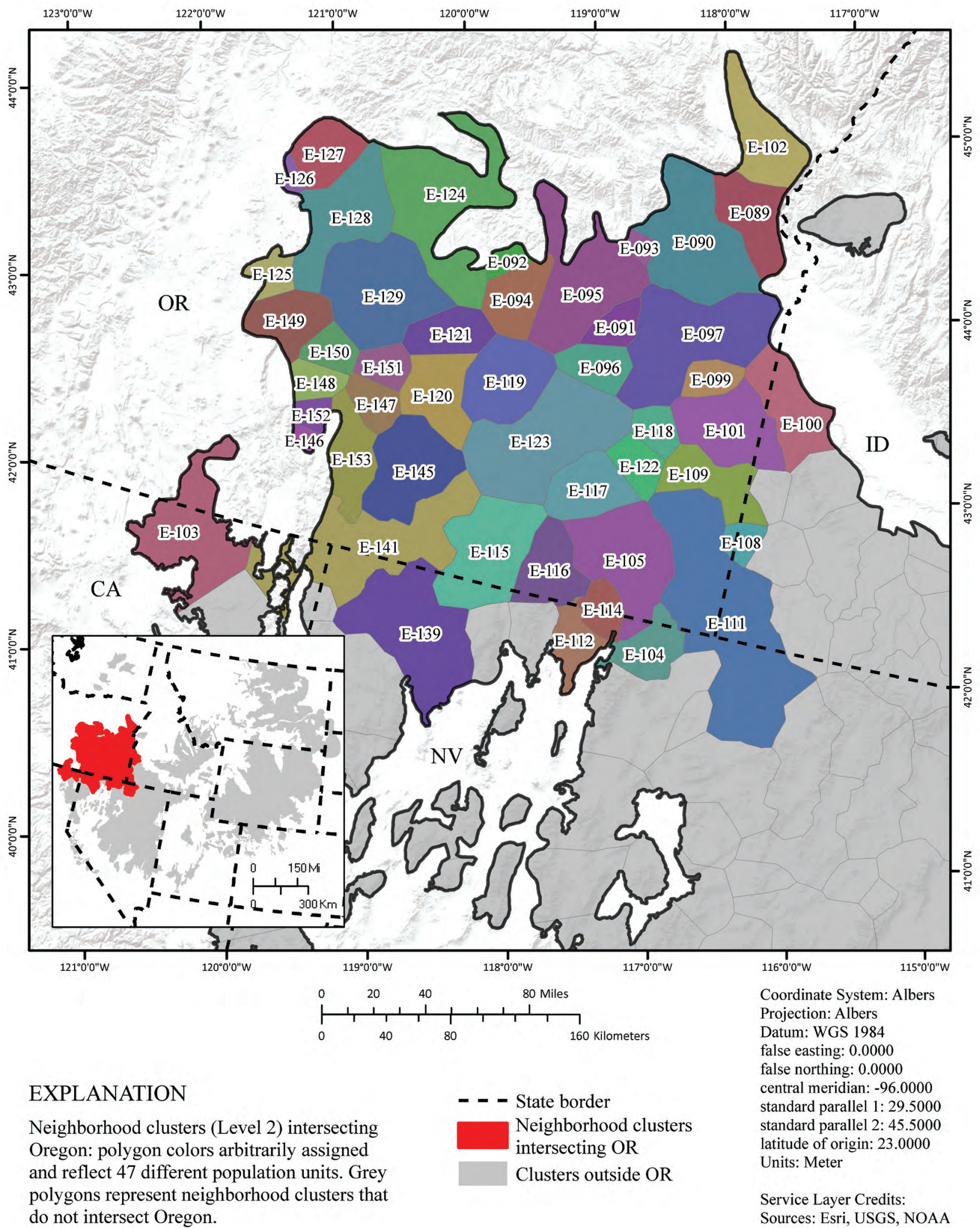

Figure 4.8. Greater sage-grouse (Centrocercus urophasianus) hierarchical population monitoring framework for neighborhood clusters that intersect the state of Oregon. Map image is the intellectual property of Esri and is used herein under license. Copyright () 2020 Esri and its licensors. All rights reserved. 
Table 4.3. Greater sage-grouse (Centrocercus urophasianus) average annual rate of population change $(\hat{\lambda})$ across six periods of oscillation in abundance that represent temporal scales for each climate cluster within the state of Oregon.

[CC, climate cluster; E, Great Basin area]

\begin{tabular}{|c|c|c|c|c|c|c|c|c|c|}
\hline \multirow[b]{2}{*}{ CC } & \multirow[b]{2}{*}{$\begin{array}{l}\text { Percent } \\
\text { CC1 }\end{array}$} & \multicolumn{6}{|c|}{ Temporal scales² } & \multirow[b]{2}{*}{ Number of leks 3} & \multirow[b]{2}{*}{$\begin{array}{l}\text { Average } \\
\text { count/lek }\end{array}$} \\
\hline & & Long & Medium/Long & Medium & $\begin{array}{c}\text { Short/ } \\
\text { Medium }\end{array}$ & Short & Recent & & \\
\hline
\end{tabular}

1The percent of each climate cluster that intersects the state.

${ }^{2}$ Temporal scales were estimated from present to each major population abundance nadir (trough) since 1960. Number of temporal scales were used to estimate population trends across six different temporal scales from approximately 10 to approximately 60 years.

${ }^{3}$ Number of leks in database. In parentheses from left to right is (1) total number in cluster were used in trend analysis, (2) number used in trend analysis within state boundary, (3) total number in cluster were used in the targeted annual warning system (TAWS) analysis, and (4) number used in TAWS analysis within state boundary.

Table 4.4. Greater sage-grouse (Centrocercus urophasianus) average annual rate of population change $(\hat{\lambda})$ across six periods of oscillation in abundance that represent temporal scales for each neighborhood cluster within the state of Oregon.

[NC, neighborhood cluster; NA, not applicable]

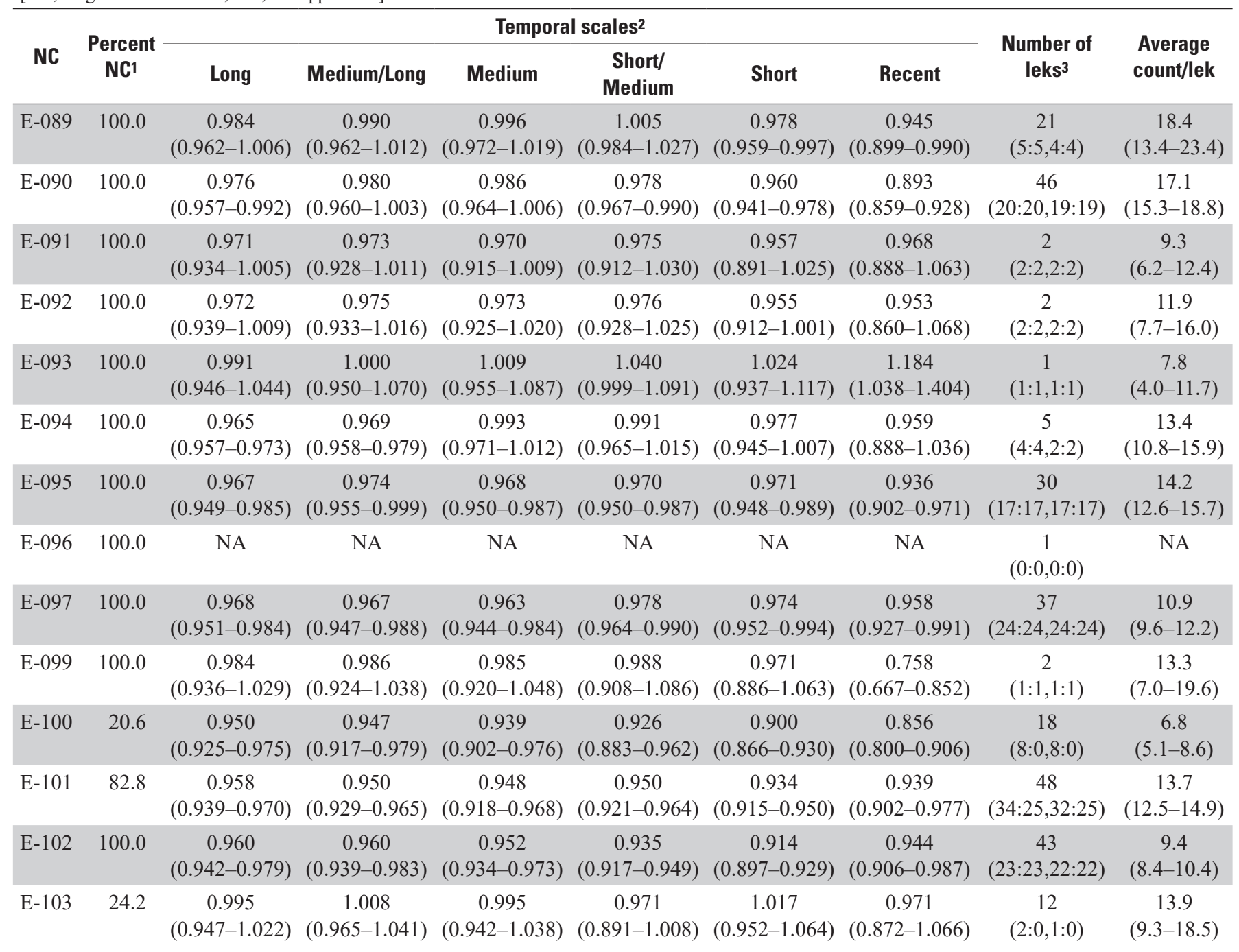


Table 4.4. Greater sage-grouse (Centrocercus urophasianus) average annual rate of population change $(\hat{\lambda})$ across six periods of oscillation in abundance that represent temporal scales for each neighborhood cluster within the state of Oregon.-Continued

[NC, neighborhood cluster; NA, not applicable]

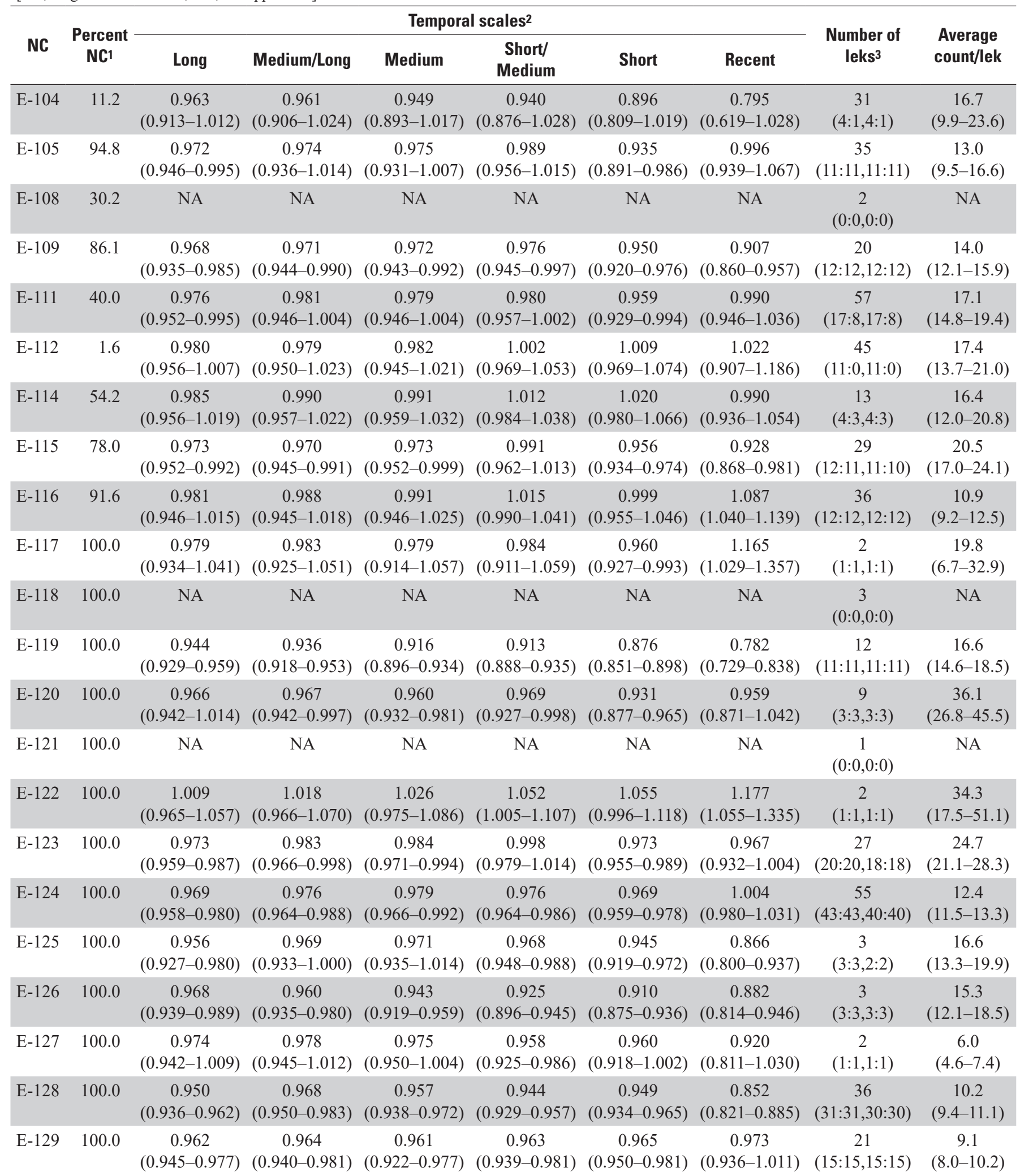


Table 4.4. Greater sage-grouse (Centrocercus urophasianus) average annual rate of population change $(\hat{\lambda})$ across six periods of oscillation in abundance that represent temporal scales for each neighborhood cluster within the state of Oregon.-Continued

[NC, neighborhood cluster; NA, not applicable]

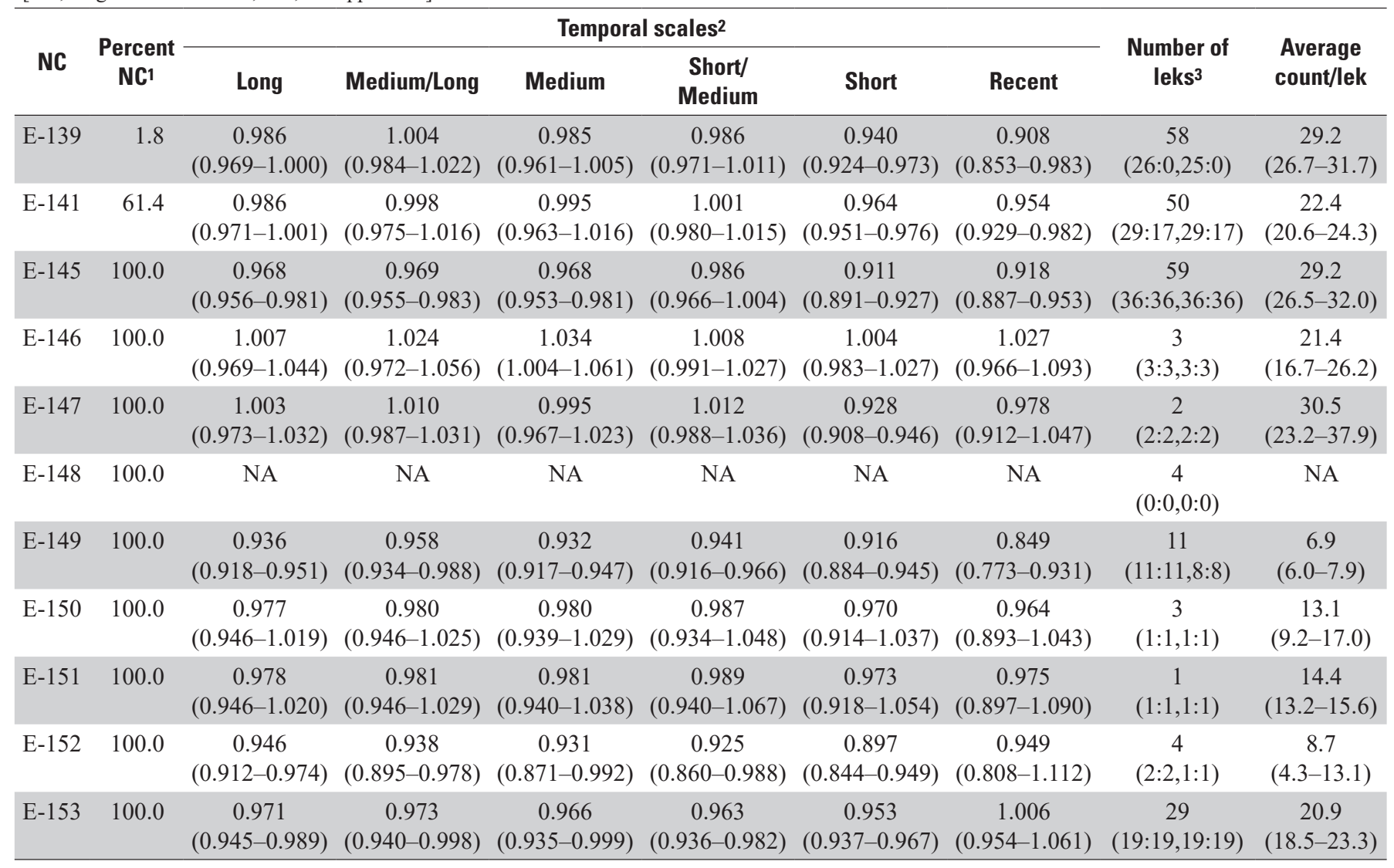

1The percent of each neighborhood cluster that intersects the state.

2Temporal scales were estimated from present to each major population abundance nadir

(trough) since 1960. Number of temporal scales were used to estimate population trends across six different temporal scales from approximately 10 to approximately 60 years.

${ }^{3}$ Number of leks in database. In parentheses from left to right is

(1) total number in cluster were used in trend analysis,

(2) number used in trend analysis within state boundary,

(3) total number in cluster were used in the targeted annual warning system

(TAWS) analysis, and

(4) number used in TAWS analysis within state boundary. 

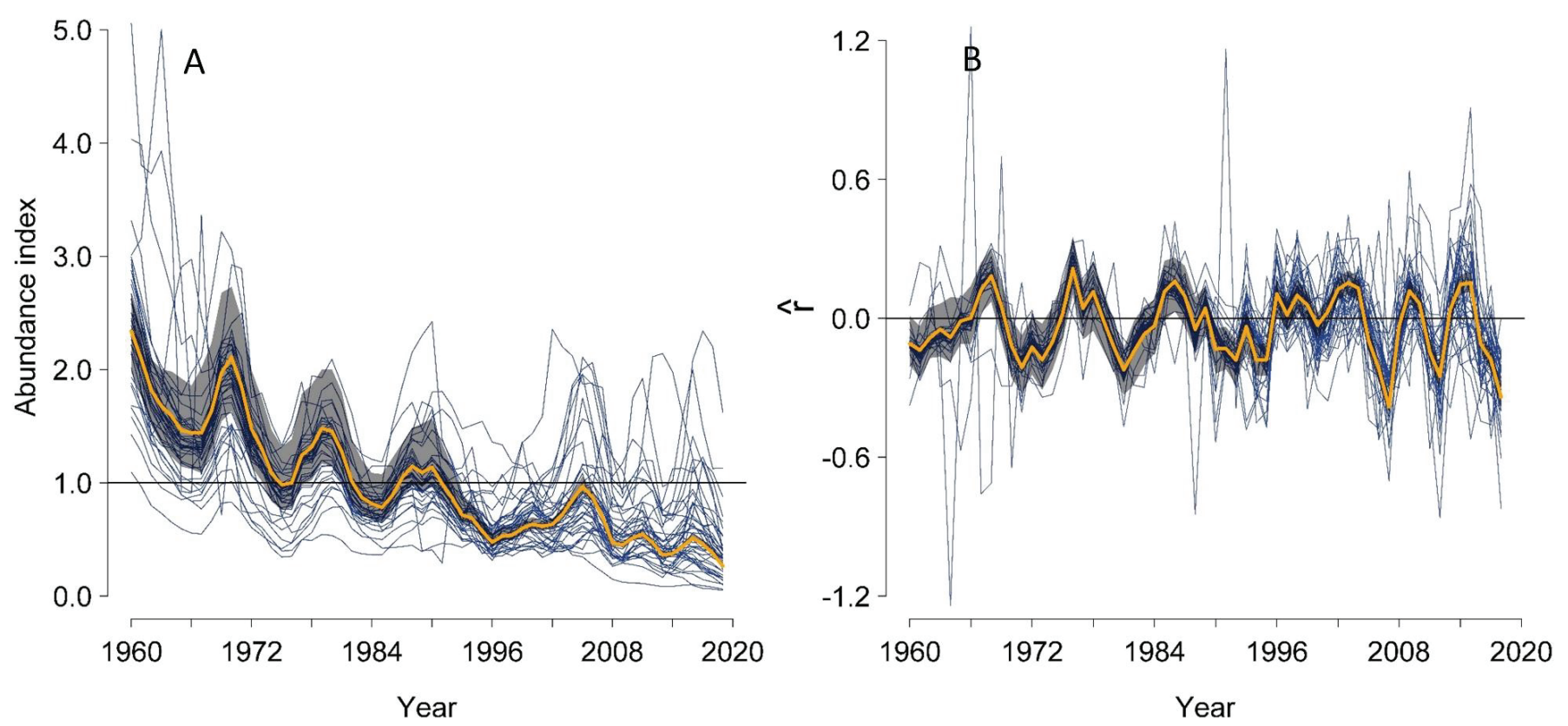

Figure 4.9. A, Abundance index (calculated as $\hat{N}$ divided by 60 -year mean of $\hat{N}$ ); and $B$, intrinsic rate of population change $(\hat{r})$ of greater sage-grouse (Centrocercus urophasianus) within the state of Oregon from 1960 to 2019. Thick yellow line represents median estimates across all leks. Shaded areas represent 95-percent credible limits. Thin blue lines represent median values for neighborhood clusters. Black horizontal line (abundance index $=1.0$ ) represents 60 -year average. 

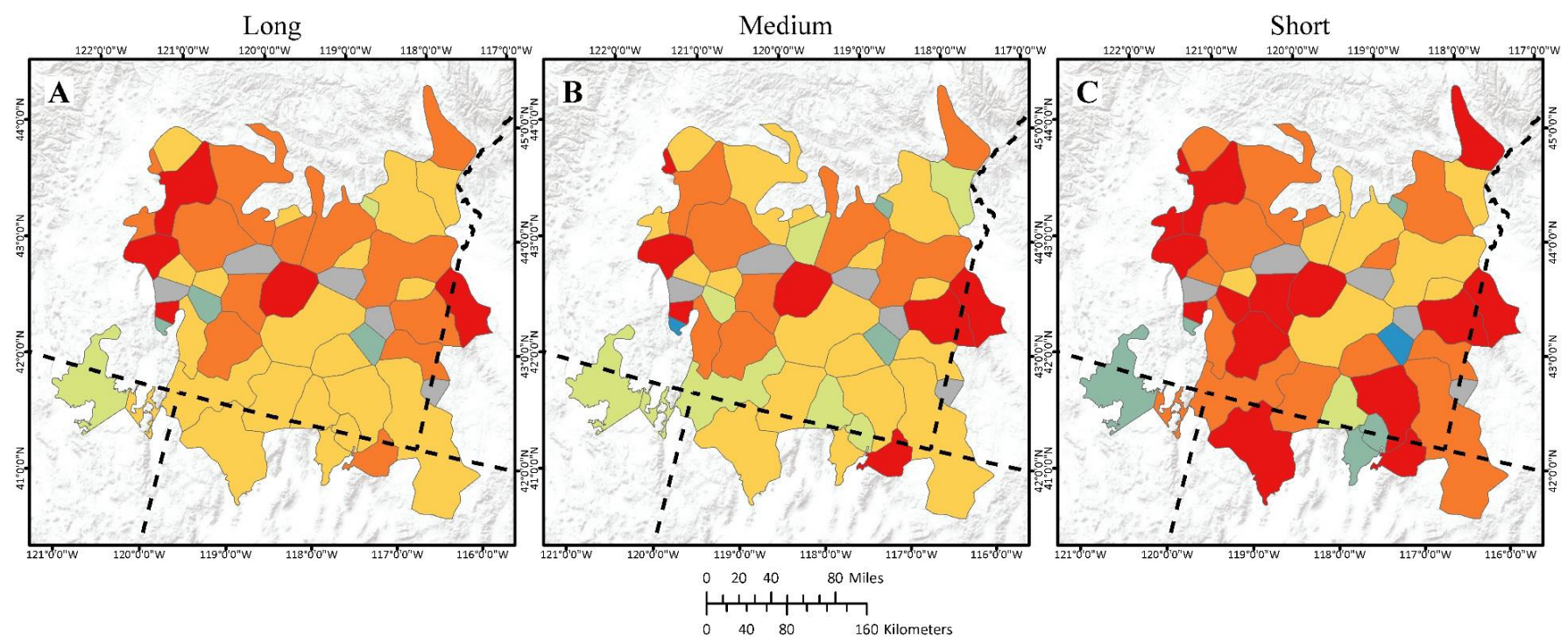

\section{EXPLANATION}

Neighborhood clusters (Level 2) intersecting Oregon: polygon colors represent average lambda values for sage-grouse populations over varying lengths of time (long, medium, short). Symbols are based on a range-wide distribution of values and all may not be represented here.

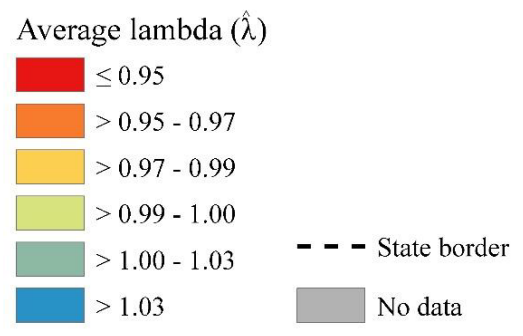

Coordinate System: Albers Projection: Albers Datum: WGS 1984 false easting: 0.0000 false northing: 0.0000 central meridian: -96.0000 standard parallel $1: 29.5000$ standard parallel 2: 45.5000 latitude of origin: 23.0000 Units: Meter

Service Layer Credits: Sources: Esri, USGS, NOAA

Figure 4.10. Spatial estimates of population trends across three temporal scales based on periods of oscillation (short, medium, and long) while accounting for periods of oscillation of greater sage-grouse (Centrocercus urophasianus) at different neighborhood clusters within the state of Oregon. Map images are the intellectual property of Esri and are used herein under license. Copyright (C) 2020 Esri and its licensors. All rights reserved. 

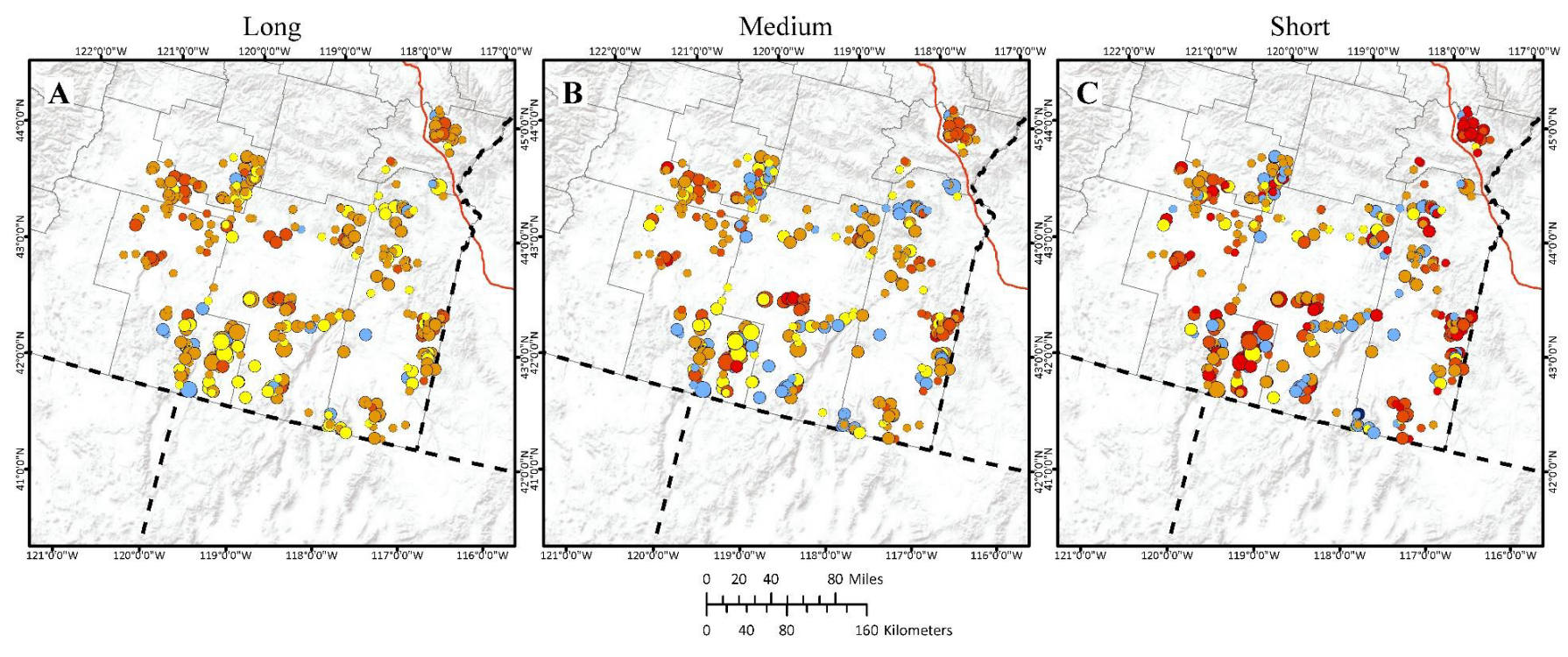

\section{EXPLANATION}

Leks within Oregon: colors represent average lambda $(\hat{\lambda})$ values of sage-grouse populations over various time scales (long, medium, short). The size of the symbol represents the maximum population size of the lek over the years of the analysis, relativized to the range-wide maximum population size across all leks. Symbols are based on a range-wide distribution of values and all may not be represented here.

$$
\begin{aligned}
\text { Average lambda }(\hat{\lambda}) \\
\quad \leq 0.90 \\
\quad>0.90-0.94 \\
\quad>0.94-0.98 \\
\quad>0.98-1.00 \\
\quad>1.00-1.10 \\
\quad>1.10
\end{aligned}
$$$$
\longrightarrow \text { Road }
$$

Coordinate System: Albers Projection: Albers Datum: WGS 1984 false easting: 0.0000 false northing: 0.0000 central meridian: -96.0000 standard parallel 1:29.5000 standard parallel $2: 45.5000$ latitude of origin: 23.0000 Units: Meter

Service Layer Credits: Sources: Esri, USGS, NOAA

Figure 4.11. Spatial estimates of population trends across three temporal scales based on periods of oscillation (short, medium, and long) while accounting for periods of oscillation of greater sage-grouse (Centrocercus urophasianus) at lek sites within the state of Oregon. Map images are the intellectual property of Esri and are used herein under license. Copyright $(2020$ Esri and its licensors. All rights reserved. 


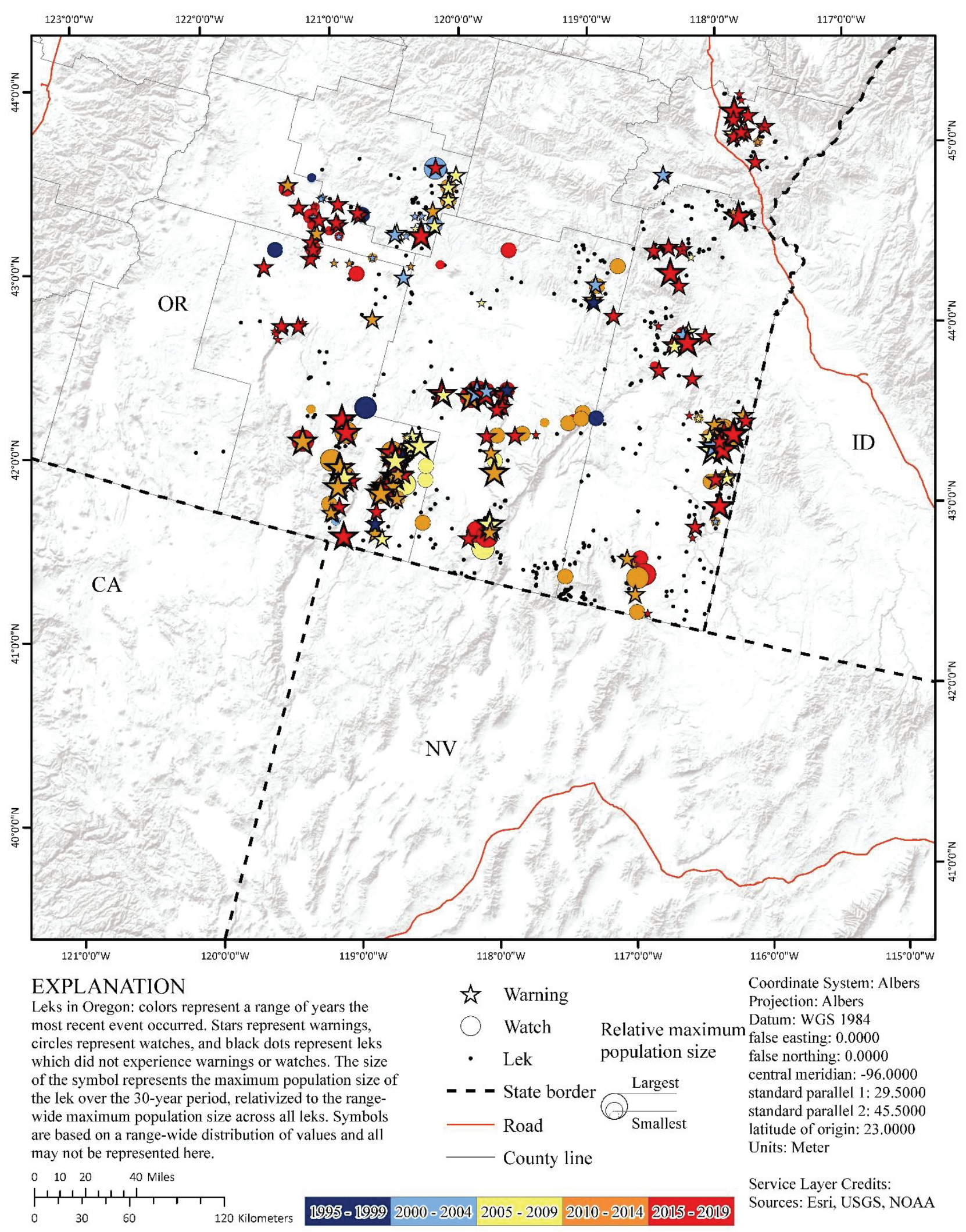

Figure 4.12. Spatial and temporal depiction of watches and warnings of greater sage-grouse (Centrocercus urophasianus) population declines at the lek scale within the state of Oregon from 1990 to 2019. Map image is the intellectual property of Esri and is used herein under license. Copyright $\left({ }^{2} 2020\right.$ Esri and its licensors. All rights reserved. 


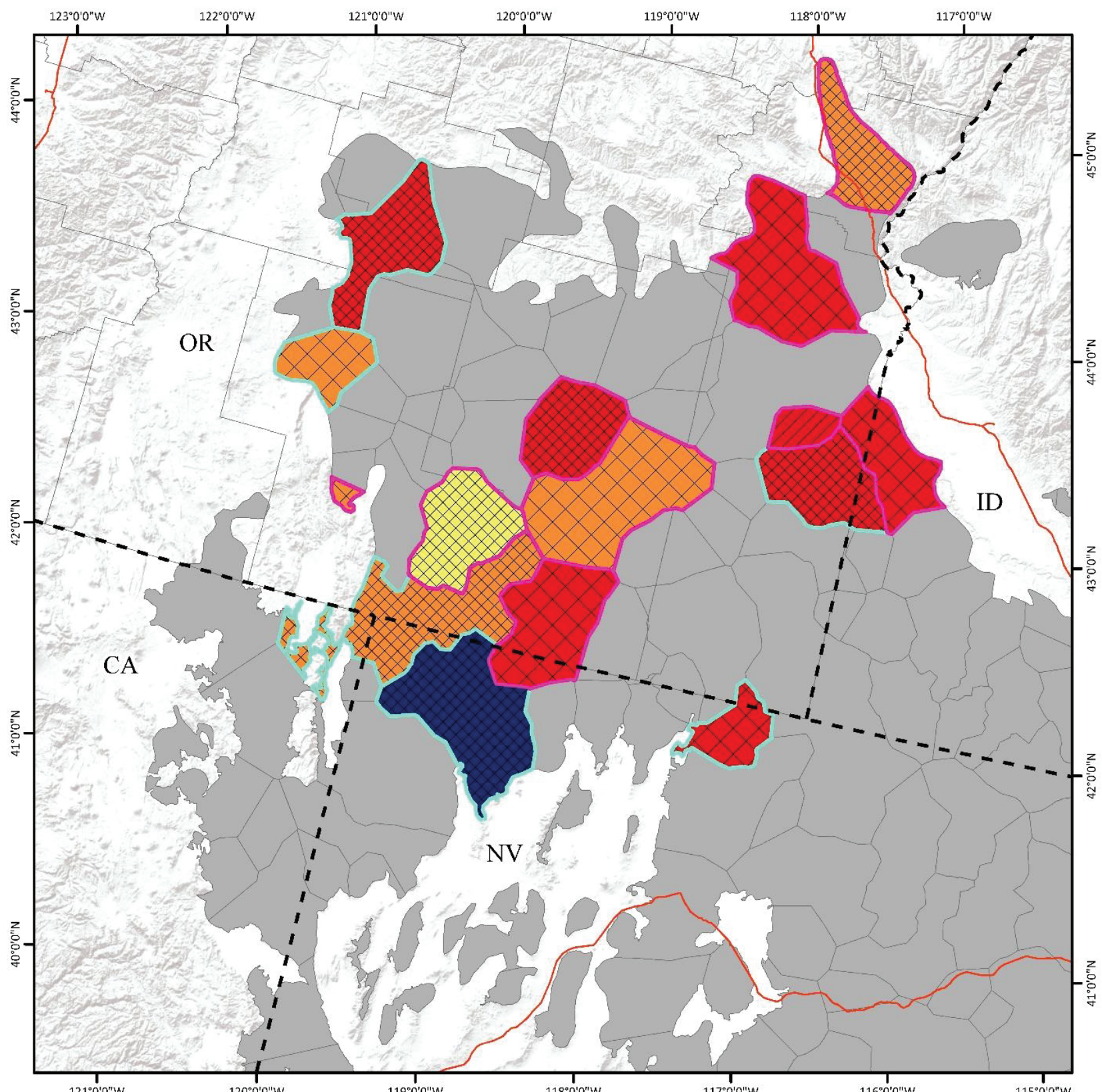

EXPLANATION

Neighborhood clusters (Level 2) in Oregon: polygon colors represent a range of years the most recent event occurred. Outline colors indicate whether the most recent event was a watch or warning. The hatching represents the maximum population size of the cluster over the 30 -year period, relativized to the range-wide maximum population size across all neighborhood clusters. Symbols are based on a range-wide distribution of values and all may not be represented here. $\begin{array}{llll}0 & 10 & 20 & 40 \\ & \text { Miles }\end{array}$ |, $0 \quad 20 \quad 40 \quad 80$ Kilometers 1995-1999 2000-2004 2005- 20092010 - 20142015 -2019
- - - State border

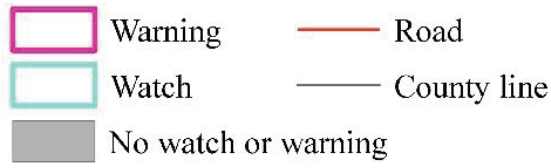

Smallest
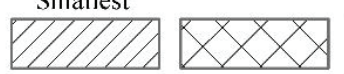

Largest
Coordinate System: Albers Projection: Albers Datum: WGS 1984 false easting: 0.0000 false northing: 0.0000 central meridian: -96.0000 standard parallel 1: 29.5000 standard parallel 2: 45.5000 latitude of origin: 23.0000 Units: Meter

Service Layer Credits: Sources: Esri, USGS, NOAA

Figure 4.13. Spatial and temporal depiction of watches and warnings of greater sage-grouse (Centrocercus urophasianus) population declines at neighborhood cluster within the state of Oregon from 1990 to 2019. Map image is the intellectual property of Esri and is used herein under license. Copyright (c) 2020 Esri and its licensors. All rights reserved. 


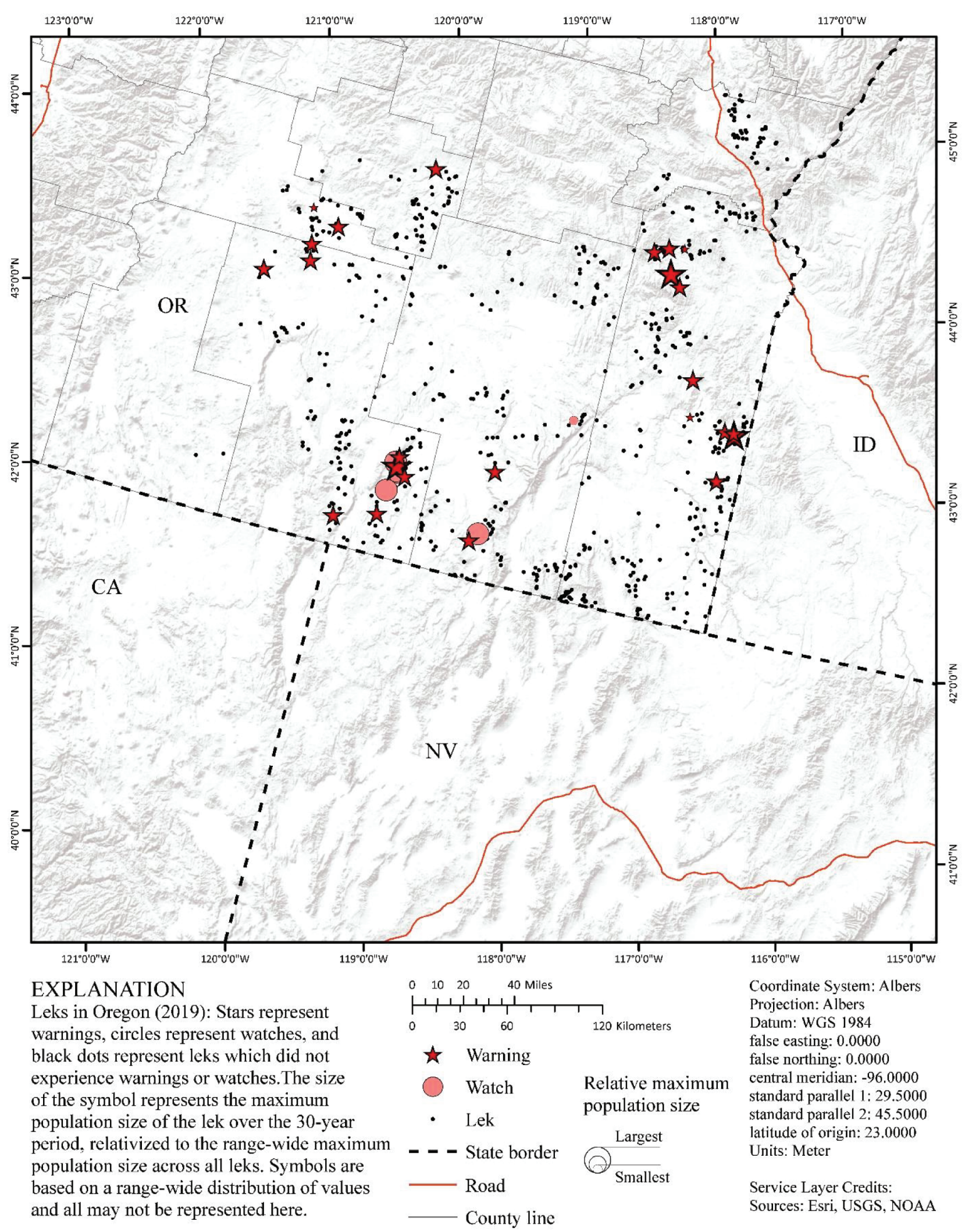

Figure 4.14. Spatial and temporal depiction of watches and warnings of greater sage-grouse (Centrocercus urophasianus) population declines at the lek scale within the state of Oregon during 2019. Map image is the intellectual property of Esri and is used herein under license. Copyright (c) 2020 Esri and its licensors. All rights reserved. 


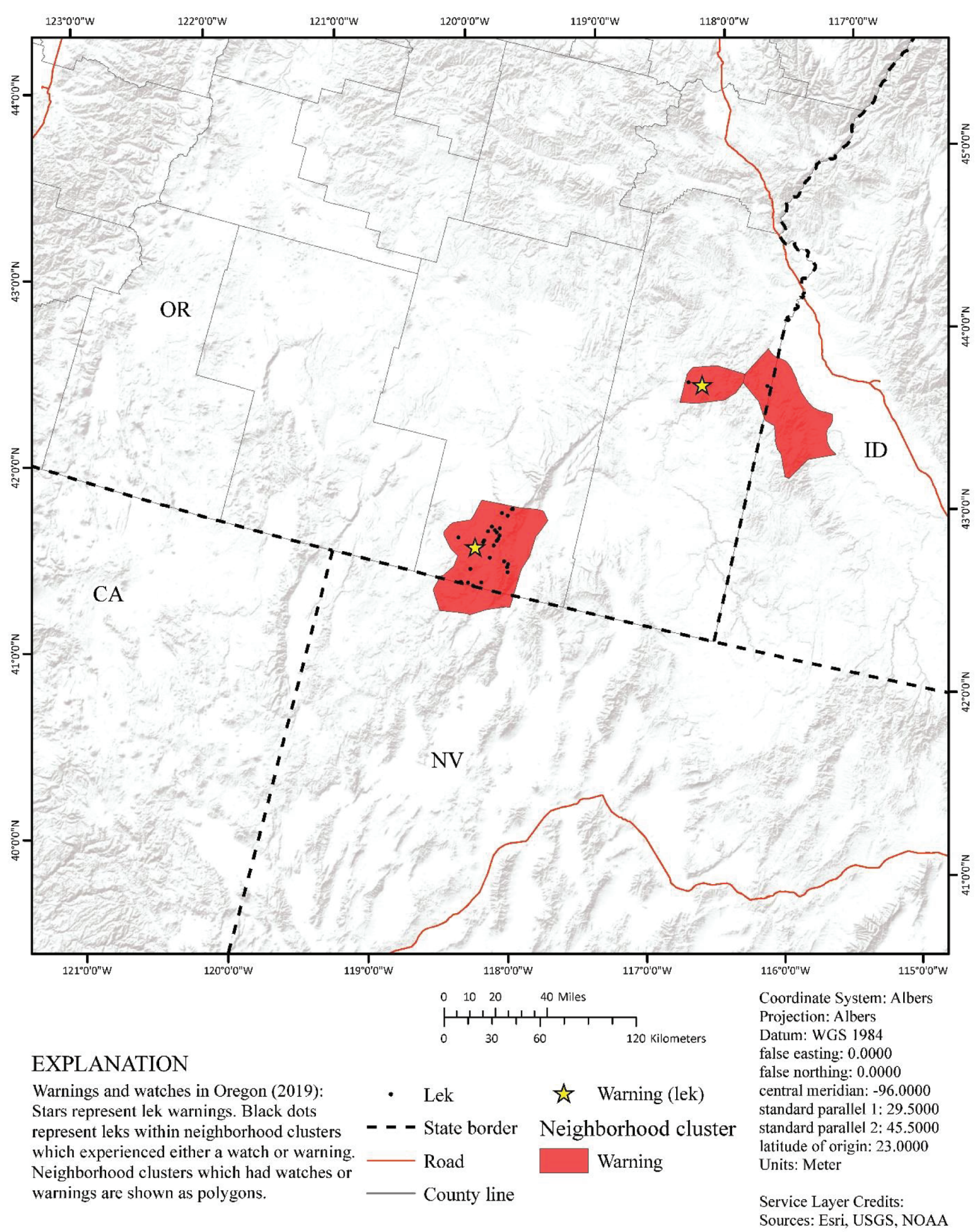

Figure 4.15. Spatial and temporal depiction of watches and warnings of greater sage-grouse (Centrocercus urophasianus) population declines at the neighborhood cluster scale within the state of Oregon during 2019. All leks within watch and warning boundaries were assigned as watch and warning, respectively. Yellow stars represent leks that reached warning independently. Map image is the intellectual property of Esri and is used herein under license. Copyright $@ 2020$ Esri and its licensors. All rights reserved. 


\section{Washington Results}

\section{Washington Lek Data and Clusters (Objectives 1 and 2)}

All of Washington's leks fell inside CC-B, which contained or intersected four different NCs (fig. 4.16). The total area of the four NCs within Washington was $1,139,955$ ha. Three of these clusters were used in the analysis. One NC was omitted because of limitations in sample sizes. These areas consisted of 108 leks and represented 1.28 percent of the range-wide lek database. After extensive QA/QC, we used 70 leks in the state-space model for population trend estimation (tables 4.5 and 4.6), totaling 1,141 individual lek count surveys. Mean male count was 7.51 (95-percent confidence interval $=7.29-7.73$ ) for leks within the state of Washington.

\section{Washington Population Trend Analysis (Objective 3)}

For CC-B, we estimated six population abundance nadirs (troughs) that dated back to 1960. Each of these population abundance nadirs represent between one and six complete periods of oscillation. We used these nadirs to estimate population trends across three different temporal scales that represented two, four, and six complete periods for the state (for instance, second, fourth, and sixth nadir). We estimated the average annual finite rate of population change $(\hat{\lambda})$ at the short (two periods), medium (four periods), and long (six periods) temporal scales as $0.966(95$-percent $\mathrm{CRI}=0.936-$ 0.998), 0.946 (95-percent $\mathrm{CRI}=0.907-0.966)$, and 0.956 (95-percent CRI=0.944-0.973), respectively (fig. 4.17). For all NCs that were modeled and intersected Washington, we estimated median $\hat{\lambda}$ to be less than 1.0 for all clusters across short, medium, and long temporal scales (table 4.6). We estimated median $\hat{\lambda}$ to be less than 1.0 for $84.3,97.1$, and 98.6 percent of all modeled leks within Washington across short, medium, and long temporal scales, respectively. We reported spatial and temporal variation in average annual $\hat{\lambda}$ across different NCs (fig. 4.18) and leks (fig. 4.19).

\section{Washington Targeted Annual Warning System Analysis (Objective 4)}

During 1990-2019, the TAWS for sage-grouse populations in Washington activated a total of eight and six leks as watches and warnings, respectively (fig. 4.20), which was 16.0 and 12.0 percent of the sampled leks used in the analysis. On average, across the 29 years, approximately 1.2 and 0.5 percent of leks per year experienced watches and warnings, respectively. The higher percentage for watches corresponds to repetitive activation. During 2019, the TAWS activated zero watches and zero warnings at leks and NCs. 

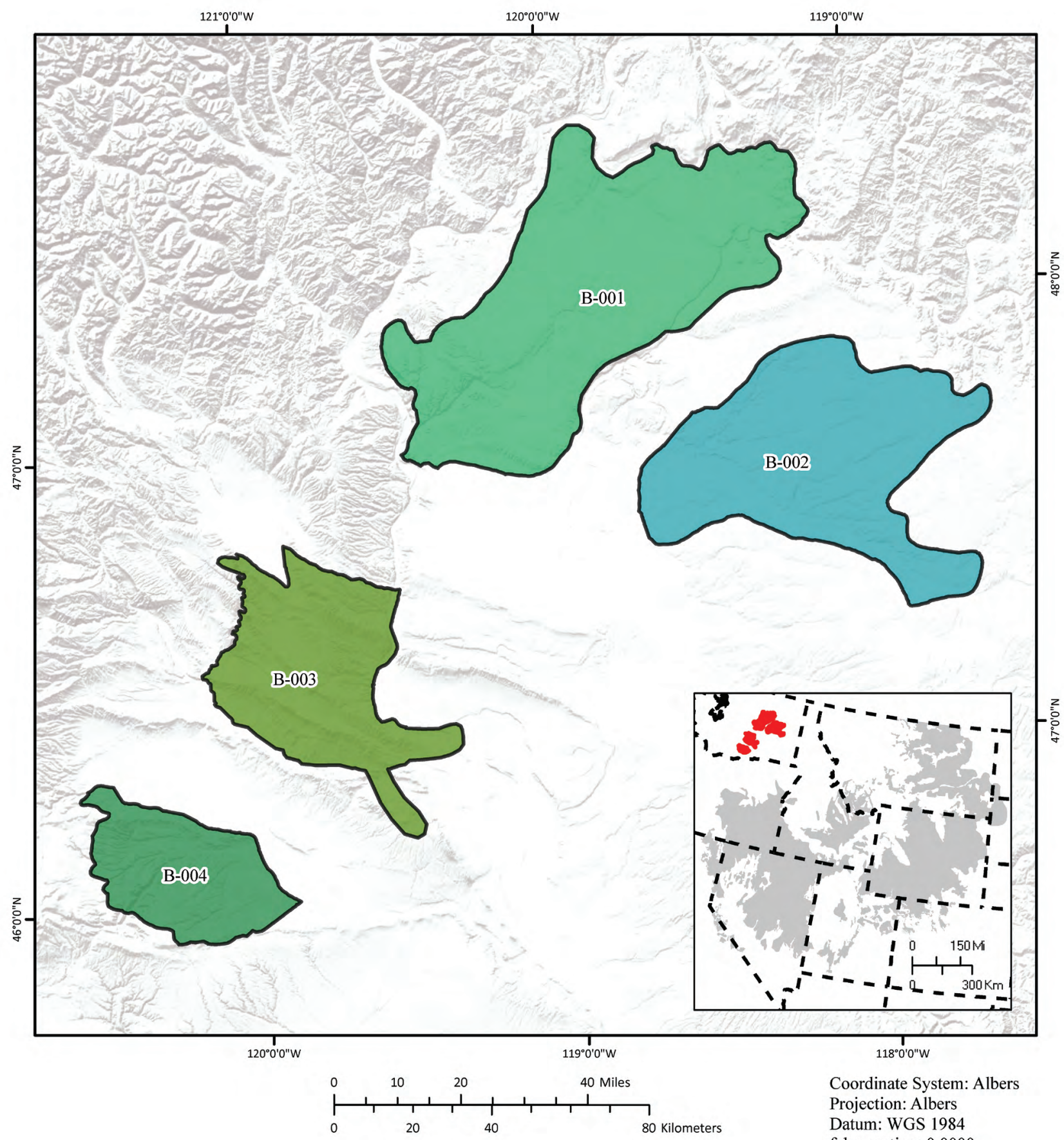

Coordinate System: Albers Projection: Albers Datum: WGS 1984 false easting: 0.0000 false northing: 0.0000

\section{EXPLANATION}

Neighborhood clusters (Level 2) intersecting Washington: polygon colors arbitrarily assigned and reflect 4 different population units. Grey polygons represent neighborhood clusters that do not intersect Washington. central meridian: -96.0000 standard parallel $1: 29.5000$

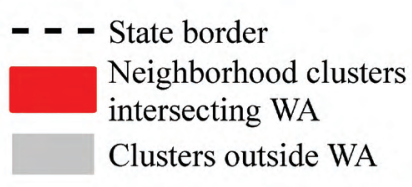

standard parallel 2: 45.5000

latitude of origin: 23.0000

Units: Meter

Service Layer Credits: Sources: Esri, USGS, NOAA

Figure 4.16. Greater sage-grouse (Centrocercus urophasianus) hierarchical population monitoring framework for neighborhood clusters that intersect the state of Washington. Map image is the intellectual property of Esri and is used herein under license. Copyright (c) 2020 Esri and its licensors. All rights reserved. 
Table 4.5. Table of greater sage-grouse (Centrocercus urophasianus) average annual rate of population change ( $\hat{\lambda})$ across six periods of oscillation in abundance that represent temporal scales for each climate cluster within the state of Washington. Estimates were derived from leks within the entire climate cluster.

[CC, climate cluster; B, Washington area]

\begin{tabular}{|c|c|c|c|c|c|c|c|c|c|}
\hline \multirow{2}{*}{ CC } & \multirow{2}{*}{$\begin{array}{l}\text { Percent } \\
\text { CC1 }\end{array}$} & \multicolumn{6}{|c|}{ Temporal scales ${ }^{2}$} & \multirow{2}{*}{$\begin{array}{l}\text { Number of } \\
\text { leks }^{3}\end{array}$} & \multirow{2}{*}{$\begin{array}{l}\text { Average } \\
\text { count/lek }\end{array}$} \\
\hline & & Long & Medium/Long & Medium & Short/Medium & Short & Recent & & \\
\hline B & 100.0 & $\begin{array}{c}0.956 \\
(0.944-0.973)\end{array}$ & $\begin{array}{c}0.949 \\
(0.928-0.968)\end{array}$ & $\begin{array}{c}0.946 \\
(0.907-0.966)\end{array}$ & $\begin{array}{c}0.956 \\
(0.909-0.981)\end{array}$ & $\begin{array}{c}0.966 \\
(0.936-0.998)\end{array}$ & $\begin{array}{c}0.957 \\
(0.932-1.000)\end{array}$ & $\begin{array}{c}108 \\
(70: 70,50: 50)\end{array}$ & $\begin{array}{c}14.0 \\
(13.2-14.9)\end{array}$ \\
\hline
\end{tabular}

\footnotetext{
${ }^{1}$ The percent of each climate cluster that intersects the state.

2 Temporal scales were estimated from present to each major population abundance nadir (trough) since 1960. Number of temporal scales were used to estimate population trends across six different temporal scales from approximately 10 to approximately 60 years.

${ }^{3}$ Number of leks in database. In parentheses from left to right is (1) total number in cluster were used in trend analysis, (2) number used in trend analysis within state boundary, (3) total number in cluster were used in the targeted annual warning system (TAWS) analysis, and (4) number used in TAWS analysis within state boundary.
}

Table 4.6. Table of greater sage-grouse (Centrocercus urophasianus) average annual rate of population change ( $\hat{\lambda})$ across six periods of oscillation in abundance that represent temporal scales for each neighborhood cluster within the state of Washington. Estimates were derived from leks within the entire neighborhood cluster.

[NC, neighborhood cluster; NA, not applicable]

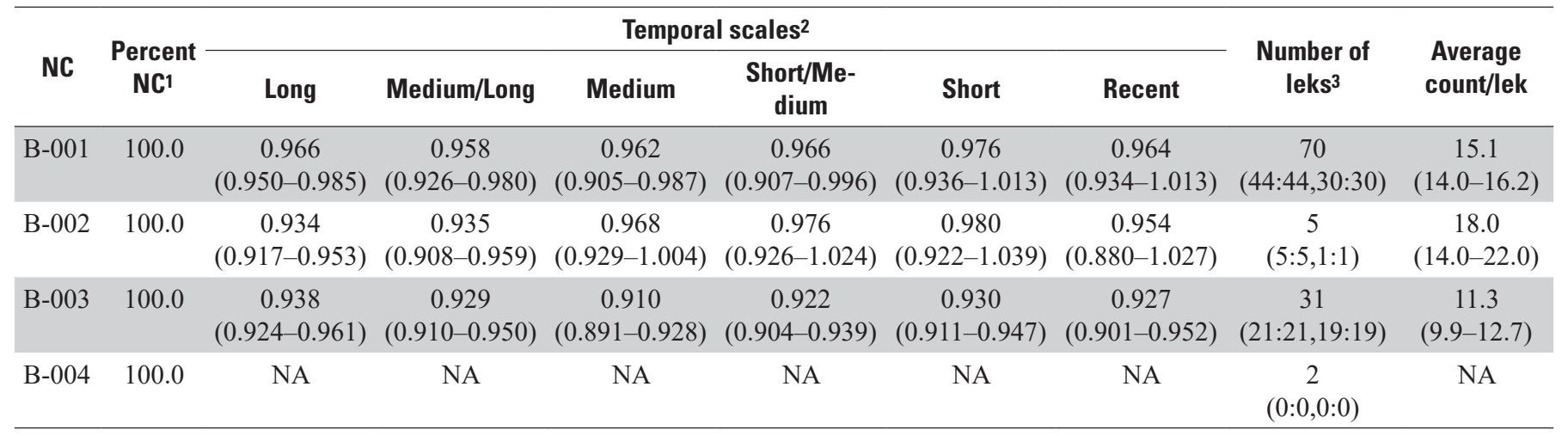

1The percent of each neighborhood cluster that intersects the state.

${ }^{2}$ Temporal scales were estimated from present to each major population abundance nadir (trough) since 1960. Number of temporal scales were used to estimate population trends across six different temporal scales from approximately 10 to approximately 60 years.

${ }^{3}$ Number of leks in database. In parentheses from left to right is (1) total number in cluster were used in trend analysis, (2) number used in trend analysis within state boundary, (3) total number in cluster were used in the targeted annual warning system (TAWS) analysis, and (4) number used in TAWS analysis within state boundary. 

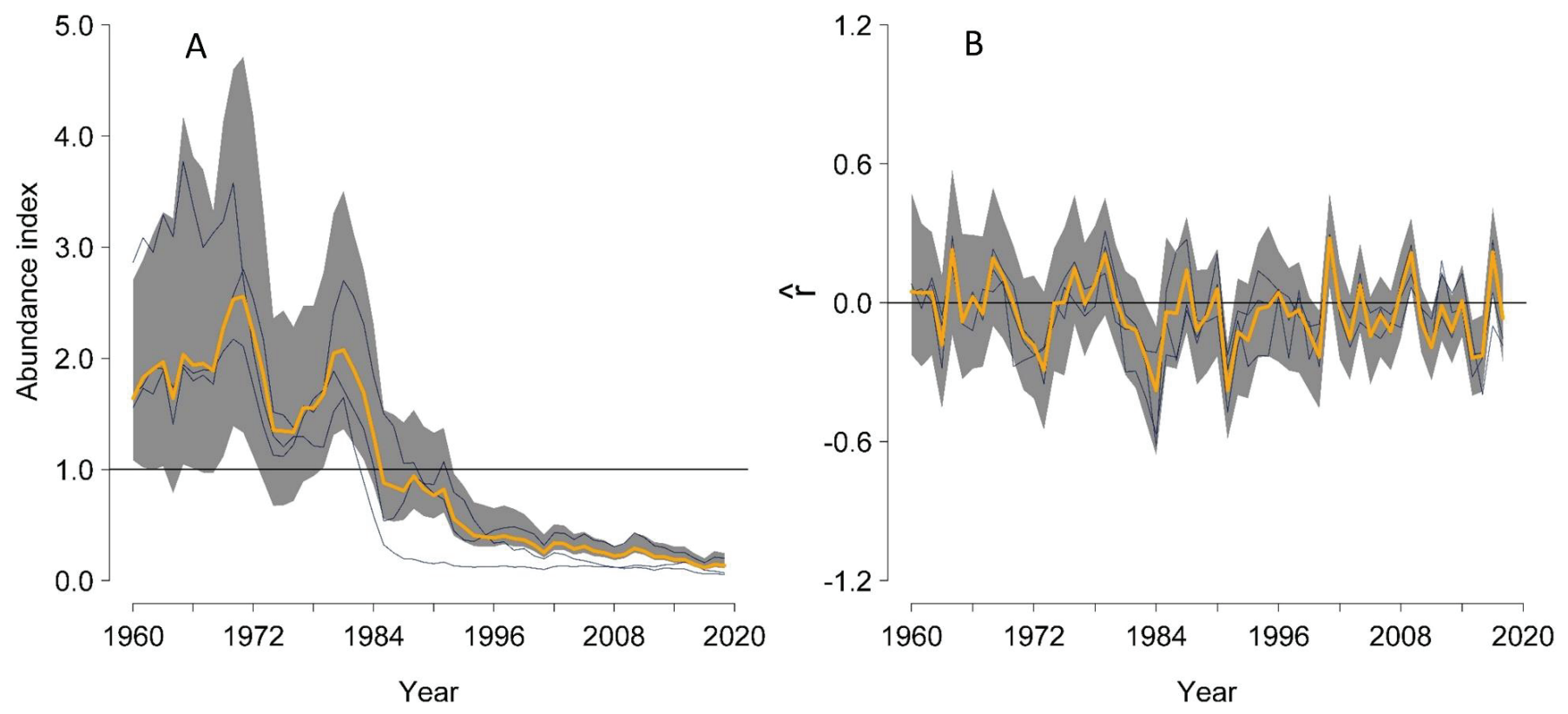

Figure 4.17. A, Estimated abundance index; and $B$, intrinsic rate of population change $(\hat{r})$ of greater sage-grouse (Centrocercus urophasianus) within the state of Washington from 1960 to 2019. Thick yellow line represents median estimates across all leks. Shaded areas represent 95 -percent credible limits. Thin blue lines represent median values for neighborhood clusters. Black horizontal line (abundance index $=1.0$ ) represents 60-year average. Vertical red arrows represented population abundance nadirs (troughs), and solid lines correspond to estimates of long, medium, and short (left to right) temporal scales. 


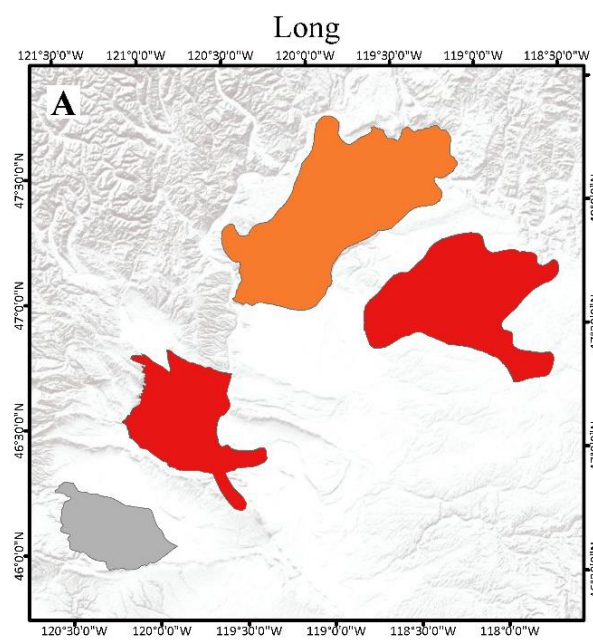

\section{EXPLANATION}

Neighborhood clusters (Level 2) intersecting Washington: polygon colors represent average lambda values for sage-grouse populations over varying lengths of time (long, medium, short). Symbols are based on a range-wide distribution of values and all may not be represented here.
Medium

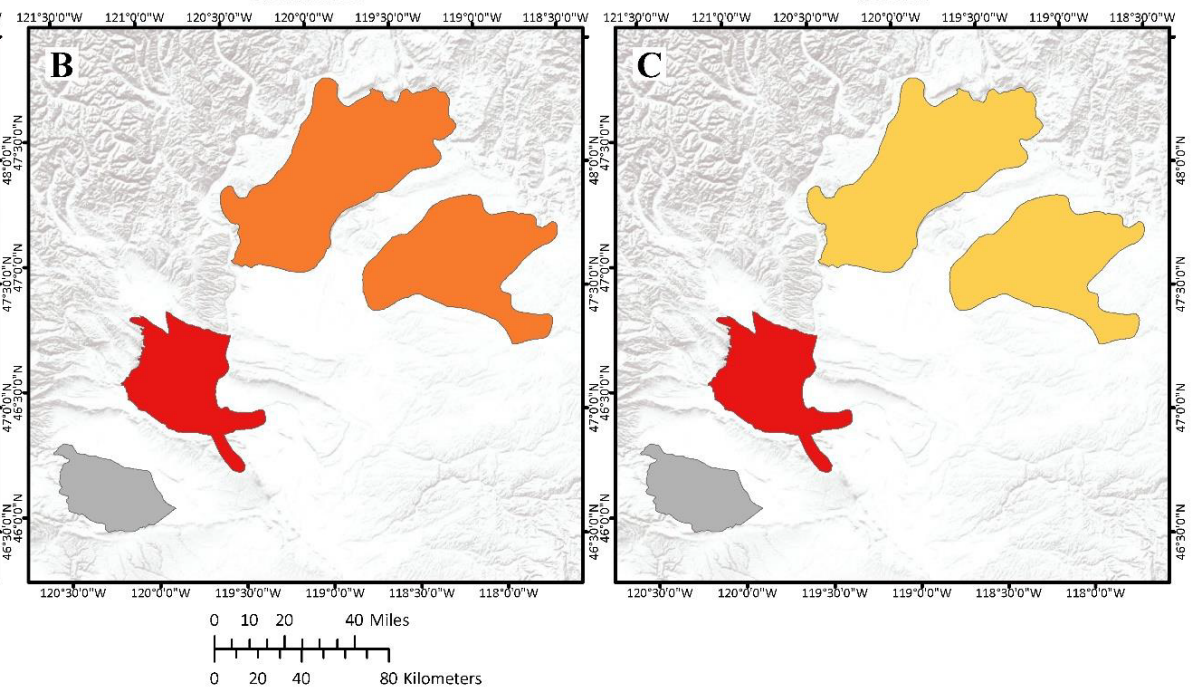

Average lambda $(\hat{\lambda})$

$\begin{aligned} & \leq 0.95 \\ & >0.95-0.97 \\ \square & >0.97-0.99 \\ \square & >0.99-1.00 \\ & >1.00-1.03 \\ & >1.03\end{aligned}$

Coordinate System: Albers Projection: Albers

Datum: WGS 1984

false easting: 0.0000

false northing: 0.0000

central meridian: -96.0000

standard parallel 1:29.5000

standard parallel 2: 45.5000

Units: Meter latitude of origin: 23.0000

Figure 4.18. Spatial estimates of population trends across three temporal scales based on periods of oscillation (short, medium, and long) while accounting for periods of oscillation of greater sage-grouse (Centrocercus urophasianus) at different neighborhood clusters within the state of Washington. Map images are the intellectual property of Esri and are used herein under license. Copyright (c) 2020 Esri and its licensors. All rights reserved. 
Long

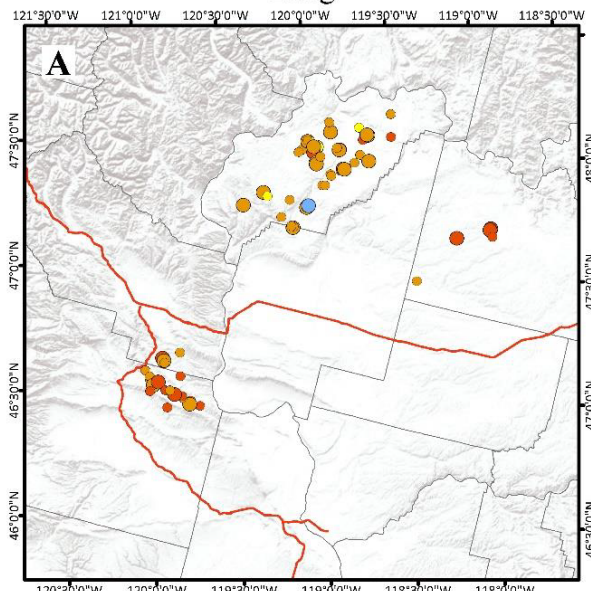

EXPLANATION

Leks within Washington: colors represent average lambda $(\hat{\lambda})$ values of sage-grouse populations over various time scales (long, medium, short). The size of the symbol represents the maximum population size of the lek over the years of the analysis, relativized to the range-wide maximum population size across all leks. Symbols are based on a rangewide distribution of values and all may not be represented here.
Medium

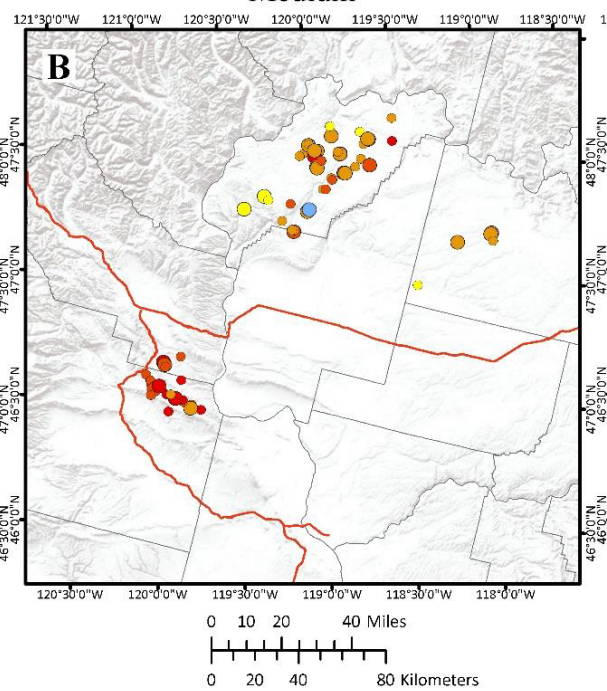

Average lambda $(\hat{\lambda})$

- $\leq 0.90$

- $>0.90-0.94$

- $>0.94-0.98$

$>0.98-1.00$

$>1.00-1.10$

- $>1.10$
Short

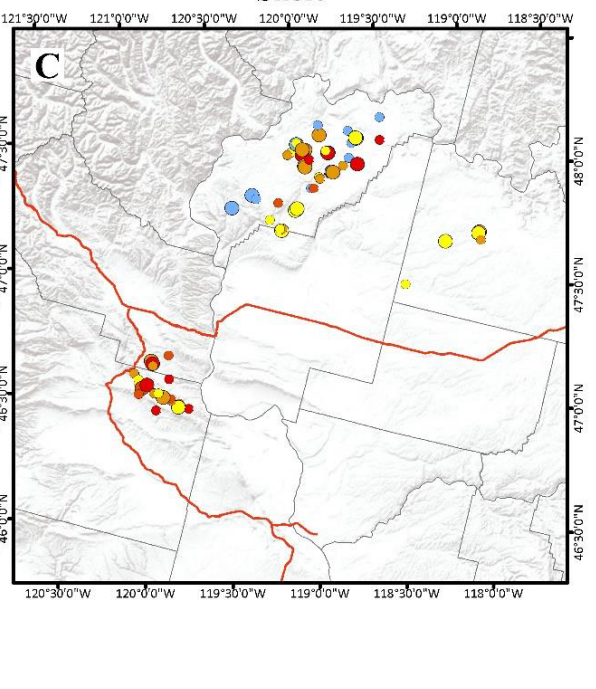

Figure 4.19. Spatial estimates of population trends across three temporal scales based on periods of oscillation (short, medium, and long) while accounting for periods of oscillation of greater sage-grouse (Centrocercus urophasianus) at lek sites within the state of Washington. Map images are the intellectual property of Esri and are used herein under license. Copyright (C) 2020 Esri and its licensors. All rights reserved. 


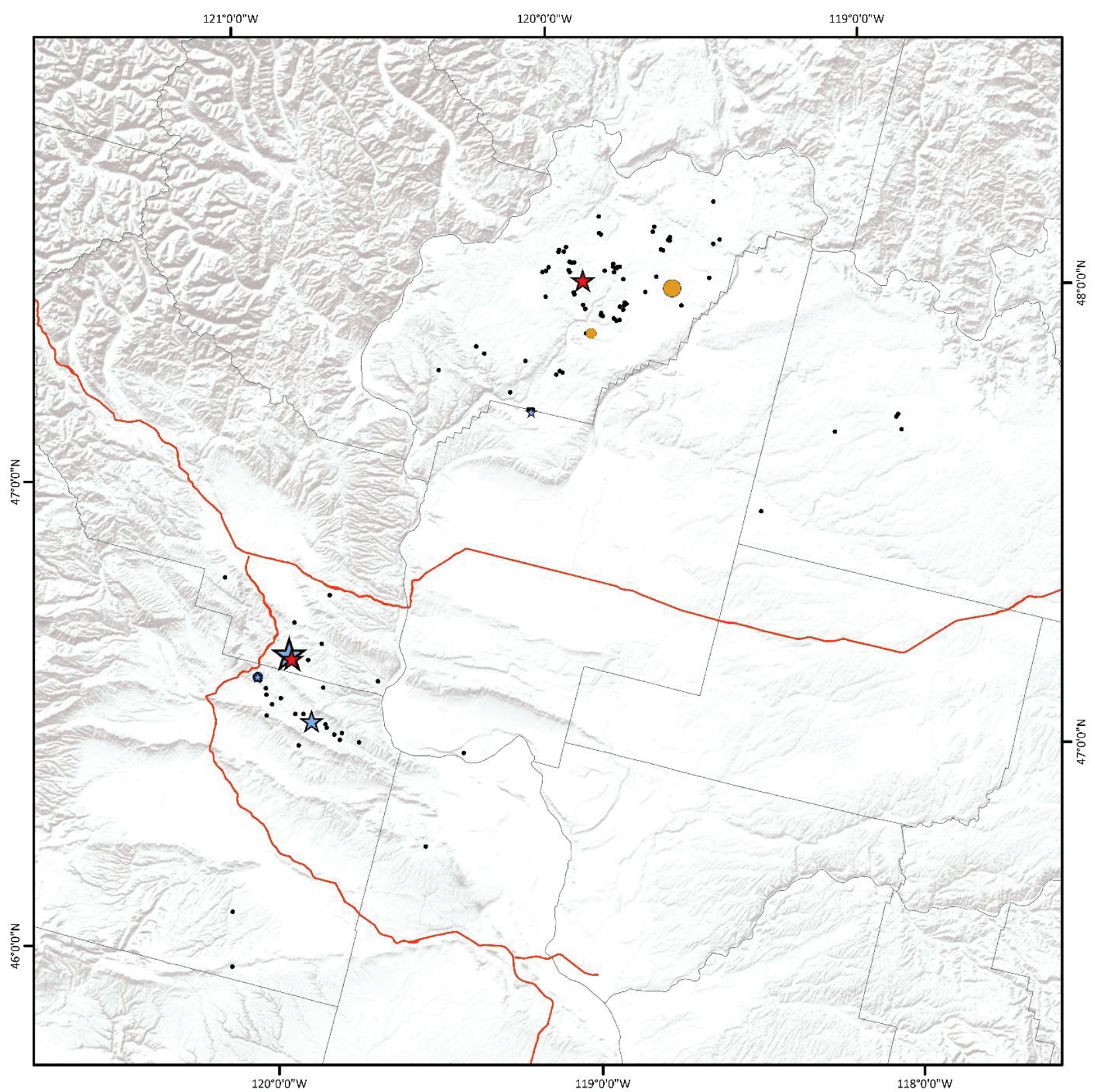

\section{EXPLANATION}

Leks in Washington: colors represent a range of years the most recent event occurred. Stars represent warnings, circles represent watches, and black dots represent leks which did not experience warnings or watches. The size of the symbol represents the maximum population size of the lek over the 30 -year period, relativized to the rangewide maximum population size across all leks. Symbols are based on a range-wide distribution of values and all may not be represented here.

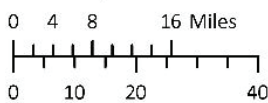

$\begin{array}{llll}0 & 10 \quad 20 \quad 40 \text { Kilometers }\end{array}$

\section{放 Warning}

$\bigcirc$ Watch

- Lek

Road
County line

Coordinate System: Albers Projection: Albers

Relative maximum $\begin{aligned} & \text { Datum: WGS } 1984 \\ & \text { false easting: } 0.0000\end{aligned}$ population size

false northing: 0.0000 central meridian: -96.0000 standard parallel 1: 29.5000 standard parallel 2: 45.5000 latitude of origin: 23.0000 Units: Meter

Service Layer Credits: 1993-1999 2000-2004 2005-2009 2010-2014 2015-2019 Sources: Esri, USGS, NOAA

Figure 4.20. Spatial and temporal depiction of watches and warnings of greater sage-grouse (Centrocercus urophasianus) population declines at the lek scale within the state of Washington from 1990-2019. Map image is the intellectual property of Esri and is used herein under license. Copyright $(2020$ Esri and its licensors. All rights reserved. 


\section{Nevada Results}

\section{Nevada Lek Data and Clusters (Objectives 1 and 2)}

Nevada was within CC-A and CC-E and contained or intersected 124 different NCs (fig. 4.21). The total area of the 124 NCs within Nevada was 15,938,591 ha. There were 110 clusters used in the analysis after, but 14 were omitted because of limitations in sample sizes. These areas consisted of 1,938 leks, 1,369 of which were located within the Nevada state boundary and represented 16.26 percent of the rangewide lek database. After extensive QA/QC, we used 618 leks from Nevada in the SSM for population trend estimation (tables 4.7 and 4.8), totaling 9,435 individual lek count surveys. Mean male count was 13.79 (95-percent confidence interval=13.59-13.99) for leks within NCs that were within or overlapped Nevada. Mean male count for leks within Nevada was 12.79 (95-percent confidence interval=12.55-13.02).

\section{Nevada Population Trend Analysis (Objective 3)}

The state of Nevada intersected CC-A and CC-E. Most of the sage-grouse range within Nevada ( 98.3 percent) fell within CC-E. For CC-E, we estimated six population abundance nadirs (troughs) that dated back to 1960. Each of these population abundance nadirs represent between one and six complete periods. We used these nadirs to estimate population trends across three different temporal scales that represented two, four, and six complete periods for the state (for instance, second, fourth, and sixth nadir). We estimated the average annual finite rate of population change $(\hat{\lambda})$ at the short (two periods), medium (four periods), and long (six periods) temporal scales as 0.983 (95-percent $\mathrm{CRI}=0.978-0.989)$, 0.981 (95-percent CRI $=0.973-0.986)$, and 0.977 (95-percent $\mathrm{CRI}=0.974-0.982$ ), respectively (fig. 4.22). For all NCs that were included in model and intersected Nevada, we estimated median $\hat{\lambda}$ to be less than 1.0 for $71.8,82.7$, and 93.6 percent across short, medium, and long temporal scales, respectively (fig. 4.23; table 4.8). We estimated median $\hat{\lambda}$ to be less than 1.0 for $68.8,74.6$, and 80.1 percent of all modeled leks within Nevada across short, medium, and long temporal scales, respectively (fig. 4.25). We reported spatial and temporal variation in average annual $\hat{\lambda}$ across different NCs (fig. 4.23) and leks (fig. 4.24).

\section{Nevada Targeted Annual Warning System Analysis (Objective 4)}

During 1990-2019, the TAWS for sage-grouse populations in Nevada activated a total of 290 and 179 leks as watches and warnings, respectively (fig. 4.25), which was 55.2 and 34.1 percent of the sampled leks used in the analysis. On average, across the 29 years, approximately 4.9 and 1.4 percent of leks per year experienced watches and warnings, respectively. The higher percentage for watches corresponds to repetitive activation. During the 29 years, the TAWS also activated a total of 33 and 22 NCs as watches and warnings, respectively (fig. 4.26), which was 34.4 and 22.9 percent of the sampled clusters used in the analysis. On average, across the 29 years, approximately 2.1 and 0.9 percent of NCs per year experienced watches and warnings, respectively. The higher percentage for watches corresponds to repetitive activation. During 2019, the TAWS activated 20 (first $=4$ ) watches and 17 (first=1) warnings at leks (fig. 4.27), as well as 0 (first=0) watches and 2 (first=0) warnings at NCs, respectively (fig. 4.28). 

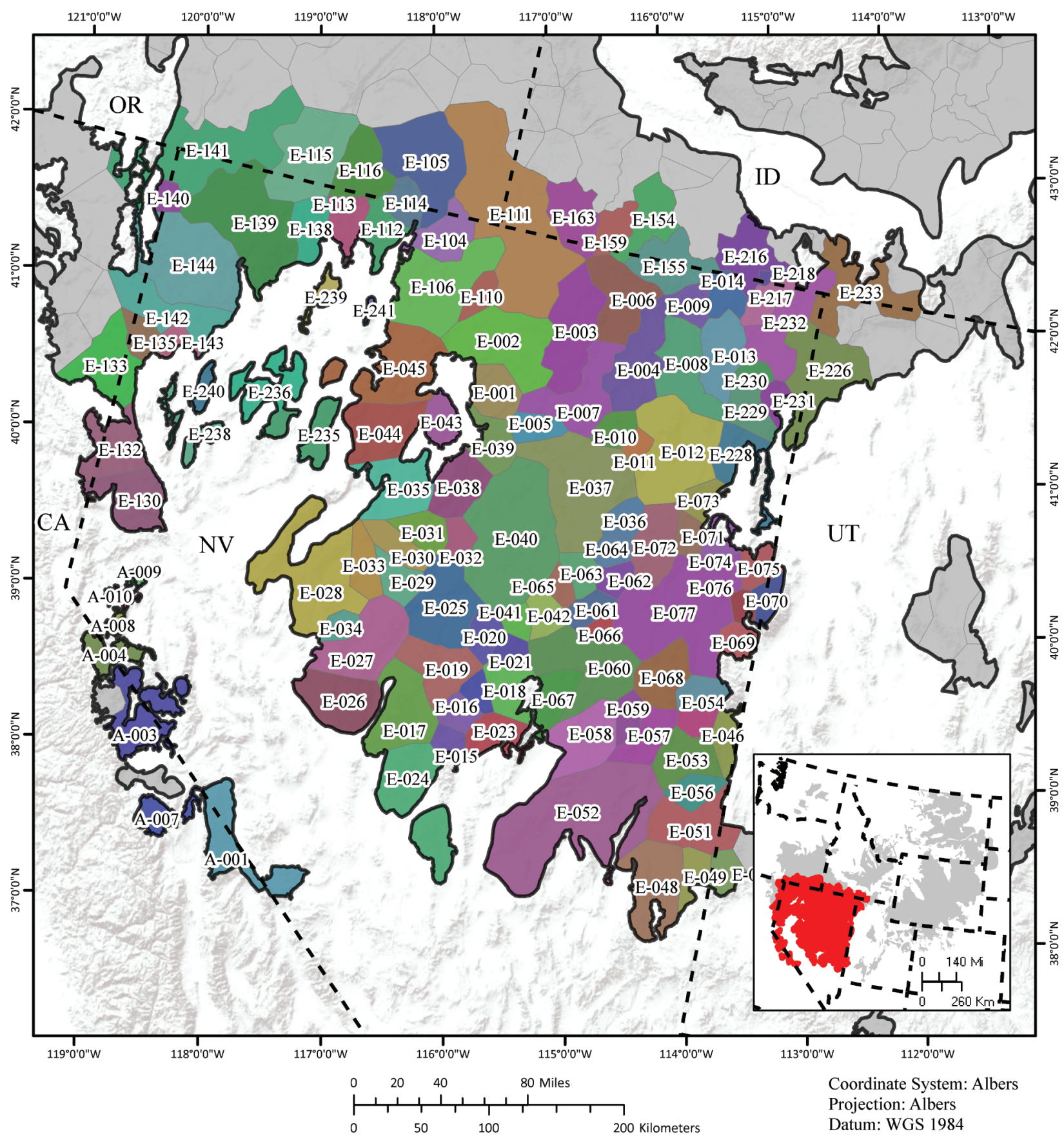

\section{EXPLANATION}

Neighborhood clusters (Level 2) intersecting Nevada: polygon colors arbitrarily assigned and reflect 124 different population units. Grey polygons represent neighborhood clusters that do not intersect Nevada.

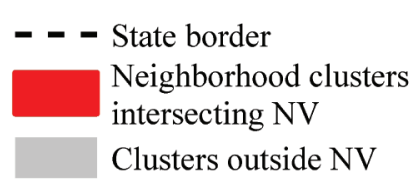

Coordinate System: Albers Projection: Albers Datum: WGS 1984 false easting: 0.0000 false northing: 0.0000 central meridian: -96.0000 standard parallel 1: 29.5000 standard parallel 2: 45.5000 latitude of origin: 23.0000 Units: Meter

Service Layer Credits: Sources: Esri, USGS, NOAA

Figure 4.21. Greater sage-grouse (Centrocercus urophasianus) hierarchical population monitoring framework for neighborhood clusters that intersect the state of Nevada. Map image is the intellectual property of Esri and is used herein under license. Copyright (C) 2020 Esri and its licensors. All rights reserved. 
Table 4.7. Table of greater sage-grouse (Centrocercus urophasianus) average annual rate of population change $(\hat{\lambda})$ across six periods of oscillation in abundance that represent temporal scales for each climate cluster within the state of Nevada. Estimates were derived from leks within the entire climate cluster.

[CC, climate cluster; A, Bi-state area; E, Great Basin area]

\begin{tabular}{|c|c|c|c|c|c|c|c|c|c|}
\hline \multirow[b]{2}{*}{ CC } & \multirow[b]{2}{*}{$\begin{array}{l}\text { Percent } \\
\text { CC1 }\end{array}$} & \multicolumn{6}{|c|}{ Temporal scales ${ }^{2}$} & \multirow[b]{2}{*}{ Number of leks 3} & \multirow[b]{2}{*}{$\begin{array}{l}\text { Average } \\
\text { count/lek }\end{array}$} \\
\hline & & Long & Medium/Long & Medium & $\begin{array}{c}\text { Short/ } \\
\text { Medium }\end{array}$ & Short & Recent & & \\
\hline E & 45.2 & $\begin{array}{c}0.971 \\
(0.967-0.976)\end{array}$ & $\begin{array}{c}0.973 \\
(0.967-0.978)\end{array}$ & $\begin{array}{c}0.974 \\
(0.963-0.979)\end{array}$ & $\begin{array}{c}0.986 \\
(0.981-0.990)\end{array}$ & $\begin{array}{c}0.968 \\
(0.964-0.971)\end{array}$ & $\begin{array}{c}0.949 \\
(0.944-0.955)\end{array}$ & $\begin{array}{c}4,012 \\
(2,187: 604 \\
1,908: 514)\end{array}$ & $\begin{array}{c}17.3 \\
(17.1-17.5)\end{array}$ \\
\hline
\end{tabular}

\footnotetext{
${ }^{1}$ The percent of each climate cluster that intersects the state.

2 Temporal scales were estimated from present to each major population abundance nadir (trough) since 1960. Number of temporal scales were used to estimate population trends across six different temporal scales from approximately 10 to approximately 60 years.

${ }^{3}$ Number of leks in database. In parentheses from left to right is (1) total number in cluster were used in trend analysis, (2) number used in trend analysis within state boundary, (3) total number in cluster were used in the targeted annual warning system (TAWS) analysis, and (4) number used in TAWS analysis within state boundary.
}

Table 4.8. Table of greater sage-grouse (Centrocercus urophasianus) average annual rate of population change $(\hat{\lambda})$ across six periods of oscillation in abundance that represent temporal scales for each neighborhood cluster within the state of Nevada. Estimates were derived from leks within the entire neighborhood cluster.

[NC, neighborhood cluster; NA, not applicable]

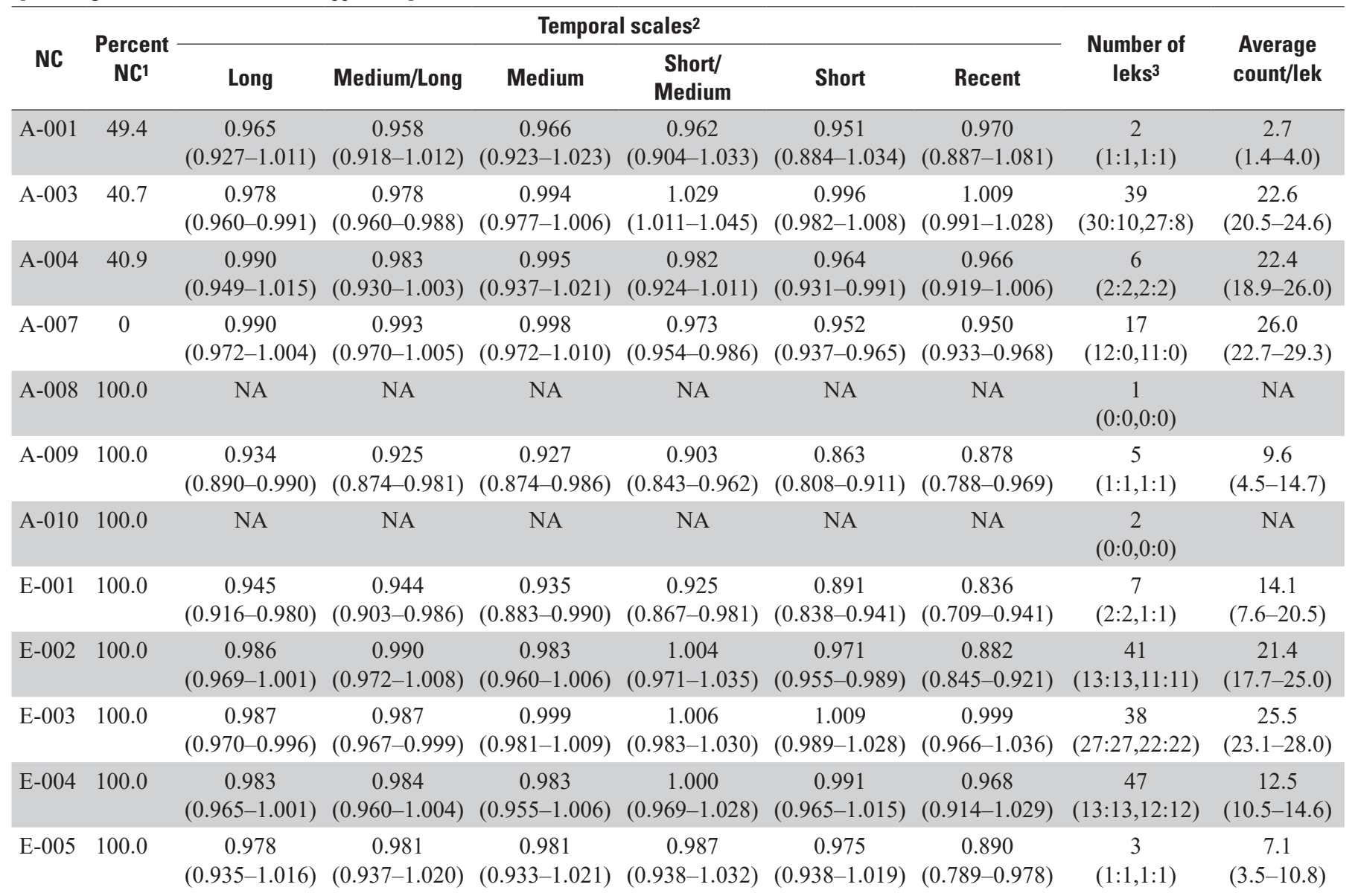


Table 4.8. Table of greater sage-grouse (Centrocercus urophasianus) average annual rate of population change $(\hat{\lambda})$ across six periods of oscillation in abundance that represent temporal scales for each neighborhood cluster within the state of Nevada. Estimates were derived from leks within the entire neighborhood cluster.-Continued

[NC, neighborhood cluster; NA, not applicable]

\begin{tabular}{|c|c|c|c|c|c|c|c|c|c|}
\hline \multirow[b]{2}{*}{ NC } & \multirow{2}{*}{$\begin{array}{l}\text { Percent } \\
\text { NC1 }\end{array}$} & \multicolumn{6}{|c|}{ Temporal scales² } & \multirow{2}{*}{$\begin{array}{l}\text { Number of } \\
\text { leks }^{3}\end{array}$} & \multirow{2}{*}{$\begin{array}{l}\text { Average } \\
\text { count/lek }\end{array}$} \\
\hline & & Long & Medium/Long & Medium & $\begin{array}{c}\text { Short/ } \\
\text { Medium }\end{array}$ & Short & Recent & & \\
\hline E-007 & 100.0 & $\begin{array}{c}0.974 \\
(0.958-0.992)\end{array}$ & $\begin{array}{c}0.974 \\
(0.959-0.989)\end{array}$ & $\begin{array}{c}0.972 \\
(0.958-0.985)\end{array}$ & $\begin{array}{c}1.002 \\
(0.976-1.022)\end{array}$ & $\begin{array}{c}0.996 \\
(0.980-1.013)\end{array}$ & $\begin{array}{c}0.970 \\
(0.939-1.003)\end{array}$ & $\begin{array}{c}50 \\
(31: 31,20: 20)\end{array}$ & $\begin{array}{c}16.0 \\
(14.5-17.5)\end{array}$ \\
\hline E-009 & 100.0 & $\begin{array}{c}0.973 \\
(0.959-0.983)\end{array}$ & $\begin{array}{c}0.972 \\
(0.955-0.988)\end{array}$ & $\begin{array}{c}0.962 \\
(0.947-0.975)\end{array}$ & $\begin{array}{c}0.990 \\
(0.964-1.017)\end{array}$ & $\begin{array}{c}0.993 \\
(0.973-1.015)\end{array}$ & $\begin{array}{c}0.852 \\
(0.800-0.904)\end{array}$ & $\begin{array}{c}28 \\
(12: 12,8: 8)\end{array}$ & $\begin{array}{c}21.2 \\
(18.4-24.0)\end{array}$ \\
\hline E-010 & 100.0 & $\begin{array}{c}0.943 \\
(0.911-0.974)\end{array}$ & $\begin{array}{c}1.007 \\
(0.964-1.051)\end{array}$ & $\begin{array}{c}1.021 \\
(0.946-1.095)\end{array}$ & $\begin{array}{c}0.991 \\
(0.874-1.132)\end{array}$ & $\begin{array}{c}0.937 \\
(0.802-1.084)\end{array}$ & $\begin{array}{c}0.881 \\
(0.641-1.199)\end{array}$ & $\begin{array}{c}1 \\
(1: 1,1: 1)\end{array}$ & $\begin{array}{c}29.8 \\
(18.7-41.0)\end{array}$ \\
\hline E-013 & 100.0 & $\begin{array}{c}0.985 \\
(0.964-0.998)\end{array}$ & $\begin{array}{c}0.996 \\
(0.957-1.011)\end{array}$ & $\begin{array}{c}0.976 \\
(0.926-0.996)\end{array}$ & $\begin{array}{c}1.003 \\
(0.967-1.034)\end{array}$ & $\begin{array}{c}1.002 \\
(0.983-1.021)\end{array}$ & $\begin{array}{c}0.976 \\
(0.926-1.028)\end{array}$ & $\begin{array}{c}36 \\
(16: 16,13: 13)\end{array}$ & $\begin{array}{c}19.9 \\
(16.6-23.2)\end{array}$ \\
\hline E-014 & 97.1 & $\begin{array}{c}1.005 \\
(0.955-1.054)\end{array}$ & $\begin{array}{c}1.014 \\
(0.958-1.070)\end{array}$ & $\begin{array}{c}1.022 \\
(0.970-1.083)\end{array}$ & $\begin{array}{c}1.017 \\
(0.982-1.050)\end{array}$ & $\begin{array}{c}1.108 \\
(1.042-1.193)\end{array}$ & $\begin{array}{c}1.080 \\
(0.942-1.242)\end{array}$ & $\begin{array}{c}28 \\
(1: 1,1: 1)\end{array}$ & $\begin{array}{c}6.6 \\
(2.2-11.0)\end{array}$ \\
\hline E-015 & 100.0 & $\begin{array}{c}0.967 \\
(0.930-1.007)\end{array}$ & $\begin{array}{c}0.968 \\
(0.923-1.013)\end{array}$ & $\begin{array}{c}0.965 \\
(0.907-1.015)\end{array}$ & $\begin{array}{c}0.966 \\
(0.901-1.023)\end{array}$ & $\begin{array}{c}0.948 \\
(0.878-1.006)\end{array}$ & $\begin{array}{c}0.904 \\
(0.783-1.017)\end{array}$ & $\begin{array}{c}3 \\
(2: 2,2: 2)\end{array}$ & $\begin{array}{c}9.4 \\
(5.6-13.3)\end{array}$ \\
\hline E-016 & 100.0 & $\begin{array}{c}0.989 \\
(0.956-1.018)\end{array}$ & $\begin{array}{c}0.983 \\
(0.954-1.012)\end{array}$ & $\begin{array}{c}0.988 \\
(0.944-1.038)\end{array}$ & $\begin{array}{c}1.019 \\
(0.976-1.064)\end{array}$ & $\begin{array}{c}0.998 \\
(0.970-1.025)\end{array}$ & $\begin{array}{c}0.959 \\
(0.897-1.026)\end{array}$ & $\begin{array}{c}8 \\
(3: 3,2: 2)\end{array}$ & $\begin{array}{c}18.4 \\
(14.9-21.9)\end{array}$ \\
\hline E-017 & 100.0 & $\begin{array}{c}0.980 \\
(0.963-0.999)\end{array}$ & $\begin{array}{c}0.991 \\
(0.962-1.015)\end{array}$ & $\begin{array}{c}0.983 \\
(0.952-1.008)\end{array}$ & $\begin{array}{c}0.993 \\
(0.959-1.026)\end{array}$ & $\begin{array}{c}0.977 \\
(0.951-0.995)\end{array}$ & $\begin{array}{c}0.907 \\
(0.871-0.942)\end{array}$ & $\begin{array}{c}15 \\
(6: 6,5: 5)\end{array}$ & $\begin{array}{c}34.3 \\
(28.6-40.0)\end{array}$ \\
\hline E-021 & 100.0 & $\begin{array}{c}0.993 \\
(0.950-1.036)\end{array}$ & $\begin{array}{c}0.997 \\
(0.955-1.044)\end{array}$ & $\begin{array}{c}1.001 \\
(0.957-1.051)\end{array}$ & $\begin{array}{c}1.017 \\
(0.971-1.070)\end{array}$ & $\begin{array}{c}1.014 \\
(0.977-1.051)\end{array}$ & $\begin{array}{c}1.071 \\
(0.969-1.188)\end{array}$ & $\begin{array}{c}1 \\
(1: 1,1: 1)\end{array}$ & $\begin{array}{c}7.9 \\
(5.9-10.0)\end{array}$ \\
\hline E-022 & 100.0 & $\begin{array}{c}0.991 \\
(0.977-1.005)\end{array}$ & $\begin{array}{c}1.004 \\
(0.993-1.015)\end{array}$ & $\begin{array}{c}1.008 \\
(0.984-1.040)\end{array}$ & $\begin{array}{c}1.026 \\
(0.999-1.057)\end{array}$ & $\begin{array}{c}1.015 \\
(0.991-1.039)\end{array}$ & $\begin{array}{c}0.985 \\
(0.922-1.051)\end{array}$ & $\begin{array}{c}3 \\
(1: 1,1: 1)\end{array}$ & $\begin{array}{c}29.1 \\
(24.2-34.0)\end{array}$ \\
\hline E-023 & 100.0 & NA & NA & NA & NA & NA & NA & $\begin{array}{c}1 \\
(0: 0,0: 0)\end{array}$ & NA \\
\hline E-024 & 100.0 & $\begin{array}{c}0.937 \\
(0.902-0.978)\end{array}$ & $\begin{array}{c}0.934 \\
(0.891-0.980)\end{array}$ & $\begin{array}{c}0.921 \\
(0.875-0.967)\end{array}$ & $\begin{array}{c}0.902 \\
(0.855-0.947)\end{array}$ & $\begin{array}{c}0.887 \\
(0.845-0.928)\end{array}$ & $\begin{array}{c}0.900 \\
(0.796-1.031)\end{array}$ & $\begin{array}{c}2 \\
(2: 2,2: 2)\end{array}$ & $\begin{array}{c}3.9 \\
(2.3-5.5)\end{array}$ \\
\hline E-025 & 100.0 & $\begin{array}{c}0.991 \\
(0.975-1.007)\end{array}$ & $\begin{array}{c}0.984 \\
(0.963-1.000)\end{array}$ & $\begin{array}{c}0.987 \\
(0.956-1.004)\end{array}$ & $\begin{array}{c}1.022 \\
(0.994-1.045)\end{array}$ & $\begin{array}{c}1.009 \\
(0.986-1.028)\end{array}$ & $\begin{array}{c}0.918 \\
(0.883-0.951)\end{array}$ & $\begin{array}{c}34 \\
(16: 16,14: 14)\end{array}$ & $\begin{array}{c}17.3 \\
(15.6-19.0)\end{array}$ \\
\hline E-026 & 100.0 & $\begin{array}{c}1.011 \\
(0.986-1.039)\end{array}$ & $\begin{array}{c}1.009 \\
(0.987-1.025)\end{array}$ & $\begin{array}{c}1.028 \\
(0.977-1.060)\end{array}$ & $\begin{array}{c}1.082 \\
(1.037-1.125)\end{array}$ & $\begin{array}{c}1.006 \\
(0.988-1.026)\end{array}$ & $\begin{array}{c}0.961 \\
(0.916-1.012)\end{array}$ & $\begin{array}{c}4 \\
(1: 1,1: 1)\end{array}$ & $\begin{array}{c}68.6 \\
(57.8-79.4)\end{array}$ \\
\hline E-027 & 100.0 & $\begin{array}{c}1.006 \\
(0.981-1.022)\end{array}$ & $\begin{array}{c}1.023 \\
(0.995-1.042)\end{array}$ & $\begin{array}{c}1.025 \\
(1.002-1.058)\end{array}$ & $\begin{array}{c}1.048 \\
(1.016-1.076)\end{array}$ & $\begin{array}{c}1.029 \\
(1.015-1.043)\end{array}$ & $\begin{array}{c}1.026 \\
(0.989-1.065)\end{array}$ & $\begin{array}{c}14 \\
(8: 8,8: 8)\end{array}$ & $\begin{array}{c}22.9 \\
(19.0-26.8)\end{array}$ \\
\hline
\end{tabular}


Table 4.8. Table of greater sage-grouse (Centrocercus urophasianus) average annual rate of population change $(\hat{\lambda})$ across six periods of oscillation in abundance that represent temporal scales for each neighborhood cluster within the state of Nevada. Estimates were derived from leks within the entire neighborhood cluster.-Continued

[NC, neighborhood cluster; NA, not applicable]

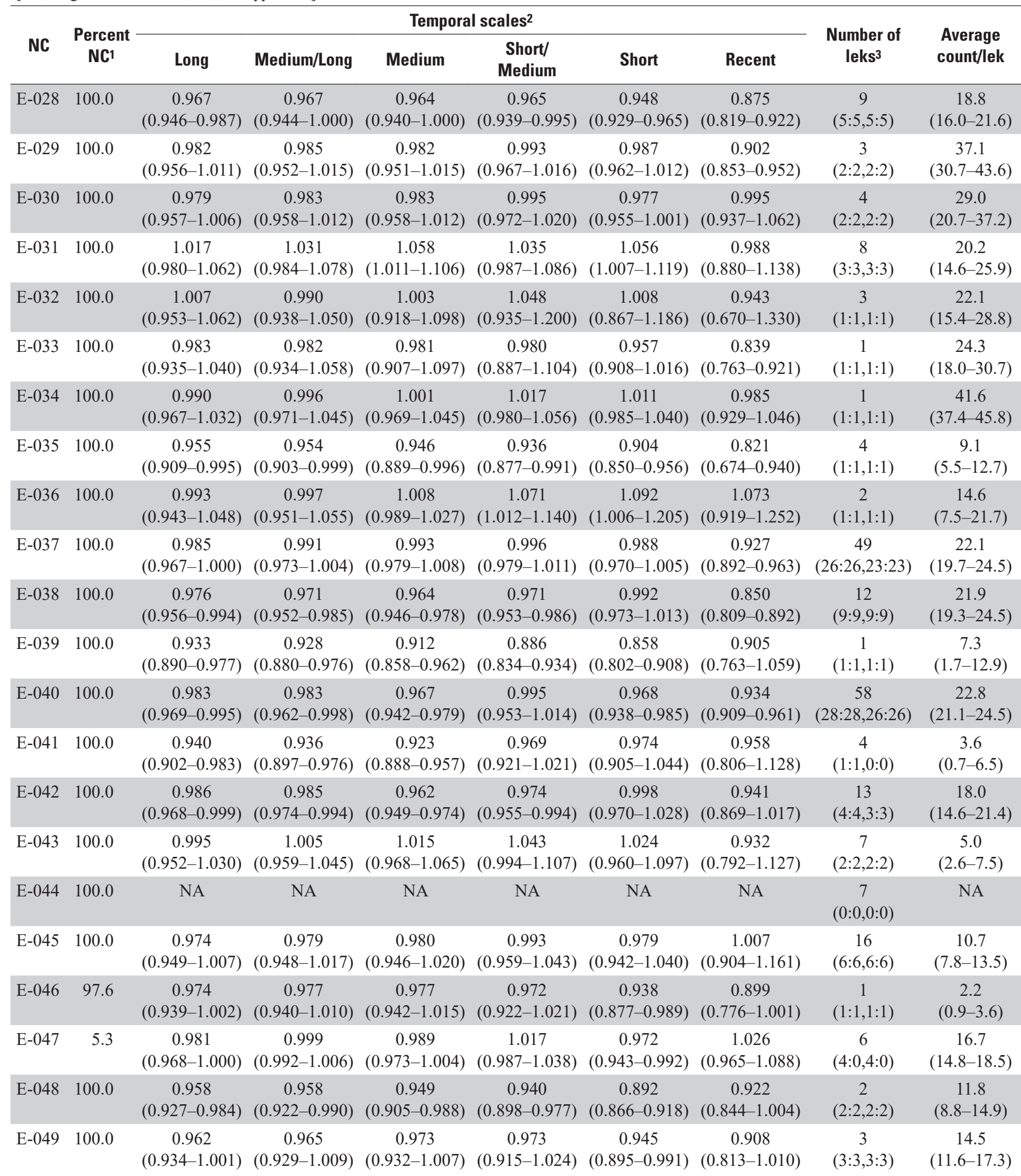


Table 4.8. Table of greater sage-grouse (Centrocercus urophasianus) average annual rate of population change $(\hat{\lambda})$ across six periods of oscillation in abundance that represent temporal scales for each neighborhood cluster within the state of Nevada. Estimates were derived from leks within the entire neighborhood cluster.-Continued

[NC, neighborhood cluster; NA, not applicable]

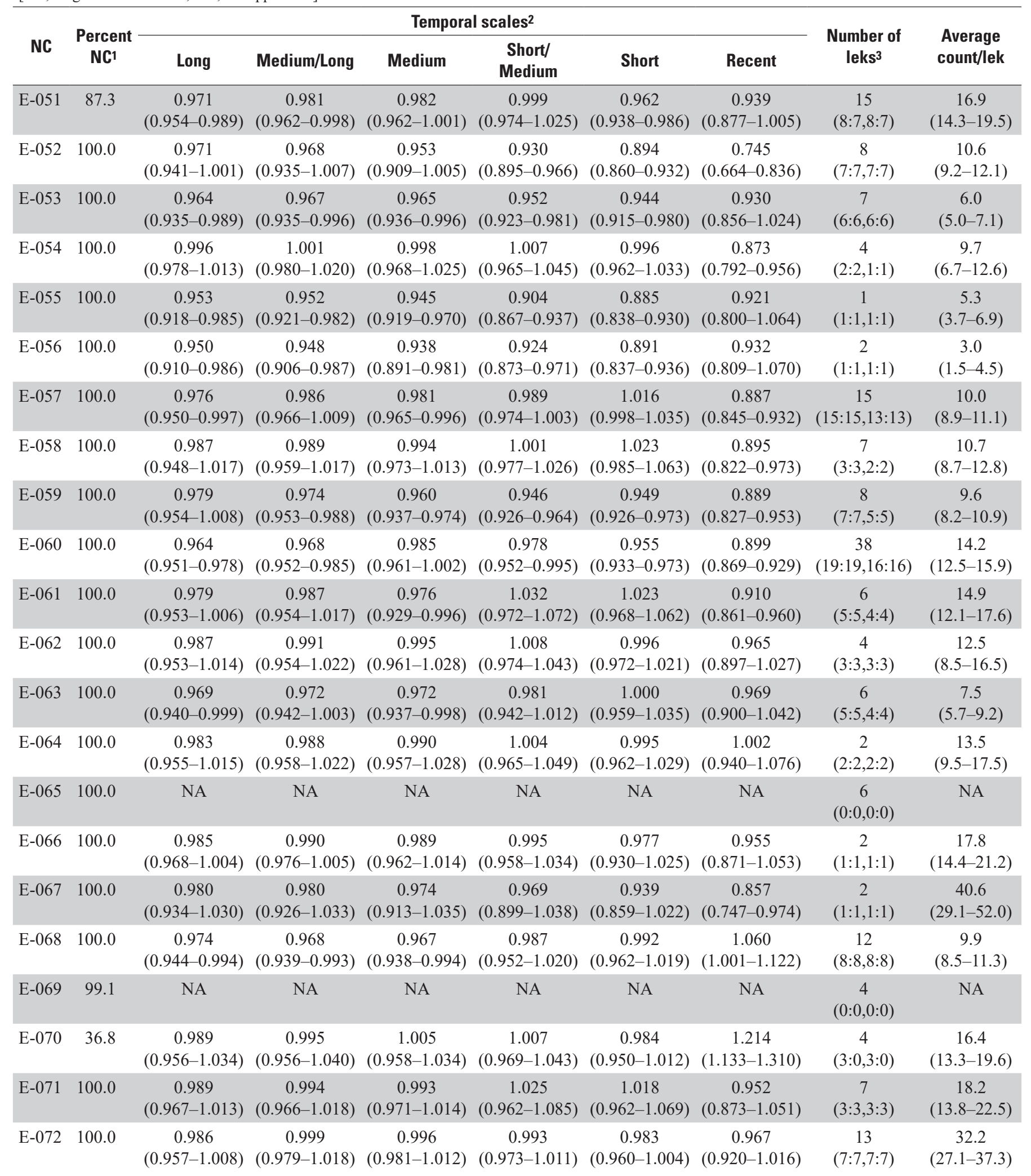


Table 4.8. Table of greater sage-grouse (Centrocercus urophasianus) average annual rate of population change $(\hat{\lambda})$ across six periods of oscillation in abundance that represent temporal scales for each neighborhood cluster within the state of Nevada. Estimates were derived from leks within the entire neighborhood cluster.-Continued

[NC, neighborhood cluster; NA, not applicable]

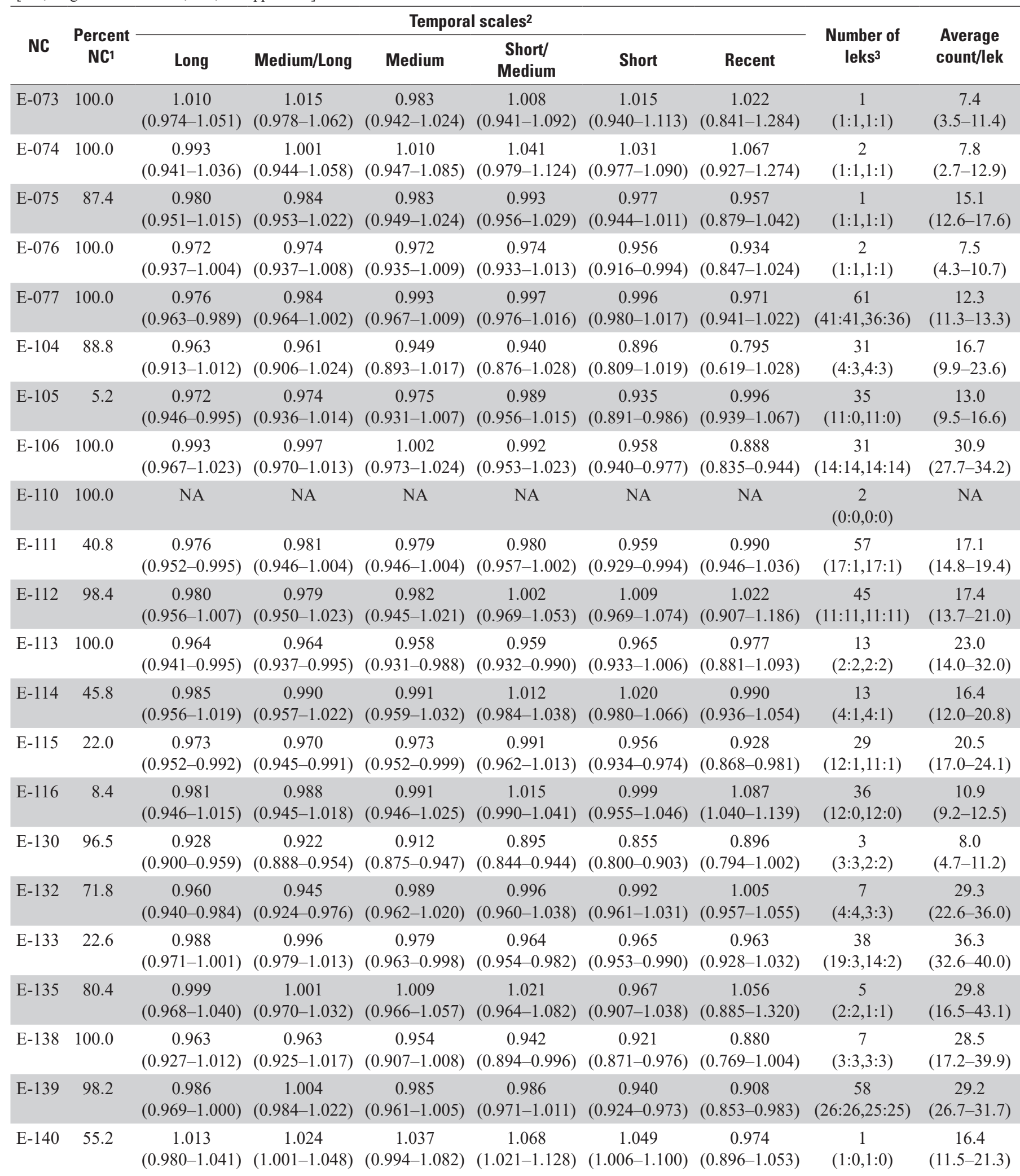


Table 4.8. Table of greater sage-grouse (Centrocercus urophasianus) average annual rate of population change $(\hat{\lambda})$ across six periods of oscillation in abundance that represent temporal scales for each neighborhood cluster within the state of Nevada. Estimates were derived from leks within the entire neighborhood cluster.-Continued

[NC, neighborhood cluster; NA, not applicable]

\begin{tabular}{|c|c|c|c|c|c|c|c|c|c|}
\hline \multirow[b]{2}{*}{ NC } & \multirow{2}{*}{$\begin{array}{c}\text { Percent } \\
\text { NC1 }\end{array}$} & \multicolumn{6}{|c|}{ Temporal scales 2} & \multirow{2}{*}{$\begin{array}{l}\text { Number of } \\
\text { leks }^{3}\end{array}$} & \multirow{2}{*}{$\begin{array}{l}\text { Average } \\
\text { count/lek }\end{array}$} \\
\hline & & Long & Medium/Long & Medium & $\begin{array}{c}\text { Short/ } \\
\text { Medium }\end{array}$ & Short & Recent & & \\
\hline E-142 & 73.0 & $\begin{array}{c}0.992 \\
(0.970-1.017)\end{array}$ & $\begin{array}{c}0.990 \\
(0.969-1.013)\end{array}$ & $\begin{array}{c}0.994 \\
(0.958-1.027)\end{array}$ & $\begin{array}{c}1.030 \\
(0.977-1.077)\end{array}$ & $\begin{array}{c}1.042 \\
(1.000-1.095)\end{array}$ & $\begin{array}{c}1.091 \\
(0.969-1.251)\end{array}$ & $\begin{array}{c}17 \\
(6: 4,5: 3)\end{array}$ & $\begin{array}{c}24.6 \\
(18.2-31.1)\end{array}$ \\
\hline E-144 & 92.5 & $\begin{array}{c}0.973 \\
(0.956-0.988)\end{array}$ & $\begin{array}{c}0.973 \\
(0.955-0.988)\end{array}$ & $\begin{array}{c}0.982 \\
(0.959-0.996)\end{array}$ & $\begin{array}{c}0.979 \\
(0.956-1.001)\end{array}$ & $\begin{array}{c}0.960 \\
(0.940-0.986)\end{array}$ & $\begin{array}{c}0.983 \\
(0.935-1.058)\end{array}$ & $\begin{array}{c}35 \\
(22: 22,20: 20)\end{array}$ & $\begin{array}{c}31.6 \\
(28.6-34.7)\end{array}$ \\
\hline E-154 & 5.7 & $\begin{array}{c}0.986 \\
(0.971-1.004)\end{array}$ & $\begin{array}{c}0.988 \\
(0.965-1.013)\end{array}$ & $\begin{array}{c}0.990 \\
(0.956-1.017)\end{array}$ & $\begin{array}{c}1.025 \\
(1.001-1.048)\end{array}$ & $\begin{array}{c}1.027 \\
(1.010-1.042)\end{array}$ & $\begin{array}{c}1.063 \\
(1.027-1.099)\end{array}$ & $\begin{array}{c}29 \\
(19: 0,18: 0)\end{array}$ & $\begin{array}{c}18.9 \\
(16.8-21.1)\end{array}$ \\
\hline E-163 & 11.6 & $\begin{array}{c}0.980 \\
(0.954-1.001)\end{array}$ & $\begin{array}{c}0.980 \\
(0.946-1.009)\end{array}$ & $\begin{array}{c}0.979 \\
(0.944-1.015)\end{array}$ & $\begin{array}{c}0.977 \\
(0.951-1.017)\end{array}$ & $\begin{array}{c}0.963 \\
(0.940-0.987)\end{array}$ & $\begin{array}{c}0.968 \\
(0.924-1.009)\end{array}$ & $\begin{array}{c}23 \\
(19: 0,19: 0)\end{array}$ & $\begin{array}{c}20.8 \\
(17.0-24.6)\end{array}$ \\
\hline E-216 & 11.4 & $\begin{array}{c}0.986 \\
(0.971-0.998)\end{array}$ & $\begin{array}{c}1.006 \\
(0.989-1.021)\end{array}$ & $\begin{array}{c}0.995 \\
(0.974-1.009)\end{array}$ & $\begin{array}{c}1.009 \\
(0.997-1.021)\end{array}$ & $\begin{array}{c}1.011 \\
(0.994-1.024)\end{array}$ & $\begin{array}{c}1.044 \\
(1.018-1.071)\end{array}$ & $\begin{array}{c}75 \\
(34: 1,31: 1)\end{array}$ & $\begin{array}{c}16.1 \\
(14.8-17.4)\end{array}$ \\
\hline E-217 & 81.5 & $\begin{array}{c}0.991 \\
(0.973-1.002)\end{array}$ & $\begin{array}{c}0.957 \\
(0.935-0.983)\end{array}$ & $\begin{array}{c}0.967 \\
(0.943-0.999)\end{array}$ & $\begin{array}{c}1.003 \\
(0.981-1.024)\end{array}$ & $\begin{array}{c}0.992 \\
(0.971-1.011)\end{array}$ & $\begin{array}{c}1.043 \\
(0.992-1.098)\end{array}$ & $\begin{array}{c}51 \\
(15: 10,10: 5)\end{array}$ & $\begin{array}{c}15.6 \\
(13.1-18.1)\end{array}$ \\
\hline E-218 & 9.3 & $\begin{array}{c}0.991 \\
(0.965-1.002)\end{array}$ & $\begin{array}{c}1.005 \\
(0.965-1.021)\end{array}$ & $\begin{array}{c}1.003 \\
(0.960-1.019)\end{array}$ & $\begin{array}{c}1.033 \\
(1.009-1.051)\end{array}$ & $\begin{array}{c}1.021 \\
(1.002-1.039)\end{array}$ & $\begin{array}{c}1.076 \\
(1.039-1.122)\end{array}$ & $\begin{array}{c}15 \\
(10: 0,10: 0)\end{array}$ & $\begin{array}{c}20.9 \\
(18.2-23.7)\end{array}$ \\
\hline E-226 & 35.3 & $\begin{array}{c}0.991 \\
(0.969-1.003)\end{array}$ & $\begin{array}{c}0.991 \\
(0.971-1.000)\end{array}$ & $\begin{array}{c}0.994 \\
(0.982-1.004)\end{array}$ & $\begin{array}{c}0.997 \\
(0.982-1.011)\end{array}$ & $\begin{array}{c}0.958 \\
(0.945-0.973)\end{array}$ & $\begin{array}{c}0.972 \\
(0.935-1.017)\end{array}$ & $\begin{array}{c}34 \\
(16: 2,16: 2)\end{array}$ & $\begin{array}{c}23.2 \\
(19.8-26.6)\end{array}$ \\
\hline E-231 & 100.0 & $\begin{array}{c}0.978 \\
(0.937-1.017)\end{array}$ & $\begin{array}{c}0.981 \\
(0.936-1.026)\end{array}$ & $\begin{array}{c}0.981 \\
(0.933-1.033)\end{array}$ & $\begin{array}{c}0.991 \\
(0.943-1.046)\end{array}$ & $\begin{array}{c}0.977 \\
(0.933-1.032)\end{array}$ & $\begin{array}{c}0.925 \\
(0.805-1.026)\end{array}$ & $\begin{array}{c}1 \\
(1: 1,1: 1)\end{array}$ & $\begin{array}{c}4.1 \\
(2.2-6.0)\end{array}$ \\
\hline E-232 & 85.3 & $\begin{array}{c}0.978 \\
(0.956-0.999)\end{array}$ & $\begin{array}{c}0.976 \\
(0.947-1.000)\end{array}$ & $\begin{array}{c}0.965 \\
(0.937-0.990)\end{array}$ & $\begin{array}{c}0.973 \\
(0.943-1.009)\end{array}$ & $\begin{array}{c}0.953 \\
(0.924-0.994)\end{array}$ & $\begin{array}{c}1.017 \\
(0.932-1.154)\end{array}$ & $\begin{array}{c}29 \\
(17: 12,14: 11)\end{array}$ & $\begin{array}{c}16.7 \\
(13.6-19.8)\end{array}$ \\
\hline E-233 & 6.0 & $\begin{array}{c}0.966 \\
(0.954-0.978)\end{array}$ & $\begin{array}{c}0.967 \\
(0.939-0.982)\end{array}$ & $\begin{array}{c}0.970 \\
(0.937-0.984)\end{array}$ & $\begin{array}{c}0.980 \\
(0.963-0.993)\end{array}$ & $\begin{array}{c}0.952 \\
(0.940-0.963)\end{array}$ & $\begin{array}{c}0.915 \\
(0.890-0.941)\end{array}$ & $\begin{array}{c}60 \\
(44: 0,42: 0)\end{array}$ & $\begin{array}{c}10.2 \\
(9.5-10.9)\end{array}$ \\
\hline E-235 & 100.0 & $\begin{array}{c}0.951 \\
(0.903-0.998)\end{array}$ & $\begin{array}{c}0.949 \\
(0.899-1.003)\end{array}$ & $\begin{array}{c}0.940 \\
(0.883-1.002)\end{array}$ & $\begin{array}{c}0.929 \\
(0.869-0.991)\end{array}$ & $\begin{array}{c}0.904 \\
(0.849-0.958)\end{array}$ & $\begin{array}{c}0.900 \\
(0.765-1.049)\end{array}$ & $\begin{array}{c}5 \\
(1: 1,1: 1)\end{array}$ & $\begin{array}{c}3.4 \\
(0.6-6.1)\end{array}$ \\
\hline E-236 & 100.0 & NA & NA & NA & NA & NA & NA & $\begin{array}{c}4 \\
(0: 0,0: 0)\end{array}$ & NA \\
\hline E-237 & 100.0 & NA & NA & NA & NA & NA & NA & $\begin{array}{c}0 \\
(0: 0,0: 0)\end{array}$ & NA \\
\hline E-238 & 100.0 & NA & NA & NA & NA & NA & NA & $\begin{array}{c}0 \\
(0: 0,0: 0)\end{array}$ & NA \\
\hline
\end{tabular}


Table 4.8. Table of greater sage-grouse (Centrocercus urophasianus) average annual rate of population change $(\hat{\lambda})$ across six periods of oscillation in abundance that represent temporal scales for each neighborhood cluster within the state of Nevada. Estimates were derived from leks within the entire neighborhood cluster.-Continued

[NC, neighborhood cluster; NA, not applicable]

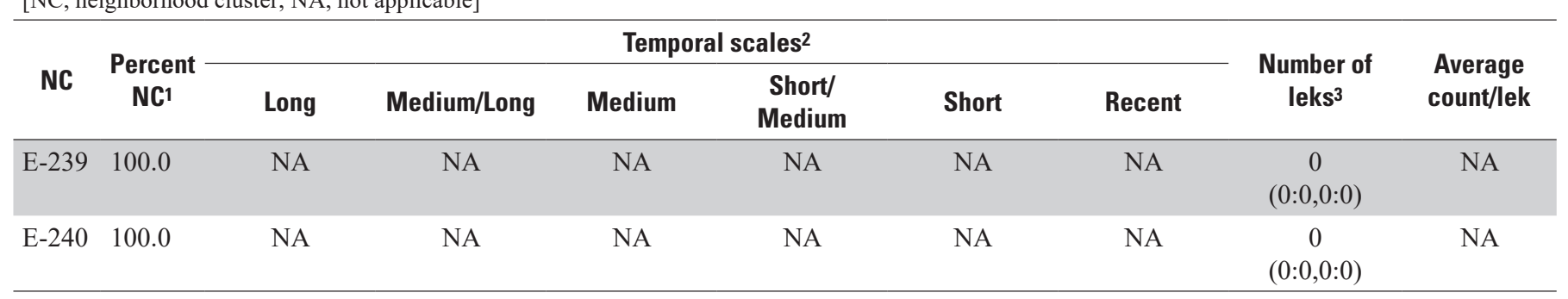

1The percent of each neighborhood cluster that intersects the state.

2Temporal scales were estimated from present to each major population abundance nadir (trough) since 1960 . Number of temporal scales were used to estimate population trends across six different temporal scales from approximately 10 to approximately 60 years.

${ }^{3}$ Number of leks in database. In parentheses from left to right is (1) total number in cluster were used in trend analysis, (2) number used in trend analysis within state boundary, (3) total number in cluster were used in the targeted annual warning system (TAWS) analysis, and (4) number used in TAWS analysis within state boundary.
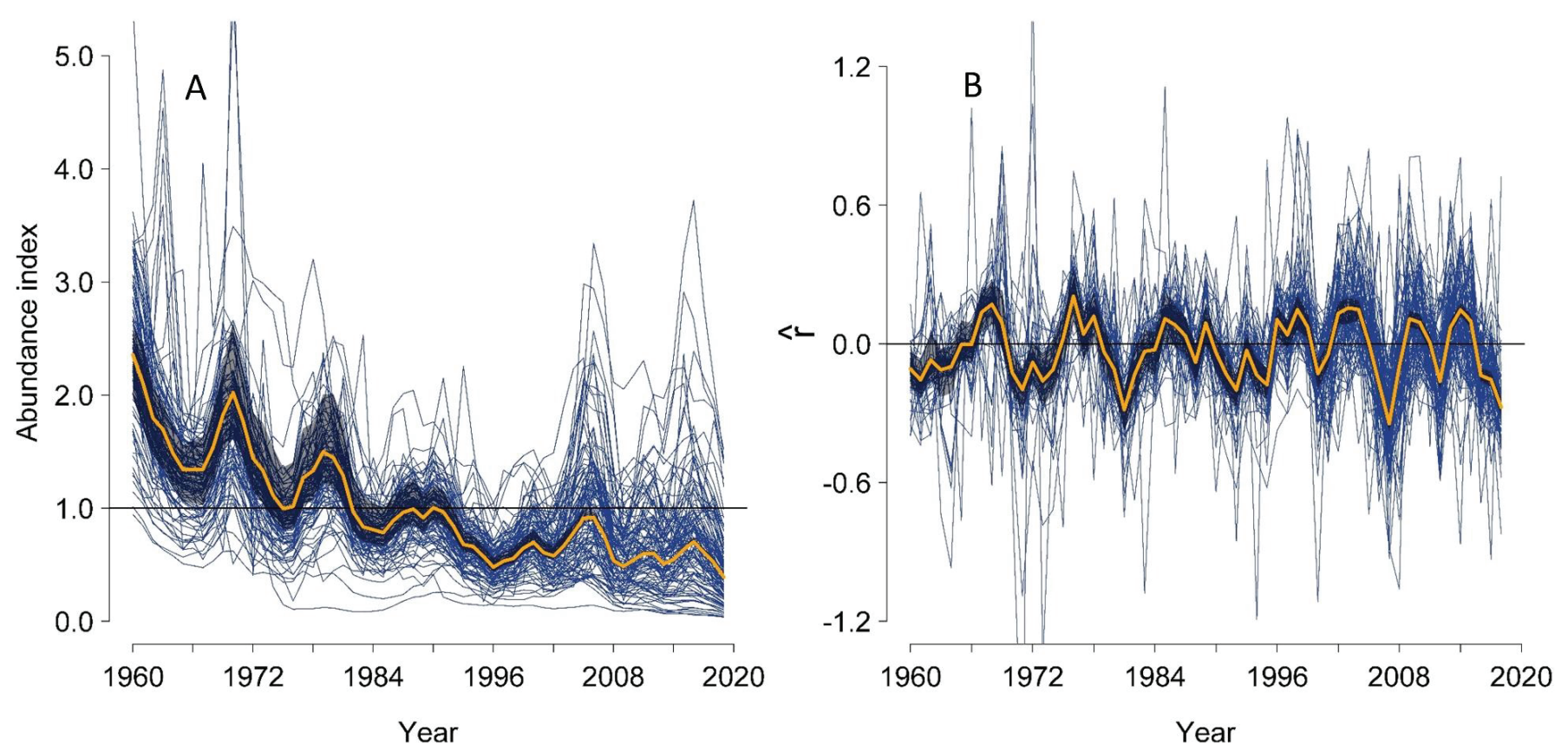

Figure 4.22. A, Abundance index (calculated as $\hat{N}$ divided by 60 -year mean of $\hat{N}$ ); and $B$, intrinsic rate of population change $(\hat{r})$ of greater sage-grouse (Centrocercus urophasianus) within the state of Nevada from 1960-2019. Thick yellow line represents median estimates across all leks. Shaded areas represent 95 -percent credible limits. Thin blue lines represent median values for neighborhood clusters. Black horizontal line (abundance index=1.0) represents 60-year average. 


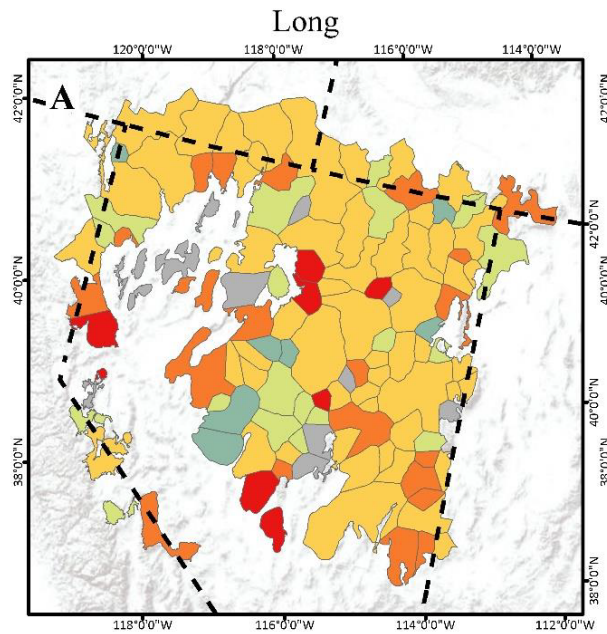

EXPLANATION

Neighborhood clusters (Level 2) intersecting Nevada: polygon colors represent average lambda values for sage-grouse populations over varying lengths of time (long, medium, short). Symbols are based on a range-wide distribution of values and all may not be represented here.
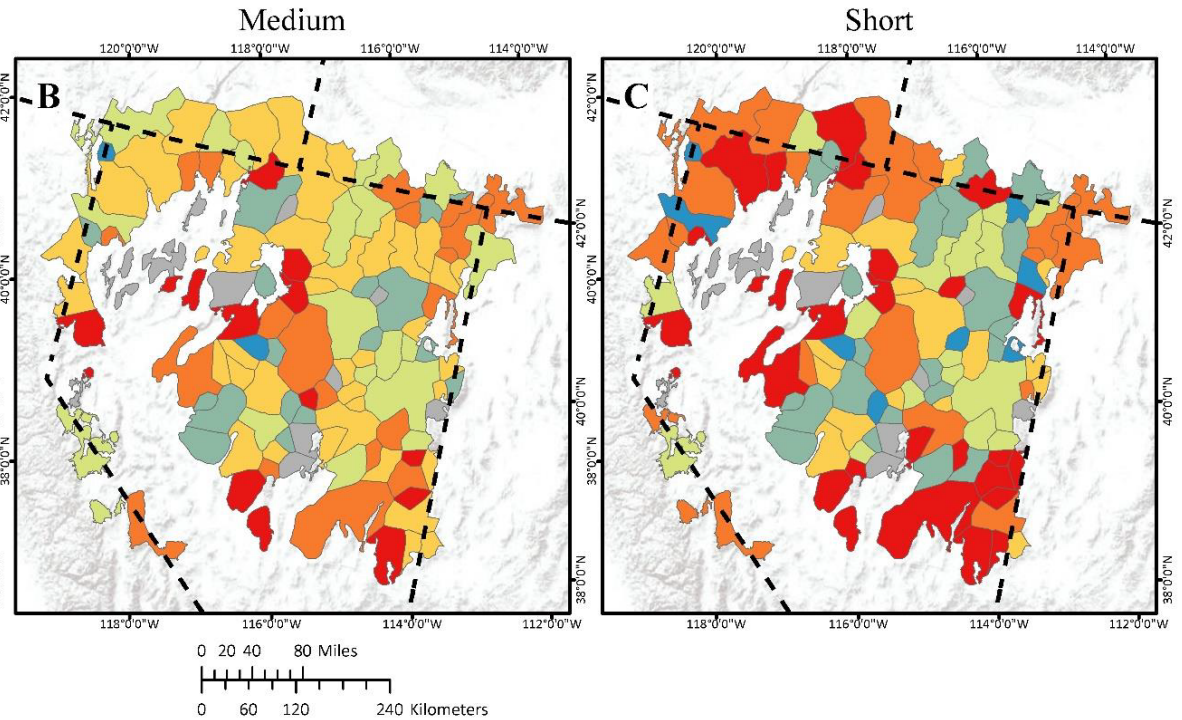

Average lambda $(\hat{\lambda})$

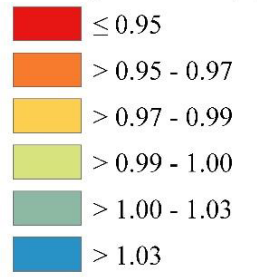

Coordinate System: Albers Projection: Albers Datum: WGS 1984 false easting: 0.0000 central meridian: -96.0000 standard parallel $1: 29.5000$ standard parallel 2: 45.5000 latitude of origin: 23.0000 Units: Meter

Service Layer Credits: Sources: Esri, USGS, NOAA false northing: 0.0000

Figure 4.23. Spatial estimates of population trends across three temporal scales based on periods of oscillation (short, medium, and long) while accounting for periods of oscillation of greater sage-grouse (Centrocercus urophasianus) at different neighborhood clusters within the state of Nevada. Map images are the intellectual property of Esri and are used herein under license. Copyright @ 2020 Esri and its licensors. All rights reserved. 


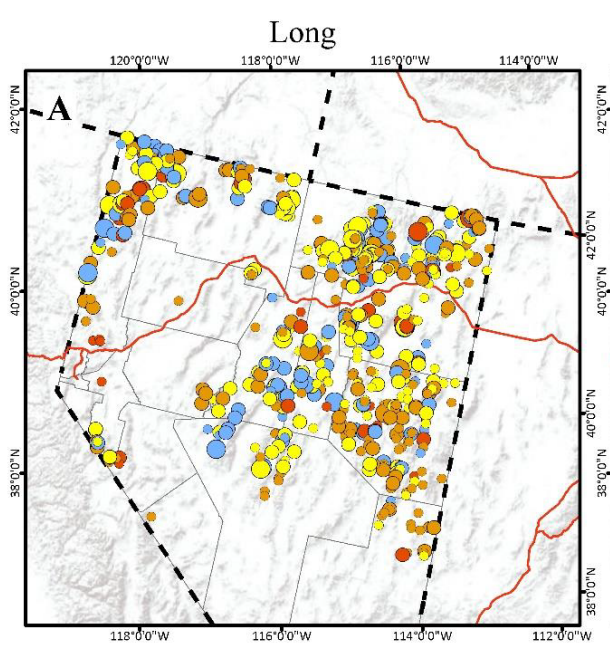

EXPLANATION

Leks within Nevada: colors represent average lambda $(\hat{\lambda})$ values of sage-grouse populations over various time scales (long, medium, short). The size of the symbol represents the maximum population size of the lek over the years of the analysis, relativized to the range-wide maximum population size across all leks. Symbols are based on a range-wide distribution of values and all may not be represented here.
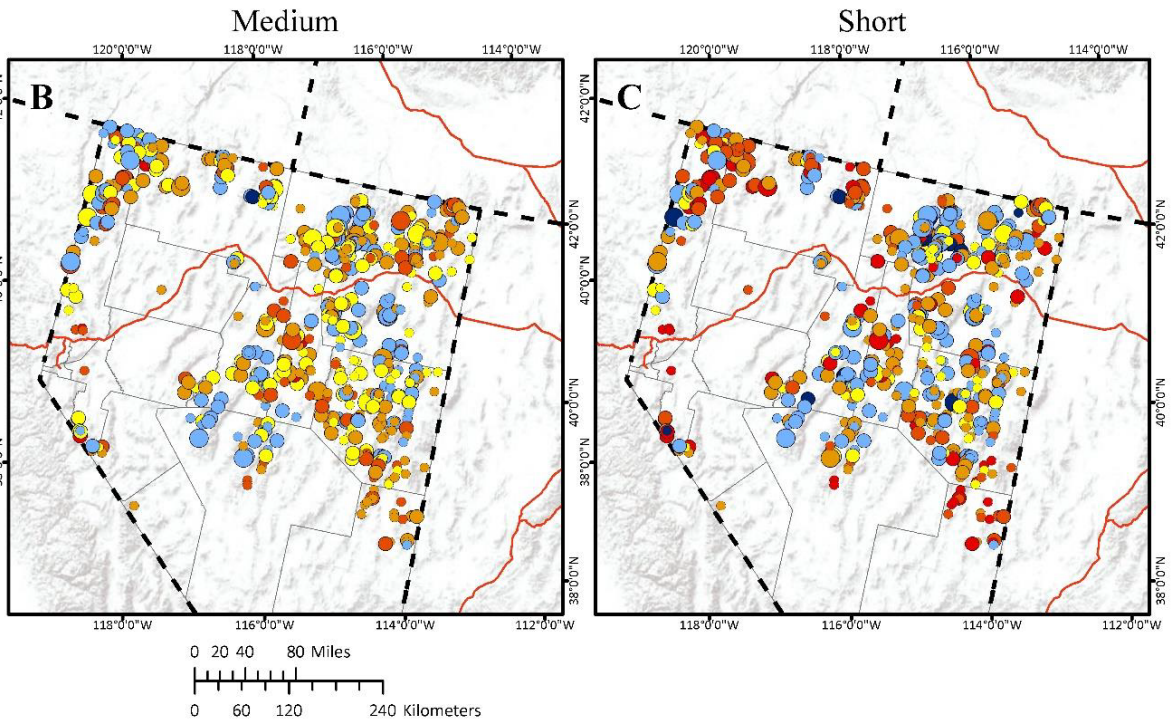

$$
\begin{aligned}
\text { Average lambda }(\hat{\lambda}) \\
\quad \leq 0.90 \\
\quad>0.90-0.94 \\
\quad>0.94-0.98 \\
\quad>0.98-1.00 \\
\quad>1.00-1.10 \\
\quad>1.10
\end{aligned}
$$$$
\longrightarrow \text { Road }
$$

Coordinate System: Albers Projection: Albers Datum: WGS 1984 false easting: 0.0000 false northing: 0.0000 central meridian: -96.0000 standard parallel 1: 29.5000 standard parallel 2: 45.5000 latitude of origin: 23.0000 Units: Meter

Service Layer Credits: Sources: Esri, USGS, NOAA

Figure 4.24. Spatial estimates of population trends across three temporal scales based on periods of oscillation (short, medium, and long) while accounting for periods of oscillation of greater sage-grouse (Centrocercus urophasianus) at lek sites within the state of Nevada. Map images are the intellectual property of Esri and are used herein under license. Copyright $@ 2020$ Esri and its licensors. All rights reserved. 


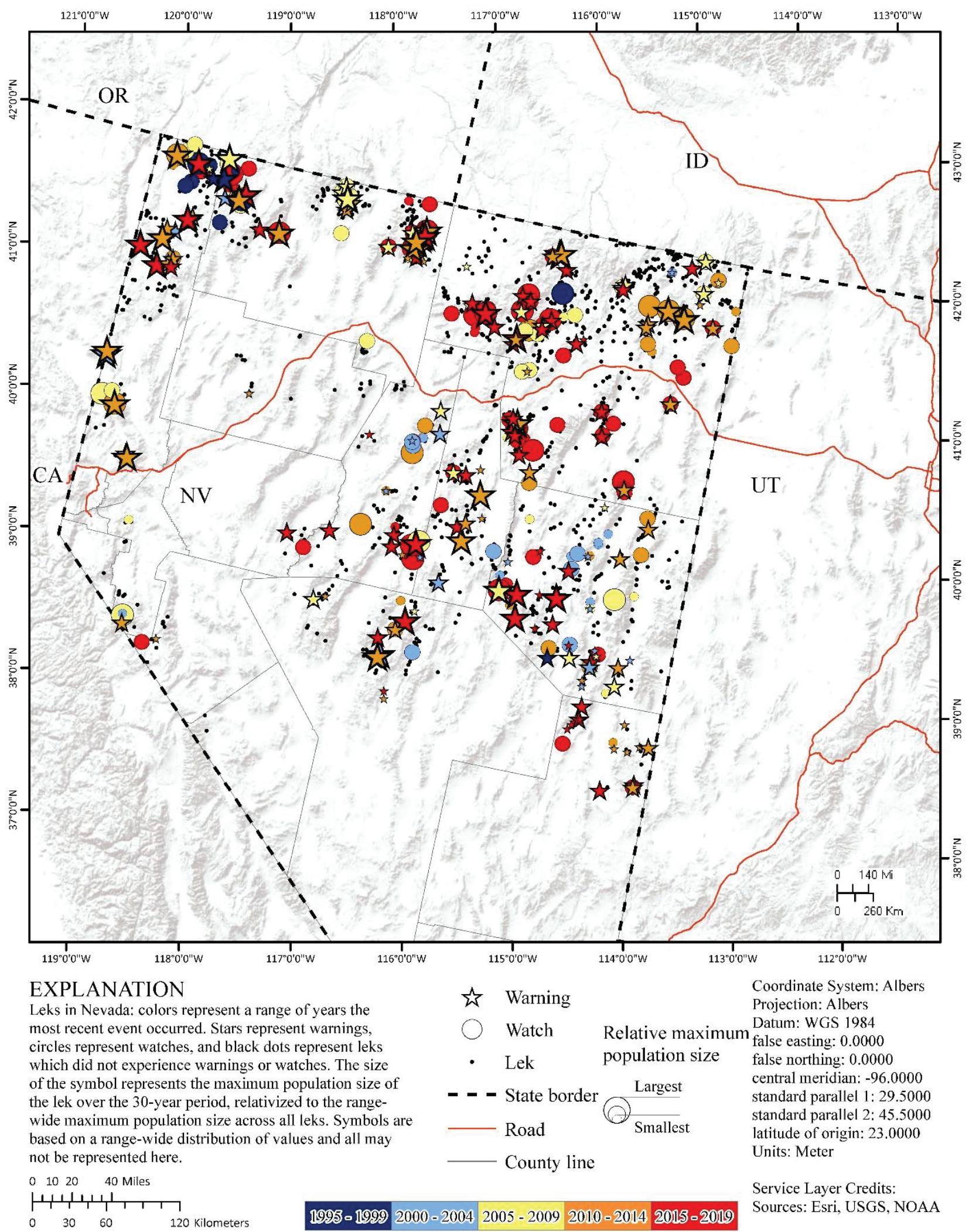

Figure 4.25. Spatial and temporal depiction of watches and warnings of greater sage-grouse (Centrocercus urophasianus) population declines at the lek scale within the state of Nevada from 1990 to 2019. Map image is the intellectual property of Esri and is used herein under license. Copyright (c) 2020 Esri and its licensors. All rights reserved. 


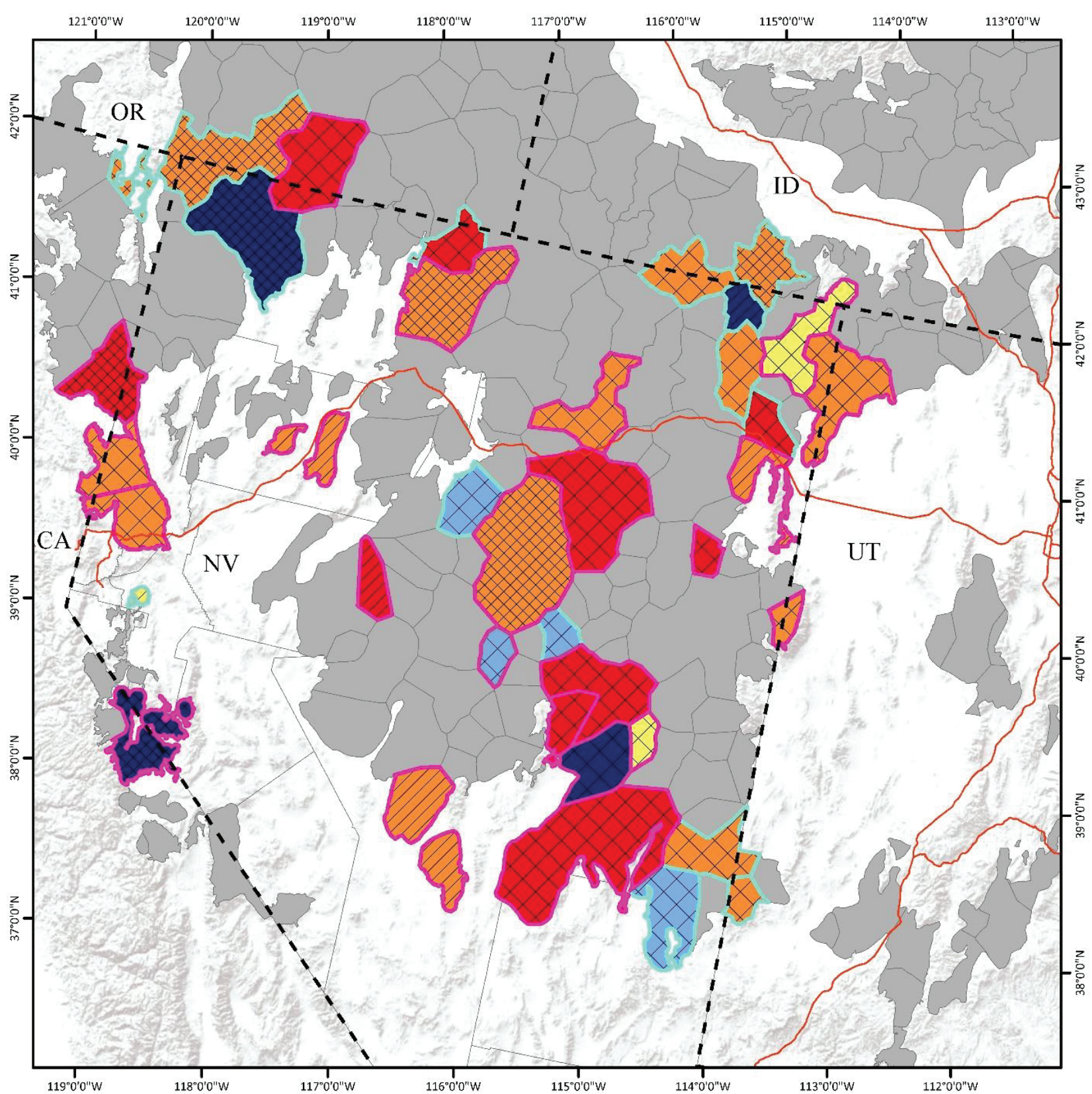

EXPLANATION

Neighborhood clusters (Level 2) in Nevada: polygon colors represent a range of years the most recent event occurred. Outline colors indicate whether the most recent event was a watch or warning. The hatching represents the maximum population size of the cluster over the 30-year period, relativized to the range-wide maximum population size across all neighborhood clusters. Symbols are based on a rangewide distribution of values and all may not be represented here $0 \quad 1020 \quad 40$ Miles | - $30 \quad 60 \quad 120$ Kilometers 1995-1999 2000-2004 2005- $20092010-20142015-2019$

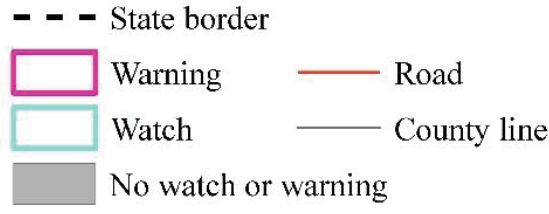

Largest

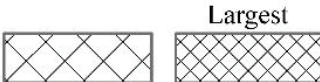

Coordinate System: Albers Projection: Albers Datum: WGS 1984 false easting: 0.0000 false northing: 0.0000 central meridian: -96.0000 standard parallel 1: 29.5000 standard parallel 2: 45.5000 latitude of origin: 23.0000 Units: Meter

Service Layer Credits: Sources: Esri, USGS, NOAA

Figure 4.26. Spatial and temporal depiction of watches and warnings of greater sage-grouse (Centrocercus urophasianus) population declines at neighborhood cluster within the state of Nevada from 1990 to 2019. Map image is the intellectual property of Esri and is used herein under license. Copyright (C) 2020 Esri and its licensors. All rights reserved. 


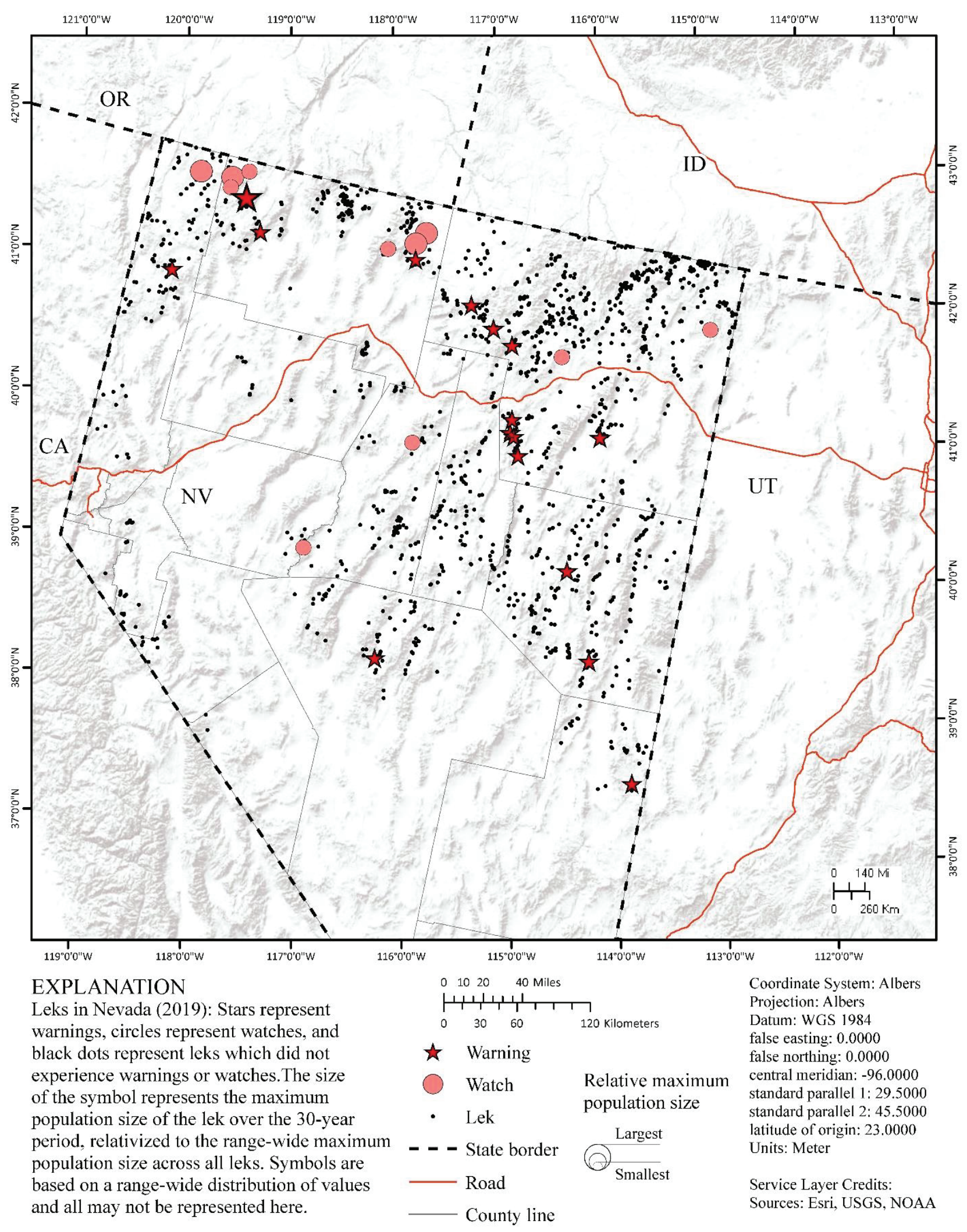

Figure 4.27. Spatial and temporal depiction of watches and warnings of greater sage-grouse (Centrocercus urophasianus) population declines at the lek scale within the state of Nevada during 2019. Map image is the intellectual property of Esri and is used herein under license. Copyright () 2020 Esri and its licensors. All rights reserved. 


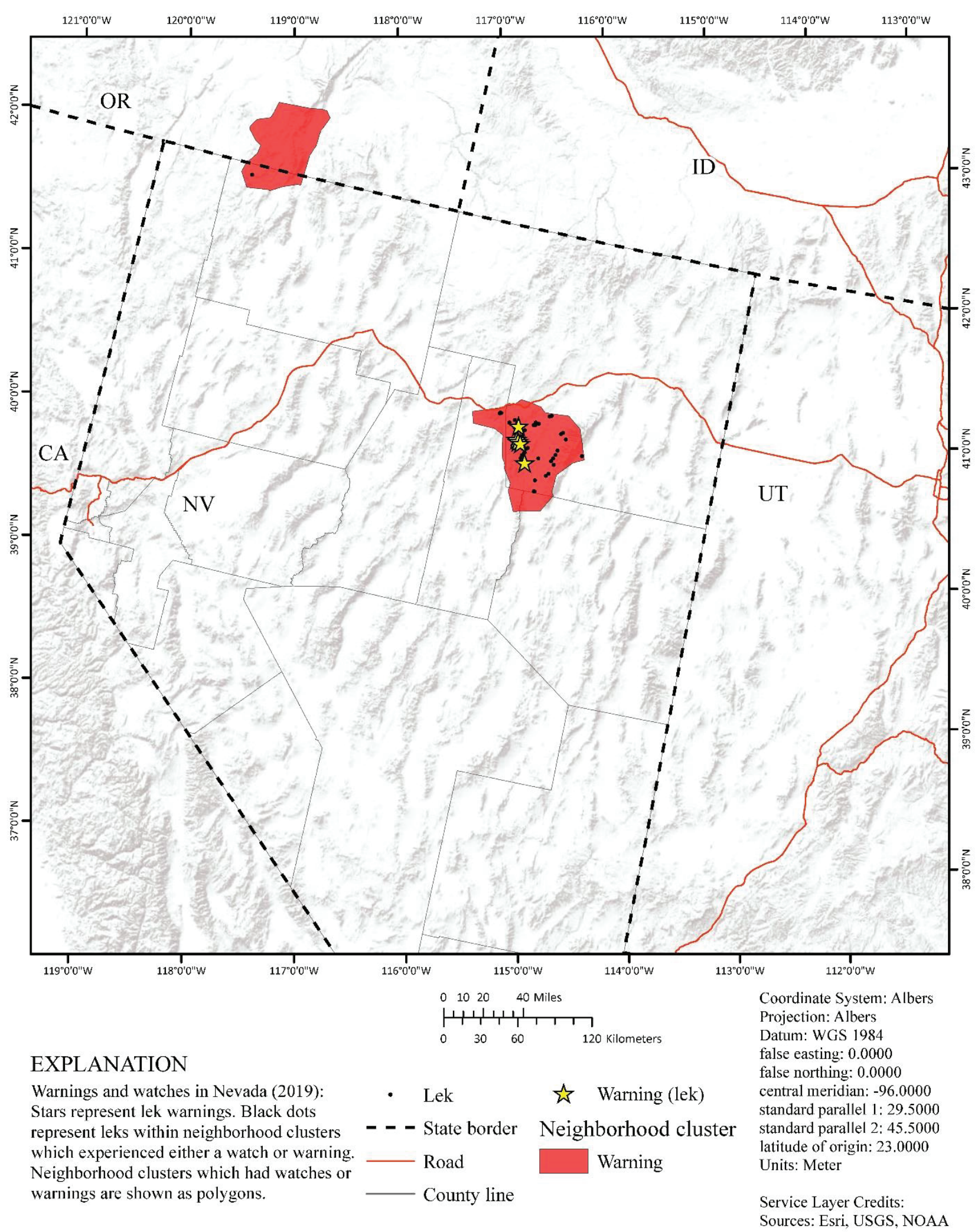

Figure 4.28. Spatial and temporal depiction of watches and warnings of greater sage-grouse (Centrocercus urophasianus) population declines at the neighborhood cluster scale within the state of Nevada during 2019. All leks within watch and warning boundaries were assigned as watch and warning, respectively. Yellow stars represent leks that reached warning independently. Map image is the intellectual property of Esri and is used herein under license. Copyright $₫ 2020$ Esri and its licensors. All rights reserved. 


\section{Idaho Results}

\section{Idaho Lek Data and Clusters (Objectives 1 and 2)}

Idaho intersected CC-E and CC-F and contained or intersected 77 different NCs (fig. 4.29). Total area of the 77 NCs within Idaho was 7,084,233. There were 71 of these clusters used in the analysis after, 6 were omitted because of limitations in sample sizes. These areas consisted of 1,953 leks, of which 1,601 were located within the Idaho state boundary and represented 19.01 percent of the range-wide lek database. After extensive QA/QC, we used 944 leks from Idaho in the SSM for population trend estimation (tables 4.9 and 4.10), totaling 17,554 individual lek count surveys. Mean male count was 10.81 (95-percent confidence level=10.6610.96) for leks within NCs that were within or overlapped Idaho. Mean male count for leks within Idaho was 10.69 (95-percent confidence level=10.53-10.85).

\section{Idaho Population Trend Analysis (Objective 3)}

Although Idaho intersected CC-E and CC-F, a large majority of area (98.1 percent) fell within CC-E. For CC-E, we estimated six population abundance nadirs (troughs) that dated back to 1960. Each of these population abundance nadirs represent between one and six complete periods of oscillation. We used these nadirs to estimate population trends across three different temporal scales that represented two, four, and six complete periods for the state (for instance, second, fourth, and sixth nadir). We estimated the average annual finite rate of population change $(\hat{\lambda})$ at the short (two periods), medium (four periods), and long (six periods) temporal scales as 0.970 (95-percent $\mathrm{CRI}=0.960-0.974), 0.971$ (95-percent $\mathrm{CRI}=0.956-0.978$ ), and 0.969 (95-percent $\mathrm{CRI}=0.964-0.973$ ), respectively (fig. 4.30). For all NCs that were included in the model and intersected Idaho, we estimated median $\hat{\lambda}$ to be less than 1.0 for $81.7,87.3$, and 94.4 percent across short, medium, and long temporal scales, respectively (table 4.10). We estimated median $\hat{\lambda}$ to be less than 1.0 for $71.8,74.6$, and 87.0 percent of all modeled leks within Idaho across short, medium, and long temporal scales, respectively. We reported spatial and temporal variation in average annual $\hat{\lambda}$ across different NCs (fig. 4.31) and leks (fig. 4.32).

\section{Idaho Targeted Annual Warning System Analysis (Objective 4)}

During 1990-2019, the TAWS for sage-grouse populations in Idaho activated a total of 486 and 353 leks as watches and warnings, respectively (fig. 4.33), which was 61.1 and 44.4 percent of the sampled leks used in the analysis. On average, across the 29 years, approximately 5.7 and 1.8 percent of leks per year experienced watches and warnings, respectively. The higher percentage for watches corresponds to repetitive activation. During this time frame, the TAWS also activated a total of 30 and $18 \mathrm{NCs}$ as watches and warnings, respectively (fig. 4.34), which was 46.2 and 27.7 percent of the sampled clusters used in the analysis. On average, approximately 2.3 and 1.1 percent of clusters per year experienced watches and warnings, respectively. Similar to leks, the higher percentage for watches corresponds to repetitive activation. During 2019, the TAWS activated 65 (first $=26$ ) watches and 77 (first $=31$ ) warnings at leks (fig. 4.35), as well as 2 (first=2) watches and $8($ first $=2)$ warnings at NCs (fig. 4.36). 


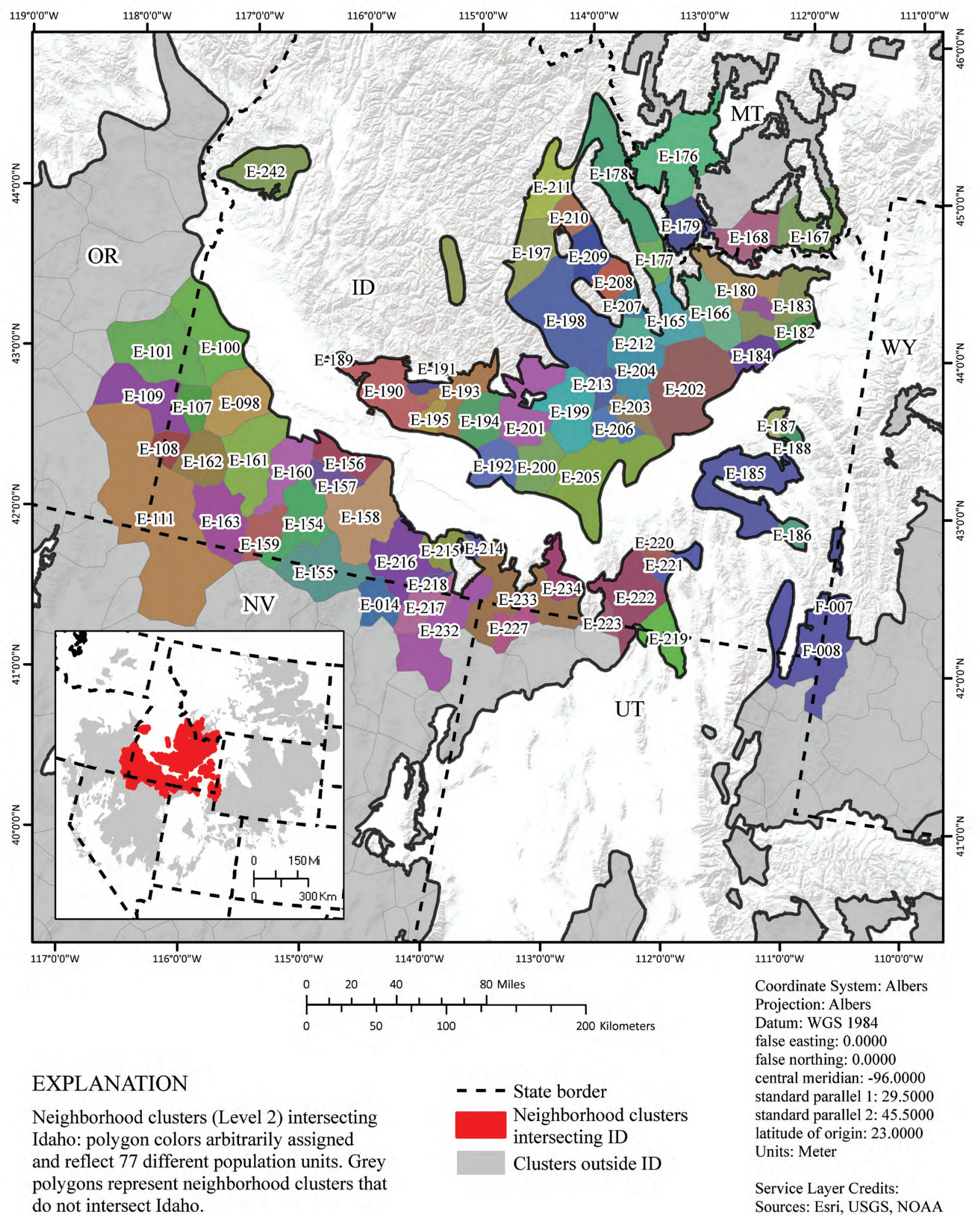

Figure 4.29. Greater sage-grouse (Centrocercus urophasianus) hierarchical population monitoring framework for neighborhood clusters that intersect the state of Idaho. Map image is the intellectual property of Esri and is used herein under license. Copyright (C) 2020 Esri and its licensors. All rights reserved. 
Table 4.9. Table of greater sage-grouse (Centrocercus urophasianus) average annual rate of population change $(\hat{\lambda})$ across six periods of oscillation in abundance that represent temporal scales for each neighborhood cluster within the state of Idaho. Estimates were derived from leks within the entire climate cluster.

[CC, climate cluster; E, Great Basin area; F, Wyoming]

\begin{tabular}{|c|c|c|c|c|c|c|c|c|c|}
\hline \multirow[b]{2}{*}{ CC } & \multirow{2}{*}{$\begin{array}{l}\text { Percent } \\
\text { CC1 }\end{array}$} & \multicolumn{6}{|c|}{ Temporal scales 2} & \multirow[b]{2}{*}{ Number of leks ${ }^{3}$} & \multirow{2}{*}{$\begin{array}{l}\text { Average } \\
\text { count/lek }\end{array}$} \\
\hline & & Long & Medium/Long & Medium & $\begin{array}{c}\text { Short/ } \\
\text { Medium }\end{array}$ & Short & Recent & & \\
\hline $\mathrm{F}$ & 1.5 & $\begin{array}{c}0.980 \\
(0.975-0.987)\end{array}$ & $\begin{array}{c}0.976 \\
(0.969-0.984)\end{array}$ & $\begin{array}{c}0.976 \\
(0.966-0.980)\end{array}$ & $\begin{array}{c}1.003 \\
(0.997-1.008)\end{array}$ & $\begin{array}{c}0.991 \\
(0.988-0.995)\end{array}$ & $\begin{array}{c}1.016 \\
(1.011-1.023)\end{array}$ & $\begin{array}{c}1,253 \\
(974: 14,892: 13)\end{array}$ & $\begin{array}{c}23.7 \\
(23.3-24.2)\end{array}$ \\
\hline
\end{tabular}

${ }^{1}$ The percent of each climate cluster that intersects the state.

2Temporal scales were estimated from present to each major population abundance nadir (trough) since 1960. Number of temporal scales were used to estimate population trends across six different temporal scales from approximately 10 to approximately 60 years.

${ }^{3}$ Number of leks in database. In parentheses from left to right is (1) total number in cluster were used in trend analysis, (2) number used in trend analysis within state boundary, (3) total number in cluster were used in the targeted annual warning system (TAWS) analysis, and (4) number used in TAWS analysis within state boundary.

Table 4.10. Table of greater sage-grouse (Centrocercus urophasianus) average annual rate of population change $(\hat{\lambda})$ across six periods of oscillation in abundance that represent temporal scales for each neighborhood cluster within the state of Idaho. Estimates were derived from leks within the entire neighborhood cluster.

[NC, neighborhood cluster; NA, not applicable]

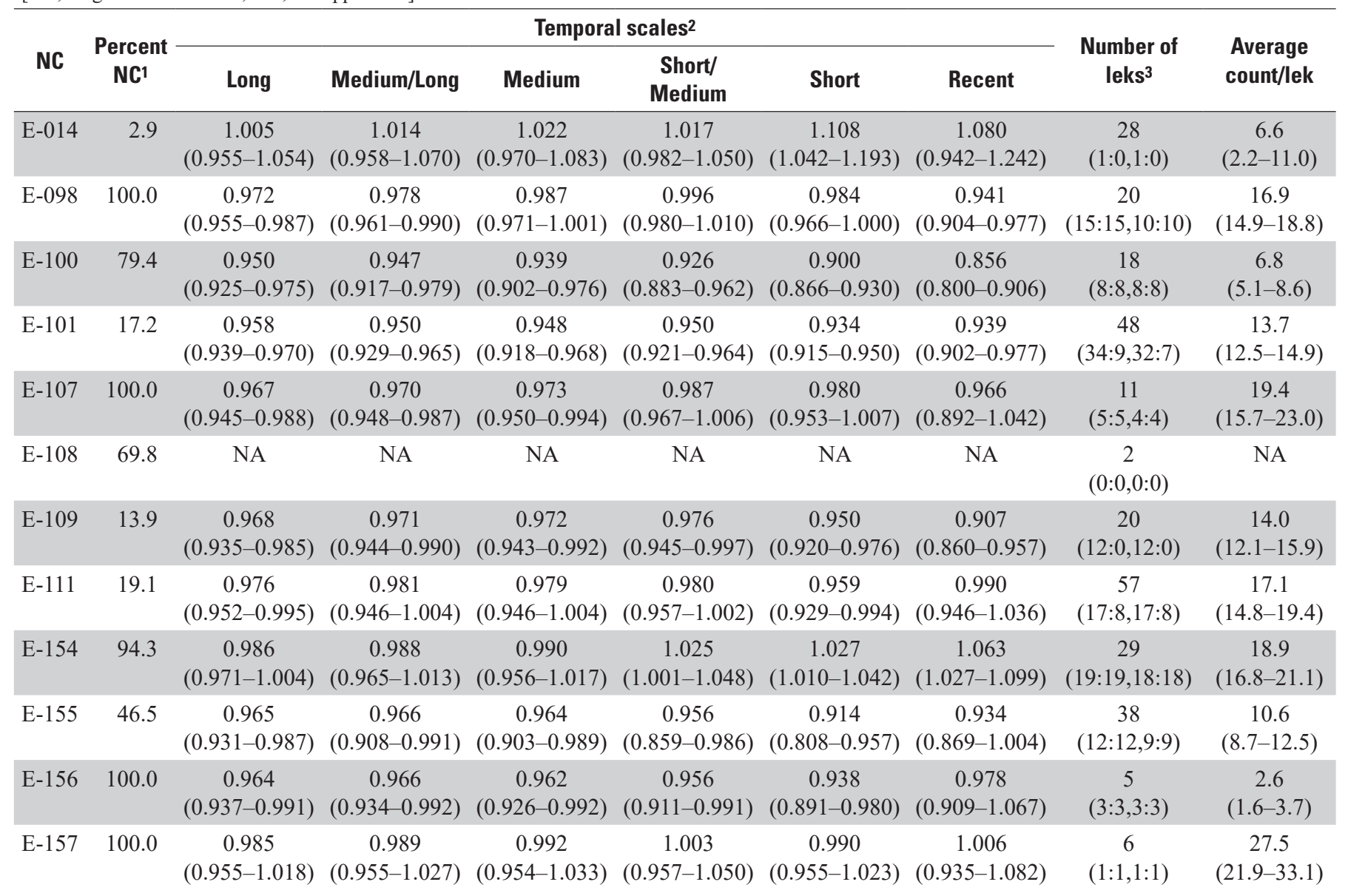


Table 4.10. Table of greater sage-grouse (Centrocercus urophasianus) average annual rate of population change $(\hat{\lambda})$ across six periods of oscillation in abundance that represent temporal scales for each neighborhood cluster within the state of Idaho. Estimates were derived from leks within the entire neighborhood cluster.-Continued

[NC, neighborhood cluster; NA, not applicable]

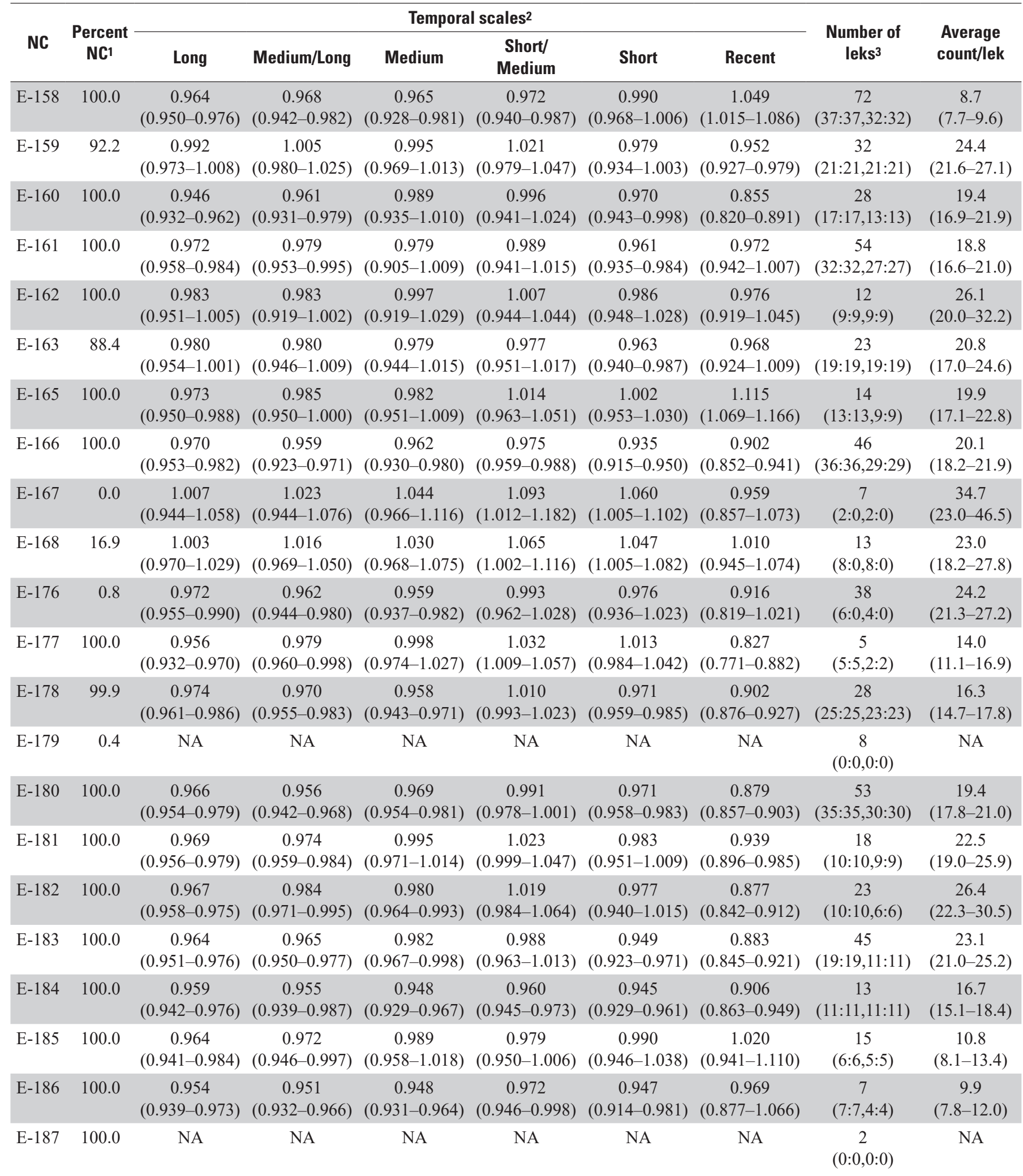


Table 4.10. Table of greater sage-grouse (Centrocercus urophasianus) average annual rate of population change $(\hat{\lambda})$ across six periods of oscillation in abundance that represent temporal scales for each neighborhood cluster within the state of Idaho. Estimates were derived from leks within the entire neighborhood cluster.-Continued

[NC, neighborhood cluster; NA, not applicable]

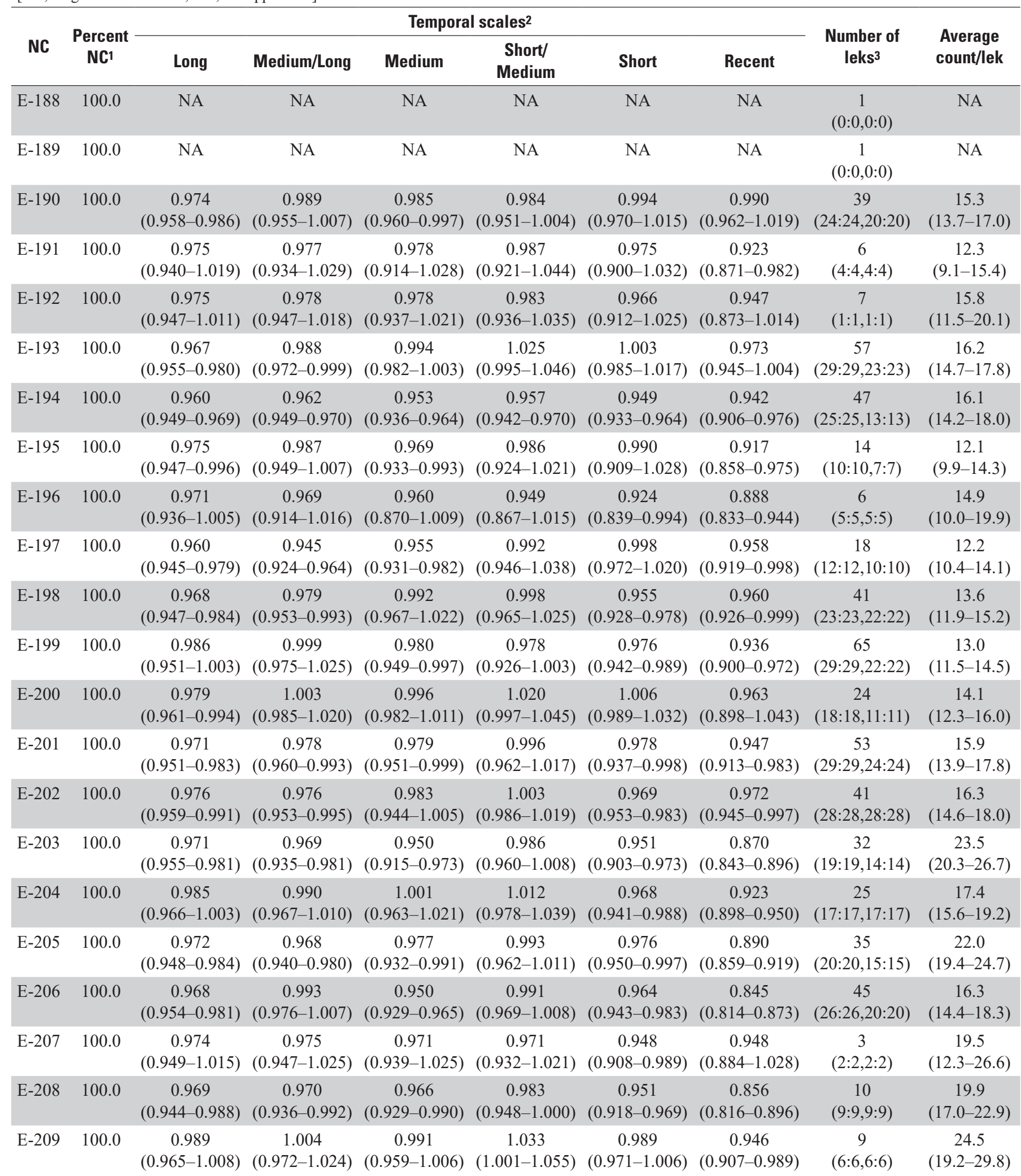


Table 4.10. Table of greater sage-grouse (Centrocercus urophasianus) average annual rate of population change $(\hat{\lambda})$ across six periods of oscillation in abundance that represent temporal scales for each neighborhood cluster within the state of Idaho. Estimates were derived from leks within the entire neighborhood cluster.-Continued

[NC, neighborhood cluster; NA, not applicable]

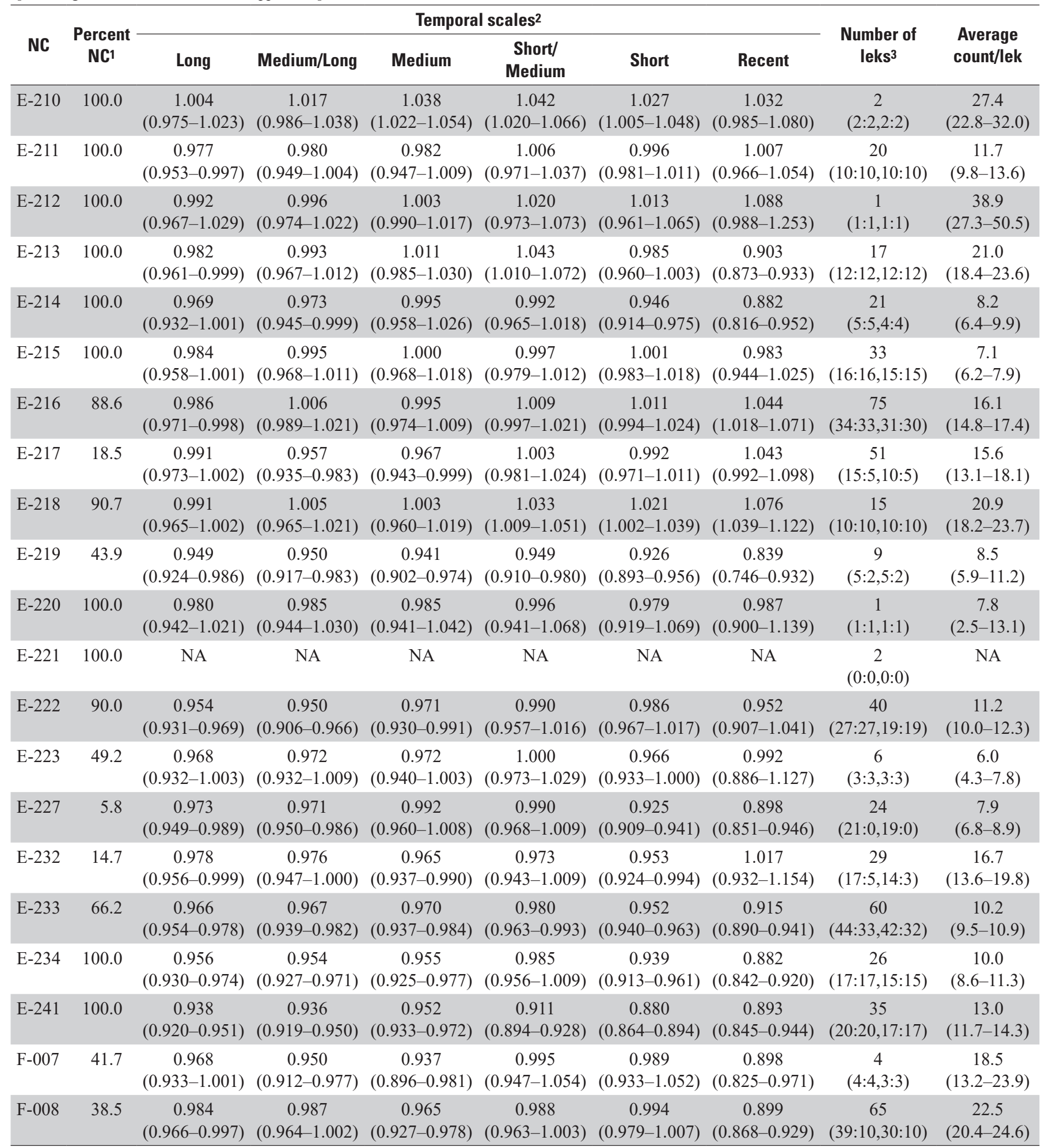



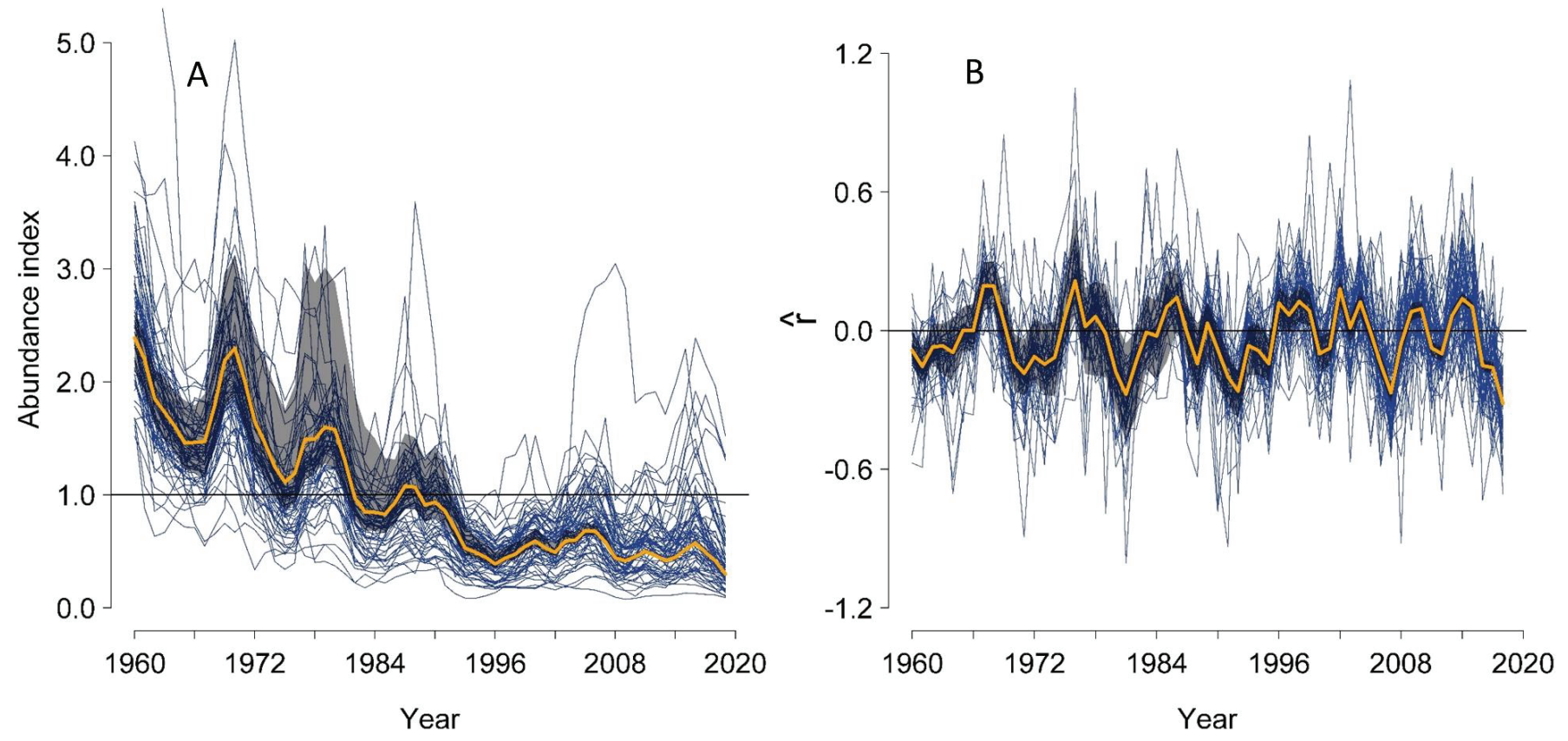

Figure 4.30. $A$, Abundance index (calculated as $\hat{N}$ divided by 60 -year mean of $\hat{N}$ ); and $B$, intrinsic rate of population change ( $\hat{r}$ ) of greater sage-grouse (Centrocercus urophasianus) within the state of Idaho from 1960-2019. Thick yellow line represents median estimates across all leks. Shaded areas represent 95-percent credible limits. Thin blue lines represent median values for neighborhood clusters. Black horizontal line (abundance index $=1.0$ ) represents 60 -year average. 

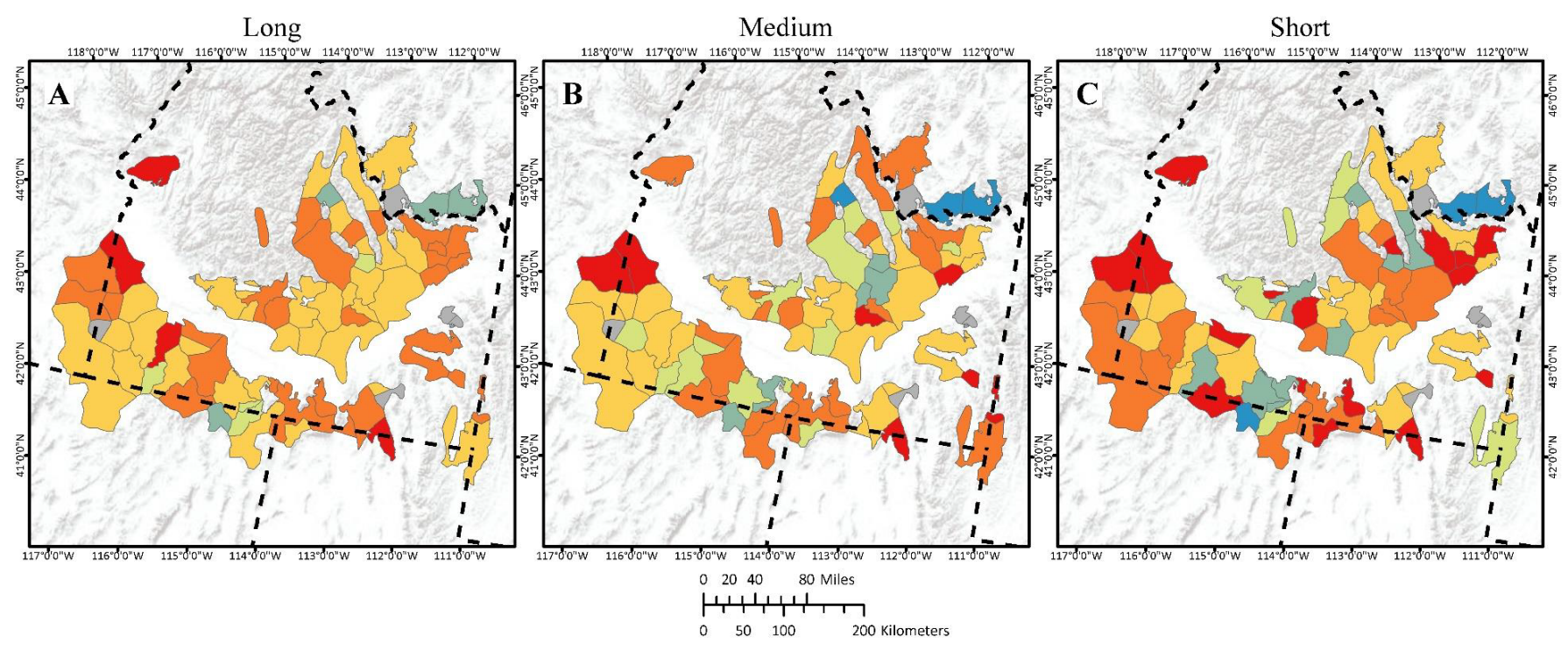

\section{EXPLANATION}

Neighborhood clusters (Level 2) intersecting Idaho: polygon colors represent average lambda values for sage-grouse populations over varying lengths of time (long, medium, short). Symbols are based on a range-wide distribution of values and all may not be represented here.
Average lambda $(\hat{\lambda})$

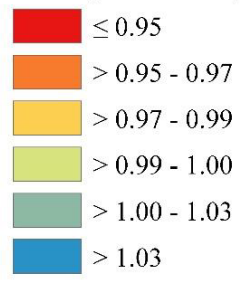

Coordinate System: Albers Projection: Albers Datum: WGS 1984 false easting: 0.0000 false northing: 0.0000 central meridian: -96.0000 standard parallel $1: 29.5000$ standard parallel 2: 45.5000 latitude of origin: 23.0000 Units: Meter

Service Layer Credits: Sources: Esri, USGS, NOAA

Figure 4.31. Spatial estimates of population trends across three temporal scales based on periods of oscillation (short, medium, and long) while accounting for periods of oscillation of greater sage-grouse (Centrocercus urophasianus) at different neighborhood clusters within the state of Idaho. Map images are the intellectual property of Esri and are used herein under license. Copyright $(2020$ Esri and its licensors. All rights reserved. 


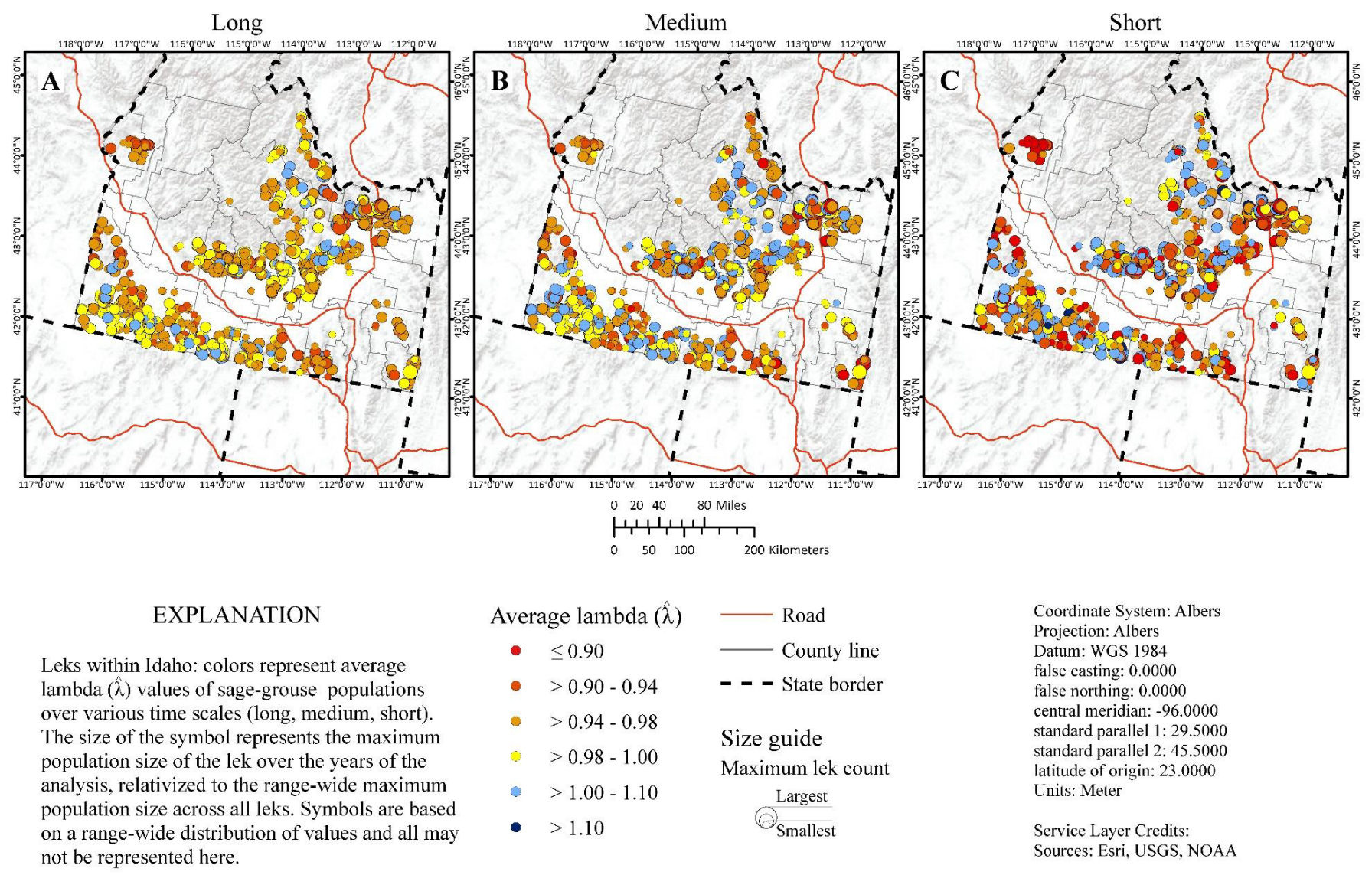

Figure 4.32. Spatial estimates of population trends across three temporal scales based on periods of oscillation (short, medium, and long) while accounting for periods of oscillation of greater sage-grouse (Centrocercus urophasianus) at lek sites within the state of Idaho. Map images are the intellectual property of Esri and are used herein under license. Copyright (C) 2020 Esri and its licensors. All rights reserved. 


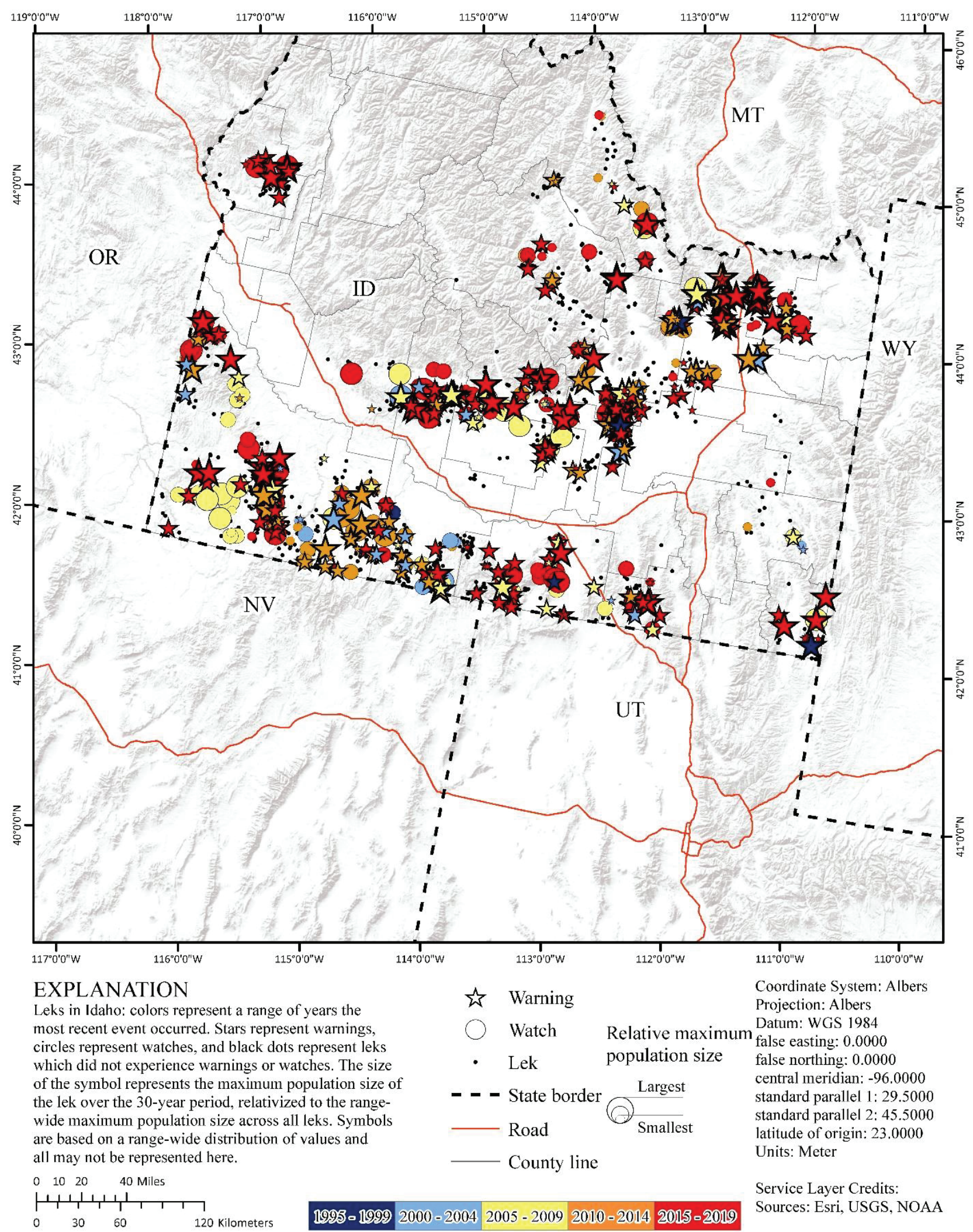

Figure 4.33. Spatial and temporal depiction of watches and warnings of greater sage-grouse (Centrocercus urophasianus) population declines at the lek scale within the state of Idaho from 1990 to 2019. Map image is the intellectual property of Esri and is used herein under license. Copyright $(C 2020$ Esri and its licensors. All rights reserved. 


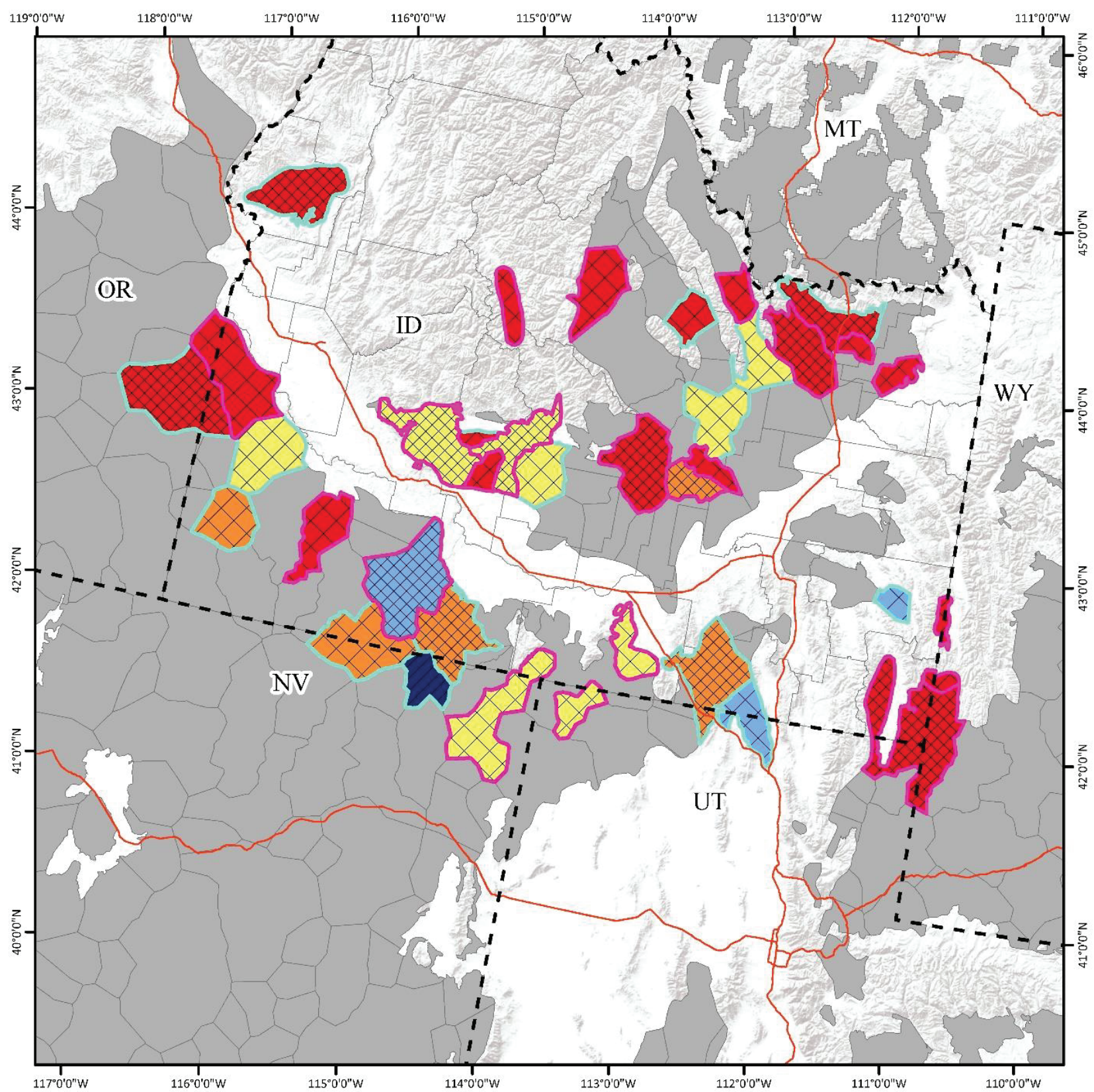

\section{EXPLANATION}

Neighborhood clusters (Level 2) in Idaho: polygon colors represent a range of years the most recent event occurred. Outline colors indicate whether the most recent event was a watch or warning. The hatching represents the maximum population size of the cluster over the 30 -year period, relativized to the range-wide maximum population size across all neighborhood clusters. Symbols are based on a range-wide distribution of values and all may not be represented here. 051020 Miles |ा, $0 \quad 20 \quad 40 \quad 80$ Kilometers 1995-1999 2000-2004 2005- $20092010-20142015-2019$
- - - State border
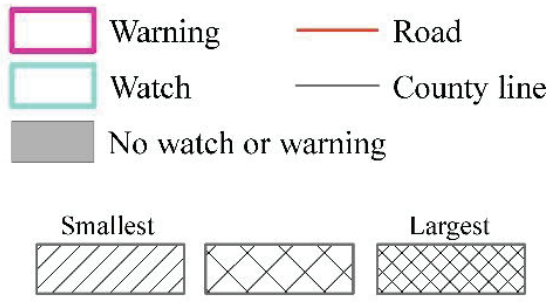

Coordinate System: Albers Projection: Albers Datum: WGS 1984 false easting: 0.0000 false northing: 0.0000 central meridian: -96.0000 standard parallel $1: 29.5000$ standard parallel 2: 45.5000 latitude of origin: 23.0000 Units: Meter

Service Layer Credits: Sources: Esri, USGS, NOAA

Figure 4.34. Spatial and temporal depiction of watches and warnings of greater sage-grouse (Centrocercus urophasianus) population declines at neighborhood cluster within the state of Idaho from 1990 to 2019. Map image is the intellectual property of Esri and is used herein under license. Copyright (C) 2020 Esri and its licensors. All rights reserved. 


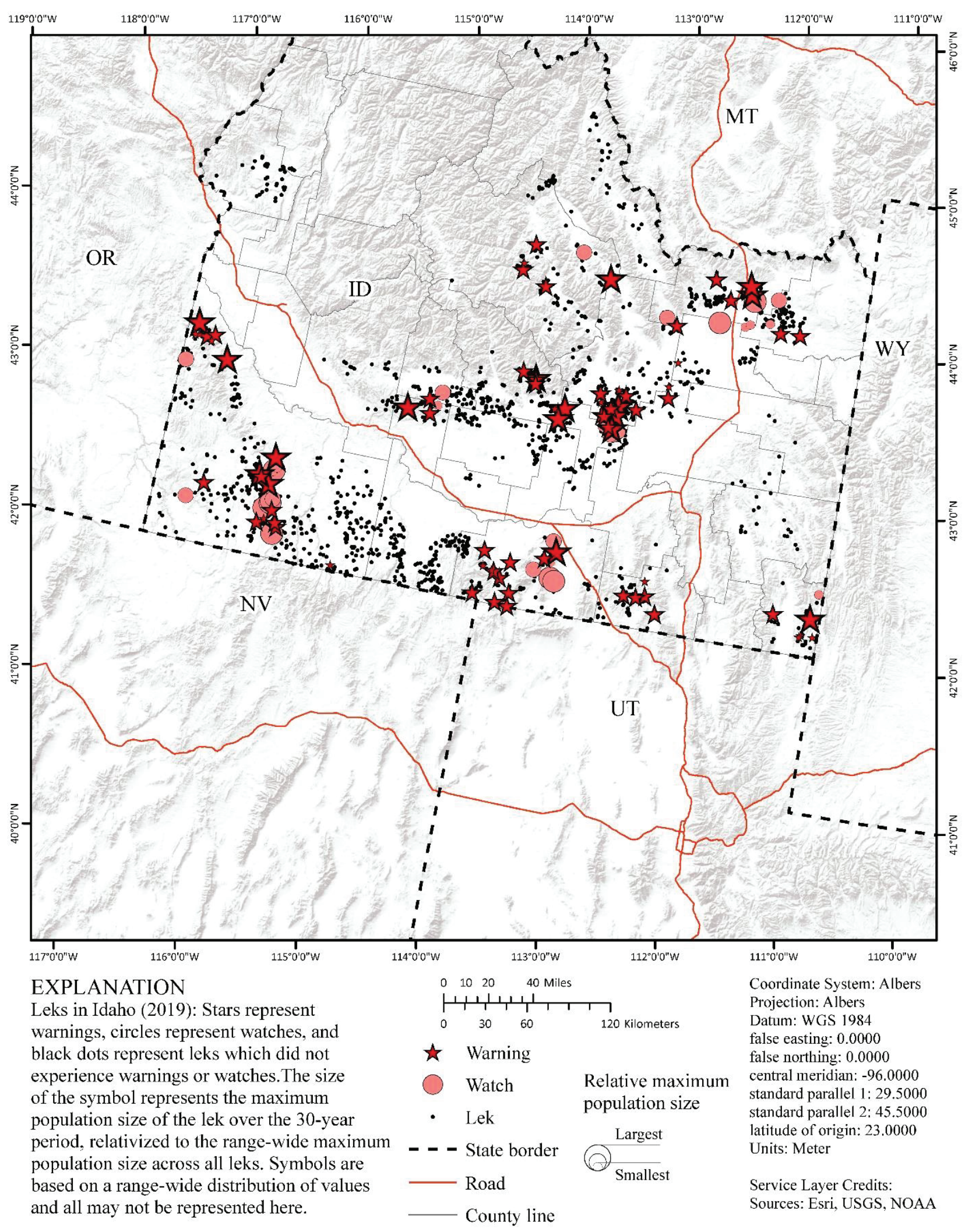

Figure 4.35. Spatial and temporal depiction of watches and warnings of greater sage-grouse (Centrocercus urophasianus) population declines at the lek and cluster scale within the state of Idaho during 2019. Map image is the intellectual property of Esri and is used herein under license. Copyright (c) 2020 Esri and its licensors. All rights reserved. 


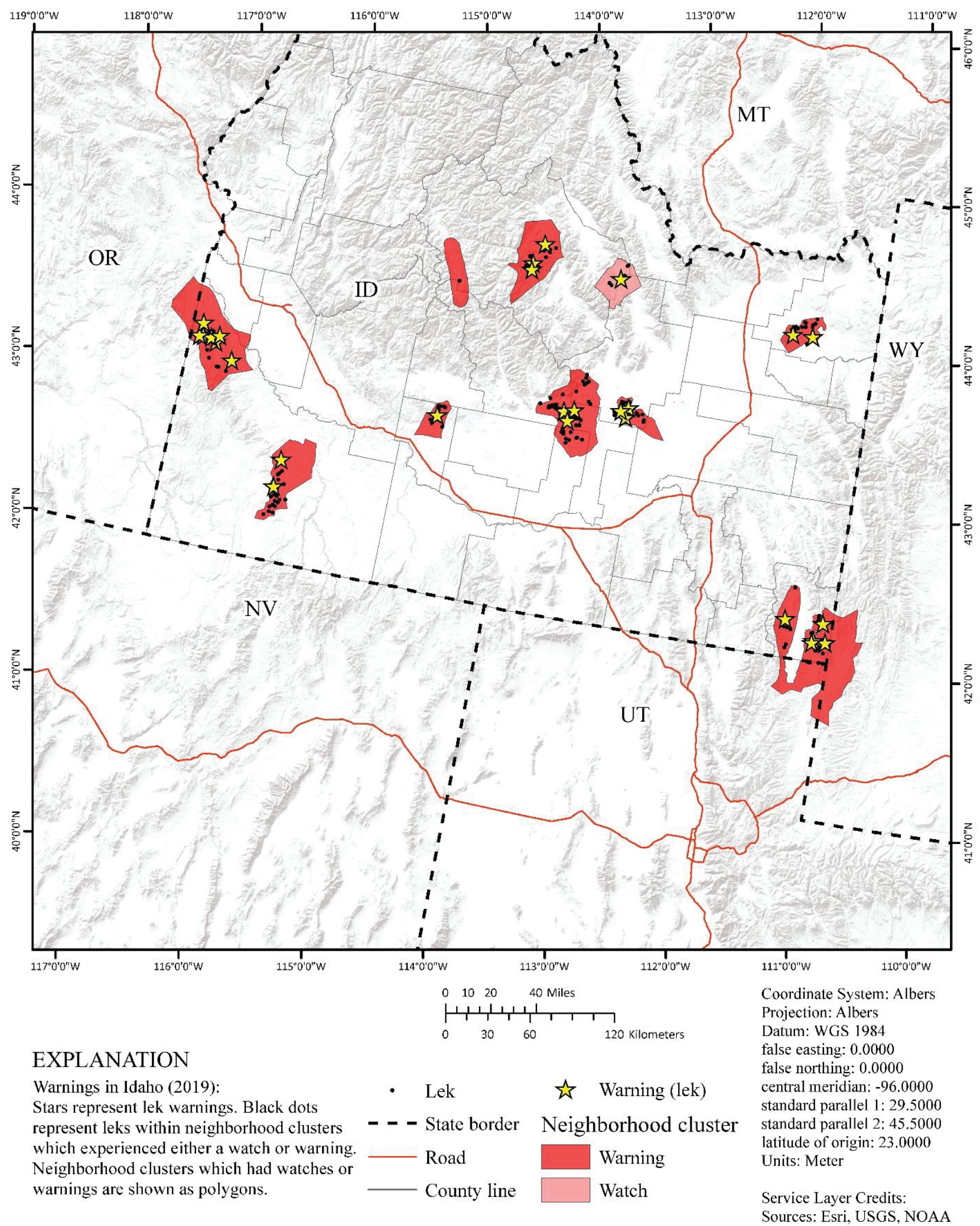

Figure 4.36. Spatial and temporal depiction of watches and warnings of greater sage-grouse (Centrocercus urophasianus) population declines at the neighborhood cluster scale within the state of Idaho during 2019. All leks within watch and warning boundaries were assigned as watch and warning, respectively. Yellow stars represent leks that reached warning independently. Map image is the intellectual property of Esri and is used herein under license. Copyright (c) 2020 Esri and its licensors. All rights reserved. 


\section{Utah Results}

\section{Utah Lek Data and Clusters (Objectives 1 and 2)}

Utah intersected CC-D, CC-E, and CC-F and contained or intersected 60 different NCs (fig. 4.37). The total area of the $60 \mathrm{NCs}$ within Utah was 4,284,583 ha. There were 56 clusters used in the analysis, after $4 \mathrm{NCs}$ were omitted because of limitations in sample sizes. These areas consisted of 728 leks, 457 of which were located within the Utah state boundary representing 5.43 percent of the range-wide lek database. After extensive QA/QC, we used 368 leks from Utah in the SSM for population trend estimation (tables 4.11 and 4.12), totaling 8,954 individual lek counts. Mean male lek count was 14.31 (95-percent confidence interval=13.99-14.63) for leks within NCs that were within or overlapped Utah. Mean male count for leks within Utah was 15.65 (95-percent confidence interval $=15.20-16.11$ ).

\section{Utah Population Trend Analysis (Objective 3)}

Although Utah interested three CCs, the cluster that consisted of the most area (42.8 percent) was CC-E. For CC-E, we estimated six population abundance nadirs (troughs) that dated back to 1960. Each of these population abundance nadirs represent between one and six complete periods of oscillation. We used these nadirs to estimate population trends across three different temporal scales that represented two, four, and six complete periods for the state (for instance, second, fourth, and sixth nadir). We estimated the average annual finite rate of population change $(\hat{\lambda})$ at the short (two periods), medium (four periods), and long (six periods) temporal scales as 0.955 (95-percent $\mathrm{CRI}=0.949-0.963), 0.974$ (95-percent $\mathrm{CRI}=0.965-0.979)$, and 0.966 (95-percent CRI $=0.960-0.973$ ), respectively (fig. 4.38). We estimated median $\hat{\lambda}$ to be less than 1.0 for $82.1,82.1$, and 89.3 percent of all modeled leks within Utah across short, medium, and long temporal scales, respectively. For all NCs that were modeled and intersected Utah, we estimated median $\hat{\lambda}$ to be less than 1.0 for 83.4 , 79.3 , and 87.8 percent across short, medium, and long temporal scales, respectively. We reported spatial and temporal variation in average annual $\hat{\lambda}$ across different NCs (fig. 4.39) and leks (fig. 4.40).

\section{Utah Targeted Annual Warning System Analysis (Objective 4)}

During 1990-2019, the TAWS for sage-grouse populations in Utah activated a total of 251 and 193 leks as watches and warnings, respectively (fig. 4.41), which was 78.2 and 60.1 percent of the sampled leks used in the analysis. On average, across the 29 years, approximately 7.4 and 2.4 percent of leks per year experienced watches and warnings, respectively. The higher percentage for watches corresponds to repetitive activation. During this time frame, the TAWS also activated a total of 29 and $15 \mathrm{NCs}$ as watches and warnings, respectively (fig. 4.42), which was 61.7 and 31.9 percent of the sampled clusters used in the analysis. On average, across the 29 years, approximately 3.6 and 1.3 percent of clusters per year experienced watches and warnings, respectively. Similar to leks, the higher percentage for watches corresponds to repetitive activation. During 2019 only, the TAWS activated 64 (first=11) watches and 61 (first=12) warnings at leks (fig. 4.43), as well as 2 (first $=0$ ) watches and 4 (first=1) warning at NCs (fig. 4.44). 

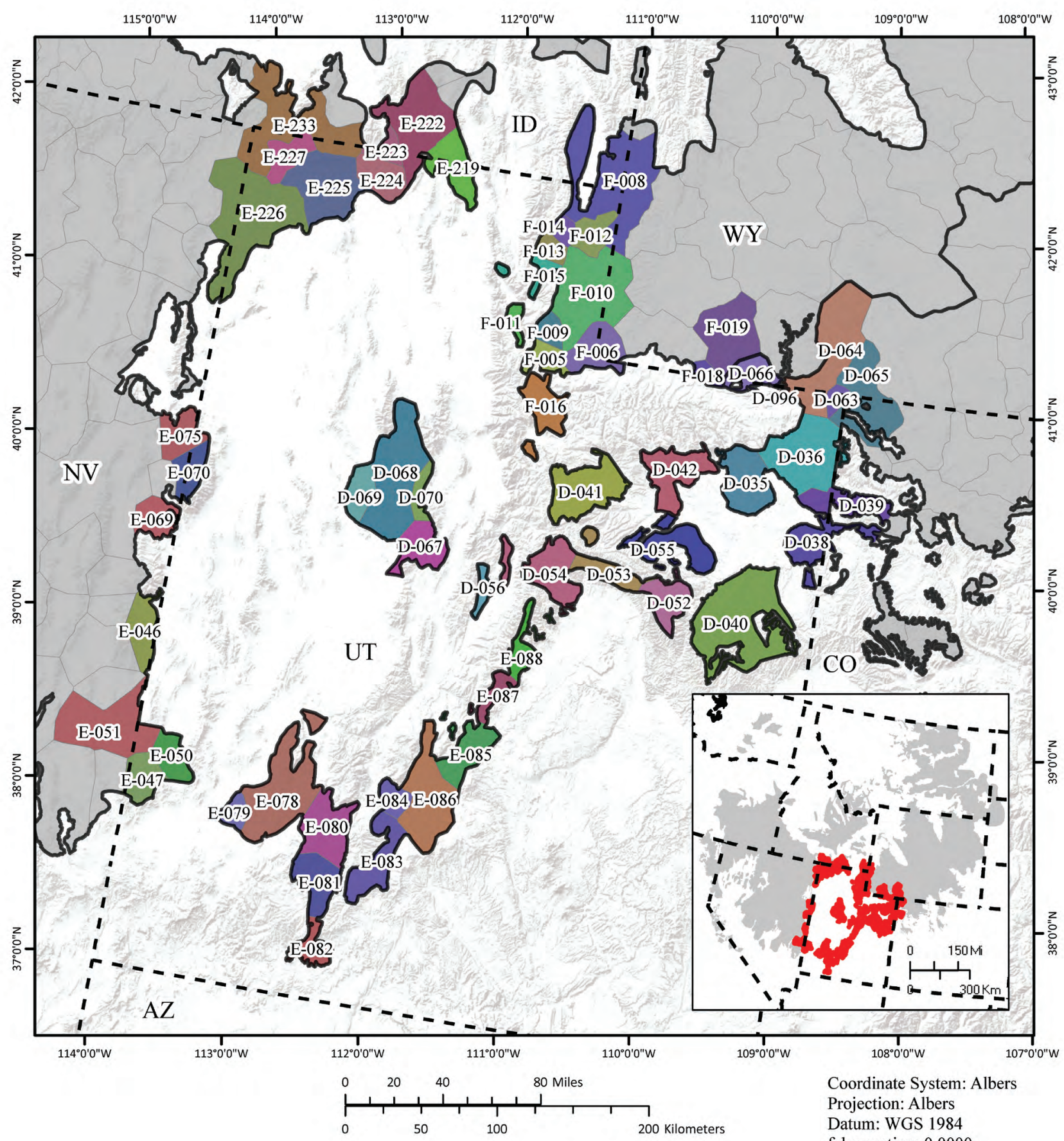

Coordinate System: Albers Projection: Albers Datum: WGS 1984 false easting: 0.0000 false northing: 0.0000

Neighborhood clusters (Level 2) intersecting Utah: polygon colors arbitrarily assigned and reflect 60 different population units. Grey polygons represent neighborhood clusters that do not intersect Utah. central meridian: -96.0000 standard parallel 1: 29.5000 standard parallel $2: 45.5000$ latitude of origin: 23.0000 Units: Meter

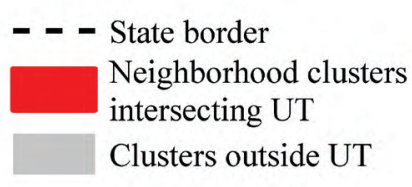

Service Layer Credits: Sources: Esri, USGS, NOAA

Figure 4.37. Greater sage-grouse (Centrocercus urophasianus) hierarchical population monitoring framework for neighborhood clusters that intersect the state of Utah. Map image is the intellectual property of Esri and is used herein under license. Copyright (C) 2020 Esri and its licensors. All rights reserved. 
Table 4.11. Table of greater sage-grouse (Centrocercus urophasianus) average annual rate of population change $(\hat{\lambda})$ across six periods of oscillation in abundance that represent temporal scales for each climate cluster within the state of Utah. Estimates were derived from leks within the entire climate cluster.

[CC, climate cluster; D, eastern area; E, Great Basin area; F, Wyoming]

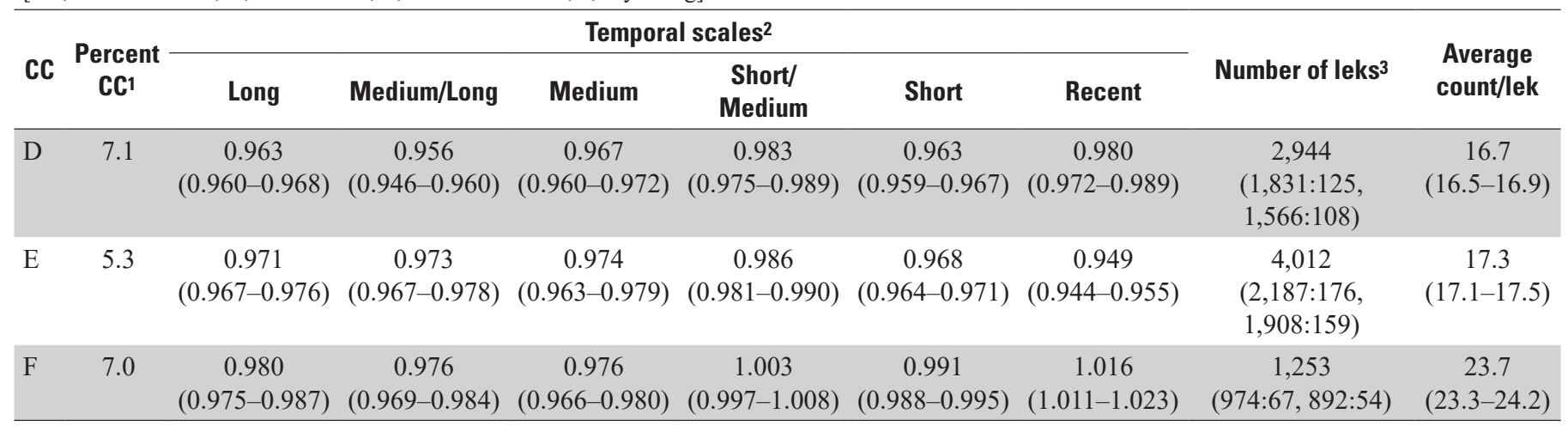

1The percent of each climate cluster that intersects the state.

${ }^{2}$ Temporal scales were estimated from present to each major population abundance nadir (trough) since 1960. Number of temporal scales were used to estimate population trends across six different temporal scales from approximately 10 to approximately 60 years.

${ }^{3}$ Number of leks in database. In parentheses from left to right is (1) total number in cluster were used in trend analysis, (2) number used in trend analysis within state boundary, (3) total number in cluster were used in the targeted annual warning system (TAWS) analysis, and (4) number used in TAWS analysis within state boundary.

Table 4.12. Table of greater sage-grouse (Centrocercus urophasianus) average annual rate of population change $(\hat{\lambda})$ across six periods of oscillation in abundance that represent temporal scales for each neighborhood cluster within the state of Utah. Estimates were derived from leks within the entire neighborhood cluster.

[NC, neighborhood cluster; NA, not applicable]

\begin{tabular}{|c|c|c|c|c|c|c|c|c|c|}
\hline \multirow[b]{2}{*}{ NC } & \multirow{2}{*}{$\begin{array}{c}\text { Percent } \\
\text { NC1 }\end{array}$} & \multicolumn{6}{|c|}{ Temporal scales² } & \multirow{2}{*}{$\begin{array}{c}\text { Number of } \\
\text { leks }^{3}\end{array}$} & \multirow{2}{*}{$\begin{array}{l}\text { Average } \\
\text { count/lek }\end{array}$} \\
\hline & & Long & Medium/Long & Medium & $\begin{array}{c}\text { Short/ } \\
\text { Medium }\end{array}$ & Short & Recent & & \\
\hline D-036 & 96.7 & $\begin{array}{c}0.952 \\
(0.938-0.962)\end{array}$ & $\begin{array}{c}0.950 \\
(0.930-0.964)\end{array}$ & $\begin{array}{c}0.969 \\
(0.947-0.984)\end{array}$ & $\begin{array}{c}0.965 \\
(0.947-0.980)\end{array}$ & $\begin{array}{c}0.919 \\
(0.903-0.932)\end{array}$ & $\begin{array}{c}0.734 \\
(0.711-0.759)\end{array}$ & $\begin{array}{c}48 \\
(38: 36,36: 34)\end{array}$ & $\begin{array}{c}18.3 \\
(16.0-20.6)\end{array}$ \\
\hline D-039 & 35.8 & $\begin{array}{c}0.954 \\
(0.930-0.979)\end{array}$ & $\begin{array}{c}0.928 \\
(0.898-0.968)\end{array}$ & $\begin{array}{c}0.932 \\
(0.884-0.972)\end{array}$ & $\begin{array}{c}0.947 \\
(0.905-0.976)\end{array}$ & $\begin{array}{c}0.874 \\
(0.833-0.905)\end{array}$ & $\begin{array}{c}0.763 \\
(0.726-0.798)\end{array}$ & $\begin{array}{c}17 \\
(13: 5,13: 5)\end{array}$ & $\begin{array}{c}21.9 \\
(17.5-26.3)\end{array}$ \\
\hline D-040 & 100.0 & $\begin{array}{c}0.933 \\
(0.903-0.957)\end{array}$ & $\begin{array}{c}0.916 \\
(0.893-0.938)\end{array}$ & $\begin{array}{c}0.911 \\
(0.894-0.928)\end{array}$ & $\begin{array}{c}0.884 \\
(0.859-0.910)\end{array}$ & $\begin{array}{c}0.881 \\
(0.844-0.920)\end{array}$ & $\begin{array}{c}0.952 \\
(0.839-1.085)\end{array}$ & $\begin{array}{c}8 \\
(4: 4,3: 3)\end{array}$ & $\begin{array}{c}8.5 \\
(6.2-10.8)\end{array}$ \\
\hline D-052 & 100.0 & $\begin{array}{c}1.007 \\
(0.989-1.022)\end{array}$ & $\begin{array}{c}0.997 \\
(0.975-1.013)\end{array}$ & $\begin{array}{c}1.012 \\
(0.975-1.047)\end{array}$ & $\begin{array}{c}1.031 \\
(0.999-1.062)\end{array}$ & $\begin{array}{c}0.986 \\
(0.964-1.009)\end{array}$ & $\begin{array}{c}0.894 \\
(0.837-0.948)\end{array}$ & $\begin{array}{c}7 \\
(5: 5,5: 5)\end{array}$ & $\begin{array}{c}11.0 \\
(8.7-13.3)\end{array}$ \\
\hline D-053 & 100.0 & $\begin{array}{c}0.968 \\
(0.943-1.002)\end{array}$ & $\begin{array}{c}0.965 \\
(0.946-0.977)\end{array}$ & $\begin{array}{c}0.977 \\
(0.949-1.002)\end{array}$ & $\begin{array}{c}1.000 \\
(0.982-1.018)\end{array}$ & $\begin{array}{c}0.982 \\
(0.961-1.002)\end{array}$ & $\begin{array}{c}0.933 \\
(0.879-0.993)\end{array}$ & $\begin{array}{c}11 \\
(10: 10,9: 9)\end{array}$ & $\begin{array}{c}12.0 \\
(10.2-13.9)\end{array}$ \\
\hline D-054 & 100.0 & $\begin{array}{c}0.973 \\
(0.952-0.998)\end{array}$ & $\begin{array}{c}0.969 \\
(0.939-0.999)\end{array}$ & $\begin{array}{c}0.983 \\
(0.948-1.018)\end{array}$ & $\begin{array}{c}0.996 \\
(0.951-1.041)\end{array}$ & $\begin{array}{c}0.978 \\
(0.926-1.033)\end{array}$ & $\begin{array}{c}0.949 \\
(0.891-1.007)\end{array}$ & $\begin{array}{c}5 \\
(5: 5,3: 3)\end{array}$ & $\begin{array}{c}9.0 \\
(6.3-11.7)\end{array}$ \\
\hline D-055 & 100.0 & $\begin{array}{c}0.978 \\
(0.946-1.010)\end{array}$ & $\begin{array}{c}0.969 \\
(0.940-0.991)\end{array}$ & $\begin{array}{c}1.002 \\
(0.968-1.032)\end{array}$ & $\begin{array}{c}0.977 \\
(0.927-1.025)\end{array}$ & $\begin{array}{c}0.959 \\
(0.917-0.996)\end{array}$ & $\begin{array}{c}0.900 \\
(0.832-0.971)\end{array}$ & $\begin{array}{c}9 \\
(6: 6,6: 6)\end{array}$ & $\begin{array}{c}6.7 \\
(5.1-8.3)\end{array}$ \\
\hline
\end{tabular}


Table 4.12. Table of greater sage-grouse (Centrocercus urophasianus) average annual rate of population change $(\hat{\lambda})$ across six periods of oscillation in abundance that represent temporal scales for each neighborhood cluster within the state of Utah. Estimates were derived from leks within the entire neighborhood cluster.-Continued

[NC, neighborhood cluster; NA, not applicable]

\begin{tabular}{|c|c|c|c|c|c|c|c|c|c|}
\hline \multirow[b]{2}{*}{ NC } & \multirow{2}{*}{$\begin{array}{c}\text { Percent } \\
\text { NC1 }\end{array}$} & \multicolumn{6}{|c|}{ Temporal scales } & \multirow{2}{*}{$\begin{array}{c}\text { Number of } \\
\text { leks }^{3}\end{array}$} & \multirow{2}{*}{$\begin{array}{l}\text { Average } \\
\text { count/lek }\end{array}$} \\
\hline & & Long & Medium/Long & Medium & $\begin{array}{c}\text { Short/ } \\
\text { Medium }\end{array}$ & Short & Recent & & \\
\hline D-063 & 63.4 & $\begin{array}{c}0.944 \\
(0.914-0.978)\end{array}$ & $\begin{array}{c}0.931 \\
(0.908-0.955)\end{array}$ & $\begin{array}{c}0.941 \\
(0.912-0.970)\end{array}$ & $\begin{array}{c}0.992 \\
(0.947-1.043)\end{array}$ & $\begin{array}{c}0.856 \\
(0.803-0.910)\end{array}$ & $\begin{array}{c}0.979 \\
(0.801-1.207)\end{array}$ & $\begin{array}{c}3 \\
(2: 1,1: 1)\end{array}$ & $\begin{array}{c}6.3 \\
(3.8-8.9)\end{array}$ \\
\hline D-065 & 4.7 & $\begin{array}{c}0.975 \\
(0.955-0.997)\end{array}$ & $\begin{array}{c}0.961 \\
(0.941-0.984)\end{array}$ & $\begin{array}{c}0.980 \\
(0.955-1.003)\end{array}$ & $\begin{array}{c}0.983 \\
(0.957-1.000)\end{array}$ & $\begin{array}{c}1.019 \\
(0.993-1.046)\end{array}$ & $\begin{array}{c}0.920 \\
(0.891-0.946)\end{array}$ & $\begin{array}{c}24 \\
(18: 0,18: 0)\end{array}$ & $\begin{array}{c}26.7 \\
(23.8-29.7)\end{array}$ \\
\hline D-066 & 18.7 & $\begin{array}{c}0.957 \\
(0.894-1.010)\end{array}$ & $\begin{array}{c}0.956 \\
(0.889-1.016)\end{array}$ & $\begin{array}{c}0.962 \\
(0.894-1.027)\end{array}$ & $\begin{array}{c}0.919 \\
(0.866-0.963)\end{array}$ & $\begin{array}{c}0.871 \\
(0.815-0.922)\end{array}$ & $\begin{array}{c}0.748 \\
(0.621-0.884)\end{array}$ & $\begin{array}{c}2 \\
(2: 0,2: 0)\end{array}$ & $\begin{array}{c}9.1 \\
(5.7-12.4)\end{array}$ \\
\hline D-069 & 100.0 & $\begin{array}{c}0.983 \\
(0.941-1.034)\end{array}$ & $\begin{array}{c}0.973 \\
(0.919-1.033)\end{array}$ & $\begin{array}{c}0.987 \\
(0.924-1.050)\end{array}$ & $\begin{array}{c}0.998 \\
(0.937-1.078)\end{array}$ & $\begin{array}{c}0.984 \\
(0.924-1.044)\end{array}$ & $\begin{array}{c}1.041 \\
(0.940-1.148)\end{array}$ & $\begin{array}{c}1 \\
(1: 1,1: 1)\end{array}$ & $\begin{array}{c}15.6 \\
(10.7-20.5)\end{array}$ \\
\hline D-070 & 100.0 & NA & NA & NA & NA & NA & NA & $\begin{array}{c}1 \\
(0: 0,0: 0)\end{array}$ & NA \\
\hline D-096 & 100.0 & NA & NA & NA & NA & NA & NA & $\begin{array}{c}0 \\
(0: 0,0: 0)\end{array}$ & NA \\
\hline E-046 & 2.4 & $\begin{array}{c}0.974 \\
(0.939-1.002)\end{array}$ & $\begin{array}{c}0.977 \\
(0.940-1.010)\end{array}$ & $\begin{array}{c}0.977 \\
(0.942-1.015)\end{array}$ & $\begin{array}{c}0.972 \\
(0.922-1.021)\end{array}$ & $\begin{array}{c}0.938 \\
(0.877-0.989)\end{array}$ & $\begin{array}{c}0.899 \\
(0.776-1.001)\end{array}$ & $\begin{array}{c}1 \\
(1: 0,1: 0)\end{array}$ & $\begin{array}{c}2.2 \\
(0.9-3.6)\end{array}$ \\
\hline E-047 & 94.7 & $\begin{array}{c}0.981 \\
(0.968-1.000)\end{array}$ & $\begin{array}{c}0.999 \\
(0.992-1.006)\end{array}$ & $\begin{array}{c}0.989 \\
(0.973-1.004)\end{array}$ & $\begin{array}{c}1.017 \\
(0.987-1.038)\end{array}$ & $\begin{array}{c}0.972 \\
(0.943-0.992)\end{array}$ & $\begin{array}{c}1.026 \\
(0.965-1.088)\end{array}$ & $\begin{array}{c}6 \\
(4: 4,4: 4)\end{array}$ & $\begin{array}{c}16.7 \\
(14.8-18.5)\end{array}$ \\
\hline E-070 & 63.2 & $\begin{array}{c}0.989 \\
(0.956-1.034)\end{array}$ & $\begin{array}{c}0.995 \\
(0.956-1.040)\end{array}$ & $\begin{array}{c}1.005 \\
(0.958-1.034)\end{array}$ & $\begin{array}{c}1.007 \\
(0.969-1.043)\end{array}$ & $\begin{array}{c}0.984 \\
(0.950-1.012)\end{array}$ & $\begin{array}{c}1.214 \\
(1.133-1.310)\end{array}$ & $\begin{array}{c}4 \\
(3: 3,3: 3)\end{array}$ & $\begin{array}{c}16.4 \\
(13.3-19.6)\end{array}$ \\
\hline E-075 & 12.6 & $\begin{array}{c}0.980 \\
(0.951-1.015)\end{array}$ & $\begin{array}{c}0.984 \\
(0.953-1.022)\end{array}$ & $\begin{array}{c}0.983 \\
(0.949-1.024)\end{array}$ & $\begin{array}{c}0.993 \\
(0.956-1.029)\end{array}$ & $\begin{array}{c}0.977 \\
(0.944-1.011)\end{array}$ & $\begin{array}{c}0.957 \\
(0.879-1.042)\end{array}$ & $\begin{array}{c}1 \\
(1: 0,1: 0)\end{array}$ & $\begin{array}{c}15.1 \\
(12.6-17.6)\end{array}$ \\
\hline E-078 & 100.0 & $\begin{array}{c}0.966 \\
(0.948-0.983)\end{array}$ & $\begin{array}{c}0.963 \\
(0.940-0.981)\end{array}$ & $\begin{array}{c}0.968 \\
(0.948-0.983)\end{array}$ & $\begin{array}{c}0.999 \\
(0.976-1.017)\end{array}$ & $\begin{array}{c}0.982 \\
(0.957-1.005)\end{array}$ & $\begin{array}{c}0.914 \\
(0.875-0.952)\end{array}$ & $\begin{array}{c}18 \\
(15: 15,11: 11)\end{array}$ & $\begin{array}{c}13.2 \\
(11.6-14.8)\end{array}$ \\
\hline E-079 & 100.0 & $\begin{array}{c}0.978 \\
(0.949-1.023)\end{array}$ & $\begin{array}{c}0.981 \\
(0.945-1.026)\end{array}$ & $\begin{array}{c}0.980 \\
(0.934-1.034)\end{array}$ & $\begin{array}{c}0.988 \\
(0.937-1.055)\end{array}$ & $\begin{array}{c}0.972 \\
(0.920-1.037)\end{array}$ & $\begin{array}{c}0.929 \\
(0.858-0.991)\end{array}$ & $\begin{array}{c}1 \\
(1: 1,1: 1)\end{array}$ & $\begin{array}{c}19.4 \\
(14.7-24.1)\end{array}$ \\
\hline E-080 & 100.0 & $\begin{array}{c}0.973 \\
(0.953-0.994)\end{array}$ & $\begin{array}{c}0.988 \\
(0.962-1.005)\end{array}$ & $\begin{array}{c}0.975 \\
(0.945-0.996)\end{array}$ & $\begin{array}{c}0.991 \\
(0.957-1.020)\end{array}$ & $\begin{array}{c}0.975 \\
(0.945-1.002)\end{array}$ & $\begin{array}{c}0.931 \\
(0.895-0.967)\end{array}$ & $\begin{array}{c}14 \\
(11: 11,10: 10)\end{array}$ & $\begin{array}{c}23.0 \\
(19.6-26.5)\end{array}$ \\
\hline E-081 & 100.0 & $\begin{array}{c}1.004 \\
(0.982-1.018)\end{array}$ & $\begin{array}{c}0.987 \\
(0.960-0.998)\end{array}$ & $\begin{array}{c}0.996 \\
(0.972-1.009)\end{array}$ & $\begin{array}{c}1.009 \\
(0.994-1.023)\end{array}$ & $\begin{array}{c}0.981 \\
(0.966-0.996)\end{array}$ & $\begin{array}{c}0.973 \\
(0.934-1.016)\end{array}$ & $\begin{array}{c}8 \\
(7: 7,6: 6)\end{array}$ & $\begin{array}{c}21.8 \\
(18.6-25.0)\end{array}$ \\
\hline E-082 & 100.0 & $\begin{array}{c}1.000 \\
(0.957-1.047)\end{array}$ & $\begin{array}{c}1.007 \\
(0.962-1.049)\end{array}$ & $\begin{array}{c}1.013 \\
(0.982-1.042)\end{array}$ & $\begin{array}{c}1.064 \\
(1.032-1.103)\end{array}$ & $\begin{array}{c}1.013 \\
(0.978-1.048)\end{array}$ & $\begin{array}{c}1.065 \\
(0.960-1.179)\end{array}$ & $\begin{array}{c}1 \\
(1: 1,1: 1)\end{array}$ & $\begin{array}{c}8.8 \\
(6.4-11.2)\end{array}$ \\
\hline
\end{tabular}


Table 4.12. Table of greater sage-grouse (Centrocercus urophasianus) average annual rate of population change $(\hat{\lambda})$ across six periods of oscillation in abundance that represent temporal scales for each neighborhood cluster within the state of Utah. Estimates were derived from leks within the entire neighborhood cluster.-Continued

[NC, neighborhood cluster; NA, not applicable]

\begin{tabular}{|c|c|c|c|c|c|c|c|c|c|}
\hline \multirow[b]{2}{*}{ NC } & \multirow[b]{2}{*}{$\begin{array}{c}\text { Percent } \\
\text { NC1 }\end{array}$} & \multicolumn{6}{|c|}{ Temporal scales ${ }^{2}$} & \multirow[b]{2}{*}{$\begin{array}{l}\text { Number of } \\
\text { leks }^{3}\end{array}$} & \multirow[b]{2}{*}{$\begin{array}{l}\text { Average } \\
\text { count/lek }\end{array}$} \\
\hline & & Long & Medium/Long & Medium & $\begin{array}{c}\text { Short/ } \\
\text { Medium }\end{array}$ & Short & Recent & & \\
\hline E-084 & 100.0 & $\begin{array}{c}0.993 \\
(0.960-1.023)\end{array}$ & $\begin{array}{c}0.994 \\
(0.957-1.031)\end{array}$ & $\begin{array}{c}0.994 \\
(0.950-1.032)\end{array}$ & $\begin{array}{c}0.985 \\
(0.957-1.007)\end{array}$ & $\begin{array}{c}0.949 \\
(0.919-0.974)\end{array}$ & $\begin{array}{c}1.008 \\
(0.938-1.077)\end{array}$ & $(3: 3,3: 3)$ & $\begin{array}{c}25.3 \\
(19.7-30.8)\end{array}$ \\
\hline E-086 & 100.0 & $\begin{array}{c}0.971 \\
(0.955-0.981)\end{array}$ & $\begin{array}{c}0.979 \\
(0.963-0.989)\end{array}$ & $\begin{array}{c}0.990 \\
(0.970-1.004)\end{array}$ & $\begin{array}{c}0.977 \\
(0.953-0.995)\end{array}$ & $\begin{array}{c}0.936 \\
(0.919-0.948)\end{array}$ & $\begin{array}{c}0.879 \\
(0.859-0.899)\end{array}$ & $\begin{array}{c}39 \\
(35: 35,33: 33)\end{array}$ & $\begin{array}{c}23.5 \\
(21.3-25.7)\end{array}$ \\
\hline E-087 & 100.0 & $\begin{array}{c}0.983 \\
(0.942-1.014)\end{array}$ & $\begin{array}{c}0.985 \\
(0.945-1.026)\end{array}$ & $\begin{array}{c}0.987 \\
(0.942-1.026)\end{array}$ & $\begin{array}{c}0.991 \\
(0.955-1.017)\end{array}$ & $\begin{array}{c}1.020 \\
(0.975-1.059)\end{array}$ & $\begin{array}{c}0.897 \\
(0.826-0.969)\end{array}$ & $\begin{array}{c}2 \\
(2: 2,2: 2)\end{array}$ & $\begin{array}{c}15.3 \\
(11.6-18.9)\end{array}$ \\
\hline E-222 & 10.0 & $\begin{array}{c}0.954 \\
(0.931-0.969)\end{array}$ & $\begin{array}{c}0.950 \\
(0.906-0.966)\end{array}$ & $\begin{array}{c}0.971 \\
(0.930-0.991)\end{array}$ & $\begin{array}{c}0.990 \\
(0.957-1.016)\end{array}$ & $\begin{array}{c}0.986 \\
(0.967-1.017)\end{array}$ & $\begin{array}{c}0.952 \\
(0.907-1.041)\end{array}$ & $\begin{array}{c}40 \\
(27: 0,19: 0)\end{array}$ & $\begin{array}{c}11.2 \\
(10.0-12.3)\end{array}$ \\
\hline E-223 & 50.8 & $\begin{array}{c}0.968 \\
(0.932-1.003)\end{array}$ & $\begin{array}{c}0.972 \\
(0.932-1.009)\end{array}$ & $\begin{array}{c}0.972 \\
(0.940-1.003)\end{array}$ & $\begin{array}{c}1.000 \\
(0.973-1.029)\end{array}$ & $\begin{array}{c}0.966 \\
(0.933-1.000)\end{array}$ & $\begin{array}{c}0.992 \\
(0.886-1.127)\end{array}$ & $\begin{array}{c}6 \\
(3: 0,3: 0)\end{array}$ & $\begin{array}{c}6.0 \\
(4.3-7.8)\end{array}$ \\
\hline E-224 & 100.0 & $\begin{array}{c}0.939 \\
(0.925-0.952)\end{array}$ & $\begin{array}{c}0.945 \\
(0.929-0.960)\end{array}$ & $\begin{array}{c}0.955 \\
(0.922-0.989)\end{array}$ & $\begin{array}{c}0.955 \\
(0.907-1.000)\end{array}$ & $\begin{array}{c}0.925 \\
(0.880-0.968)\end{array}$ & $\begin{array}{c}0.828 \\
(0.718-0.944)\end{array}$ & $\begin{array}{c}6 \\
(3: 3,1: 1)\end{array}$ & $\begin{array}{c}14.1 \\
(10.8-17.5)\end{array}$ \\
\hline E-225 & 100.0 & $\begin{array}{c}0.978 \\
(0.960-0.996)\end{array}$ & $\begin{array}{c}0.973 \\
(0.952-0.987)\end{array}$ & $\begin{array}{c}0.972 \\
(0.938-0.989)\end{array}$ & $\begin{array}{c}0.960 \\
(0.937-0.983)\end{array}$ & $\begin{array}{c}0.943 \\
(0.915-0.965)\end{array}$ & $\begin{array}{c}0.917 \\
(0.885-0.952)\end{array}$ & $\begin{array}{c}29 \\
(23: 23,22: 22)\end{array}$ & $\begin{array}{c}17.0 \\
(15.0-18.9)\end{array}$ \\
\hline E-226 & 64.7 & $\begin{array}{c}0.991 \\
(0.969-1.003)\end{array}$ & $\begin{array}{c}0.991 \\
(0.971-1.000)\end{array}$ & $\begin{array}{c}0.994 \\
(0.982-1.004)\end{array}$ & $\begin{array}{c}0.997 \\
(0.982-1.011)\end{array}$ & $\begin{array}{c}0.958 \\
(0.945-0.973)\end{array}$ & $\begin{array}{c}0.972 \\
(0.935-1.017)\end{array}$ & $\begin{array}{c}34 \\
(16: 14,16: 14)\end{array}$ & $\begin{array}{c}23.2 \\
(19.8-26.6)\end{array}$ \\
\hline F-006 & 60.9 & $\begin{array}{c}1.002 \\
(0.965-1.048)\end{array}$ & $\begin{array}{c}1.004 \\
(0.944-1.056)\end{array}$ & $\begin{array}{c}1.025 \\
(0.976-1.070)\end{array}$ & $\begin{array}{c}1.026 \\
(0.981-1.069)\end{array}$ & $\begin{array}{c}1.018 \\
(0.982-1.050)\end{array}$ & $\begin{array}{c}1.080 \\
(0.998-1.159)\end{array}$ & $\begin{array}{c}4 \\
(2: 1,2: 1)\end{array}$ & $\begin{array}{c}17.2 \\
(13.5-20.9)\end{array}$ \\
\hline F-008 & 20.4 & $\begin{array}{c}0.984 \\
(0.966-0.997)\end{array}$ & $\begin{array}{c}0.987 \\
(0.964-1.002)\end{array}$ & $\begin{array}{c}0.965 \\
(0.927-0.978)\end{array}$ & $\begin{array}{c}0.988 \\
(0.963-1.003)\end{array}$ & $\begin{array}{c}0.994 \\
(0.979-1.007)\end{array}$ & $\begin{array}{c}0.899 \\
(0.868-0.929)\end{array}$ & $\begin{array}{c}65 \\
(39: 6,30: 5)\end{array}$ & $\begin{array}{c}22.5 \\
(20.4-24.6)\end{array}$ \\
\hline F-009 & 100.0 & $\begin{array}{c}0.972 \\
(0.928-1.015)\end{array}$ & $\begin{array}{c}0.967 \\
(0.915-1.014)\end{array}$ & $\begin{array}{c}0.958 \\
(0.887-1.020)\end{array}$ & $\begin{array}{c}0.966 \\
(0.881-1.040)\end{array}$ & $\begin{array}{c}0.942 \\
(0.853-1.024)\end{array}$ & $\begin{array}{c}0.973 \\
(0.825-1.151)\end{array}$ & $\begin{array}{c}1 \\
(1: 1,1: 1)\end{array}$ & $\begin{array}{c}7.0 \\
(1.1-12.9)\end{array}$ \\
\hline F-010 & 71.9 & $\begin{array}{c}0.965 \\
(0.952-0.977)\end{array}$ & $\begin{array}{c}0.965 \\
(0.945-0.978)\end{array}$ & $\begin{array}{c}0.976 \\
(0.960-0.991)\end{array}$ & $\begin{array}{c}0.986 \\
(0.970-0.998)\end{array}$ & $\begin{array}{c}0.935 \\
(0.918-0.947)\end{array}$ & $\begin{array}{c}0.948 \\
(0.917-0.980)\end{array}$ & $\begin{array}{c}38 \\
(34: 31,30: 28)\end{array}$ & $\begin{array}{c}25.2 \\
(23.7-26.8)\end{array}$ \\
\hline F-011 & 100.0 & $\begin{array}{c}0.976 \\
(0.952-1.001)\end{array}$ & $\begin{array}{c}0.976 \\
(0.958-0.993)\end{array}$ & $\begin{array}{c}1.009 \\
(0.980-1.043)\end{array}$ & $\begin{array}{c}1.034 \\
(0.977-1.103)\end{array}$ & $\begin{array}{c}1.038 \\
(0.983-1.105)\end{array}$ & $\begin{array}{c}1.010 \\
(0.873-1.167)\end{array}$ & $\begin{array}{c}3 \\
(2: 2,1: 1)\end{array}$ & $\begin{array}{c}6.9 \\
(5.3-8.6)\end{array}$ \\
\hline F-012 & 100.0 & $\begin{array}{c}0.960 \\
(0.939-0.980)\end{array}$ & $\begin{array}{c}0.955 \\
(0.937-0.967)\end{array}$ & $\begin{array}{c}0.930 \\
(0.907-0.943)\end{array}$ & $\begin{array}{c}0.976 \\
(0.942-0.995)\end{array}$ & $\begin{array}{c}0.934 \\
(0.898-0.956)\end{array}$ & $\begin{array}{c}0.810 \\
(0.746-0.869)\end{array}$ & $\begin{array}{c}15 \\
(10: 10,7: 7)\end{array}$ & $\begin{array}{c}22.9 \\
(19.7-26.0)\end{array}$ \\
\hline F-013 & 100.0 & $\begin{array}{c}0.982 \\
(0.972-0.992)\end{array}$ & $\begin{array}{c}0.969 \\
(0.959-0.980)\end{array}$ & $\begin{array}{c}1.008 \\
(0.987-1.029)\end{array}$ & $\begin{array}{c}1.006 \\
(0.982-1.033)\end{array}$ & $\begin{array}{c}0.976 \\
(0.944-1.006)\end{array}$ & $\begin{array}{c}0.957 \\
(0.851-1.062)\end{array}$ & $\begin{array}{c}3 \\
(3: 3,2: 2)\end{array}$ & $\begin{array}{c}12.4 \\
(9.9-15.0)\end{array}$ \\
\hline
\end{tabular}


Table 4.12. Table of greater sage-grouse (Centrocercus urophasianus) average annual rate of population change $(\hat{\lambda})$ across six periods of oscillation in abundance that represent temporal scales for each neighborhood cluster within the state of Utah. Estimates were derived from leks within the entire neighborhood cluster.-Continued

[NC, neighborhood cluster; NA, not applicable]

\begin{tabular}{|c|c|c|c|c|c|c|c|c|c|}
\hline \multirow[b]{2}{*}{ NC } & \multirow[b]{2}{*}{$\begin{array}{c}\text { Percent } \\
\text { NC1 }\end{array}$} & \multicolumn{6}{|c|}{ Temporal scales } & \multirow{2}{*}{$\begin{array}{c}\text { Number of } \\
\text { leks }^{3}\end{array}$} & \multirow{2}{*}{$\begin{array}{l}\text { Average } \\
\text { count/lek }\end{array}$} \\
\hline & & Long & Medium/Long & Medium & $\begin{array}{c}\text { Short/ } \\
\text { Medium }\end{array}$ & Short & Recent & & \\
\hline F-015 & 100.0 & $\begin{array}{c}1.010 \\
(0.964-1.064)\end{array}$ & $\begin{array}{c}1.010 \\
(0.949-1.064)\end{array}$ & $\begin{array}{c}1.020 \\
(0.958-1.104)\end{array}$ & $\begin{array}{c}1.056 \\
(0.956-1.163)\end{array}$ & $\begin{array}{c}1.066 \\
(0.943-1.195)\end{array}$ & $\begin{array}{c}1.104 \\
(0.915-1.314)\end{array}$ & $\begin{array}{c}1 \\
(1: 1,1: 1)\end{array}$ & $\begin{array}{c}22.1 \\
(11.9-32.3)\end{array}$ \\
\hline F-018 & 35.4 & $\begin{array}{c}0.986 \\
(0.939-1.034)\end{array}$ & $\begin{array}{c}0.981 \\
(0.931-1.032)\end{array}$ & $\begin{array}{c}0.965 \\
(0.941-0.987)\end{array}$ & $\begin{array}{c}0.970 \\
(0.937-1.002)\end{array}$ & $\begin{array}{c}1.022 \\
(0.960-1.105)\end{array}$ & $\begin{array}{c}1.319 \\
(1.106-1.611)\end{array}$ & $\begin{array}{c}1 \\
(1: 0,1: 0)\end{array}$ & $\begin{array}{c}6.1 \\
(3.1-9.1)\end{array}$ \\
\hline F-019 & 0 & $\begin{array}{c}0.980 \\
(0.961-0.995)\end{array}$ & $\begin{array}{c}0.975 \\
(0.953-0.995)\end{array}$ & $\begin{array}{c}0.981 \\
(0.959-0.997)\end{array}$ & $\begin{array}{c}0.993 \\
(0.972-1.005)\end{array}$ & $\begin{array}{c}0.988 \\
(0.972-1.001)\end{array}$ & $\begin{array}{c}1.052 \\
(1.012-1.094)\end{array}$ & $\begin{array}{c}18 \\
(15: 0,15: 0)\end{array}$ & $\begin{array}{c}19.3 \\
(16.9-21.6)\end{array}$ \\
\hline
\end{tabular}

1The percent of each neighborhood cluster that intersects the state.

2Temporal scales were estimated from present to each major population abundance nadir (trough) since 1960. Number of temporal scales were used to estimate population trends across six different temporal scales from approximately 10 to approximately 60 years.

${ }^{3}$ Number of leks in database. In parentheses from left to right is (1) total number in cluster were used in trend analysis, (2) number used in trend analysis within state boundary, (3) total number in cluster were used in the targeted annual warning system (TAWS) analysis, and (4) number used in TAWS analysis within state boundary.
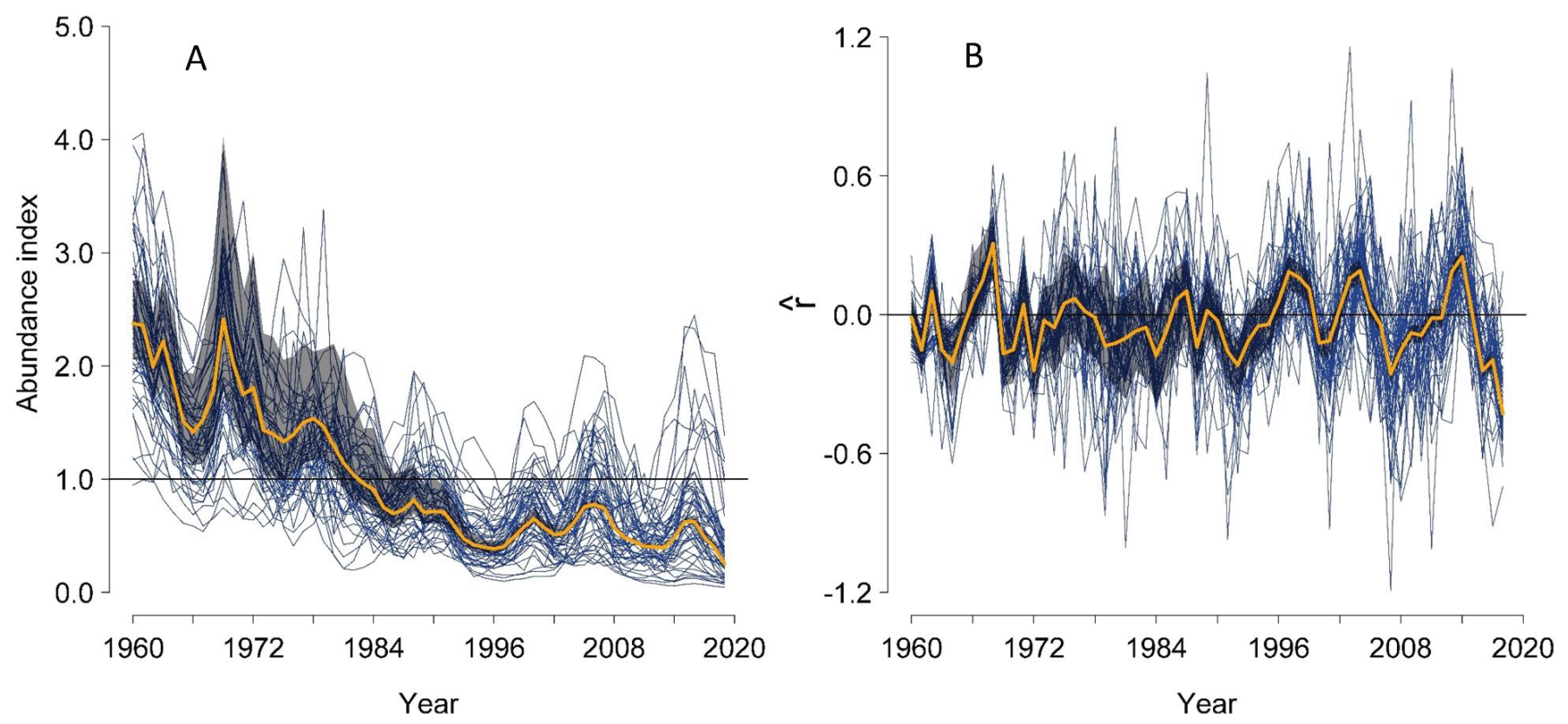

Figure 4.38. A, Abundance index (calculated as $\hat{N}$ divided by 60-year mean of $\hat{N}$ ); and $B$, intrinsic rate of population change $(\hat{r})$ of greater sage-grouse (Centrocercus urophasianus) within the state of Utah from 1960-2019. Thick yellow line represents median estimates across all leks. Shaded areas represent 95-percent credible limits. Thin blue lines represent median values for neighborhood clusters. Black horizontal line (abundance index $=1.0$ ) represents 60 -year average. 

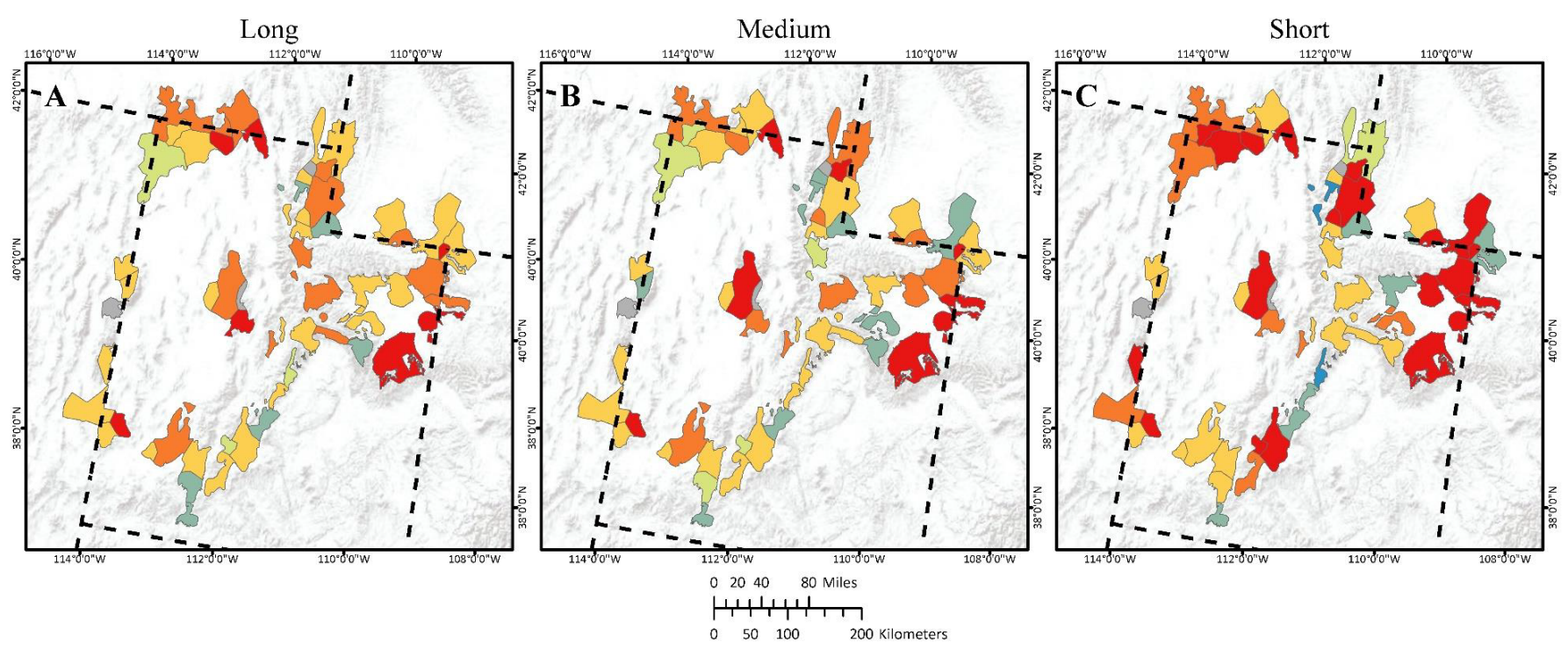

\section{EXPLANATION}

Neighborhood clusters (Level 2) intersecting Utah: polygon colors represent average lambda values for sage-grouse populations

over varying lengths of time (long, medium, short). Symbols are based on a range-wide distribution of values and all may not be represented here.
Average lambda $(\hat{\lambda})$

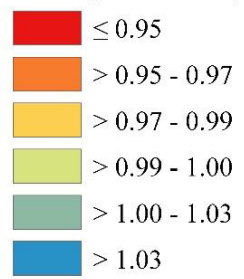

Coordinate System: Albers Projection: Albers Datum: WGS 1984

false easting: 0.0000 false northing: 0.0000 central meridian: -96.0000 standard parallel 1: 29.5000 standard parallel 2: 45.5000 latitude of origin: 23.0000 Units: Meter

Service Layer Credits: Sources: Esri, USGS, NOAA

Figure 4.39. Spatial estimates of population trends across three temporal scales based on periods of oscillation (short, medium, and long) while accounting for periods of oscillation of greater sage-grouse (Centrocercus urophasianus) at different neighborhood clusters within the state of Utah. Map images are the intellectual property of Esri and are used herein under license. Copyright (C) 2020 Esri and its licensors. All rights reserved. 

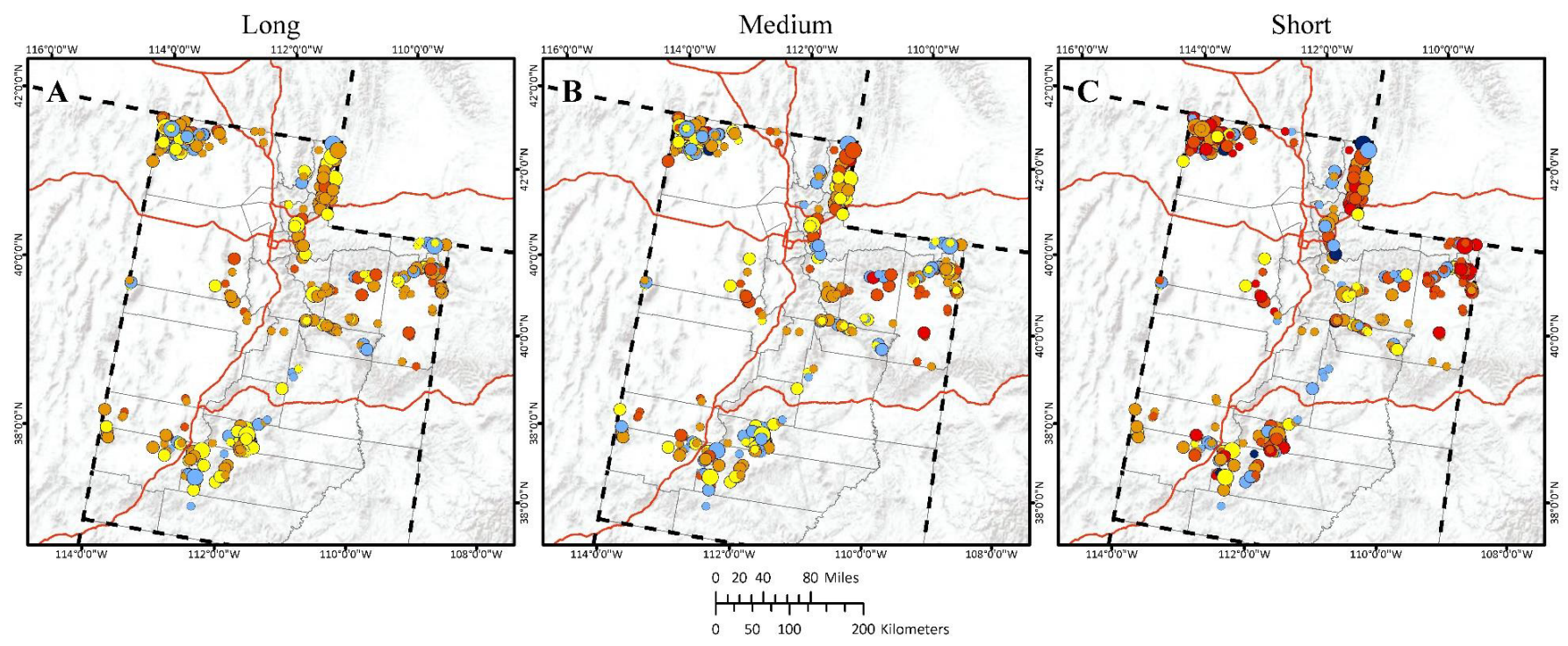

\section{EXPLANATION}

Leks within Utah: colors represent average lambda $(\hat{\lambda})$ values of sage-grouse populations over various time scales (long, medium, short) The size of the symbol represents the maximum population size of the lek over the years of the analysis, relativized to the range-wide maximum population size across all leks. Symbols are based on a range-wide distribution of values and all may not be represented here.

$$
\begin{aligned}
\text { Average lambda }(\hat{\lambda}) \\
\quad \leq 0.90 \\
-\quad>0.90-0.94 \\
\quad>0.94-0.98 \\
\quad>0.98-1.00 \\
\quad>1.00-1.10 \\
\quad>1.10
\end{aligned}
$$

Road
- County line
- - State border
Size guide
Maximum lek count
Largest
$\oint_{\text {Smallest }}$

Coordinate System: Albers Projection: Albers Datum: WGS 1984 false easting: 0.0000 false northing: 0.0000 central meridian: -96.0000 standard parallel $1: 29.5000$ standard parallel $2: 45.5000$ latitude of origin: 23.0000 Units: Meter

Service Layer Credits: Sources: Esri, USGS, NOAA

Figure 4.40. Spatial estimates of population trends across three temporal scales based on periods of oscillation (short, medium, and long) while accounting for periods of oscillation of greater sage-grouse (Centrocercus urophasianus) at lek sites within the state of Utah. Map images are the intellectual property of Esri and are used herein under license. Copyright $(\subset) 2020$ Esri and its licensors. All rights reserved. 


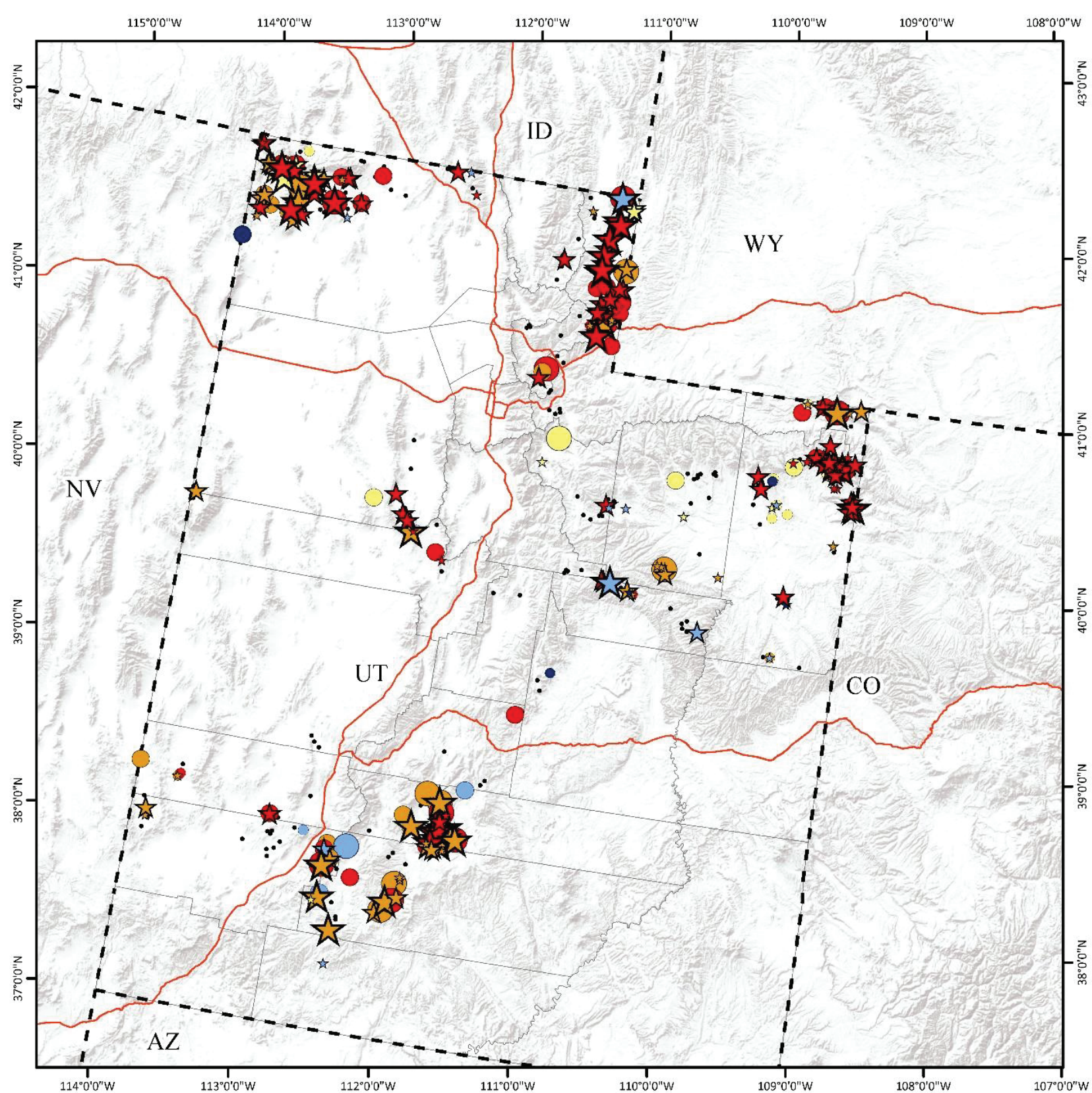

\section{EXPLANATION}

Leks in Utah: colors represent a range of years the most recent event occurred. Stars represent warnings, circles represent watches, and black dots represent leks which did not experience warnings or watches. The size of the symbol represents the maximum population size of the lek over the 30-year period, relativized to the rangewide maximum population size across all leks. Symbols are based on a range-wide distribution of values and all may not be represented here.

$\begin{array}{llll}0 & 10 & 20 & 40 \text { Miles }\end{array}$

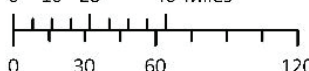

$\begin{array}{llll}0 & 30 \quad 60 \quad 120 \text { Kilometers }\end{array}$ is Warning

$\bigcirc$ Watch

- Lek

- - - State border

Road County line
Coordinate System: Albers Projection: Albers

Relative maximum $\begin{aligned} & \text { Datum: WGS } 1984 \\ & \text { false easting: } 0.0000\end{aligned}$ population size

false northing: 0.0000 central meridian: -96.0000 standard parallel $1: 29.5000$ standard parallel 2: 45.5000 latitude of origin: 23.0000 Units: Meter

Service Layer Credits: Sources: Esri, USGS, NOAA

Figure 4.41. Spatial and temporal depiction of watches and warnings of greater sage-grouse (Centrocercus urophasianus) population declines at the lek scale within the state of Utah from 1990 to 2019. Map image is the intellectual property of Esri and is used herein under license. Copyright (C) 2020 Esri and its licensors. All rights reserved. 


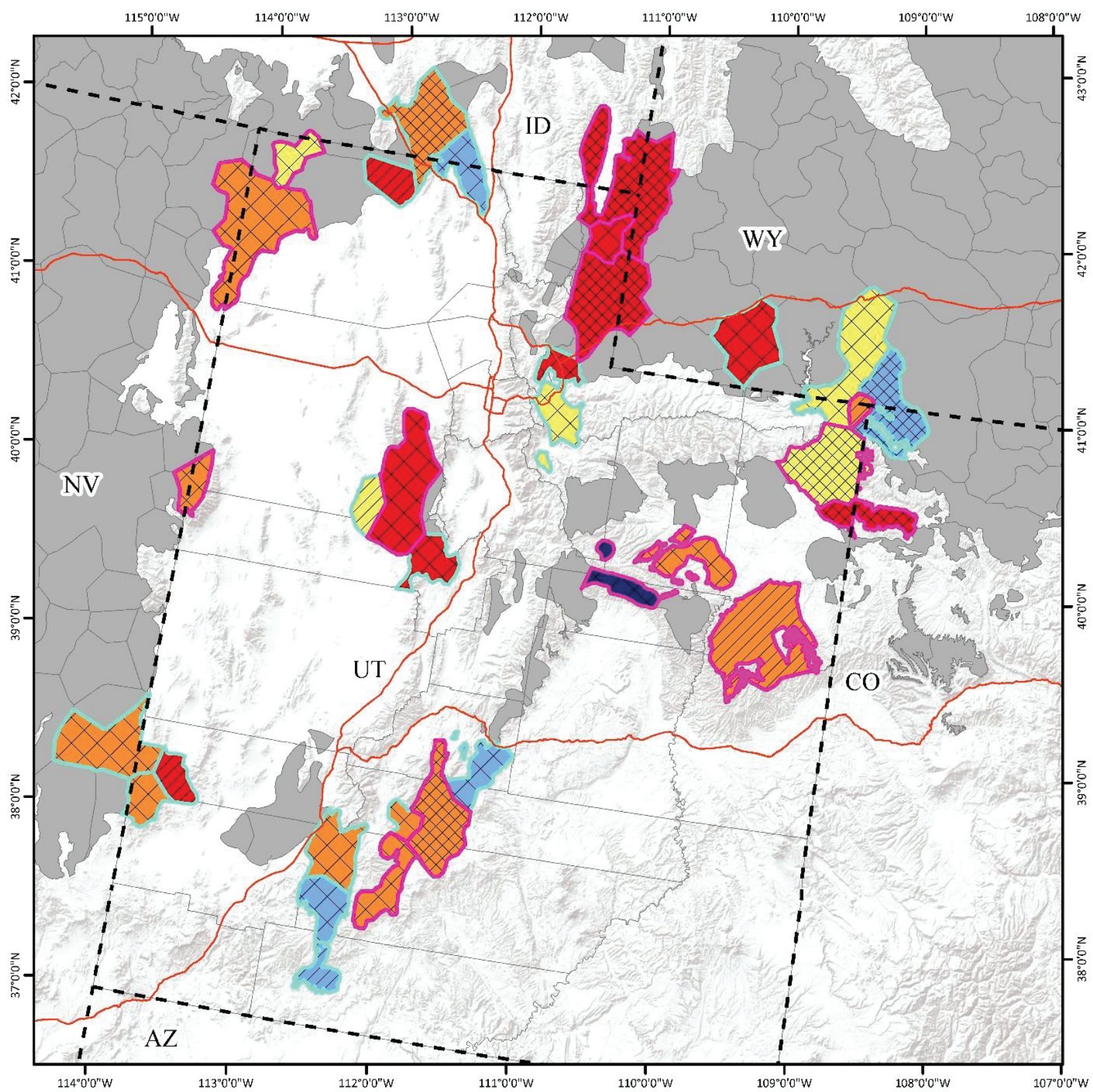

\section{EXPLANATION}

Neighborhood clusters (Level 2) in Utah: polygon colors represent a range of years the most recent event occurred. Oulline colors indicate whether the most recent event was a watch or warning. The hatching represents the maximum population size of the cluster over the 30 -year period, relativized to the range-wide maximum population size across all neighborhood clusters. Symbols are based on a range-wide distribution of values and all may not be represented here. $\begin{array}{llll}0 & 10 & 20 & 40 \text { Miles }\end{array}$ קובוד| - $20 \quad 40 \quad 80$ Kilometers $1995 \circ 1999$
- - State border
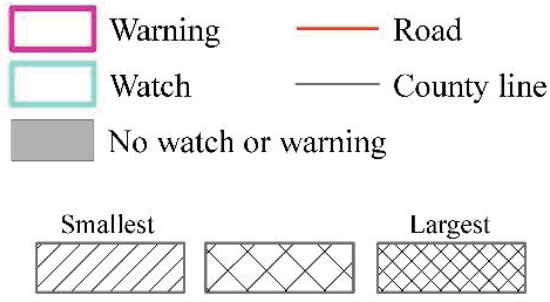

$2000-20042005-20092010-20142015-2019$
Coordinate System: Albers Projection: Albers Datum: WGS 1984 false easting: 0.0000 false northing: 0.0000 central meridian: -96.0000 standard parallel $1: 29.5000$ standard parallel 2: 45.5000 latitude of origin: 23.0000 Units: Meter

Service Layer Credits: Sources: Esri, USGS, NOAA

Figure 4.42. Spatial and temporal depiction of watches and warnings of greater sage-grouse (Centrocercus urophasianus) population declines at neighborhood cluster within the state of Utah from 1990 to 2019. Map image is the intellectual property of Esri and is used herein under license. Copyright (C) 2020 Esri and its licensors. All rights reserved. 


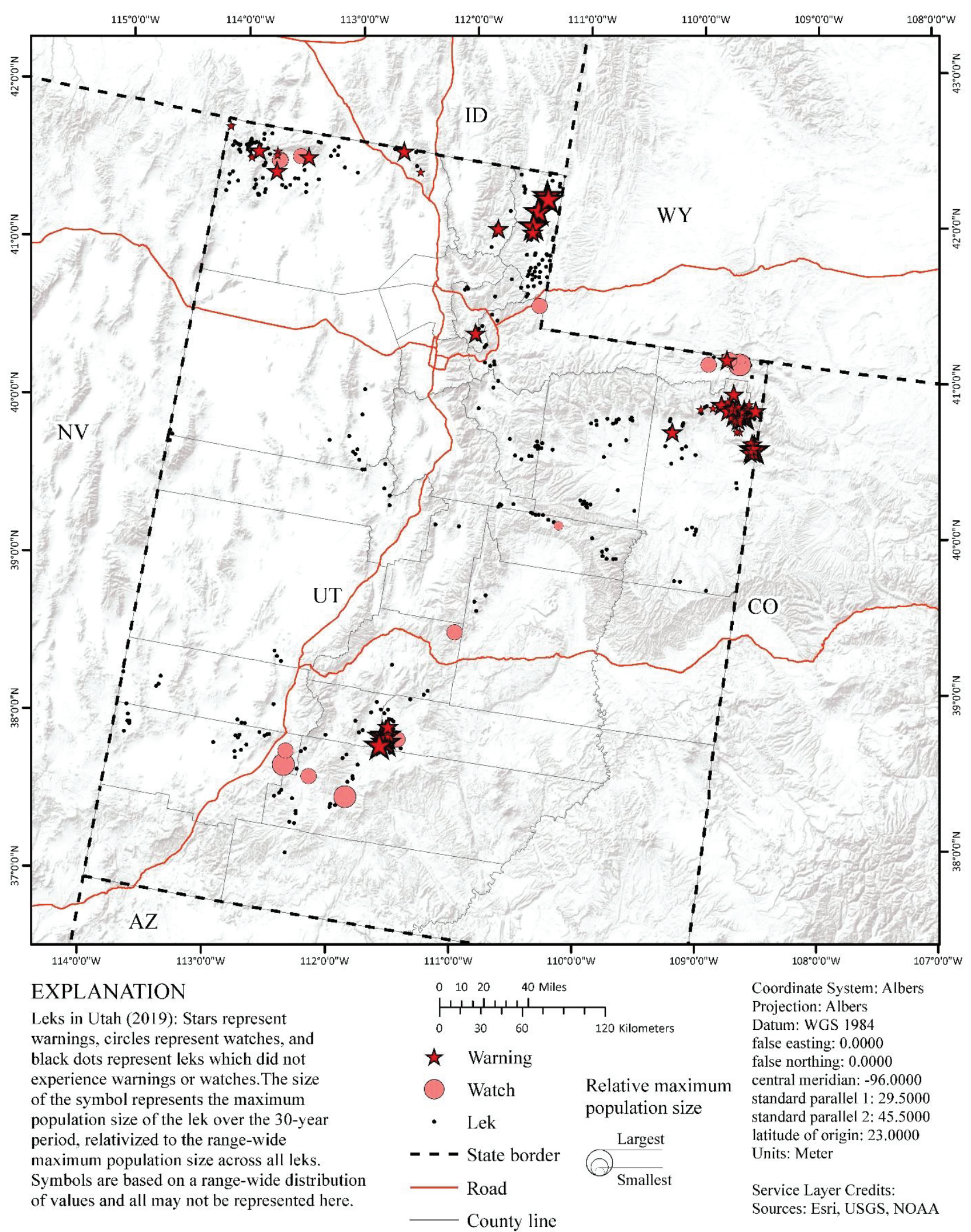

Figure 4.43. Spatial and temporal depiction of watches and warnings of greater sage-grouse (Centrocercus urophasianus) population declines at the lek and cluster scale within the state of Utah during 2019. Map image is the intellectual property of Esri and is used herein under license. Copyright (c) 2020 Esri and its licensors. All rights reserved. 


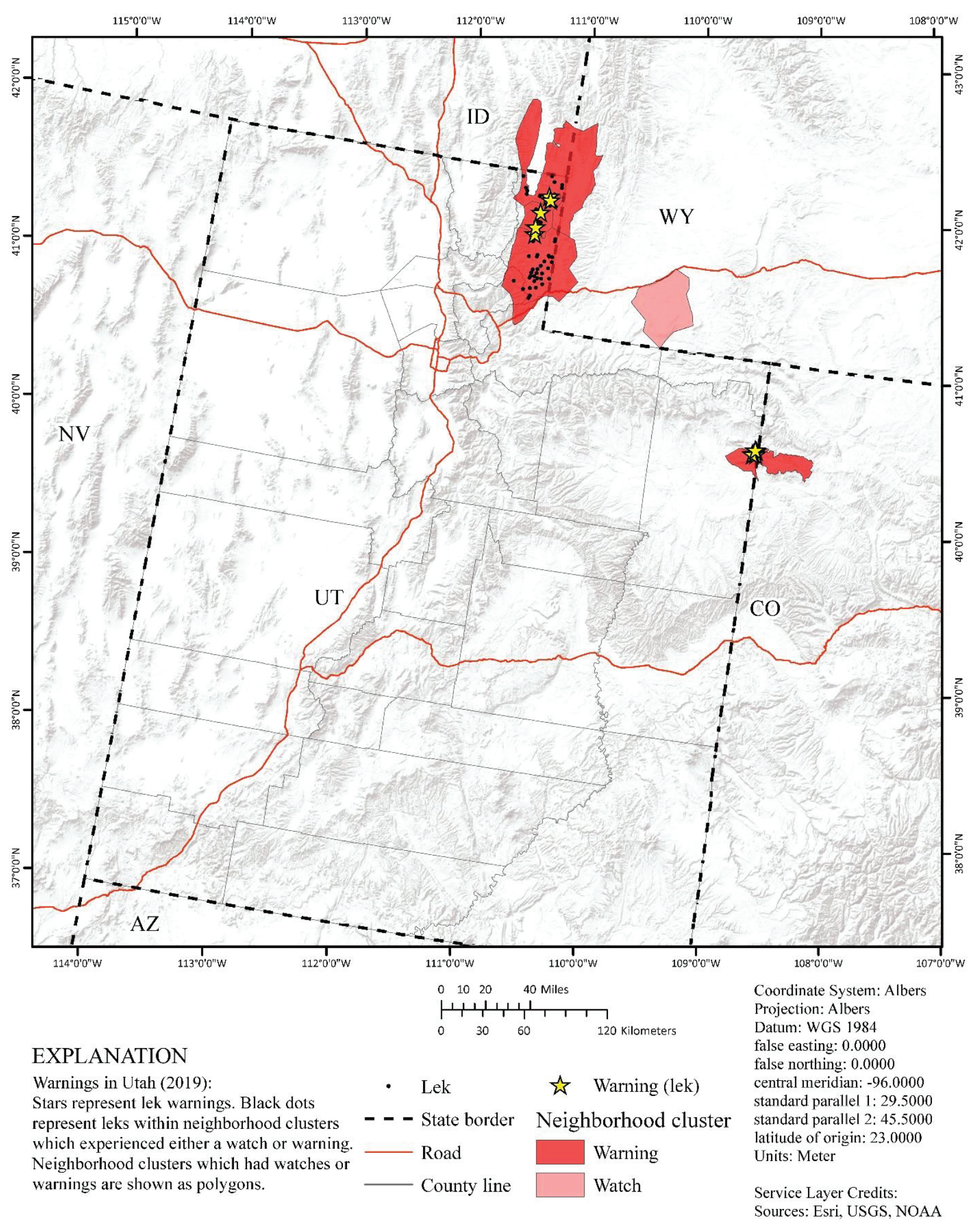

Figure 4.44. Spatial and temporal depiction of watches and warnings of greater sage-grouse (Centrocercus urophasianus) population declines at the neighborhood cluster scale within the state of Utah during 2019. All leks within watch and warning boundaries were assigned as watch and warning, respectively. Yellow stars represent leks that reached warning independently. Map image is the intellectual property of Esri and is used herein under license. Copyright (c) 2020 Esri and its licensors. All rights reserved. 


\section{Wyoming Results}

\section{Wyoming Lek Data and Clusters (Objectives 1 and 2)}

Wyoming intersected CC-C, CC-D, CC-F and contained or intersected 114 different NCs (fig. 4.45). Total area of the 114 NCs within Wyoming was 17,419,738 ha. One-hundred and nine of these clusters were used in the analysis and five were omitted because of limitations in sample sizes. These areas consisted of 2,610 leks, of which 2,342 were located within the Wyoming state boundary and represented 27.81 percent of the range-wide lek database. After extensive QA/QC, we used 1,790 leks from Wyoming in the SSM for population trend estimation (tables 4.13 and 4.14), totaling 36,623 individual lek counts. Mean male lek count was 15.66 (95-percent confidence level=15.52-15.80) for leks within NCs that were within or overlapped Wyoming. Mean male count for leks within Wyoming was 15.74 (95-percent confidence interval=15.59-15.89).

\section{Wyoming Population Trend Analysis (Objective 3)}

Although Wyoming intersected three CCs, the CC with the strongest representation (54.5 percent) was CC-D. For this $\mathrm{CC}$, we estimated six population abundance nadirs (troughs) that dated back to 1960. Each of these population abundance nadirs represent between one and six complete periods of oscillation. We used these nadirs to estimate population trends across three different temporal scales that represented two, four, and six complete periods for the state (for instance, second, fourth, and sixth nadir). We estimated the average annual finite rate of population change $(\hat{\lambda})$ at the short (two periods), medium (four periods), and long (six periods) temporal scales as 0.973 (95-percent CRI $=0.971-0.975$ ), 0.969 (95-percent CRI=0.960-0.974), and 0.971 (95-percent CRI $=0.967-0.976$ ), respectively (fig. 4.46). Climate cluster estimates, which included leks in adjacent states to Wyoming, were slightly different than estimates generated from leks only within Wyoming. For all NCs that were modeled and intersected Wyoming, we estimated median $\hat{\lambda}$ to be less than 1.0 for $78.9,90.8$, and 93.6 percent across short, medium, and long temporal scales, respectively (fig. 4.47; table 4.14). We estimated median $\hat{\lambda}$ to be less than 1.0 for $70.2,77.4$, and 81.9 percent of all modeled leks within Wyoming across short, medium, and long temporal scales, respectively (fig. 4.48). We reported spatial and temporal variation in average annual across different NCs (fig. 4.47) and leks (fig. 4.48).

\section{Wyoming Targeted Annual Warning System Analysis (Objective 4)}

During 1990-2019, the TAWS for sage-grouse populations in Wyoming activated a total of 1,149 and 875 leks as watches and warnings, respectively (fig. 4.49), which was 72.1 and 54.9 percent of the sampled leks used in the analysis. On average, across the 29 years, approximately 6.9 and 2.2 percent of leks per year experienced watches and warnings, respectively. The higher percentage for watches corresponds to repetitive activation. During this time frame, the TAWS also activated a total of 59 and $39 \mathrm{NCs}$ as watches and warnings, respectively (fig. 4.50), which was 56.7 and 37.5 percent of the sampled clusters used in the analysis. On average, approximately 3.3 and 1.5 percent of clusters per year experienced watches and warnings, respectively. Similar to leks, the higher percentage for watches corresponds to repetitive activation. During 2019 only, the TAWS activated 165 (first $=34$ ) watches and 148 (first $=52$ ) warnings at leks (fig. 4.51) as well as 5 (first=3) watches and 7 (first=3) warnings at NCs (fig. 4.52). 

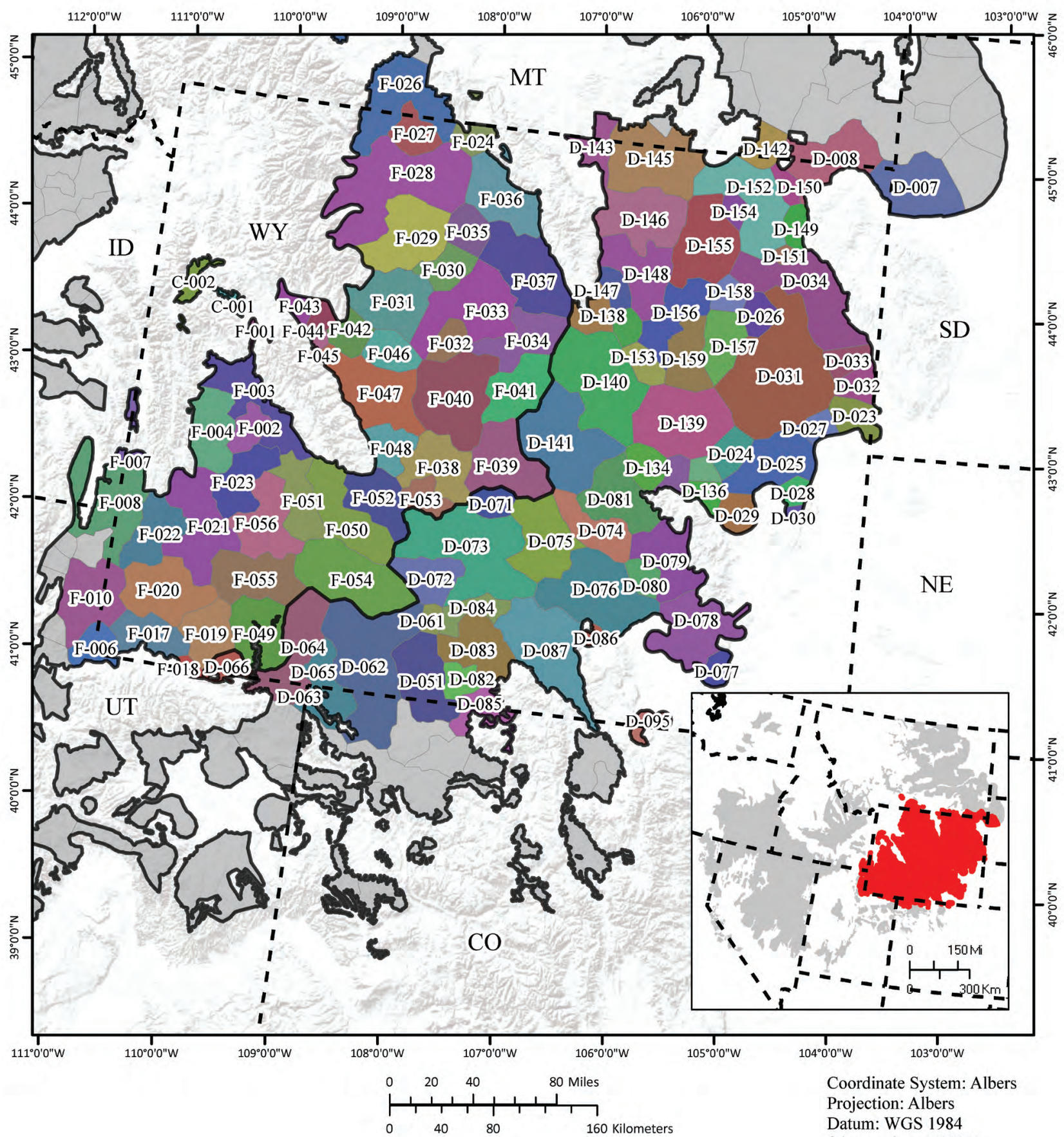

Coordinate System: Albers Projection: Albers Datum: WGS 1984 false easting: 0.0000 false northing: 0.0000 central meridian: -96.0000 standard parallel $1: 29.5000$ standard parallel 2: 45.5000 latitude of origin: 23.0000 Units: Meter Wyoming: polygon colors arbitrarily assigned and reflect 114 different population units. Grey polygons represent neighborhood clusters that Service Layer Credits: do not intersect Wyoming.

Sources: Esri, USGS, NOAA

Figure 4.45. Greater sage-grouse (Centrocercus urophasianus) hierarchical population monitoring framework for neighborhood clusters that intersect the state of Wyoming. Map image is the intellectual property of Esri and is used herein under license. Copyright (C) 2020 Esri and its licensors. All rights reserved. 
Table 4.13. Table of greater sage-grouse (Centrocercus urophasianus) average annual rate of population change $(\hat{\lambda})$ across six periods of oscillation in abundance that represent temporal scales for each climate cluster within the state of Wyoming. Estimates were derived from leks within the entire climate cluster.

[CC, climate cluster; C, Jackson Hole, Wyoming area; D, eastern area; F, Wyoming]

\begin{tabular}{|c|c|c|c|c|c|c|c|c|c|}
\hline \multirow[b]{2}{*}{ CC } & \multirow{2}{*}{$\begin{array}{l}\text { Percent } \\
\text { CC1 }\end{array}$} & \multicolumn{6}{|c|}{ Temporal scales ${ }^{2}$} & \multirow[b]{2}{*}{ Number of leks ${ }^{3}$} & \multirow{2}{*}{$\begin{array}{l}\text { Average } \\
\text { count/lek }\end{array}$} \\
\hline & & Long & Medium/Long & Medium & $\begin{array}{c}\text { Short/ } \\
\text { Medium }\end{array}$ & Short & Recent & & \\
\hline $\mathrm{D}$ & 36.6 & $\begin{array}{c}0.963 \\
(0.960-0.968)\end{array}$ & $\begin{array}{c}0.956 \\
(0.946-0.960)\end{array}$ & $\begin{array}{c}0.967 \\
(0.960-0.972)\end{array}$ & $\begin{array}{c}0.983 \\
(0.975-0.989)\end{array}$ & $\begin{array}{c}0.963 \\
(0.959-0.967)\end{array}$ & $\begin{array}{c}0.980 \\
(0.972-0.989)\end{array}$ & $\begin{array}{c}2,944 \\
(1,831: 903 \\
1,566: 773)\end{array}$ & $\begin{array}{c}16.7 \\
(16.5-16.9)\end{array}$ \\
\hline $\mathrm{F}$ & 88.4 & $\begin{array}{c}0.980 \\
(0.975-0.987)\end{array}$ & $\begin{array}{c}0.976 \\
(0.969-0.984)\end{array}$ & $\begin{array}{c}0.976 \\
(0.966-0.980)\end{array}$ & $\begin{array}{c}1.003 \\
(0.997-1.008)\end{array}$ & $\begin{array}{c}0.991 \\
(0.988-0.995)\end{array}$ & $\begin{array}{c}1.016 \\
(1.011-1.023)\end{array}$ & $\begin{array}{c}1,253 \\
(974: 873,892: 806)\end{array}$ & $\begin{array}{c}23.7 \\
(23.3-24.2)\end{array}$ \\
\hline
\end{tabular}

${ }^{1}$ The percent of each climate cluster that intersects the state.

2Temporal scales were estimated from present to each major population abundance nadir (trough) since 1960. Number of temporal scales were used to estimate population trends across six different temporal scales from approximately 10 to approximately 60 years.

${ }^{3}$ Number of leks in database. In parentheses from left to right is (1) total number in cluster were used in trend analysis, (2) number used in trend analysis within state boundary, (3) total number in cluster were used in the targeted annual warning system (TAWS) analysis, and (4) number used in TAWS analysis within state boundary.

Table 4.14. Table of greater sage-grouse (Centrocercus urophasianus) average annual rate of population change $(\hat{\lambda})$ across six periods of oscillation in abundance that represent temporal scales for each neighborhood cluster within the state of Wyoming. Estimates were derived from leks within the entire neighborhood cluster.

[NC, neighborhood cluster; NA, not applicable]

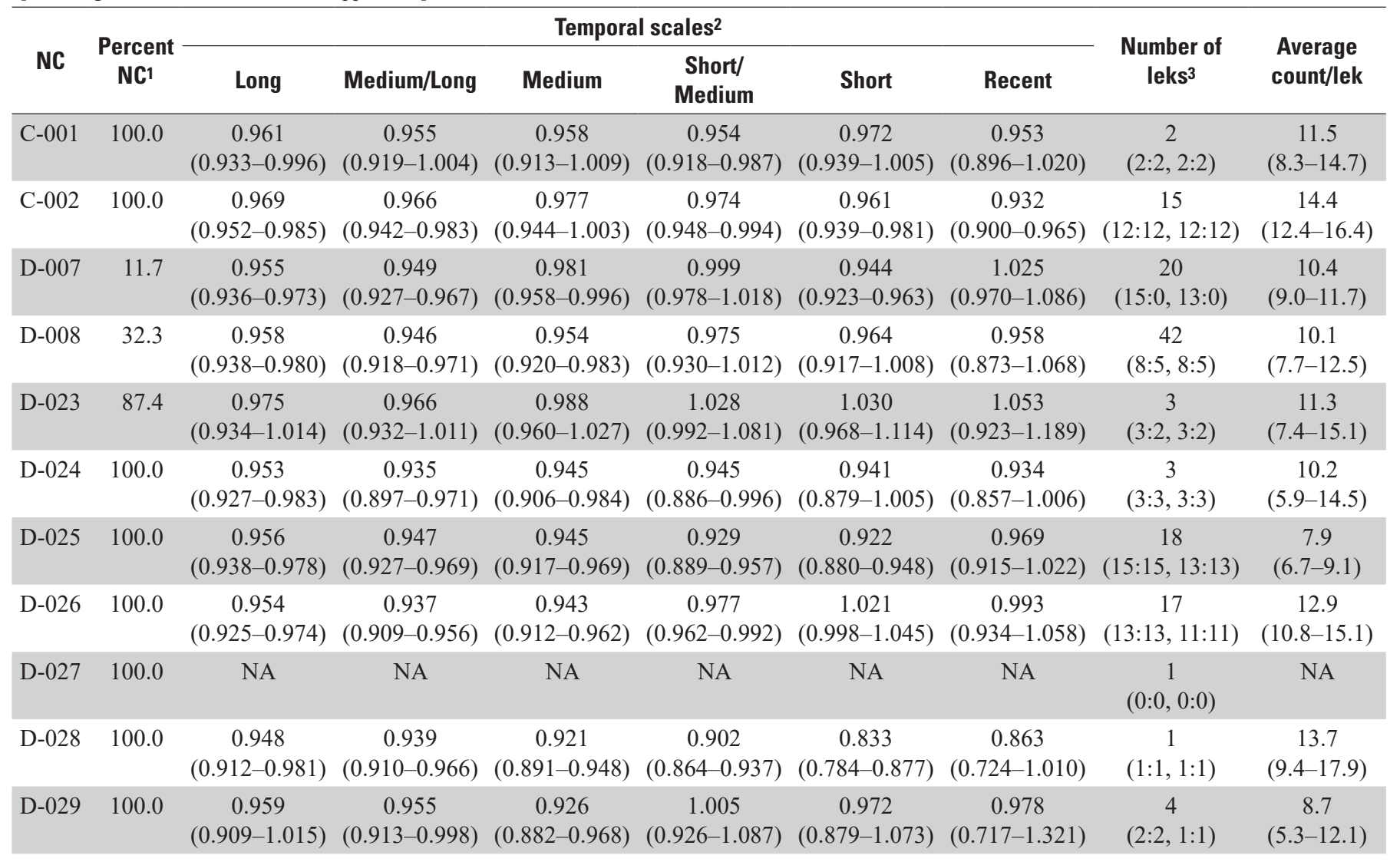


Table 4.14. Table of greater sage-grouse (Centrocercus urophasianus) average annual rate of population change $(\hat{\lambda})$ across six periods of oscillation in abundance that represent temporal scales for each neighborhood cluster within the state of Wyoming. Estimates were derived from leks within the entire neighborhood cluster.-Continued

[NC, neighborhood cluster; NA, not applicable]

\begin{tabular}{|c|c|c|c|c|c|c|c|c|c|}
\hline \multirow[b]{2}{*}{ NC } & \multirow{2}{*}{$\begin{array}{l}\text { Percent } \\
\text { NC1 }\end{array}$} & \multicolumn{6}{|c|}{ Temporal scales² } & \multirow{2}{*}{$\begin{array}{c}\text { Number of } \\
\text { leks }^{3}\end{array}$} & \multirow{2}{*}{$\begin{array}{l}\text { Average } \\
\text { count/lek }\end{array}$} \\
\hline & & Long & Medium/Long & Medium & $\begin{array}{c}\text { Short/ } \\
\text { Medium }\end{array}$ & Short & Recent & & \\
\hline D-031 & 100.0 & $\begin{array}{c}0.957 \\
(0.938-0.974)\end{array}$ & $\begin{array}{c}0.940 \\
(0.918-0.961)\end{array}$ & $\begin{array}{c}0.945 \\
(0.923-0.968)\end{array}$ & $\begin{array}{c}0.945 \\
(0.925-0.965)\end{array}$ & $\begin{array}{c}0.924 \\
(0.910-0.938)\end{array}$ & $\begin{array}{c}0.973 \\
(0.935-1.011)\end{array}$ & $\begin{array}{c}62 \\
(40: 40,37: 37)\end{array}$ & $\begin{array}{c}10.8 \\
(9.7-11.8)\end{array}$ \\
\hline D-033 & 100.0 & $\begin{array}{c}0.940 \\
(0.899-0.978)\end{array}$ & $\begin{array}{c}0.925 \\
(0.877-0.967)\end{array}$ & $\begin{array}{c}0.927 \\
(0.872-0.976)\end{array}$ & $\begin{array}{c}0.920 \\
(0.856-0.975)\end{array}$ & $\begin{array}{c}0.880 \\
(0.814-0.935)\end{array}$ & $\begin{array}{c}0.897 \\
(0.760-1.030)\end{array}$ & $\begin{array}{c}3 \\
(1: 1,1: 1)\end{array}$ & $\begin{array}{c}3.8 \\
(1.3-6.2)\end{array}$ \\
\hline D-034 & 100.0 & $\begin{array}{c}0.956 \\
(0.942-0.966)\end{array}$ & $\begin{array}{c}0.946 \\
(0.930-0.959)\end{array}$ & $\begin{array}{c}0.952 \\
(0.937-0.968)\end{array}$ & $\begin{array}{c}0.968 \\
(0.948-0.985)\end{array}$ & $\begin{array}{c}0.969 \\
(0.955-0.982)\end{array}$ & $\begin{array}{c}1.015 \\
(0.981-1.054)\end{array}$ & $\begin{array}{c}44 \\
(34: 34,25: 25)\end{array}$ & $\begin{array}{c}12.1 \\
(11.0-13.3)\end{array}$ \\
\hline D-062 & 74.2 & $\begin{array}{c}0.974 \\
(0.959-0.984)\end{array}$ & $\begin{array}{c}0.961 \\
(0.944-0.972)\end{array}$ & $\begin{array}{c}0.979 \\
(0.958-0.993)\end{array}$ & $\begin{array}{c}0.992 \\
(0.967-1.011)\end{array}$ & $\begin{array}{c}1.008 \\
(0.990-1.028)\end{array}$ & $\begin{array}{c}0.994 \\
(0.964-1.035)\end{array}$ & $\begin{array}{c}41 \\
(33: 25,32: 24)\end{array}$ & $\begin{array}{c}25.1 \\
(23.1-27.0)\end{array}$ \\
\hline D-063 & 32.6 & $\begin{array}{c}0.944 \\
(0.914-0.978)\end{array}$ & $\begin{array}{c}0.931 \\
(0.908-0.955)\end{array}$ & $\begin{array}{c}0.941 \\
(0.912-0.970)\end{array}$ & $\begin{array}{c}0.992 \\
(0.947-1.043)\end{array}$ & $\begin{array}{c}0.856 \\
(0.803-0.910)\end{array}$ & $\begin{array}{c}0.979 \\
(0.801-1.207)\end{array}$ & $\begin{array}{c}3 \\
(2: 1,1: 0)\end{array}$ & $\begin{array}{c}6.3 \\
(3.8-8.9)\end{array}$ \\
\hline D-064 & 81.7 & $\begin{array}{c}0.985 \\
(0.965-1.009)\end{array}$ & $\begin{array}{c}0.966 \\
(0.948-0.983)\end{array}$ & $\begin{array}{c}1.003 \\
(0.990-1.015)\end{array}$ & $\begin{array}{c}1.005 \\
(0.982-1.029)\end{array}$ & $\begin{array}{c}0.937 \\
(0.919-0.960)\end{array}$ & $\begin{array}{c}0.910 \\
(0.860-0.974)\end{array}$ & $\begin{array}{c}25 \\
(17: 10,15: 8)\end{array}$ & $\begin{array}{c}18.8 \\
(16.8-20.8)\end{array}$ \\
\hline D-065 & 45.6 & $\begin{array}{c}0.975 \\
(0.955-0.997)\end{array}$ & $\begin{array}{c}0.961 \\
(0.941-0.984)\end{array}$ & $\begin{array}{c}0.980 \\
(0.955-1.003)\end{array}$ & $\begin{array}{c}0.983 \\
(0.957-1.000)\end{array}$ & $\begin{array}{c}1.019 \\
(0.993-1.046)\end{array}$ & $\begin{array}{c}0.920 \\
(0.891-0.946)\end{array}$ & $\begin{array}{c}24 \\
(18: 11,18: 11)\end{array}$ & $\begin{array}{c}26.7 \\
(23.8-29.7)\end{array}$ \\
\hline D-066 & 81.3 & $\begin{array}{c}0.957 \\
(0.894-1.010)\end{array}$ & $\begin{array}{c}0.956 \\
(0.889-1.016)\end{array}$ & $\begin{array}{c}0.962 \\
(0.894-1.027)\end{array}$ & $\begin{array}{c}0.919 \\
(0.866-0.963)\end{array}$ & $\begin{array}{c}0.871 \\
(0.815-0.922)\end{array}$ & $\begin{array}{c}0.748 \\
(0.621-0.884)\end{array}$ & $\begin{array}{c}2 \\
(2: 2,2: 2)\end{array}$ & $\begin{array}{c}9.1 \\
(5.7-12.4)\end{array}$ \\
\hline D-074 & 100.0 & $\begin{array}{c}0.983 \\
(0.967-1.000)\end{array}$ & $\begin{array}{c}1.043 \\
(1.009-1.075)\end{array}$ & $\begin{array}{c}0.995 \\
(0.968-1.008)\end{array}$ & $\begin{array}{c}1.076 \\
(1.049-1.112)\end{array}$ & $\begin{array}{c}0.970 \\
(0.950-0.985)\end{array}$ & $\begin{array}{c}1.011 \\
(0.958-1.065)\end{array}$ & $\begin{array}{c}22 \\
(18: 18,15: 15)\end{array}$ & $\begin{array}{c}18.9 \\
(16.5-21.2)\end{array}$ \\
\hline D-075 & 100.0 & $\begin{array}{c}0.964 \\
(0.949-0.979)\end{array}$ & $\begin{array}{c}0.956 \\
(0.926-0.972)\end{array}$ & $\begin{array}{c}0.968 \\
(0.931-0.980)\end{array}$ & $\begin{array}{c}0.996 \\
(0.973-1.007)\end{array}$ & $\begin{array}{c}0.953 \\
(0.939-0.969)\end{array}$ & $\begin{array}{c}0.970 \\
(0.933-1.013)\end{array}$ & $\begin{array}{c}34 \\
(28: 28,28: 28)\end{array}$ & $\begin{array}{c}21.1 \\
(19.5-22.8)\end{array}$ \\
\hline D-076 & 100.0 & $\begin{array}{c}0.959 \\
(0.941-0.971)\end{array}$ & $\begin{array}{c}1.006 \\
(0.994-1.019)\end{array}$ & $\begin{array}{c}1.011 \\
(0.998-1.024)\end{array}$ & $\begin{array}{c}1.030 \\
(1.017-1.049)\end{array}$ & $\begin{array}{c}0.970 \\
(0.950-0.997)\end{array}$ & $\begin{array}{c}1.018 \\
(0.973-1.097)\end{array}$ & $\begin{array}{c}65 \\
(54: 54,44: 44)\end{array}$ & $\begin{array}{c}14.7 \\
(13.6-15.8)\end{array}$ \\
\hline D-077 & 100.0 & $\begin{array}{c}0.956 \\
(0.928-0.994)\end{array}$ & $\begin{array}{c}0.946 \\
(0.912-0.983)\end{array}$ & $\begin{array}{c}0.970 \\
(0.925-1.018)\end{array}$ & $\begin{array}{c}0.991 \\
(0.931-1.059)\end{array}$ & $\begin{array}{c}0.975 \\
(0.903-1.060)\end{array}$ & $\begin{array}{c}0.949 \\
(0.802-1.110)\end{array}$ & $\begin{array}{c}5 \\
(2: 2,1: 1)\end{array}$ & $\begin{array}{c}5.9 \\
(2.5-9.3)\end{array}$ \\
\hline D-078 & 100.0 & $\begin{array}{c}0.963 \\
(0.947-0.978)\end{array}$ & $\begin{array}{c}0.970 \\
(0.946-0.993)\end{array}$ & $\begin{array}{c}0.961 \\
(0.939-0.984)\end{array}$ & $\begin{array}{c}0.997 \\
(0.970-1.030)\end{array}$ & $\begin{array}{c}0.941 \\
(0.921-0.976)\end{array}$ & $\begin{array}{c}0.941 \\
(0.888-1.050)\end{array}$ & $\begin{array}{c}35 \\
(20: 20,10: 10)\end{array}$ & $\begin{array}{c}27.1 \\
(23.1-31.1)\end{array}$ \\
\hline D-079 & 100.0 & $\begin{array}{c}0.961 \\
(0.949-0.975)\end{array}$ & $\begin{array}{c}0.972 \\
(0.951-0.985)\end{array}$ & $\begin{array}{c}0.979 \\
(0.956-0.997)\end{array}$ & $\begin{array}{c}1.005 \\
(0.964-1.045)\end{array}$ & $\begin{array}{c}1.041 \\
(0.998-1.082)\end{array}$ & $\begin{array}{c}1.065 \\
(1.030-1.103)\end{array}$ & $\begin{array}{c}29 \\
(20: 20,12: 12)\end{array}$ & $\begin{array}{c}18.8 \\
(16.1-21.4)\end{array}$ \\
\hline D-080 & 100.0 & $\begin{array}{c}0.917 \\
(0.893-0.939)\end{array}$ & $\begin{array}{c}0.940 \\
(0.906-0.972)\end{array}$ & $\begin{array}{c}0.911 \\
(0.881-0.936)\end{array}$ & $\begin{array}{c}0.953 \\
(0.913-0.993)\end{array}$ & $\begin{array}{c}0.815 \\
(0.770-0.859)\end{array}$ & $\begin{array}{c}0.987 \\
(0.798-1.185)\end{array}$ & $\begin{array}{c}9 \\
(4: 4,3: 3)\end{array}$ & $\begin{array}{c}13.6 \\
(9.1-18.0)\end{array}$ \\
\hline
\end{tabular}


Table 4.14. Table of greater sage-grouse (Centrocercus urophasianus) average annual rate of population change $(\hat{\lambda})$ across six periods of oscillation in abundance that represent temporal scales for each neighborhood cluster within the state of Wyoming. Estimates were derived from leks within the entire neighborhood cluster-Continued

[NC, neighborhood cluster; NA, not applicable]

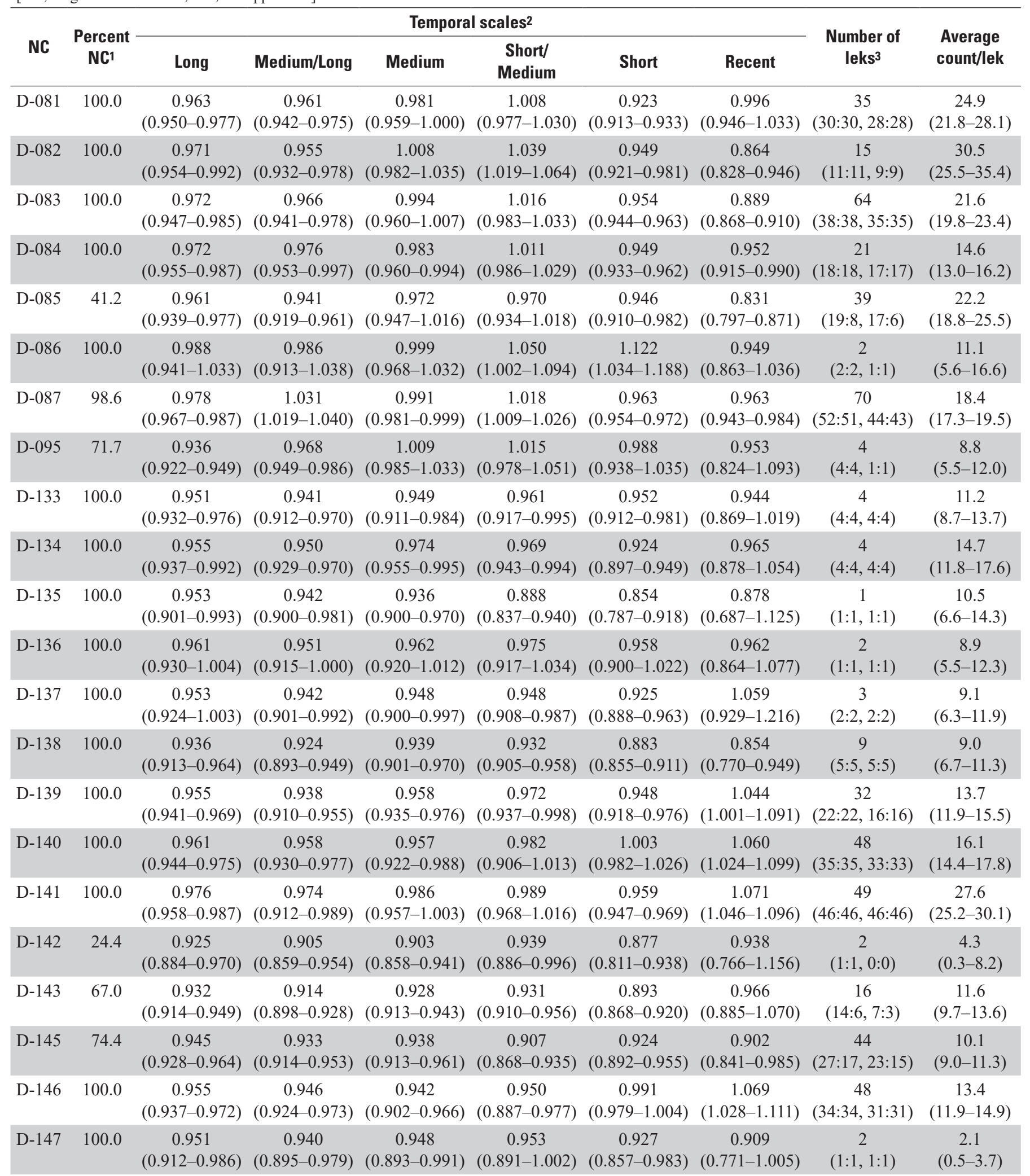


Table 4.14. Table of greater sage-grouse (Centrocercus urophasianus) average annual rate of population change $(\hat{\lambda})$ across six periods of oscillation in abundance that represent temporal scales for each neighborhood cluster within the state of Wyoming. Estimates were derived from leks within the entire neighborhood cluster.-Continued

[NC, neighborhood cluster; NA, not applicable]

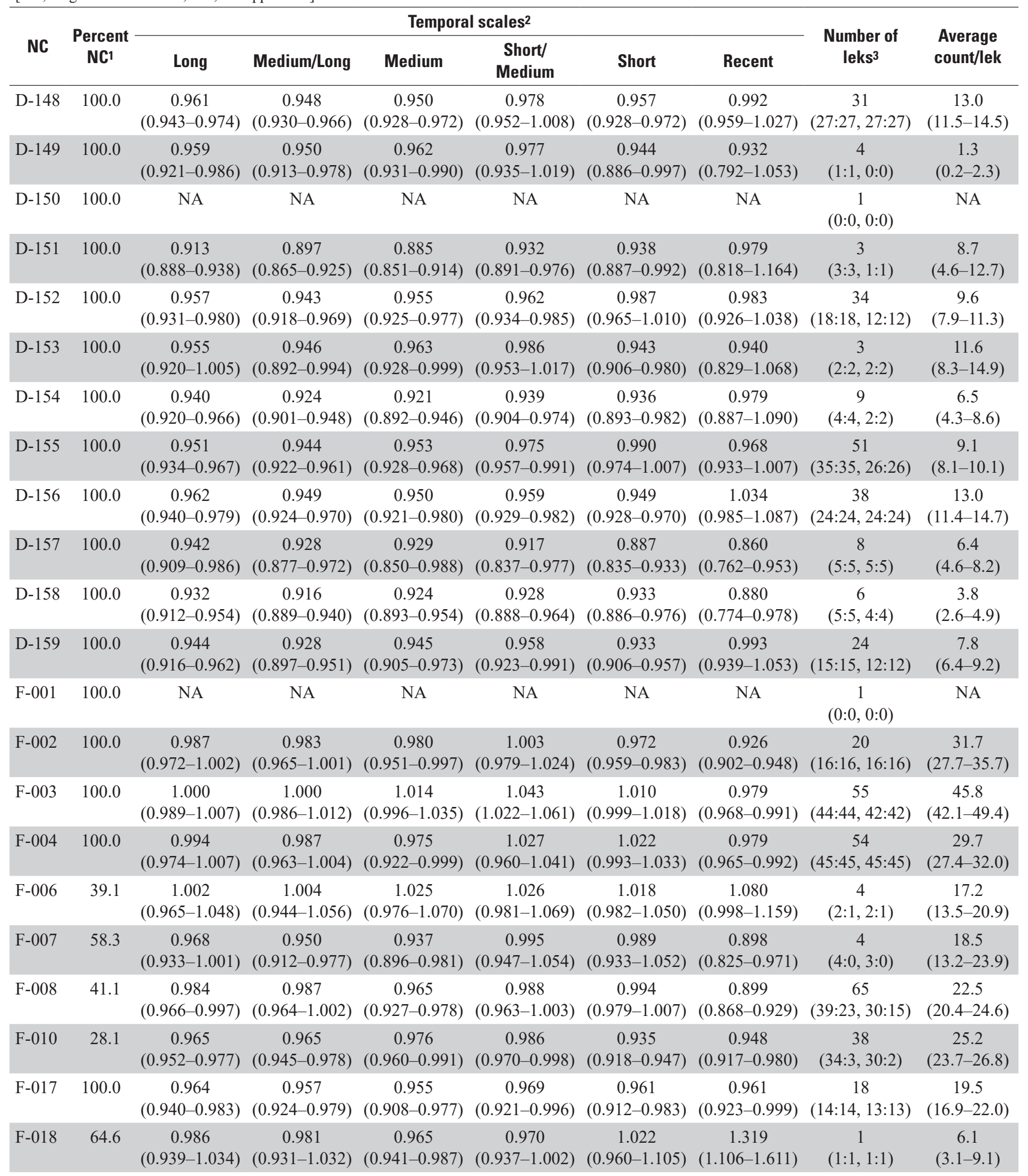


Table 4.14. Table of greater sage-grouse (Centrocercus urophasianus) average annual rate of population change $(\hat{\lambda})$ across six periods of oscillation in abundance that represent temporal scales for each neighborhood cluster within the state of Wyoming. Estimates were derived from leks within the entire neighborhood cluster.-Continued

[NC, neighborhood cluster; NA, not applicable]

\begin{tabular}{|c|c|c|c|c|c|c|c|c|c|}
\hline \multirow[b]{2}{*}{ NC } & \multirow{2}{*}{$\begin{array}{l}\text { Percent } \\
\text { NC1 }\end{array}$} & \multicolumn{6}{|c|}{ Temporal scales ${ }^{2}$} & \multirow{2}{*}{$\begin{array}{l}\text { Number of } \\
\text { leks }^{3}\end{array}$} & \multirow{2}{*}{$\begin{array}{l}\text { Average } \\
\text { count/lek }\end{array}$} \\
\hline & & Long & Medium/Long & Medium & $\begin{array}{c}\text { Short/ } \\
\text { Medium }\end{array}$ & Short & Recent & & \\
\hline F-020 & 100.0 & $\begin{array}{c}0.973 \\
(0.961-0.991)\end{array}$ & $\begin{array}{c}0.964 \\
(0.946-0.986)\end{array}$ & $\begin{array}{c}0.953 \\
(0.939-0.966)\end{array}$ & $\begin{array}{c}0.972 \\
(0.954-0.990)\end{array}$ & $\begin{array}{c}0.961 \\
(0.947-0.977)\end{array}$ & $\begin{array}{c}0.900 \\
(0.868-0.935)\end{array}$ & $\begin{array}{c}36 \\
(31: 31,25: 25)\end{array}$ & $\begin{array}{c}22.8 \\
(20.2-25.3)\end{array}$ \\
\hline F-022 & 100.0 & $\begin{array}{c}0.979 \\
(0.965-0.993)\end{array}$ & $\begin{array}{c}0.978 \\
(0.959-0.989)\end{array}$ & $\begin{array}{c}0.972 \\
(0.947-0.991)\end{array}$ & $\begin{array}{c}0.983 \\
(0.955-1.003)\end{array}$ & $\begin{array}{c}0.995 \\
(0.961-1.012)\end{array}$ & $\begin{array}{c}0.948 \\
(0.922-0.977)\end{array}$ & $\begin{array}{c}41 \\
(30: 30,30: 30)\end{array}$ & $\begin{array}{c}21.4 \\
(19.0-23.8)\end{array}$ \\
\hline F-023 & 100.0 & $\begin{array}{c}0.975 \\
(0.960-0.996)\end{array}$ & $\begin{array}{c}0.969 \\
(0.949-0.991)\end{array}$ & $\begin{array}{c}0.970 \\
(0.945-0.994)\end{array}$ & $\begin{array}{c}0.985 \\
(0.959-1.006)\end{array}$ & $\begin{array}{c}0.984 \\
(0.961-1.003)\end{array}$ & $\begin{array}{c}1.049 \\
(1.024-1.073)\end{array}$ & $\begin{array}{c}24 \\
(21: 21,20: 20)\end{array}$ & $\begin{array}{c}22.2 \\
(20.0-24.5)\end{array}$ \\
\hline F-027 & 89.0 & $\begin{array}{c}1.007 \\
(0.985-1.033)\end{array}$ & $\begin{array}{c}1.008 \\
(0.987-1.033)\end{array}$ & $\begin{array}{c}1.003 \\
(0.978-1.034)\end{array}$ & $\begin{array}{c}0.992 \\
(0.968-1.034)\end{array}$ & $\begin{array}{c}0.976 \\
(0.950-1.029)\end{array}$ & $\begin{array}{c}1.027 \\
(0.975-1.115)\end{array}$ & $\begin{array}{c}19 \\
(14: 12,13: 11)\end{array}$ & $\begin{array}{c}26.0 \\
(22.7-29.4)\end{array}$ \\
\hline F-028 & 100.0 & $\begin{array}{c}1.005 \\
(0.990-1.022)\end{array}$ & $\begin{array}{c}0.998 \\
(0.978-1.012)\end{array}$ & $\begin{array}{c}0.996 \\
(0.978-1.006)\end{array}$ & $\begin{array}{c}1.007 \\
(0.984-1.022)\end{array}$ & $\begin{array}{c}0.983 \\
(0.962-0.998)\end{array}$ & $\begin{array}{c}1.108 \\
(1.074-1.146)\end{array}$ & $\begin{array}{c}48 \\
(41: 41,39: 39)\end{array}$ & $\begin{array}{c}11.9 \\
(10.9-13.0)\end{array}$ \\
\hline F-029 & 100.0 & $\begin{array}{c}0.977 \\
(0.967-0.988)\end{array}$ & $\begin{array}{c}0.979 \\
(0.968-0.989)\end{array}$ & $\begin{array}{c}0.989 \\
(0.978-0.997)\end{array}$ & $\begin{array}{c}0.993 \\
(0.981-1.001)\end{array}$ & $\begin{array}{c}0.990 \\
(0.978-0.999)\end{array}$ & $\begin{array}{c}1.028 \\
(1.000-1.056)\end{array}$ & $\begin{array}{c}42 \\
(33: 33,29: 29)\end{array}$ & $\begin{array}{c}13.4 \\
(12.4-14.4)\end{array}$ \\
\hline F-030 & 100.0 & $\begin{array}{c}0.996 \\
(0.972-1.012)\end{array}$ & $\begin{array}{c}0.998 \\
(0.969-1.017)\end{array}$ & $\begin{array}{c}0.982 \\
(0.965-0.998)\end{array}$ & $\begin{array}{c}0.991 \\
(0.974-1.004)\end{array}$ & $\begin{array}{c}0.996 \\
(0.979-1.013)\end{array}$ & $\begin{array}{c}0.959 \\
(0.921-0.997)\end{array}$ & $\begin{array}{c}11 \\
(11: 11,11: 11)\end{array}$ & $\begin{array}{c}15.2 \\
(13.1-17.4)\end{array}$ \\
\hline F-031 & 100.0 & $\begin{array}{c}0.983 \\
(0.968-0.999)\end{array}$ & $\begin{array}{c}0.977 \\
(0.951-0.993)\end{array}$ & $\begin{array}{c}0.969 \\
(0.938-0.984)\end{array}$ & $\begin{array}{c}0.995 \\
(0.965-1.014)\end{array}$ & $\begin{array}{c}0.987 \\
(0.965-1.009)\end{array}$ & $\begin{array}{c}0.997 \\
(0.956-1.047)\end{array}$ & $\begin{array}{c}24 \\
(20: 20,18: 18)\end{array}$ & $\begin{array}{c}12.3 \\
(10.9-13.7)\end{array}$ \\
\hline F-035 & 100.0 & $\begin{array}{c}0.965 \\
(0.925-0.997)\end{array}$ & $\begin{array}{c}0.959 \\
(0.921-0.995)\end{array}$ & $\begin{array}{c}0.950 \\
(0.918-0.986)\end{array}$ & $\begin{array}{c}0.930 \\
(0.886-0.973)\end{array}$ & $\begin{array}{c}0.908 \\
(0.851-0.957)\end{array}$ & $\begin{array}{c}0.975 \\
(0.843-1.128)\end{array}$ & $(1: 1,1: 1)$ & $\begin{array}{c}1.8 \\
(0.8-2.8)\end{array}$ \\
\hline F-036 & 100.0 & $\begin{array}{c}0.984 \\
(0.963-1.006)\end{array}$ & $\begin{array}{c}0.984 \\
(0.968-0.999)\end{array}$ & $\begin{array}{c}0.990 \\
(0.968-0.999)\end{array}$ & $\begin{array}{c}1.013 \\
(0.987-1.025)\end{array}$ & $\begin{array}{c}1.006 \\
(0.988-1.020)\end{array}$ & $\begin{array}{c}1.037 \\
(1.007-1.070)\end{array}$ & $\begin{array}{c}23 \\
(20: 20,17: 17)\end{array}$ & $\begin{array}{c}11.9 \\
(10.8-13.0)\end{array}$ \\
\hline F-037 & 100.0 & $\begin{array}{c}0.978 \\
(0.962-0.992)\end{array}$ & $\begin{array}{c}0.972 \\
(0.958-0.986)\end{array}$ & $\begin{array}{c}0.982 \\
(0.965-0.992)\end{array}$ & $\begin{array}{c}0.992 \\
(0.972-1.004)\end{array}$ & $\begin{array}{c}0.976 \\
(0.954-0.991)\end{array}$ & $\begin{array}{c}1.025 \\
(0.991-1.062)\end{array}$ & $\begin{array}{c}43 \\
(37: 37,34: 34)\end{array}$ & $\begin{array}{c}14.1 \\
(13.1-15.1)\end{array}$ \\
\hline F-038 & 100.0 & $\begin{array}{c}1.005 \\
(0.990-1.016)\end{array}$ & $\begin{array}{c}1.002 \\
(0.987-1.016)\end{array}$ & $\begin{array}{c}0.985 \\
(0.972-1.001)\end{array}$ & $\begin{array}{c}1.027 \\
(1.012-1.042)\end{array}$ & $\begin{array}{c}1.015 \\
(1.004-1.025)\end{array}$ & $\begin{array}{c}1.057 \\
(1.037-1.077)\end{array}$ & $\begin{array}{c}34 \\
(29: 29,28: 28)\end{array}$ & $\begin{array}{c}30.3 \\
(27.8-32.8)\end{array}$ \\
\hline F-039 & 100.0 & $\begin{array}{c}0.973 \\
(0.956-0.983)\end{array}$ & $\begin{array}{c}0.977 \\
(0.957-0.994)\end{array}$ & $\begin{array}{c}0.977 \\
(0.954-1.000)\end{array}$ & $\begin{array}{c}1.032 \\
(1.011-1.050)\end{array}$ & $\begin{array}{c}0.999 \\
(0.980-1.013)\end{array}$ & $\begin{array}{c}1.006 \\
(0.987-1.027)\end{array}$ & $\begin{array}{c}33 \\
(27: 27,25: 25)\end{array}$ & $\begin{array}{c}25.7 \\
(23.0-28.4)\end{array}$ \\
\hline F-040 & 100.0 & $\begin{array}{c}0.967 \\
(0.952-0.984)\end{array}$ & $\begin{array}{c}0.957 \\
(0.931-0.972)\end{array}$ & $\begin{array}{c}0.969 \\
(0.935-0.986)\end{array}$ & $\begin{array}{c}1.011 \\
(0.980-1.033)\end{array}$ & $\begin{array}{c}0.987 \\
(0.975-0.997)\end{array}$ & $\begin{array}{c}1.071 \\
(1.038-1.104)\end{array}$ & $\begin{array}{c}35 \\
(29: 29,25: 25)\end{array}$ & $\begin{array}{c}14.8 \\
(13.4-16.2)\end{array}$ \\
\hline F-041 & 100.0 & $\begin{array}{c}0.986 \\
(0.971-1.009)\end{array}$ & $\begin{array}{c}0.987 \\
(0.965-1.008)\end{array}$ & $\begin{array}{c}1.000 \\
(0.974-1.018)\end{array}$ & $\begin{array}{c}1.026 \\
(1.002-1.044)\end{array}$ & $\begin{array}{c}1.001 \\
(0.974-1.022)\end{array}$ & $\begin{array}{c}1.040 \\
(1.004-1.073)\end{array}$ & $\begin{array}{c}21 \\
(17: 17,17: 17)\end{array}$ & $\begin{array}{c}17.4 \\
(15.4-19.3)\end{array}$ \\
\hline
\end{tabular}


Table 4.14. Table of greater sage-grouse (Centrocercus urophasianus) average annual rate of population change $(\hat{\lambda})$ across six periods of oscillation in abundance that represent temporal scales for each neighborhood cluster within the state of Wyoming. Estimates were derived from leks within the entire neighborhood cluster.-Continued

[NC, neighborhood cluster; NA, not applicable]

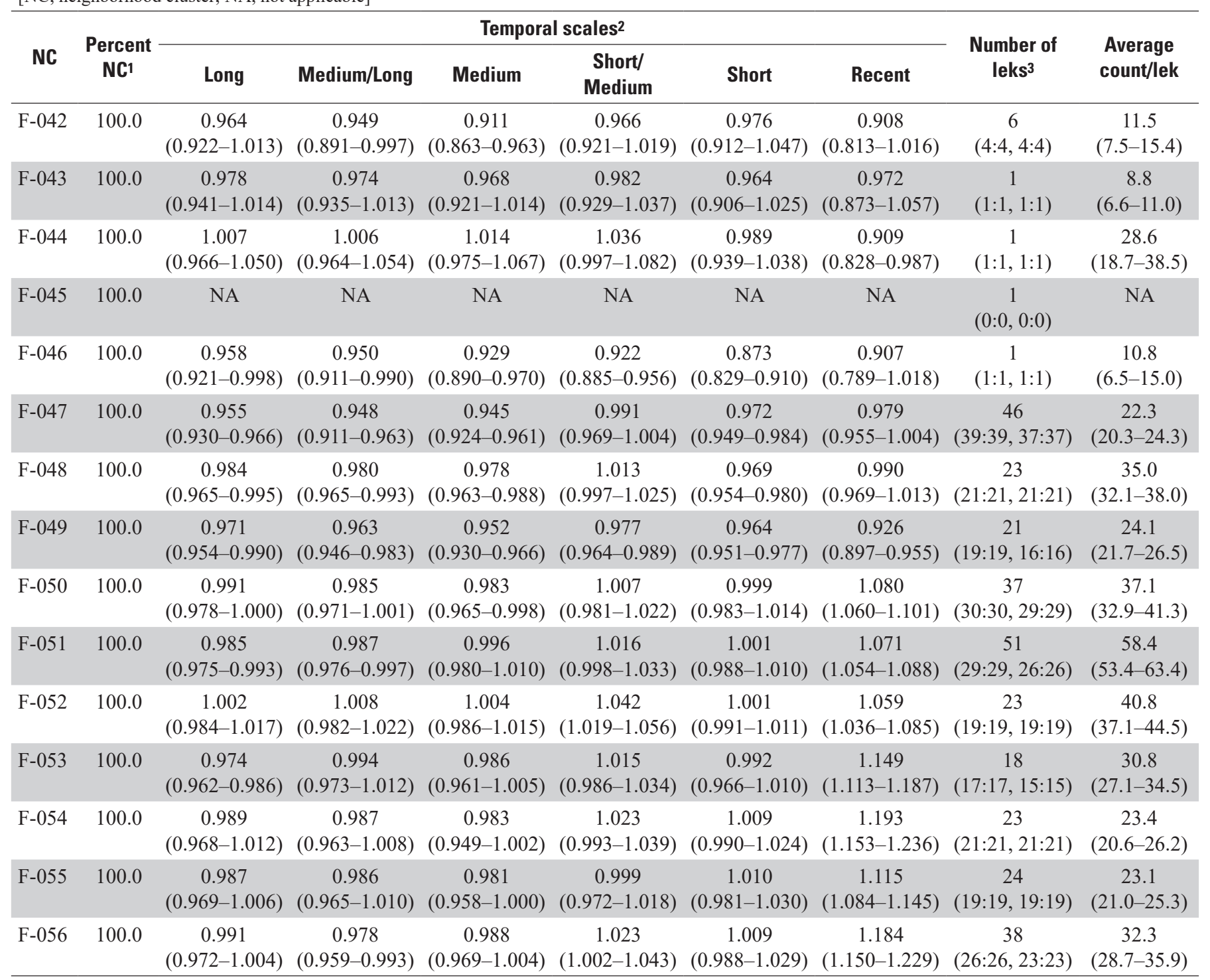

1The percent of each neighborhood cluster that intersects the state.

${ }^{2}$ Temporal scales were estimated from present to each major population abundance nadir (trough) since 1960. Number of temporal scales were used to estimate population trends across six different temporal scales from approximately 10 to approximately 60 years.

${ }^{3}$ Number of leks in database. In parentheses from left to right is (1) total number in cluster were used in trend analysis, (2) number used in trend analysis within state boundary, (3) total number in cluster were used in the targeted annual warning system (TAWS) analysis, and (4) number used in TAWS analysis within state boundary. 

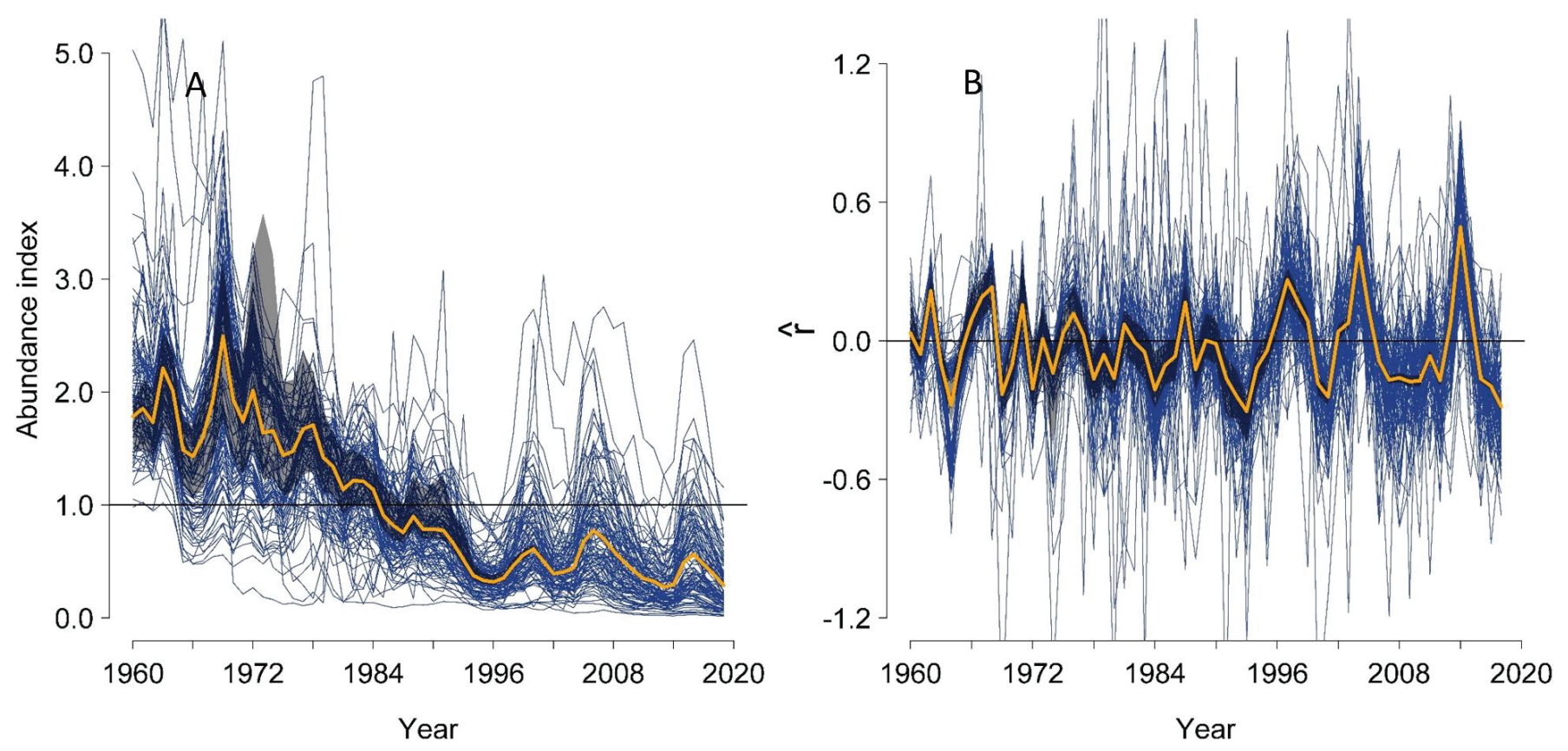

Figure 4.46. A, Abundance index (calculated as $\hat{N}$ divided by 60 -year mean of $\hat{N}$ ); and $B$, intrinsic rate of population change $(\hat{r})$ of greater sage-grouse (Centrocercus urophasianus) within the state of Wyoming from 1960-2019. Thick yellow line represents median estimates across all leks. Shaded areas represent 95 -percent credible limits. Thin blue lines represent median values for neighborhood clusters. Black horizontal line (abundance index=1.0) represents 60 -year average. 

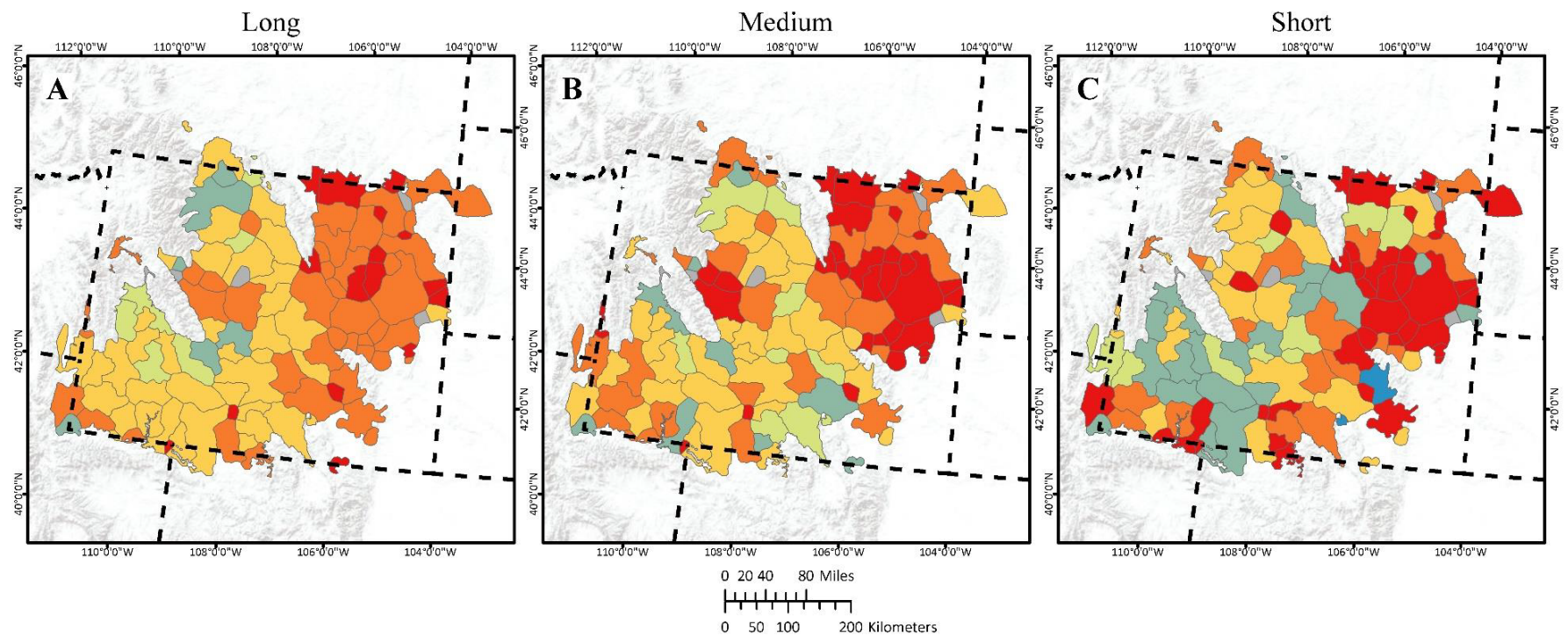

\section{EXPLANATION}

Neighborhood clusters (Level 2) intersecting Wyoming: polygon colors represent average lambda values for sage-grouse populations over varying lengths of time (long, medium, short). Symbols are based on a range-wide distribution of values and all may not be represented here.

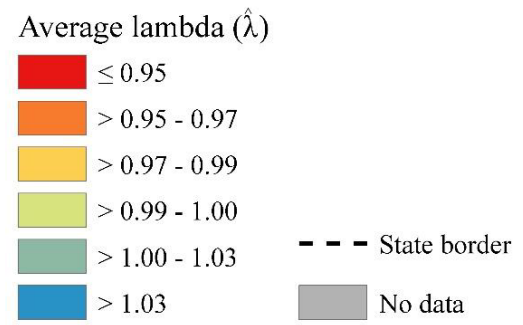

Coordinate System: Albers Projection: Albers Datum: WGS 1984 false easting: 0.0000 false northing: 0.0000 central meridian: -96.0000 standard parallel $1: 29.5000$ standard parallel 2: 45.5000 latitude of origin: 23.0000 Units: Meter

Service Layer Credits: Sources: Esri, USGS, NOAA

Figure 4.47. Spatial estimates of population trends across three temporal scales based on periods of oscillation (short, medium, and long) while accounting for fluctuations of greater sage-grouse (Centrocercus urophasianus) abundance across different neighborhood clusters within the state of Wyoming. Map images are the intellectual property of Esri and are used herein under license. Copyright (C) 2020 Esri and its licensors. All rights reserved. 

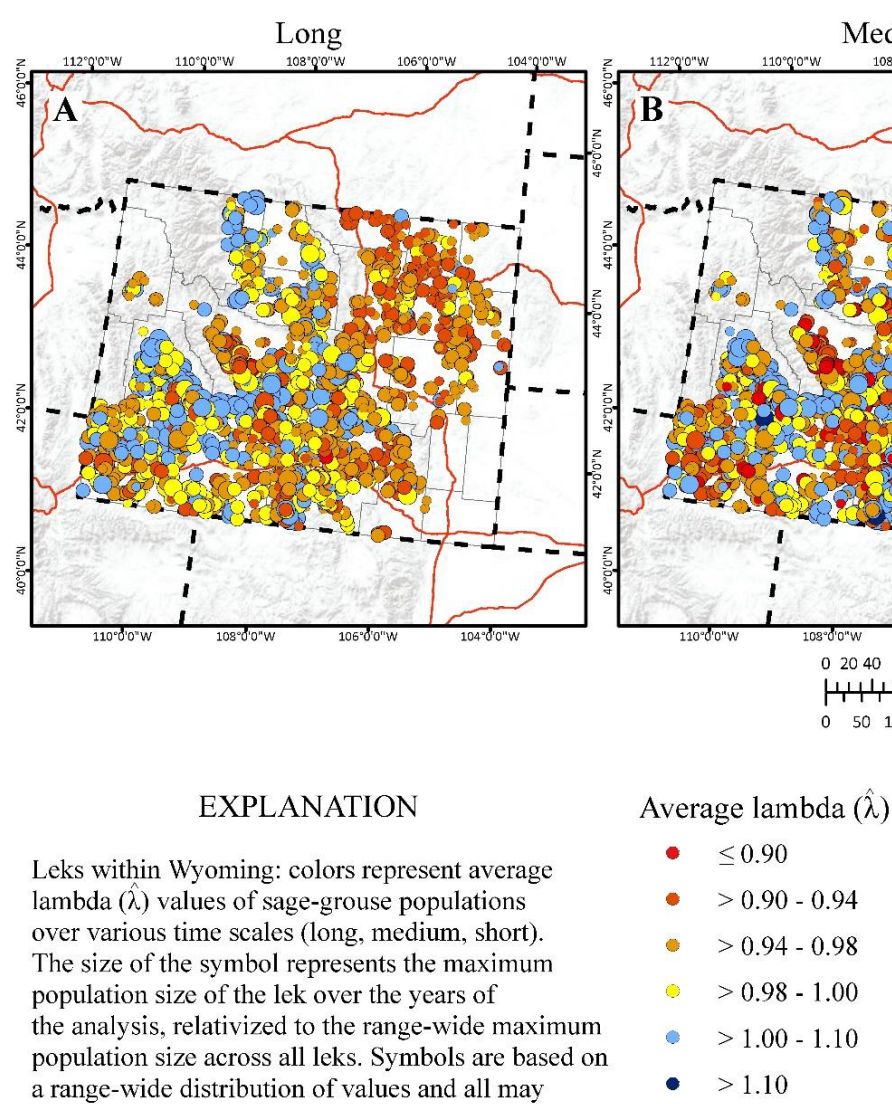

Medium
um

${ }_{1060} \log ^{\circ} \mathrm{w}$

${ }_{104^{\circ} 0^{\circ} 10^{\circ} \mathrm{N}}$

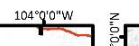

Short

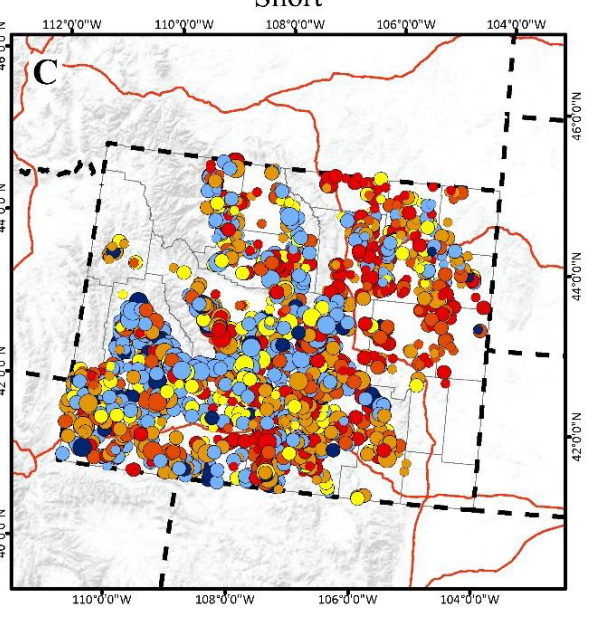

$2040 \quad 80$ Miles

50100200 Kilometers

\section{EXPLANATION}

Leks within Wyoming: colors represent average lambda $(\hat{\lambda})$ values of sage-grouse populations over various time scales (long, medium, short). The size of the symbol represents the maximum population size of the lek over the years of the analysis, relativized to the range-wide maximum population size across all leks. Symbols are based on a range-wide distribution of values and all may not be represented here.

$$
\text { Average lambda }(\hat{\lambda})
$$$$
\longrightarrow \text { Road }
$$$$
\text { - County line }
$$$$
\text { - - - State border }
$$$$
\text { Size guide }
$$$$
\text { Maximum lek count }
$$$$
\text { Largest }
$$$$
Q_{\text {Smallest }}
$$

\author{
Coordinate System: Albers \\ Projection: Albers \\ Datum: WGS 1984 \\ false easting: 0.0000 \\ false northing: 0.0000 \\ central meridian: -96.0000 \\ standard parallel 1: 29.5000 \\ standard parallel $2: 45.5000$ \\ latitude of origin: 23.0000 \\ Units: Meter \\ Service Layer Credits: \\ Sources: Esri, USGS, NOAA
}

Figure 4.48. Spatial estimates of population trends across three temporal scales based on periods of oscillation (short, medium, and long) while accounting for fluctuations of greater sage-grouse (Centrocercus urophasianus) abundance across lek sites within the state of Wyoming. Map images are the intellectual property of Esri and are used herein under license. Copyright (C) 2020 Esri and its licensors. All rights reserved. 


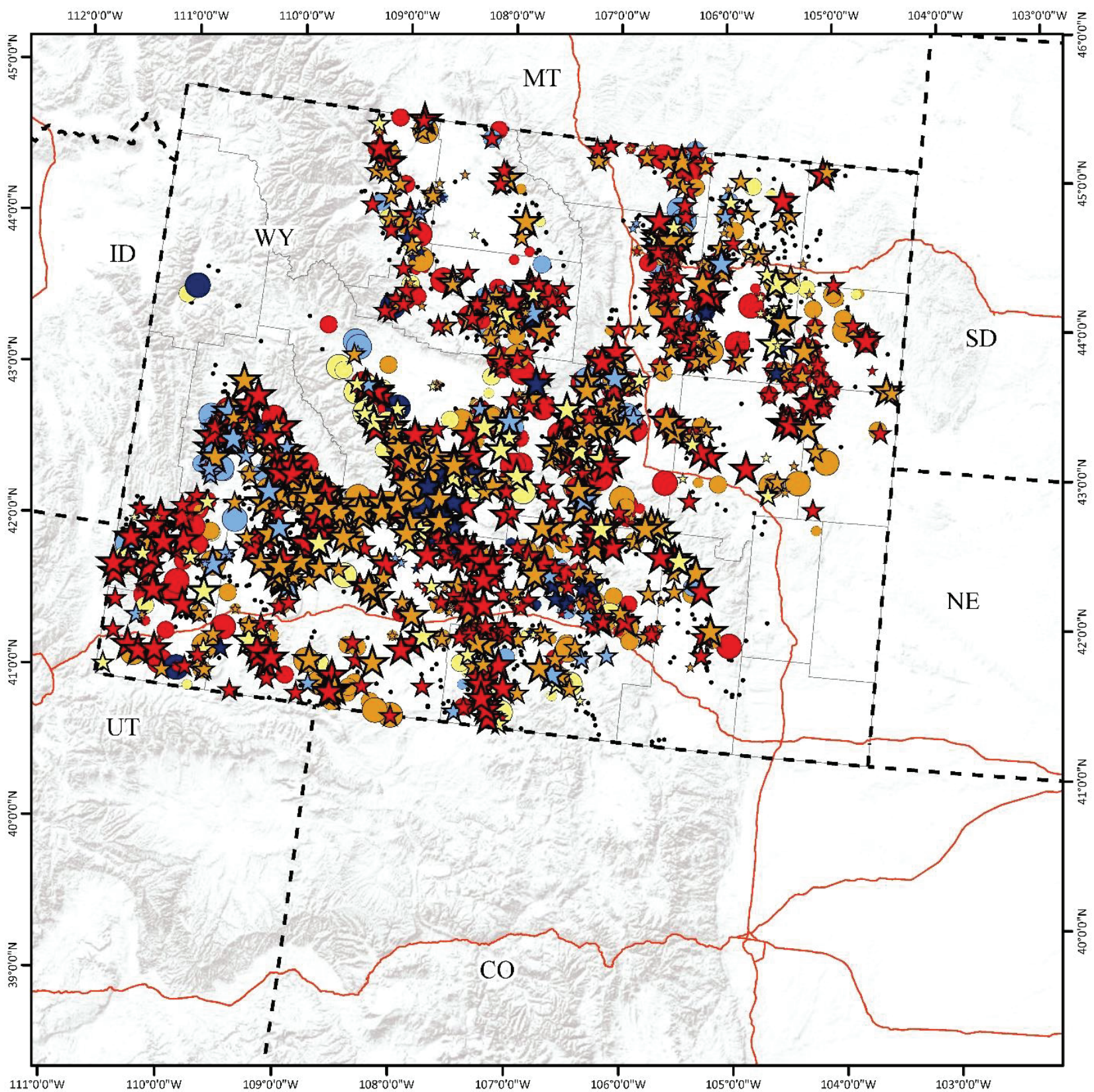

EXPLANATION

Leks in Wyoming: colors represent a range of years the most recent event occurred. Stars represent warnings, circles represent watches, and black dots represent leks which did not experience warnings or watches. The size of the symbol represents the maximum population size of the lek over the 30-year period, relativized to the rangewide maximum population size across all leks. Symbols are based on a range-wide distribution of values and all may not be represented here.

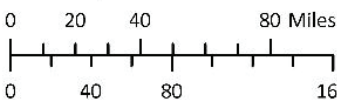

80 Miles
Coordinate System: Albers Projection: Albers

$\bigcirc$ Watch Relative maximum $\begin{aligned} & \text { Datum: WGS } 1984 \\ & \text { false easting: } 0.0000\end{aligned}$

- Lek population size false northing: 0.0000

- - - State border Largest $\quad$ central meridian: -96.0000 standard parallel 2: 45.5000 latitude of origin: 23.0000 Units: Meter

Service Layer Credits: Sources: Esri, USGS, NOAA

Figure 4.49. Spatial and temporal depiction of watches and warnings of greater sage-grouse (Centrocercus urophasianus) population declines at the lek scale within the state of Wyoming from 1990 to 2019. Map image is the intellectual property of Esri and is used herein under license. Copyright $(2020$ Esri and its licensors. All rights reserved. 


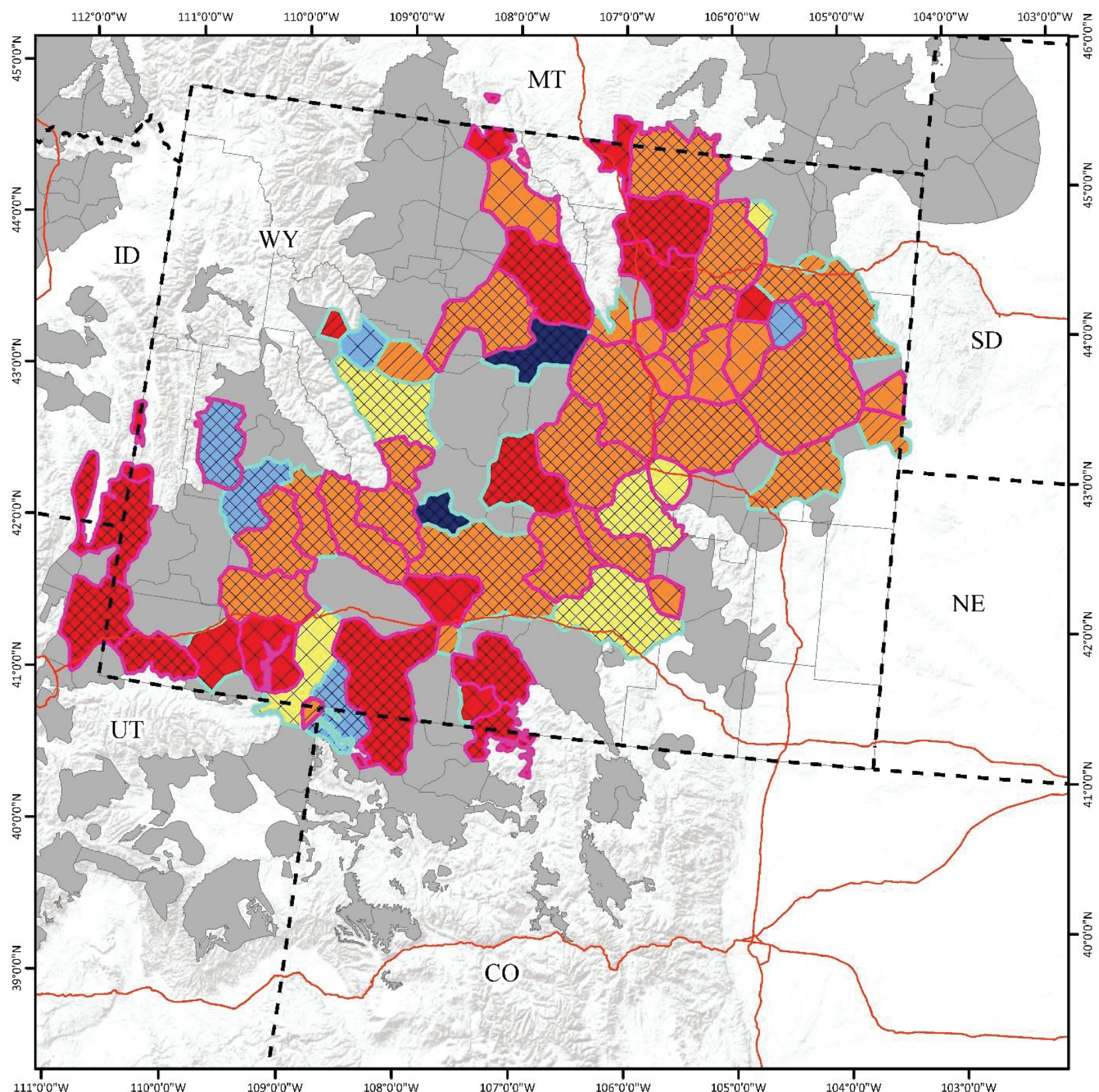

EXPLANATION

Neighborhood clusters (Level 2) in Wyoming: polygon colors represent a range of years the most recent event occurred. Oulline colors indicate whether the most recent event was a watch or warning. The hatching represents the maximum population size of the cluster over the 30 -year period, relativized to the range-wide maximum population size across all neighborhood clusters. Symbols are based on a range-wide distribution of values and all may not be represented here. $01020 \quad 40$ Miles

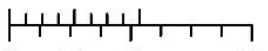
o $30 \quad 60 \quad 120$ Kilometers
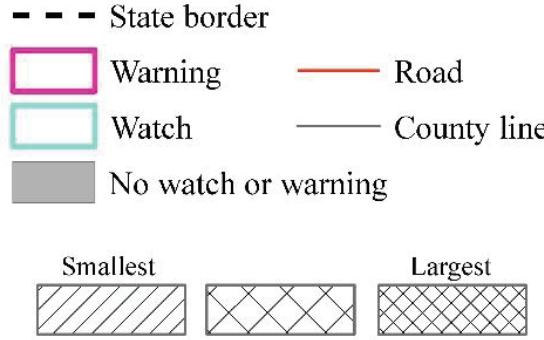

1995-1999 2000-2004 2005- $2009 \quad 2010-20142015-2019$
Coordinate System: Albers Projection: Albers Datum: WGS 1984 false easting: 0.0000 false northing: 0.0000 central meridian: -96.0000 standard parallel 1: 29.5000 standard parallel 2: 45.5000 latitude of origin: 23.0000 Units: Meter

Service Layer Credits: Sources: Esri, USGS, NOAA

Figure 4.50. Spatial and temporal depiction of watches and warnings of greater sage-grouse (Centrocercus urophasianus) population declines at neighborhood clusters within the state of Wyoming from 1990 to 2019. Map image is the intellectual property of Esri and is used herein under license. Copyright (C) 2020 Esri and its licensors. All rights reserved. 


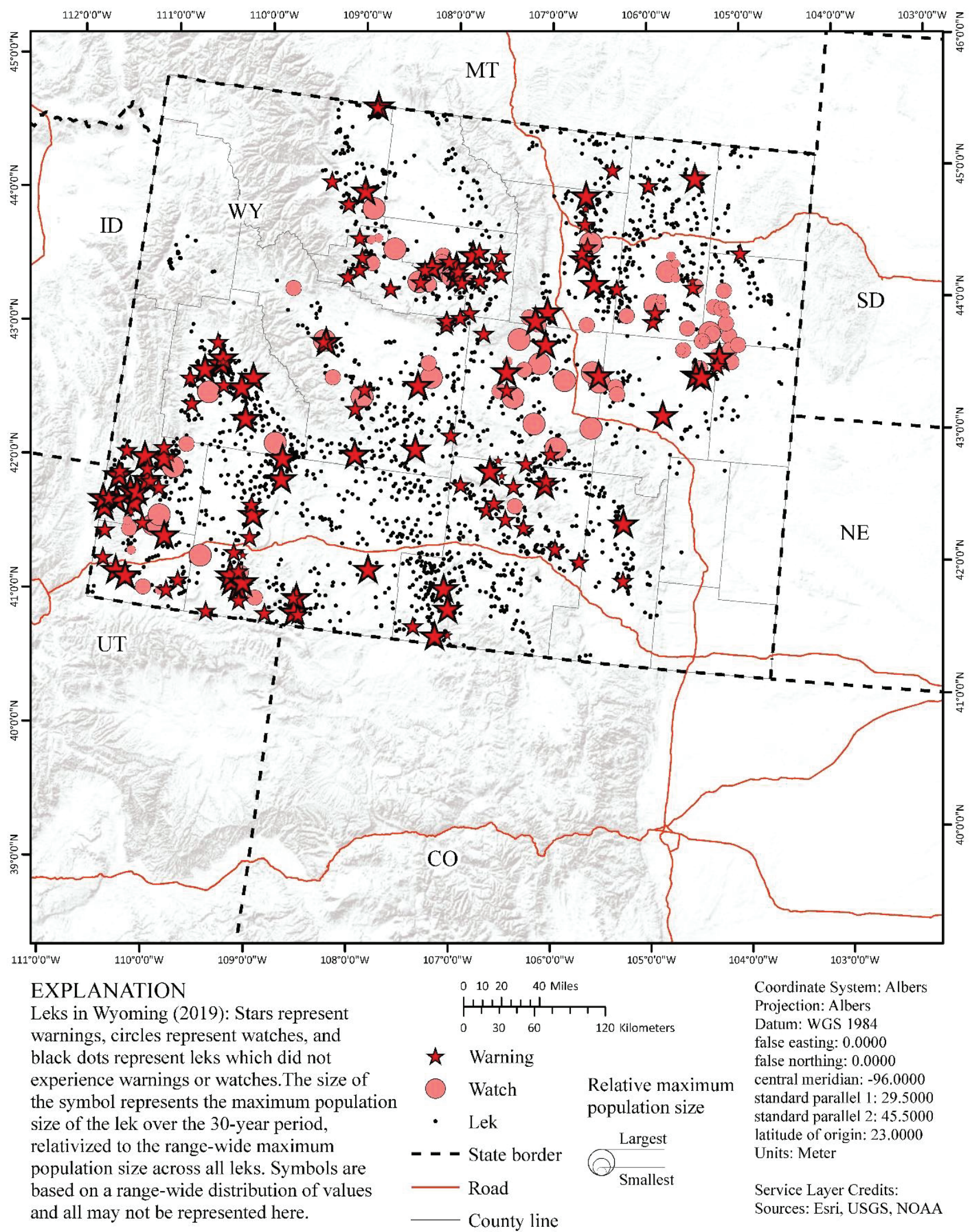

Figure 4.51. Spatial and temporal depiction of watches and warnings of greater sage-grouse (Centrocercus urophasianus) population declines at the lek and neighborhood cluster scale within the state of Wyoming during 2019. Map image is the intellectual property of Esri and is used herein under license. Copyright () 2020 Esri and its licensors. All rights reserved. 


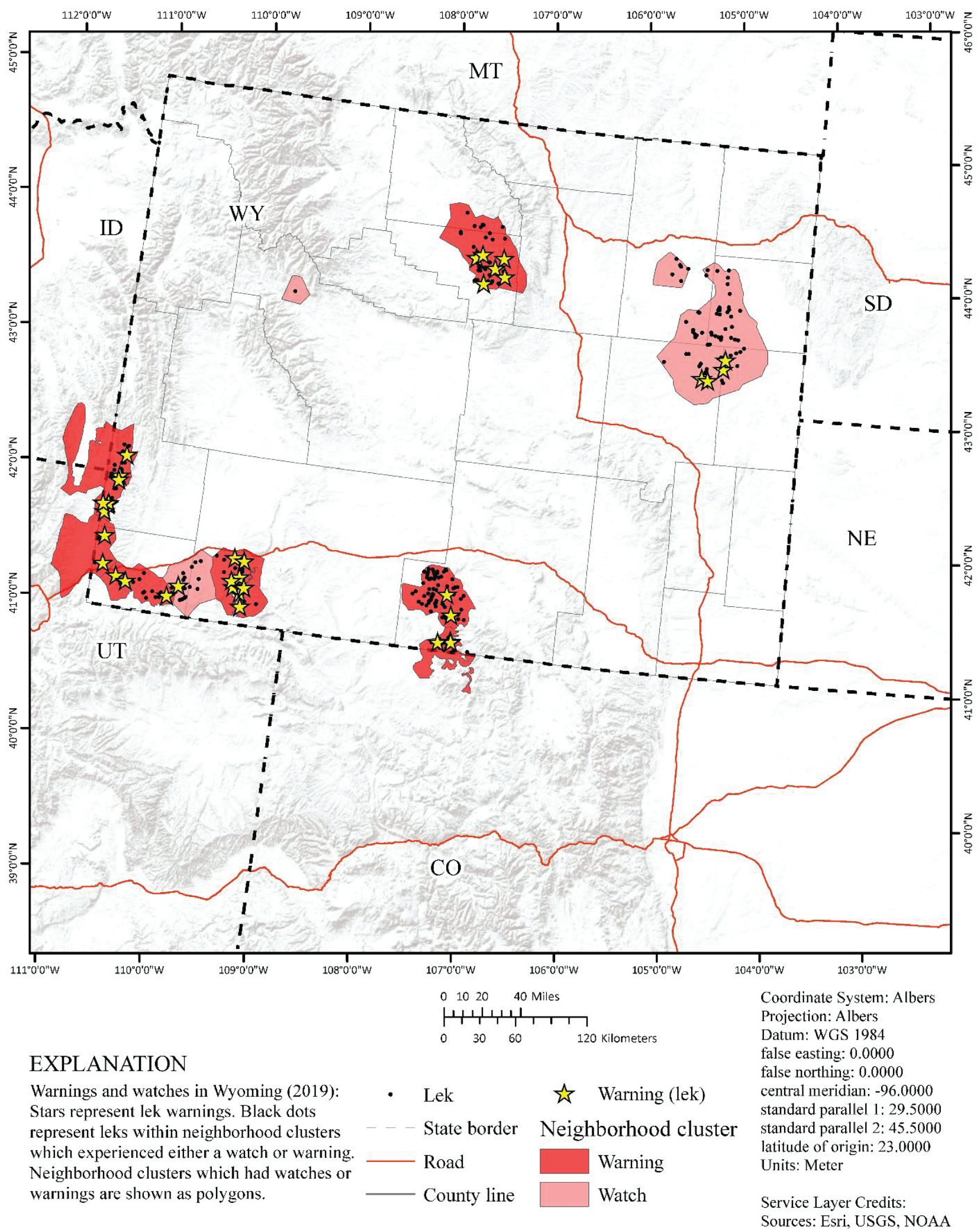

Figure 4.52. Spatial and temporal depiction of watches and warnings of greater sage-grouse (Centrocercus urophasianus) population declines at the neighborhood cluster scale within the state of Wyoming during 2019. All leks within watch and warning boundaries were assigned as watch and warning, respectively. Yellow stars represent leks that reached warning independently. Map image is the intellectual property of Esri and is used herein under license. Copyright $\Subset 2020$ Esri and its licensors. All rights reserved. 


\section{Montana Results}

\section{Montana Lek Data and Clusters (Objectives 1 and 2)}

Montana intersected CC-D, CC-E, and CC-F and contained or intersected 83 different NCs (fig. 4.53). Total area of the $83 \mathrm{NCs}$ within Montana was 13,266,902 ha. Of these clusters, 58 were used in the analysis after, 25 were omitted because of limitations in sample sizes. These areas consisted of 1,459 leks, of which 1,272 were located within the Montana state boundary, representing 15.11 percent of the range-wide lek database. After extensive QA/QC, we used 564 leks from Montana in the SSM for population trend estimation (tables 4.15 and 4.16), totaling 8,137 individual lek counts. Mean male count was 12.57 (95-percent confidence interval $=12.41-12.74$ ) for leks within NCs that were within or overlapped Montana. Mean male count for leks within Montana was 13.26 (95-percent confidence interval=13.07-13.46).

\section{Montana Population Trend Analysis (Objective 3)}

Although Montana intersected three CCs, the largest part (88.5 percent) of the state falls within CC-D. For CC-D we estimated six population abundance nadirs (troughs) that dated back to 1960. Each of these population abundance nadirs represent between one and six complete periods of oscillation. We used these nadirs to estimate population trends across three different temporal scales that represented two, four, and six complete periods for the state (for instance, second, fourth, and sixth nadir). We estimated the average annual finite rate of population change $(\hat{\lambda})$ at the short (two periods), medium (four periods), and long (six periods) temporal scales as 0.967 (95-percent $\mathrm{CRI}=0.960-0.977$ ), 0.975 (95-percent CRI $=0.967-0.982$ ), and 0.968 (95-percent $\mathrm{CRI}=0.962-0.973$ ), respectively (fig. 4.54). Climate cluster estimates, which included leks in adjacent states to Montana, were slightly different than estimates generated from leks only within Montana. For all NCs that were modeled and intersected Montana, we estimated median $\hat{\lambda}$ to be less than 1.0 for $89.7,87.9$, and 94.8 percent across short, medium, and long temporal scales, respectively (fig. 4.55; table 4.16). We estimated median $\hat{\lambda}$ to be less than 1.0 for $82.8,82.4$, and 91.8 percent of all modeled leks within Montana across short, medium, and long temporal scales, respectively (fig. 4.56). We reported spatial and temporal variation in average annual $\hat{\lambda}$ across different NCs (fig. 4.55) and leks (fig. 4.56).

\section{Montana Targeted Annual Warning System Analysis (Objective 4)}

During 1990-2019, the TAWS for sage-grouse populations in Montana activated a total of 202 and 124 leks as watches and warnings, respectively (fig. 4.57), which was 43.5 and 26.7 percent of the sampled leks used in the analysis. On average, across the 29 years, approximately 3.8 and 1.1 percent of leks per year experienced watches and warnings, respectively. The higher percentage for watches corresponds to repetitive activation. During this time frame, TAWS also activated a total of 10 and 8 NCs as watches and warnings, respectively (fig. 4.58), which was 19.2 and 15.4 percent of the sampled clusters used in the analysis. On average, across the 29 years, approximately 1.3 and 0.6 percent of clusters per year experienced watches and warnings, respectively. The higher percentage for watches corresponds to repetitive activation. During 2019, the TAWS activated eight (first $=3$ ) watches and five (first=2) warnings at leks (fig. 4.59) but no watches or warnings at NC scale. 


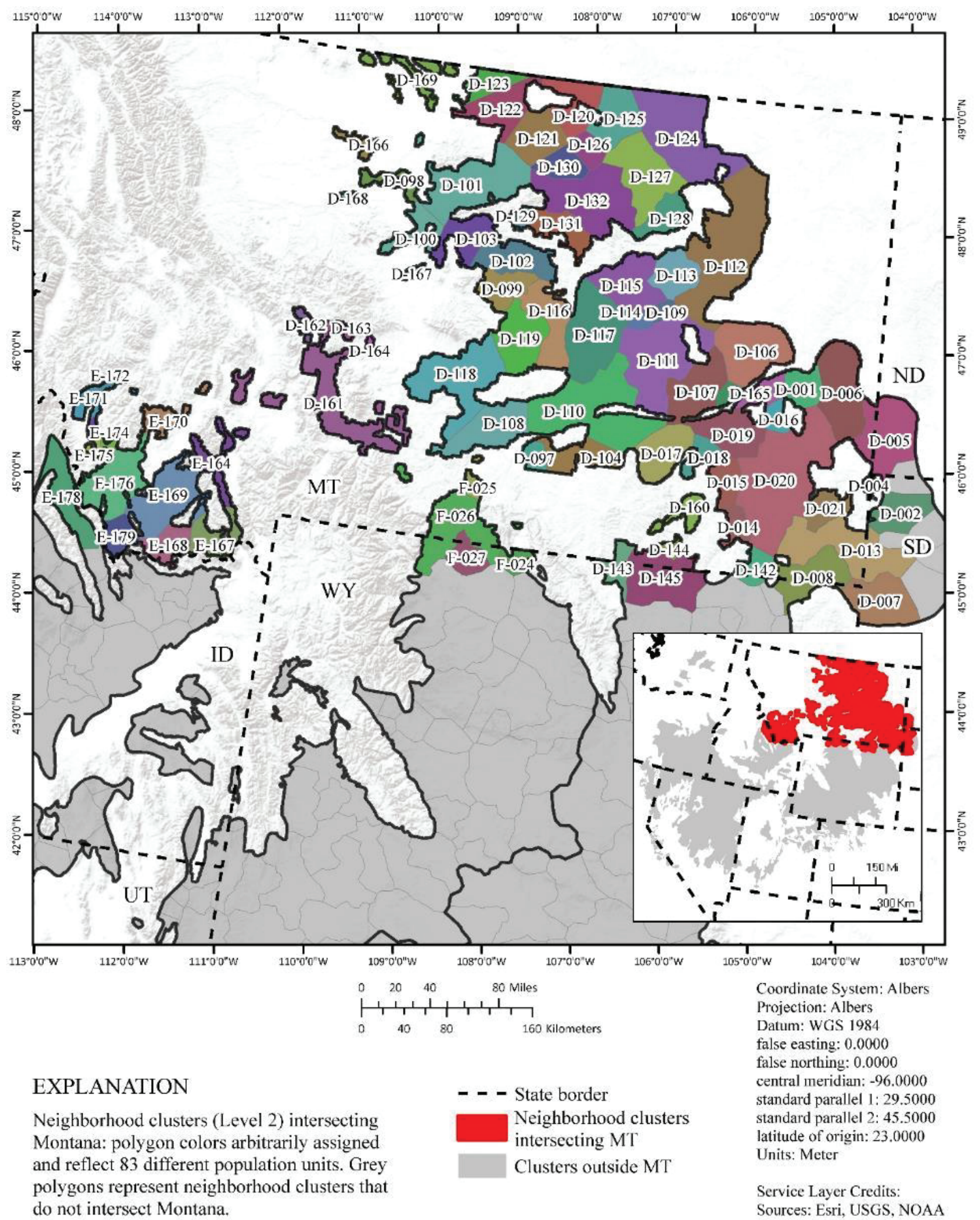

Figure 4.53. Greater sage-grouse (Centrocercus urophasianus) hierarchical population monitoring framework for neighborhood clusters that intersect the state of Montana. Map image is the intellectual property of Esri and is used herein under license. Copyright $(\subset)$ 2020 Esri and its licensors. All rights reserved. 
Table 4.15. Table of greater sage-grouse (Centrocercus urophasianus) average annual rate of population change ( $\hat{\lambda})$ across six periods of oscillation in abundance that represent temporal scales for each climate cluster within the state of Montana. Estimates were derived from leks within the entire climate cluster.

[CC, climate cluster; D, eastern area; E, Great Basin area; F, Wyoming]

\begin{tabular}{|c|c|c|c|c|c|c|c|c|c|}
\hline \multirow[b]{2}{*}{ CC } & \multirow{2}{*}{$\begin{array}{l}\text { Percent } \\
\text { CC1 }\end{array}$} & \multicolumn{6}{|c|}{ Temporal scales 2} & \multirow[b]{2}{*}{ Number of leks ${ }^{3}$} & \multirow{2}{*}{$\begin{array}{l}\text { Average } \\
\text { count/lek }\end{array}$} \\
\hline & & Long & Medium/Long & Medium & $\begin{array}{c}\text { Short/ } \\
\text { Medium }\end{array}$ & Short & Recent & & \\
\hline $\mathrm{E}$ & 3.6 & $\begin{array}{c}0.971 \\
(0.967-0.976)\end{array}$ & $\begin{array}{c}0.973 \\
(0.967-0.978)\end{array}$ & $\begin{array}{c}0.974 \\
(0.963-0.979)\end{array}$ & $\begin{array}{c}0.986 \\
(0.981-0.990)\end{array}$ & $\begin{array}{c}0.968 \\
(0.964-0.971)\end{array}$ & $\begin{array}{c}0.949 \\
(0.944-0.955)\end{array}$ & $\begin{array}{c}4,012 \\
(2,187: 33 \\
1,908: 31)\end{array}$ & $\begin{array}{c}17.3 \\
(17.1-17.5)\end{array}$ \\
\hline $\mathrm{F}$ & 3.2 & $\begin{array}{c}0.980 \\
(0.975-0.987)\end{array}$ & $\begin{array}{c}0.976 \\
(0.969-0.984)\end{array}$ & $\begin{array}{c}0.976 \\
(0.966-0.980)\end{array}$ & $\begin{array}{c}1.003 \\
(0.997-1.008)\end{array}$ & $\begin{array}{c}0.991 \\
(0.988-0.995)\end{array}$ & $\begin{array}{c}1.016 \\
(1.011-1.023)\end{array}$ & $\begin{array}{c}1,253 \\
(974: 20,892: 19)\end{array}$ & $\begin{array}{c}23.7 \\
(23.3-24.2)\end{array}$ \\
\hline
\end{tabular}

1The percent of each climate cluster that intersects the state.

2Temporal scales were estimated from present to each major population abundance nadir (trough) since 1960. Number of temporal scales were used to estimate population trends across six different temporal scales from approximately 10 to approximately 60 years.

${ }^{3}$ Number of leks in database. In parentheses from left to right is (1) total number in cluster were used in trend analysis, (2) number used in trend analysis within state boundary, (3) total number in cluster were used in the targeted annual warning system (TAWS) analysis, and (4) number used in TAWS analysis within state boundary.

Table 4.16. Table of greater sage-grouse (Centrocercus urophasianus) average annual rate of population change $(\hat{\lambda})$ across six periods of oscillation in abundance that represent temporal scales for each neighborhood cluster within the state of Montana. Estimates were derived from leks within the entire neighborhood cluster.

[NC, neighborhood cluster; NA, not applicable]

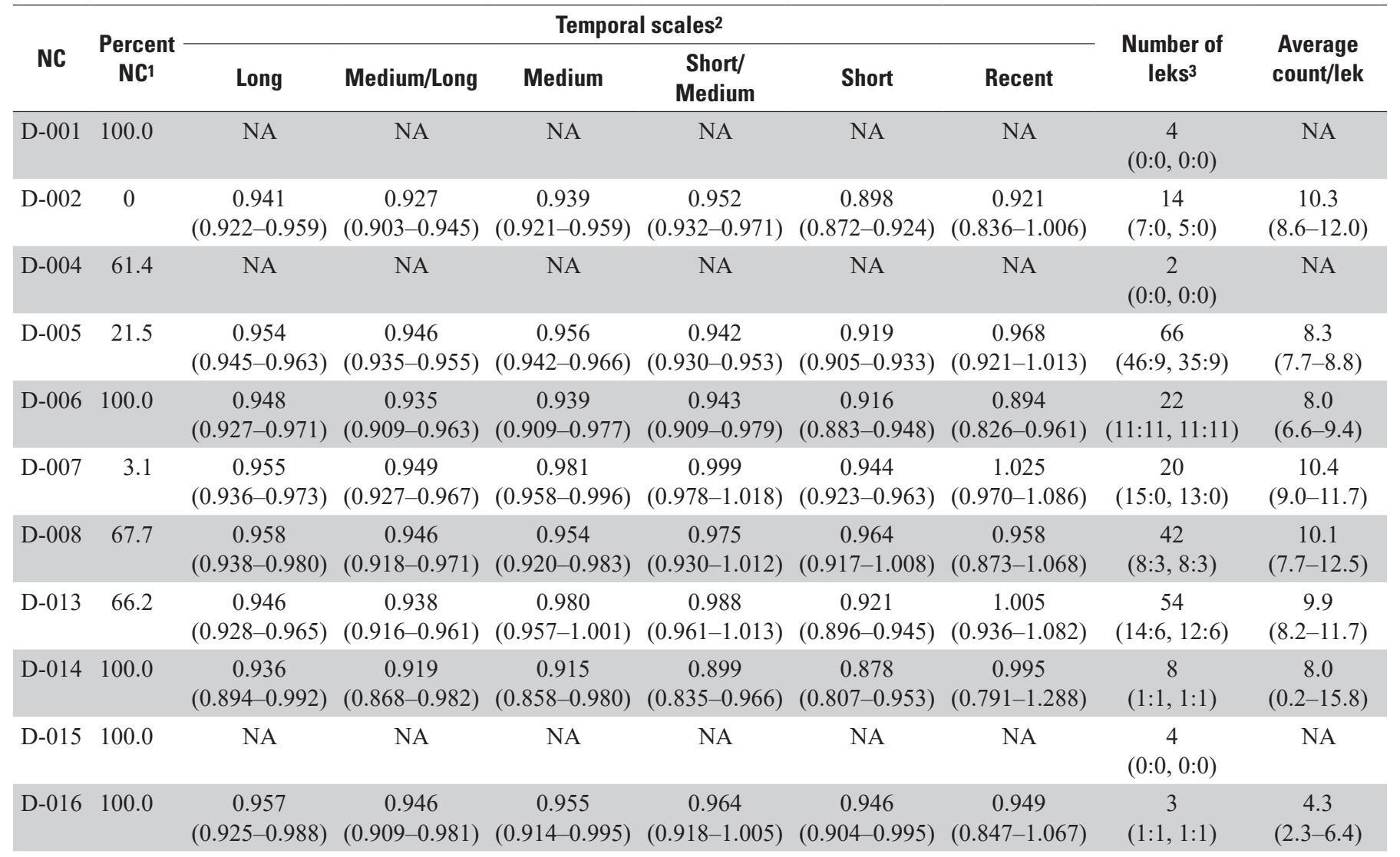


Table 4.16. Table of greater sage-grouse (Centrocercus urophasianus) average annual rate of population change $(\hat{\lambda})$ across six periods of oscillation in abundance that represent temporal scales for each neighborhood cluster within the state of Montana. Estimates were derived from leks within the entire neighborhood cluster.-Continued

[NC, neighborhood cluster; NA, not applicable]

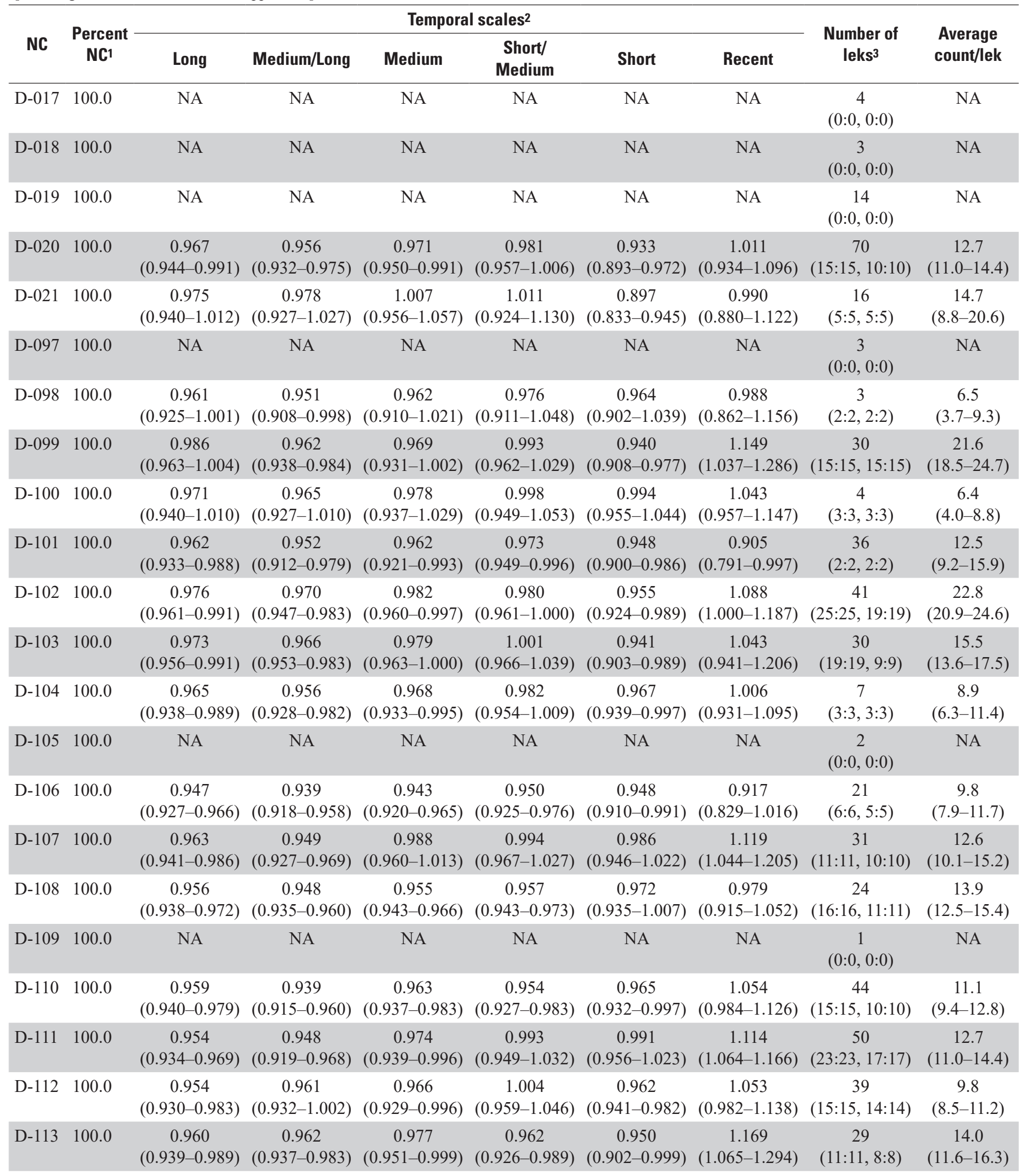


Table 4.16. Table of greater sage-grouse (Centrocercus urophasianus) average annual rate of population change $(\hat{\lambda})$ across six periods of oscillation in abundance that represent temporal scales for each neighborhood cluster within the state of Montana. Estimates were derived from leks within the entire neighborhood cluster.-Continued

[NC, neighborhood cluster; NA, not applicable]

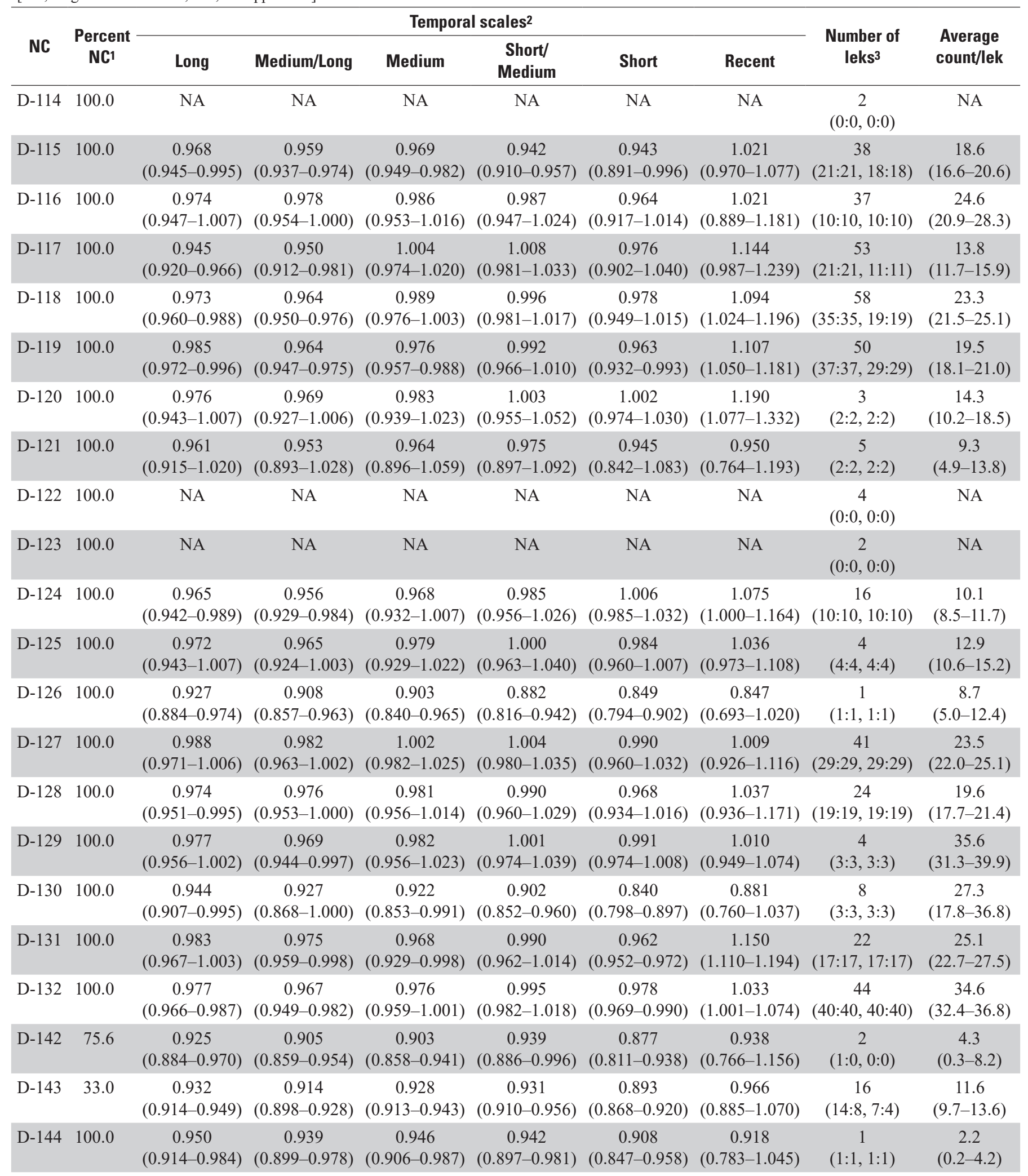


Table 4.16. Table of greater sage-grouse (Centrocercus urophasianus) average annual rate of population change $(\hat{\lambda})$ across six periods of oscillation in abundance that represent temporal scales for each neighborhood cluster within the state of Montana. Estimates were derived from leks within the entire neighborhood cluster.-Continued

[NC, neighborhood cluster; NA, not applicable]

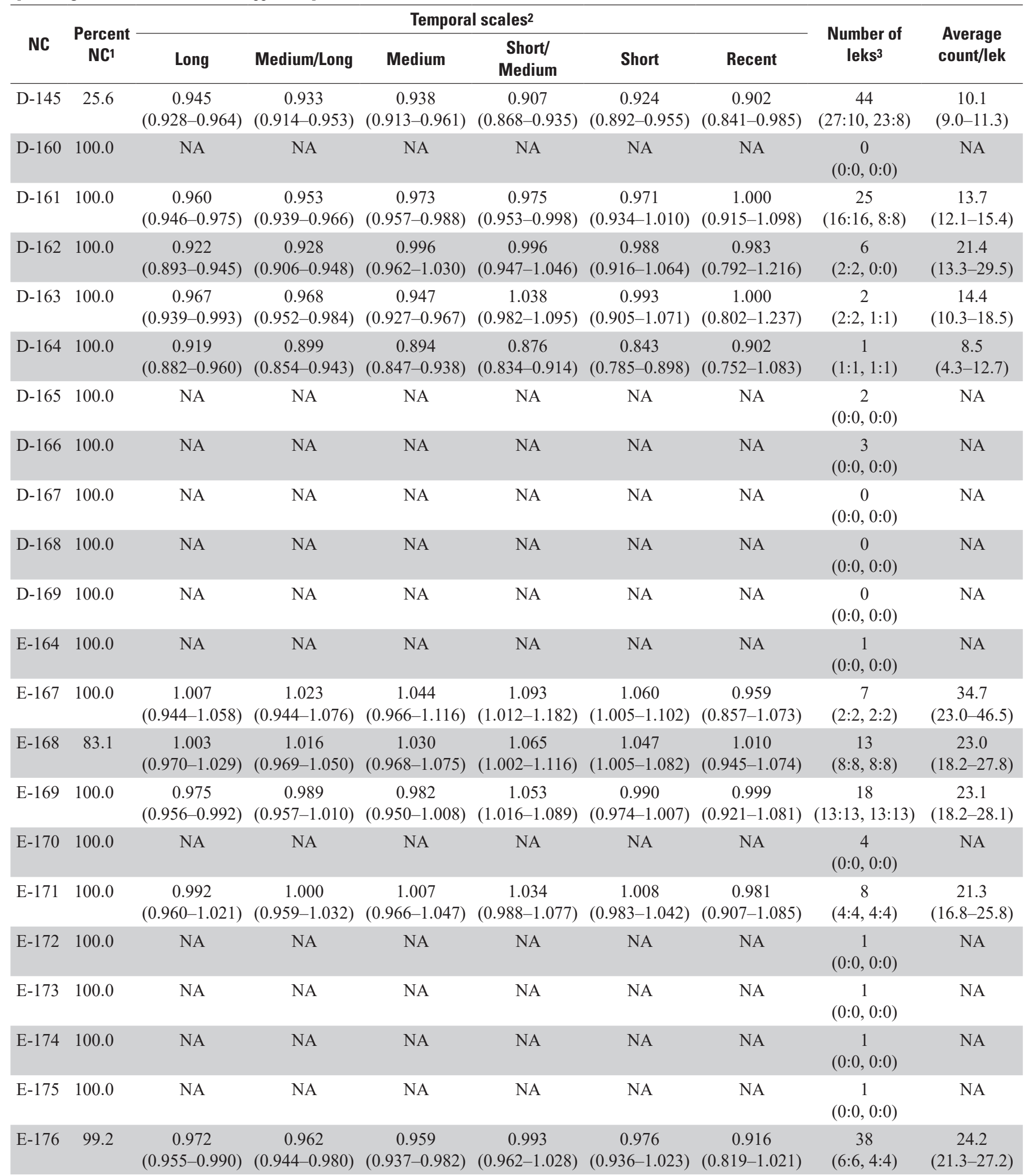


Table 4.16. Table of greater sage-grouse (Centrocercus urophasianus) average annual rate of population change $(\hat{\lambda})$ across six periods of oscillation in abundance that represent temporal scales for each neighborhood cluster within the state of Montana. Estimates were derived from leks within the entire neighborhood cluster.-Continued

[NC, neighborhood cluster; NA, not applicable]

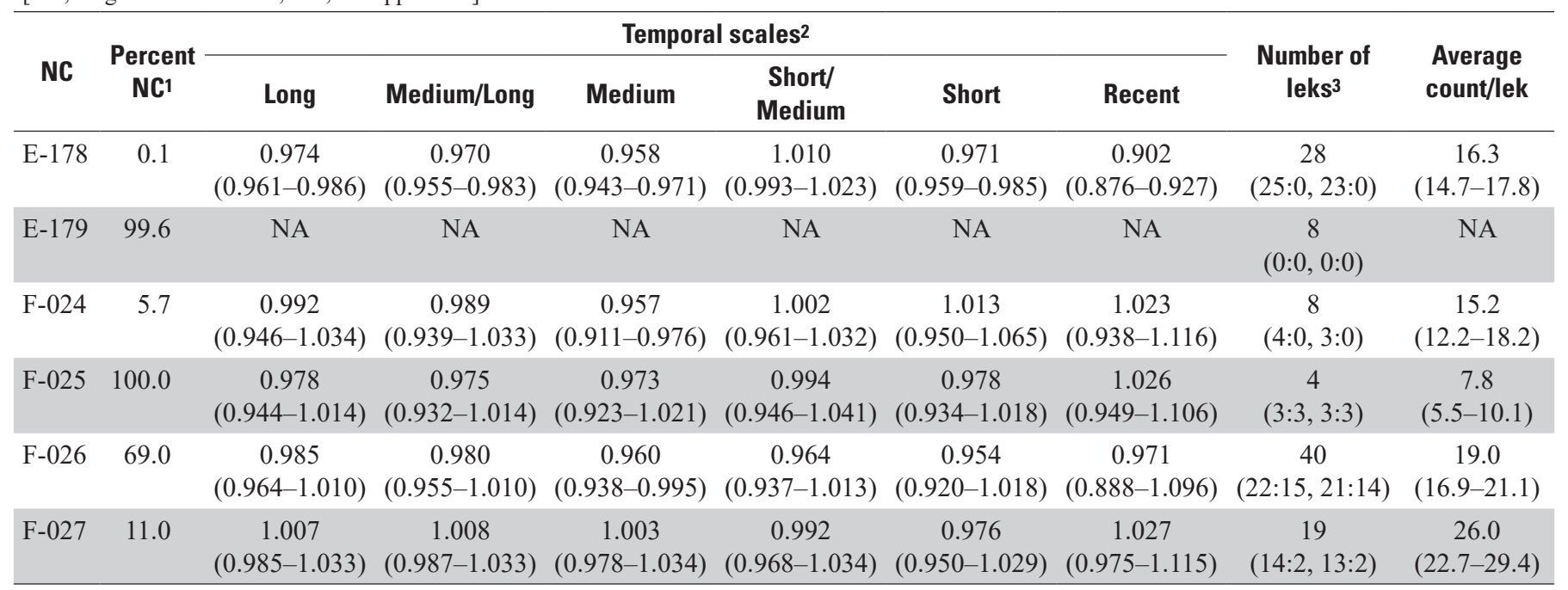

1The percent of each neighborhood cluster that intersects the state.

2Temporal scales were estimated from present to each major population abundance nadir (trough) since 1960. Number of temporal scales were used to estimate population trends across six different temporal scales from approximately 10 to approximately 60 years.

${ }^{3}$ Number of leks in database. In parentheses from left to right is (1) total number in cluster were used in trend analysis, (2) number used in trend analysis within state boundary, (3) total number in cluster were used in the targeted annual warning system (TAWS) analysis, and (4) number used in TAWS analysis within state boundary.
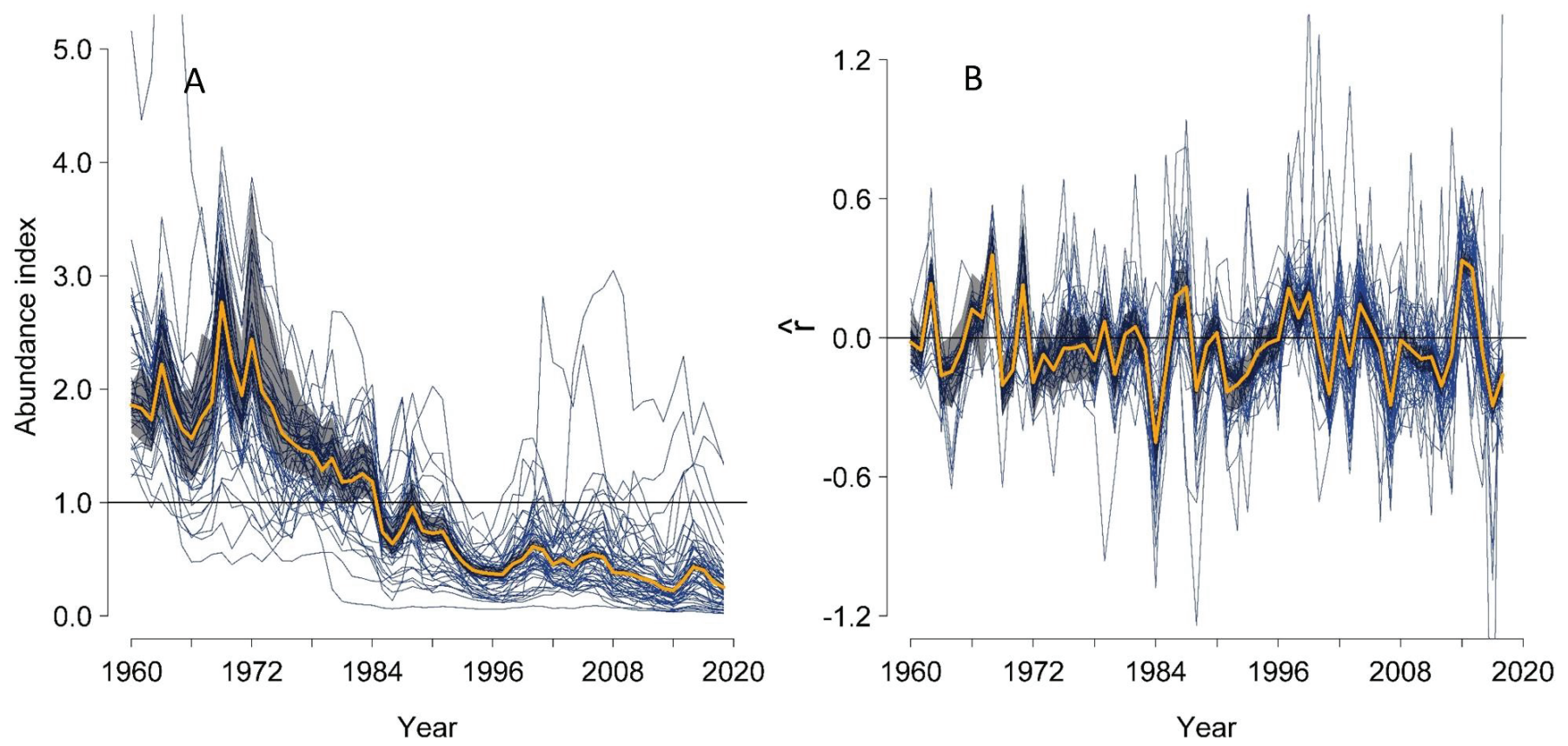

Figure 4.54. $A$, Abundance index (calculated as $\hat{N}$ divided by 60-year mean of $\hat{N}$ ); and $B$, intrinsic rate of population change ( $\hat{r}$ ) of greater sage-grouse (Centrocercus urophasianus) within the state of Montana from 1960-2019. Thick yellow line represents median estimates across all leks. Shaded areas represent 95-percent credible limits. Thin blue lines represent median values for neighborhood clusters. Black horizontal line (abundance index=1.0) represents 60-year average. 

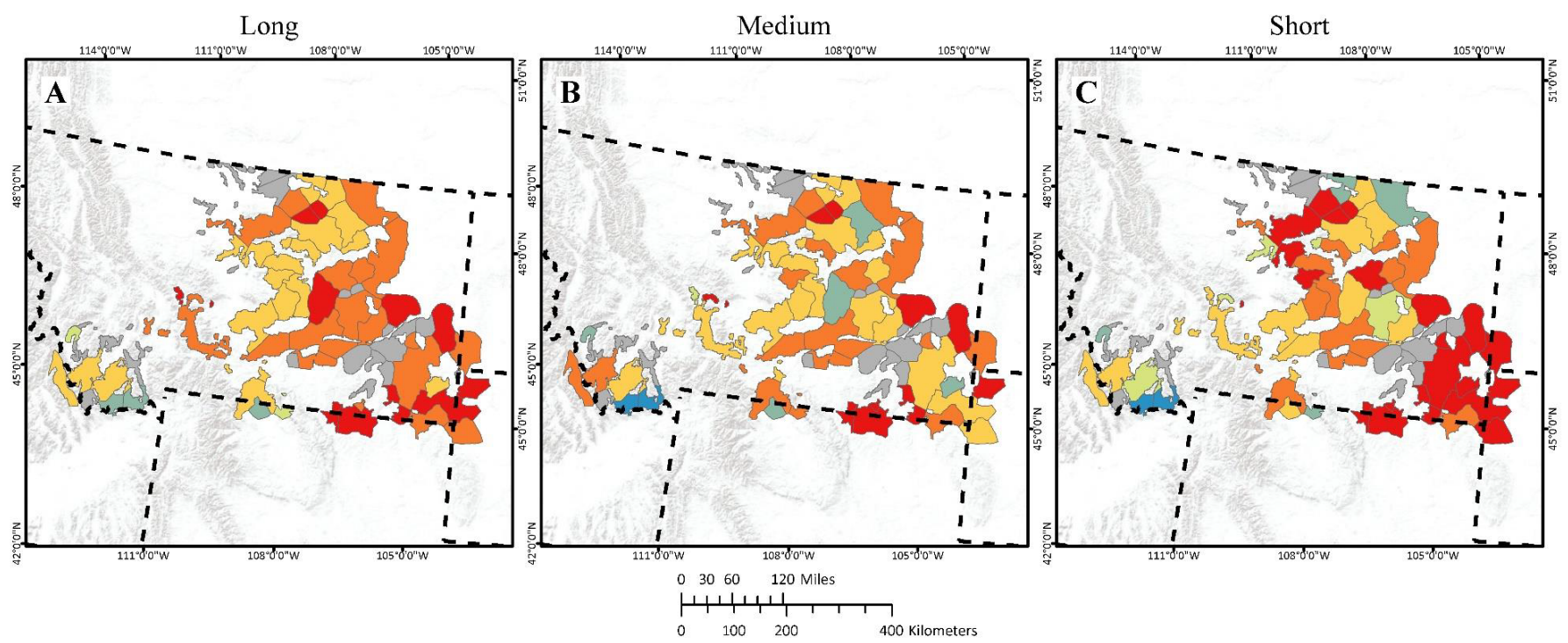

\section{EXPLANATION}

Neighborhood clusters (Level 2) intersecting Montana: polygon colors represent average lambda values for sage-grouse populations over varying lengths of time (long, medium, short). Symbols are based on a range-wide distribution of values and all may not be represented here.

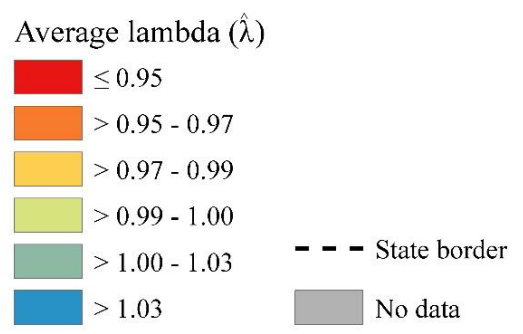

Coordinate System: Albers Projection: Albers Datum: WGS 1984 false easting: 0.0000 false northing: 0.0000 central meridian: -96.0000 standard parallel 1: 29.5000 standard parallel 2: 45.5000 latitude of origin: 23.0000 Units: Meter

Service Layer Credits: Sources: Esri, USGS, NOAA

Figure 4.55. Spatial estimates of population trends across three temporal scales based on periods of oscillation (short, medium, and long) while accounting for fluctuations of greater sage-grouse (Centrocercus urophasianus) abundance across different neighborhood clusters within the state of Montana. Map images are the intellectual property of Esri and are used herein under license. Copyright (C) 2020 Esri and its licensors. All rights reserved. 


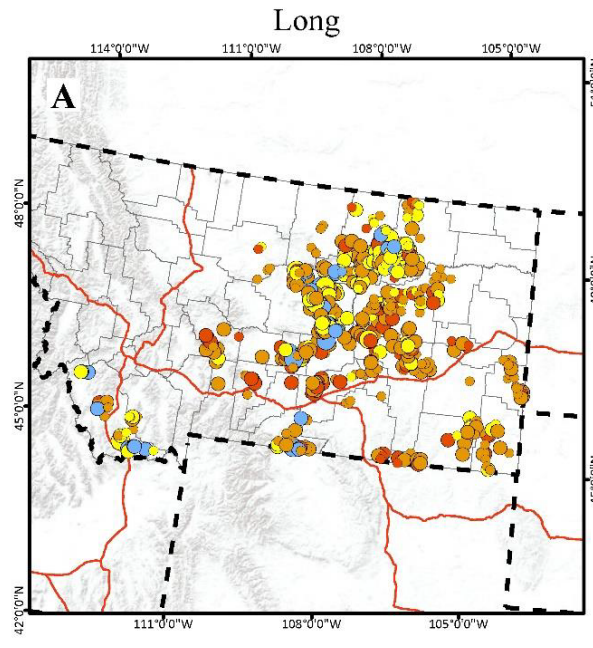

EXPLANATION

Leks within Montana: colors represent average lambda $(\hat{\lambda})$ values of sage-grouse populations over various time scales (long, medium, short). The size of the symbol represents the maximum population size of the lek over the years of the analysis, relativized to the range-wide maximum population size across all leks. Symbols are based on a rangewide distribution of values and all may not be represented here.
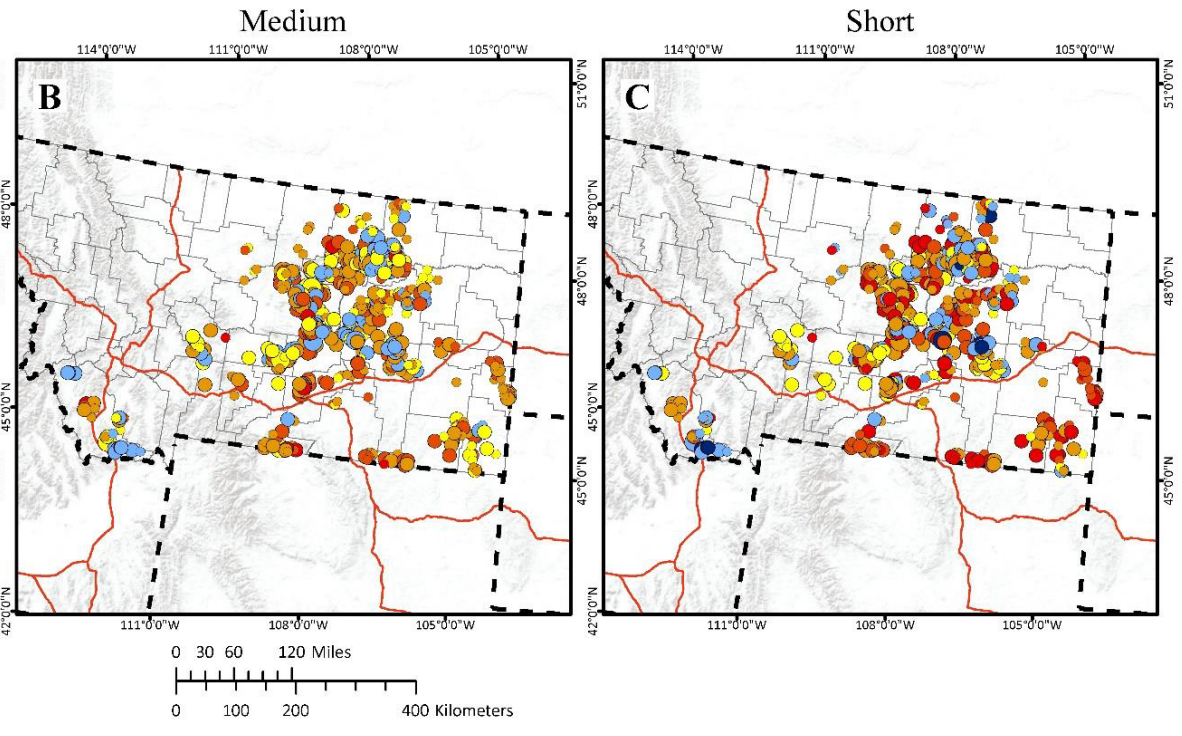

$$
\begin{aligned}
\text { Average lambda }(\hat{\lambda}) \\
\quad \leq 0.90 \\
\quad>0.90-0.94 \\
\quad>0.94-0.98 \\
\quad>0.98-1.00 \\
\quad>1.00-1.10 \\
\quad>1.10
\end{aligned}
$$$$
\longrightarrow \text { Road }
$$$$
\text { County line }
$$

Coordinate System: Albers Projection: Albers Datum: WGS 1984 false easting: 0.0000 false northing: 0.0000 central meridian: -96.0000 standard parallel $1: 29.5000$ standard parallel 2: 45.5000 latitude of origin: 23.0000 Units: Meter

Service Layer Credits: Sources: Esri, USGS, NOAA

Figure 4.56. Spatial estimates of population trends across three temporal scales based on periods of oscillation (short, medium, and long) while accounting for fluctuations of greater sage-grouse (Centrocercus urophasianus) abundance across lek sites within the state of Montana. Map images are the intellectual property of Esri and are used herein under license. Copyright (C) 2020 Esri and its licensors. All rights reserved. 


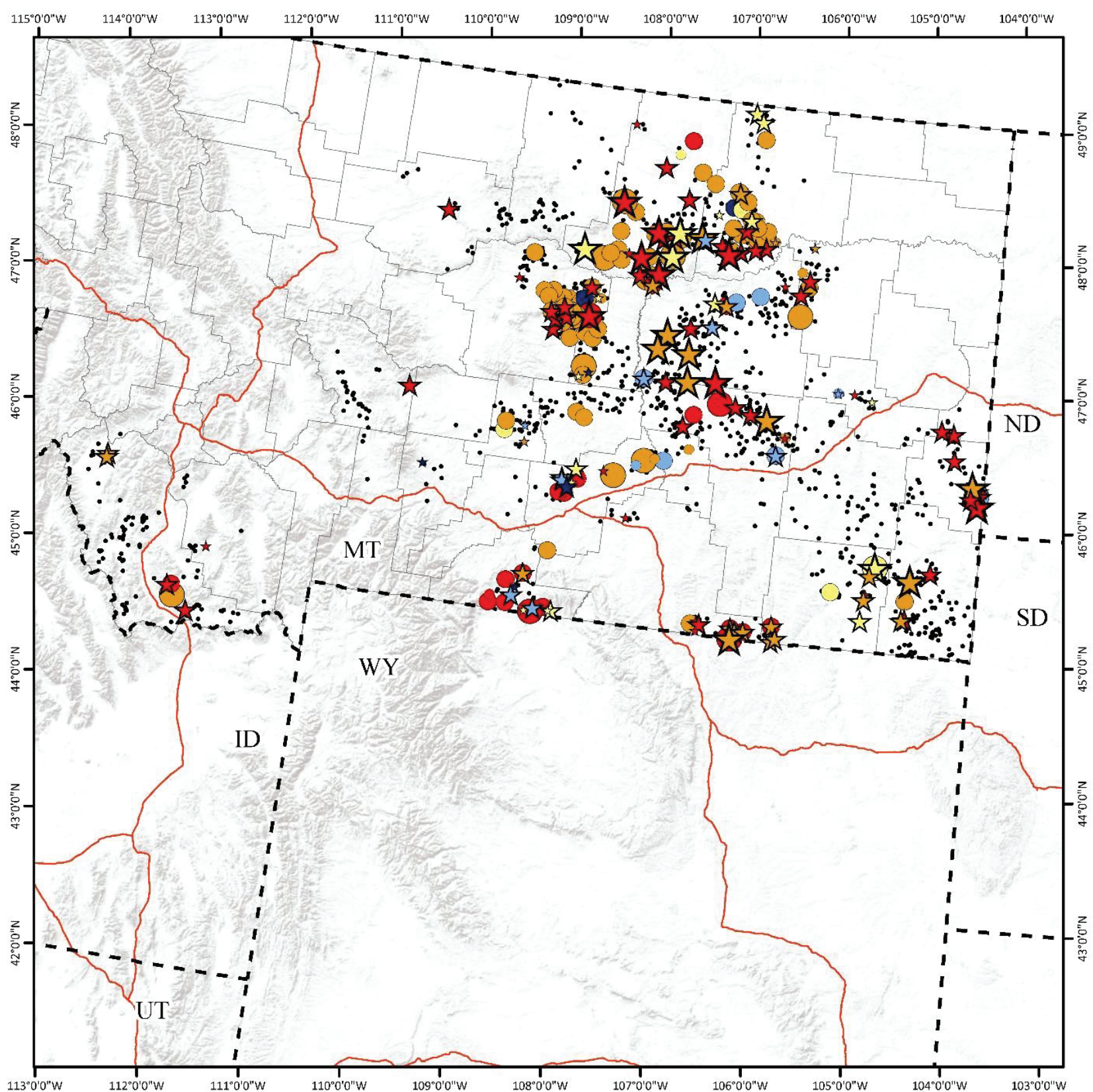

EXPLANATION

Leks in Montana: colors represent a range of years the most recent event occurred. Stars represent warnings, circles represent watches, and black dots represent leks which did not experience warnings or watches. The size of the symbol represents the maximum population size of the lek over the 30 -year period, relativized to the rangewide maximum population size across all leks. Symbols are based on a range-wide distribution of values and all may not be represented here.

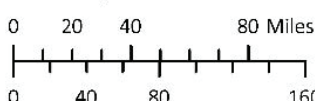

色 Warning

$\bigcirc$ Watch

- Lek

- - - State border

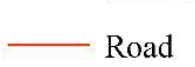

Road

County line
Coordinate System: Albers Projection: Albers

Relative maximum $\begin{aligned} & \text { Datum: WGS } 1984 \\ & \text { false easting: } 0.0000\end{aligned}$ population size

false northing: 0.0000 central meridian: -96.0000 standard parallel 1: 29.5000 standard parallel 2: 45.5000 latitude of origin: 23.0000 Units: Meter

Service Layer Credits: Sources: Esri, USGS, NOAA

Figure 4.57. Spatial and temporal depiction of watches and warnings of greater sage-grouse (Centrocercus urophasianus) population declines at the lek scale within the state of Montana from 1990 to 2019. Map image is the intellectual property of Esri and is used herein under license. Copyright $@ 2020$ Esri and its licensors. All rights reserved. 


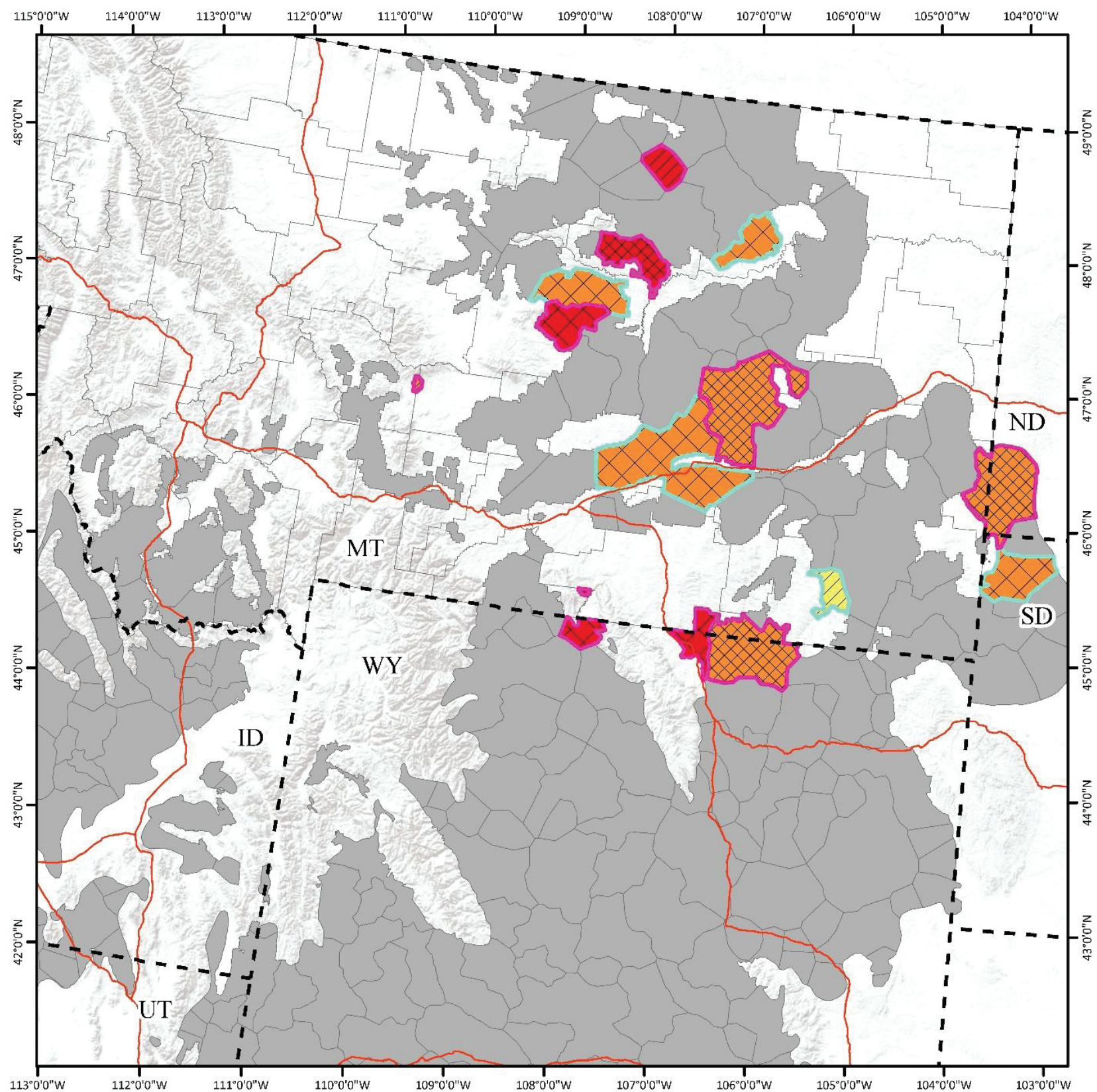

\section{EXPLANATION}

Neighborhood clusters (Level 2) in Montana: polygon colors represent a range of years the most recent event occurred. Oulline colors indicate whether the most recent event was a watch or warning. The hatching represents the maximum population size of the cluster over the 30 -year period, relativized to the range-wide maximum population size across all neighborhood clusters. Symbols are based on a range-wide distribution of values and all may not be represented here. $01020 \quad 40$ Miles | 0 $30 \quad 60 \quad 120$ Kilometers $1995 \circ 199$
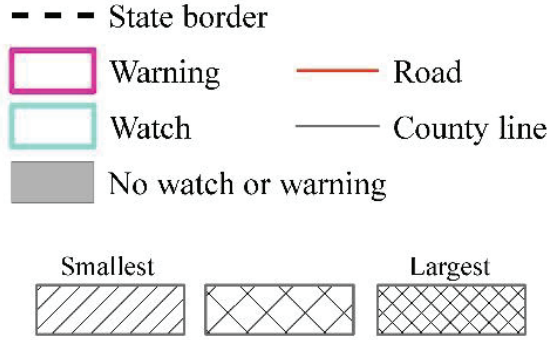

$2000-20042005-20092010-20142015-2019$
Coordinate System: Albers Projection: Albers Datum: WGS 1984 false easting: 0.0000 false northing: 0.0000 central meridian: -96.0000 standard parallel 1: 29.5000 standard parallel 2: 45.5000 latitude of origin: 23.0000 Units: Meter

Service Layer Credits: Sources: Esri, USGS, NOAA

Figure 4.58. Spatial and temporal depiction of watches and warnings of greater sage-grouse (Centrocercus urophasianus) population declines at neighborhood cluster within the state of Montana from 1990 to 2019. Map image is the intellectual property of Esri and is used herein under license. Copyright () 2020 Esri and its licensors. All rights reserved. 


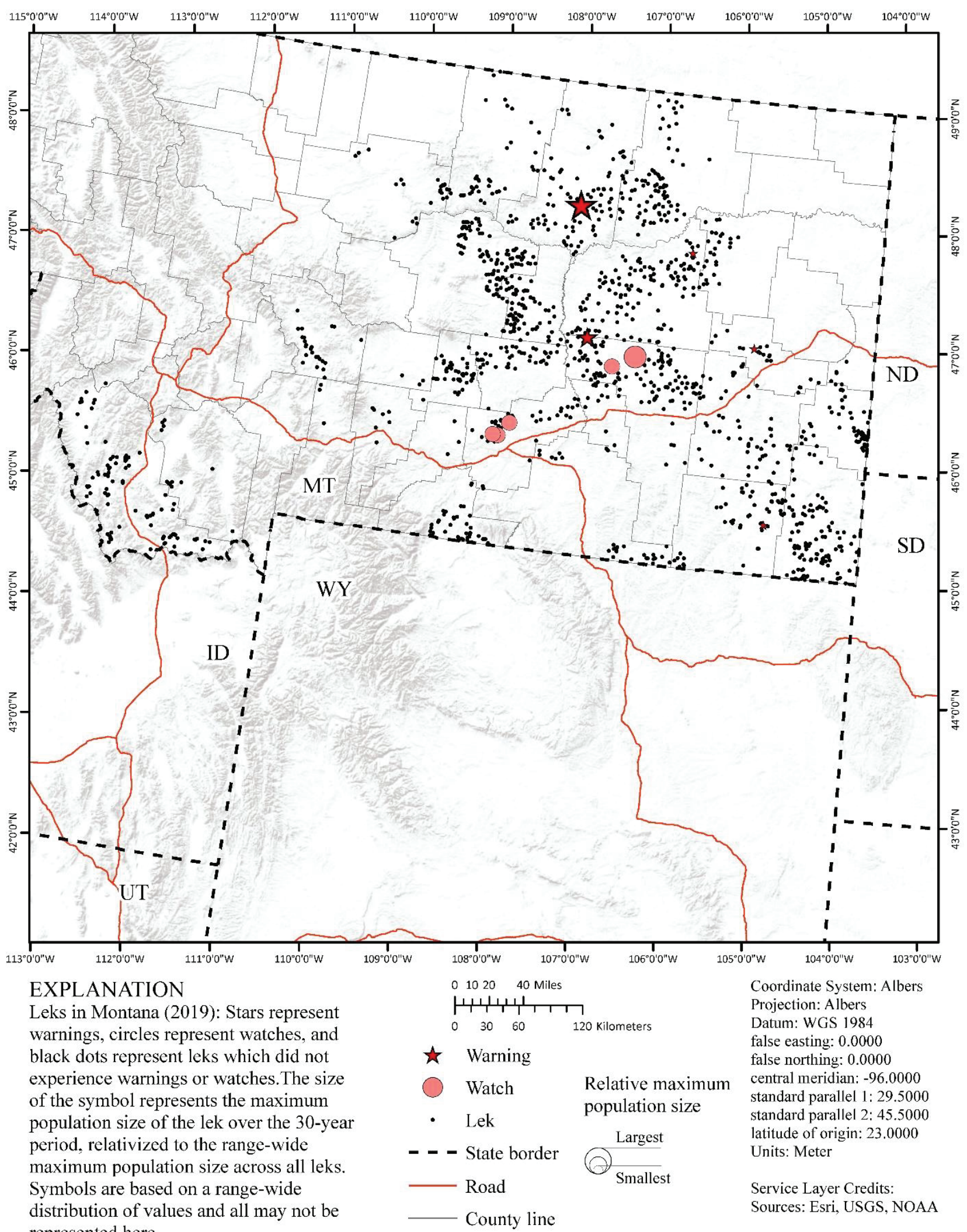

Figure 4.59. Spatial and temporal depiction of watches and warnings of greater sage-grouse (Centrocercus urophasianus) population declines at the lek and neighborhood cluster scale within the state of Montana during 2019. Map image is the intellectual property of Esri and is used herein under license. Copyright $@ 2020$ Esri and its licensors. All rights reserved. 


\section{Colorado Results}

Colorado intersected CC-D and contained or intersected 30 different NCs (fig. 4.60). Total area of the $30 \mathrm{NCs}$ within Colorado was 1,575,974 ha. Of the 23 clusters used in the analysis, 3 were omitted because of limitations in sample sizes. These areas consisted of 523 leks, of which 321 were located within the Colorado state boundary, representing 3.81 percent of the range-wide lek database. After extensive QA/QC, we used 213 leks from Colorado in the SSM for population trend estimation (tables 4.17 and 4.18), totaling 2,631 individual lek counts. Mean male count was 14.43 (95-percent confidence interval $=14.07-14.79$ ) for leks within NCs that were within or overlapped Colorado. Mean male count for leks within Colorado was 13.80 (95-percent confidence interval $=13.27-14.32$ ).

\section{Colorado Population Trend Analysis (Objective 3)}

For CC-D, we estimated six population abundance nadirs (troughs) that dated back to 1960. Each of these population abundance nadirs represent between one and six complete periods of oscillation. We used these nadirs to estimate population trends across three different temporal scales that represented two, four, and six complete periods for the state (for instance, second, fourth, and sixth nadir). We estimated the average annual finite rate of population change $(\hat{\lambda})$ at the short (two periods), medium (four periods), and long (six periods) temporal scales as 0.961 (95-percent $\mathrm{CRI}=0.948-0.971), 0.962$ (95-percent $\mathrm{CRI}=0.947-0.973)$, and 0.963 (95-percent CRI=0.956-0.971), respectively (fig. 4.61). Climate cluster estimates, which included leks in adjacent states to Colorado, were slightly different than estimates generated from leks only within Colorado. For all NCs that were modeled and intersected Colorado, we estimated median to be less than 1.0 for $88.9,96.3$, and 100.0 percent across short, medium, and long temporal scales, respectively (fig. 4.62; table 4.18). We estimated median $\hat{\lambda}$ to be less than 1.0 for 73.2, 89.2, and 97.2 percent of all modeled leks within Colorado across short, medium, and long temporal scales, respectively (fig. 4.63). We reported spatial and temporal variation in average annual $\hat{\lambda}$ across different neighborhood scales (fig. 4.62) and leks (fig. 4.63).

\section{Colorado Targeted Annual Warning System Analysis (Objective 4)}

During 1990-2019, the TAWS for sage-grouse populations in Colorado activated a total of 134 and 104 leks as watches and warnings, respectively (fig. 4.64), which was 62.9 and 48.8 percent of the sampled leks used in the analysis. On average, across the 29 years, approximately 5.4 and 2.0 percent of leks per year experienced watches and warnings, respectively. The higher percentage for watches corresponds to repetitive activation. During this time frame, the TAWS also activated a total of 10 and $11 \mathrm{NCs}$ as watches and warnings, respectively (fig. 4.65), which was 41.7 and 45.8 percent of the sampled clusters used in the analysis. On average, across the 29 years, approximately 2.8 and 1.8 percent of clusters per year experienced watches and warnings, respectively. Similar to leks, the higher percentage for watches corresponds to repetitive activation. During 2019 only, the TAWS activated 78 (first $=10$ ) watches and 82 (first $=41$ ) warnings at leks (fig. 4.66) and 5 (first=1) watches and 8 (first=5) warnings at NCs (fig. 4.67). 


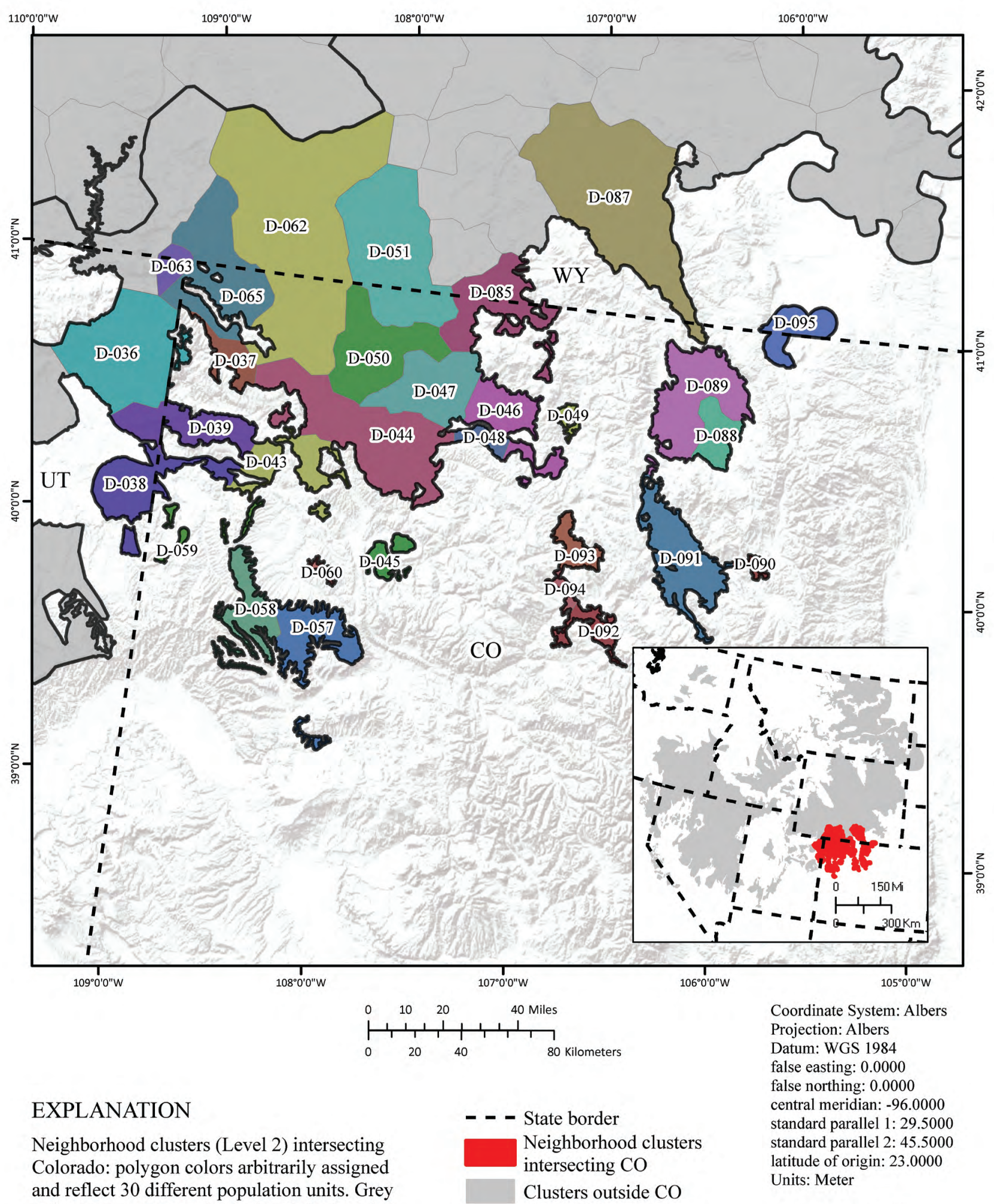
and reflect 30 different population units. Grey polygons represent neighborhood clusters that do not intersect Colorado.

Service Layer Credits: Sources: Esri, USGS, NOAA

Figure 4.60. Greater sage-grouse (Centrocercus urophasianus) hierarchical population monitoring framework for neighborhood clusters that intersect the state of Colorado. Map image is the intellectual property of Esri and is used herein under license. Copyright (C) 2020 Esri and its licensors. All rights reserved. 
Table 4.17. Table of greater sage-grouse (Centrocercus urophasianus) average annual rate of population change ( $\hat{\lambda})$ across six periods of oscillation in abundance that represent temporal scales for each climate cluster within the state of Colorado. Estimates were derived from leks within the entire climate cluster.

[CC, climate cluster; D, eastern area]

\begin{tabular}{|c|c|c|c|c|c|c|c|c|c|}
\hline \multirow[b]{2}{*}{ CC } & \multirow{2}{*}{$\begin{array}{c}\text { Percent } \\
\text { CC1 }\end{array}$} & \multicolumn{6}{|c|}{ Temporal scales 2} & \multirow[b]{2}{*}{ Number of leks ${ }^{3}$} & \multirow{2}{*}{$\begin{array}{l}\text { Average } \\
\text { count/lek }\end{array}$} \\
\hline & & Long & Medium/Long & Medium & $\begin{array}{c}\text { Short/ } \\
\text { Medium }\end{array}$ & Short & Recent & & \\
\hline
\end{tabular}

\footnotetext{
${ }^{1}$ The percent of each climate cluster that intersects the state.

${ }^{2}$ Temporal scales were estimated from present to each major population abundance nadir (trough) since 1960. Number of temporal scales were used to estimate population trends across six different temporal scales from approximately 10 to approximately 60 years.

${ }^{3}$ Number of leks in database. In parentheses from left to right is (1) total number in cluster were used in trend analysis, (2) number used in trend analysis within state boundary, (3) total number in cluster were used in the targeted annual warning system (TAWS) analysis, and (4) number used in TAWS analysis within state boundary.
}

Table 4.18. Table of greater sage-grouse (Centrocercus urophasianus) average annual rate of population change $(\hat{\lambda})$ across six periods of oscillation in abundance that represent temporal scales for each neighborhood cluster within the state of Colorado. Estimates were derived from leks within the entire neighborhood cluster.

[NC, neighborhood cluster; NA, not applicable]

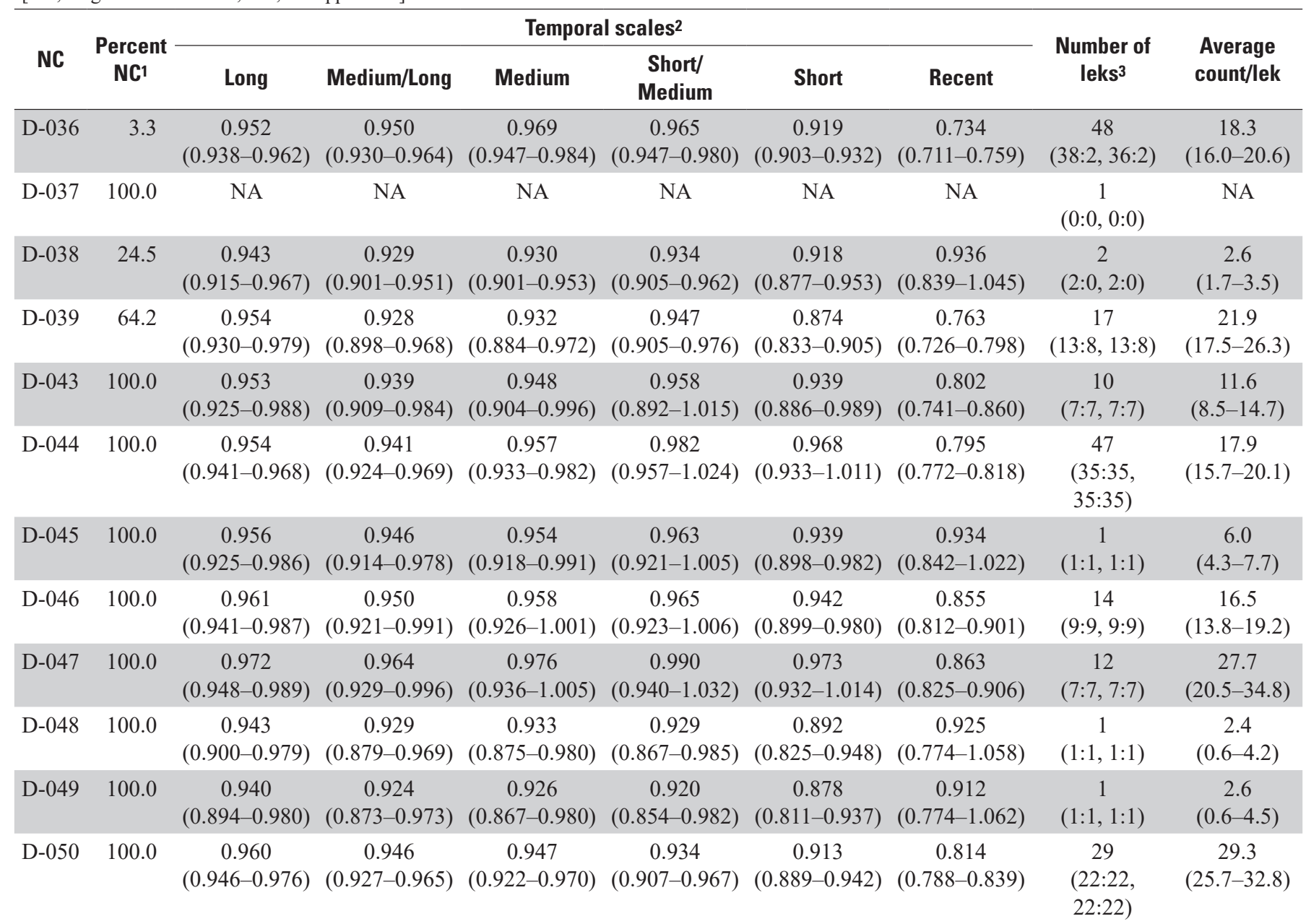


Table 4.18. Table of greater sage-grouse (Centrocercus urophasianus) average annual rate of population change $(\hat{\lambda})$ across six periods of oscillation in abundance that represent temporal scales for each neighborhood cluster within the state of Colorado. Estimates were derived from leks within the entire neighborhood cluster-Continued

[NC, neighborhood cluster; NA, not applicable]

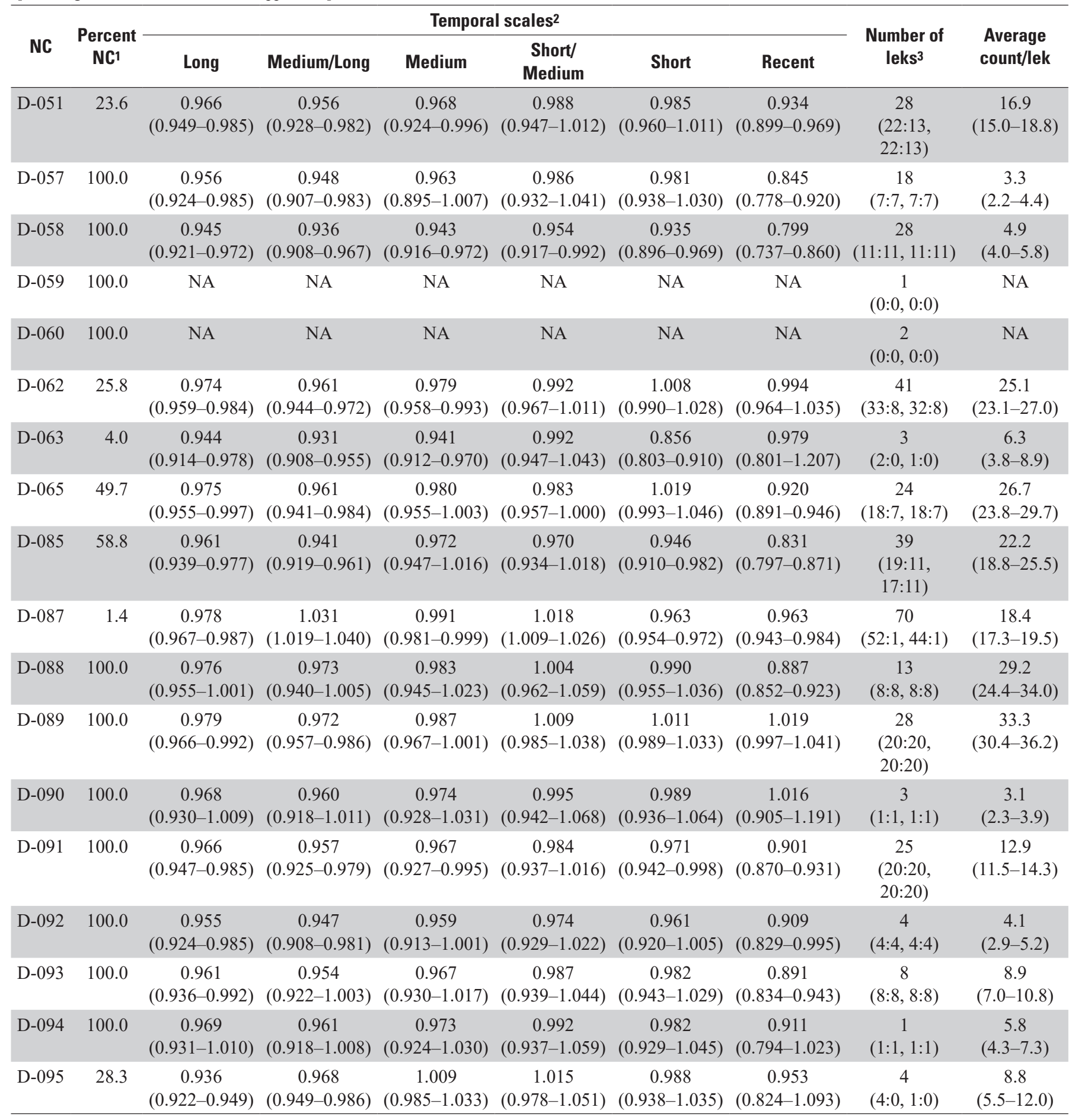

${ }^{1}$ The percent of each neighborhood cluster that intersects the state.

${ }^{2}$ Temporal scales were estimated from present to each major population abundance nadir (trough) since 1960. Number of temporal scales were used to estimate population trends across six different temporal scales from approximately 10 to approximately 60 years.

${ }^{3}$ Number of leks in database. In parentheses from left to right is (1) total number in cluster were used in trend analysis, (2) number used in trend analysis within state boundary, (3) total number in cluster were used in the targeted annual warning system (TAWS) analysis, and (4) number used in TAWS analysis within state boundary. 

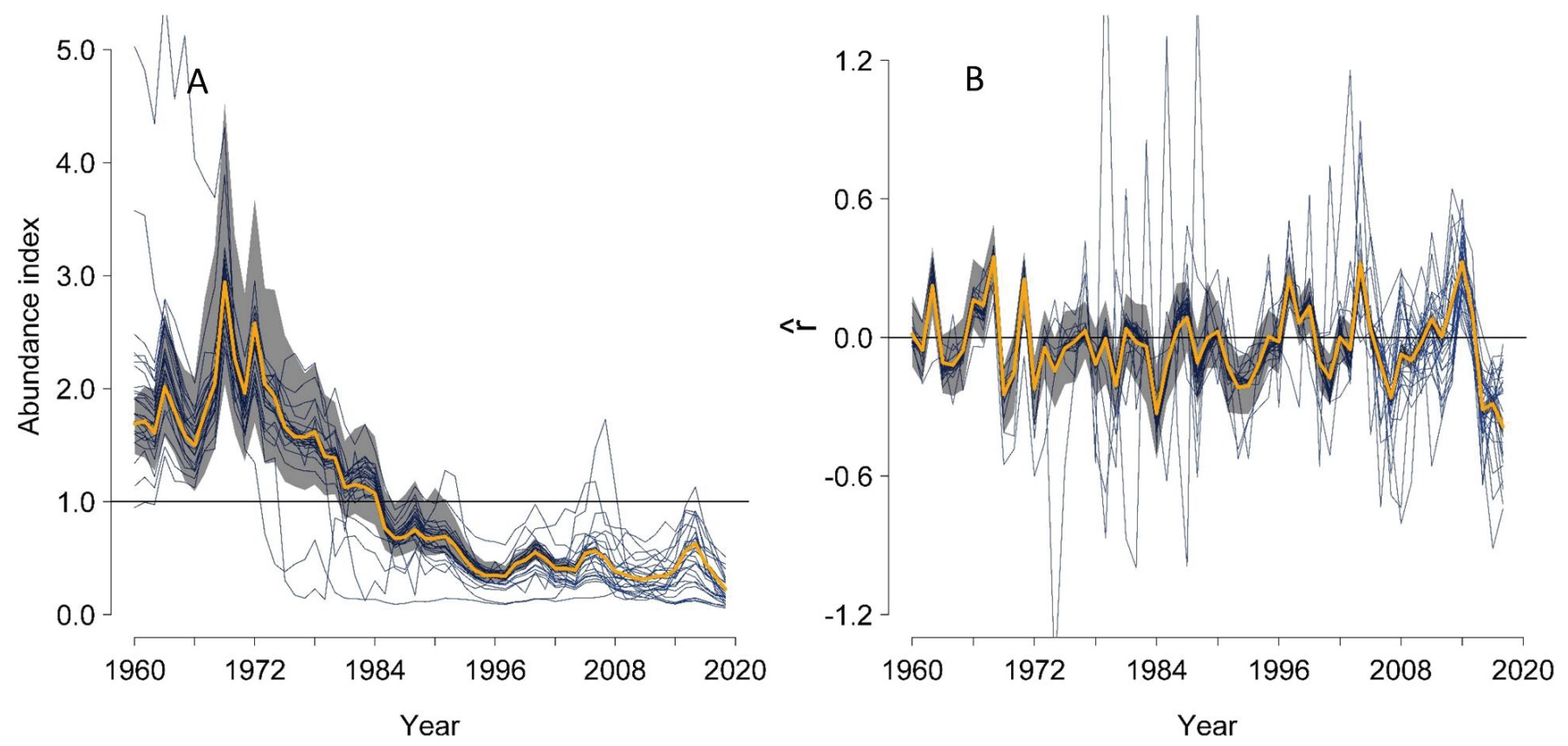

Figure 4.61. A, Abundance index (calculated as $\hat{N}$ divided by 60-year mean of $\hat{N}$ ); and $B$, intrinsic rate of population change $(\hat{r})$ of greater sage-grouse (Centrocercus urophasianus) within the state of Colorado from 1960 to 2019. Thick yellow line represents median estimates across all leks. Shaded areas represent 95-percent credible limits. Thin blue lines represent median values for neighborhood clusters. Black horizontal line (abundance index $=1.0$ ) represents 60 -year average. 

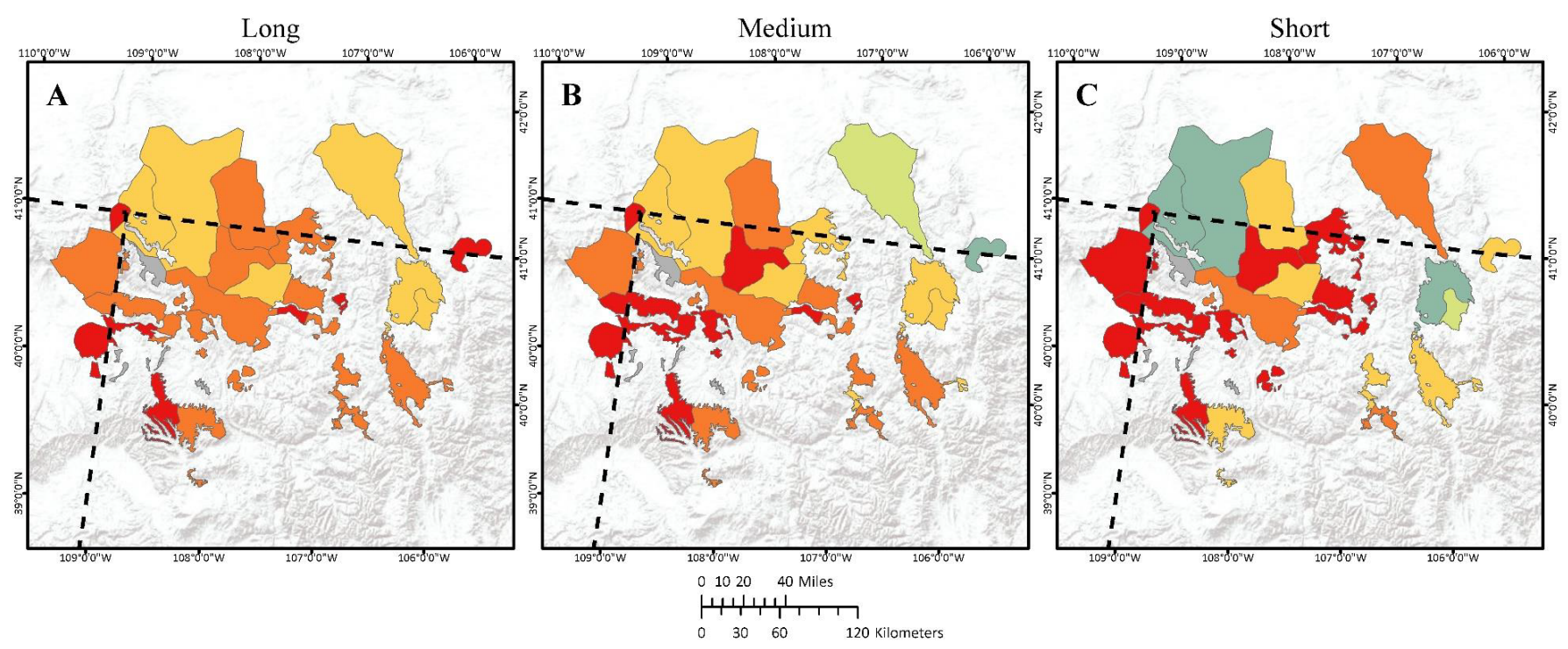

\section{EXPLANATION}

Neighborhood clusters (Level 2) intersecting Colorado: polygon colors represent average lambda values for sage-grouse populations

over varying lengths of time (long, medium, short). Symbols are based on a range-wide distribution of values and all may not be represented here.
Average lambda $(\hat{\lambda})$

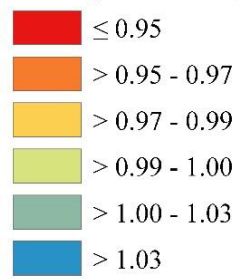

Coordinate System: Albers Projection: Albers Datum: WGS 1984

false easting: 0.0000 false northing: 0.0000 central meridian: -96.0000 standard parallel $1: 29.5000$ standard parallel 2: 45.5000 latitude of origin: 23.0000 Units: Meter

Service Layer Credits: Sources: Esri, USGS, NOAA

Figure 4.62. Spatial estimates of population trends across three temporal scales based on periods of oscillation (short, medium, and long) while accounting for fluctuations of greater sage-grouse (Centrocercus urophasianus) abundance across different neighborhood clusters within the state of Colorado. Map images are the intellectual property of Esri and are used herein under license. Copyright ( $)$ 2020 Esri and its licensors. All rights reserved. 


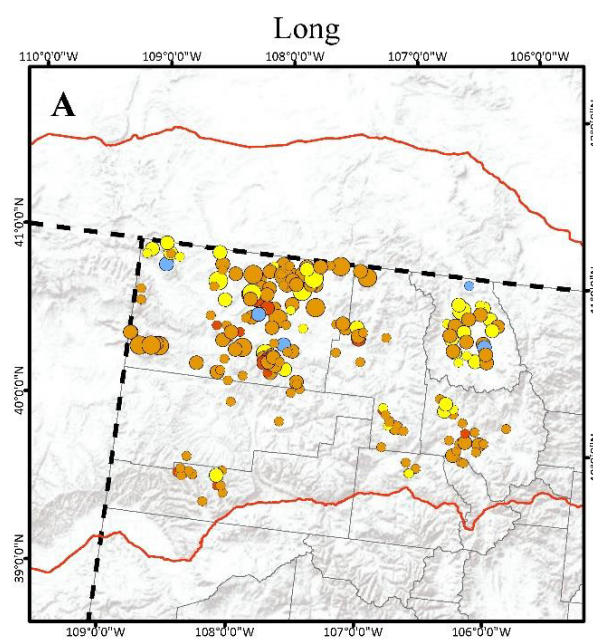

EXPLANATION

Leks within Colorado: colors represent average lambda $(\hat{\lambda})$ values of sage-grouse populations over various time scales (long, medium, short). The size of the symbol represents the maximum population size of the lek over the years of the analysis, relativized to the range-wide maximum population size across all leks. Symbols are based on a rangewide distribution of values and all may not be represented here.
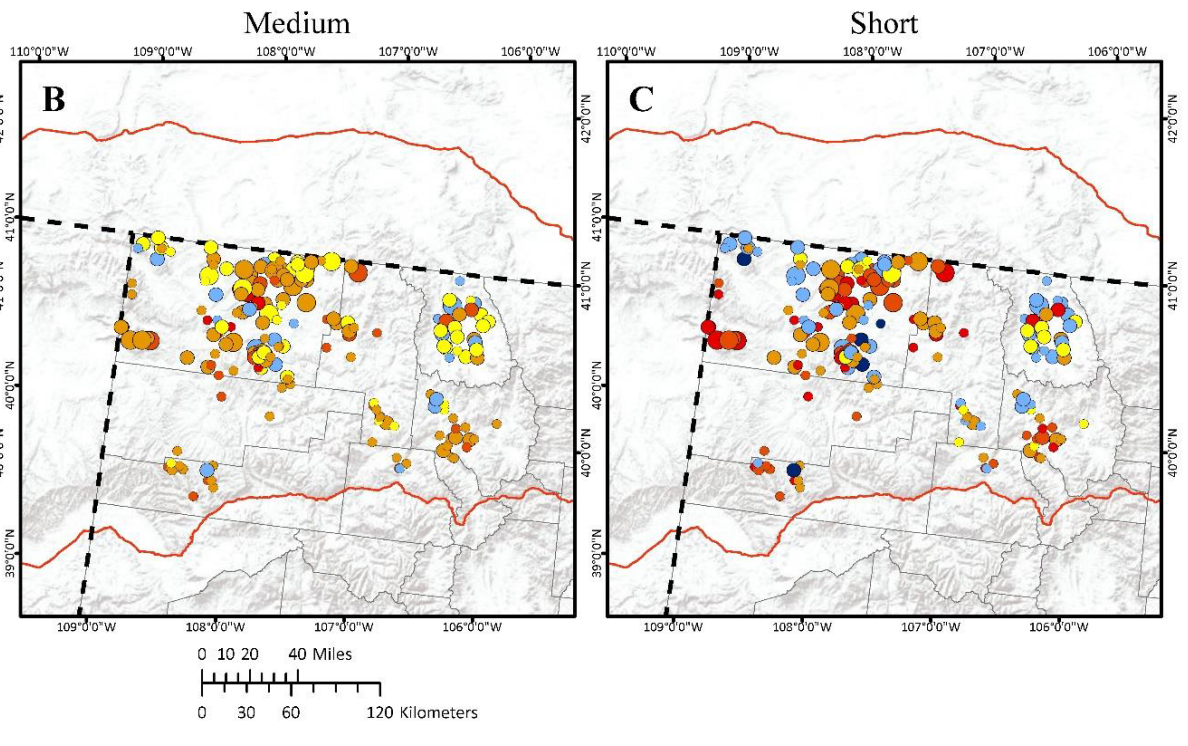

$$
\begin{aligned}
\text { Average lambda }(\hat{\lambda}) \\
\quad \leq 0.90 \\
\quad>0.90-0.94 \\
\quad>0.94-0.98 \\
\quad>0.98-1.00 \\
\quad>1.00-1.10 \\
\quad>1.10
\end{aligned}
$$$$
\text { Road }
$$$$
\text { County line }
$$

Coordinate System: Albers Projection: Albers Datum: WGS 1984 false easting: 0.0000 false northing: 0.0000 central meridian: -96.0000 standard parallel 1: 29.5000 standard parallel 2: 45.5000 latitude of origin: 23.0000 Units: Meter

Service Layer Credits: Sources: Esri, USGS, NOAA

Figure 4.63. Spatial estimates of population trends across three temporal scales based on periods of oscillation (short, medium, and long) while accounting for fluctuations of greater sage-grouse (Centrocercus urophasianus) abundance across lek sites within the state of Colorado. Map images are the intellectual property of Esri and are used herein under license. Copyright (C) 2020 Esri and its licensors. All rights reserved. 


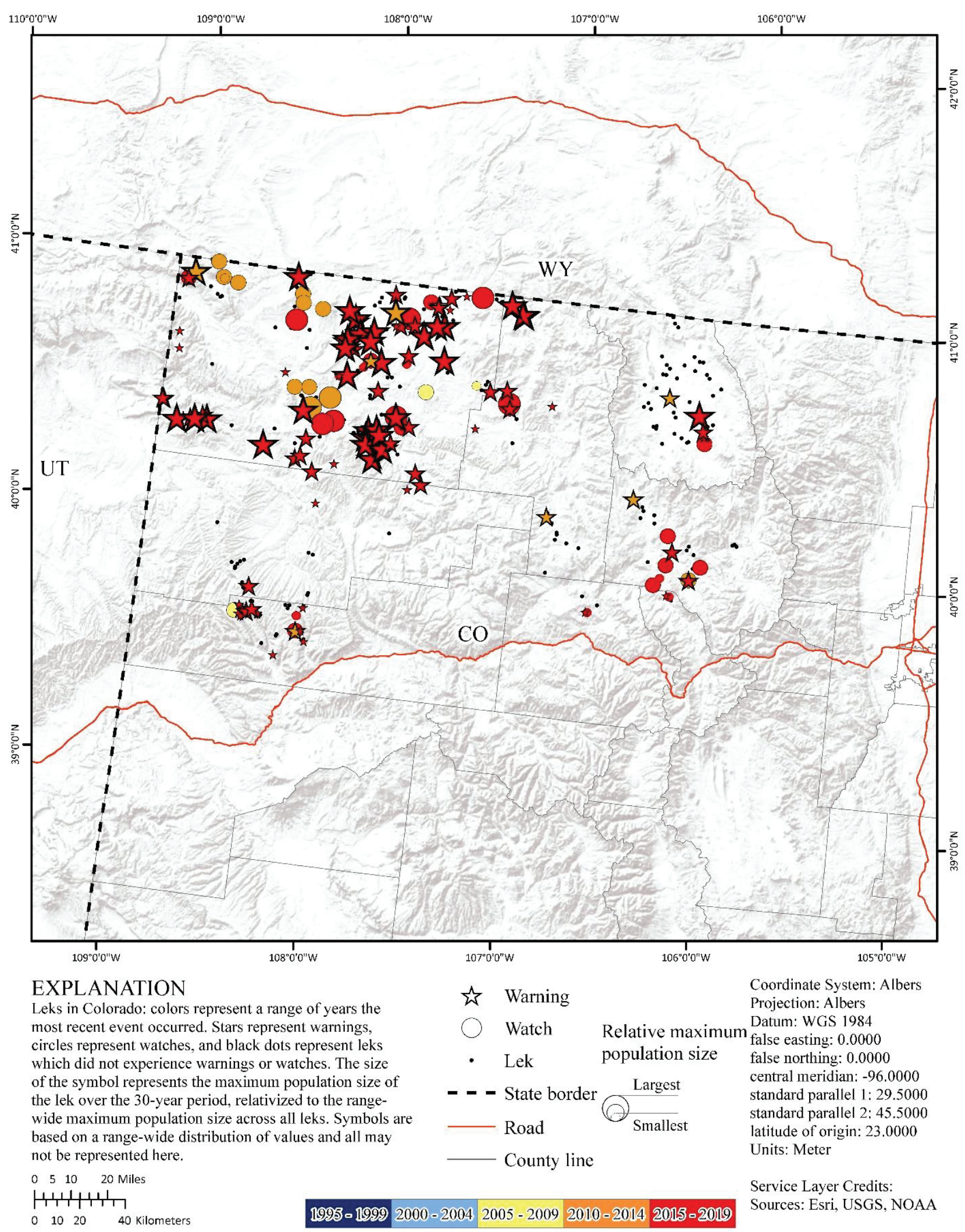

Figure 4.64. Spatial and temporal depiction of watches and warnings of greater sage-grouse (Centrocercus urophasianus) population declines at the lek scale within the state of Colorado from 1990 to 2019. Map image is the intellectual property of Esri and is used herein under license. Copyright (C) 2020 Esri and its licensors. All rights reserved. 


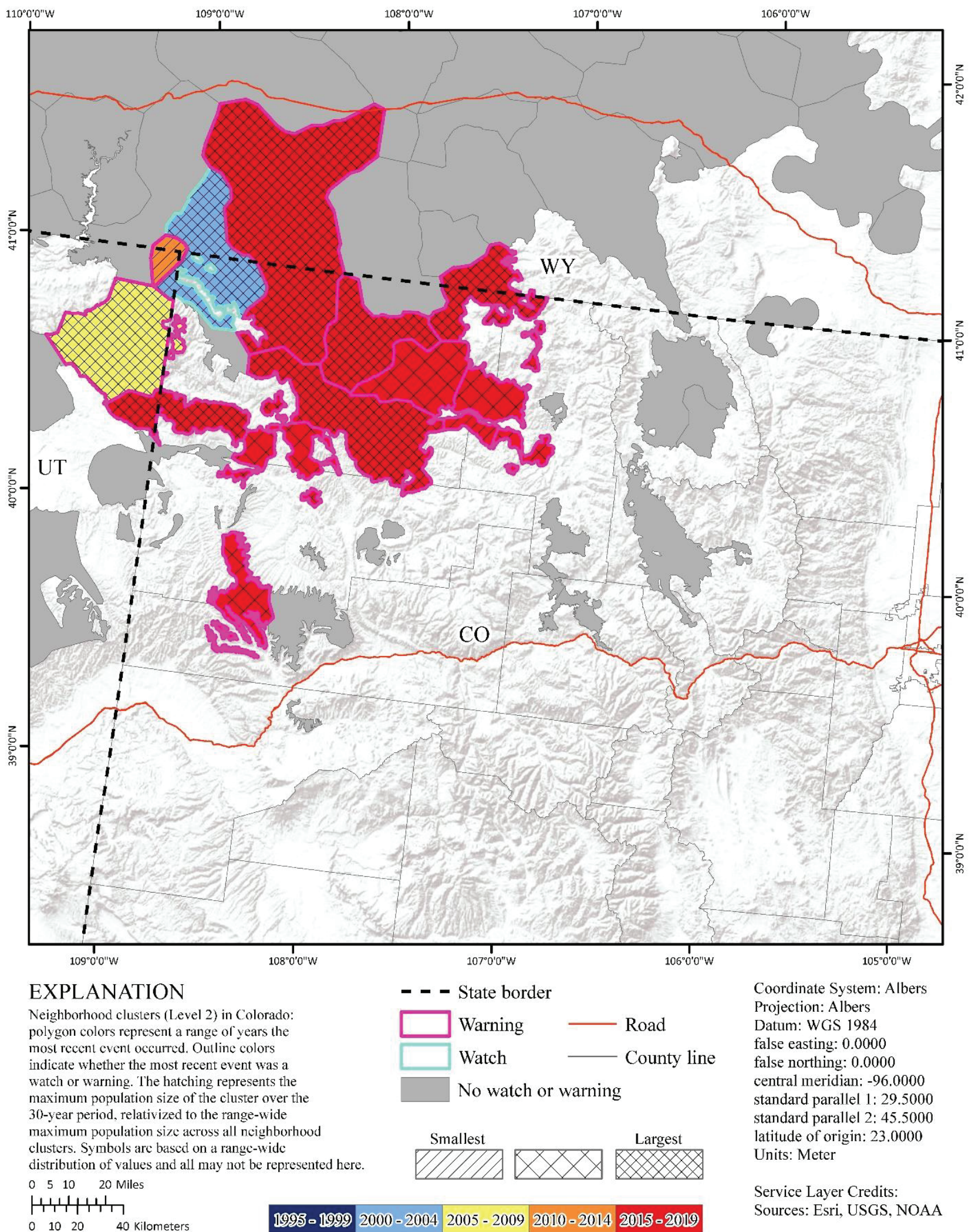

Figure 4.65. Spatial and temporal depiction of watches and warnings of greater sage-grouse (Centrocercus urophasianus) population declines at neighborhood cluster within the state of Colorado from 1990 to 2019. Map image is the intellectual property of Esri and is used herein under license. Copyright (C) 2020 Esri and its licensors. All rights reserved. 


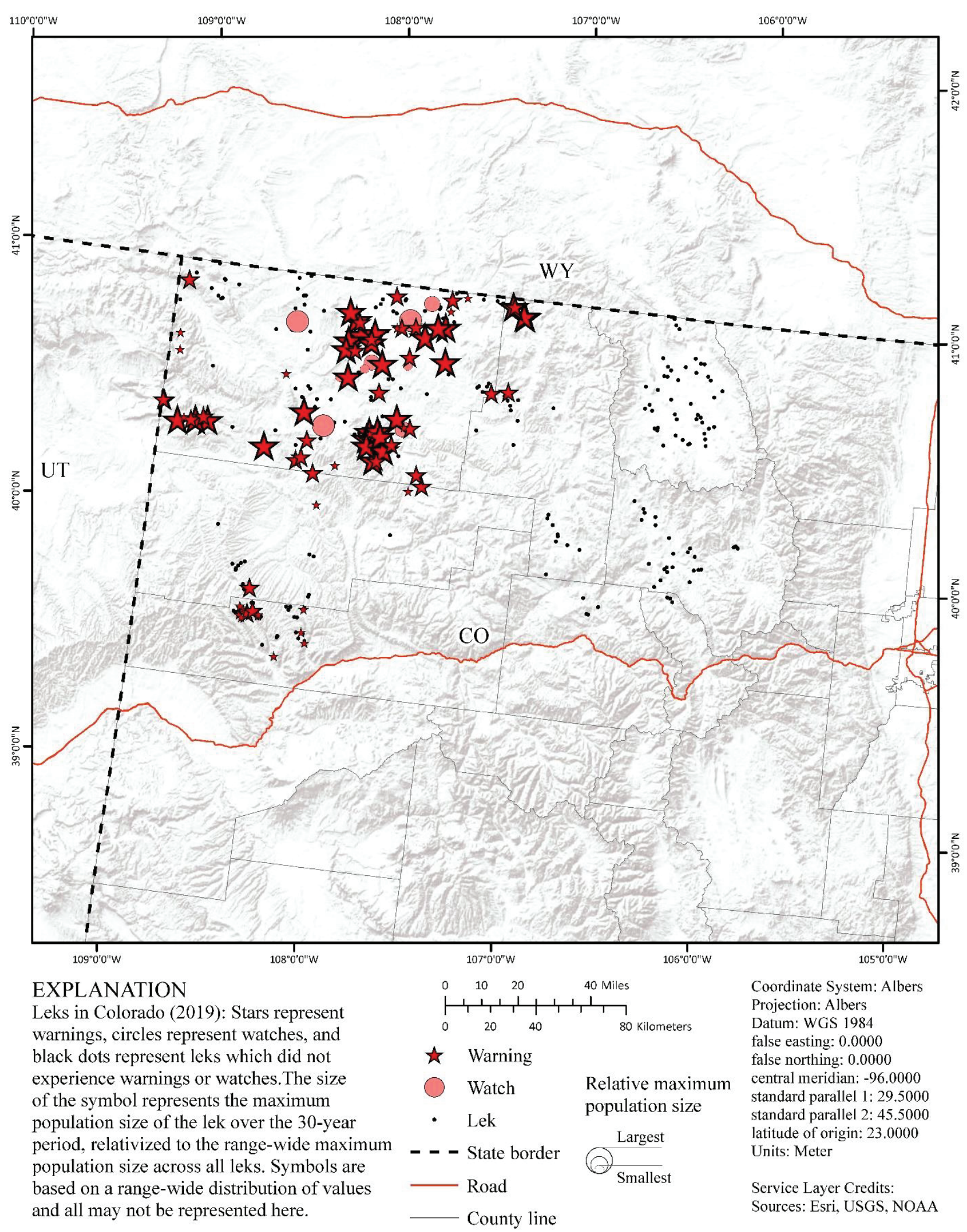

Figure 4.66. Spatial and temporal depiction of watches and warnings of greater sage-grouse (Centrocercus urophasianus) population declines at the lek and neighborhood cluster scale within the state of Colorado during 2019. Map image is the intellectual property of Esri and is used herein under license. Copyright $@ 2020$ Esri and its licensors. All rights reserved. 


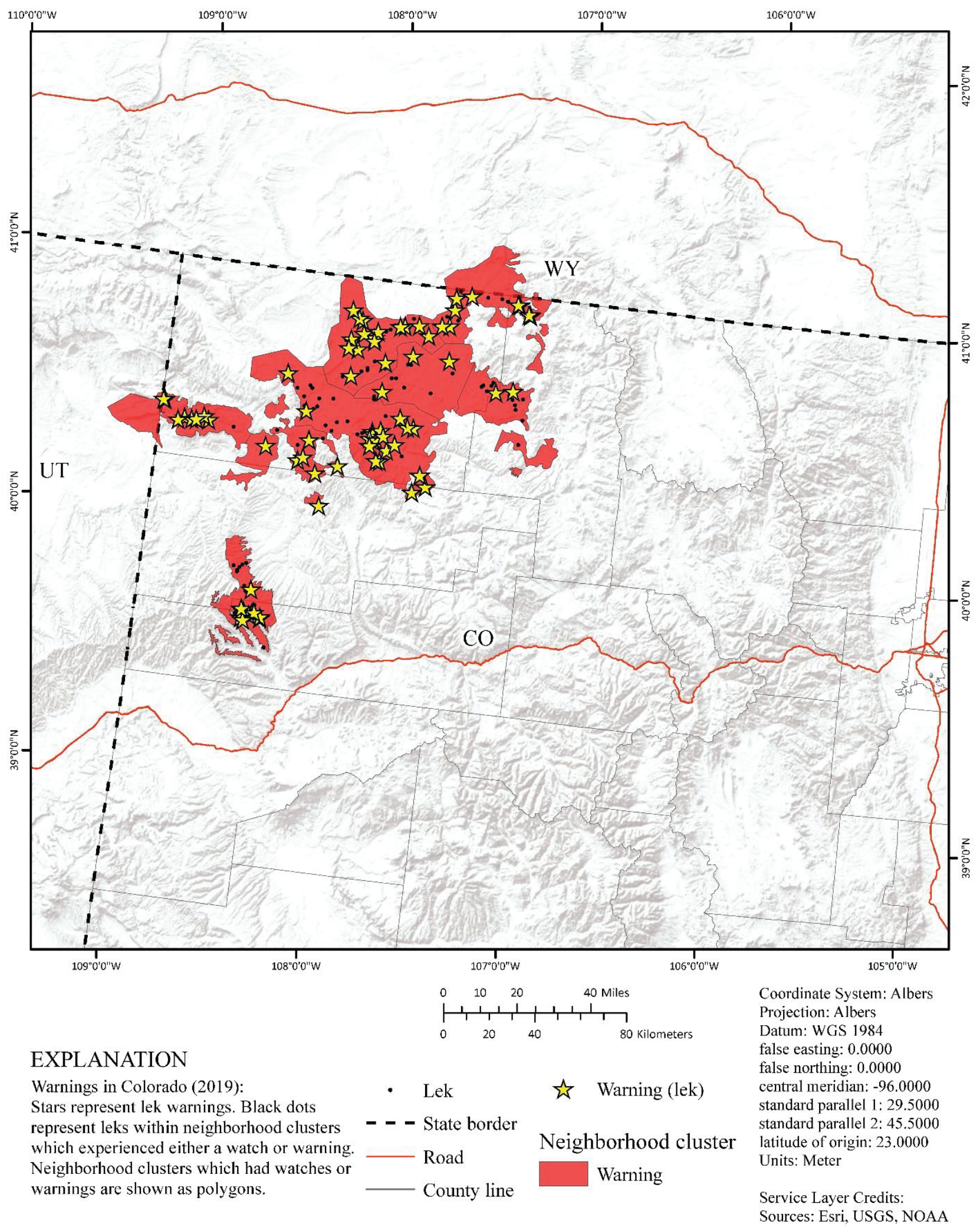

Figure 4.67. Spatial and temporal depiction of watches and warnings of greater sage-grouse (Centrocercus urophasianus) population declines at the neighborhood cluster scale within the state of Colorado during 2019. All leks within watch and warning boundaries were assigned as watch and warning, respectively. Yellow stars represent leks that reached warning independently. Map image is the intellectual property of Esri and is used herein under license. Copyright (C) 2020 Esri and its licensors. All rights reserved. 


\section{South Dakota Results}

\section{South Dakota Lek Data and Clusters (Objectives 1 and 2)}

South Dakota intersected CC-D and contained or intersected 13 different NCs (fig. 4.68). The total area of the 13 NCs within South Dakota was 1,001,220 ha. There were 11 clusters used in the analysis after 2 were omitted because of limitations in sample sizes. These areas consisted of 179 leks, of which 59 were located within the South Dakota state boundary representing 0.70 percent of the range-wide lek database. After extensive QA/QC, we used 40 leks from South Dakota in the SSM for population trend estimation (tables 4.19 and 4.20), totaling 717 individual lek counts. Mean male lek count was 6.46 (95-percent confidence interval $=6.18-6.73$ ) for leks within NCs that were within or overlapped South Dakota. Mean male count for leks within South Dakota was 6.66 (95-percent confidence interval $=6.23-7.10)$.

\section{South Dakota Population Trend Analysis (Objective 3)}

For CC-D, we estimated six population abundance nadirs (troughs) that dated back to 1960. Each of these population abundance nadirs represent between one and six complete periods of oscillation. We used these nadirs to estimate population trends across three different temporal scales that represented two, four, and six complete periods for the state (for instance, second, fourth, and sixth nadir). We estimated the average annual finite rate of population change $(\hat{\lambda})$ at the short (two periods), medium (four periods), and long (six periods) temporal scales as 0.927 (95-percent
CRI $=0913-0.947), 0.965$ (95-percent CRI $=0.950-0.977$ ), and 0.947 (95-percent $\mathrm{CRI}=0.934-0.959$ ), respectively (fig. 4.69). Climate cluster estimates, which included leks in adjacent states to South Dakota, were slightly different than estimates generated from leks only within South Dakota. For all NCs that were modeled and intersected South Dakota, we estimated median $\hat{\lambda}$ to be less than 1.0 for $81.8,100.0$, and 100.0 percent across short, medium, and long temporal scales, respectively (fig. 4.70 ; table 4.20 ). We estimated median $\hat{\lambda}$ to be less than 1.0 for $92.5,92.5$, and 97.5 percent of all modeled leks within South Dakota across short, medium, and long temporal scales, respectively (fig. 4.71). We reported spatial and temporal variation in average annual $\hat{\lambda}$ across different NCs (fig. 4.70) and leks (fig. 4.71).

\section{South Dakota Targeted Annual Warning System Analysis (Objective 4)}

During 1990-2019, the TAWS for sage-grouse populations in South Dakota activated a total of 27 and 18 leks as watches and warnings, respectively (fig. 4.72), which was 84.4 and 56.2 percent of the sampled leks used in the analysis. On average, across the 29 years, approximately 8.9 and 2.2 percent of leks per year experienced watches and warnings, respectively. The higher percentage for watches corresponds to repetitive activation. During this time frame, the TAWS also activated a total of five and three NCs as watches and warnings, respectively (fig. 4.73), which was 55.6 and 33.3 percent of the sampled clusters used in the analysis. On average, across the 29 years, approximately 4.9 and 1.3 percent of clusters per year experienced watches and warnings, respectively. Similar to leks, the higher percentage for watches corresponds to repetitive activation. During 2019, the TAWS did not activate any watches or warnings for NCs or leks. 


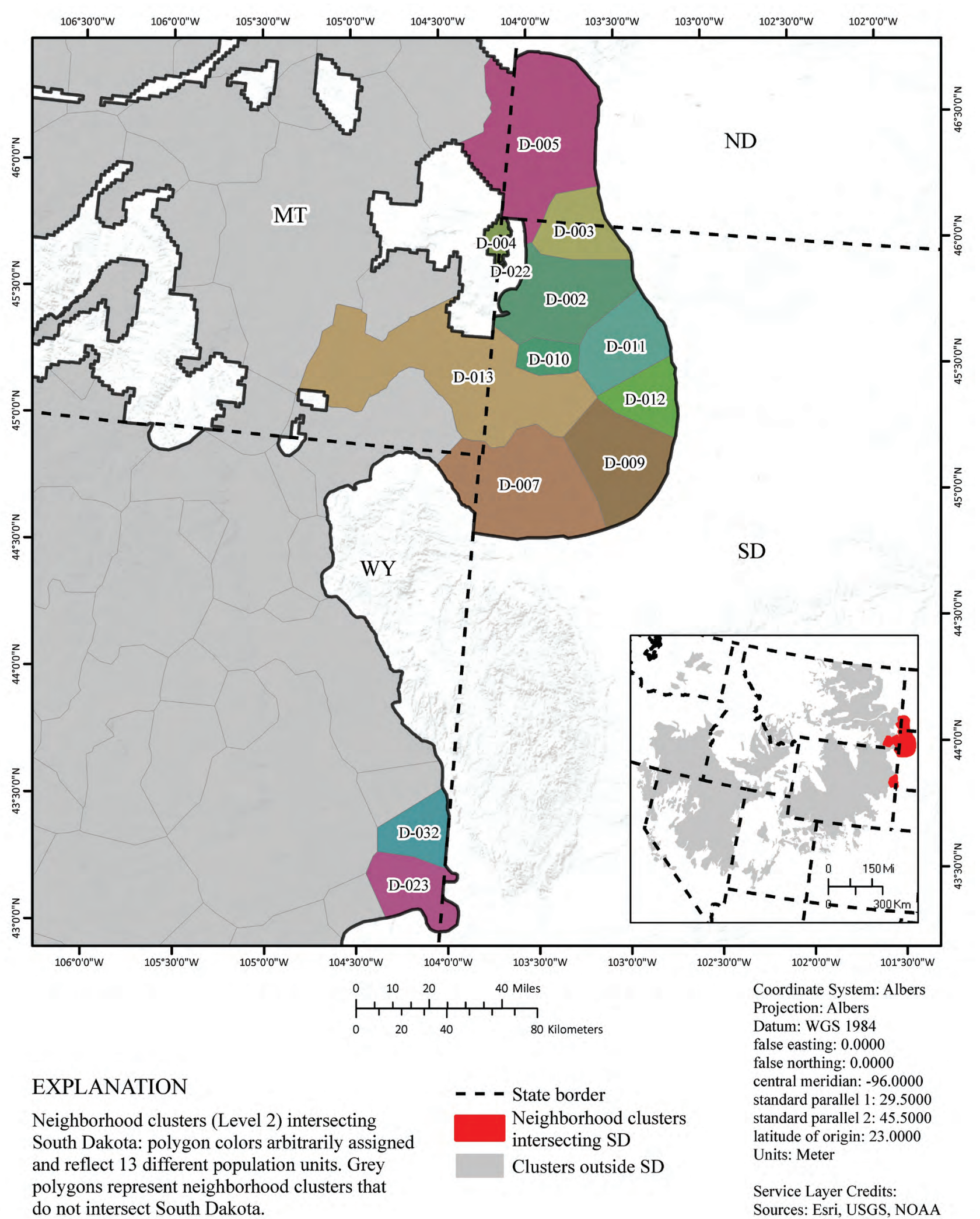

Figure 4.68. Greater sage-grouse (Centrocercus urophasianus) hierarchical population monitoring framework for neighborhood clusters that intersect the state of South Dakota. Map image is the intellectual property of Esri and is used herein under license. Copyright @ 2020 Esri and its licensors. All rights reserved. 
Table 4.19. Table of greater sage-grouse (Centrocercus urophasianus) average annual rate of population change $(\hat{\lambda})$ across six periods of oscillation in abundance that represent temporal scales for each climate cluster within the state of South Dakota. Estimates were derived from leks within the entire climate cluster.

[CC, climate cluster; D, eastern area]

\begin{tabular}{|c|c|c|c|c|c|c|c|c|c|}
\hline \multirow{2}{*}{ CC } & \multirow{2}{*}{$\begin{array}{l}\text { Percent } \\
\text { CC1 }\end{array}$} & \multicolumn{6}{|c|}{ Temporal scales ${ }^{2}$} & \multirow{2}{*}{$\begin{array}{l}\text { Number of } \\
\text { leks }^{3}\end{array}$} & \multirow{2}{*}{$\begin{array}{c}\text { Average } \\
\text { count/lek }\end{array}$} \\
\hline & & Long & Medium/Long & Medium & Short/Medium & Short & Recent & & \\
\hline $\mathrm{D}$ & 3.9 & $\begin{array}{c}0.963 \\
(0.960-0.968)\end{array}$ & $\begin{array}{c}0.956 \\
(0.946-0.960)\end{array}$ & $\begin{array}{c}0.967 \\
(0.960-0.972)\end{array}$ & $\begin{array}{c}0.983 \\
(0.975-0.989)\end{array}$ & $\begin{array}{c}0.963 \\
(0.959-0.967)\end{array}$ & $\begin{array}{c}0.980 \\
(0.972-0.989)\end{array}$ & $\begin{array}{c}2,944 \\
(1,831: 40 \\
1,566: 32)\end{array}$ & $\begin{array}{c}16.7 \\
(16.5-16.9)\end{array}$ \\
\hline
\end{tabular}

\footnotetext{
1The percent of each climate cluster that intersects the state.

${ }^{2}$ Temporal scales were estimated from present to each major population abundance nadir (trough) since 1960. Number of temporal scales were used to estimate population trends across six different temporal scales from approximately 10 to approximately 60 years.

3 Number of leks in database. In parentheses from left to right is (1) total number in cluster were used in trend analysis, (2) number used in trend analysis within state boundary, (3) total number in cluster were used in the targeted annual warning system (TAWS) analysis, and (4) number used in TAWS analysis within state boundary.
}

Table 4.20. Table of greater sage-grouse (Centrocercus urophasianus) average annual rate of population change $(\hat{\lambda})$ across six periods of oscillation in abundance that represent temporal scales for each neighborhood cluster within the state of South Dakota. Estimates were derived from leks within the entire neighborhood cluster.

[NC, neighborhood cluster; NA, not applicable]

\begin{tabular}{|c|c|c|c|c|c|c|c|c|c|}
\hline \multirow[b]{2}{*}{ NC } & \multirow[b]{2}{*}{$\begin{array}{l}\text { Percent } \\
\text { NC1 }\end{array}$} & \multicolumn{6}{|c|}{ Temporal scales² } & \multirow[b]{2}{*}{$\begin{array}{c}\text { Number of } \\
\text { leks }^{3}\end{array}$} & \multirow[b]{2}{*}{$\begin{array}{l}\text { Average } \\
\text { count/lek }\end{array}$} \\
\hline & & Long & Medium/Long & Medium & $\begin{array}{c}\text { Short/ } \\
\text { Medium }\end{array}$ & Short & Recent & & \\
\hline D-002 & 100.0 & $\begin{array}{c}0.941 \\
(0.922-0.959)\end{array}$ & $\begin{array}{c}0.927 \\
(0.903-0.945)\end{array}$ & $\begin{array}{c}0.939 \\
(0.921-0.959)\end{array}$ & $\begin{array}{c}0.952 \\
(0.932-0.971)\end{array}$ & $\begin{array}{c}0.898 \\
(0.872-0.924)\end{array}$ & $\begin{array}{c}0.921 \\
(0.836-1.006)\end{array}$ & $\begin{array}{c}14 \\
(7: 7,5: 5)\end{array}$ & $\begin{array}{c}10.3 \\
(8.6-12.0)\end{array}$ \\
\hline D-003 & 62.0 & $\begin{array}{c}0.952 \\
(0.926-0.968)\end{array}$ & $\begin{array}{c}0.956 \\
(0.925-0.982)\end{array}$ & $\begin{array}{c}0.971 \\
(0.932-1.008)\end{array}$ & $\begin{array}{c}0.977 \\
(0.931-1.027)\end{array}$ & $\begin{array}{c}0.960 \\
(0.913-1.012)\end{array}$ & $\begin{array}{c}0.936 \\
(0.829-1.051)\end{array}$ & $\begin{array}{c}5 \\
(3: 1,1: 1)\end{array}$ & $\begin{array}{c}10.7 \\
(8.0-13.5)\end{array}$ \\
\hline D-005 & 1.9 & $\begin{array}{c}0.954 \\
(0.945-0.963)\end{array}$ & $\begin{array}{c}0.946 \\
(0.935-0.955)\end{array}$ & $\begin{array}{c}0.956 \\
(0.942-0.966)\end{array}$ & $\begin{array}{c}0.942 \\
(0.930-0.953)\end{array}$ & $\begin{array}{c}0.919 \\
(0.905-0.933)\end{array}$ & $\begin{array}{c}0.968 \\
(0.921-1.013)\end{array}$ & $\begin{array}{c}66 \\
(46: 0,35: 0)\end{array}$ & $\begin{array}{c}8.3 \\
(7.7-8.8)\end{array}$ \\
\hline D-007 & 85.2 & $\begin{array}{c}0.955 \\
(0.936-0.973)\end{array}$ & $\begin{array}{c}0.949 \\
(0.927-0.967)\end{array}$ & $\begin{array}{c}0.981 \\
(0.958-0.996)\end{array}$ & $\begin{array}{c}0.999 \\
(0.978-1.018)\end{array}$ & $\begin{array}{c}0.944 \\
(0.923-0.963)\end{array}$ & $\begin{array}{c}1.025 \\
(0.970-1.086)\end{array}$ & $\begin{array}{c}20 \\
(15: 15,13: 13)\end{array}$ & $\begin{array}{c}10.4 \\
(9.0-11.7)\end{array}$ \\
\hline D-009 & 100.0 & $\begin{array}{c}0.925 \\
(0.885-0.982)\end{array}$ & $\begin{array}{c}0.906 \\
(0.860-0.950)\end{array}$ & $\begin{array}{c}0.923 \\
(0.867-0.985)\end{array}$ & $\begin{array}{c}0.908 \\
(0.839-0.981)\end{array}$ & $\begin{array}{c}0.866 \\
(0.805-0.937)\end{array}$ & $\begin{array}{c}0.919 \\
(0.757-1.118)\end{array}$ & $\begin{array}{c}4 \\
(3: 3,2: 2)\end{array}$ & $\begin{array}{c}6.8 \\
(3.5-10.0)\end{array}$ \\
\hline D-011 & 100.0 & $\begin{array}{c}0.957 \\
(0.916-0.990)\end{array}$ & $\begin{array}{c}0.965 \\
(0.941-0.987)\end{array}$ & $\begin{array}{c}0.945 \\
(0.906-0.979)\end{array}$ & $\begin{array}{c}0.943 \\
(0.897-0.990)\end{array}$ & $\begin{array}{c}0.889 \\
(0.829-0.944)\end{array}$ & $\begin{array}{c}0.770 \\
(0.631-0.930)\end{array}$ & $\begin{array}{c}4 \\
(3: 3,2: 2)\end{array}$ & $\begin{array}{c}6.3 \\
(4.5-8.1)\end{array}$ \\
\hline D-012 & 100.0 & $\begin{array}{c}0.932 \\
(0.892-0.978)\end{array}$ & $\begin{array}{c}0.913 \\
(0.868-0.956)\end{array}$ & $\begin{array}{c}0.908 \\
(0.867-0.948)\end{array}$ & $\begin{array}{c}0.878 \\
(0.834-0.916)\end{array}$ & $\begin{array}{c}0.813 \\
(0.752-0.864)\end{array}$ & $\begin{array}{c}0.876 \\
(0.711-1.067)\end{array}$ & $\begin{array}{c}1 \\
(1: 1,1: 1)\end{array}$ & $\begin{array}{c}13.4 \\
(9.5-17.3)\end{array}$ \\
\hline D-013 & 33.8 & $\begin{array}{c}0.946 \\
(0.928-0.965)\end{array}$ & $\begin{array}{c}0.938 \\
(0.916-0.961)\end{array}$ & $\begin{array}{c}0.980 \\
(0.957-1.001)\end{array}$ & $\begin{array}{c}0.988 \\
(0.961-1.013)\end{array}$ & $\begin{array}{c}0.921 \\
(0.896-0.945)\end{array}$ & $\begin{array}{c}1.005 \\
(0.936-1.082)\end{array}$ & $\begin{array}{c}54 \\
(14: 8,12: 6)\end{array}$ & $\begin{array}{c}9.9 \\
(8.2-11.7)\end{array}$ \\
\hline D-022 & 100.0 & $\begin{array}{c}0.978 \\
(0.943-1.015)\end{array}$ & $\begin{array}{c}0.972 \\
(0.941-1.007)\end{array}$ & $\begin{array}{c}0.988 \\
(0.964-1.017)\end{array}$ & $\begin{array}{c}1.019 \\
(0.996-1.047)\end{array}$ & $\begin{array}{c}1.017 \\
(0.983-1.060)\end{array}$ & $\begin{array}{c}0.974 \\
(0.887-1.075)\end{array}$ & $\begin{array}{c}1 \\
(1: 1,1: 1)\end{array}$ & $\begin{array}{c}13.6 \\
(11.6-15.6)\end{array}$ \\
\hline D-023 & 12.6 & $\begin{array}{c}0.975 \\
(0.934-1.014)\end{array}$ & $\begin{array}{c}0.966 \\
(0.932-1.011)\end{array}$ & $\begin{array}{c}0.988 \\
(0.960-1.027)\end{array}$ & $\begin{array}{c}1.028 \\
(0.992-1.081)\end{array}$ & $\begin{array}{c}1.030 \\
(0.968-1.114)\end{array}$ & $\begin{array}{c}1.053 \\
(0.923-1.189)\end{array}$ & $\begin{array}{c}3 \\
(3: 1,3: 1)\end{array}$ & $\begin{array}{c}11.3 \\
(7.4-15.1)\end{array}$ \\
\hline
\end{tabular}

${ }^{1}$ The percent of each neighborhood cluster that intersects the state.

${ }^{2}$ Temporal scales were estimated from present to each major population abundance nadir (trough) since 1960. Number of temporal scales were used to estimate population trends across six different temporal scales from approximately 10 to approximately 60 years.

${ }^{3}$ Number of leks in database. In parentheses from left to right is (1) total number in cluster were used in trend analysis, (2) number used in trend analysis within state boundary, (3) total number in cluster were used in the targeted annual warning system (TAWS) analysis, and (4) number used in TAWS analysis within state boundary. 

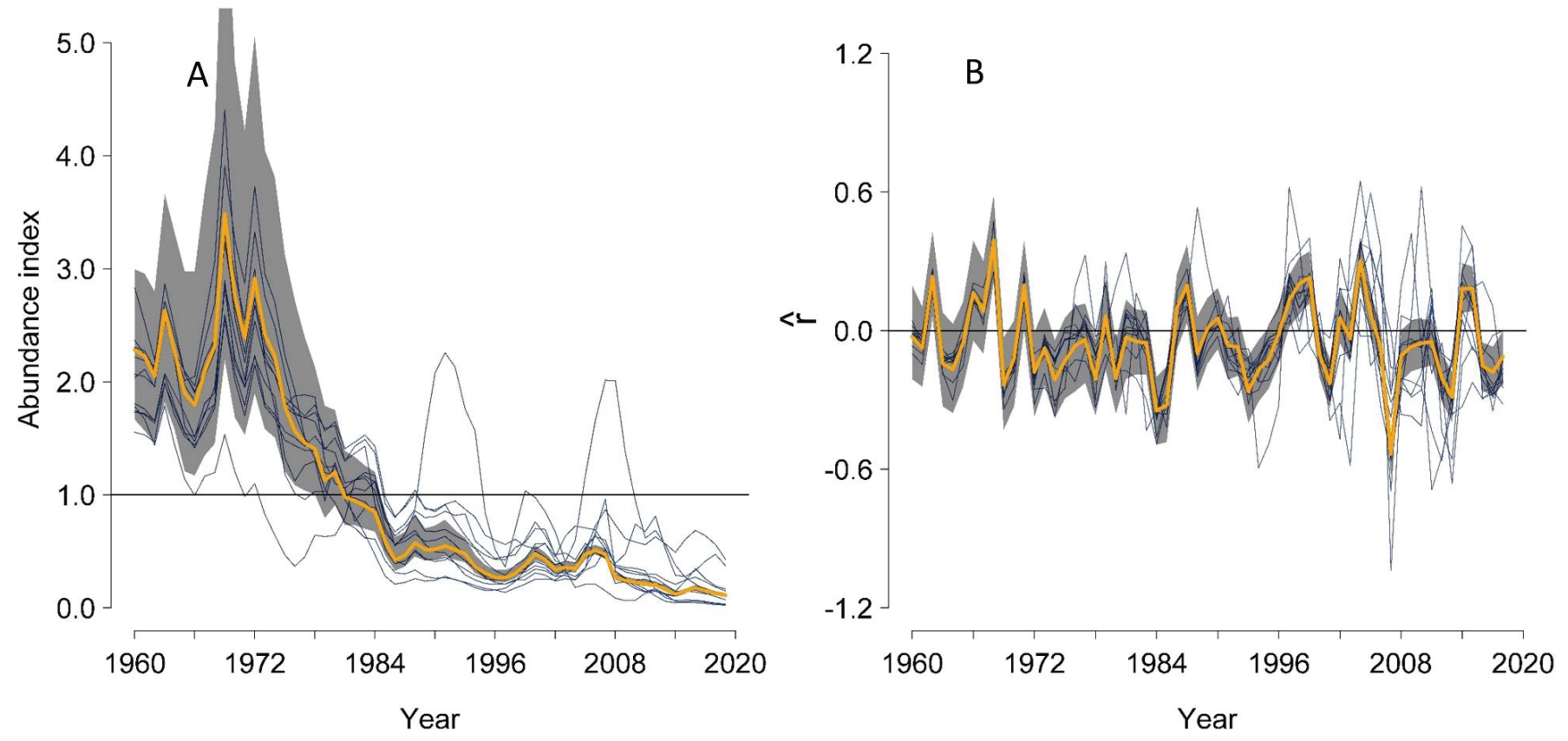

Figure 4.69. $A$, Abundance index (calculated as $\hat{N}$ divided by 60 -year mean of $\hat{N}$ ); and $B$, intrinsic rate of population change ( $\hat{r}$ ) of greater sage-grouse (Centrocercus urophasianus) within the state of South Dakota from 1960 to 2019 . Thick yellow line represents median estimates across all leks. Shaded areas represent 95-percent credible limits. Thin blue lines represent median values for neighborhood clusters. Black horizontal line (abundance index $=1.0$ ) represents 60 -year average. 


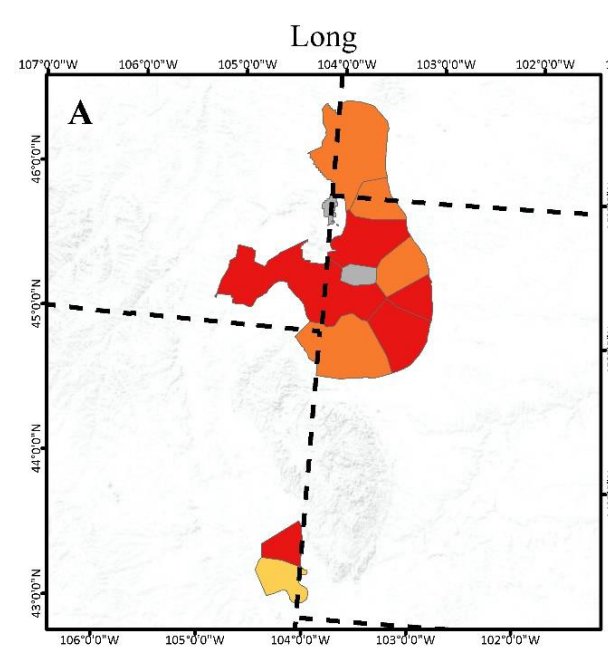

EXPLANATION

Neighborhood clusters (Level 2) intersecting South Dakota: polygon colors represent average lambda values for sage-grouse populations

over varying lengths of time (long, medium, short). Symbols are based on a range-wide distribution of values and all may not be represented here.
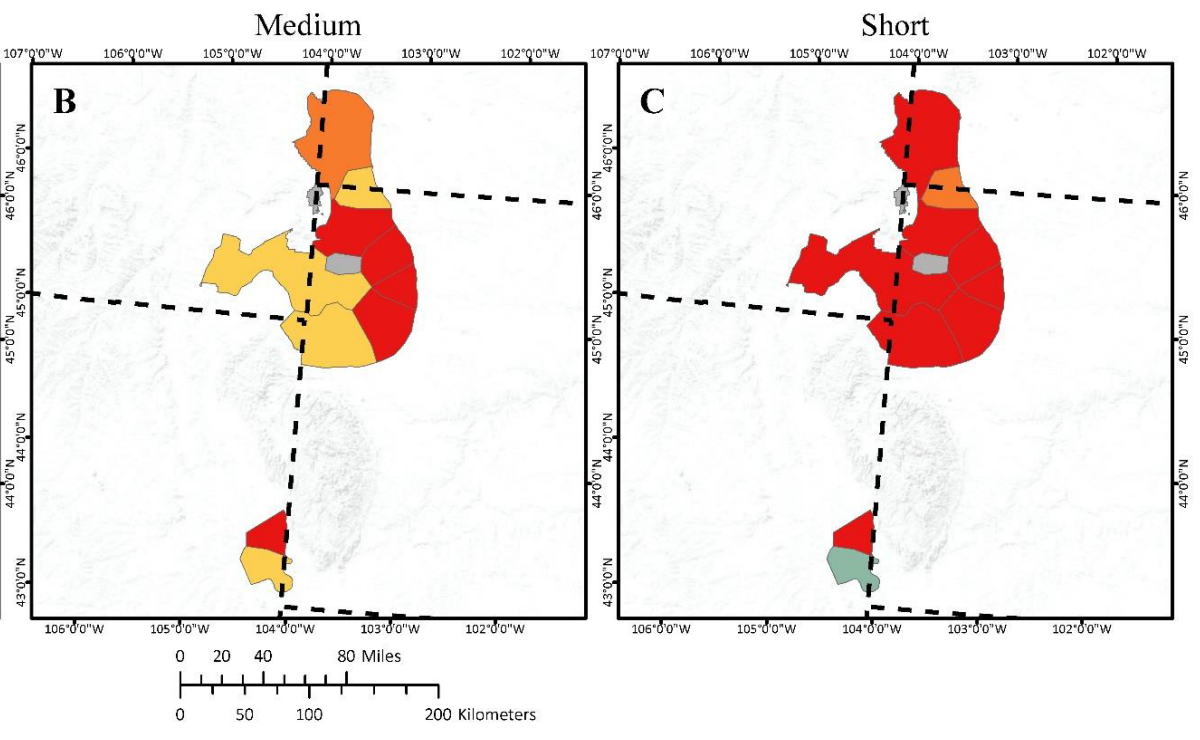

Average lambda $(\hat{\lambda})$

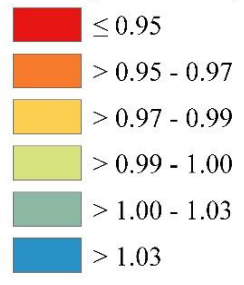

Coordinate System: Albers Projection: Albers Datum: WGS 1984

false easting: 0.0000 false northing: 0.0000 central meridian: -96.0000 standard parallel $1: 29.5000$ standard parallel 2: 45.5000 latitude of origin: 23.0000 Units: Meter

Service Layer Credits: Sources: Esri, USGS, NOAA

Figure 4.70. Spatial estimates of population trends across three temporal scales based on periods of oscillation (short, medium, and long) while accounting for fluctuations of greater sage-grouse (Centrocercus urophasianus) abundance across different neighborhood clusters within the state of South Dakota. Map images are the intellectual property of Esri and are used herein under license. Copyright (C) 2020 Esri and its licensors. All rights reserved. 


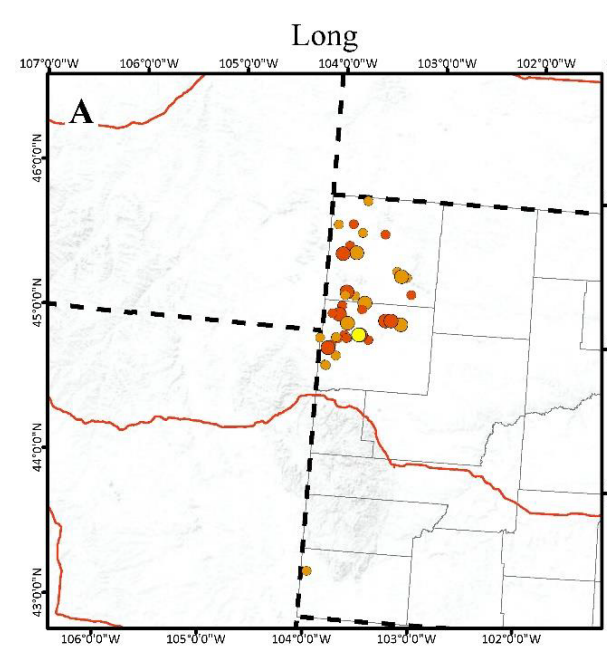

\section{EXPLANATION}

Leks within South Dakota: colors represent average lambda $(\hat{\lambda})$ values of sage-grouse populations over various time scales (long, medium, short). The size of the symbol represents the maximum population size of the lek over the years of the analysis, relativized to the range-wide maximum population size across all leks. Symbols are based on a range-wide distribution of values and all may not be represented here.

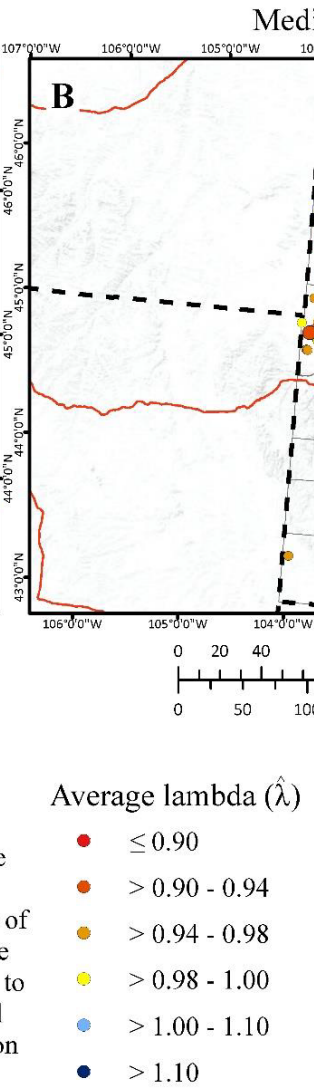

Medium

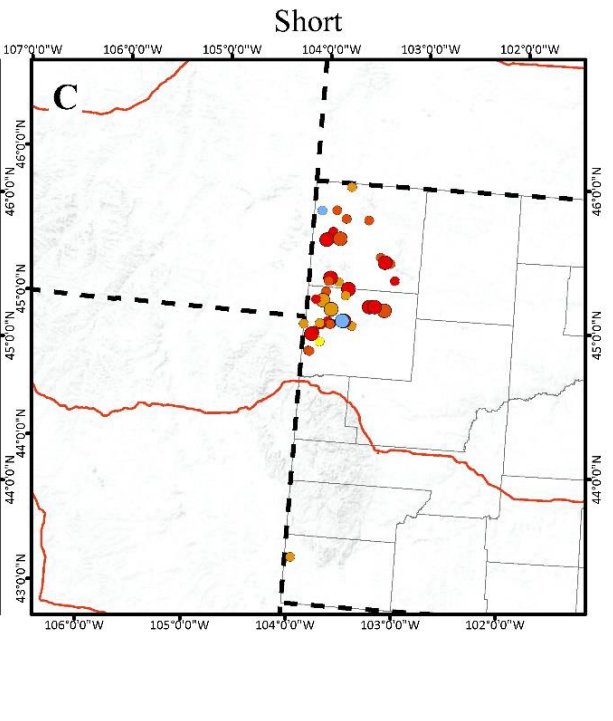

200 Kilometers

Road
- County line
- - - State border

Size guide

Maximum lek count

$\bigodot_{\text {Smallest }}^{\text {Largest }}$
Coordinate System: Albers

Projection: Albers

Datum: WGS 1984

false easting: 0.0000

false northing: 0.0000

central meridian: -96.0000

standard parallel 1: 29.5000

standard parallel 2: 45.5000

latitude of origin: 23.0000

Units: Meter

Service Layer Credits: Sources: Esri, USGS, NOAA

Figure 4.71. Spatial estimates of population trends across three temporal scales based on periods of oscillation (short, medium, and long) while accounting for fluctuations of greater sage-grouse (Centrocercus urophasianus) abundance across lek sites within the state of South Dakota. Map images are the intellectual property of Esri and are used herein under license. Copyright @ 2020 Esri and its licensors. All rights reserved. 


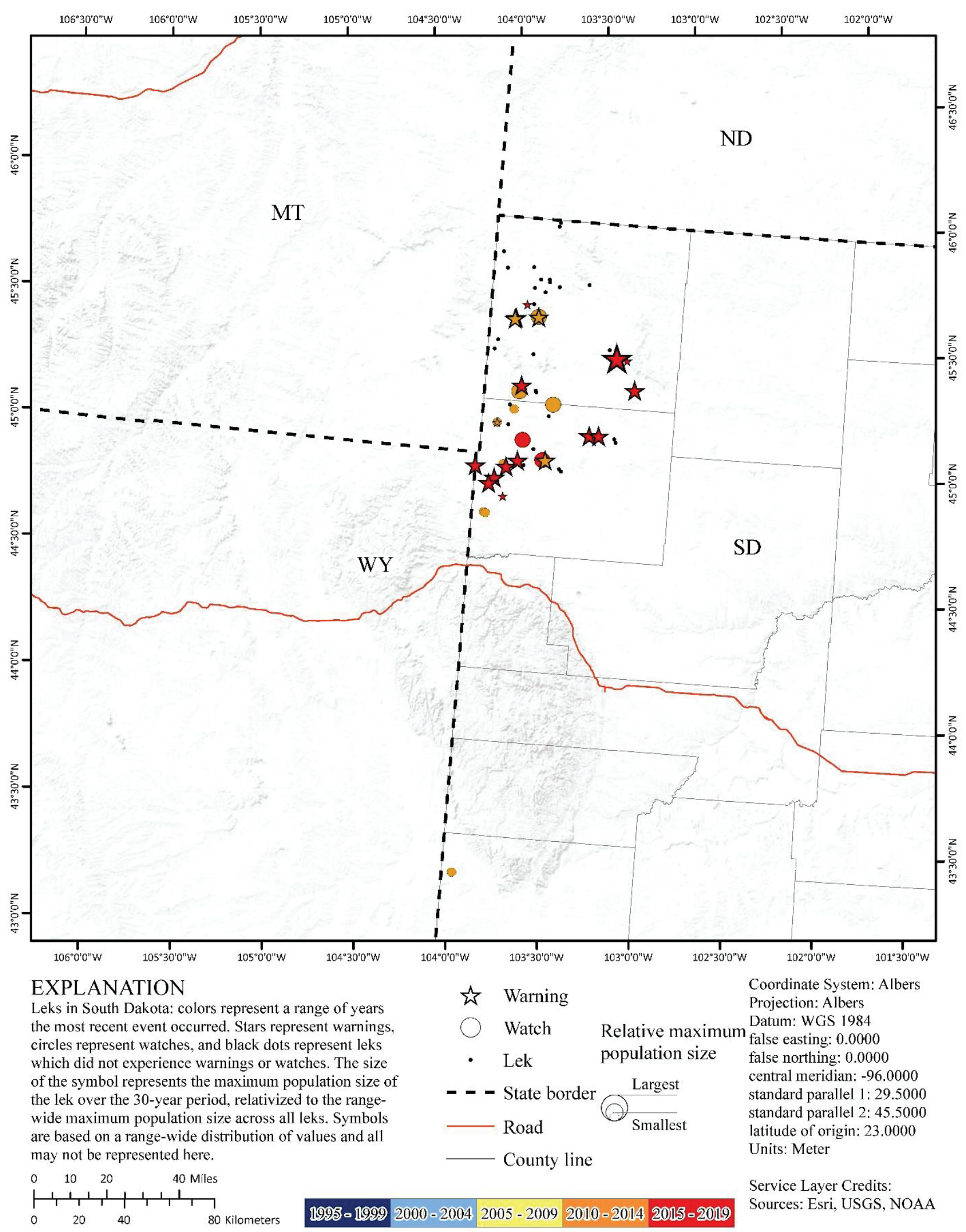

Figure 4.72. Spatial and temporal depiction of watches and warnings of greater sage-grouse (Centrocercus urophasianus) population declines at the lek scale within the state of South Dakota from 1990 to 2019. Map image is the intellectual property of Esri and is used herein under license. Copyright (C) 2020 Esri and its licensors. All rights reserved. 


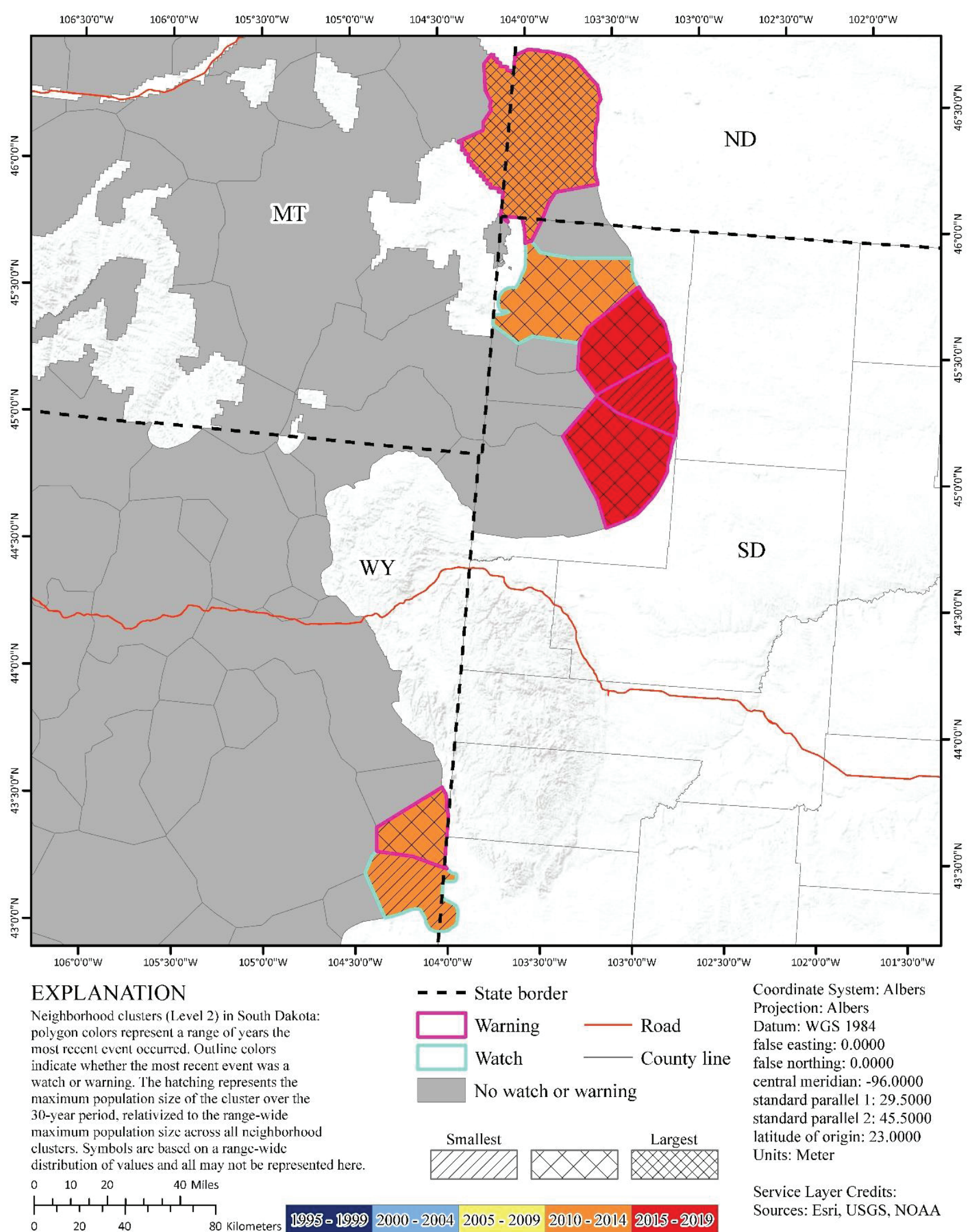

Figure 4.73. Spatial and temporal depiction of watches and warnings of greater sage-grouse (Centrocercus urophasianus) population declines at neighborhood cluster within the state of South Dakota from 1990 to 2019. Map image is the intellectual property of Esri and is used herein under license. Copyright $(\complement 2020$ Esri and its licensors. All rights reserved. 


\section{North Dakota Results}

\section{North Dakota Lek Data and Clusters (Objectives 1 and 2)}

North Dakota intersected CC-D and contained or intersected two different NCs (fig. 4.74). The total area of the two NCs within North Dakota was 284,697 ha. These areas consisted of 71 leks, 43 of which were located within the North Dakota state boundary representing 0.51 percent of the range-wide lek database. After extensive QA/QC, we used 39 leks from North Dakota in the SSM for population trend estimation (tables 4.21 and 4.22), totaling 1,317 individual lek counts. Mean male lek count was 6.70 (95-percent confidence interval $=6.33-7.08$ ) for leks within NCs that were within or overlapped North Dakota. Mean male count for leks within North Dakota was 7.69 (95-percent confidence interval $=7.15-8.24)$.

\section{North Dakota Population Trend Analysis (Objective 3)}

For CC-D, we estimated six population abundance nadirs (troughs) that dated back to 1960. Each of these population abundance nadirs represent between one and six complete periods of oscillation. We used these nadirs to estimate population trends across three different temporal scales that represented two, four, and six complete periods for the state (for instance, second, fourth, and sixth nadir). We estimated the average annual finite rate of population change $(\hat{\lambda})$ at the short (two periods), medium (four periods), and long (six periods) temporal scales as 0.917 (95-percent $\mathrm{CRI}=0.899-0.932), 0.954$ (95-percent $\mathrm{CRI}=0.943-0.963$ ), and 0.953 (95-percent $\mathrm{CRI}=0.943-0.962$ ), respectively (fig. 4.75). Climate cluster estimates, which included leks in adjacent states to North Dakota, were slightly different than estimates generated from leks only within North Dakota. For all NCs that were modeled and intersected North Dakota, we estimated median $\hat{\lambda}$ to be less than 1.0 for all temporal scales, respectively (fig. 4.76; table 4.22). We estimated median $\hat{\lambda}$ to be less than 1.0 for $97.4,92.3$, and 100.0 percent of all modeled leks within North Dakota across short, medium, and long temporal scales, respectively (fig. 4.77). We reported spatial and temporal variation in average annual $\hat{\lambda}$ across different NCs (fig. 4.76) and leks (fig. 4.77).

\section{North Dakota Targeted Annual Warning System Analysis (Objective 4)}

During 1990-2019, the TAWS for sage-grouse populations in North Dakota activated a total of 15 watches and 22 warnings (fig. 4.78), which was 57.7 and 84.6 percent of the sampled leks used in the analysis. On average, across the 29 years, approximately 4.6 and 3.4 percent of leks per year experienced watches and warnings, respectively. The higher percentage for watches corresponds to repetitive activation. During this time frame, the TAWS also activated a total of zero watches and one warning at the NC scale (fig. 4.79). On average, approximately 4.0 percent of clusters per year experienced warnings. During 2019, the TAWS activated no watches or warnings at leks or NCs. 

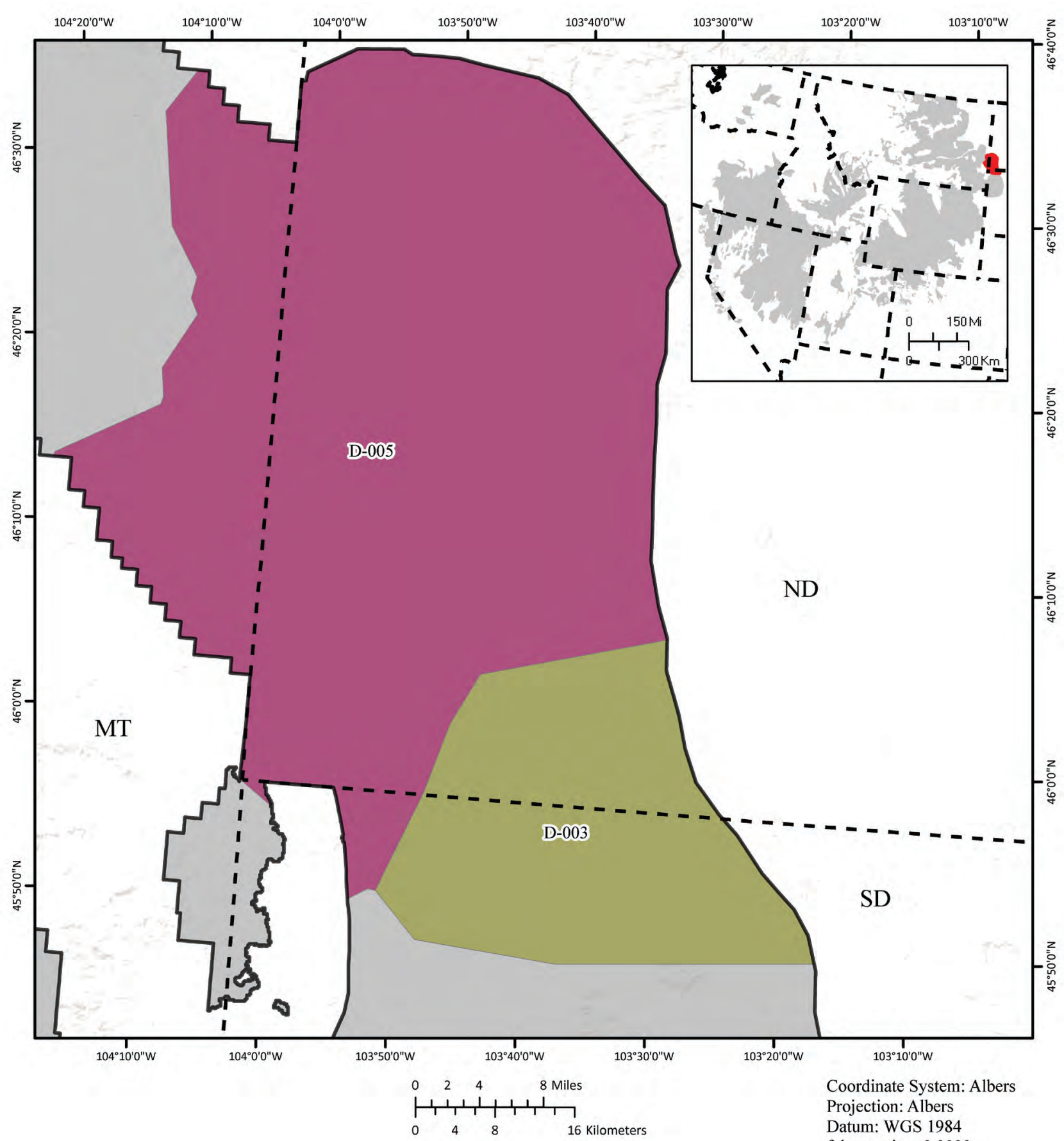

Coordinate System: Albers Projection: Albers Datum: WGS 1984 false easting: 0.0000 false northing: 0.0000

\section{EXPLANATION}

Neighborhood clusters (Level 2) intersecting North Dakota: polygon colors arbitrarily assigned and reflect 2 different population units. Grey polygons represent neighborhood clusters that do not intersect North Dakota. central meridian: -96.0000

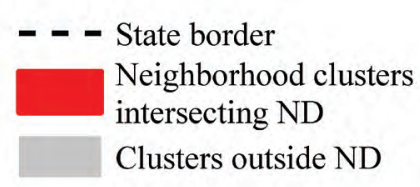

standard parallel 1: 29.5000 standard parallel 2: 45.5000 latitude of origin: 23.0000 Units: Meter

Service Layer Credits: Sources: Esri, USGS, NOAA

Figure 4.74. Greater sage-grouse (Centrocercus urophasianus) hierarchical population monitoring framework for neighborhood clusters that intersect the state of North Dakota. Map image is the intellectual property of Esri and is used herein under license. Copyright (C) 2020 Esri and its licensors. All rights reserved. 
Table 4.21. Table of greater sage-grouse (Centrocercus urophasianus) average annual rate of population change $(\hat{\lambda})$ across six periods of oscillation in abundance that represent temporal scales for each climate cluster within the state of North Dakota. Estimates were derived from leks within the entire climate cluster.

[CC, climate cluster; D, eastern area]

\begin{tabular}{|c|c|c|c|c|c|c|c|c|c|}
\hline \multirow[b]{2}{*}{ CC } & \multirow{2}{*}{$\begin{array}{l}\text { Percent } \\
\text { CC1 }\end{array}$} & \multicolumn{6}{|c|}{ Temporal scales² } & \multirow{2}{*}{$\begin{array}{c}\text { Number of } \\
\text { leks }^{3}\end{array}$} & \multirow{2}{*}{$\begin{array}{l}\text { Average } \\
\text { count/lek }\end{array}$} \\
\hline & & Long & Medium/Long & Medium & $\begin{array}{c}\text { Short/ } \\
\text { Medium }\end{array}$ & Short & Recent & & \\
\hline
\end{tabular}

1The percent of each climate cluster that intersects the state.

2Temporal scales were estimated from present to each major population abundance nadir (trough) since 1960. Number of temporal scales were used to estimate population trends across six different temporal scales from approximately 10 to approximately 60 years.

${ }^{3}$ Number of leks in database. In parentheses from left to right is (1) total number in cluster were used in trend analysis, (2) number used in trend analysis within state boundary, (3) total number in cluster were used in the targeted annual warning system (TAWS) analysis, and (4) number used in TAWS analysis within state boundary.

Table 4.22. Table of greater sage-grouse (Centrocercus urophasianus) average annual rate of population change $(\hat{\lambda})$ across six periods of oscillation in abundance that represent temporal scales for each neighborhood cluster within the state of North Dakota. Estimates were derived from leks within the entire neighborhood cluster.

[NC, neighborhood cluster; NA, not applicable]

\begin{tabular}{|c|c|c|c|c|c|c|c|c|c|}
\hline \multirow{2}{*}{ NC } & \multirow{2}{*}{$\begin{array}{l}\text { Percent } \\
\text { NC1 }\end{array}$} & \multicolumn{6}{|c|}{ Temporal scales 2} & \multirow{2}{*}{$\begin{array}{l}\text { Number of } \\
\text { leks }^{3}\end{array}$} & \multirow{2}{*}{$\begin{array}{l}\text { Average } \\
\text { count/lek }\end{array}$} \\
\hline & & Long & Medium/Long & Medium & Short/Medium & Short & Recent & & \\
\hline D-003 & 38 & $\begin{array}{c}0.952 \\
(0.926-0.968)\end{array}$ & $\begin{array}{c}0.956 \\
(0.925-0.982)\end{array}$ & $\begin{array}{c}0.971 \\
(0.932-1.008)\end{array}$ & $\begin{array}{c}0.977 \\
(0.931-1.027)\end{array}$ & $\begin{array}{c}0.960 \\
(0.913-1.012)\end{array}$ & $\begin{array}{c}0.936 \\
(0.829-1.051)\end{array}$ & $\begin{array}{c}5 \\
(3: 2,1: 0)\end{array}$ & $\begin{array}{c}10.7 \\
(8.0-13.5)\end{array}$ \\
\hline D-005 & 76.6 & $\begin{array}{c}0.954 \\
(0.945-0.963)\end{array}$ & $\begin{array}{c}0.946 \\
(0.935-0.955)\end{array}$ & $\begin{array}{c}0.956 \\
(0.942-0.966)\end{array}$ & $\begin{array}{c}0.942 \\
(0.930-0.953)\end{array}$ & $\begin{array}{c}0.919 \\
(0.905-0.933)\end{array}$ & $\begin{array}{c}0.968 \\
(0.921-1.013)\end{array}$ & $\begin{array}{c}66 \\
(46: 37,35: 26)\end{array}$ & $\begin{array}{c}8.3 \\
(7.7-8.8)\end{array}$ \\
\hline
\end{tabular}

1The percent of each neighborhood cluster that intersects the state.

${ }^{2}$ Temporal scales were estimated from present to each major population abundance nadir (trough) since 1960. Number of temporal scales were used to estimate population trends across six different temporal scales from approximately 10 to approximately 60 years.

${ }_{3}^{3}$ Number of leks in database. In parentheses from left to right is (1) total number in cluster were used in trend analysis, (2) number used in trend analysis within state boundary, (3) total number in cluster were used in the targeted annual warning system (TAWS) analysis, and (4) number used in TAWS analysis within state boundary. 

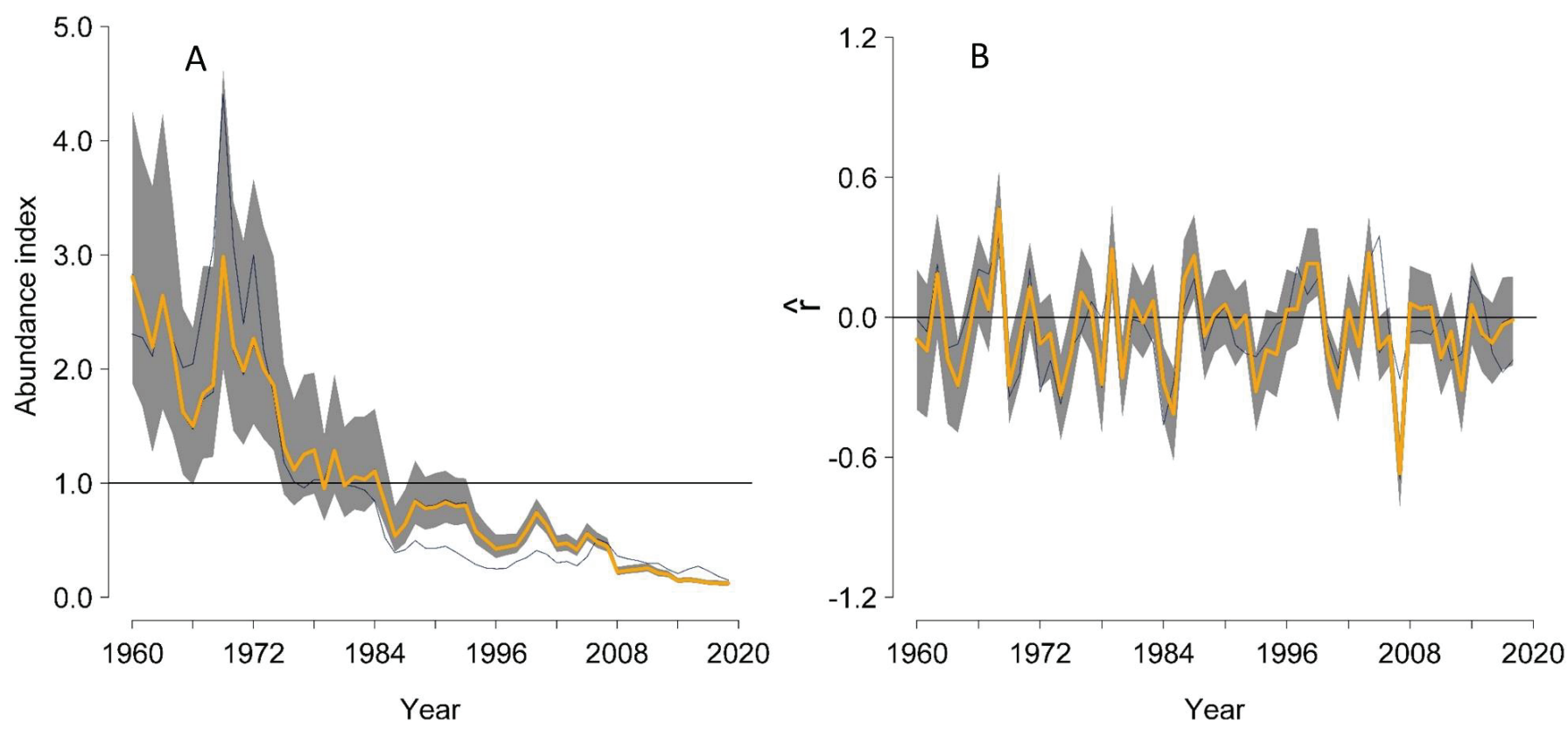

Figure 4.75. A, Abundance index (calculated as $\hat{N}$ divided by 60-year mean of $\hat{N}$ ); and $B$, intrinsic rate of population change ( $\hat{r}$ ) of greater sage-grouse (Centrocercus urophasianus) within the state of North Dakota from 1960 to 2019 . Thick yellow line represents median estimates across all leks. Shaded areas represent 95-percent credible limits. Black horizontal line (abundance index=1.0) represents 60-year average. 


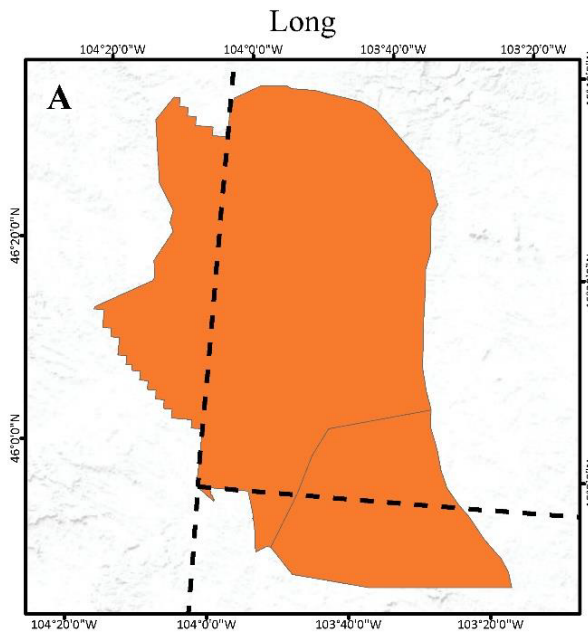

EXPLANATION

Neighborhood clusters (Level 2) intersecting North Dakota: polygon colors represent average lambda values for sage-grouse populations

over varying lengths of time (long, medium, short). Symbols are based on a range-wide distribution of values and all may not be represented here.
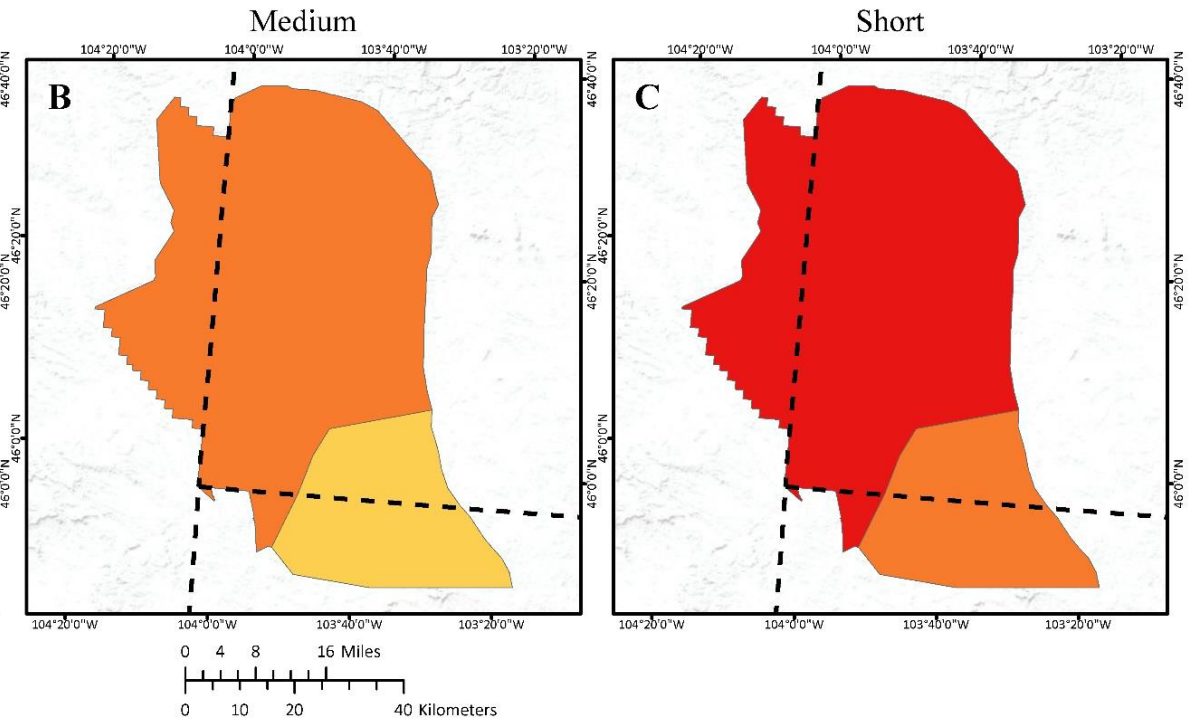

Average lambda $(\hat{\lambda})$

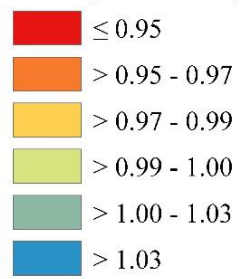

Coordinate System: Albers Projection: Albers Datum: WGS 1984

false easting: 0.0000 false northing: 0.0000 central meridian: -96.0000 standard parallel $1: 29.5000$ standard parallel 2: 45.5000 latitude of origin: 23.0000 Units: Meter

Service Layer Credits: Sources: Esri, USGS, NOAA

Figure 4.76. Spatial estimates of population trends across three temporal scales based on periods of oscillation (short, medium, and long) while accounting for fluctuations of greater sage-grouse (Centrocercus urophasianus) abundance across different neighborhood clusters within the state of North Dakota. Map images are the intellectual property of Esri and are used herein under license. Copyright (C) 2020 Esri and its licensors. All rights reserved. 


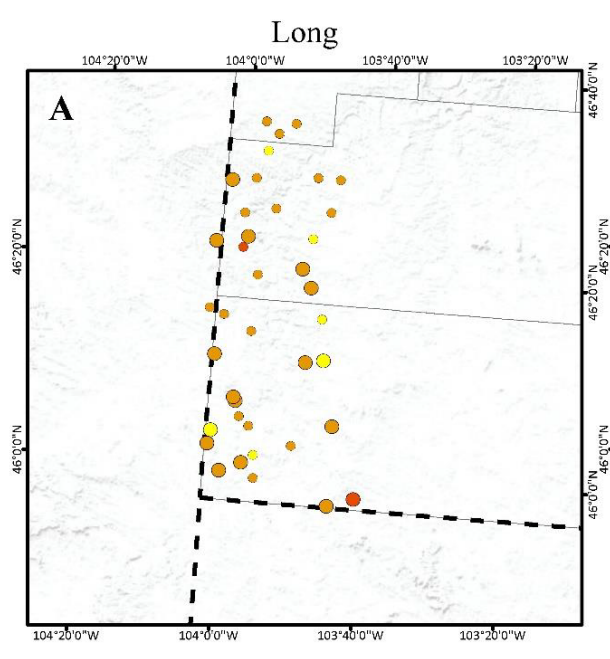

EXPLANATION

Leks withinNorth Dakota: colors represent average lambda $(\hat{\lambda})$ values of sage-grouse populations over various time scales (long, medium, short). The size of the symbol represents the maximum population size of the lek over the years of the analysis, relativized to the range-wide maximum population size across all leks. Symbols are based on a range-wide distribution of values and all may not be represented here.
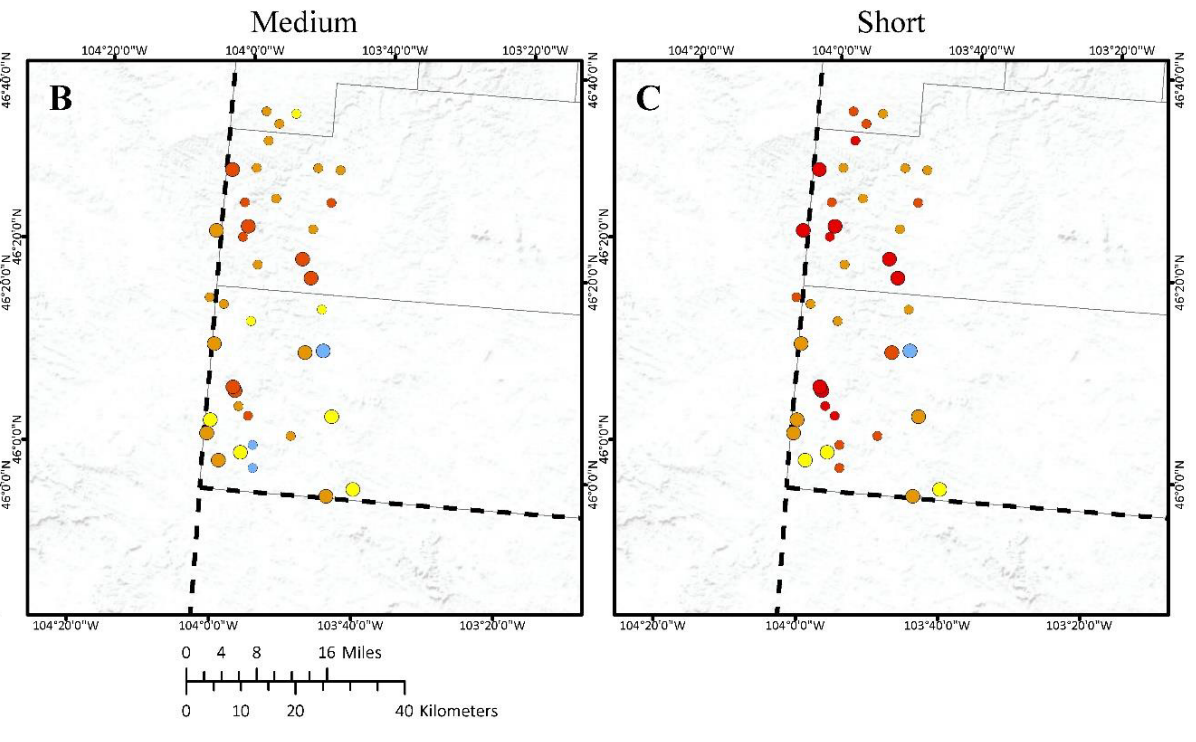
Average lambda $(\hat{\lambda})$
- $\leq 0.90$
- $>0.90-0.94$
- $>0.94-0.98$
$>0.98-1.00$
- $>1.00-1.10$
- $>1.10$
- Road
$\begin{aligned} & \text { County line } \\ & \text { - } \text { - - State border }\end{aligned}$
Size guide
Maximum lek count
Largest

Coordinate System: Albers

Projection: Albers

Datum: WGS 1984

false easting: 0.0000

false northing: 0.0000

central meridian: -96.0000

standard parallel $1: 29.5000$

standard parallel $2: 45.5000$

latitude of origin: 23.0000

Units: Meter

Service Layer Credits:

Sources: Esri, USGS, NOAA

Figure 4.77. Spatial estimates of population trends across three temporal scales based on periods of oscillation (short, medium, and long) while accounting for fluctuations of greater sage-grouse (Centrocercus urophasianus) abundance across lek sites within the state of North Dakota. Map images are the intellectual property of Esri and are used herein under license. Copyright $(2020$ Esri and its licensors. All rights reserved. 


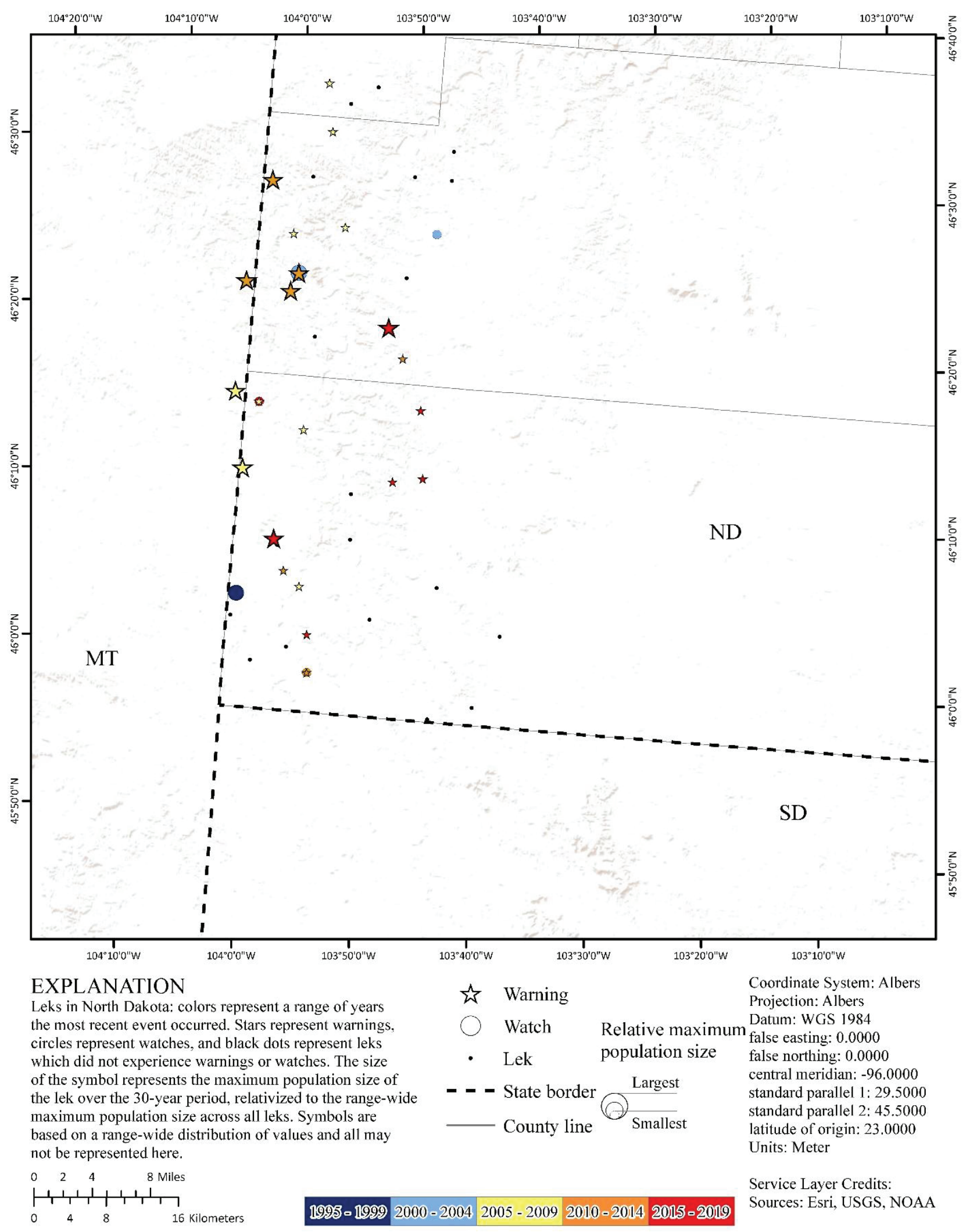

Figure 4.78. Spatial and temporal depiction of watches and warnings of greater sage-grouse (Centrocercus urophasianus) population declines at the lek scale within the state of North Dakota from 1990 to 2019. Map image is the intellectual property of Esri and is used herein under license. Copyright (c) 2020 Esri and its licensors. All rights reserved. 


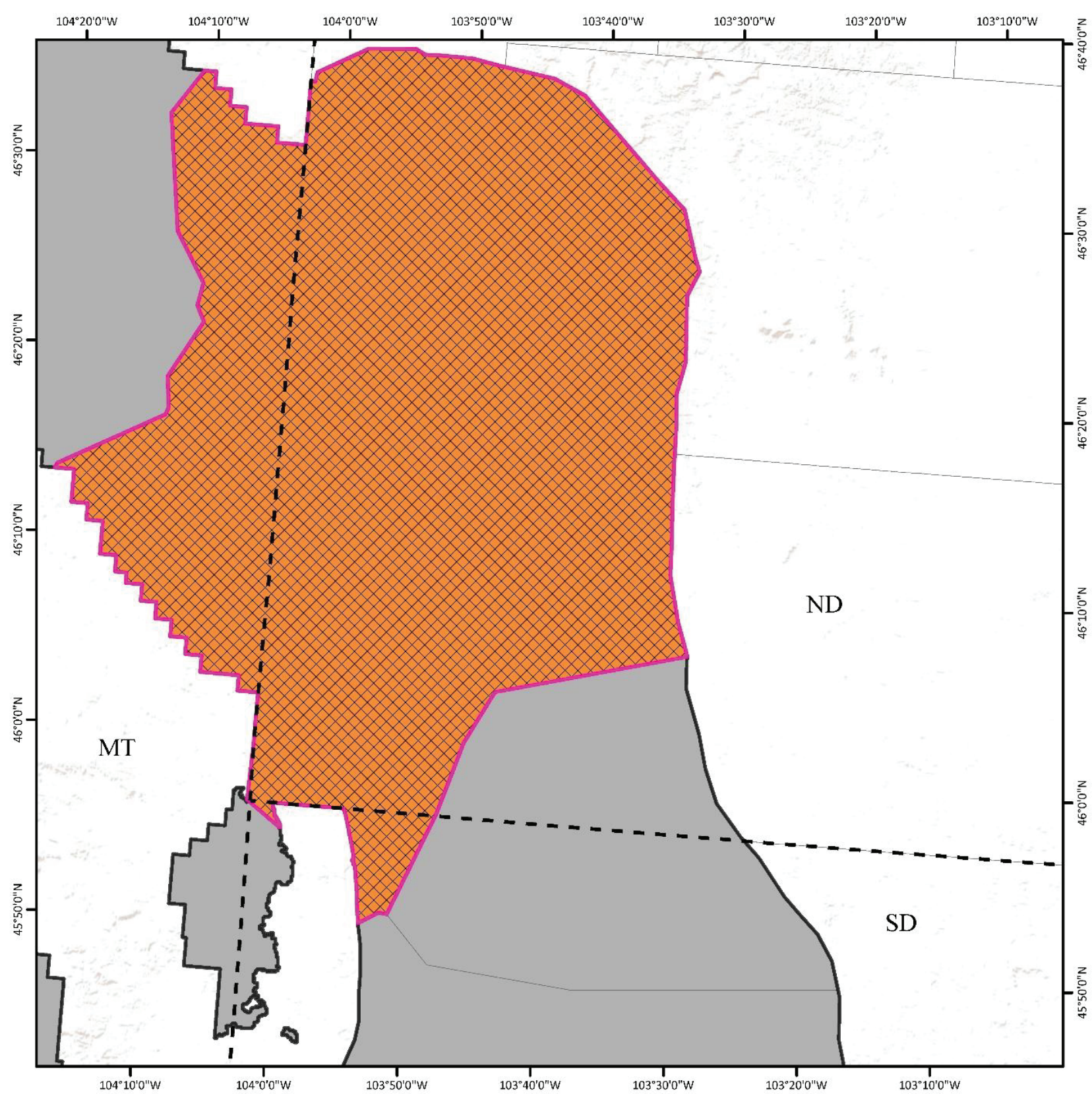

\section{EXPLANATION}

Neighborhood clusters (Level 2) in North Dakota: polygon colors represent a range of years the most recent event occurred. Oulline colors indicate whether the most recent event was a watch or warning. The hatching represents the maximum population size of the cluster over the 30 -year period, relativized to the range-wide maximum population size across all neighborhood clusters. Symbols are based on a range-wide distribution of values and all may not be represented here. $\begin{array}{llll}0 & 2 & 4 & \text { Miles }\end{array}$

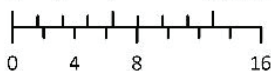
$\begin{array}{llll}0 & 4 & 8 & 16 \text { Kilometers }\end{array}$ 1995 -1999 2000-2004 2005- 20092010 - 20142015 -2019

- - - State border County line

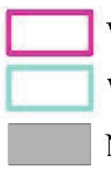
Warning Watch No watch or warning
Smallest
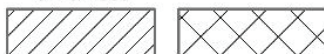

Largest

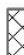
$\$ 28 \times$
Coordinate System: Albers Projection: Albers Datum: WGS 1984 false easting: 0.0000 false northing: 0.0000 central meridian: -96.0000 standard parallel 1: 29.5000 standard parallel 2: 45.5000 latitude of origin: 23.0000 Units: Meter

Service Layer Credits: Sources: Esri, USGS, NOAA

Figure 4.79. Spatial and temporal depiction of watches and warnings of greater sage-grouse (Centrocercus urophasianus) population declines at neighborhood clusters within the state of North Dakota from 1990 to 2019. Map image is the intellectual property of Esri and is used herein under license. Copyright (C) 2020 Esri and its licensors. All rights reserved. 


\section{Appendix 5. Simulation to Establish Thresholds for a Targeted Annual Warning System and Omission and Commission Errors}

\section{Optimal Threshold Simulation}

We carried out the following steps to determine threshold values that identified declining populations performing below the climate cluster scale for the Targeted Annual Warning System (TAWS). Step 1: We used a state-space model with nested random effects to estimate posterior distributions (PD) of $\hat{r}$ for each lek (that is, smallest spatial scale; point), neighborhood cluster (NC; that is, intermediate spatial scale; polygon), and climate cluster (CC; that is, largest spatial scale; polygon) from 1990 to 2019. Step 2: We developed a method for describing the relationship between two PDs of $\hat{r}$, calculated at spatiotemporally nested scales (for example, a 2002 comparison of a lek against its CC), by using the log odds ratio (LOR) of the two PDs. Comparisons of $\hat{r}$ PDs only were made within the same year and between a lek and its CC or a NC and its CC. The steps required to derive the LOR for the lek are described in the next paragraph.

The density of the PD of $\hat{r}$ for the lek $\left(\rho \hat{r}_{l}\right)$ was broken up into $\mathrm{n}(\mathrm{n}=1-4)$ distinct polygons (fig. 39 in the main text). Those four polygons were described in terms of the relationship between $\rho \hat{r}_{l}$ and the density of the PD of $\hat{r}$ for the CC $\left(\rho \hat{r}_{c}\right)$, stability, and the median of $\hat{r}$ for the $\mathrm{CC}\left(\widetilde{X}_{c}\right)$, namely (1) lower than the $\mathrm{CC}$ and decreasing (LD); (2) similar to the $\mathrm{CC}$, decreasing, and $<\widetilde{X}_{c}$ (SDL); (3) similar, decreasing, and $>\widetilde{X}_{c}$ (SDG); and (4) stable or increasing (SI). Once isolated and identified, we measured the area under the curve (AUC) for each of $n=4$ polygons that made up the $\rho \hat{r}_{l}$ using the 'overlap' function from the "overlapping" package (Pastore, 2018) in R (R Core Team, 2018). Polygons that were missing received an AUC value of 0 .

Using the four AUC values described previously, we defined the log-odds ratio (LOR) as evidence of decrease (EOD) divided by evidence against decrease (EAD), which took the form:

$\mathrm{LOR}=\log \left(\frac{E O D}{E A D}\right)=\log \left(\frac{A U C(L D)}{A U C(S D L)+A U C(S D G)+A U C(S I)}\right)$

For our purposes, the LD AUC was the only area that warranted management consideration because it corresponded to the proportion of the $\rho \hat{r}_{l}$ that was below stability $(\hat{r}<0)$ and lower than the $\mathrm{CC}$ within which it was spatially nested. In other words, the LD AUC was the only proportion of the $\rho \hat{r}_{l}$ that provided evidence of a decreasing population not associated with large-scale processes. Therefore, the LD AUC was assigned to the EOD category. The SDL and SDG AUCs were similar to the LD AUC in that they were below stability $(\hat{r}<0)$, but unlike the LD AUC, they were trending with or outperforming the $\mathrm{CC}$ to which they were nested. For that reason, EAD comprised AUCs for SDL, SDG, and SI.

Step 3: We developed a method that would identify, based on LOR values, whether leks were declining slowly or precipitously (a finding used to alert possible management attention) by using an iterative process in program $\mathrm{R}$ (and is described in the following paragraph). Specifically, we created two identical 100-element long vectors of threshold values that spanned -4.595 to 4.595 on the LOR scale. These values are equivalent to a 100-element long vector that spans $0.01-0.99$ on the probability (here, probability of decrease) scale. We referred to these vectors as the slow-signal and fast-signal threshold vectors, where the first element in both vectors corresponded to the minimum slow and fast threshold values, respectively. Likewise, the hundredth element from each vector corresponded to the maximum slow and fast threshold values, respectively.

We iterated through every possible combination $(\mathrm{n}=10,000$ iterations) of slow and fast-signal threshold values ( $T_{s}$ and $T_{f}$ ) and compared the LOR value (calculated in step 2) for every lek (or NC) and year combination to the $T_{s}$ and $T_{f}$ values selected during iteration $i$. For example, during iteration $i=1$, we compared the LOR for all leks, across all years, against the $T_{s}$ and $T_{f}$ combination of -4.595 and -4.595 (that is, 0.01 and 0.01 on the probability of decrease scale), and during iteration $i=2$, we compared the LOR for all leks, across all years, against the $T_{s}$ and $T_{f}$ combination of -4.595 and -3.892 (that is, 0.01 and 0.02 on the probability of decrease scale). Instances where the LOR value for a given lek (or NC) in a given year were greater than or equal to $T_{S}$ that lek would receive an indicator value $\left(I_{s}\right)$ of 1 , otherwise it received a value of 0 . Likewise, if the LOR value for that lek in that year was greater than or equal to $T_{f}$ it received an indicator value $\left(I_{f}\right)$ of 1 , otherwise it received a value of 0 . The binary indicators for $I_{s}$ and $I_{f}$ were kept separate, so that a lek in a given year could possess four distinct $I_{s}$ and $I_{f}$ codes $(0$ and 0,1 and 0,0 and 1, or 1 and 1). These binary indicators were equivalent to signals for warnings. For example, when a lek had an $I_{s}$ binary indicator of one in 3 out of 4 consecutive years (for example, 1995-1998 was 0-1-1-1; 1-0-1-1; 1-1-0-1; or 1-1-1-0), regardless of $I_{f}$ values over the same time frame, that lek would be 'marked' to receive simulated management action. When a lek had an $I_{f}$ binary indicator of one in 2 out of 3 consecutive years, regardless of $I_{s}$ values over the same time frame, we would similarly mark it to receive simulated management action. Very briefly, simulated management action consisted of improving the $\hat{r}$ values of marked leks (or NC) for all years following the marking event. For a more detailed explanation of the steps taken to simulate management action, please see the "Simulated Management" section. 
At the end of each iteration ( $i$ ), an evaluation process took place, using an optimization index $\left(O_{i}\right)$, which took the form:

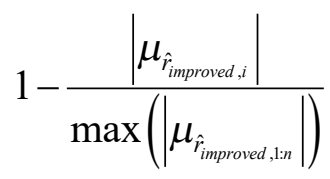

where $n$ represents the total number of iterations run for the simulation, and $\mu_{\hat{r}_{\text {improved }, i} \text { represents the weighted }}$ average (based on lek size) of $\hat{r}$, calculated across all leks and years, during iteration $(i)$. Within a given iteration, values of $\hat{r}$ for each year consisted of a combination of leks that were never marked (that is, the original modeled values), leks that were marked, but before marking took place (that is, the original modeled values), and leks that were 1 or more years beyond a marking event (that is, sampled values). Leks that were 1 or more years beyond a marking event represented the only instance where $\hat{r}$ values could be updated, and, therefore the only potential source for improvement over the observed (that is, modeled) parameter estimates. Iterations that resulted in $\mu_{\hat{r}_{\text {improved }}}=1.1$ (that is, 10-percent mean annual growth) and 0.9 (that is, 10-percent mean annual decline) would be ranked equally in terms of their optimization index. The rationale for this type of index was that a management scenario that did not result in population stability was inadequate, whereas a management scenario that went beyond stability was too aggressive. In addition to the optimization index, we also kept track of the number of leks that signaled during every iteration of the simulation, and the percent improvement ( $\mu_{\hat{r}_{\text {improved }}}$ ) over the observed (that is, modeled) weighted average rate of change in abundance. Multiple iterations produced $\mu_{\hat{r}_{\text {in }}}$ values $=1$. However, the number of warnings activated across scenarios that produced $\mu_{\hat{r}_{\text {improved }}}$ values=1 varied. In other words, some iterations that resulted in population stability required a greater number of leks to achieve that objective. With the goal of balancing type I and type II errors (see "Omission and Commission Error for Targeted Annual Warning System" section), we chose the iteration (that is, $T_{s}$ and $T_{f}$ combination) that resulted in an average number of warnings. We refer to the $T_{s}$ and $T_{f}$ pair with the highest $\mu_{\hat{r}_{\text {improved }}}$ value and average number of warnings as the optimal combinatorial threshold pair (fig. 5.1A). The same process was applied to NCs for determining the optimal $T_{s}$ and $T_{f}$ pair at that scale with one important distinction. When a $\mathrm{NC}$ was marked for simulated management, all leks that fell within its boundaries would receive updated $\hat{r}$ and abundance values. The optimal $T_{s}$ and $T_{f}$ pair for leks and NCs were used to determine the location and timing of signals, watches, and warnings across the range from 1990 to 2019. 

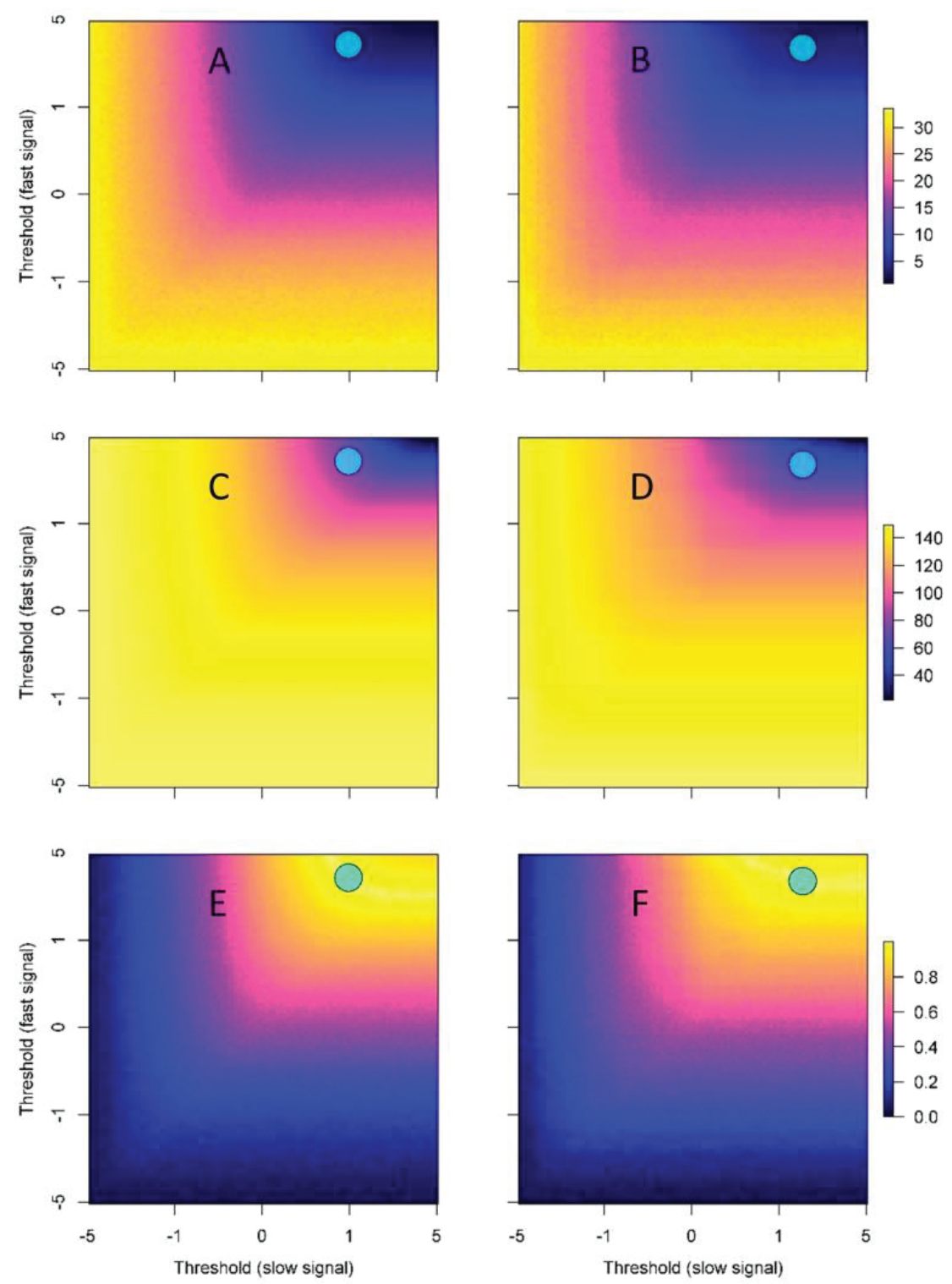

Figure 5.1. $\quad A$, Percent change in population rate of change for leks (percent change $=3.768$ ); $B$, percent change in population rate of change for neighborhood clusters (percent change $=3.726) ; C$, mean number of annual signals for leks (mean=69.8); $D$, mean number of annual signals for neighborhood clusters (mean=60.3); $E$, optimization index for leks (index=1); and $F$, optimization index for neighborhood clusters (index=1), given optimized values for slow-fast signal combination (blue circle) for leks (slow=1.059 and fast=2.452) and neighborhood clusters (slow=1.462 and fast=2.324), respectively. Optimized values for slow-fast signal combinations determined signals used for watches and warnings within a targeted annual warning system. 


\section{Simulated Management}

Simulated management consisted of replacing the observed $\hat{r}$ for marked leks with an $\hat{r}$ value sampled from a distribution of values formed from the entire population (range-wide) of $\hat{r}$ values during the same year. To simulate a non-linear relationship between management action and population performance through time, the percentile of the distribution that was sampled would increase every year (fig. 5.2). Recovery rates were assigned to each lek, based on the underlying index of resilience and resistance (R\&R; Maestas and others, 2016) and whether the decline was probabilistically associated with sagebrush loss or an unmeasured disturbance. Additionally, we evaluated sagebrush trends (1985-2018; 33 years, because 2012 imagery was unavailable) at each lek based on back in time estimates (Shi and others, 2018; Rigge and others, 2019, 2020). This assessment of sagebrush trends coincided with the duration of the lek count data used in the population analysis. We categorized the percent change in sagebrush for each lek as either decreasing or having experienced no change/increase. Similarly, we categorized the long-term (for example, 30 year) rate of change in abundance for each lek as either decreasing $(\bar{r}<0)$ or stable/increasing $(\bar{r} \geq 0)$. The combination of categorical sagebrush change and categorical rate of change in abundance were summarized across the three R\&R classes. The proportion of leks that landed within the combinatorial category of losing sagebrush and experiencing declining rate of change served as our probability of assignment to a slow recovery rate. Those probabilities were 0.51 (low R\&R), 0.41 (moderate R\&R), and 0.36 (high R\&R). In other words, 51 percent of marked leks in low R\&R would be assigned to slow recovery, 41 percent of marked leks in moderate R\&R to slow recovery, and 36 percent of marked leks in high R\&R to slow recovery.

The complimentary percentages for each R\&R class were used to assign leks to a fast recovery rate. Fast recovery rates were assumed to coincide with remedial measures (for example, sound/light abatement and road closures during lekking season) that would lead to full recovery potential in 3 years. Slow recovery rates, on the other hand, were associated with sagebrush loss and remedial actions that would take longer to positively influence sage-grouse populations. Based on a meta-analysis of average recovery rates for the three dominant sagebrush communities (Wyoming big sagebrush Artemisia tridentata wyomingensis; mountain big sagebrush A. tridentata vaseyana; and low sagebrush A. nova, A. arbuscular) across sage-grouse range, we determined full recovery potentials of 117 years for marked leks in low R\&R (Watts and Wambolt, 1996; Wambolt and others, 2001; Baker, 2006; Lesica and others, 2007; Cooper and others, 2011; Miller and others, 2013), 59 years for marked leks in moderate R\&R (Miller and others, 2013), and 27 years for marked leks in high R\&R (Baker, 2006; Lesica and others, 2007; Ziegenhagen and Miller, 2009; Miller and others, 2013). All recovery rates (that is, slow and fast) took on a sigmoidal curve based on the assumption of relatively slow recovery in the beginning of the corresponding time frame, rapid recovery in the middle, and a slow tapering to a recovery ceiling (that is, full recovery potential) toward the later years. As an example, a lek that received a warning in 1995 was located in high R\&R and was assigned to the slow recovery rate, would have its $\hat{r}$ value replaced in 1996 (that is, 1 -year later) with the approximately 30 th percentile of the population level $\hat{r}$ from 1996. In 2012 (that is, 17-years later), its $\hat{r}$ value would be replaced with the approximately 56th percentile of the population level $\hat{r}$ from 2012, and in 2019 (27-years later) it's $\hat{r}$ value would be replaced with the approximately 70th percentile of the population level $\hat{r}$ from 2019. Once a lek activated a warning, it would remain in the warning state and continue to receive management induced improvements to rate of change in abundance for the remainder of the time series. As such, a lek that signaled in 2007 would receive management induced improvements to rate of change in abundance from 2008 to 2019 (that is, 12 years). This does not imply that active management occur every year of that time frame. It is possible that management action taking place in 2008 could perpetuate through to 2019; a one-time management action with carry-over effects. By sampling $\hat{r}$ from a distribution formed from all leks and within the same year, we improved the accuracy in estimating management induced rate of change in abundance over choosing an arbitrary value (for example, $\hat{r}=0$; stability), which could underperform or outperform model estimates over the same time frame. That said, the percentile of the distribution sampled is a subjective choice and changing those values could change the results of the simulation (that is, optimal $T_{s}$ and $T_{f}$ value combination chosen). For that reason, we decided to pick a conservative range of values (that is, 30-70th percentile), so that when implemented annually, simulated improvements in rate of change in abundance would be much less likely to overestimate management efficacy. 


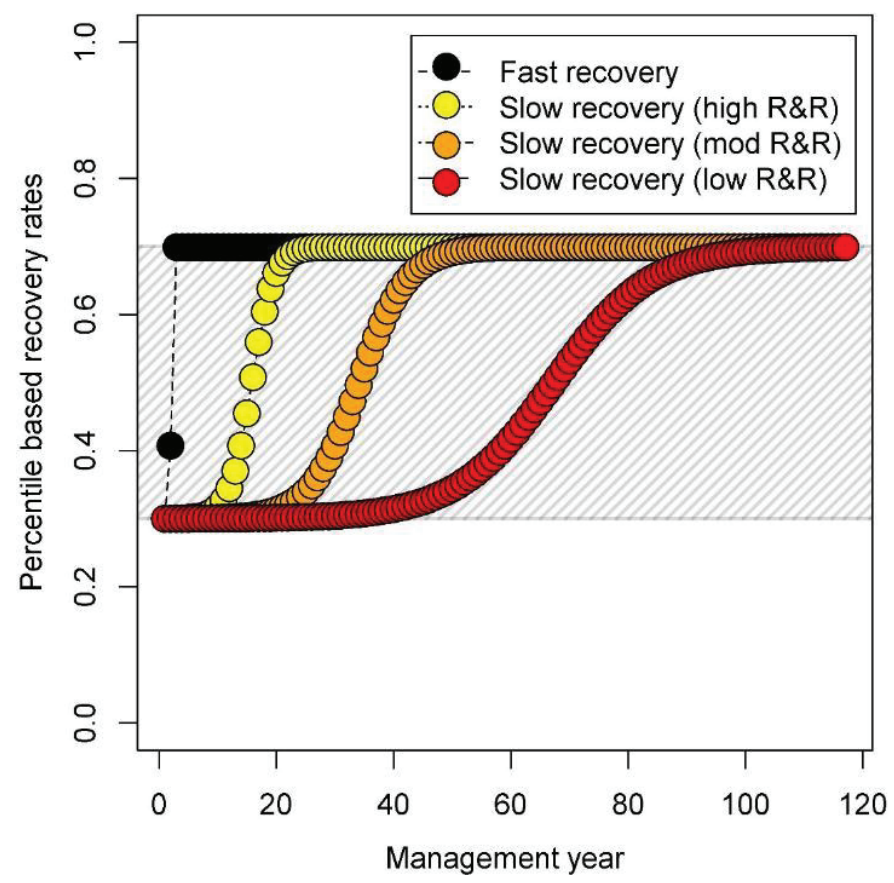

Figure 5.2. Recovery curves for leks that weed for simulated management intervention. Black circles represent fast recovery following management actions such as predator removal and noise abatement. Yellow, orange, and red circles represent slow recovery for leks occupying areas of high, moderate, and low resilience and resistance. Slow recovery scenarios are based on management actions that reverse sagebrush related impacts to sage-grouse populations (for example, fire).

\section{Omission and Commission Error for Targeted Annual Warning System}

We assessed type I and type II error rates for the TAWS using a subset of leks identified as best (commission dataset) and worst (omission dataset) performers. The commission dataset was used to evaluate type I errors (false positives), and the omission dataset was used to evaluate type II errors (false negatives). Leks chosen for the commission dataset needed to demonstrate better performance during the latter portion of the time series (2010-2019; after period) compared to the early portion of the time series (1990-2009; before period), which we assessed using summary statistics of abundance ( $\hat{N})$ and population growth $(\hat{r})$. Because a lek selected for the commission dataset was expected to perform better in the after period than before, we should not expect warnings to occur during the after period. Any warnings that occurred at commission leks were scored as false positives. Leks selected for the omission dataset were expected to perform poorly during the before or after period. Any leks within the omission dataset that did not receive a warning were scored as false negatives based on a clear pattern of decreasing trend with strong evidence of local extirpation.

To create our commission dataset, we developed five rules. First, we retained leks that had a stable or positive average $\hat{r}$ during the after period (Rule 1; stable or growing after). We imposed this rule to eliminate leks that were experiencing long-term declines. Second, we chose leks with a minimum of two or more males counted in 2 or more years during the before period (Rule 2; active before), which was to ensure that leks were active and monitored during the before period when warnings were assessed. Third, the averaged $\hat{N}$ in the after period was required to exceed averaged $\hat{N}$ during the before period (Rule 3; greater $\hat{N}$ after), which was intended to guard against an anomalous spike in $\hat{N}$ during the after period. Fourth, the $\hat{N}$ in the final year of the time series (that is, 2019) was required to exceed the average $\hat{N}$ in the before period (Rule 4; greater $2019 \hat{N}$ ), which eliminated leks that experienced a sudden and precipitous drop at the end of the time series. Last, we ensured that average $\hat{N}$ in the after period was greater than five males (Rule 5; greater than five after), allowing large enough leks to be used to draw conclusions of long-term averages of $\hat{r}$.

Similarly, we created five rules for the omission dataset, which follow. First we retained leks that had a negative average $\hat{r}$ during the after period (Rule 1; declining after) to insure that leks did not show signs of recovery during this period, which might imply a lack of need for active management. Second, leks must have met a minimum of two or more males counted in 2 or more years during the before period (Rule 2; active before) and therefore could be considered for a warning. Third, leks were required to have an average $\hat{N}$ in the after period that was less than the average $\hat{N}$ in the before period (Rule 3 ; lower $\hat{N}$ after). This rule was intended to guard against a precipitous increase in $\hat{N}$ at the end of the before period/beginning of the after period, which could mask/misinterpret Rule 1 as a relaxation in $\hat{r}$ and a return of $\hat{N}$ to a stable state during the after period. Fourth, we required the average $\hat{N}$ in the before period to be greater than five males (Rule 4; greater than five before) which we determined large enough to warrant attention. Last, the lek experienced an extirpation event that lasted a minimum of 10 years (Rule 5; extirpation) to reflect a clear sign of sustained loss of activity at that lek. 
To calculate type I and type II error rates, we compared the list of leks within the omission and commission datasets to the list of leks that received warnings through the targeted annual warning system. Because leks could receive warnings one of two ways, either at the lek level or at the neighborhood cluster (NC) level, we evaluated type I and type II errors by creating lists of leks that received warnings at each level independently (lek warning versus $\mathrm{NC}$ warning) and under an either or scenario (either a lek or NC warning). Error rates were calculated as the proportion of leks misidentified within the omission or commission dataset divided by the total number of leks within the respective dataset. Misidentification of a lek within the commission dataset was defined as any lek within that dataset that also existed in the warning dataset. Misidentification of a lek within the omission dataset was defined as any lek within that dataset that did not exist in the warning dataset.

Omission (type II; 0.36) and commission (type I; 0.19) error rates were highest using warnings identified at the $\mathrm{NC}$ level, and nearly five (0.08) and four (0.05) times greater than those calculated at the lek level. The lowest error rate observed was the omission error using warnings at both levels combined (table 5.1). However, commission error rates were considerably higher under the scenario that considered both lek and $\mathrm{NC}$ level warnings. Warnings that are activated at the NC level are likely to occur in response to large scale impacts such as wildfire. When wildfires, or other landscape level disturbances, occur within NCs they are likely to affect a proportion of the leks within it, but not all. Therefore, it is reasonable to have higher rates of commission errors based on warnings activated at the $\mathrm{NC}$ level, because not every lek that is within the NC will be affected by the disturbance event. Interestingly, omission error rates were considerably higher than commission error rates when considering warnings activated at the $\mathrm{NC}$ level. This might suggest that a larger proportion of disturbance events that exist on the landscape are the result of point sources as opposed to impacts that possess larger footprints. This is further supported by the relatively similar rates among omission and commission errors observed at the lek level.

Table 5.1. Omission and commission errors of warnings at leks and neighborhood clusters (NC) for greater sage-grouse (Centrocercus urophasianus) targeted annual warning system using estimates from state-space models of annual rate of change in population abundance across their range from 1990 to 2019 in western United States.

\begin{tabular}{lccc}
\hline $\begin{array}{c}\text { Spatial } \\
\text { scale }\end{array}$ & $\begin{array}{c}\text { Omission } \\
\text { error rate }\end{array}$ & $\begin{array}{c}\text { Commission } \\
\text { error rate }\end{array}$ & $\begin{array}{c}\text { Average } \\
\text { error rate }\end{array}$ \\
\hline Lek & 0.080 & 0.047 & 0.064 \\
NC & 0.360 & 0.185 & 0.272 \\
Both & 0.070 & 0.205 & 0.138 \\
\hline
\end{tabular}




\section{References Cited}

Baker, W.L., 2006, Fire and restoration of sagebrush ecosystems: Wildlife Society Bulletin, v. 34, no. 1, p. 177185, https://doi.org/10.2193/0091-7648(2006)34[177:FARO $\mathrm{SE}] 2.0 . \mathrm{CO} ; 2$.

Cooper, S.V., Lesica, P., and Kudray, G.M., 2011, Post-fire recovery of Wyoming big sagebrush steppe in central and southeast Montana: Natural Resources and Environmental Issues, v. 16, no. 12, 9 p., https://digitalcommons.usu.edu/ cgi/viewcontent.cgi? article $=1723 \&$ context $=$ nrei.

Lesica, P., Cooper, S.V., and Kudray, G., 2007, Recovery of big sagebrush following fire in southwest Montana: Rangeland Ecology and Management, v. 60, no. 3, p. 261269, https://doi.org/10.2111/1551-5028(2007)60[261:ROBS FF]2.0.CO;2.

Maestas, J.D., Campbell, S.B., Chambers, J.C., Pellant, M., and Miller, R.F., 2016, Tapping soil survey information for rapid assessment of sagebrush ecosystem resilience and resistance: Rangelands, v. 38, no. 3, p. 120-128, https://doi.org/10.1016/j.rala.2016.02.002.

Miller, R.F., Chambers, J.C., Pyke, D.A., Pierson, F.B., and Williams, C.J., 2013, A review of fire effects on vegetation and soils in the Great Basin Region-Response and ecological site characteristics: Fort Collins, Colo., U.S. Department of Agriculture, Forest Service, Rocky Mountain Research Station General Technical Report RMRSGTR-308, 126 p., https://pdfs.semanticscholar.org/08da/ af5351a4047a27cad7662677a0185d48957b.pdf.

Pastore, M., 2018, Overlapping-A R package for estimating overlapping in empirical distributions: The Journal of Open Source Software, v. 3, no. 32, p. 1023, https://doi.org/10.21105/joss.01023.
R-Core Team, 2018, R-A language and environment for statistical computing: Vienna, Austria, R Foundation for Statistical Computing, accessed February 2, 2018, at http://www.R-project.org/.

Rigge, M., Shi, H., Homer, C., Danielson, P., and Granneman, B., 2019, Long-term trajectories of fractional component change in the Northern Great Basin, USA: Ecosphere, v. 10, no. 6, 24 p., https://doi.org/10.1002/ecs2.2762.

Rigge, M., Homer, C., Cleeves, L., Meyer, D.K., Bunde, B., Shi, H., Xian, G., Schell, S., and Bobo, M., 2020, Quantifying western U.S. rangelands as fractional components with multi-resolution remote sensing and in situ data: Remote Sensing, v. 12, no. 3, 26 p., https://doi.org/10.3390/rs12030412.

Shi, H., Rigge, M., Homer, C.G., Xian, G., Meyer, D.K., and Bunde, B., 2018, Historical cover trends in a sagebrush steppe ecosystem from 1985 to 2013 - Links with climate, disturbance, and management: Ecosystems, v. 21, no. 5, p. 913-929, https://doi.org/10.1007/s10021-017-0191-3.

Wambolt, C.L., Walhof, K.S., and Frisina, M.R., 2001, Recovery of big sagebrush communities after burning in south-western Montana: Journal of Environmental Management, v. 61, no. 3, p. 243-252, https://doi.org/10.1006/jema.2000.0411.

Watts, M.J., and Wambolt, C.L., 1996, Long-term recovery of Wyoming big sagebrush after four treatments: Journal of Environmental Management, v. 46, no. 1, p. 95-102, https://doi.org/10.1006/jema.1996.0009.

Ziegenhagen, L.L., and Miller, R.F., 2009, Postfire recovery of two shrubs in the interiors of large burns in the intermountain West, USA: Western North American Naturalist, v. 69, no. 2, p. 195-205, https://doi.org/10.3398/064.069.0208. 


\section{Appendix 6. Case Comparison of a State-Space Model and $\mathbf{N}$-Mixture Estimates}

During 2020, the state of Montana carried out an $N$-mixture modeling approach to estimate abundance $(\hat{N})$ and population growth $(\hat{\lambda})$ at the state-wide scale from 2002 to 2020 using within-season repeated measurements at leks. For comparative purposes, we constrained the Montana state $N$-mixture model estimates from 2002 to 2019 , which resulted in an average annual $\hat{\lambda}$ of 0.966 . This estimate was identical to the state-space model (SSM) average annual estimate ( $\hat{\lambda}=0.966)$ generated from our models over the same time frame (fig. 6.1). Thus, we conclude that the SSM based on maximum counts provides very similar estimates as those from an $\mathrm{N}$-mixture model, which rely heavily on repeated counts. These similarities in trends should be expected under the assumption of constant or random error in detection through time (Monroe and others, 2019). One advantage to $N$-mixture models is explicit adjustment for imperfect detection to more reliably estimate true $\hat{N}$ rather than an apparent $\hat{N}$ that is likely biased low. Nevertheless, if the goal of land and wildlife resource managers is to estimate $\hat{\lambda}$ and not necessarily $\hat{N}$, then extra time and expense associated with repeated counts may not be necessary.

\section{Reference Cited}

Monroe, A.P., Wann, G.T., Aldridge, C.L., and Coates, P.S., 2019, The importance of simulation assumptions when evaluating detectability in population models: Ecosphere, v. 10, no. 7, 16 p., https://doi.org/10.1002/ecs2.2791.

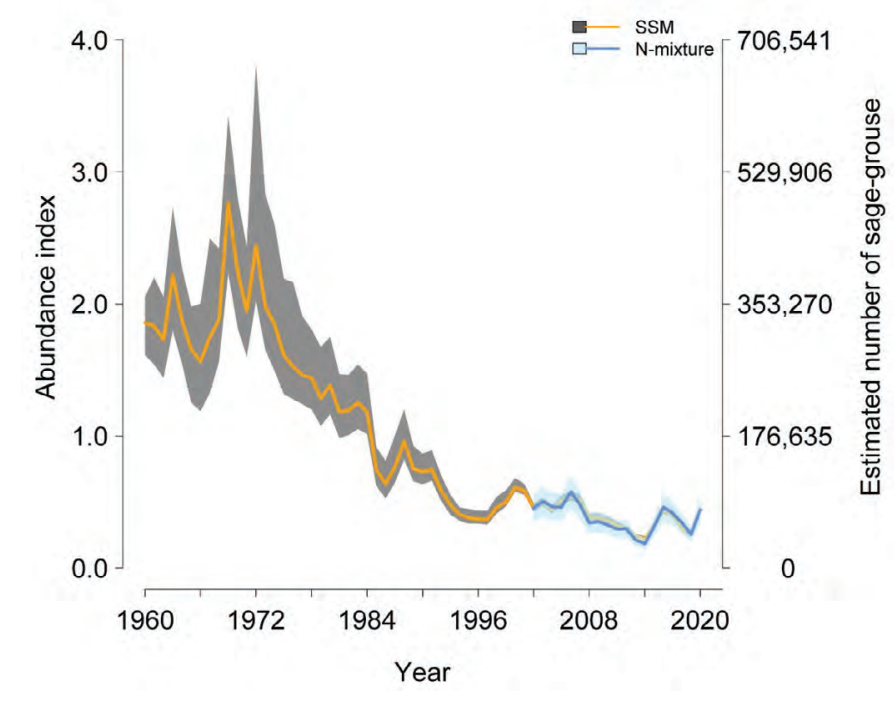

Figure 6.1. Comparison between $\mathrm{N}$-mixture model estimates of estimated number of sage-grouse and state-space model (SSM) estimates of abundance index between years 2002 and 2019 for the state of Montana. The SSM estimates were developed using maximum lek count data ranging from 1960 to 2019. $\mathrm{N}$-mixture models rely on repeated counts within the year and ranged from 2002 to 2020. 


\section{Appendix 7. Example of Targeted Annual Warning System at Pueblo Fire in Southeast Oregon}

To demonstrate the utility of the targeted annual warning system (TAWS) as a tool for managers to identify when and where populations (that is, leks and neighborhood clusters; $\mathrm{NCs}$ ) are declining below trends at the climate cluster (CC) scale, we provide a real-world example using the Pueblo Fire, which ignited on August 21, 2006, (fig. 7.1B) and burned approximately 27,500 hectares (ha) in southeast Oregon (Eidenshink and others, 2007). The Pueblo Fire was entirely contained within a single NC (E-115) located along the border of southeast Oregon and northwest Nevada. Based on data compiled from state lek count databases, there were a total of 29 known leks located within NC E-115. Due to data limitations at individual leks over the period of inference (1990-2019), only 11 leks were modeled and subsequently used within the TAWS. Ten leks were located within the state of Oregon and one within the state of California. Of the 10 leks located in Oregon, 6 were located inside the fire perimeter and 4 outside the fire perimeter. The average distance for leks located outside the fire perimeter and within the Oregon side of NC E-115 was 6.8 kilometers $(\mathrm{km} ; \mathrm{SD}=2.5)$. The single lek located in California was approximately $41 \mathrm{~km}$ from the nearest edge of the fire perimeter. In 2007, 1 year after the Pueblo Fire, six leks (three outside the fire perimeter, three inside the fire perimeter) received a watch (moderate evidence for declining below the CC) and two leks (inside the fire perimeter) received a warning (strong evidence for declining below the CC; fig. 7.1C). In 2008, 2 years after the Pueblo Fire, two leks inside the fire perimeter which had received a watch the previous year were upgraded to warnings (fig. 7.1D). Due to the overwhelming evidence that occurred at the lek level, the $\mathrm{NC}$ received its first warning in 2007, and again in 2008 .

\section{Reference Cited}

Eidenshink, J., Schwind, B., Brewer, K., Zhu, Z., Quayle, B., and Howard, S., 2007, A project for monitoring trends in burn severity: Fire ecology, v. 3, no. 1, p. 3-21, https://doi.org/10.4996/fireecology.0301003.
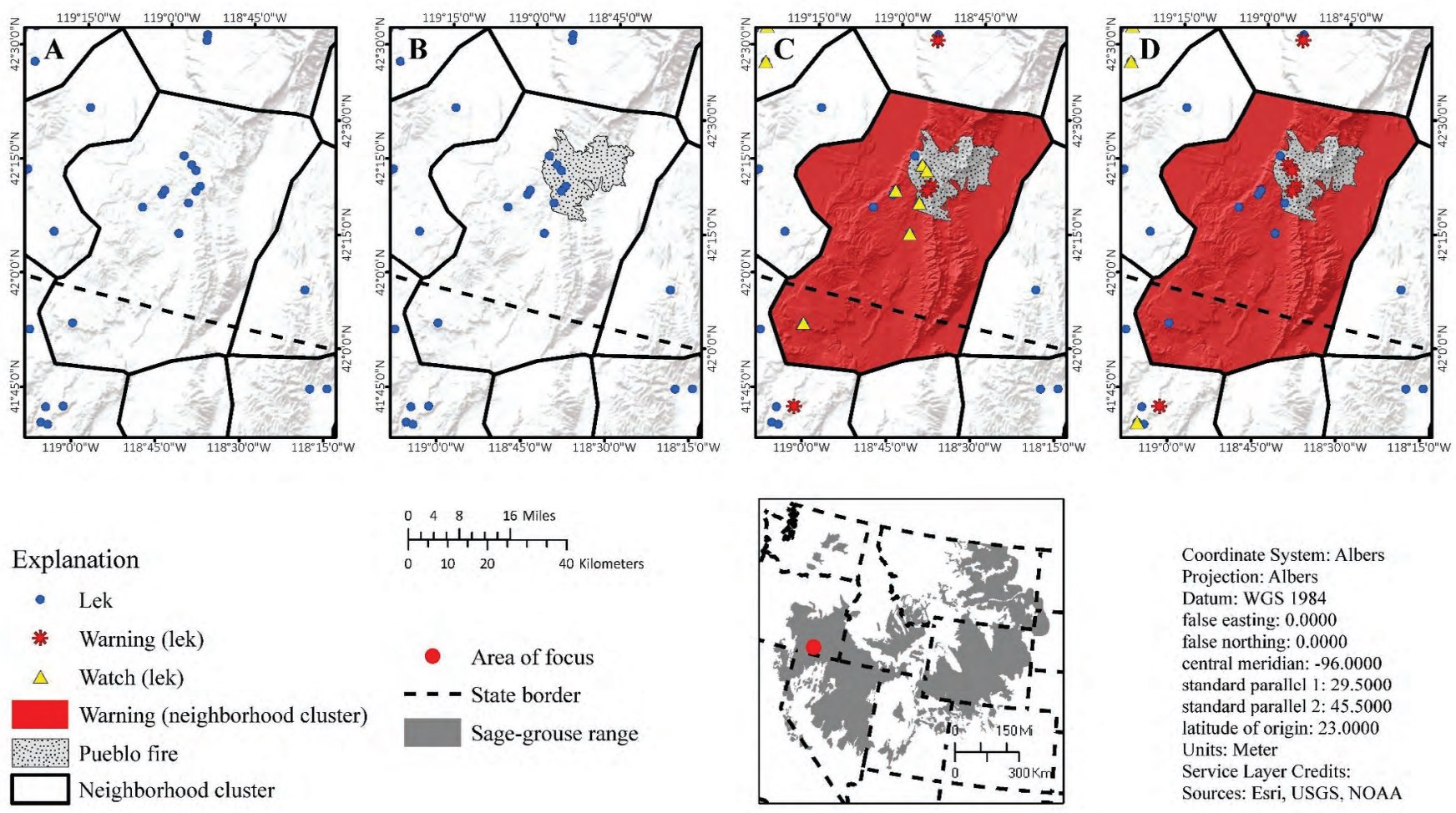

Figure 7.1. Example of Targeted Annual Warning System in southeast Oregon during A, 2005 (pre-wildfire); B, 2006 (wildfire occurs); $C, 2007$; and $D, 2008$, which illustrates activation of watches and warnings at the lek and NC level immediately following the Pueblo Fire. Map images are the intellectual property of Esri and are used herein under license. Copyright ( 2020 Esri and its licensors. All rights reserved. 


\section{Appendix 8. Development of Targeted Annual Warning System for Climate Cluster Stability}

We carried out similar steps as described in appendix 5 to determine optimal threshold values from slow-fast signal combinations that identified declining populations performing below the climate cluster (CC) scale. This example analysis differed from the appendix 5 simulations such that threshold values were allowed to vary by CC. Different slow-fast signal combinations for each CC focused stability on the CC scale as opposed to the range-wide scale. Activation of warnings from independent thresholds at each CC likely provides more support than a single threshold across the species' range for fundamental conservation biology principles of resiliency, redundancy, and representation. However, rangewide thresholds still likely serve a broader goal of optimizing population level stability across the species' range within the western United States.
Table 8.1. Optimized values for slow-fast signal combinations used for watches and warnings evaluated at the lek and neighborhood cluster scales within a targeted annual warning system.

[Separate slow-fast threshold combinations were developed for each climate cluster (A-F) using state-space model estimates for greater sage-grouse (Centrocercus urophasianus) across their range in the western United States during 1990-2019. Abbreviations: CC, climate cluster; NC, neighborhood cluster; A, Bi-state area; B, Washington area; C, Jackson Hole, Wyoming, area; D, eastern area; E, Great Basin area; F, Wyoming]

\begin{tabular}{ccccc}
\hline CC & lek (slow) & lek (fast) & NC (slow) & NC (fast) \\
\hline A & -0.219 & 3.483 & -0.591 & 2.596 \\
B & 0.299 & 0.634 & -0.678 & 0.02 \\
C & -0.179 & 0.814 & -1.398 & -0.634 \\
D & 1.528 & 2.324 & 2.761 & 3.186 \\
E & 1.059 & 2.207 & 0.634 & 3.483 \\
F & 1.059 & 2.452 & 1.278 & 3.483 \\
\hline
\end{tabular}


Table 8.2. Watches and warnings identified at greater sage-grouse (Centrocercus urophasianus) leks and neighborhood clusters (NC) across climate clusters (A-F) using state-space model estimates within a targeted annual warning system developed for climate cluster stability in the western United States during 1990-2019 (all years) and 2019 alone (most recent year).

[Number of watches and warnings that include repeat (r), only first time (f), and proportion of leks (p) are reported for different spatial (lek, NC) and temporal (all years, most recent year) scales. Abbreviations: CC, climate cluster; NC, neighborhood cluster; A, Bi-state area; B, Washington area; C, Jackson Hole, Wyoming, area; D, eastern area; E, Great Basin area; F, Wyoming]

\begin{tabular}{|c|c|c|c|c|c|c|c|}
\hline CC & r.watch & f.watch & p.watch & r.warning & f.warning & p.warning & Levels \\
\hline \multicolumn{8}{|c|}{ lek } \\
\hline \multicolumn{8}{|c|}{ All years } \\
\hline A & 179 & 41 & 0.854 & 143 & 31 & 0.646 & 48 \\
\hline B & 63 & 22 & 0.440 & 86 & 21 & 0.420 & 50 \\
\hline $\mathrm{C}$ & 32 & 8 & 0.571 & 27 & 6 & 0.429 & 14 \\
\hline $\mathrm{D}$ & 1,475 & 789 & 0.504 & 1,748 & 659 & 0.421 & 1,566 \\
\hline $\mathrm{E}$ & 2,834 & 1,163 & 0.610 & 2,851 & 859 & 0.450 & 1,908 \\
\hline $\mathrm{F}$ & 706 & 415 & 0.465 & 886 & 351 & 0.393 & 892 \\
\hline Total & 5,289 & 2,438 & 0.544 & 5,741 & 1,927 & 0.430 & 4,478 \\
\hline \multicolumn{8}{|c|}{ Most recent year } \\
\hline A & 11 & 0 & 0 & 15 & 3 & 0.063 & 48 \\
\hline B & 0 & 0 & 0 & 1 & 0 & 0 & 50 \\
\hline $\mathrm{C}$ & 1 & 0 & 0 & 0 & 0 & 0 & 14 \\
\hline $\mathrm{D}$ & 135 & 32 & 0.020 & 134 & 52 & 0.033 & 1,566 \\
\hline $\mathrm{E}$ & 115 & 36 & 0.019 & 139 & 32 & 0.017 & 1,908 \\
\hline $\mathrm{F}$ & 51 & 18 & 0.020 & 71 & 33 & 0.037 & 892 \\
\hline Total & 313 & 86 & 0.019 & 360 & 120 & 0.027 & 4,478 \\
\hline \multicolumn{8}{|c|}{ NC } \\
\hline \multicolumn{8}{|c|}{ All years } \\
\hline A & 37 & 9 & 1.000 & 22 & 5 & 0.556 & 9 \\
\hline B & 3 & 2 & 0.667 & 2 & 1 & 0.333 & 3 \\
\hline $\mathrm{C}$ & 5 & 1 & 0.500 & 3 & 1 & 0.500 & 2 \\
\hline $\mathrm{D}$ & 30 & 26 & 0.187 & 52 & 27 & 0.194 & 139 \\
\hline $\mathrm{E}$ & 383 & 145 & 0.687 & 263 & 88 & 0.417 & 211 \\
\hline $\mathrm{F}$ & 47 & 31 & 0.596 & 23 & 13 & 0.250 & 52 \\
\hline Total & 505 & 214 & 0.514 & 365 & 135 & 0.325 & 416 \\
\hline \multicolumn{8}{|c|}{ Most recent year } \\
\hline A & 0 & 0 & 0 & 1 & 0 & 0 & 9 \\
\hline B & 1 & 0 & 0 & 1 & 0 & 0 & 3 \\
\hline $\mathrm{C}$ & 0 & 0 & 0 & 0 & 0 & 0 & 2 \\
\hline $\mathrm{D}$ & 3 & 1 & 0.007 & 7 & 4 & 0.029 & 139 \\
\hline $\mathrm{E}$ & 21 & 3 & 0.014 & 26 & 11 & 0.052 & 211 \\
\hline $\mathrm{F}$ & 5 & 2 & 0.038 & 4 & 3 & 0.058 & 52 \\
\hline Total & 30 & 6 & 0.014 & 39 & 18 & 0.043 & 416 \\
\hline
\end{tabular}




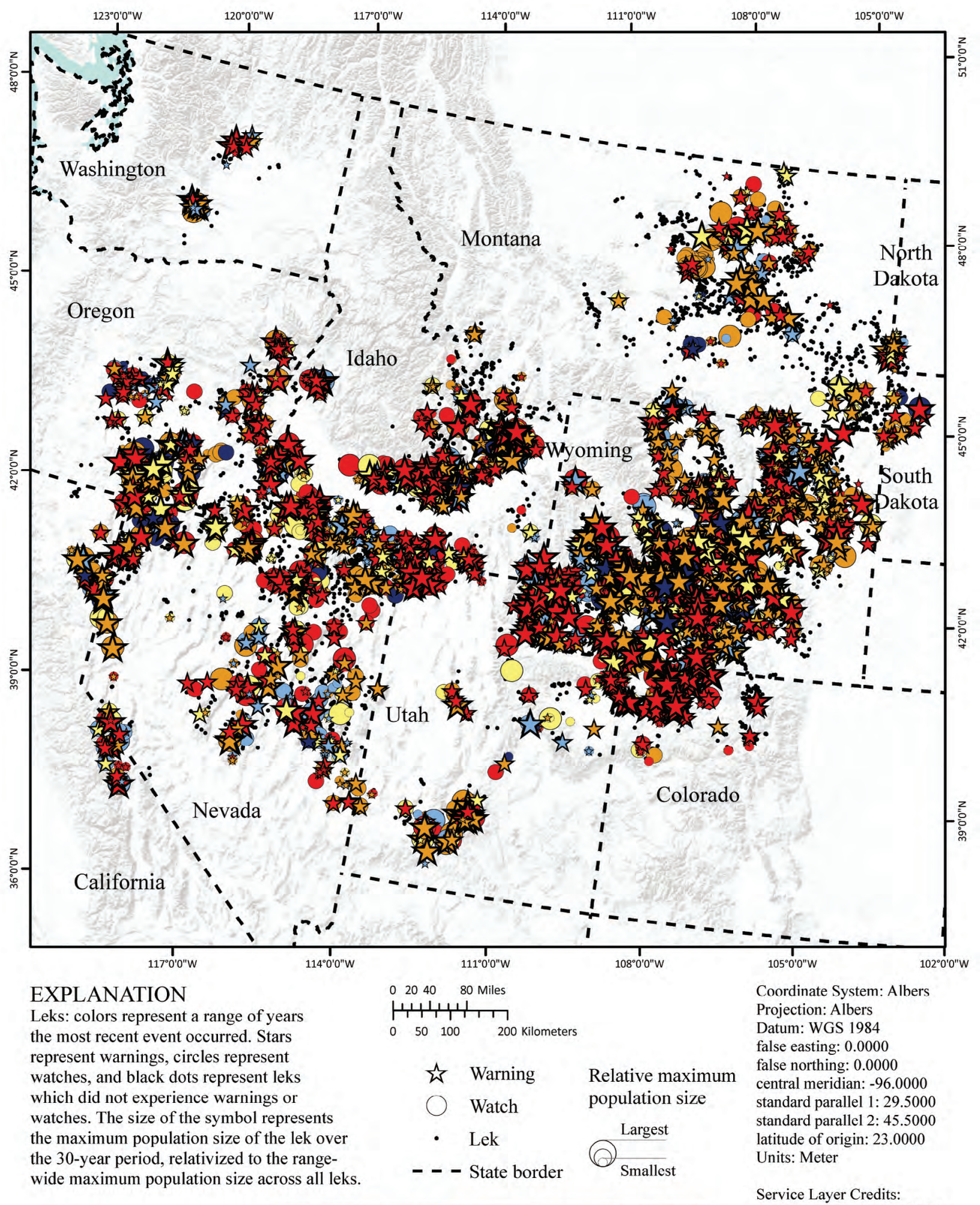

1995 -1999 2000-2004 2005-2009 2010-2014 2015-2019

Service Layer Credits: Sources: Esri, USGS, NOAA

Figure 8.1. Spatial and temporal depiction of range-wide watches and warnings of greater sage-grouse (Centrocercus urophasianus) population declines at the lek scale using a targeted annual warning system that was developed for climate cluster stability within the western United States from 1990 to 2019. Map image is the intellectual property of Esri and is used herein under license. Copyright (C) 2020 Esri and its licensors. All rights reserved. 


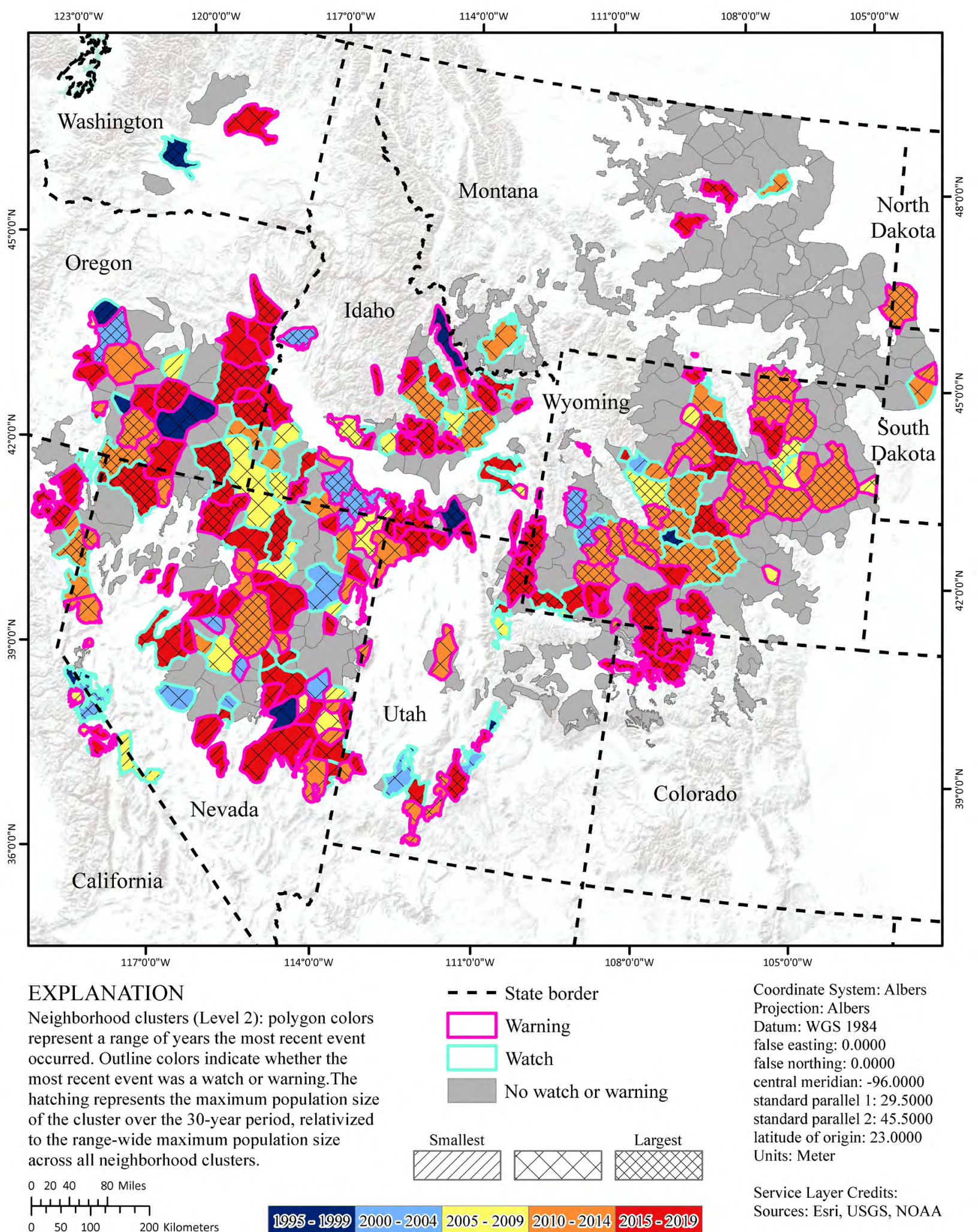

Figure 8.2. Spatial and temporal depiction of range-wide watches and warnings of greater sage-grouse (Centrocercus urophasianus) population declines at the neighborhood cluster scale using a targeted annual warning system that was developed for climate cluster stability within the western United States from 1990 to 2019. Map image is the intellectual property of Esri and is used herein under license. Copyright (C) 2020 Esri and its licensors. All rights reserved. 

For more information concerning the research in this report, contact the Director, Western Ecological Research Center

U.S. Geological Survey

3020 State University Drive East

Sacramento, California 95819

https://www.usgs.gov/centers/werc

Publishing support provided by the U.S. Geological Survey Science Publishing Network, Sacramento Publishing Service Center 


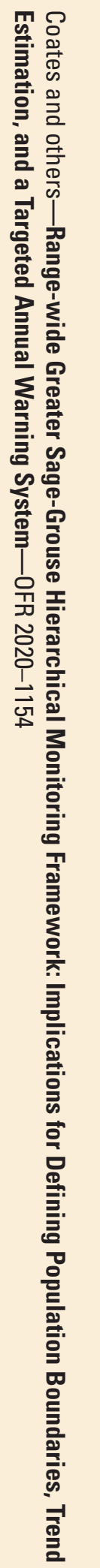

